\title{
NWCF Evaporator Tank System 2001 Offgas Emissions Inventory
}

R. D. Boardman

K. M. Lamb

L. A. Matejka

J. A. Nenni

February 2002

Idaho National Engineering and Environmental Laboratory Bechtel BWXT Idaho, LLC 
INEEL/EXT-02-00198

\section{NWCF Evaporator Tank System 2001 Offgas Emissions Inventory}

R. D. Boardman

K. M. Lamb

L. A. Matejka

J. A. Nenni

February 2002

Idaho National Engineering and Environmental Laboratory High Level Waste Program Idaho Falls, Idaho 83415

Prepared for the U.S. Department of Energy

Assistant Secretary for Environmental Management

Under DOE Idaho Operations Office

Contract DE-AC07-99ID13727 


\begin{abstract}
An offgas emissions inventory and liquid stream characterization of the Idaho New Waste Calcining Facility (NWCF) Evaporator Tank System (ETS), formerly known as the High Level Liquid Waste Evaporator (HLLWE), has been completed. The emissions rates of volatile and semi-volatile organic compounds, multiple metals, particulate, and hydrochloric acid $(\mathrm{HCl}) / \mathrm{Cl}_{2}$ were measured in accordance with an approved Quality Assurance Project Plan (QAPjP) and Test Plan that invoked U.S. Environmental Protection Agency (EPA) standard sample collection and analysis procedures. Offgas samples were collected during the start up and at the end of evaporator batches when it was hypothesized the emissions would be at peak rates. Corresponding collection of samples from the evaporator feed, overhead condensate, and bottoms was made at approximately the same time as the emissions inventory to support material balance determinations for the evaporator process. The data indicate that organic compound emissions are slightly higher at the beginning of the batch while metals emissions, including mercury, are slightly higher at the end of the evaporator batch. The maximum emissions concentrations are low for all constituents of primary concern. Mercury emissions were less than $5 \mathrm{ppbv}(<40 \mu \mathrm{g} / \mathrm{dscm})$, while the sum of $\mathrm{HCl}$ and $\mathrm{Cl}_{2}$ emissions was less than 1 ppmv. The sum of all organic emissions also was less than 1 ppmv. The estimated hazardous quotient (HQ) for the evaporator was 6.2e-6 as compared to 0.25 for the EPA target criteria. The cancer risk was 1.3e-10 compared to an EPA target of 1e-5.
\end{abstract}




\section{SUMMARY}

This report presents the 2001 effluent gas emissions inventory data for the NWCF Evaporator Tank System (ETS) operated at the INTEC. Liquid wastes generated from decontamination activities are stored in the INTEC High Level Waste Tank Farm Facility (TFF). The Tank Farm wastes are currently being concentrated using the NWCF ETS (formally known as High Level Liquid Waste Evaporator, or HLLWE). The NWCF ETS currently operates under Resource Conservation and Recovery Act (RCRA) interim status. A RCRA Part B permit application for this unit is being prepared and will be submitted in FY-2003. In order to support the permitting activities, the feed and output streams were characterized during evaporator operations in May and June, 2001. During this time, the NWCF ETS was being used to reduce the volume of a blend of two parts by volume of solution from WM-184 and one part by volume of solution from WM-181. Both of these tanks contained sodium-bearing waste (SBW).

The NWCF ETS is a single-stage, thermal siphon, batch evaporator. Dilute Tank Farm liquid wastes are semi-continuously fed to the evaporator to maintain a constant level in the evaporator. The system consists of a feed tank (VES-NCC-152), a flash column (VES-NCC-150), a reboiler (HE-NCC-350), and a condenser (VES-NCC-151). Blended tank farm wastes are added to the flash column via the feed tank. When the level in the flash column reaches its normal operating level, steam is introduced into the shell side of the evaporator reboiler. Once the desired specific gravity is reached, the concentrated solution is cooled and returned to the Tank Farm. Each batch generally requires $10-16$ hours to process, followed by 10-16 hours to refill the feed tanks and to attend to waste transfers back to the Tank Farm.

The NWCF ETS overhead vapor is condensed and sent to the INTEC Process Equipment Waste Evaporator (PEWE) to be re-evaporated. Non condensable and purge gasses are vented from the feed tank and the condenser to the NWCF equipment vent system. The combined offgas passes through the NWCF high-efficiency particulate air (HEPA) filters and then through the Atmospheric Protection System (APS) before being discharged from the main INTEC stack with other vessel offgas and building ventilation air. The offgas tie-in sample location previously used to sample the NWCF Calciner offgas stream was determined to be the best location for sampling the NWCF ETS emissions.

\section{Scope and Approach}

The Tank Farm wastes are highly acidic (mainly nitric acid) and contain several RCRA metals, including mercury, and trace amounts of volatile and semi-volatile hazardous organic compounds which were introduced into the Tank Farm Facility by previous disposal of laboratory analytical wastes, NWCF Calciner scrub solution recycle, and organic solvent cleaning. Those components with low boiling points are released to the NWCF ETS offgas system during waste transfers, mixing, and evaporation. Trace amounts of heavy, nonvolatile hydrocarbons may also exist in the waste feed solutions; however, emissions of the nonvolatile hydrocarbons and also the nonvolatile metals may occur due to aerosol entrainment from the evaporator. The majority of the entrained droplets are believed to be collected in the offgas condensers, mist eliminators, and HEPA filters. 
The scope of the NWCF ETS process effluent gas emissions inventory included:

- Measurement of the NWCF offgas duct velocity, temperature, and flowrate during operation of the NWCF ETS

- Manual offgas sampling and analysis for particulate matter (PM), $\mathrm{HCl}, \mathrm{Cl}_{2}$, selected metals, volatile organic compounds (VOCs), and semi-volatile organic compounds (SVOCs)

- Measurement of oxygen concentrations

Standard EPA sample collection and analysis methods were used to collect the offgas samples. Sampling was conducted following standard EPA methodology for emissions compliance testing, with attention being given to the following:

- Development and adherence to an approved project quality assurance/quality control plan

- Implementation of chain-of-custody (COC)/requests-for-analysis (RFA) and master sample collection lists that utilize and implement an in-field sample tracking and sample identification number verification

- Development of target analyte lists (TAL) and precision, accuracy, representativeness, completeness, and comparability (PARCC) data quality objectives

- Collection of samples using checklists to record train set up, sample collection data, and sample recovery steps

- Collection and analysis of reagent blanks, trip blanks, and field blanks to achieve prescribed data quality objectives

- Sample collection monitoring by a Project Quality Assurance Officer (PQAO)

- Application of EPA Solid Waste (SW)-846 and 40 Code of Federal Regulations (CFR) 60 Appendix A reference methods for sample analysis.

- Multiple reviews and verifications of field data, analytical data, process data, and resultant calculations of emissions rates

Samples of the feed were analyzed prior to initiation of processing the blend in the NWCF ETS to ensure that the chemistry of the feed solution was compatible with the process equipment. Samples of the condensed overheads and the concentrated bottoms from the first several batches processed were analyzed to ensure that the chemistry of those streams was compatible with down-stream process equipment. The results of these analyses have been included in this report to provide a resource for process permitting discussions and planning.

At the beginning of the offgas sample collection period, the vertical gas velocity profile and swirl angle in the duct were measured to determine an appropriate fixed-point location to collect the offgas samples. Sample contamination survey trains and routine 
radiological surveys and screenings were completed throughout the sample collection period to ensure that the samples shipped to the contract analytical laboratory met the labs radioactive materials license criteria. At the end of the sample collection period, the sample probe was removed from the duct and rinsed with acetone and nitric acid. The acetone and nitric acid probe rinses were analyzed for PM and metals.

A set of two runs was completed for each EPA sample train configuration at the beginning and another at the end of evaporator batches. This provided a total of four runs for each method to compare emissions trends at the beginning and end of the evaporator batches. Oxygen concentrations were monitored during each sample train run. The oxygen concentrations in the duct were consistently found to be similar to ambient air conditions. Therefore, it was not necessary to constantly operate the oxygen monitor.

Moisture levels in the offgas were determined from gravimetric and/or volumetric changes in the sample train resins and impingers, respectively. The offgas moisture level was typically less than the dew point of the sample gas passing through the sample collection train condensers. At the most, only 1-2 mL net condensate was collected in any of the condensate knockout impingers.

\section{Data Quality Assessment}

All of the planned emissions inventory samples data and associated quality assurance/quality control (QA/QC) samples were collected in accordance with the test plan (PLN-879) and Quality Assurance Project Plan (PLN-880) which were developed and approved for this project. An extensive discussion is provided in the report body regarding conformance of the sample collection activities with the procedures and EPA Method requirements, performance of the QA/QC samples, sampling surrogates, and internal standards.

Although an independent review of the data by the INEEL Sample Management Office (SMO) was not completed, the analytical data reports and data reduction calculations were reviewed by the contract laboratory Quality Officer, the Project Technical Leads, and the BBWI Project Quality Assurance Officer. All of the analytical data and offgas emissions results are judged to be useful for their intended purpose of completing an emissions inventory for the NWCF ETS system. The results are applicable to, and bounded by, the 2:1 volumetric blend of Tank WM-184 and Tank WM-181 feed composition, and NWCF ETS process operating parameters and conditions corresponding to the offgas sampling period.

\section{Emissions Results}

The concentration levels of the 20 highest VOC compounds emitted from the evaporator are plotted in Figure S-1. In general, volatile organic emissions are slightly higher at the start of an evaporator batch. The two highest volatile organics emitted from 
the NWCF ETS were dodecane and acetone, which on a volumetric basis are only $50 \mathrm{ppbv}$ and $30 \mathrm{ppbv}$, respectively. Acetone was also detected in the feed to the NWCF Calciner. Dodecane was not a target analyte for the liquid feed and therefore was not measured.

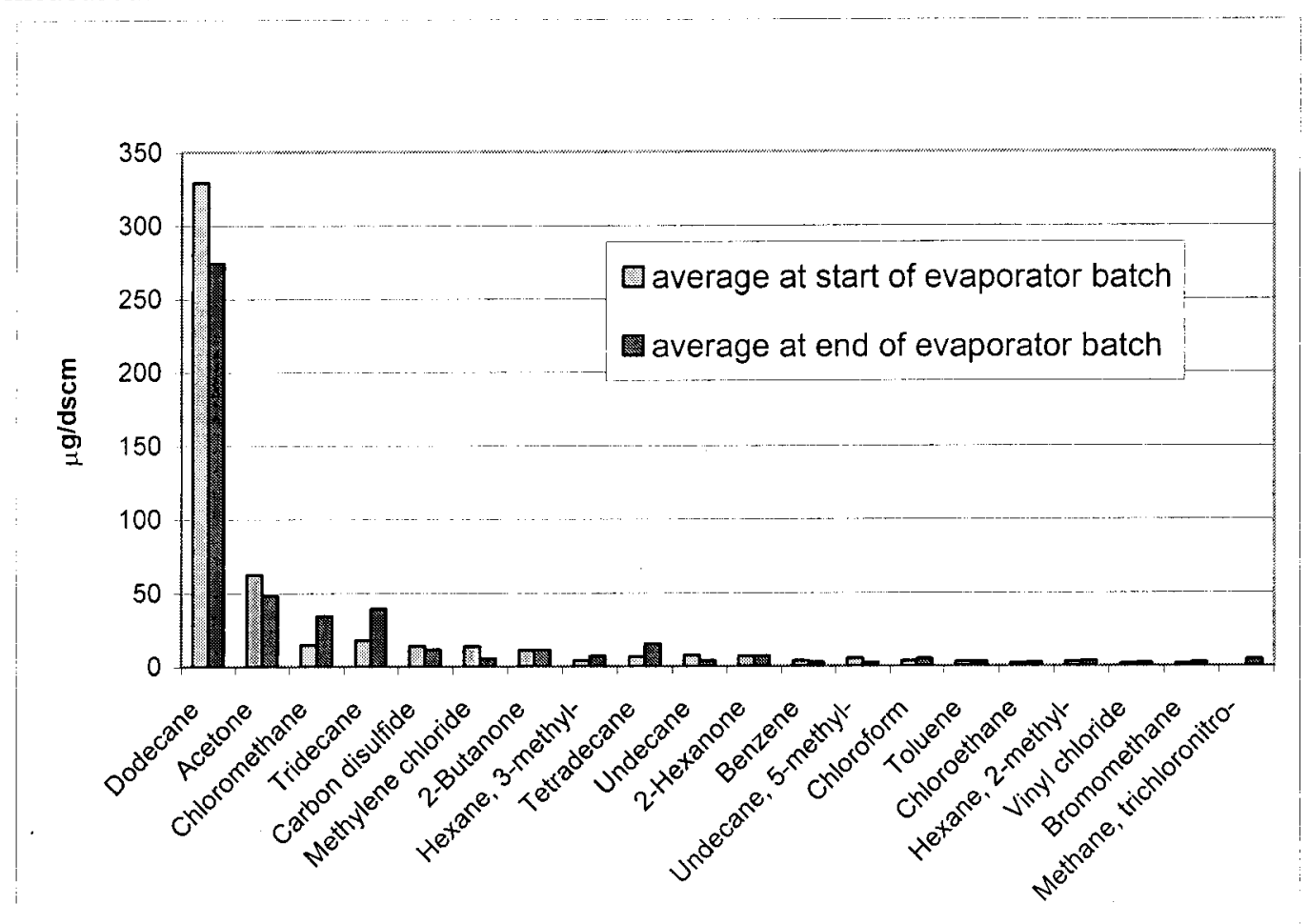

Figure S-1. Comparison of volatile organic emissions at the beginning and end of evaporator batches.

The top 20 SVOCs measured in the offgas stream are plotted in Figure S-2. SVOC emissions also appear to be slightly higher at the start of the evaporator batch. Benzoic acid (a target analyte) and benzaldehyde (a tentatively identified compound) were the two most prevalent semi-volatile organics emitted during operation of the NWCF ETS. The maximum emissions concentrations for benzoic acid and benzaldehyde were $310 \mathrm{ppbv}$ and $80 \mathrm{ppbv}$, respectively.

Nearly all of the compounds are derivatives of benzene or other cyclic compounds and are possibly the products of incomplete combustion of the kerosene used to heat the Calciner. With the exception of benzoic acid, all of the SVOC species emitted from the evaporator were also detected during the NWCF Calciner offgas emissions inventory (Boardman 2001). It is therefore postulated that these compounds entered the Tank Farm System when Calciner scrub was recycled to the tank farm. They could also be formed by oxidation of benzene and toluene in the acidic waste solutions.

On a volumetric basis, the sum of all volatile and semi-volatile organics is less than $1 \mathrm{ppm}$. The hourly total emissions rate for all volatile and semi-volatile organic emissions was less than $0.02 \mathrm{lbs} / \mathrm{hr}$. 


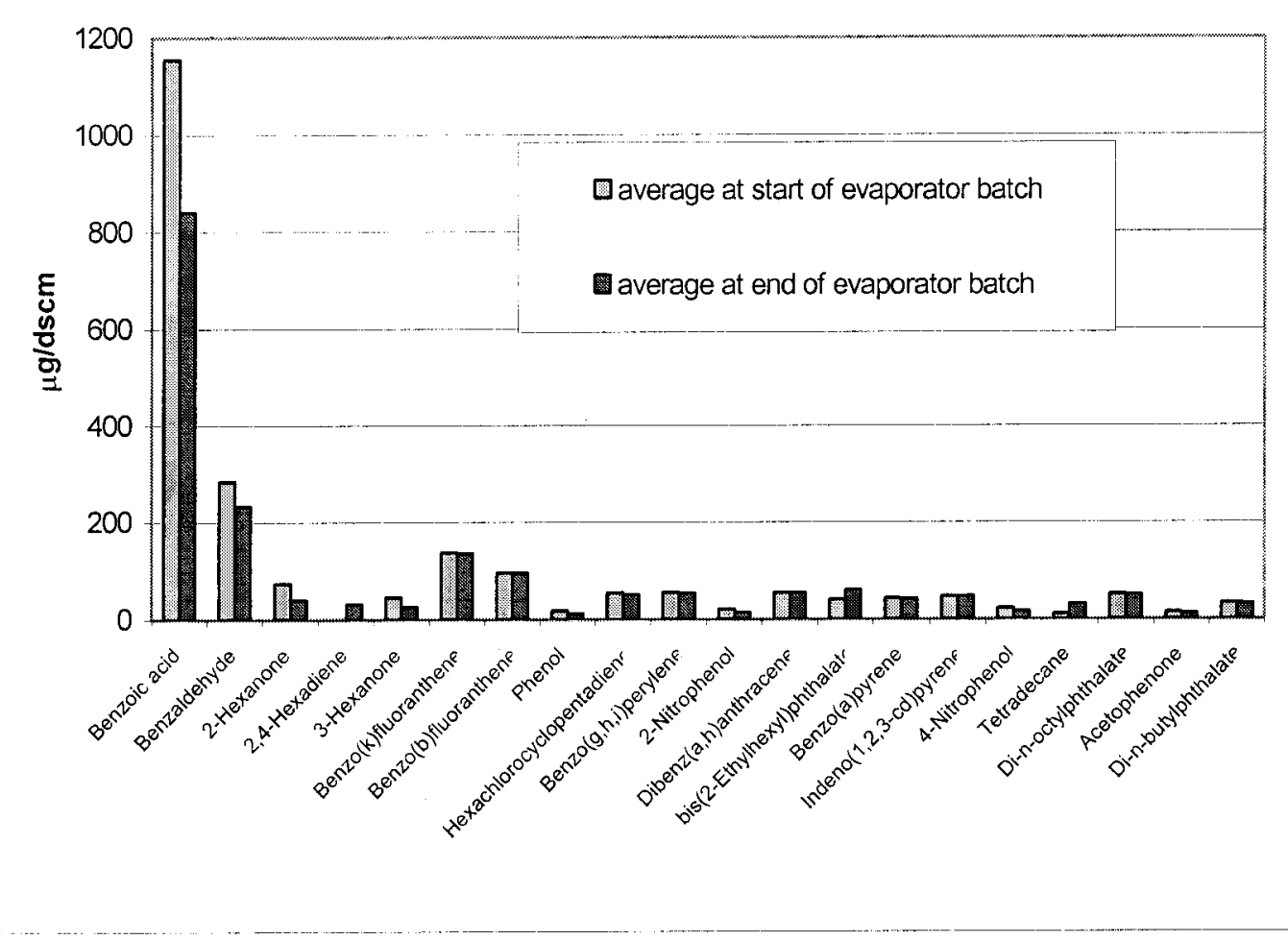

Figure S-2. Comparison of semi-volatile organic emissions at the beginning and end of evaporator batches.

The average metals emissions rates at the beginning and end of evaporator batches are plotted in Figure S-3. As anticipated, metals emissions, including mercury, were typically higher at the end of an evaporator batch when the evaporator solution reached its maximum density. The emissions of all metals species appear to correlate with the solution density.

Total particulate and chloride emissions rate averages at the start and end of two evaporator batches were very low. The sum of chloride emission contributions from $\mathrm{HCl}$ and $\mathrm{Cl}_{2}$ was less than 1 ppmv. Particulate emissions were slightly higher at the beginning of the batch which followed the trend of the semi-volatile organic species emissions. 


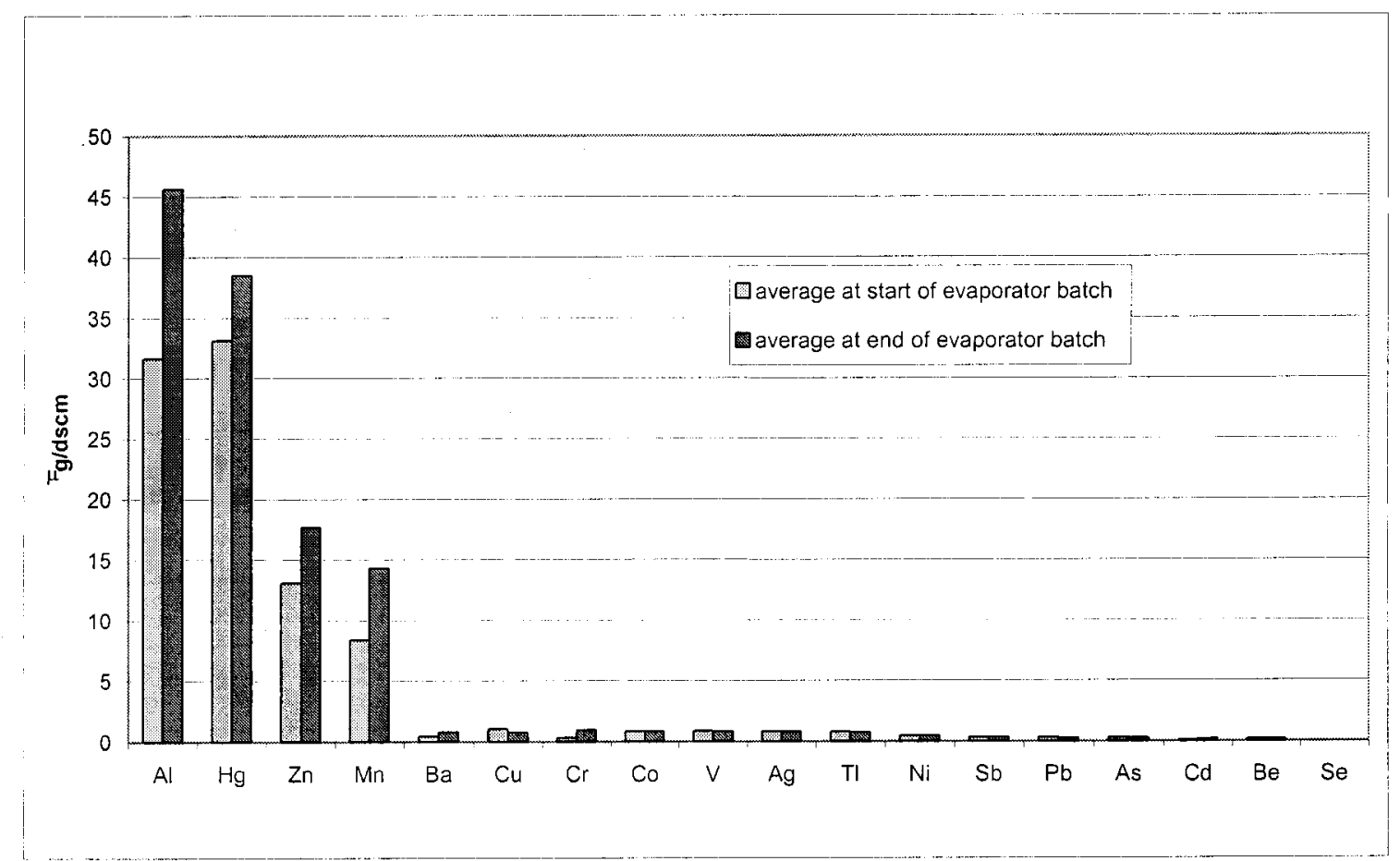

Figure S-3. Comparison of metals emissions at the beginning and end of evaporator batches (not including final probe rinse species apportionment).

\section{Emissions Risk}

The emission rate measurements were used to calculate the risk to human health. Pollutants from the NWCF ETS are released from the same point (i.e., the INTEC main stack) and under the same conditions as NWCF Calciner emissions. Therefore, to a close approximation, the NWCF ETS hazards and risks can be scaled using the risk terms previously determined for the NWCF Calciner operations (Boardman 2001).

It was observed that the emissions rates, and hence component-specific risk contributions, were generally much lower from the NWCF ETS than from the NWCF Calciner. Most of the materials "found" were present at levels below the analytical laboratory reporting limits. The summed hazardous quotient (HQ) for all emissions from the NWCF ETS was 6.2e-6 as compared to the EPA target criteria of 0.25 . The cancer risk was $1.3 \mathrm{e}-10$ compared to an EPA target of 1e-5. The semi-volatiles were the largest contributor to the HQ and the Risk. The most significant species was a phthalate (bis $(2$ ethylhexyl)phthalate) which is a common contaminate from plastics present in laboratory and sampling areas.

In conclusion the measured emissions from the NWCF ETS are extremely low for all categories of pollutants. The estimated cancer risk and health hazard quotient are each several orders less than the limit normally allowed by EPA. 


\section{ACKNOWLEDGEMENTS}

This report is the result of a concerted effort on the part of several Company support organizations. The authors wish to acknowledge C. N. Woodall, Sr. Technical Specialist, for set up of the sample collection equipment and support during sample collection and shipping, and for chemical and waste management. R.M. Gifford is recognized for administrative support, training coordination, records management, and preparation of this report. The project manager for both the offgas and liquid sampling activities was L. J. Young, who supported technical planning, equipment and sample collection area setup, work authorization prerequisites, and ultimately, the sample collection activities. 


\section{CONTENTS}

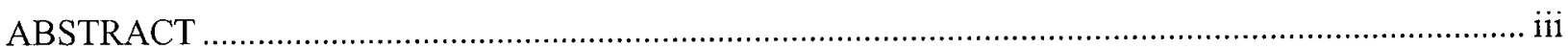

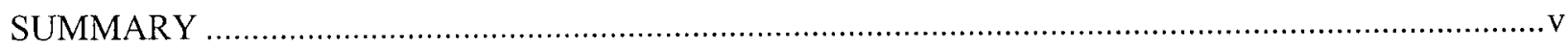

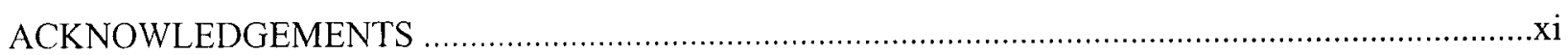

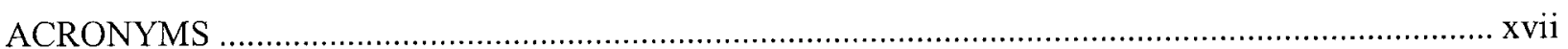

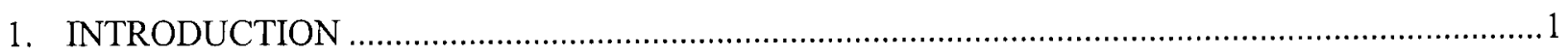

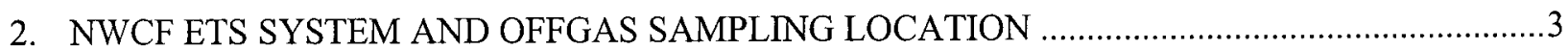

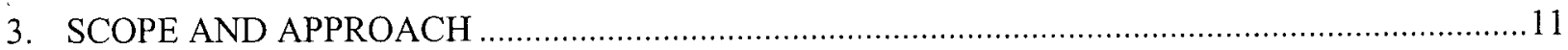

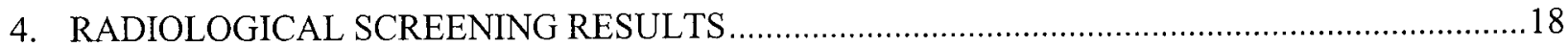

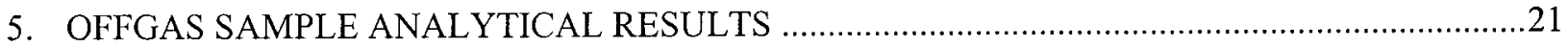

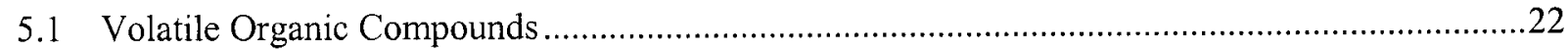

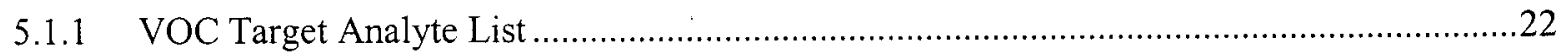

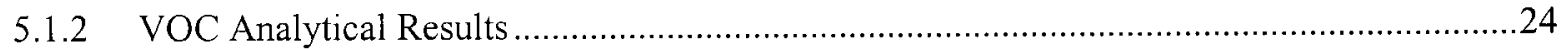

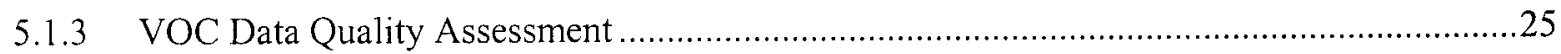

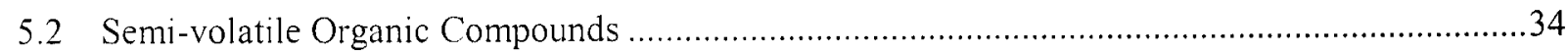

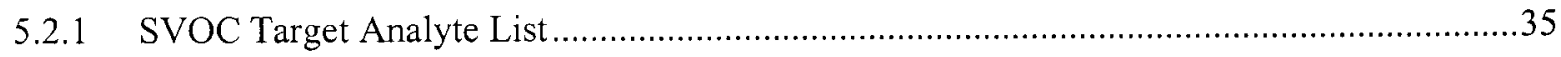

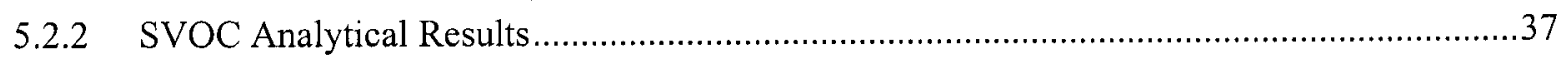

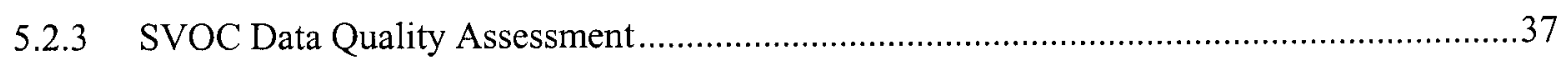

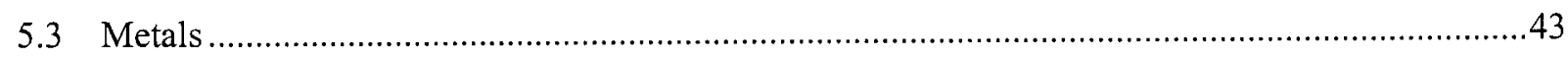

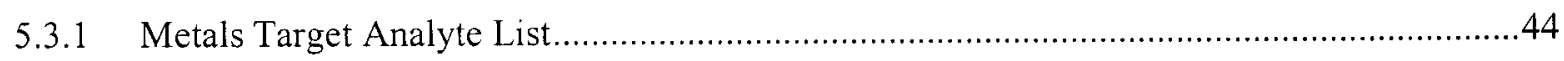

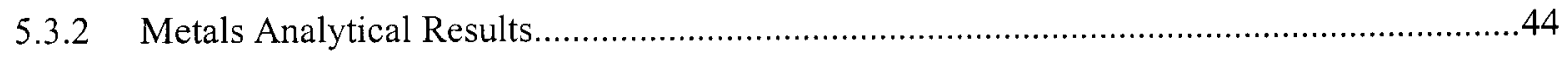

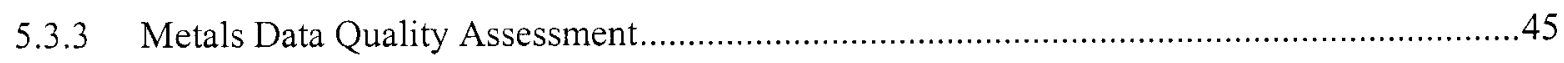

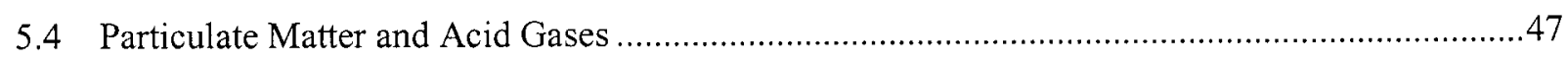

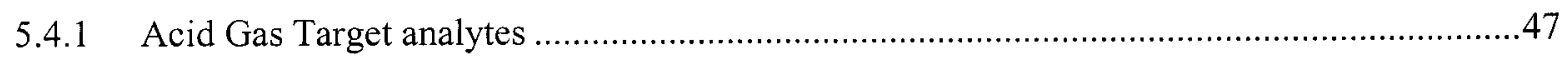

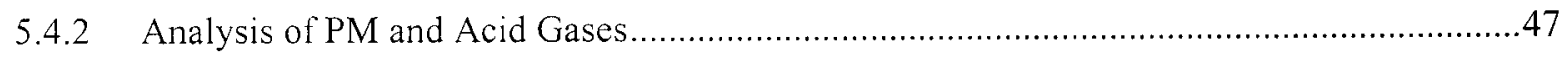

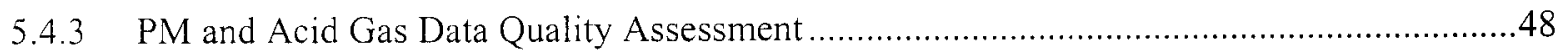




\section{CONTENTS}

6. PROCESS STREAM CHARACTERIZATION RESULTS .......................................................50

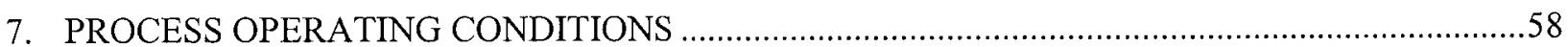

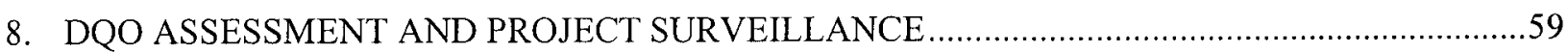

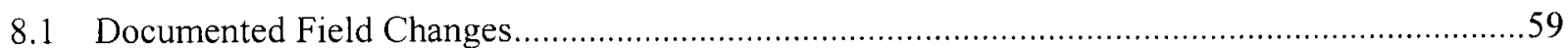

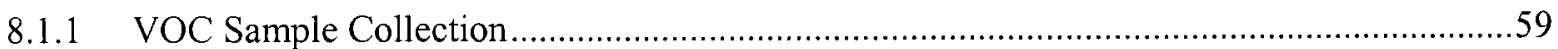

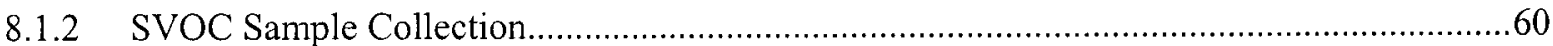

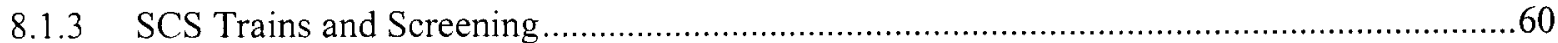

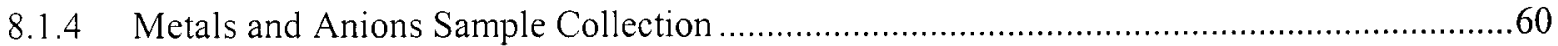

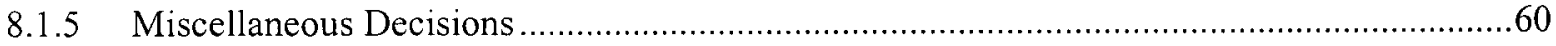

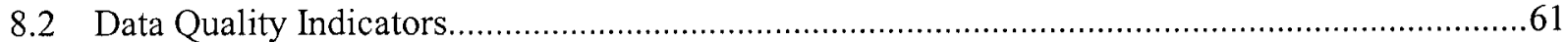

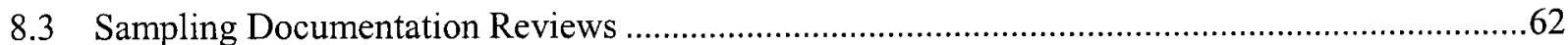

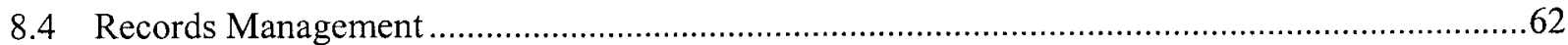

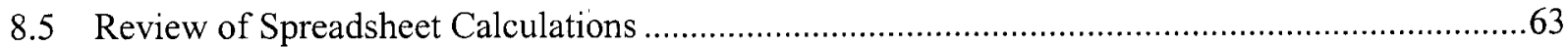

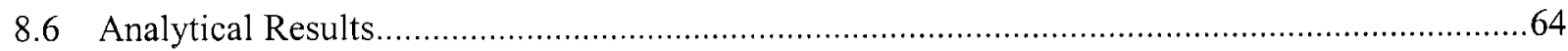

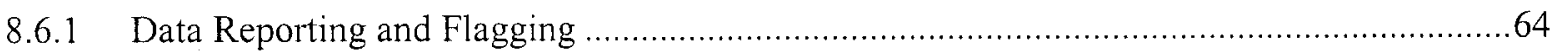

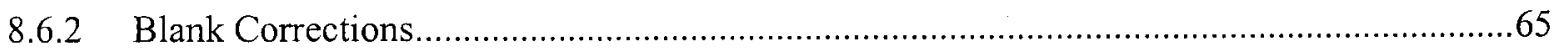

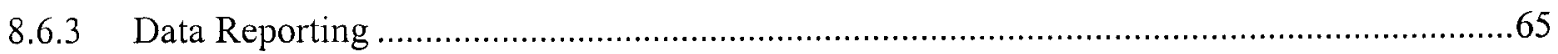

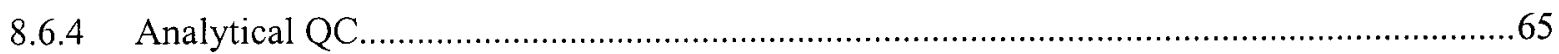

8.7 Request for Analysis and Chain-of-Custody Forms ....................................................67

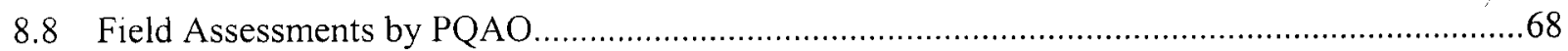

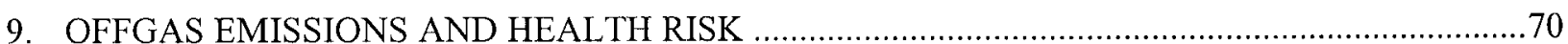

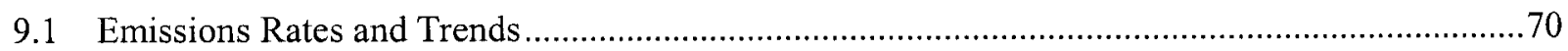

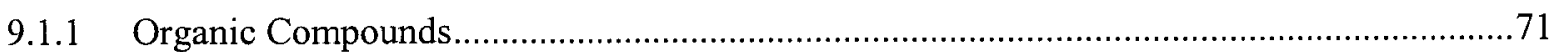

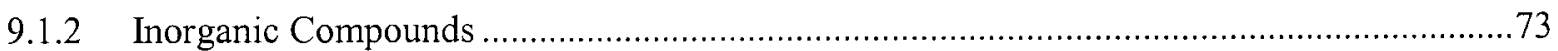

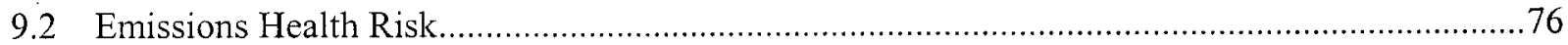




\section{CONTENTS}

10. CONCLUSIONS

11. REFERENCES

Appendix A. Analytical Data Summaries

Appendix B. Offgas Sampling Data

Appendix C. Process Stream Sampling Data

Appendix D. DCS Data

\section{FIGURES}

S-1. Comparison of volatile organic emissions at the beginning and end of evaporator batches ........... viii

$\mathrm{S}-2$. Comparison of semi-volatile organic emissions at the beginning and end of evaporator batches .... ix

S-3. Comparison of metals emissions at the beginning and end of evaporator batches (not including final

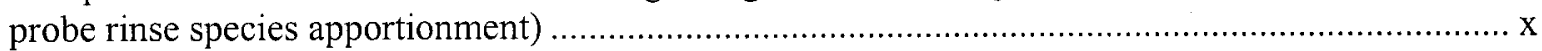

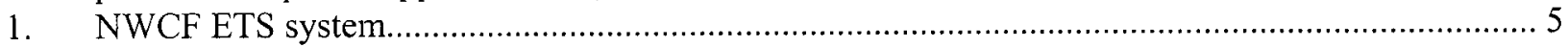

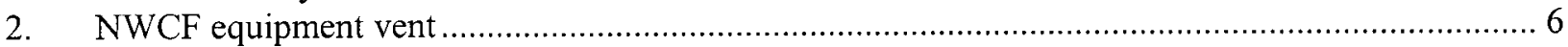

3. Offgas pipe axial view of the offgas tie-in sample location ..................................................... 7

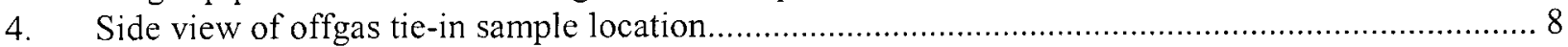

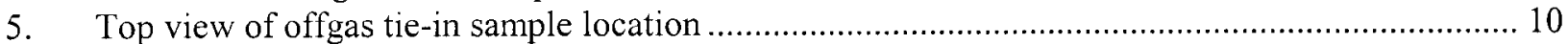

6. Average evaporator vessel temperature comparison for SVOC runs at the start and end on the

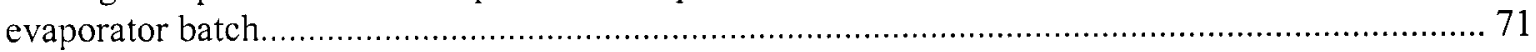

7. Comparison of volatile organic emissions at the beginning and end of evaporator batches ........... 72

8. Comparison of semi-volatile organic emissions at the beginning and end of evaporator batches ... 73

9. Comparison of metals emissions at the beginning and end of evaporator batches (not including final probe rinse species apportionment) ............................................................................. 74

10. Comparison of chloride emissions at the beginning and end of evaporator batches ...................... 75

11. Comparison of particulate emissions at the beginning and end of the evaporator run with probe

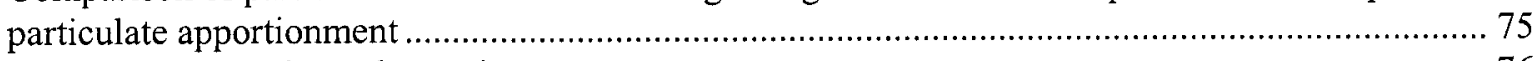

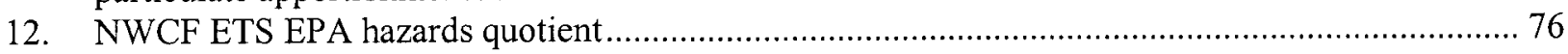

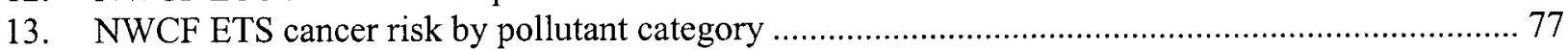

\section{TABLES}

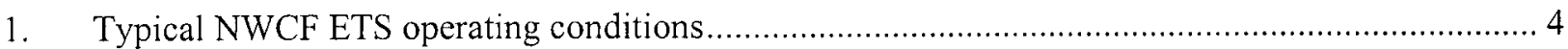

2. Summary of NWCF ETS offgas sample collection and analysis methods................................ 12

3. Summary of samples collected in support of the INEEL NWCF ETS Effluent Gas..................... 15

4. Sample contamination survey train radio-assay results ...................................................... 19

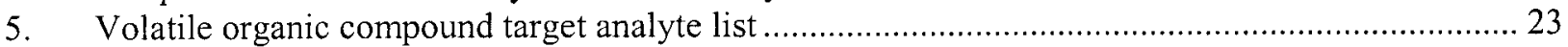

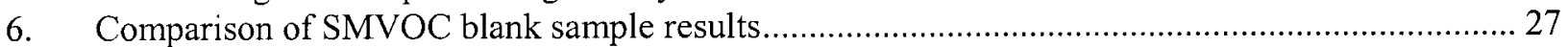

7. Volatile Organic Compound (VOC) internal standard recoveries............................................. 30

8. Volatile Organic Compound (VOC) surrogate compound recoveries.......................................... 32

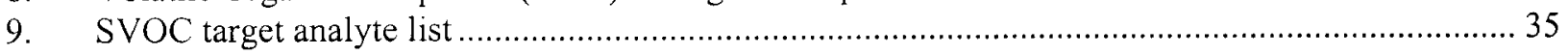




\section{CONTENTS}

10. SVOC train sample internal standard compound recoveries ................................................. 39

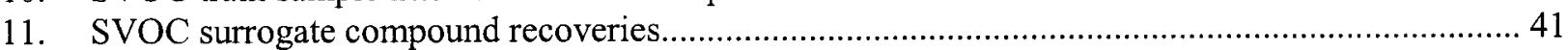

12. Metals target analyte list................................................................................................ 44

13. Inorganic analyses of feed streams processed during NWCF ETS off-gas emissions sampling...... 51

14. Inorganic analyses of bottoms streams during NWCF ETS off-gas emissions sampling .............. 53

15. Inorganic analyses of condensate streams during NWCF ETS off-gas emissions sampling........... 54

16. VOC analyses of NWCF ETS streams during NWCF ETS off-gas emissions sampling............... 55

17. SVOC analyses of NWCF ETS streams during NWCF ETS off-gas emissions sampling ............. 56

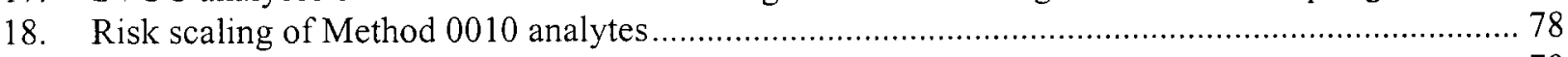

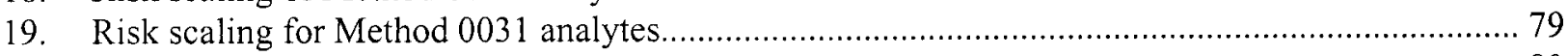

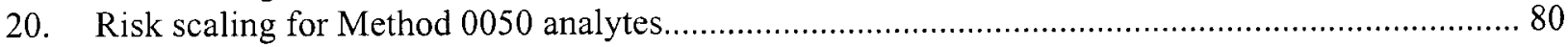

21. Risk scaling for Method 0060 analytes................................................................................ 80 


\section{ACRONYMS}

ACS

ALD

APS

CAS

CEMS continuous emissions monitoring system

CFL central file location

CFR Code of Federal Regulations

COC chain of custody

CVAAS cold vapor atomic absorption spectroscopy

DF decontamination factor

$\mathrm{D} / \mathrm{F} \quad$ dioxins and furans

DCS Distributive control system

D.I. deionized

DOT Department of Transportation

DQOs data quality objectives

DWSD Drinking Water Standards Division

EDD electronic data deliverables

EMSL Environmental Monitoring Systems Laboratory

EPA U. S. Environmental Protection Agency

GC/MS gas chromatography/mass spectrometry

$\mathrm{HCl}$ hydrochloric acid

HEPA high-efficiency particulate air (filter)

HQ hazardous quotient

HRGC/HRMS high resolution gas chromatography/high resolution mass spectrometry

INEEL Idaho National Engineering and Environmental Laboratory 


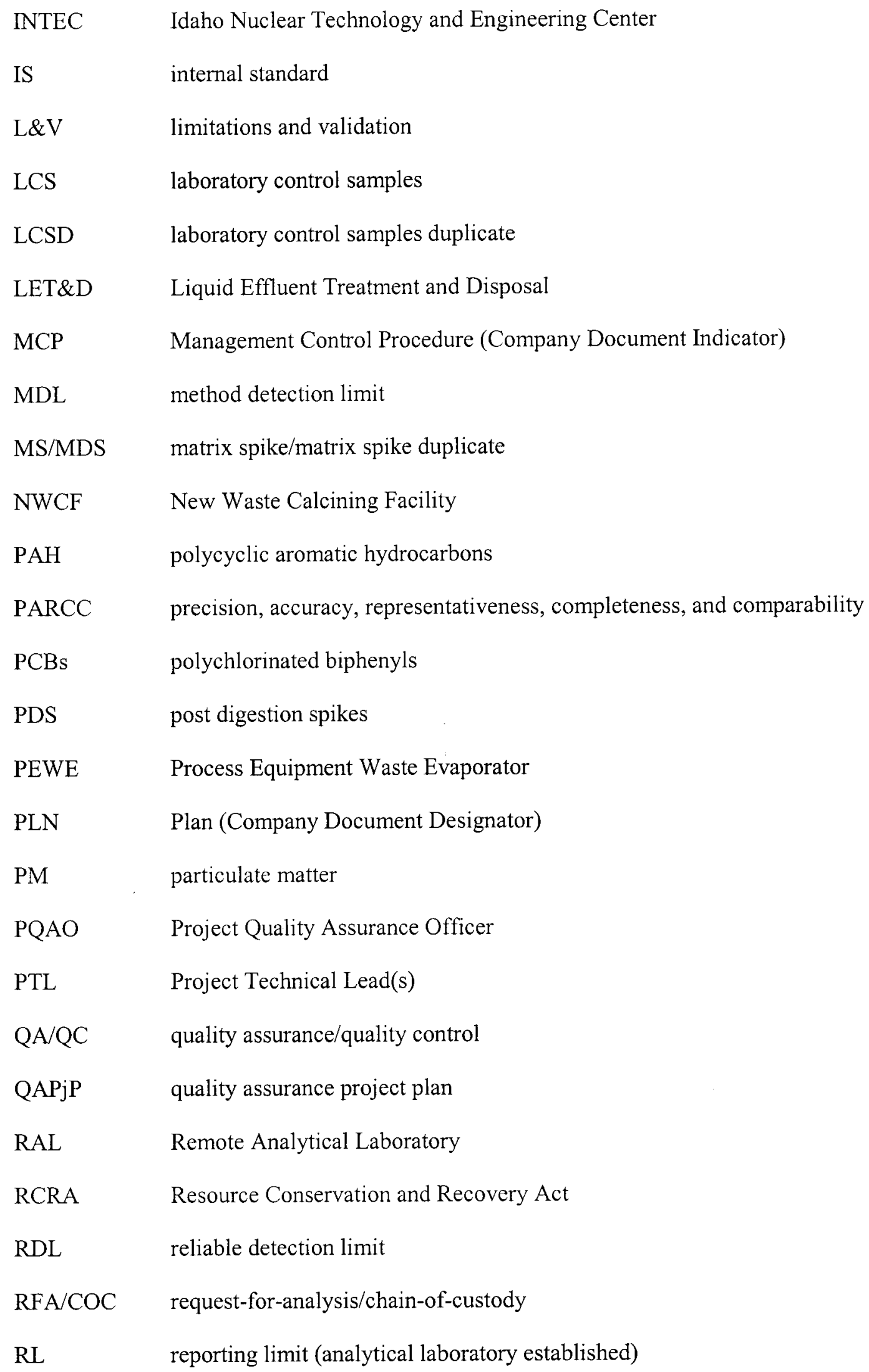




\begin{tabular}{ll} 
RPD & relative percent difference \\
RPF & relative potency factor \\
SAIC & Science Applications International Corporation \\
SDG & sample delivery group \\
SMO & Sample Management Office \\
SMVOC & sampling method for volatile organic compounds \\
SOW & statement of work \\
STL & Severn-Trent Laboratory- (Knoxville, Tennessee) \\
SVOC & semi-volatile organic compound \\
SW & Solid Waste \\
TAL & target analyte list \\
TFF & Tank Farm Facility \\
TICs & tentatively identified compounds \\
TOC & total organic carbon \\
TOS & task order specific (statement of work) \\
TPR & Technical Requirements Procedure (Company Document Designator) \\
VOC & volatile organic compound \\
\hline
\end{tabular}




\section{NWCF Evaporator Tank System 2001 Offgas Emissions Inventory}

\section{INTRODUCTION}

Liquid wastes generated by fuel reprocessing and decontamination activities are stored in the Idaho Nuclear Technology and Engineering Center (INTEC) Tank Farm Facility. The Tank Farm wastes are currently being concentrated using the INTEC New Waste Calcining Facility (NWCF) Evaporator Tank System (ETS) (formally know as High Level Liquid Waste Evaporator HLLWE). The NWCF ETS currently operates under Resource Conservation and Recovery Act (RCRA) interim status. A RCRA Part B permit application for this unit is being prepared and is planned to be submitted in FY-2003. In order to support the permitting activities, the feed and output streams were characterized during evaporator operations in May and June, 2001. Characterization of the NWCF ETS process gaseous emissions were completed in conjunction with liquid feed and concentrated effluent analyses.

A detailed test plan (Test Plan for the HLLWE Effluent Gas Emissions Inventory, PLN-879) and quality assurance project plan (QAPjP- INTEC Quality Assurance Project Plan for the HLLWE Offgas Emissions Inventory Project, PLN-880) were developed for this project. The test plan discusses project organization, training requirements, safety implementation plans, sample collection objectives, and potential NWCF ETS offgas emissions. The QAPjP specifies the quality assurance and quality control (QA/QC) requirements, applicable quality standards, and both Idaho National Engineering and Environmental Laboratory (INEEL) and project-specific procedures for collecting, packaging, preserving, shipping, and analyzing the NWCF ETS offgas samples. The sample collection and analysis methods and procedures adhere to U.S. Environmental Protection Agency (EPA) protocol and technical requirements.

Science Applications International Corporation, Idaho Falls, Idaho (herein referred to as SAIC) was subcontracted to collect and recover the samples using the EPA prescribed procedures and equipment. SAIC also assisted BBWI in calculation of the air emissions rates using the data collected in the field and the sample analytical results. SAIC is recognized for its training and experience as a sample collection team. They previously supported the NWCF Calciner offgas emissions inventory project. Sample collection was performed using checklists and field data sheets.

Severn-Trent Laboratories, Knoxville, Tennessee (herein referred to as STL) performed the offgas sample preparations and analyses. The samples sent to STL were accompanied by a Request-forAnalysis Form (RFA), which documents the project-specific analytical specifications and quality control instructions to the laboratory. As part of the RFA documentation, a Chain-of-Custody (COC) and tractability record was maintained for all sample transfers to the laboratory. An analytical report for the final analytical data (STL 2001) was provided by STL. The analytical report includes a description of the analytical procedures that were used to acquire the data generated in support of this project.

Liquid feed streams and effluents associated with the NWCF ETS were collected in conjunction with the offgas sampling and were analyzed to complete mass balance and emissions inventory calculations. The samples were collected and analyzed under the Balance of Plant Sampling and Analysis Plan (inputs to Process Equipment Waste Evaporator (PEWE) and Liquid Effluent Treatment and Disposal (LET\&D)). The liquid stream samples were collected and analyzed remotely to reduce operator and analyst exposure to radiation. Liquid sample collection was performed by the NWCF ETS operators 
using double-needle sample collection system. The samples were sent the INTEC Remote Analytical Laboratory (RAL) for analyses.

The purpose of this report is to document and discuss the NWCF ETS offgas emissions inventory results and liquid feed stream analytical results. A technical description of the facility is followed by a description of the sample collection matrix and results. The risk associated with the offgas emissions has also been calculated and is presented herein. 


\section{NWCF ETS SYSTEM AND OFFGAS SAMPLING LOCATION}

The NWCF ETS is a single-stage, thermal siphon, batch evaporator. Dilute Tank Farm liquid wastes are semi-continuously fed to the evaporator to maintain a constant level in the evaporator. A schematic of the NWCF ETS process is shown in Figure 1. The system consists of a feed tank (VESNCC-152), a flash column (VES-NCC-150), a reboiler (HE-NCC-350), and a condenser (VES-NCC151). Blended tank farm wastes are added to the flash column via the feed tank. When the level in the flash column reaches its normal operating level, steam is introduced into the shell side of the evaporator reboiler. As the evaporator solution temperature increases, its density decreases and the solution starts to rise. Steam bubbles form and further decrease the solution density. This draws the liquid from the bottom of the flash column into the tubes of the reboiler and creates a thermosiphon. The steam from the reboiler rises through a demister mesh and proceeds to the condenser. Typical NWCF ETS process operating conditions during sampling collection and analysis is shown in Table 1.

Once the desired specific gravity is reached, the concentrated solution is cooled and returned to the Tank Farm. The NWCF ETS overhead vapor is condensed in a total condenser and sent to the INTEC Process Equipment Waste Evaporator (PEWE) to be re-evaporated. Non condensable and purge gasses are vented from the feed tank and the condenser to the NWCF equipment vent system as shown in Figure 2. The equipment vent gasses join with the main process off-gas steam from the NWCF prior to the system high-efficiency particulate air (HEPA) filters.

Each batch generally requires $10-16$ hours to process, followed by $10-16$ hours to refill the feed tanks and to attend to waste transfers back to the Tanks Farm. Operating conditions that were monitored during emissions inventory testing are shown in discussed in Section 7. Normal operating conditions were maintained to provide the most stable and representative conditions throughout the sample collection period. All operating conditions are routinely recorded by the NWCF and Atmospheric Protection System (APS) control systems. These records are maintained by INTEC High Level Waste operations.

The offgas tie-in sample location used previously to sample the NWCF Calciner offgas stream was determined to be the best location for sample collection and offgas measurements for the scope and objectives of this project. The existing offgas tie-in location is downstream of the NWCF compressors and upstream of the APS. At this location, the offgas pipe is underground. The estimated offgas conditions at this location are listed in Table 1. Figure 3 shows the 12 -inch ID pipe placement $9 \mathrm{ft}$ underground, contained inside a larger 20 inch pipe encasement, which is inside a concrete encasement. The encasements provide the necessary physical protection and radiation shielding as the offgas flows to the APS.

Figure 4 shows a side view of the offgas tie-in location. This location is over $10 \mathrm{ft}$ (10 pipe diameters) or more upstream and downstream of flow interference, so the flow should be reasonably straight (except for any disruption caused by the 12-inch tee). A 12-inch ID tee topped with a flange provides access through a manhole to the offgas pipe. Several penetrations (shown in top view in Figure 5) through the flange enable sample probe access and sample extraction.

The two-inch diameter port (line 2" POG-AR-156513) was used exclusively for the NWCF ETS offgas sample collection. This port is located at the centerline of the offgas duct cross section, allowing a vertical traverse of the duct to be made. A custom heated Method 5 probe (1.75 inch outside diameter) for was fabricated for sampling at this location. The sample probe was equipped with a compression fitting to provide a seal on the outer sheath of the sample probe. Pressurized air is used to continuously purge the annulus between the port inner wall and the probe sheath. 
Table 1. Typical NWCF ETS operating conditions.

\begin{tabular}{|c|c|c|}
\hline Parameter & DCS Identification Number & Value (a) \\
\hline \multicolumn{3}{|c|}{ HEPA filters } \\
\hline Evaporator temperature & T150-1 through T150-10 & $95-110^{\circ} \mathrm{C}$ \\
\hline Steam to evaporator & $\mathrm{F} 350-1 \mathrm{C}$ & $1500-2000 \mathrm{lbs} / \mathrm{hr}$ \\
\hline Evaporator level & $\mathrm{L} 150-1 \mathrm{C}$ & $100-140$ inches \\
\hline Evaporator density & & $1.0-1.35 \mathrm{~g} / \mathrm{mL}$ \\
\hline $\begin{array}{l}\text { Superheater (HE-NCC-335) outlet } \\
\text { offgas temperature (HEPA filter bank } \\
\text { inlet temperature) }\end{array}$ & $\mathrm{T} 335-2 \mathrm{C}$ & $150-205^{\circ} \mathrm{F}$ \\
\hline HEPA filter inlet pressure & P130-2C & 30 to 100 in. $\mathrm{H}_{2} \mathrm{O}$ \\
\hline HEPA filter stage 1 differential pressure & $\begin{array}{l}\text { PD130-1-1C, }-2-1 C,-3-1 C \text {, } \\
-4-1 C\end{array}$ & $\begin{array}{l}0.5-10 \text { in. } \mathrm{H}_{2} \mathrm{O} \text { (when online) } \\
0-0.5 \text { in. } \mathrm{H}_{2} \mathrm{O} \text { (when offline) }\end{array}$ \\
\hline $\begin{array}{l}\text { Total differential pressure across HEPA } \\
\text { filter stages } 1-3\end{array}$ & PD130-1C & $2-18$ in. $\mathrm{H}_{2} \mathrm{O}$ \\
\hline HEPA filter stage 3 outlet temperature & $\begin{array}{l}\mathrm{T} 130-1-1 \mathrm{C},-2-1 \mathrm{C},-3-1 \mathrm{C} \\
-4-1 \mathrm{C}\end{array}$ & $80-150{ }^{\circ} \mathrm{F}$ \\
\hline $\begin{array}{l}\text { NWCF process offgas flowrate (HEPA } \\
\text { filter outlet offgas flowrate) }\end{array}$ & F130-1C & $50-1,000 \mathrm{scfm}$ \\
\hline \multicolumn{3}{|c|}{ Equipment Vent Conditions } \\
\hline Offgas flow & F136-1C & $500-1200 \mathrm{scfm}$ \\
\hline Offgas temperature & $\mathrm{T} 336-1 \mathrm{C}$ & $60^{\circ}-80^{\circ} \mathrm{F}$ \\
\hline Offgas to APS pressure & P122-1 & $6-12$ in. $\mathrm{H}_{2} \mathrm{O}$ vacuum \\
\hline \multicolumn{3}{|c|}{$\begin{array}{l}\text { Atmospheric Protection System (APS) and Other Equipment Downstream of the Offgas Tie-in Sample } \\
\text { Location }\end{array}$} \\
\hline $\begin{array}{l}\text { APS inlet offgas temperature (process } \\
\text { offgas condenser outlet gas temperature }\end{array}$ & T-OGF-104 & $180-200^{\circ} \mathrm{F}$ \\
\hline APS inlet flowrate (process offgas flow) & F-OGF-2 & $1000-2000 \mathrm{scfm}$ \\
\hline APS inlet offgas pressure & P-OGF-22 & Negative $5-15$ in. $\mathrm{H}_{2} \mathrm{O}$ (c) \\
\hline Main stack offgas flowrate & F-OGF-4/5 & $80,000-100,000 \mathrm{scfm}$ \\
\hline Main stack offgas temperature & T-OGF-4-1, -5-1 & $70-100^{\circ} \mathrm{F}$ \\
\hline \multicolumn{3}{|c|}{$\begin{array}{l}\text { a) If the value for an operating parameter drifts outside of he indicated value range, or outside }+/-10 \% \text { of the } \\
\text { range is shown, then the test team leader must determine if sample collection should discontinue until NWCF } \\
\text { operation is modified to correct the value. }\end{array}$} \\
\hline \multicolumn{3}{|c|}{ b) Standard temperature and pressure is $60^{\circ} \mathrm{F}, 1$ atmosphere. } \\
\hline \multicolumn{3}{|c|}{$\begin{array}{l}\text { c) This pressure is controlled using dampers on offgas blowers BLO-OGS-213 and }-214 \text {, and can be adjusted to } \\
\text { control the static pressure at the Offgas Tie-in location }\end{array}$} \\
\hline
\end{tabular}




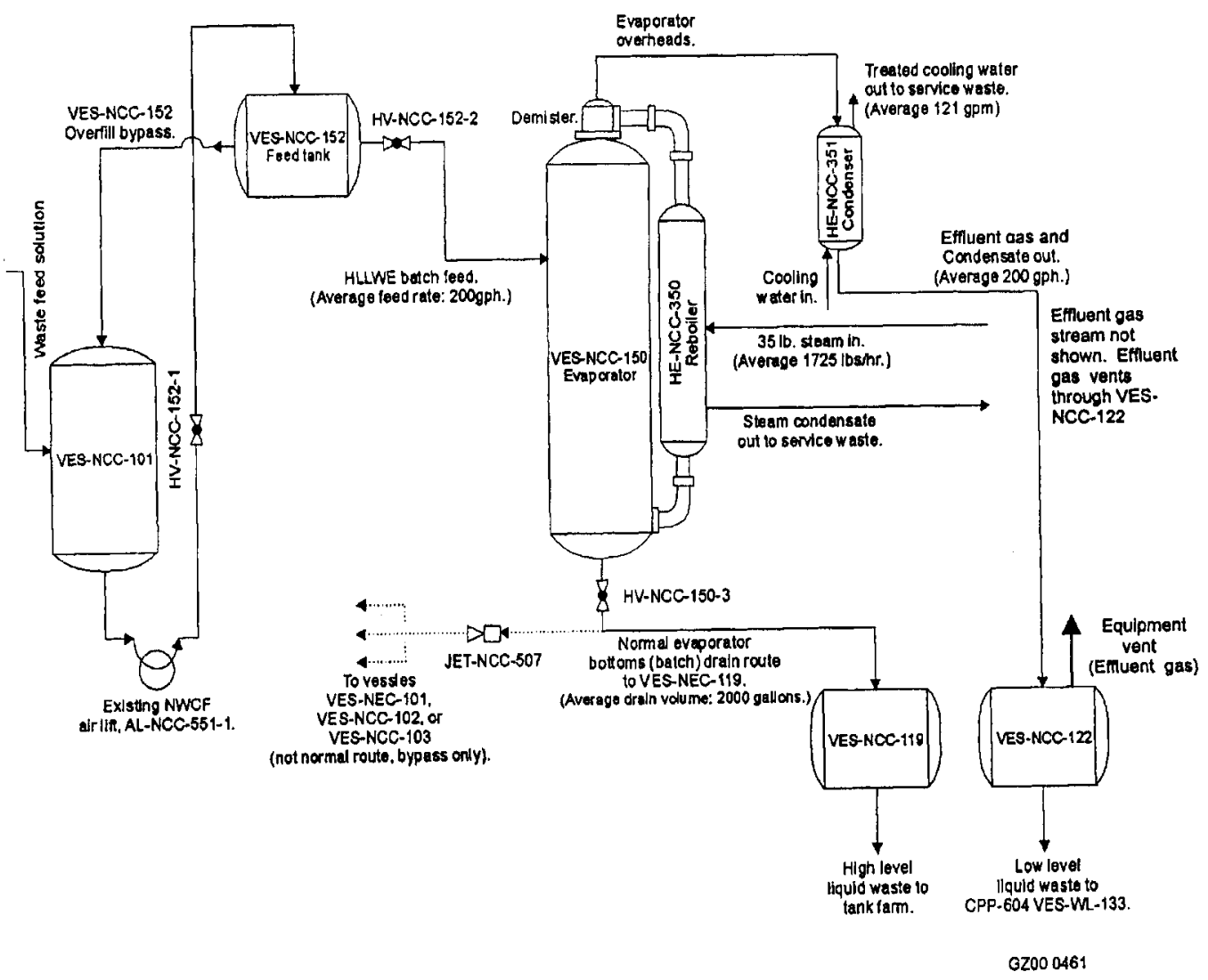

Figure 1. NWCF ETS system. 


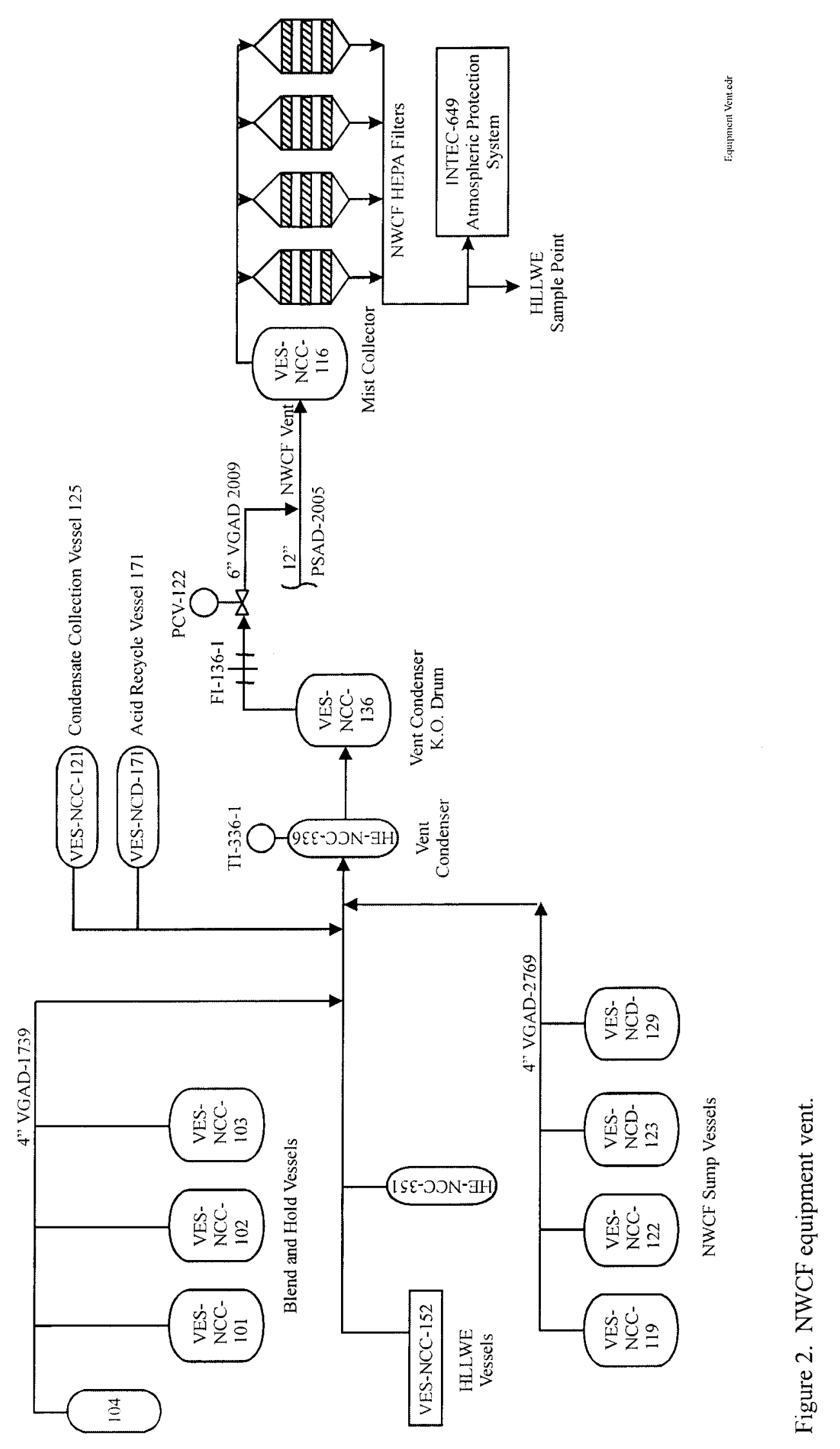




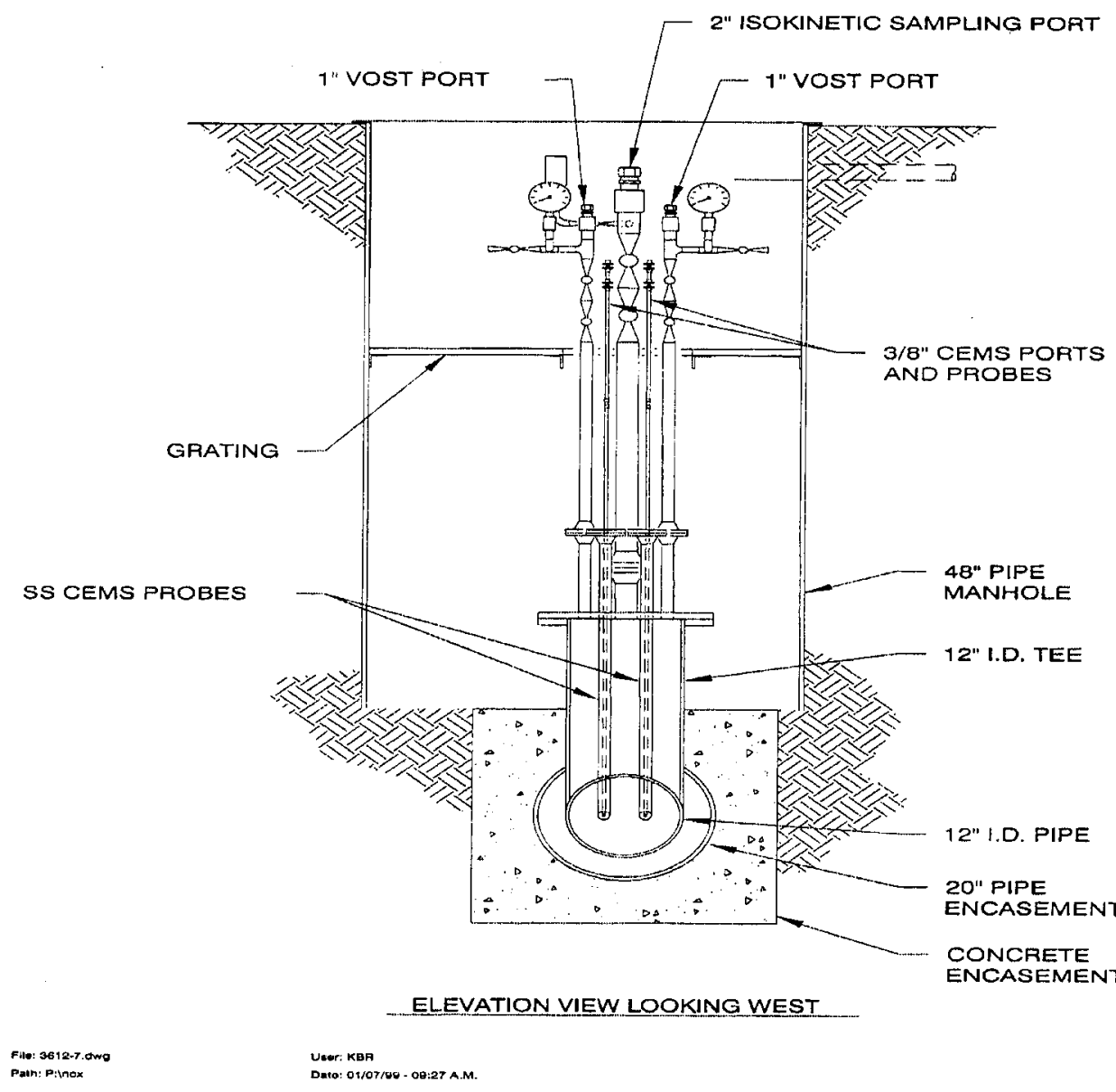

Figure 3. Offgas pipe axial view of the offgas tie-in sample location. 


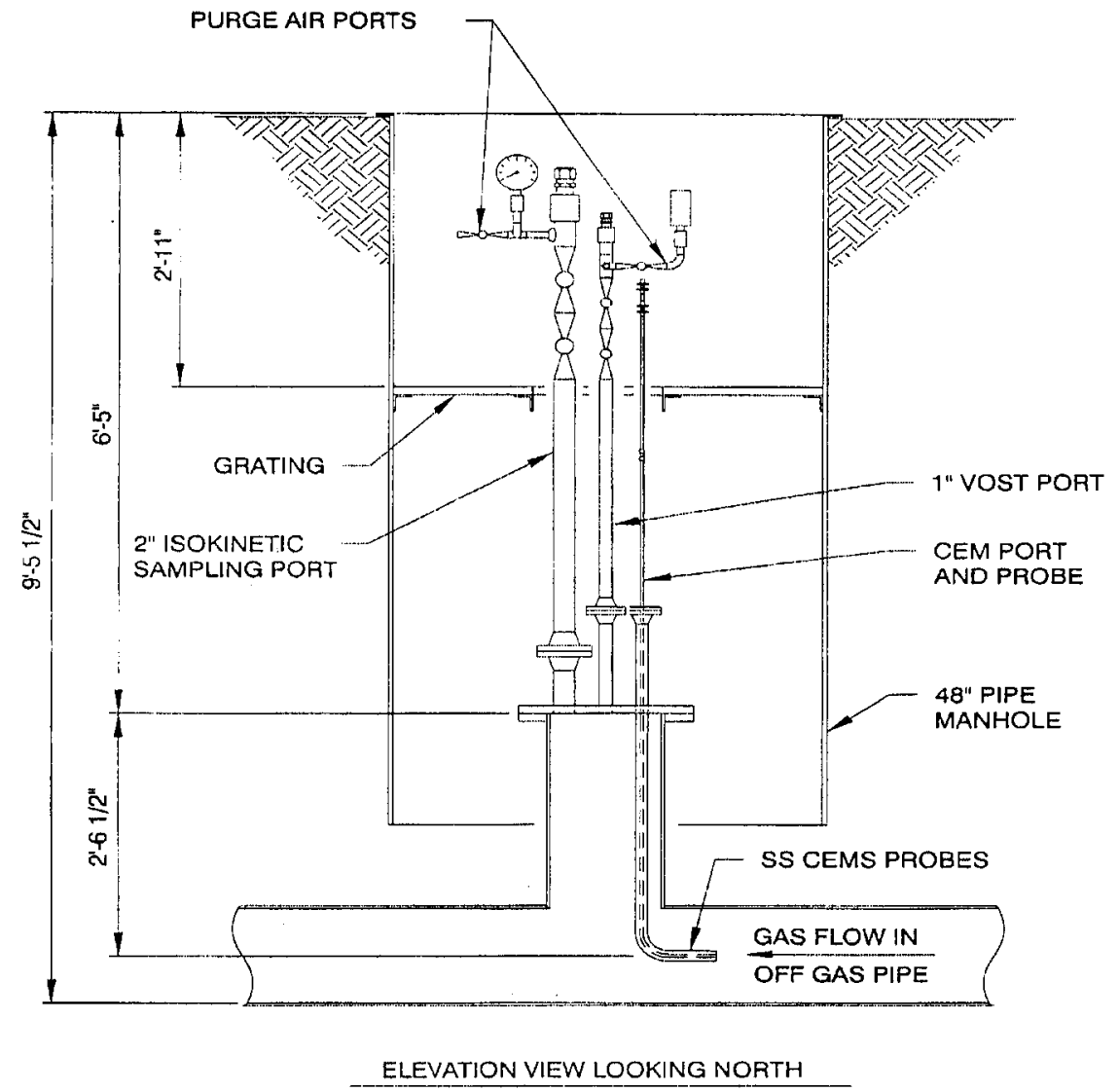

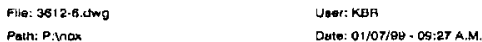

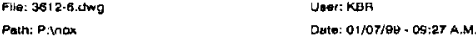

Figure 4. Side view of offgas tie-in sample location. 
This sample collection location does not meet all specified EPA requirements for offgas sample collection (40 Code of Federal Regulations (CFR) 60 Appendix A, Methods 1 and 2) since it is not possible to traverse the duct at two orthogonal positions. There may also be some mutual disturbance of the offgas flow pattern caused by the $3 / 8$-inch tubes that are slightly upstream of the 2 -inch access port as shown in a top view of the sample tie in (Figure 5). Fortunately, interference between sample ports is minimized as there is a clear path to oncoming gas flow as shown in the cross sectional view.

Another possible limitation to the sampling location is the presence of radionuclide contamination in the NWCF offgas duct. The procedure for inserting the $12 \mathrm{ft}$ probe into the duct required donning of anti-contamination clothing and active monitoring by a Radiological Control Technician and Industrial Hygienist. The fragile probe tip can be easily damaged, and possibly could fall into the NWCF offgas duct- an event that is undesirable because it would introduction foreign material into the duct upstream of the Atmospheric Protect System. In addition, any potential presence of loose contamination in the duct could result in the spread of radiological contamination and possible exposure to the sample collection attendants. Hence, it was determined that the probe would be placed in the duct at a fixed point and not disturbed until the NWCF ETS offgas measurements were concluded. The probe was only articulated at the beginning of the sample collection tests in order to measure the vertical velocity profile in the duct. 


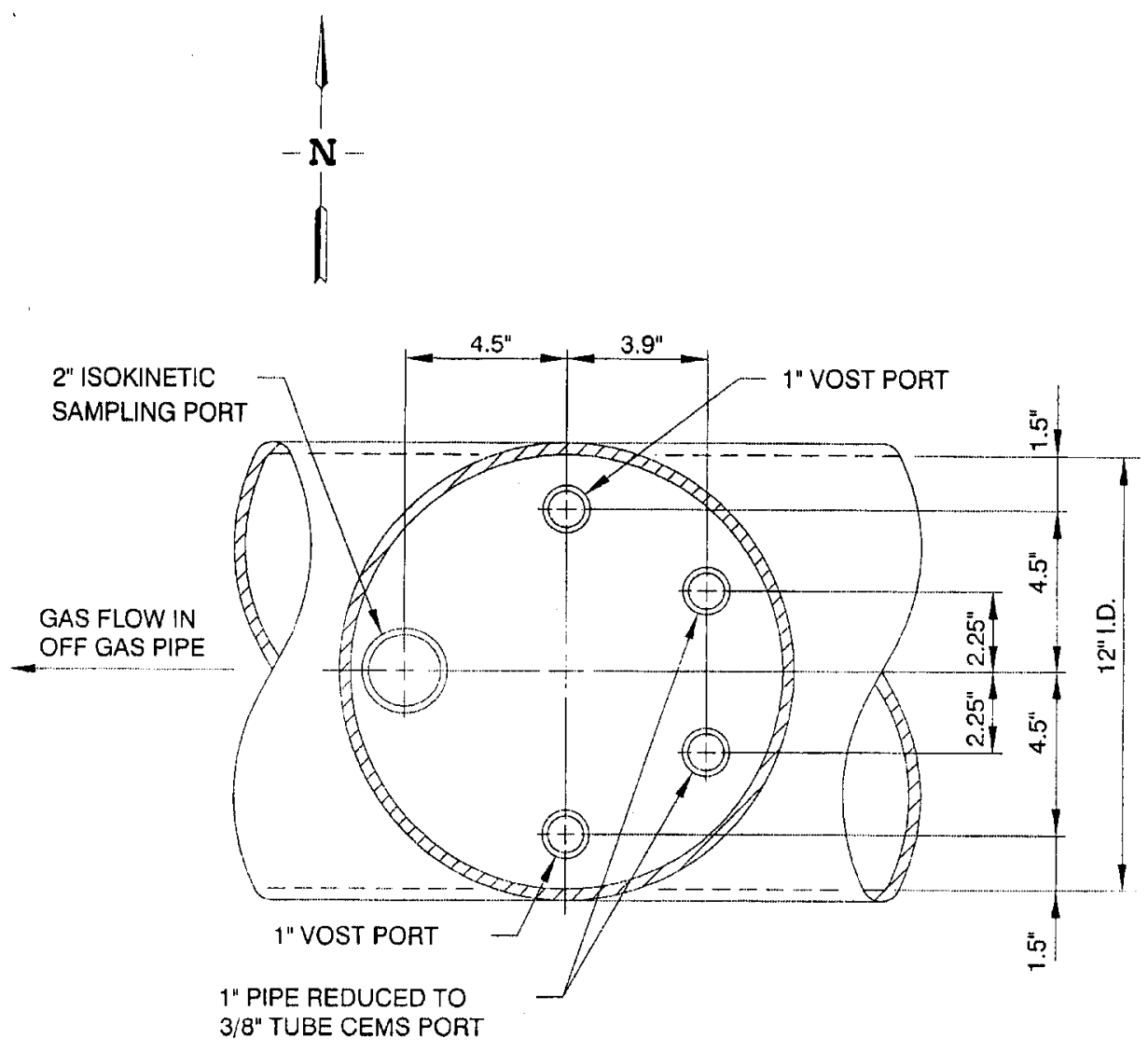

TOP VIEW OF SAMPLING TEE AT NWCF OFF-GAS TIE IN LOCATION

$\begin{array}{ll}\text { File: } 3612-5 . \text { dwg } & \text { Usar: KBR } \\ \text { Path: P:Inox } & \text { Date: 01/0799 - 09:26 R.M. }\end{array}$

Figure 5. Top view of offgas tie-in sample location. 


\section{SCOPE AND APPROACH}

The purpose of this activity is to characterize the NWCF ETS process effluent gas emissions. The Tank Farm wastes are highly acidic (mainly nitric acid) inorganic salt solutions and contain several RCRA metals, including mercury, and trace amounts of volatile and semi-volatile hazardous organic compounds. Those components with low boiling points are released to the NWCF ETS offgas system during waste transfers, mixing, and evaporation. Trace amounts of heavy, nonvolatile hydrocarbons may also exist in the waste feed solutions; however, emissions of the nonvolatile hydrocarbons and also the nonvolatile metals is theorized to mainly occur by aerosol entrainment from the evaporator. The majority of the entrained droplets are believed to be collected in the offgas condensers, mist eliminators, and HEPA filters.

The scope of the NWCF ETS process effluent gas emissions inventory includes:

- Measurement of the NWCF offgas duct velocity, temperature, and flowrate during operation of the NWCF ETS

- Manual offgas sampling and analysis for particulate matter (PM), hydrochloric acid ( $\mathrm{HCl}), \mathrm{Cl}_{2}$, selected metals including Hg, volatile organic compounds (VOCs), and semi-volatile organic compounds (SVOCs)

- Measurement of radiological contaminate concentrations in sampling media using sample collection trains that are representative of the EPA sample collection trains

- Measurement of oxygen concentrations

- Measurement of probe rinseate for apportionment of metals and PM adsorbed on the probe to the respective train totals

Standard EPA sample collection and analysis methods were used to characterize the measure target analytes for each of the categories listed in Table 2. Measurements of moisture content and offgas temperature, velocity, and flowrate are included in each of the isokinetic sample train measurements. Sampling was conducted following EPA methodology with attention being given to the following:

- Development and adherence to a project quality assurance/quality control plan

- Implementation of sample chain-of-custody/requests-for-analysis, master sample lists, and sample labeling and tracking which assured in-field verifications of correctness of sample identifiers

- Development of target analyte lists (TAL) and precision, accuracy, representativeness, completeness, and comparability (PARCC) data quality indicators and objectives

- Collection of samples using checklists to record train set up, sample collection data, and sample recovery steps

- Collection and analysis of reagent blanks, trip blanks, and field blanks to assess prescribed data quality objectives

- Sample collection and documentation by a Project Quality Assurance Officer (PQAO) 
- Application of EPA Solid Waste (SW)-846 and 40CFR 60 Appendix A reference methods for sample analysis

- Multiple reviews and verifications of field data, analytical data, process data, and resulting calculations of emissions rates

Table 2. Summary of NWCF ETS offgas sample collection and analysis methods.

\begin{tabular}{|c|c|c|c|}
\hline $\begin{array}{l}\text { Sample train } \\
\text { procedure or } \\
\text { Method }\end{array}$ & Measurement & Train description & Analytical procedures \\
\hline 2 & $\begin{array}{l}\text { Gas velocity, } \\
\text { temp., swirl } \\
\text { angle }\end{array}$ & $\begin{array}{l}\text { S-type pitot, incline } \\
\text { manometer, thermocouple }\end{array}$ & --- \\
\hline 0010 & SVOCs & $\begin{array}{l}\text { Isokinetic single-point, } \\
\text { glass-lined probe, heated } \\
\text { filter, XAD-2 }{ }^{\circledR} \text { sorbent, } \\
\text { impingers }\end{array}$ & $\begin{array}{l}3542 / 8270 \mathrm{C} \text { GC/MS (SVOCs) } \\
\text { STL SOP KNOX-ID-0012 }\end{array}$ \\
\hline 0060 & $\begin{array}{l}\text { Multiple metals } \\
\text { including } \mathrm{Hg}\end{array}$ & $\begin{array}{l}\text { Isokinetic single-point, } \\
\text { glass-lined probe, heated } \\
\text { filter, impingers that contain } \\
\text { sorbent solutions }\end{array}$ & $\begin{array}{l}\text { 6010A (ICAP) for multiple } \\
\text { metals, } 7470 \text { (CVAAS) for } \\
\text { mercury }\end{array}$ \\
\hline $\begin{array}{l}0050 \text { modified } \\
\text { for PM }\end{array}$ & $\mathrm{HCl}, \mathrm{Cl}_{2}, \mathrm{PM}$ & & $\begin{array}{l}9056 / 9057 \text { (IC for } \mathrm{HCl}, \mathrm{Cl}_{2} \text {, and } \\
\mathrm{F} \text { ); and Method } 5 \text { (gravimetric for } \\
\text { PM), STL SOP KNOX-MS-0011 }\end{array}$ \\
\hline $\begin{array}{l}0031 \\
\text { SMVOC }\end{array}$ & VOCs & $\begin{array}{l}\text { Single point, non-isokinetic, } \\
\text { three sorbent tubes in series } \\
-\left(\text { Tenax }^{\circledR} / \text { Tenax }^{\circledR}\right. \\
\left.\text { /Anasorb }{ }^{\circledR} 747\right) \text { and } \\
\text { condensate trap }\end{array}$ & $5041 \mathrm{~A} / 8260 \mathrm{GC} / \mathrm{MS}$ \\
\hline $\begin{array}{l}3 \mathrm{~A} \text { or other (as } \\
\text { requested by } \\
\text { project lead) }\end{array}$ & $\mathrm{O}_{2}$ & $\begin{array}{l}\text { Single point, nonisokinetic, } \\
\text { heated sample line }\end{array}$ & Paramagnetism \\
\hline
\end{tabular}

The level of organics in the acidic Tank Farm waste solutions is very low and the NWCT ETS is operated at a much lower temperature than the NWCF Calciner. Therefore, because temperature and chemical precursors are not there in the system, it was determined that separate analysis of PCBs, and $\mathrm{D} / \mathrm{Fs}$ was not necessary. It was determined that the results of the SVOCs for the target PAH compounds would be sufficient for risk assessment calculations. The offgas results presented in this report demonstrate that SVOC emissions are indeed negligible, as are precursors to PAHs and also higher 
molecular weight compounds, including PAHs, PCBs, and D/Fs. Hence, the scope of the NWCF ETS offgas emissions inventory was limited to those methods shown in Table 2.

During the recent NWCF Calciner offgas emissions inventory (Boardman 2001), sample collection runs were conducted for analysis of 24 of the highly toxic semi-volatile polynuclear aromatic hydrocarbons (PAH), polychlorinated biphenyl compounds $(\mathrm{PCB})$ and dioxins and furans $(\mathrm{D} / \mathrm{Fs})$, as well as SVOCs. This required separate runs with EPA Method 0010 and EPA Method 0023A trains. The samples are extracted and concentrated for subsequent analysis by high resolution gas chromatography/high resolution mass spectroscopy (HRGC/HRMS). Isotope dilution is used for each target analyte; thus, it was possible to achieve method detection limit concentrations for those analytes that were typically one-three orders of magnitude less than MDLS for the current reported project, where standard EPA Method $8270 \mathrm{C}$ gas chromatography/mass spectrometry (GC/MS) was specified. Lower detection limits for these compounds were desired to assist in the analysis of the Calciner performance, and also to provide the best possible data for Calciner emissions health risk assessment.

At the beginning of the sample collection period, the vertical gas velocity profile and swirl angle in the duct was measured to determine an appropriate fixed-point location to collect the offgas samples. Fixed-point sampling was necessary to avoid potential spread of contamination and possible damage to the glass probe tip. Two separate traverses were made to enhance accuracy of the velocity measurements. The minimum number of traverse points per Method 1 on the single (vertical) traverse for particulate and nonparticulate traverses (4) plus the pipe centerline were included in the traverses. The swirl angle at each traverse point and the average swirl angle per EPA Method 1, Section 2.4 were also determined. Subsequently, the probe was fixed at the point of maximum flow which corresponds EPA prescribed sample position at four inches from the pipe wall.

A set of two samples trains (referred to herein as "runs") were collected for each category of pollutants at the beginning and also at the end of evaporator runs. This provided a total of four runs for each method and brackets the emissions over the entire batch. One blank train (field blank) was also collected during the period that the four trains for each method were being run. Trip blanks and reagent blanks also were collected, as required by the QAPjP.

Oxygen concentrations were periodically recorded from the digital readout of the oxygen monitor located in the sampling tent during the manual sample collection operations. Because the off-gas sampled was essentially air supplied to ventilate NWCF vessels it was supposed and confirmed that the oxygen concentrations in the duct were similar to ambient air conditions. Therefore, it was determined that there was no need to constantly operate the oxygen monitor.

Moisture levels in the offgas were determined from the gravimetric and volumetric changes in the sample train resins and impingers, respectively. The moisture level was typically less than the dew point of the sample gas passing through the sample collection train condensers. At the most, only $1-2 \mathrm{~mL}$ of condensate was collected in any of the condensate knockout impingers. This is consistant with the use of a total condenser on the process and the addition of dry instrument air.

Finally, sample contamination survey trains were collected at the beginning of the tests in accordance with an INTEC management control procedure (MCP-1173, Package and Ship NWCF Offgas Emissions Samples Offsite for Analysis, Revision 2). Radioanlytical results from these trains were used to bracket the expected radioactivity range in the offgas samples to ensure that sample shipments to STL were in accordance with their radioactive materials license. Additional screening was performed routinely throughout the sample collection period. Every sample, as a minimum, was screened for gamma/beta emissions using a micro- $\mathrm{R}$ radiation detector, which is approximately 10 times more sensitive than the hand-held friskers used in the field by the Radiological Control Technicians. All of the 
Method 5 filters were also submitted to the INTEC Radiochemical Laboratory for an extended duration (typically 12 hours) gamma count.

At the end of the sample collection period, the sample probe was removed from the duct and rinsed with acetone and nitric acid. These samples were surveyed for radiological contamination before being shipped to the analytical laboratory for measurement of PM and metals that were adsorbed on the probe. The results of these samples were used to address the technical acceptability of leaving the probe at a fixed position. It was assumed that the level of particulate in the duct would be negligible as a result of the sample location being downsteam of the NWCF HEPA filter banks. This was shown to be an appropriate assumption for the NWCF Calciner offgas emissions inventory project (Boardman 2001) and for the NWCF ETS as discussed later in this report. When a measurement of any target analyte in the probe rinsate was greater than the method detection limits, the result was apportioned to the metals and PM trains results.

Table 3 summarizes the sample collection trains, blank trains (field blanks), trip and reagent blanks, probe rinses, and radiological survey trains. Also listed is the sample collection date, time, and volume of offgas that was pulled through the train. 


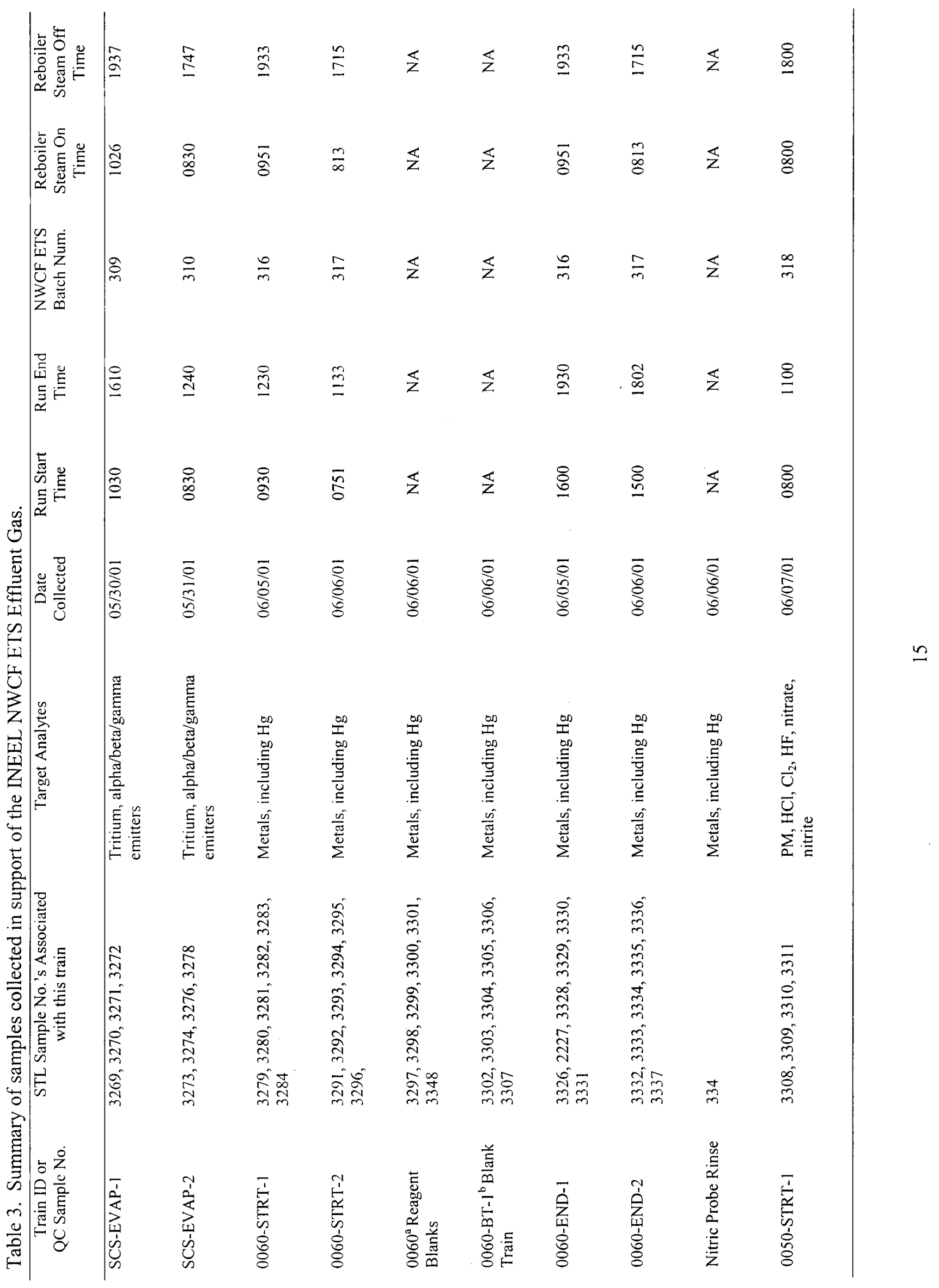




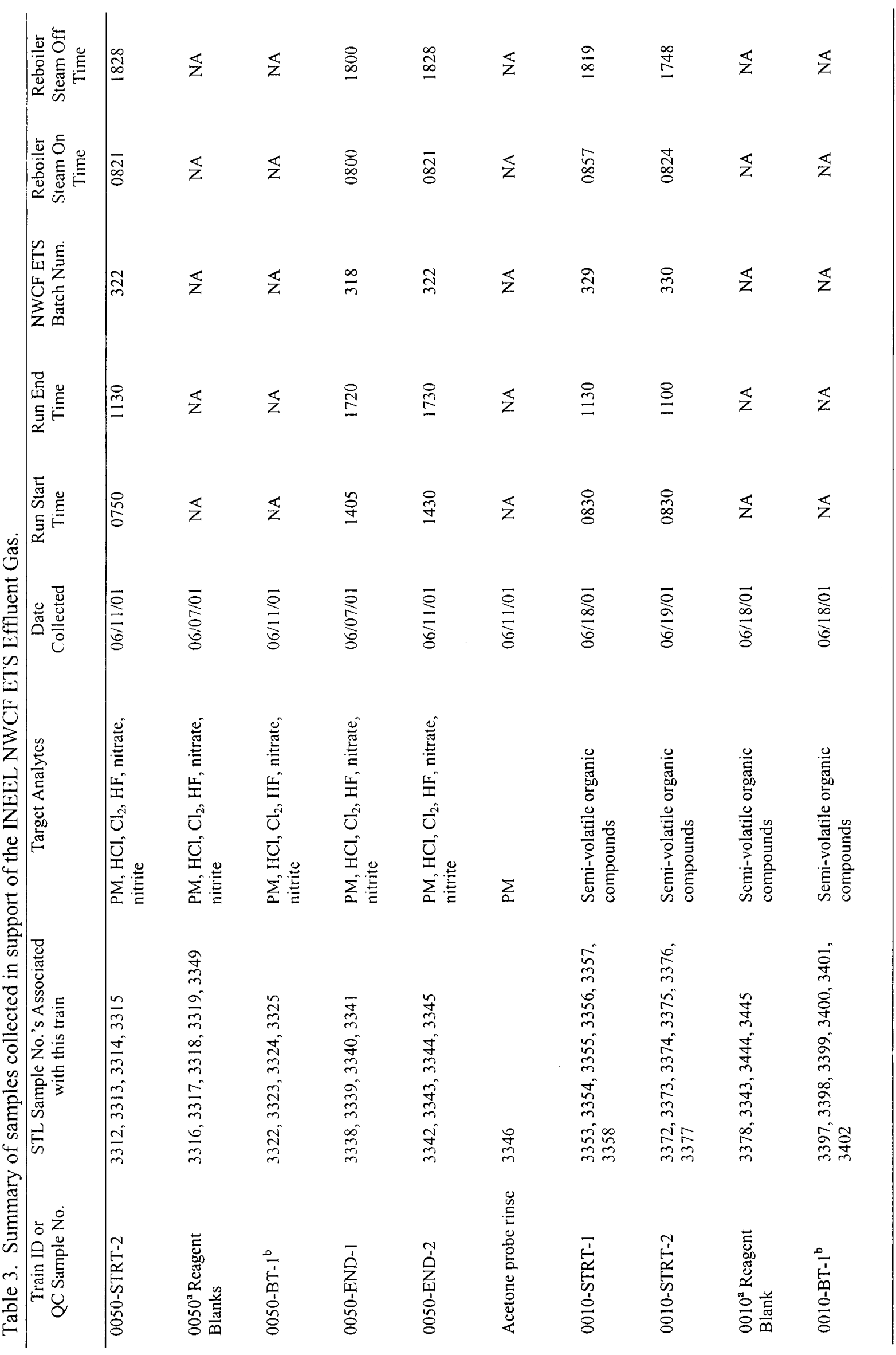




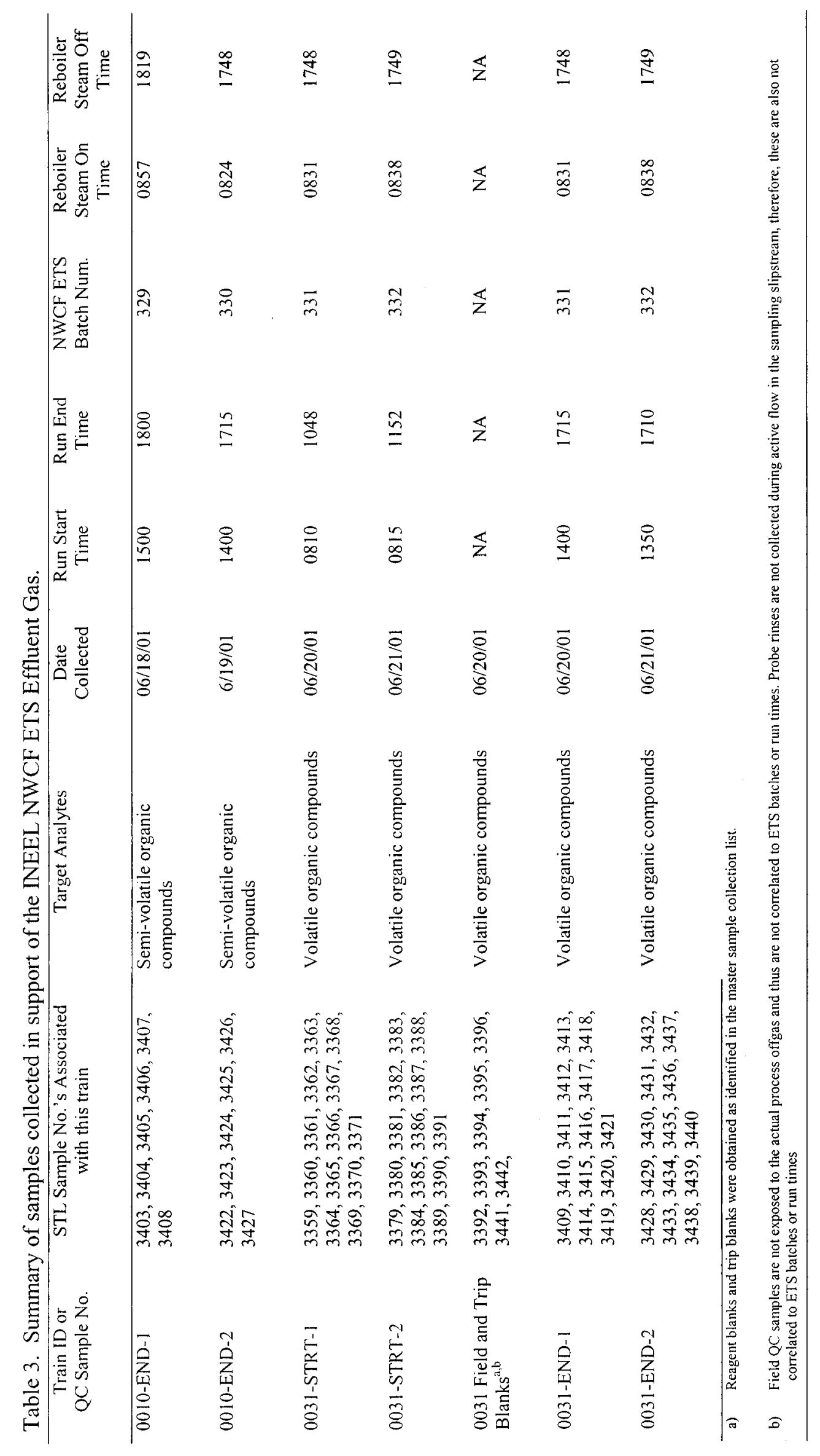




\section{RADIOLOGICAL SCREENING RESULTS}

Two radiological contamination survey trains were collected at the beginning of the NWCF ETS offgas emissions inventory to establish the level of radiological contamination that could be uptaken by the EPA sample collection trains. The first train, identified as SCS-EVAP-1, was a hybrid of the Method 0060 for metals and Method 0050 for anions. The configuration of this train included a particle filter, followed by a condenser and condensate collection impinger, and then a pair of nitric acid/hydrogen peroxide impingers from the Method 0060 and a pair of sodium hydroxide impingers from the Method 0050 train. The acid and hydroxide impingers were used to capture the particulate and volatile radionuclides that are not disengaged by the filter and condensate trap. A gas volume of $3 \mathrm{dscm}$ (dry, standard cubic meters) of gas was collected to match the volume of gas that was collected by the Method 0060 and 0050 sampling runs. Less than $2 \mathrm{~mL}$ of condensate was collected by this train- an insufficient amount for accurate analysis. Therefore, the condensate was added to the nitric/peroxide impinger solution.

The second radiological contamination train, identified as SCS-EVAP-2, was simply a standard Method 0010 train for semi-volatile organic collection, consisting of a particle filter, condenser, XAD- ${ }^{\circledR}$ resin tube, a condensate trap and two organic-free water impingers. This train was mainly used to establish the level of contamination that could be potentially captured by the XAD- ${ }^{\circledR}$ resin tube. The sample line, train glassware and filter housing were rinsed with acetone and methylene chloride. These rinses were composited into single sample for radiochemical analysis. The volume of gas collected was 3 $\mathrm{dscm}$. The amount of condensate collected by the train was also very low (approximately $2 \mathrm{~mL}$ ) for this train, indicating the offgas was essentially dry. The small amount of condensate was added to the organic-free impingers. Following the 12 -hr gamma scan of the XAD- $2^{\circledR}$ resin, the upper section of the resin bed, which first contract the sample gas and condensate, was extracted and prepped for gross alpha/gross beta counting.

Method 0031 for VOCs collection requires only $20 \mathrm{dsL}$ (dry, standard liters) total, and only $5 \mathrm{dsL}$ for each set of tubes. Therefore, the contamination levels established by the reference survey trains, at a total volume of $3000 \mathrm{dsL}$, clearly bounded the potential contamination picked up on the Tenax ${ }^{\mathbb{B}}$ resin tubes used in the Sampling Method for Volatile Organic Compounds (SMVOC) runs.

Analysis of the contamination survey train samples was completed by the INTEC Radiochemical Laboratory. Appropriate standards were prepared and used to provide quantitative results for the various sample collection media. Each sample was first analyzed by a non-intrusive gamma scan to measure gamma-emitting nuclides such as $\mathrm{Ba}^{137}$ (which is the short lived daughter product of $\mathrm{Cs}^{137}$ ). Since $\mathrm{Cs}^{137}$ is the most abundant non-volatile radionuclide in the waste, it is a convenient marker for the non-volatile radionuclides that could be present in the offgas samples, including $\mathrm{Sr}^{90}$ and actinide isotopes. Therefore, an accurate gamma scan provides a basis for identifying the potential presence of $\mathrm{Ba}^{137}$, and hence $\mathrm{Cs}^{137}$, $\mathrm{Sr}^{90}$, and other fission products and actinides that may be present in the samples. A 12-hr gamma scan was performed to provide the most accurate analysis possible.

Following the gamma scan, the samples were prepared for gross alpha/gross beta counting. This required that the solid sample media be digested and then dried to obtain a valid measure of the particle emissions. The activity of tritium was determined by beta scintillation of an aliquot of the up-front liquid impinger contents to which the small amount of condensate was added. These fraction also absorb the largest percentage of the non-condensable water vapor.

Table 4 summarizes the radio-assay results for SCS-EVAP-1 and SCS-EVAP-1. Only an ultra low level of gross beta and gross alpha emissions was detected in the samples. The sample contamination 
levels are conservatively less than the analytical laboratory sample screening acceptance criteria for Category I samples.

Table 4. Sample contamination survey train radio-assay results.

\begin{tabular}{|c|c|c|c|c|c|c|}
\hline Train ID & $\begin{array}{l}\text { Sample } \\
\text { ID }\end{array}$ & Sample Media & Gamma & $\begin{array}{c}\text { Gross } \\
\text { Beta }\end{array}$ & $\begin{array}{l}\text { Gross } \\
\text { Alpha }\end{array}$ & Tritium \\
\hline \multirow{3}{*}{ SCS-EVAP-1 } & 3269 & Particle filter & $\begin{array}{l}\text { No nuclides } \\
\text { identified }\end{array}$ & $\begin{array}{l}5.7 \mathrm{E}+00 \\
\pm 1.4 \mathrm{E}+00 \\
\mathrm{pCi}\end{array}$ & $\begin{array}{l}9.8 \mathrm{E}-01 \\
\pm 6.7 \mathrm{E}-01 \\
\mathrm{pCi}\end{array}$ & $\begin{array}{l}\text { NA } \\
\text { (dry sample) }\end{array}$ \\
\hline & $\begin{array}{l}3270 / 3271 \\
\text { composite }\end{array}$ & $\begin{array}{l}\text { Condensate and } \\
\text { nitric/peroxide } \\
\text { impinger contents }\end{array}$ & $\begin{array}{l}\text { No nuclides } \\
\text { identified }\end{array}$ & $\begin{array}{l}4.0 \mathrm{E}-02 \\
\pm 2.1 \mathrm{E}-02 \\
\mathrm{pCi} / \mathrm{mL}\end{array}$ & Not detected & $\begin{array}{l}0.17 \\
\mu \mathrm{Ci} / \text { sample }\end{array}$ \\
\hline & 3272 & $\begin{array}{l}\text { Hydrogen peroxide } \\
\text { impinger contents }\end{array}$ & $\begin{array}{l}\text { No nuclides } \\
\text { identified }\end{array}$ & $\begin{array}{l}4.3 \mathrm{E}+00 \\
\pm 2.1 \mathrm{E}-01 \\
\mathrm{pCi} / \mathrm{mL}\end{array}$ & $\begin{array}{l}3.4 \mathrm{E}-02 \\
\pm 4.0 \mathrm{E}-02 \\
\mathrm{pCi} / \mathrm{mL}\end{array}$ & $\begin{array}{l}\text { NA } \\
\text { (negligible } \\
\text { condensate } \\
\text { in sample) }\end{array}$ \\
\hline \multirow{4}{*}{ SCS-EVAP-2 } & 3273 & Particle filter & $\begin{array}{l}\text { No nuclides } \\
\text { identified }\end{array}$ & $\begin{array}{l}1.85 \mathrm{E}+01 \\
\pm 4.0 \mathrm{E}+00 \\
\mathrm{pCi}\end{array}$ & $\begin{array}{l}6.0 \mathrm{E}+00 \\
\pm 2.6 \mathrm{E}+00 \\
\mathrm{pCi}\end{array}$ & $\begin{array}{l}\text { NA } \\
\text { (dry sample) }\end{array}$ \\
\hline & 3276 & $\mathrm{XAD}-2^{\mathrm{R}}$ resin tube & $\begin{array}{l}\text { No nuclides } \\
\text { identified }\end{array}$ & $\begin{array}{l}5.84 \mathrm{E} 00 \\
\pm 7.2 \mathrm{E}-01 \\
\mathrm{pCi} / \mathrm{g}\end{array}$ & Not detected & $\begin{array}{l}\mathrm{NA} \\
\text { (negligible } \\
\text { condensate } \\
\text { in sample) }\end{array}$ \\
\hline & 3278 & $\begin{array}{l}\text { Condensate and } \\
\text { organic-free water } \\
\text { impinger composite }\end{array}$ & $\begin{array}{l}\text { No nuclides } \\
\text { identified }\end{array}$ & $\begin{array}{l}1.6 \mathrm{E}-02 \\
\pm 1.9 \mathrm{E}-02 \\
\mathrm{pCi} / \mathrm{mL}\end{array}$ & Not detected & $\begin{array}{l}0.32 \\
\mu \mathrm{Ci} / \text { sample }\end{array}$ \\
\hline & 3274 & $\begin{array}{l}\text { Organic solvent } \\
\text { rinse composite }\end{array}$ & $\begin{array}{l}\text { No nuclides } \\
\text { identified }\end{array}$ & Not detected & Not detected & $\begin{array}{l}\text { NA } \\
\text { (negligible } \\
\text { condensate } \\
\text { in sample) }\end{array}$ \\
\hline
\end{tabular}

In order to ensure that the extremely low contamination levels in the offgas stream remained constant throughout the sample collection inventory, the particle filter for each Method 0060 , Method 0050 , and Method 0010 run, and one of the leading Tenax ${ }^{\circledR}$ tubes was submitted for a 12-hour gamma scan. This had little or no effect on sample preservation condition of the filters. Although the Tenax ${ }^{\circledR}$ was not maintained at the required temperature of $4^{\circ} \mathrm{C}$, it is not likely that the VOC analysis results were 
adversely affected since the tube was kept sealed during the gamma scan. The results of the on-going screening were consistent with the baseline results. No nuclides were identified by these extended gamma-scan analyses.

Each sample was also "smear-wiped" and counted to verify there was no detectable fugitive contamination on the surface of the sample containers. These additional screening efforts corroborated the results of the sample contamination survey trains and verified that each train did not collect any significant contamination throughout the 3 -week sample collection period.

Finally, the volume of offgas sampled, as well as the amount of condensate collected by all of the sample runs, was checked and compared to the sample contamination survey trains. This ensured that the level of condensate, and hence the estimated amount of tritium in the respective samples, was consistent with the baseline results. 


\section{OFFGAS SAMPLE ANALYTICAL RESULTS}

SAIC was responsible for setting-up, operating, and recovering the sample collection trains in the contamination containment hood. Once the samples were obtained, custody was transferred to the BBWI project principals for radiological screening and shipment to STL. The inorganic samples produced by each run were shipped to STL in Department of Transportation (DOT) approved fiber boxes with metal inner canisters. The organic samples were placed on ice and packaged in expanded, insulated coolers in order to maintain temperature preservation requirements. Chain-of-Custody and Requests-for-Analysis forms were used to track each sample. Shipments requiring preservative cooling were made using overnight delivery in order to ensure temperature preservation and analysis time limits were met. All of the samples meet the preservation and sample analysis time requirements without exception.

The results presented in this section are extracted from the Final Analytical Report provided by STL (STL 2001). Excerpts of the text and tables are included in this report to provide a single project summary document. Appendix A is a listing of the analytical lab certificates of analysis. These data were used to calculate the offgas emissions rates presented in Section 9.

STL tabulated train totals for each of the four EPA train runs that were made to characterize the NWCF ETS effluent gas emissions that are discharged through the NWCF offgas system. The results for each train component were summed to provide a run total for each target analyte. Although the laboratory data were reported down to the method detection limit (MDL), the project has implemented the reliable detection level (RDL) as the minimum value for risk calculations. The "RDL" is the detection level recommended by EPA. It is defined as 2.623 times the MDL (2.623 X MDL).

Significant figures for both the constituent fractions and the cumulative total were determined according to ASTM Standard E29-93a (1999), "Standard Practice for Using Significant Digits in Test Data to Determine Conformance with Specifications". Laboratory-assigned data qualifiers are displayed with each target analyte when required. The majority of these method-based flags are standardly defined flags among environmental laboratories. The data flags attached to the train totals represent the cumulative set of flags assigned to the result for each component that is included as part of the respective train totals. Data flags for individual component sample fractions were only carried through to the train totals when that particular train component result had an observable mathematical impact (based on significant figures as cited above) on the value of the "train totals" result for that compound.

When assigned, the "less than" $(<)$ sign indicates that at least one sample fraction result included in the run total is either a "non-detect" value that has been evaluated down to the MDL of the measurement, or an estimated "hit" value that is below the RDL. In either case, the final analyte value for any fraction that has a laboratory result below the RDL is raised to the default RDL value, and the actual value for the respective analyte is judged to be less than conservative reported value. This same logic carries through to the summation of train fractions to arrive at train totals.

Additional project-specific train total flags are applied to the run total values that are not standard EPA data flags. These project-specific flags are specific to the NWCF ETS Offgas Emissions Inventory project and are defined as follows:

- An "N" flag indicates that the compound was not measured (detected) in any of the sampling train components, or fractions.

- A "P" flag indicates that the compound was measured (detected) in one or more of the train components, or fractions, but not in all of the sampling train fractions. 
- An "A" flag indicates that the compound was measured (detected) in all of the sampling train components, or fractions.

\subsection{Volatile Organic Compounds}

The standard U.S. EPA Method 0031 SMVOC sampling train configuration was used to collect samples of the NWCF ETS offgas for the assessment of volatile compounds. Each run used four sets of volatile organic adsorption resins tubes. Each set of tubes was comprised of two Tenax ${ }^{\circledR}$ tubes and one Anasorb $747^{\mathbb{B}}$ tube in series. The resin tubes were followed by a condensate trap that was used to capture the condensate captured by all four set of tube for a given run. The volume of offgas collected across each set of tubes was $5 \mathrm{~L}$ over a time period of approximately 30-40 minutes. Hence the total volume of gas for each run was approximately $20 \mathrm{~L}$, collected over a time span of around 2.5 hours. The purpose of multiple tube sets was to integrate the sampling event over a period of time to better obtain representative data that characterizes the offgas emissions of the offgas stream sampled and is recommended by Method 0031. Each run produced 12 resin tubes and one condensate fraction that were stored on ice after they were removed from the train.

\subsubsection{VOC Target Analyte List}

The SMVOC samples were analyzed for the volatile organic compound target analytes given in Table 5. Analyses of SMVOC samples were completed using thermal desorption of the tubes onto a purge-and-trap device. SW-846 Method 5041B was implemented to carry out the thermal desorption. Method 8260B was implemented to analyze the desorbed analytes using GC/MS. The two SMVOC Tenax ${ }^{\circledR}$ tubes from a sample set were analyzed together, while the Anasorb $747^{\circledR}$ tube was separately analyzed.

A GC/MS library search was performed on each SMVOC sample (including the condensate samples) for non-target analytes, or tentatively identified compounds (TICs). The search was performed for the thirty (30) largest identifiable non-target compounds having a response that was at least $10 \%$ of the response of the nearest internal standard, which was spiked at $0.25 \mu \mathrm{g}$. The library search was conducted against the National Bureau of Standards library of mass spectral data containing an estimated 75,000 compounds. The matching criteria includes a nominal $85 \%$ match of the mass spectral features, and analyst discretion of all identities reported. 
Table 5. Volatile organic compound target analyte list.

\begin{tabular}{|c|c|}
\hline VOC Target Analyte & CAS Registry Number \\
\hline Acetone & $67-64-1$ \\
\hline Acrylonitrile & $107-13-1$ \\
\hline Benzene & $71-43-2$ \\
\hline Bromobenzene & $108-86-1$ \\
\hline Bromochloromethane & $74-97-5$ \\
\hline Bromodichloromethane & $75-27-4$ \\
\hline Bromoform & $75-25-2$ \\
\hline Bromomethane & $74-83-9$ \\
\hline 2-Butanone & $78-93-3$ \\
\hline n-Butylbenzene & $104-51-8$ \\
\hline sec-Butylbenzene & $135-98-8$ \\
\hline tert-Butylbenzene & $98-06-6$ \\
\hline Carbon disulfide & $75-15-0$ \\
\hline Carbon tetrachloride & $56-23-5$ \\
\hline Chlorobenzene & $108-90-7$ \\
\hline Chlorodibromomethane & $124-48-1$ \\
\hline Chloroethane & $75-00-3$ \\
\hline Chloroform & $67-66-3$ \\
\hline Chloromethane & $74-87-3$ \\
\hline 2-Chlorotoluene & $95-49-8$ \\
\hline 4-Chlorotoluene & $106-43-4$ \\
\hline 1,2-Dibromo-3-chloropropane & $96-12-8$ \\
\hline 1,2-Dibromoethane & $106-93-4$ \\
\hline Dibromomethane & $74-95-3$ \\
\hline 1,2-Dichlorobenzene & $95-50-1$ \\
\hline 1,3-Dichlorobenzene & $541-73-1$ \\
\hline 1,4-Dichlorobenzene & $106-46-7$ \\
\hline Dichlorodifluoromethane & $75-71-8$ \\
\hline 1,1-Dichloroethane & $75-34-3$ \\
\hline 1,2-Dichloroethane & $107-06-2$ \\
\hline 1,1-Dichloroethene & $75-35-4$ \\
\hline cis-1,2-Dichloroethene & $156-59-2$ \\
\hline trans-1,2-Dichloroethene & $156-60-5$ \\
\hline 1,2-Dichloropropane & $78-87-5$ \\
\hline 1,3-Dichloropropane & $142-28-9$ \\
\hline 2,2-Dichloropropane & $594-20-7$ \\
\hline 1,1-Dichloropropene & $563-58-6$ \\
\hline cis-1,3-Dichloropropene & $10061-01-5$ \\
\hline trans-1,3-Dichloropropene & $10061-02-6$ \\
\hline
\end{tabular}


Table 5. Volatile organic compound target analyte list.

\begin{tabular}{|c|c|}
\hline VOC Target Analyte & CAS Registry Number \\
\hline Ethylbenzene & $100-41-4$ \\
\hline Hexachlorobutadiene & $87-68-3$ \\
\hline 2-Hexanone & $591-78-6$ \\
\hline Isopropylbenzene & $98-82-8$ \\
\hline p-Isopropyltoluene & $99-87-6$ \\
\hline Methylene chloride & $75-09-2$ \\
\hline 4-Methy!-2-pentanone & $108-10-1$ \\
\hline Naphthalene & $91-20-3$ \\
\hline n-Propylbenzene & $103-65-1$ \\
\hline Styrene & $100-42-5$ \\
\hline 1,1,1,2-Tetrachloroethane & $630-20-6$ \\
\hline 1,1,2,2-Tetrachloroethane & $79-34-5$ \\
\hline Tetrachloroethene & $127-18-4$ \\
\hline Toluene & $108-88-3$ \\
\hline 1,2,3-Trichlorobenzene & $87-61-6$ \\
\hline 1,2,4-Trichlorobenzene & $120-82-1$ \\
\hline 1,1,1-Trichloroethane & $71-55-6$ \\
\hline 1,1,2-Trichloroethane & $79-00-5$ \\
\hline Trichloroethene & $79-01-6$ \\
\hline Trichlorofluoromethane & $75-69-4$ \\
\hline 1,2,3-Trichloropropane & $96-18-4$ \\
\hline 1,2,4-Trimethylbenzene & $95-63-6$ \\
\hline 1,3,5-Trimethylbenzene & $108-67-8$ \\
\hline Vinyl chloride & $75-01-4$ \\
\hline m-Xylene \& p-Xylene & $136777-61-2$ \\
\hline o-Xylene & $95-47-6$ \\
\hline
\end{tabular}

\subsubsection{VOC Analytical Results}

Tabulated data summaries for the SMVOC data are given in Appendix A. These tables have been extracted from the STL Analytical Laboratory Final Report for this project. The run total (in total $\mu \mathrm{g}$ ) for each analyte represents the sum of the amounts found in all of the SMVOC sets collected during each sampling run, including the amount of analyte found in the SMVOC condensate sample. The SMVOC condensate sample results were obtained by multiplying the observed concentration in mass/volume units $(\mu \mathrm{g} / \mathrm{L})$ by the final condensate volume $(\mathrm{L})$ collected to obtain a result in units of mass $(\mu \mathrm{g})$. 
The Method 0031 SMVOC Tube Set Total (total $\mu \mathrm{g} / \mathrm{set}$ ) result consists of the sum of the analytical results for the two Tenax ${ }^{\otimes}$ resin tube contents (analyzed together) and the analytical result for the Anasorb $747^{\circledR}$ tube contents. The calculation is conducted as follows:

$$
\begin{gathered}
\text { (Total } \left.\mu \mathrm{g} \text { on the Tenax }{ }^{\circledR} \text { Tubes } \# 1 \text { and } \# 2\right)+\left(\text { Total } \mu \mathrm{g} \text { on the Anasorb } 747^{\circledR}\right. \text { Tube) } \\
=\text { Total } \mu \mathrm{g} \text { on the Method } 0031 \text { SMVOC tube set }
\end{gathered}
$$

\subsubsection{VOC Data Quality Assessment}

The tubes were shipped to the analytical laboratory and analyzed within two week in accordance with EPA guidance and the QAPjP. The SMVOC samples were received at the laboratory in good condition. The samples were held on ice until the laboratory custodian checked the cooler temperatures and logged the samples at the laboratory.

All samples were processed through the analytical methods as planned, and analytical results were obtained for all of the expected analyses, with one exception. Low surrogate recoveries were obtained for sample A-3364 (The Anasorb $747^{\circledR}$ tube for Run 1, Set 2) and therefore the data for this sample are not usable. The results for all other samples meet the data quality objectives (DQOs) specified in the QAPjP and are therefore usable for the NWCF ETS offgas emissions inventory and risk assessment.

The toluene result for the Run 2, Set 2 Tenax ${ }^{\circledR} /$ Anasorb $747^{\circledR}$ tube appears to be an outlier. This particular result was over ten times higher than any other back-half result. Also, this result was over eight times higher than the corresponding front-half result. There were several other runs that exhibited higher toluene results for the back-half fraction than the front-half fraction. These results are not consistent with the results for other analytes, including benzene, which clearly did not break through the front-half SMVOC tubes. These inconsistent results imply that there was a source of fugitive contamination available to these tubes. Since the field and trip blanks did not generally exhibit toluene (only one fronthalf field blank and one back-half trip blank had "hits" for toluene), it appears that the sample tubes were exposed to environments containing some toluene that were not available to the field or trip blanks.

The methylene chloride results for Run 1, Set 1 and Set 2, were inconsistent with subsequent test runs. The methylene chloride result for Run 1, Set 1, was much higher than for any other analysis. The Run 1, Set 2 result was lower than the result for Run 1. The remaining results were lower in concentration, and appear to reside within three standard deviations of the mean value. Also, the acetone result for Run 1, Set 1, was the highest result for acetone that was found in any of the offgas samples. The cause of the high early results is assumed to be contamination of the sampling probe with acetone and methylene chloride. The SVOC train was used immediately prior to collection of the SMVOC samples. During this sampling event, the MM-5 probe (that was used for the SVOC train and had been rinsed with acetone and methylene chloride at the conclusion of SVOC sample collection) was used to collect the SMVOC samples. Since the same probe was rinsed with acetone and methylene chloride, it is likely that these solvents found in the offgas VOC samples originated in the equipment, not in the offgas.

The SMVOC runs results show a rapid decrease in methylene chloride in the second and third tube sets for the first run. This supports the supposition that the higher amounts of methylene chloride in the first run were an artifact of the field procedures. In spite of this supposition, the result was used when calculating the emissions health risk since the risk factor and emissions rate, although higher than actual emissions, does not impact the outcome of the cumulative emissions risk. 


\subsubsection{VOC Breakthrough Evaluation}

The analysis scheme of the three-tube configuration of Method 0031 included individual analysis of each resin sample. The historical criterion for evaluating occurrences of SMVOC system breakthrough states that less than 30 percent by weight of an analyte should be detected on the back tube relative to the total amount observed on the front two tubes. That is, the Anasorb $747 \AA$ resin tube should not contain more than 30 percent of the analyte total found on the front Tenax ${ }^{\circledR}$ resin samples. The criterion does not apply when less than 75 nanograms of an analyte are detected on the back trap. Additionally, the criterion does not apply when the analytes are the ultra-low boiling point analytes such as dichlorodifluoroethane, chloromethane, bromomethane, chloroethane, and vinyl chloride.

The SMVOC apparatus was operated under near optimum conditions during on-site sampling. The sample stream entered the first resin tube at a nominal $10^{\circ} \mathrm{C}$ as monitored by a thermocouple at the base of the condenser. A 20-liter sample was the maximum volume of gas pulled across the resin tubes, and the sampling rate was a standard SMVOC approach at approximately 0.50 liters per minute. Under these conditions the analytes were universally trapped on the Tenax ${ }^{\circledR}$ tubes except for the low boiling point analytes noted above. There are some anomalous results for acetone and toluene in which back-half fractions contained more than 30 percent of the front-half amount, and the total was more than 75 nanograms. These results appear to be derived from fugitive contamination sources, and do not represent breakthrough to the back half SMVOC tubes under these conditions. Acetone and toluene are solvents used during sampling for rinsing glassware and tubing. The reagent sources are assumed to be the source of the fugitive contamination.

\subsubsection{VOC Blank Data Assessment}

Several types of Method 0031 SMVOC blanks were evaluated during the offgas sampling analyses in order to assess the sampling and analytical environments for possible fugitive contamination sources. SMVOC field blanks were collected in order to assess the sampling train environments for possible fugitive contamination sources. Standard SMVOC trip blanks were also collected, as well as a deionized water trip blank. A comparison of the blank samples is shown in Table 6 .

The SMVOC tube field blank results do not indicate field contamination by any target analytes except the three common laboratory contaminants, acetone, methylene chloride and toluene. The data for the SMVOC tube trip blanks also exhibit the presence of these three target analytes, in addition to bromomethane that was also observed on the Tenax $®$ tubes, and dichlorodifluoromethane that was observed on the Anasorb $747 \AA$ tube. The aqueous trip blank did not exhibit general contamination. The laboratory blanks associated with these samples also exhibit the presence of acetone and methylene chloride, but at levels too low to account for the observed levels of acetone in the trip blanks. Methylene chloride, acetone, and toluene are typically considered common laboratory contaminants during data validation. The laboratory method blank and field blank results do not, however, exhibit toluene. 
Table 6. Comparison of SMVOC blank sample results.

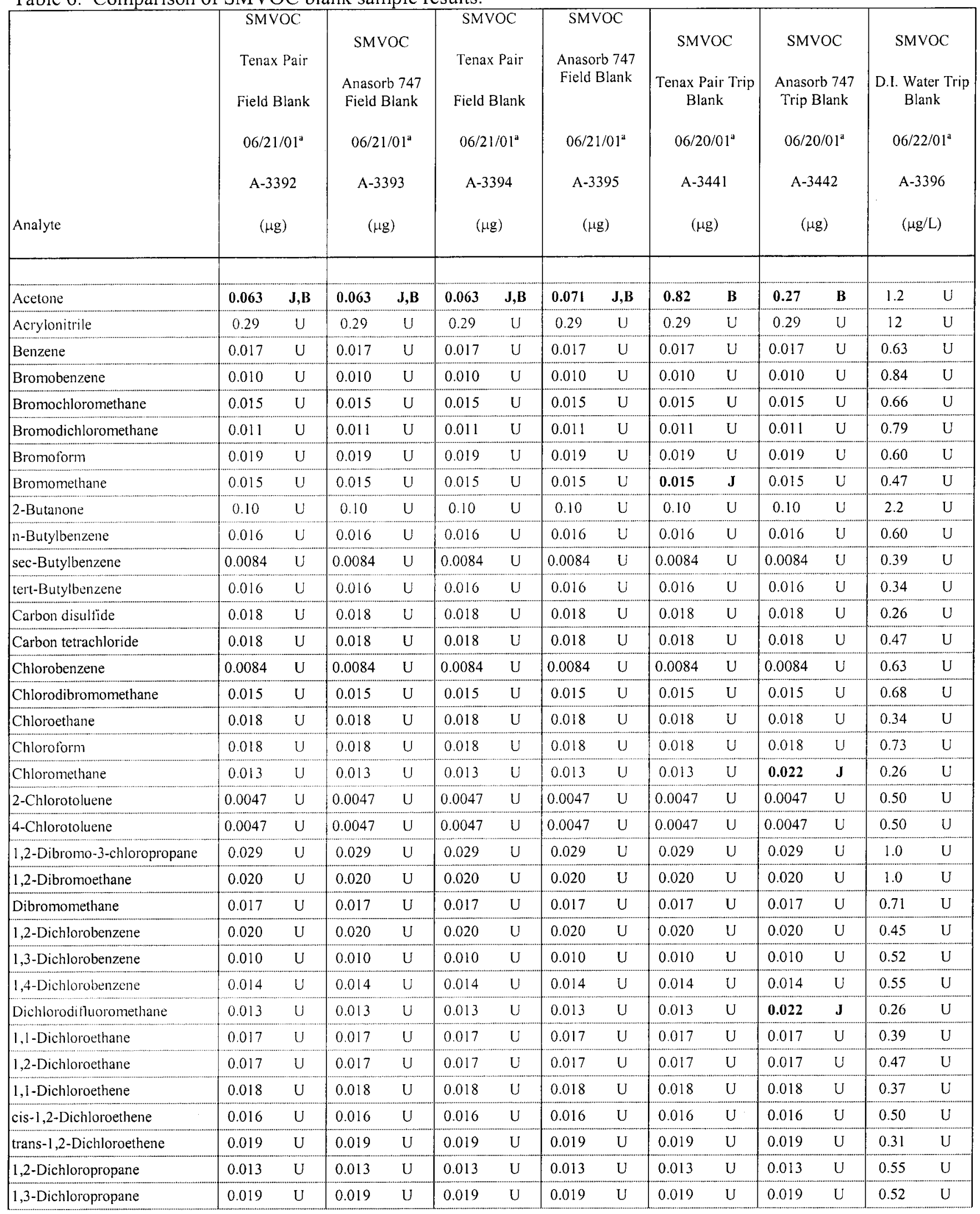


Table 6. Comparison of SMVOC blank sample results.

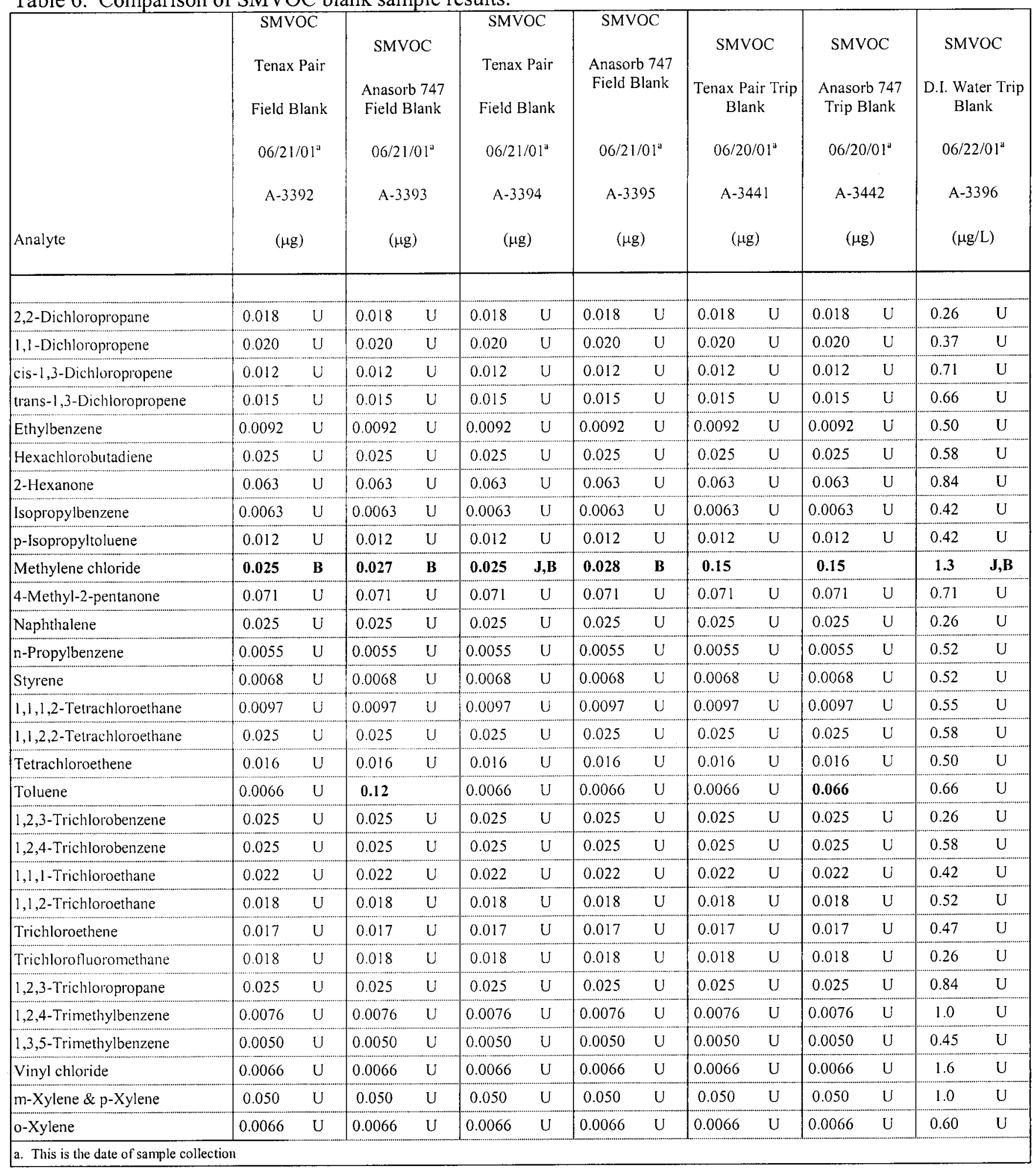


Acetone, methylene chloride, and toluene are used as rinsing solvents during Modified Method 5 sampling. These common solvents were present in the sampling area during the test series. Also, these compounds were observed in the field blanks at somewhat higher levels. Acetone was observed at a relatively high level in the SMVOC Tenax Pair Field Blank. These results indicate that fugitive contamination sources may have existed during the sampling and transport of these samples Per the SMVOC method restrictions, results from the SMVOC sampling were not blank corrected.

A practical approach was devised to use the front-half sample results for Run 1, Set 2 despite the fact that the corresponding back-half results are unusable. In order to use these results in the train total for the run, an estimate was made of the results for the corresponding back-half fraction. This estimate was obtained by averaging the results for the back halves of the remaining three sets of tubes for this run. This value is equivalent to calculating the back-half concentrations based on three (3) sets of tubes, or a total of $60 \mathrm{~L}$ of offgas. This approach has the benefit of not discarding valid data, in keeping with the general principle that all data obtained should be disclosed to the monitoring agencies.

\subsubsection{VOC Internal Standard Recovery Assessment}

Three internal standard compounds are spiked prior to thermal desportion of the SMVOC adsorbent tube. The same three internal standards were spiked into the aliquots of VOST condensate samples that were analyzed. These standards are used as a basis for the calculations of the concentrations of the target analytes and surrogates. A summary of the volatiles internal standard performance for all of the samples collected during Runs 1,2, 3, and 4 are listed in Table 7. Method SW-8260 requires internal standard recovery to be at least 50 percent but not more than 200 percent of internal standard (IS) areas for the daily standard. Internal standard performance for all samples and all matrices was well within required limits $(-50$ to +100 percent difference relative to the IS areas for the daily standard) for all samples. 
Table 7. Volatile Organic Compound (VOC) internal standard recoveries.

\begin{tabular}{|c|c|c|c|c|c|}
\hline \multirow[b]{2}{*}{ Field Sample No. } & \multirow[b]{2}{*}{ Run No. } & \multirow[b]{2}{*}{ Sample Description } & \multicolumn{3}{|c|}{ Percent Difference ${ }^{a}$} \\
\hline & & & 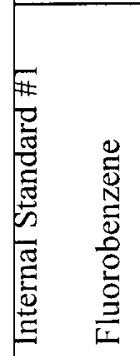 & 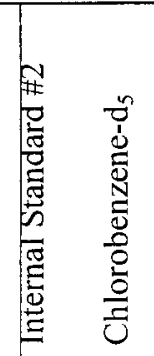 & 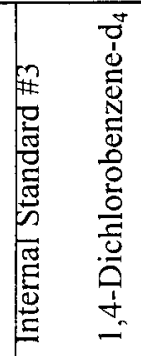 \\
\hline A-3359/A-3360 & 0031-STRT-1 & Tenax Tubes \#1 \& \#2 (Set \#1) & 1.7 & 1.8 & -4.6 \\
\hline A-3362/A-3363 & $0031-S T R T-1$ & Tenax Tubes \#1 \& \#2 (Set \#2) & 3.2 & -4.9 & -14 \\
\hline A-3365/A-3366 & $0031-S T R T-1$ & Tenax Tubes \#1 \& \#2 (Set \#3) & -2.2 & -10 & -19 \\
\hline A-3368/A-3369 & 0031-STRT-1 & Tenax Tubes \#1 \& \#2 (Set \#4) & -6.1 & -16 & -23 \\
\hline A-3379/A-3380 & $0031-$ STRT-2 & Tenax Tubes \#1 \& \#2 (Set \#1) & -6.4 & -12 & -20 \\
\hline A-3382/A-3383 & 0031-STRT-2 & Tenax Tubes \#1 \& \#2 (Set \#2) & -8.2 & -11 & -19 \\
\hline A-3385/A-3386 & $0031-S T R T-2$ & Tenax Tubes \#1 \& \#2 (Set \#3) & -14 & -23 & -32 \\
\hline A-3388/A-3389 & $0031-S T R T-2$ & Tenax Tubes \#1 \& \#2 (Set \#4) & -15 & -22 & -32 \\
\hline A-3409/A-3410 & $0031-\mathrm{END}-1$ & Tenax Tubes \#1 \& \#2 (Set \#1) & -18 & -23 & -32 \\
\hline $\mathrm{A}-3412 / \mathrm{A}-3413$ & $0031-E N D-1$ & Tenax Tubes \#1 \& \#2 (Set \#2) & -15 & -20 & -30 \\
\hline A-3415/A-3416 & 0031-END-1 & Tenax Tubes \#1 \& \#2 (Set \#3) & -6.7 & -8.1 & -15 \\
\hline A-3418/A-3419 & 0031-END-1 & Tenax Tubes \#1 \& \#2 (Set \#4) & -1.8 & -4.3 & -9.1 \\
\hline A-3428/A-3429 & 0031-END-2 & Tenax Tubes \#1 \& \#2 (Set \#1) & -25 & -34 & -37 \\
\hline A-3431/A-3432 & $0031-E N D-2$ & Tenax Tubes \#1 \& \#2 (Set \#2) & -23 & -32 & -39 \\
\hline A-3434/A-3435 & 0031 -END-2 & Tenax Tubes \#1 \& \#2 (Set \#3) & -26 & -37 & -44 \\
\hline A-3437/A-3438 & $0031-E N D-2$ & Tenax Tubes \#1 \& \#2 (Set \#4) & -27 & -38 & -43 \\
\hline A-3392 & $0031-$ STRT-2 & Tenax Tube Pair Field Blank & -22 & -30 & -38 \\
\hline A-3394 & $0031-S T R T-2$ & Tenax Tube Pair Trip Blank & -18 & -30 & -39 \\
\hline A-3441 & 0031-END-1 & Tenax Tube Pair Field Blank & -17 & -15 & -28 \\
\hline A-3361 & 0031-STRT-1 & Anasorb 747 Tube (Set \#1) & -16 & -14 & -24 \\
\hline A-3364 & 0031-STRT-1 & Anasorb 747 Tube (Set \#2) & -28 & -28 & -33 \\
\hline A-3367 & 0031-STRT-1 & Anasorb 747 Tube (Set \#3) & -28 & -31 & -36 \\
\hline A-3370 & 0031-STRT-1 & Anasorb 747 Tube (Set \#4) & -27 & -28 & -28 \\
\hline A-3381 & $0031-S T R T-2$ & Anasorb 747 Tube (Set \#1) & -36 & -42 & -46 \\
\hline A-3384 & $0031-S T R T-2$ & Anasorb 747 Tube (Set \#2) & -25 & -28 & -29 \\
\hline A-3387 & 0031-STRT-2 & Anasorb 747 Tube (Set \#3) & -24 & -26 & -28 \\
\hline A-3390 & 0031-STRT-2 & Anasorb 747 Tube (Set \#4) & -17 & -22 & -25 \\
\hline A-3411 & 0031-END-1 & Anasorb 747 Tube (Set \#1) & -13 & -11 & -17 \\
\hline A-3414 & 0031-END-1 & Anasorb 747 Tube (Set \#2) & -14 & -13 & -21 \\
\hline A-3417 & 0031-END-1 & Anasorb 747 Tube (Set \#3) & -16 & -12 & -21 \\
\hline A- 3420 & 0031-END-1 & Anasorb 747 Tube (Set \#4) & -19 & -12 & -20 \\
\hline
\end{tabular}


Table 7. Volatile Organic Compound (VOC) internal standard recoveries.

\begin{tabular}{|c|c|c|c|c|c|c|c|c|}
\hline \multirow[b]{2}{*}{ Field Sample No. } & \multirow[b]{2}{*}{ Run No. } & \multirow[b]{2}{*}{ Sample Description } & \multicolumn{6}{|c|}{ Percent Difference $^{a}$} \\
\hline & & & 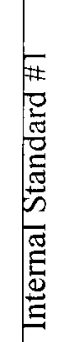 & 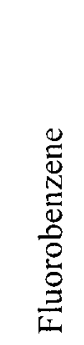 & 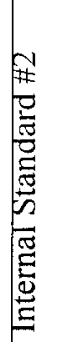 & 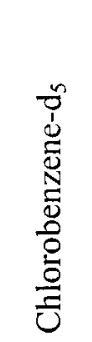 & 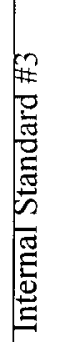 & 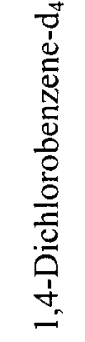 \\
\hline A-3430 & 0031-END-2 & Anasorb 747 Tube (Set \#1) & & -16 & & -25 & & -33 \\
\hline A-3433 & 0031-END-2 & Anasorb 747 Tube (Set \#2) & & -24 & & -29 & & -35 \\
\hline A-3436 & $0031-E N D-2$ & Anasorb 747 Tube (Set \#3) & & -20 & & -30 & & -38 \\
\hline A-3439 & 0031-END-2 & Anasorb 747 Tube (Set \#4) & & -24 & & -39 & & -33 \\
\hline A-3393 & 0031-STRT-2 & Anasorb 747 Field Blank & & -20 & & -28 & & -36 \\
\hline A-3395 & 0031-STRT-2 & Anasorb 747 Trip Blank & & -21 & & -25 & & -31 \\
\hline A-3442 & 0031-END-1 & Anasorb 747 Field Blank & & -19 & & -22 & & -32 \\
\hline A-3371 & 0031-STRT-1 & VOST Condensate & & -9.3 & & -7.0 & & -13 \\
\hline A-3391 & 0031-STRT-2 & VOST Condensate & & -10 & & -8.8 & & -15 \\
\hline A-3421 & 0031-END-1 & VOST Condensate & & -7.2 & & -5.0 & & -9.8 \\
\hline A- 3440 & 0031-END-2 & VOST Condensate & & -8.1 & & -5.6 & & -11 \\
\hline A-3396 & 0031-STRT-2 & VOST D.I. Water Trip Blank & & -12 & & -9.3 & & -15 \\
\hline
\end{tabular}

a. Recoveries of internal standard compounds are not typically calculated for samples analyzed by Method $8260 \mathrm{~B}$ and $8270 \mathrm{C}$. Percent Difference is calculated using the following equation.

$$
\text { Percent Difference }(\% D)=\frac{\text { Observed Value }- \text { Expected Value }}{\text { Expected Value }} \times 100 \%
$$

Where: Observed Value $=$ the area of the internal standard in the sample and

Expected Value $=$ the area of the internal standards in the daily standard 


\subsubsection{VOC Surrogate Recovery Assessment}

Four surrogate compounds were spiked onto all of the VOST samples before the thermal desorption process was initiated. The surrogate recoveries for the NWCF ETS offgas samples are presented in Table 8. Surrogate recoveries are within the targeted acceptance range (percent recovery between 50-150\%), meeting the project DQOs except for sample A-3364. Low surrogate recoveries were obtained for sample A-3364 (The Anasorb $747^{\text {B }}$ tube for Run 1, Set 2) and therefore the data for this sample are not usable. The results for Sample A-3364 indicate normal recoveries for the internal standard compounds, but very low recoveries for the surrogate compounds.

Table 8. Volatile Organic Compound (VOC) surrogate compound recoveries.

\begin{tabular}{|c|c|c|c|c|c|c|}
\hline \multirow[b]{2}{*}{ Field Sample No. } & \multirow[b]{2}{*}{ Run No. } & \multirow[b]{2}{*}{ Sample Description } & \multicolumn{4}{|c|}{ Percent Recovery ${ }^{\mathrm{a}}$} \\
\hline & & & 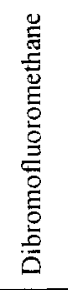 & 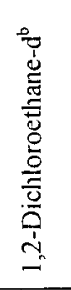 & 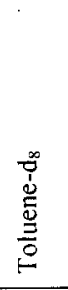 & 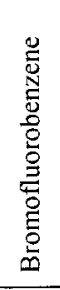 \\
\hline A-3359/A-3360 & 0031-STRT-1 & Tenax Tubes \#1 \& \#2 (Set \#1) & 84 & 81 & 101 & 83 \\
\hline$A-3362 / A-3363$ & 0031-STRT-1 & Tenax Tubes \#1 \& \#2 (Set \#2) & 90 & 85 & 114 & 99 \\
\hline A-3365/A-3366 & 0031-STRT-1 & Tenax Tubes \#1 \& \#2 (Set \#3) & 88 & 84 & 113 & 98 \\
\hline$A-3368 / A-3369$ & 003I-STRT-1 & Tenax Tubes \#1 \& \#2 (Set \#4) & 85 & 80 & 115 & 92 \\
\hline A-3379/A-3380 & 0031-STRT-2 & Tenax Tubes \#1 \& \#2 (Set \#1) & 88 & 83 & 111 & 95 \\
\hline A-3382/A-3383 & 0031-STRT-2 & Tenax Tubes \#1 \& \#2 (Set \#2) & 91 & 86 & 110 & 97 \\
\hline $\mathrm{A}-3385 / \mathrm{A}-3386$ & 003I-STRT-2 & Tenax Tubes \#1 \& \#2 (Set \#3) & 81 & 75 & 108 & 85 \\
\hline A-3388/A-3389 & 0031-STRT-2 & Tenax Tubes \#1 \& \#2 (Set \#4) & 87 & 80 & 114 & 90 \\
\hline $\mathrm{A}-3409 / \mathrm{A}-3410$ & $0031-E N D-1$ & Tenax Tubes \#1 \& \#2 (Set \#1) & 84 & 79 & 109 & 85 \\
\hline $\mathrm{A}-3412 / \mathrm{A}-3413$ & 0031-END-1 & Tenax Tubes \#1 \& \#2 (Set \#2) & 85 & 80 & 109 & 91 \\
\hline A-3415/A-3416 & 0031-END-1 & Tenax Tubes \#1 \& \#2 (Set\#3) & 83 & 78 & 101 & 82 \\
\hline$A-3418 / A-3419$ & 0031-END-1 & Tenax Tubes \#1 \& \#2 (Set \#4) & 87 & 86 & 103 & 80 \\
\hline A-3428/A-3429 & $0031-E N D-2$ & Tenax Tubes \#1 \& \#2 (Set \#1) & 87 & 79 & 116 & 87 \\
\hline A-3431/A-3432 & $0031-E N D-2$ & Tenax Tubes \#1 \& \#2 (Set \#2) & 79 & 74 & 105 & 86 \\
\hline A-3434/A-3435 & $0031-E N D-2$ & Tenax Tubes \#1 \& \#2 (Set \#3) & 83 & 77 & 117 & 90 \\
\hline A-3437/A-3438 & 0031-END-2 & Tenax Tubes \#1 \& \#2 (Set \#4) & 90 & 82 & 121 & 92 \\
\hline$A-3392$ & $0031-S T R T-2$ & Tenax Tube Pair Field Blank & 76 & 70 & 102 & 86 \\
\hline$A-3394$ & 003l-STRT-2 & Tenax Tube Pair Trip Blank & 58 & $49^{1}$ & 60 & $41^{1}$ \\
\hline A-3441 & $0031-E N D-1$ & Tenax Tube Pair Field Blank & 82 & 77 & 95 & 86 \\
\hline A-3361 & 0031-STRT-1 & Anasorb 747 Tube (Set \#1) & 83 & 79 & 94 & 77 \\
\hline A-3364 & 0031 -STRT-1 & Anasorb 747 Tube (Set \#2) & $7.9^{1}$ & $6.4^{1}$ & $2.9^{1}$ & $3.6^{1}$ \\
\hline A-3367 & $0031-S T R T-1$ & Anasorb 747 Tube (Set \#3) & 106 & 100 & 121 & 92 \\
\hline$A-3370$ & 0031-STRT-1 & Anasorb 747 Tube (Set \#4) & 86 & 83 & 99 & 82 \\
\hline A-3381 & 0031-STRT-2 & Anasorb 747 Tube (Set \#1) & 96 & 88 & 117 & 86 \\
\hline A-3384 & 0031-STRT-2 & Anasorb 747 Tube (Set \#2) & 73 & 71 & 90 & 74 \\
\hline
\end{tabular}


Table 8. Volatile Organic Compound (VOC) surrogate compound recoveries.

\begin{tabular}{|c|c|c|c|c|c|c|}
\hline \multirow[b]{2}{*}{ Field Sample No. } & \multirow[b]{2}{*}{ Run No. } & \multirow[b]{2}{*}{ Sample Description } & \multicolumn{4}{|c|}{ Percent Recovery ${ }^{\mathrm{a}}$} \\
\hline & & & 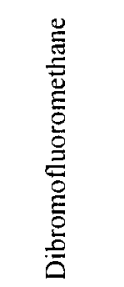 & 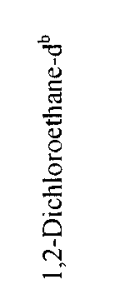 & 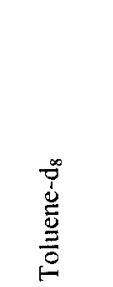 & 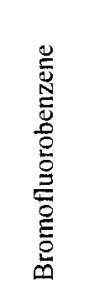 \\
\hline A-3387 & 003 I-STRT-2 & Anasorb 747 Tube (Set \#3) & 77 & 73 & 92 & 74 \\
\hline A-3390 & 0031-STRT-2 & Anasorb 747 Tube (Set \#4) & 73 & 68 & 96 & 79 \\
\hline A-3411 & 0031-END-1 & Anasorb 747 Tube (Set \#1) & 87 & 84 & 101 & 84 \\
\hline A-3414 & 0031-END-1 & Anasorb 747 Tube (Set \#2) & 91 & 86 & 107 & 86 \\
\hline$A-3417$ & 0031-END-1 & Anasorb 747 Tube (Set \#3) & 91 & 88 & 104 & 88 \\
\hline A-3420 & 0031-END-1 & Anasorb 747 Tube (Set \#4) & 78 & 75 & 96 & 81 \\
\hline A-3430 & 0031-END-2 & Anasorb 747 Tube (Set \#1) & 83 & 76 & 105 & 88 \\
\hline A-3433 & 0031-END-2 & Anasorb 747 Tube (Set \#2) & 88 & 79 & 110 & 82 \\
\hline A-3436 & $0031-E N D-2$ & Anasorb 747 Tube (Set \#3) & 82 & 75 & 108 & 85 \\
\hline A-3439 & 0031-END-2 & Anasorb 747 Tube (Set \#4) & 85 & 79 & 110 & 87 \\
\hline A-3393 & 0031-STRT-2 & Anasorb 747 Field Blank & 77 & 68 & 99 & 73 \\
\hline A-3395 & 0031-STRT-2 & Anasorb 747 Trip Blank & 82 & 74 & 92 & 75 \\
\hline A-3442 & 0031-END-1 & Anasorb 747 Field Blank & 83 & 77 & 98 & 81 \\
\hline$A-3371$ & 0031-STRT-1 & VOST Condensate & 102 & 104 & 106 & 106 \\
\hline A-3391 & 0031-STRT-2 & VOST Condensate & 100 & 103 & 107 & 106 \\
\hline A-3421 & $0031-E N D-1$ & VOST Condensate & 101 & 101 & 106 & 105 \\
\hline A-3440 & 0031-END-2 & VOST Condensate & 100 & 103 & 106 & 105 \\
\hline A-3396 & 0031-STRT-2 & VOST D.I. Water Trip Blank & 102 & 107 & 107 & 106 \\
\hline \multicolumn{3}{|c|}{ Laboratory Target Recovery Range for Tenax ${ }^{(\mathbb{B}}$ \& Anasorb $747^{(\mathbb{B})}$ Tubes: } & $50-150$ & $50-150$ & $50-150$ & $50-150$ \\
\hline \multicolumn{3}{|c|}{ Laboratory Target Recovery Range for Aqueous Samples: } & $80-120$ & $80-120$ & $80-120$ & $72-135$ \\
\hline \multicolumn{7}{|c|}{$\begin{array}{l}\text { a. Percent Recovery is calculated using the following equation: } \\
\qquad \text { Percent Re covery }(\% R)=\frac{\text { Observed Value }}{\text { Expected Value }} \times 100 \%\end{array}$} \\
\hline $\begin{array}{r}\text { Where: Observ } \\
\text { Expect } \\
\text { b. This percent rec }\end{array}$ & $\begin{array}{l}\text { Value }=\text { the meas } \\
\text { Value }=\text { the mass } \\
\text { very is outside of } t\end{array}$ & $\begin{array}{l}\text { Ired mass of the surrogate standard in the s } \\
\text { of the surrogate standard spiked into the sa } \\
\text { laboratory target recovery range. }\end{array}$ & $\begin{array}{l}\text { ample and } \\
\text { mple. }\end{array}$ & & & \\
\hline
\end{tabular}




\subsubsection{VOC Analytical Data Quality Assessment}

The sampling and analytical objectives expected for this data set were to present an acceptable characterization of the project target volatile organic compounds from the NWCF ETS offgas. The data quality indicators collectively indicate that the sampling and analytical processes for the SMVOC samples were in control during the sampling runs. Data have been collected and reviewed that allow the relative precision and accuracy to be measured for the target analytes. The data quality indicators indicate that most of the data are of acceptable quality, and that sufficient data has been obtained to characterize the project target volatile organic compounds from the NWCF ETS offgas.

There were several indications that fugitive emissions may have been present during sampling. Acetone, methylene chloride and toluene were present in at least some of the field and trip blanks. Some inconsistency of the methylene chloride results has been discussed. Toluene exhibited results that simply do not make sense, particularly with respect to higher levels of toluene that were detected in some of the back half samples. Results for these particular three constituents are not considered to demonstrate that these constituents are truly in the ETS offgas at these concentrations. These are the major classical environmental laboratory sample preparation solvents that are often detected as contaminants in sample results.

The only serious quality control deficiency was the low surrogate recovery for the Tenax ${ }^{\otimes} /$ Anasorb $747^{\circledR}$ sample for Run 1 (Set 2). This deficiency is adequately handled by the substitution of average backhalf results from the other Run 1 tube sets.

\subsection{Semi-volatile Organic Compounds}

A standard U.S. EPA Method 0010 (Modified Method 5, or MM-5) sampling train configuration was used to collect samples of the NWCF ETS offgas for the assessment of semi-volatile organic compounds (SVOCs). A total nominal volume of $3.0 \mathrm{dscm}$ of offgas was sampled in each run over 3-4 hours. The Method 0010 SVOC train configuration is comprised of six fractions:

- particulate filter

- solvent rinse of the front half of the filter holder, the sampling probe and the nozzle

- $\mathrm{XAD}-2^{\circledR}$ resin tube,

- solvent rinse of the back half of the filter holder, the coil condenser and connecting glassware,

- composite sample containing the stack gas condensate and impinger contents, and

- impinger and connecting glassware solvent rinses.

A trip/reagent blank was collected and a set of blank train (field blank) samples were analyzed to assess extraneous sources of contamination available to these samples. 


\subsubsection{SVOC Target Analyte List}

Analyses of SVOC samples were completed per SW-846 Methods 3542 and $8270 \mathrm{C}$ by first extracting the samples with methylene chloride, then analyzing the extracts using GC/MS. The SVOC target analytes are listed in Table 9.

Table 9. SVOC target analyte list.

\begin{tabular}{|c|c|}
\hline Analyte & CAS Registry Number \\
\hline Acenaphthene & $83-32-9$ \\
\hline Acenaphthylene & $208-96-8$ \\
\hline Acetophenone & $98-86-2$ \\
\hline Aniline & $62-53-3$ \\
\hline Anthracene & $120-12-7$ \\
\hline Benzidine & $92-87-5$ \\
\hline Benzoic acid & $65-85-0$ \\
\hline Benzo(a)anthracene & $56-55-3$ \\
\hline Benzo(a)pyrene & $50-32-8$ \\
\hline Benzo(b)fluoranthene & $205-99-2$ \\
\hline Benzo(ghi)perylene & $191-24-2$ \\
\hline Benzo $(k)$ fluoranthene & $207-08-9$ \\
\hline Benzyl alcohol & $100-51-6$ \\
\hline bis(2-Chloroethoxy)methane & $111-91-1$ \\
\hline bis(2-Chloroethyl)ether & $111-44-4$ \\
\hline bis(2-Ethylhexyl)phthalate & $117-81-7$ \\
\hline 4-Bromophenyl-phenylether & $101-55-3$ \\
\hline Butylbenzylphthalate & $85-68-7$ \\
\hline Carbazole & $86-74-8$ \\
\hline 4-Chloro-3-methylphenol & $59-50-7$ \\
\hline 4-Chloroaniline & $106-47-8$ \\
\hline 2-Chloronaphthalene & $91-58-7$ \\
\hline 2-Chlorophenol & $95-57-8$ \\
\hline 4-Chlorophenyl phenyl ether & $7005-72-36$ \\
\hline Chrysene & $218-01-9$ \\
\hline Di-n-butylphthalate & $84-74-2$ \\
\hline Di-n-octylphthalate & $117-84-0$ \\
\hline Dibenz(a,h)anthracene & $53-70-3$ \\
\hline Dibenzofuran & $132-64-9$ \\
\hline 1,2-Dichlorobenzene & $95-50-1$ \\
\hline 1,3-Dichlorobenzene & $541-73-1$ \\
\hline 1,4-Dichlorobenzene & $106-46-7$ \\
\hline 3,3'-Dichlorobenzidine & $91-94-1$ \\
\hline 2,4-Dichlorophenol & $120-83-2$ \\
\hline Diethylphthalate & $84-66-2$ \\
\hline Dimethyl phthalate & $131-11-3$ \\
\hline 2,4-Dimethylphenol & $105-67-9$ \\
\hline
\end{tabular}


Table 9. SVOC target analyte list.

\begin{tabular}{|c|c|}
\hline Analyte & CAS Registry Number \\
\hline 4,6-Dinitro-2-methylphenol & $534-52-1$ \\
\hline 2,4-Dinitrophenol & $51-28-5$ \\
\hline 2,4-Dinitrotoluene & $121-14-2$ \\
\hline 2,6-Dinitrotoluene & $606-20-2$ \\
\hline 1,2-Diphenylhydrazine & $122-66-7$ \\
\hline Fluoranthene & $206-44-0$ \\
\hline Fluorene & $86-73-7$ \\
\hline Hexachlorocyclopentadiene & $77-47-4$ \\
\hline Hexachiorobenzene & $118-74-1$ \\
\hline Hexachlorobutadiene & $87-68-3$ \\
\hline Hexachloroethane & $67-72-1$ \\
\hline Indeno(1,2,3-cd)pyrene & $193-39-5$ \\
\hline Isophorone & $78-59-1$ \\
\hline 2-Methylnaphthalene & $91-57-6$ \\
\hline 2-Methylphenol & $95-48-7$ \\
\hline 3-Methylphenol \& 4-Methylphenol & $65794-96-9$ \\
\hline N-Nitroso-di-n-propylamine & $621-64-7$ \\
\hline N-Nitrosodimethylamine & $62-75-9$ \\
\hline N-Nitrosodiphenylamine & $86-30-6$ \\
\hline Naphthalene & $91-20-3$ \\
\hline 2-Nitroaniline & $88-74-4$ \\
\hline 3-Nitroaniline & $99-09-2$ \\
\hline 4-Nitroaniline & $100-01-6$ \\
\hline Nitrobenzene & $98-95-1$ \\
\hline 2-Nitrophenol & $88-75-5$ \\
\hline 4-Nitrophenol & $100-02-7$ \\
\hline 2,2'-Oxybis(1-chloropropane) & $108-60-1$ \\
\hline Pentachlorobenzene & $608-93-5$ \\
\hline Pentachloronitrobenzene & $82-68-8$ \\
\hline Pentachlorophenol & $87-86-5$ \\
\hline Phenanthrene & $85-01-8$ \\
\hline Phenol & $108-95-2$ \\
\hline Pyrene & $129-00-0$ \\
\hline Pyridine & $110-86-1$ \\
\hline 1,2,4-Trichlorobenzene & $120-82-1$ \\
\hline 2,4,5-Trichlorophenol & $95-95-4$ \\
\hline 2,4,6-Trichlorophenol & $88-06-2$ \\
\hline 1,2,4,5-Tetrachlorobenzene & $95-94-3$ \\
\hline
\end{tabular}


The samples were also analyzed for non-target organic compounds as directed in the QAPjP. A GC/MS library search was performed on each SVOC sample (including the condensate samples) for TICs (tentatively identified compounds). The search was performed for the thirty largest non-target compounds that exhibited a response greater than $10 \%$ of the response of the nearest internal standard (the extract is spiked at $20 \mu \mathrm{g} / \mathrm{mL}$ ). The standard extract volume was $1.0 \mathrm{~mL}$; hence, the TICs were reported down to a level of $2 \mu \mathrm{g}$ when the original extract was not diluted (dilution factor or DF $=1$ ). The backhalf composite sample extracts for Runs 1 through 4 were analyzed at a five-fold dilution $(\mathrm{DF}=5)$; therefore, TICs in these fractions were only reported down to $10 \mu \mathrm{g}$.

The library search was conducted against the National Bureau of Standards library of mass spectral data containing an estimated 75,000 compounds. The matching criteria included a nominal $85 \%$ match of the mass spectral features, and analyst discretion of all identities reported. TICs that were derived from column bleed, surrogate addition, or aldol condensation were excluded from the report. Also, compounds that were reported as SMVOC target compounds were not reported as semi-volatile TICs because the SMVOC method provides more reliable data for these compounds.

\subsubsection{SVOC Analytical Results}

The particulate filter was combined with its associated solvent rinses to form a "front-half" composite sample. The XAD-2 resin tube was combined with its associated solvent rinses to form a "back-half" composite sample. The stack gas condensate, impinger contents and associated glassware rinses were also combined to form a composite sample. These three fractions are analyzed separately. Unique data quality control indicators are used for each fraction.

Tabulated data summaries that present the SVOC data are given in Appendix A. These tables have been extracted from the STL Analytical Laboratory Final Report for this project. For each Method 0010 offgas sampling run, the "SVOC Run Total" for each analyte (in mass units of $\mu \mathrm{g}$ ) represents the sum of the amounts found in all of the SVOC fractions collected during that run.

\subsubsection{SVOC Data Quality Assessment}

The sample fractions were sent to STL via overnight express mail to ensure that sample preservation and analysis schedules required by the QAPJP would be met. All of the samples were received by the laboratory in good condition. Sample extractions were performed within the requirements specified in the QAPjP.

On the basis of all the quality assurance indicators, all of the semi-volatile organic compound data obtained from the SVOC runs are usable and representative of the NWCF ETS offgas contents. The only deficiencies in accuracy and precision indicated by the laboratory control samples (LCS) and matrix spike samples appear to be unrelated to sample data quality.

Blank sample data indicate that sources of fugitive contamination available to the NWCF ETS offgas samples were minimized. Only phthalate esters were found in significant concentrations in the blanks. Although the back half composite containing the XAD- ${ }^{\circledR}$ sample extracts were somewhat hostile to the internal standard compounds, the analysis of these extracts at two levels of dilution appears to provide a reliable assessment of the offgas contents.

Recoveries of the surrogates indicate that the preparation and analysis processes during the SVOC sample determinations were in control with respect to all of the analytes for the offgas sample analyses. The surrogate recoveries are within the prescribed acceptance ranges and do not indicate any bias to the data. Sample dilution was required to achieve acceptable recovery of three of the six internal standards, 
and this had the affect of increasing detection limits for those analytes that are correlated to the recovery of these standards.

\subsubsection{SVOC Blank Data Assessment}

A standard SVOC trip/reagent blank (unused, sealed XAD-2® resin tube) and a blank train run samples was collected to assess potential fugitive contamination sources in the sampling environment. Review of the SVOC blank indicates that very little contamination due to fugitive emissions exists in the samples as a result of storage or transport of the sample collection media. The trip blank data exhibited low levels of acetophenone and 1,4-dichlorobenzene that are below the standard laboratory reporting limit (RL). Several tentatively identified compounds were identified in the trip blank data. Notably, benzaldehyde, methyl benzoate, and ethyl benzaldehyde were found, along with several miscellaneous hydrocarbons. These compounds were probably artifacts of the XAD-2® medium or the transport and storage of the samples, and were not found in the laboratory method blanks.

Review of the SVOC blank train results indicates that little contamination of the samples occurred as a result of sample handling or contact with the MM-5 sampling train components. Acetophenone and 1,4-dichlorbenzene were found at low levels that were similar to the trip blank, and may have originated in the sampling media, or were possibly introduced to the media during transport and storage of the samples. The target analytes found in the blank train samples also included di-n-butylphthalate, bis-2ethylhexylphthalate, and di-n-octylphthalate. The phthalate esters are considered common laboratory contaminants, and are commonly found in certain plastics and plastic tubing. These compounds were not found in the laboratory method blanks, so their origin appears to be with the sampling process. The TICs found in the back-half composite sample of the blank train were similar in identity and concentration to those found in the trip/reagent blank. Benzaldehyde, methyl benzoate, ethyl benzaldehyde, and several miscellaneous hydrocarbons were observed at levels that were similar to the trip/reagent blank results.

The origin of these contaminants may have been either the sampling media or the transport and storage of the samples. The front-half and impinger composite samples exhibited low concentrations of some additional TICs that are not found in the back-half composite samples.

\subsubsection{SVOC Internal Standard Recovery Assessment}

Internal standards are used as the basis for calculation of the concentrations of the target analytes and surrogates. Six IS compounds were spiked into all of the sample extracts prior to analysis. Method SW-8270 required limits, in terms of percent difference relative to the IS area for the lab's daily standard, are $-50 \%$ to $+100 \%$. The internal standard responses for the front-half composite sample extracts, and the condensate and impinger contents composite sample extracts were acceptable and do not indicate any deficiency in data quality. Also, the quality assurance samples exhibit acceptable recoveries of the internal standards. The only cases of significantly reduced recovery of the internal standards are noted for the back-half fractions of the offgas samples which include the XAD-2® resin. A summary of the semivolatile internal standard performance is given in Table 10 . 
Table 10. SVOC train sample internal standard compound recoveries.

\begin{tabular}{|c|c|c|c|c|c|c|c|c|c|}
\hline \multirow[b]{2}{*}{ Field Sample No. } & \multirow[b]{2}{*}{$\begin{array}{l}\text { NWCF ETS } \\
\text { Sample ID }\end{array}$} & \multirow[b]{2}{*}{ Sample Description } & \multirow[b]{2}{*}{ 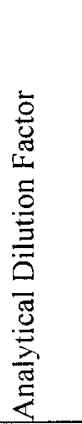 } & \multicolumn{6}{|c|}{ Percent Difference $(\%)^{\mathrm{a}}$} \\
\hline & & & & 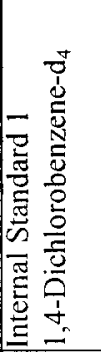 & 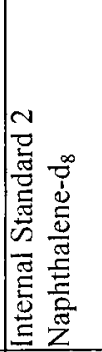 & 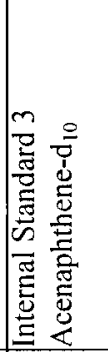 & 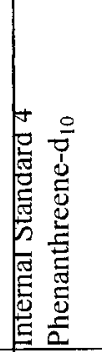 & 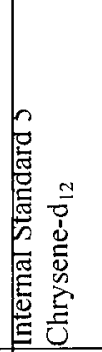 & $\begin{array}{l}\frac{a}{0} \\
\frac{d}{2} \\
\frac{2}{2} \\
0 \\
0\end{array}$ \\
\hline A-3353/A-3354 & $0010-S T R T-1$ & $\begin{array}{l}\text { Particulate Filter/Front Half of the Filter } \\
\text { Holder and Probe Solvent Rinses }\end{array}$ & 1 & 9.2 & 7.5 & -5.6 & -16 & -27 & -22 \\
\hline \multirow[t]{2}{*}{ A-3355/A-3356 } & \multirow[t]{2}{*}{ 0010-STRT-1 } & \multirow{2}{*}{$\begin{array}{l}\text { XAD-2 Resin Tube/Back Half of the } \\
\text { Filter Holder and Coil Condenser } \\
\text { Solvent Rinses }\end{array}$} & 5 & -28 & -29 & $-61^{b}$ & -38 & -57 & $-100^{b}$ \\
\hline & & & 100 & 7.0 & 3.2 & -1.6 & .0 .62 & 3.5 & -50 \\
\hline A-3357/A-3358 & $0010-S T R T-1$ & $\begin{array}{l}\text { Condensate, Impinger Contents, and } \\
\text { Glassware Solvent Rinses }\end{array}$ & 1 & 13 & 12 & 0.16 & -4.4 & -20 & -16 \\
\hline A-3372/A-3373 & 0010-STRT-2 & $\begin{array}{l}\text { Particulate Filter/Front Half of the Filter } \\
\text { Holder and Probe Solvent Rinses }\end{array}$ & 1 & 12 & 6.9 & -1.1 & -7.0 & -24 & -20 \\
\hline $\mathrm{A}-3374 / \mathrm{A}-3375$ & $0010-$ STRT-2 & $\begin{array}{l}\text { XAD-2 Resin Tube/Back Half of the } \\
\text { Filter Holder and Coil Condenser } \\
\text { Solvent Rinses }\end{array}$ & $\begin{array}{c}5 \\
100\end{array}$ & $\begin{array}{l}2.4 \\
2.4\end{array}$ & $\begin{array}{c}-5.2 \\
1.9\end{array}$ & $\begin{array}{l}-25 \\
-1.2\end{array}$ & $\begin{array}{l}-14 \\
-3.2\end{array}$ & $\begin{array}{l}-27 \\
-1.0\end{array}$ & $\begin{array}{l}-100^{b} \\
-24\end{array}$ \\
\hline$A-3376 / A-3377$ & $0010-S T R T-2$ & $\begin{array}{l}\text { Condensate, Impinger Contents, and } \\
\text { Glassware Solvent Rinses }\end{array}$ & 1 & -15 & -18 & -16 & -16 & -26 & -24 \\
\hline A-3397/A-3398 & 0010-STRT-2 & $\begin{array}{l}\text { Blank Train Particulate Filter/Front } \\
\text { Half of the Filter Holder and Probe } \\
\text { Solvent Rinses }\end{array}$ & 1 & 5.3 & 2.4 & -5.8 & -11 & -24 & -22 \\
\hline A-3399/A-3400 & 0010-STRT-2 & $\begin{array}{l}\text { Blank Train XAD-2 Resin Tube/Back } \\
\text { Half of the Filter Holder and Coil } \\
\text { Condenser Solvent Rinses }\end{array}$ & 1 & -0.69 & -7.8 & -18 & -21 & -34 & -27 \\
\hline $\mathrm{A}-3401 / \mathrm{A}-3402$ & 0010 -STRT-2 & $\begin{array}{l}\text { Blank Train Condensate, Impinger } \\
\text { Contents, and Glassware Solvent Rinses }\end{array}$ & 1 & 7.2 & 9.3 & -1.9 & -9.8 & -22 & -18 \\
\hline A-3403/A-3404 & 0010 -END-1 & $\begin{array}{l}\text { Particulate Filter/Front Half of the Filter } \\
\text { Holder and Probe Solvent Rinses }\end{array}$ & 1 & 7.6 & 9.6 & 0.92 & -13 & -26 & -20 \\
\hline A-3405/A-3406 & 0010-END-1 & $\begin{array}{l}\text { XAD-2 Resin Tube/Back Half of the } \\
\text { Filter Holder and Coil Condenser } \\
\text { Solvent Rinses }\end{array}$ & $\begin{array}{c}5 \\
100\end{array}$ & $\begin{array}{c}-6.6 \\
1.7\end{array}$ & $\begin{array}{l}-5.7 \\
1.8\end{array}$ & $\begin{array}{l}-37 \\
1.4\end{array}$ & $\begin{array}{l}-14 \\
-2.4\end{array}$ & $\begin{array}{l}-30 \\
3.1\end{array}$ & $\begin{array}{l}-100^{b} \\
-65^{b}\end{array}$ \\
\hline $\mathrm{A}-3407 / \mathrm{A}-3408$ & 0010-END-1 & $\begin{array}{l}\text { Condensate, Impinger Contents, and } \\
\text { Glassware Solvent Rinses }\end{array}$ & 1 & 0.38 & 4.2 & -2.7 & -8.3 & -22 & -20 \\
\hline A-3422/A-3423 & $0010-E N D-2$ & $\begin{array}{l}\text { Particulate Filter/Front Half of the Filter } \\
\text { Holder and Probe Solvent Rinses }\end{array}$ & 1 & 5.7 & 4.1 & -2.2 & -13 & -28 & -29 \\
\hline A-3424/A-3425 & 0010-END-2 & $\begin{array}{l}\text { XAD-2 Resin Tube/Back Half of the } \\
\text { Filter Holder and Coil Condenser } \\
\text { Solvent Rinses }\end{array}$ & $\begin{array}{c}5 \\
100\end{array}$ & $\begin{array}{c}14 \\
-2.9\end{array}$ & $\begin{array}{l}9.9 \\
-2.0\end{array}$ & $\begin{array}{l}-28 \\
-6.5\end{array}$ & $\begin{array}{l}0.05 \\
-6.0\end{array}$ & $\begin{array}{l}-19 \\
-3.7\end{array}$ & $\begin{array}{l}-100^{\mathrm{b}} \\
-66^{\mathrm{b}}\end{array}$ \\
\hline$A-3426 / A-3427$ & $0010-$ END-2 & $\begin{array}{l}\text { Condensate, Impinger Contents, and } \\
\text { Glassware Solvent Rinses }\end{array}$ & 1 & 1.8 & 4.2 & -3.1 & -8.7 & -20 & -16 \\
\hline \multirow[t]{2}{*}{ A-3378 } & $0010-S T R T-2$ & XAD-2 Resin Tube Trip/Reagent Blank & 1 & -14 & -15 & -22 & -20 & -29 & -27 \\
\hline & \multicolumn{3}{|c|}{$\begin{array}{l}\text { Internal Standard Laboratory Percent } \\
\text { Difference Acceptance Limits: }\end{array}$} & $\sum_{i}^{9} 8$ & $\stackrel{9}{9}$ & $\stackrel{8}{8} 8$ & $\begin{array}{l}9 \\
0 \\
?\end{array}$ & $\sum_{i}^{8} 8$ & $\stackrel{9}{0} 8$ \\
\hline
\end{tabular}


Table 10. SVOC train sample internal standard compound recoveries.

\begin{tabular}{|c|c|c|c|c|c|c|c|c|c|}
\hline & & & & & Per & ent Diff & rence ( & $\%)^{\mathrm{a}}$ & \\
\hline Field Sample No. & $\begin{array}{c}\text { NWCF ETS } \\
\text { Sample ID }\end{array}$ & Sample Description & 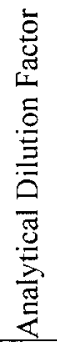 & 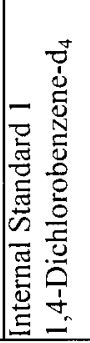 & 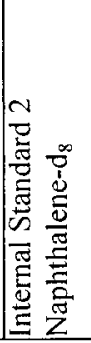 & 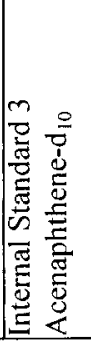 & 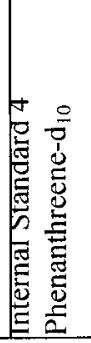 & 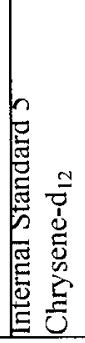 & 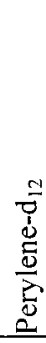 \\
\hline
\end{tabular}

a. Recoveries of internal standards are not typically calculated for samples analyzed by Method $8260 \mathrm{~B}$ and $8270 \mathrm{C}$, and internal standard recoveries are evaluated as Percent Difference from the daily standard. Percent Difference is calculated using the following equation:

$$
\text { Percent Difference }(\% D)=\frac{\text { Observed Value }- \text { Expected Value }}{\text { Expected Value }} \times 100 \%
$$

Where: Observed Value $=$ the area of the internal standard in the sample and

Expected Value $=$ the area of the internal standard in the daily standard

b. This value is outside of the laboratory and project target acceptance range.

The sample extracts of the back-half fractions of the offgas sample trains were analyzed at a dilution factor of five since analysis of the extracts without dilution gave evidence of loss of several of the internal standards. The internal standard perylene-d 12 did not recover from the extract analyses at a dilution factor of five. There was insufficient recovery of this internal standard to allow quantification of the related target analytes without applying further dilution. The internal standard compounds acenaphthene-d 10 and chrysene-d 12 also exhibited recoveries that are lower than the target acceptance criteria in the 1:5 analysis of the extract for the back-half fraction extract for Run 1. However, there was sufficient recovery of each of these internal standards to provide useful results for the related target analytes.

A second extract analysis was performed for each of the offgas back-half composite samples at a dilution factor of 100 . The back-half composite sample extracts exhibit reduced recovery of perylene-d12 at the increased dilution factor of 100 , but there was sufficient recovery of perylene-d 12 to quantify the seven target analytes that are calculated relative to it. The results based on perylene-d12 for the 1:100 dilution of the extracts are usable.

\subsubsection{SVOC Surrogate Recovery Assessment}

Six surrogate compounds were spiked onto all of the SVOC samples before extraction per SW-846 Method $8270 \mathrm{C}$. Three of the surrogates are base/neutral compounds, and three of the surrogates are acid extractable. All of the three acid extractable surrogates are phenols, which are a class of organic compounds that contain a benzene ring with the hydroxyl group attached. A sampling surrogate compound was also applied to the XAD- ${ }^{\circledR}$ resin tubes at the laboratory before sampling. This additional surrogate provides a measurement of the efficiency of the entire process, from sampling to the conclusion of the analysis. The sampling surrogate applied to the XAD- ${ }^{\circledast}$ tubes used to collect samples was ${ }^{13} \mathrm{C}_{3}$ naphthalene. This is a base-neutral compound that is distinguished from the native naphthalene by carbon-13 labeling. A summary of the semi-volatile surrogate performance is given in Table 11 . 
Table 11. SVOC surrogate compound recoveries.

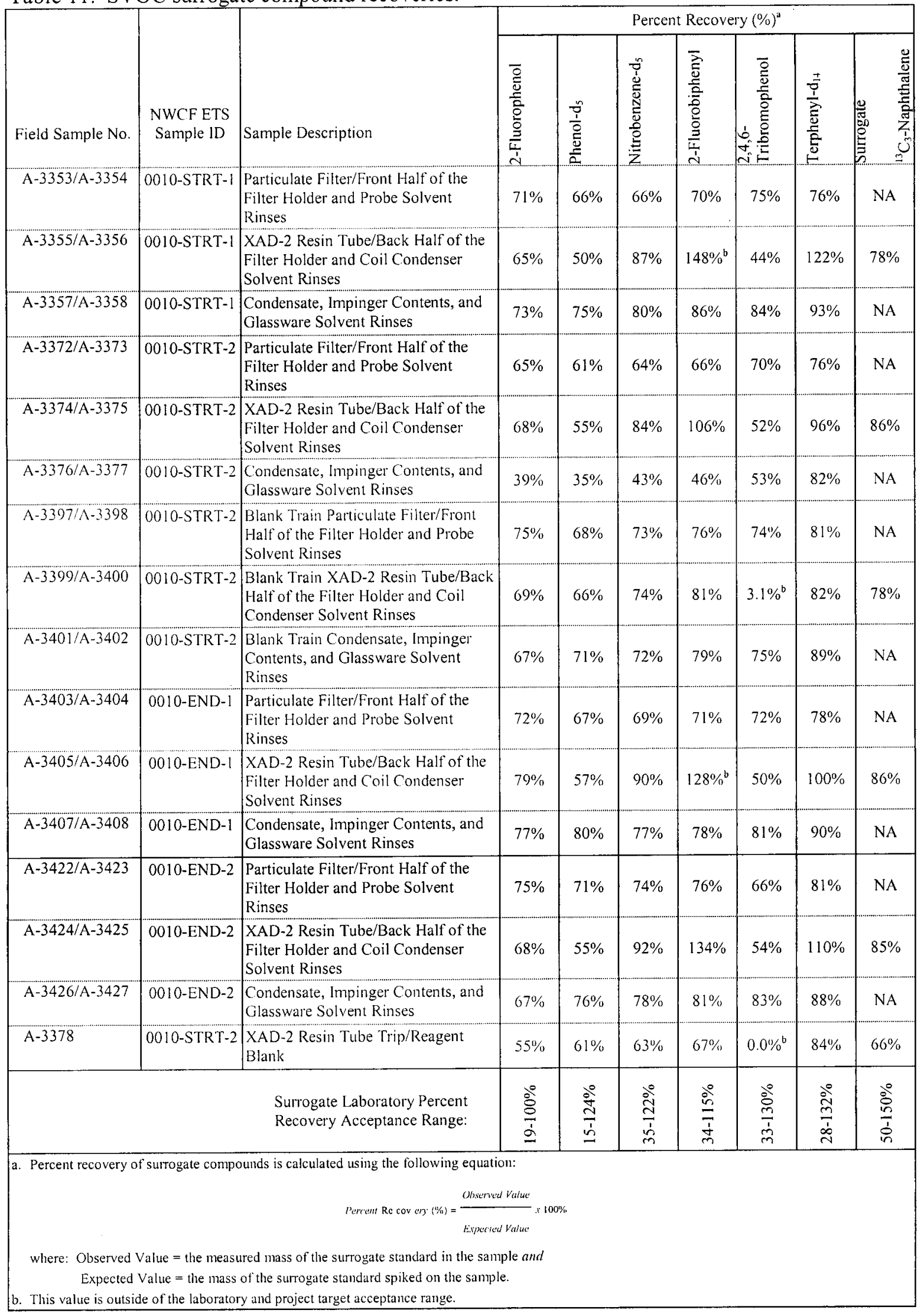


The laboratory surrogate recoveries that were obtained for the front-half composite samples are generally excellent. There are no obvious problems with the analysis of this matrix, which includes the particulate filter and solvent rinses of the front-half of the filter holder and the probe. All of the laboratory surrogate percent recovery values for the front-half samples were all within the target acceptance range. There were no significant differences between the acid surrogate performance for Runs 1 through 4 and the blank train.

The back-half samples exhibited good recoveries for both the base-neutral and the acid extractable surrogates. 2-Fluorobiphenyl recovery from the back-half fractions of Runs 1, 3 and 4 were above the target acceptance range for surrogate recovery, while the percent recovery of 2-fluorobiphenyl for Run 2 was near the upper end of the target acceptance range. The percent recovery of 2-fluorobiphenyl for both the blank train and the XAD- $2^{\circledR}$ resin tube trip/reagent blank was near the center of the target acceptance range. Reduced recovery of the related internal standard, acenapthylene- $d_{10}$ in the offgas samples appears to cause the observed high recoveries of 2-fluorobiphenyl. A high bias to the results for all of the target analytes that are calculated against acenaphthylene- $\mathrm{d}_{10}$ appears to be indicated by the increased recovery of 2-fluorobiphenyl. Inspection of the data shows that there are no positive results based on acenaphthylene- $\mathrm{d}_{10}$. Therefore, the detection limits are defensible and there is no adverse impact on data quality.

Phenol- $\mathrm{d}_{5}$ and 2-fluorophenol exhibited acceptable recovery in the blank train back-half sample and the XAD-2 trip/reagent blank, but 2,4,6-tribromophenol did not recover well. The 2,4,6-tribromophenol results were much lower than the target acceptance limits, with no recovery at all from the trip/reagent blank sample. These results were atypical of the data set in that the 2,4,6-tribromophenol recoveries in the offgas samples were well within the target acceptance range for all four runs. The associated laboratory method blank exhibited a good recovery of the 2,4,6-tribromophenol, but the laboratory control sample (LCS) and the laboratory control sample duplicate (LCSD) each exhibited a low recovery of this surrogate. Also, the LCS have low recoveries of 4-nitrophenol and pentachlorophenol, which are acidic compounds potentially sensitive to $\mathrm{pH}$ affects. There was no control of $\mathrm{pH}$ for these solid matrix samples. The surrogates were applied to the matrix in a Soxhlet extractor immediately prior to extraction. There were low levels of nitrates present in the offgas, and it is probable that the XAD-2 samples were rendered slightly acidic by contact with the offgas. Acidic samples are more likely to release the acid extractable surrogates during the extraction process. Recovery of the 2,4,6-tribromophenol was well within the target acceptance limits for the offgas samples; hence, there is no impact on the offgas data quality because the phenomenon is not observed in the offgas sample results.

On the basis of laboratory surrogate recovery, the results for the SVOC train back-half composite sample data were usable for assessment of the NWCF ETS offgas contents. All of the laboratory surrogate recoveries are consistently within the target acceptance range. The condensate, impinger contents and associated glassware rinse samples also exhibited acceptable results for all of the laboratory surrogate compounds. The sampling surrogate compound that was applied to the XAD $-2^{\circledR}$ resin tubes at the laboratory before sampling provide an independent measurement of the efficiency of the entire process, from sampling to the conclusion of the analysis. The ${ }^{13} \mathrm{C}_{3}$-naphthalene sampling surrogate recovered well from all runs. This further indicates that the data for the SVOCs are reliable.

\subsubsection{SVOC Laboratory Control Sample and Matrix Spike Sample Performance}

Laboratory control samples associated with the front half (glass fiber filter) matrix were prepared and analyzed in duplicate as required by the QAPjP. There are two spiked compounds that exhibited results that were outside the target acceptance limits. The recovery of 2,4-dinitrotoluene in the LCS was slightly below the target acceptance range. The relative percent difference (RPD) for pentachlorophenol was above the target acceptance range. However, neither of these results indicates a significant loss of 
data quality. All of the laboratory surrogates in the LCS/laboratory control samples duplicate (LCSD) analyses associated with the front-half samples are within target acceptance ranges.

Laboratory control samples associated with the back-half composite samples were prepared and analyzed in duplicate. The base-neutral surrogate recovery results for the LCS were similar to the results for the field samples, and were within the stated target acceptance range. The LCS exhibit low recoveries of 4-nitrophenol and pentachlorophenol, which are acidic compounds that are sensitive to $\mathrm{pH}$ effects. Pentachlorophenol is the most acidic of the spiked compounds, and had almost no recovery. The laboratory control sample and laboratory control sample duplicate both have low recoveries of the acidic laboratory surrogate 2,4,6-tribromophenol. This appears to be because there was no control of $\mathrm{pH}$ for these solid samples. The surrogate recoveries of similar compounds were well within target acceptance ranges for the NWCF ETS offgas samples; consequently, there is no impact on the offgas data quality as a result of these low recoveries in the LCS/LCSD.

Laboratory control samples based upon the aqueous matrix were prepared and analyzed in duplicate. The condensate and impinger contents sample for Run 2 was split into three aliquots, and a matrix spike/matrix spike duplicate pair was analyzed. All spiked analyte and surrogate percent recovery and RPD results for the laboratory control and matrix spike samples were within the stated target acceptance ranges.

\subsection{Metals}

A standard U.S. EPA Method 0060 Multi-Metals Train (MMT) configuration was used to collect NWCF ETS offgas samples for the assessment of metals (including mercury) content. An offgas sample having a nominal volume of $3.0 \mathrm{~m}^{3}$ was collected over a duration of 3-4 hours. Two sample collection runs were completed at the beginning and two at the end of consecutive evaporator batches to provide a total of four runs to characterize metals emissions. The following sample components were collected from the 0060 train after the completion of each run:

- Particle Filter

- $\quad 0.1 \mathrm{~N}$ Nitric Acid $\left(\mathrm{HNO}_{3}\right)$ Probe Rinse

- $5 \% \mathrm{HNO}_{3} / 10 \% \mathrm{H}_{2} \mathrm{O}_{2}$ Impinger Contents

- $\quad$ Empty Impinger 4 Contents

- $4 \% \mathrm{KMnO}_{4} / 10 \% \mathrm{H}_{2} \mathrm{SO}_{4}$ Impingers

- $\quad 8 \mathrm{~N} \mathrm{HCl}$ Impinger Rinses

Final nitric acid probe rinse samples were collected after the sampling was completed. The glasslined sampling probe could not be routinely removed from the offgas sampling manifold for rinsing after each run. Instead, the front-half rinse samples were limited to the connecting tubing, filter housing elements and other connecting glassware that were installed outside the manifold assembly. The glasslined probe was only removed from the offgas line after all offgas trains were completed for the test series. Probe rinse samples of the glass-lined probe were collected to assess the maximum amount of metals that adhered to the inside of the probe during testing. The probes were rinsed three times with acetone, followed by three rinses with $0.1 \mathrm{~N}$ nitric acid. These samples were combined for metals analysis after the acetone probe rinses were analyzed for PM. Since the same probe was used for all sampling runs used during the test series, a larger volume of offgas was represented by these acetone and nitric acid probe rinses than was pulled through the probe just for metals analysis. 
Field reagent and blank samples were collected in compliance with the QAPjP. Laboratory method blanks were also prepared and analyzed in support of the data, as required by SW-846 sample analysis requirements.

\subsubsection{Metals Target Analyte List}

The target analyte list for the metals is given in Table 12. These metals, except for $\mathrm{Hg}$, were analyzed by inductively coupled argon plasma spectroscopy per EPA Method 6010B. Mercury (Hg) was analyzed using cold vapor atomic absorption spectroscopy (CVAAS) Method 7470A.

Table 12. Metals target analyte list.

\begin{tabular}{|l|l|l|}
\hline Aluminum (Al) & Chromium (Cr) & Nickel (Ni) \\
\hline Antimony (Sb) & Cobalt $(\mathrm{Co})$ & Selenium (Se) \\
\hline Arsenic (As) & Copper $(\mathrm{Cu})$ & Silver (Ag) \\
\hline Barium (Ba) & Lead $(\mathrm{Pb})$ & Thallium $(\mathrm{Tl})$ \\
\hline Beryllium (Be) & Manganese (Mn) & Vanadium (V) \\
\hline Cadmium (Cd) & Mercury (Hg) & Zinc (Zn) \\
\hline
\end{tabular}

\subsubsection{Metals Analytical Results}

The compatible matrices of the MMT samples were separated into the train's front-half and backhalf samples during the sample preparation steps. The front-half samples consisted of the $0.1 \mathrm{~N}$ nitric acid probe and filter housing rinses, and the particulate filter. The combined offgas condensate, and the 5\% $\mathrm{HNO}_{3} / 10 \% \mathrm{H}_{2} \mathrm{O}_{2}$ impinger catches comprised the MMT back-half composite. The train's fourth impinger was used to separate the components of the train that contained $4 \% \mathrm{KMnO}_{4} / 10 \% \mathrm{H}_{2} \mathrm{SO}_{4}$ from the peroxide contained in the front impingers. The fourth impinger was left empty during set-up. Its contents at the completion of a run and $0.1 \mathrm{~N} \mathrm{HNO}_{3}$ collection rinses were only analyzed for mercury. The $4 \% \mathrm{KMnO}_{4} / 10 \% \mathrm{H}_{2} \mathrm{SO}_{4}$ solution is used to trap elemental mercury $(\mathrm{Hg})$. Thus, a composite sample was prepared from these impingers for the analysis of mercury, only. The internal surfaces of the backhalf impinger glassware received a final deionized (D.I.) water rinse during the train sample collections. These rinsates were collected and added to the appropriate impinger sample and included in the back-half composites. A final rinse of the potassium permanganate impingers was conducted with $8 \mathrm{~N} \mathrm{HCl}$, and was collected as a separate sample for mercury analysis.

The sample collection and analysis are outlined in the STL final report. Sample fraction and run totals are listed in Appendix A. The run totals (in $\mu \mathrm{g}$ ) are the sum of results for the front half and backhalf composite samples, and in the case of mercury, include the permanganate/sulfuric impinger composite totals. Results for the blank trains were not used to correct train totals as allowed by Method 0060 guidance. 


\subsubsection{Metals Data Quality Assessment}

Samples were shipped using overnight delivery service to the laboratory. All Method 0060 train samples that were collected during the offgas sampling program were received at the laboratory in good condition. No losses of samples due to breakage or loss of shipment occurred. Samples derived from the Method 0060 trains are not required to be refrigerated after sample collection. All of the run fractions were processed and analyzed by STL in the time requirements specified in the QAPjP.

The data quality indicators of the sampling and analytical processes for the Method 0060 train samples indicate that the metals data collected from these samples represent acceptable characterization of the offgas emissions. Sufficient data has been collected to allow accuracy and precision to be measured for these parameters. Accuracy has been measured by analyzing LCS, post digestion spikes (PDS), and a limited set of matrix spikes. Precision was measured by the preparation and analysis of laboratory control sample duplicates. The data quality indicators present sufficient evidence that the data are of acceptable quality and are usable for the NWCF ETS offgas emissions inventory.

\subsubsection{Metals Trains Reagent and Blank Data Assessment}

Laboratory method blanks were prepared and analyzed in support of the data. Review of the laboratory method blank data indicates that the laboratory did not appear to introduce significant fugitive contamination to the samples.

Reagent blanks were collected in the field and processed to assess the inherent metallic analyte background in the media being used for sampling. A representative front-half composite reagent blank (a quartz fiber particulate filter and $120 \mathrm{~mL} 0.1 \mathrm{~N} \mathrm{HNO}_{3}$ rinse reagent), a representative back-half reagent blank ( $175 \mathrm{~mL}$ of the $5 \% \mathrm{HNO}_{3} / 10 \% \mathrm{H}_{2} \mathrm{O}_{2}$ impinger solution), and a sample of the INTEC-supplied deionized water $(210 \mathrm{~mL})$ were collected and analyzed for the project target metallic analytes including mercury. Portions of the $4 \% \mathrm{KMnO}_{4} / 10 \% \mathrm{H}_{2} \mathrm{SO}_{4}$ impinger solution, and the $8 \mathrm{~N} \mathrm{HCl}$ rinse solution were collected and analyzed for mercury. Aluminum and selenium were detected in the front-half composite reagent blank at levels that were above the laboratory RL. The amounts of aluminum, antimony, barium, nickel, selenium, and zinc indicate that the reagent may have contributed to the offgas sampling train front-half results. Other metals were detected at relatively insignificant amounts. The back-half reagent blank exhibits manganese and zinc above the laboratory RL. The levels of aluminum, barium, chromium, nickel, lead, manganese, selenium, and zinc indicate that the reagent may have contributed to the offgas sampling train back-half fraction results. The INTEC deionized water did not exhibit significant levels of any metals, although aluminum was found at $5.2 \mu \mathrm{g}$.

The blank train samples exhibit similar results as those obtained for the reagent blanks, with two exceptions. Manganese was found in the blank train back-half composite sample at a level $(3700 \mu \mathrm{g})$ that far exceeded the offgas sample results. This occurrence can be traced to contamination of the back-half composite sample by $\mathrm{KMnO}_{4}$ reagent. Zinc was found at a level of $34 \mu \mathrm{g}$. This amount is roughly an order of magnitude higher that the reagent blank sample, and similar to the offgas sampling train results.

\subsubsection{Method 0060 Train Accuracy and Precision Assessment}

The quality control procedures that were implemented during the analyses of these samples for the purposes of assessing the general accuracy and precision of the analytical processes included the analysis of LCSs, LCSDs, PDS, and MS/MSD samples. Laboratory control samples test the accuracy and precision of the laboratory processes independent of stack gas matrix effects. The quantitative recovery of PDSs provides a second indicator of accuracy for the metals analysis for matrices (e.g., the front-half and back-half samples) from which matrix spikes cannot be prepared without affecting detection limits. 
These spikes act as "internal standards" and signal when problems are encountered with the digestate matrix. When acceptable quantitative recoveries are observed for a PDS, the sample introduction onto the ICP is considered to be taking place correctly and the instrument has quantified the analytes present in the digestate correctly. Low recoveries typically indicate that viscosity or matrix interference effects may have been encountered. Matrix spikes (post sampling) of mercury were applied to the four back-half fraction samples. Accurate and precise recovery of the spiked mercury indicates that the entire analytical process, including preparation, is in control.

Matrix spikes of the ICP metals were not performed for the back-half composite sample matrix because this process would raise detection levels while adequate quality control information can be otherwise obtained. In terms of compliance, SW-846 Method 0060 does not require matrix spike recovery information for the evaluation of metals train samples, while SW-846 Method 6010B requires them to be performed for each sample batch or sample delivery group (SDG). The reasoning behind the lack of matrix spike requirements in the Method 0060 relates to the difficulty of dividing a front-half train portion, which contains the particulate filter, into three equal portions of the PM sample. Dividing the filter is a precarious operation. Particulate matter may not be evenly distributed on the filter, and cutting the filter to obtain equal portions of particulate material on each portion of the filter is difficult to execute. Analysis of MS/MSD samples for the front-half composite sample in not technically advisable.

Particulate matter is the fraction of the captured stack gas sample that contributes typically the greatest level of metals to the Method 0060 samples.

Post digestion spikes were allowed in the QAPjP as an alternative QC measurement to replace MS/MSDs for both the front half and the back half train fractions. Post digestion spikes give an adequate demonstration of recovery for these samples, and are allowed by quality procedures sections of SW-846 methods for flame atomic absorption and graphite furnace atomic absorption. When coupled with laboratory control samples, and laboratory control sample duplicates, the quality of Method 0060 train sample analysis can be completely evaluated.

The LCS and PSDs corresponding to the front-half composite samples exhibited acceptable recoveries for all target metals. The recoveries of the metals, were within the target recovery range of $75 \%$ to $125 \%$, with the exception of manganese and mercury. Mercury recoveries were high for Run 1 , Run 2, Run 4 and the Final Acetone and Nitric Acid Probe Rinse. Manganese exhibited a low recovery only for Run 4.

The back-half sample fractions were also supported by laboratory control sample analysis and post digestion spike analysis. Post digestion spikes were analyzed for the back-half samples from Runs 1 and 2. The laboratory control sample results were accurate and reproducible, and indicate that the analytical processes were in control. All of the post digestion spike results were acceptable, but the mercury results have little meaning because the native levels of mercury in the samples were very high relative to the spike levels. Matrix spikes of the Run 1 back-half fractions for mercury of the impinger number 4 contents, $4 \% \mathrm{KMnO}_{4} / 10 \% \mathrm{H}_{2} \mathrm{SO}_{4}$ impinger contents, and the $8 \mathrm{~N} \mathrm{HCl}$ rinse fractions were successful. Matrix spike results for the back-half composite sample for Run 1 could not be calculated because the native level of mercury was too high, relative to the spike level.

In general, the metals laboratory control sample, post digestion spike, and matrix spike recoveries indicate acceptable performance and provide a strong indication that the analytical processes used were in control. A review of the calculated RPDs (presented in the final STL data package and archived for this project), obtained from the analysis of LCS, indicates that these analytical processes are highly reproducible. 
The data were reviewed for evidence of interelement interference because ICP (AES) analysis is subject to interelement interference from "high" levels of a few analytes. Aluminum (Al) is the only element present in the NWCF ETS samples that is in a sufficiently high concentration to be considered an interfering influence on the results for target analytes. The aluminum concentration for the Run 3 fronthalf composite sample is a potential interferent that could increase the results for vanadium. There is no effect, because the result for vanadium for this sample was non-detectable.

\subsection{Particulate Matter and Acid Gases}

The standard EPA Method $0050 \mathrm{HCl} / \mathrm{Cl}_{2} / \mathrm{Particulate}$ Train (SW-846 Method 0050) configuration was used to collect samples from the NWCF ETS offgas for the assessment of PM and acid gas vapors. An offgas sample having a nominal volume of $3.0 \mathrm{dscm}$ was isokinetically collected over 3-4 hours during each sampling run. A blank train and applicable reagent blanks were collected to support the QA/QC requirements specified in the QAPJP.

A final probe rinse sample was collected after the test runs for particulate analysis. The glass-lined sampling probe could not routinely be removed from the offgas sampling manifold for rinsing after each run for safty reasons as previously discussed. Instead, the front-half acetone rinse samples for particulate were limited to accessible tubing, filter housing elements and other connecting glassware that were installed outside the manifold and probe assembly. The glass-lined probe was only removed from the manifold after all offgas sampling trains were collected. An acetone probe rinse sample of the glass-lined probe was collected after the test runs to assess the maximum amount of particulate materials that adhered to the inside of the probe during testing. The probes were rinsed three times with acetone, and the samples were submitted for particulate analysis.

\subsubsection{Acid Gas Target analytes}

The target analytes for this method are hydrogen chloride $(\mathrm{HCl})$, chlorine $\left(\mathrm{Cl}_{2}\right)$, hydrogen fluoride $(\mathrm{HF})$, nitrate $\left(\mathrm{NO}_{3}-\right)$, nitrite $\left(\mathrm{NO}_{2}-\right)$, and $\mathrm{PM}$.

\subsubsection{Analysis of PM and Acid Gases}

The particulate samples collected included two fractions: (1) an acetone rinse of the probe and filter housing assembly, and (2) a particulate filter. Composite samples containing the contents of the first, second, and third impingers of the train were collected for $\mathrm{HCl}, \mathrm{HF}, \mathrm{HNO}_{3}$, and $\mathrm{HNO}_{2}$ analysis. The first impinger was empty at the beginning of the sampling run and served as a moisture knockout impinger. The second and third impingers were each initially charged with $100 \mathrm{~mL}$ of $0.1 \mathrm{~N} \mathrm{H}_{2} \mathrm{SO}_{4}$. These acidic impingers allowed for the dissociation and collection of $\mathrm{HCl}, \mathrm{HF}, \mathrm{HNO}_{3}$, and $\mathrm{HNO}_{2}$ from the offgas. A composite sample of the fourth and fifth impinger contents, which were each initially charged with 100 $\mathrm{mL}$ of $0.1 \mathrm{~N} \mathrm{NaOH}$, were analyzed for $\mathrm{Cl}^{-}, \mathrm{F}^{-}, \mathrm{NO}_{3}^{-}$, and $\mathrm{NO}_{2}^{-}$. Chlorine gas $\left(\mathrm{Cl}_{2}\right)$ present in the offgas does not freely dissociate in the acidic $\mathrm{H}_{2} \mathrm{SO}_{4}$ medium; therefore, it passes through the first three impingers as an unreacted gas and reacts when it comes in contact with the $\mathrm{NaOH}$ solution as follows:

$$
\mathrm{Cl}_{2}+2 \mathrm{OH}^{-} \stackrel{\mathrm{NaOH}}{\rightarrow} 2 \mathrm{OCl}^{-}+\mathrm{Cl}^{-}+\mathrm{H}_{2} \mathrm{O}
$$

At STL, the particulate filter samples were dried at $105^{\circ} \mathrm{C}$ and the acetone probe rinse samples were evaporated to dryness at room temperature. Both fractions were stored in a dessicator for 24 hours, then analyzed gravimetrically. Replicate weights were obtained until constant weights were achieved. 
The difference between pre-sampling and post-drying gravimetric measurements were then calculated for each sample.

The $0.1 \mathrm{~N} \mathrm{H}_{2} \mathrm{SO}_{4}$ and $0.1 \mathrm{~N} \mathrm{NaOH}$ impinger samples were analyzed by ion chromatography using SW-846 Methods 9056 and 9057, as implemented by STL Analytical Laboratories Method, KNOX-WC0005, Anion Analysis by Ion Chromatography, KNOX-WC-0005 (April 20,1999). The calibration range extended from $0.5 \mathrm{mg} / \mathrm{L}$ to $20 \mathrm{mg} / \mathrm{L}$ for all of the target anions. In order to quantify all of the target anions, several analyses were conducted at different dilution factors that ranged from 1 to 10 . Dilution was performed both to bring the sample concentration within the instrument calibration range and to overcome matrix effects. The optimum value was chosen for reporting, with the lowest achieved detection limits reported in each case. The final data for each anion were based on analyses that were within the calibration range of the instrument.

The tabulated data summaries provided in the STL Final Report are listed in Appendix A. Each anion result is reduced to a "Risk Result" by selecting the default value for use in risk assessment calculations in accordance with project guidelines.

\subsubsection{PM and Acid Gas Data Quality Assessment}

Samples were shipped using Federal Express overnight delivery service to the laboratory. All Method 0050 train samples that were collected during the offgas sampling program were received at the laboratory in good condition. No losses of samples due to breakage or loss of shipment occurred. All Method 0050 train fractions were processed on schedule, as required by the QAPjP, and analytical results were obtained for all of the expected analyses.

Sufficient data have been collected and reviewed to address the relative precision and accuracy of the particulate and anion target analyte measurements. The data quality indicators present sufficient evidence that the data are of acceptable quality and are usable for the NWCF ETS emissions inventory.

\subsubsection{Method 0050 Train Reagent Blank Assessment}

A particulate filter, the $0.1 \mathrm{~N} \mathrm{H}_{2} \mathrm{SO}_{4}$ and $1 \mathrm{~N} \mathrm{NaOH}$ reagents, and a sample of the INTEC supplied deionized (D.I.) water were collected during sample collection and were processed as reagent blanks in order to assess the presence of background analytes. These reagent blank samples were analyzed for the same parameters as the actual train samples, and showed minimal background levels of the target analytes. Chloride was detected in the $0.1 \mathrm{~N} \mathrm{H}_{2} \mathrm{SO}_{4}$ reagent blank at a level that was below the $\mathrm{RL}$ for the laboratory. Nitrate was detected in the $0.1 \mathrm{~N} \mathrm{NaOH}$ reagent blank and the D.I. water reagent blank at low levels that had no impact on the final results. The sample results have been presented without blank corrections with the exception of tare subtraction required for the particulate analysis. All sample calculations of offgas concentrations were performed without blank or background correction.

\subsubsection{Method 0050 Blank Train Assessment}

A set of blank train samples was collected in conjunction with the four Method 0050 runs. The blank train samples exhibited similar results as the reagent blanks. There was no evidence of significant contributions to anion contributions in the sample results as a consequence of the sample train component preparation or handling. 


\subsubsection{Laboratory Control Sample and Matrix Spike Sample Assessment}

Laboratory control sample percent recoveries indicate that the analytical process was in control. However, there were reduced recoveries of chloride in the matrix spike samples, due to apparent matrix effects. The other anions exhibit percent recoveries that are within target acceptance limits. Overall recovery of all of the target anions was sufficient to indicate that the data are useful for their intended purposes. The RPD results for both the LCS and the matrix spike samples indicate that a high level of precision is represented by this data. 


\section{PROCESS STREAM CHARACTERIZATION RESULTS}

During the NWCF ETS off-gas emissions sampling activities, the NWCF ETS was being used to process a blend of two parts by volume of solution from INTEC Tank Farm Facility (TFF) vessel WM184 and one part by volume of solution from INTEC TFF vessel WM-181. The campaign to process this blend was initiated on May 4, 2001 and continued through December 2001. At the same time the off-gas sampling was being performed, RCRA-quality liquid samples of NWCF ETS process streams were collected for analysis. These samples were collected under the protocols and QA/QC specified in sampling and analysis PLN-613 and PLN-407, the ALD QAPjP for environmental samples. These samples consisted of one each of the blended feed, condensed overheads, and concentrated bottoms. In addition, process samples of the feed were analyzed prior to initiation of processing the blend in the NWCF ETS to ensure that the chemistry of the feed solution was compatible with process equipment. Finally, samples of the condensed overheads and the concentrated bottoms from the first several batches processed were analyzed to ensure that the chemistry of those streams was compatible with down-stream process equipment. It should be noted that the non-RCRA samples were not analyzed per RCRA protocol; therefore limited QA/QC controls were evaluated.

These data obtained for the process streams may be used for component material balances around the NWCF ETS and are included in this report to provide a complete picture of the NWCF ETS and to provide a convenient location to obtain the data for subsequent system analyses. It should be noted that some limitations exist with this data. First, the INTEC Analytical Laboratory Department (ALD) that analyzed these samples has a more limited standard target analyte list for volatile organic compounds and semi-volatile organic compounds than the contract laboratory that analyzed the off-gas samples. The INTEC ALD TAL of volatile and semi-volatile organic compounds are contained in Appendix C.

Second, some of the process samples (especially those taken to ensure compatibility with the NWCF ETS and down-stream equipment) were not taken at the exact same time as the off-gas samples. However, it is reasonable to assume that the process control system provides consistent batching of the feed streams and consistent control of the process variables. Third, the samples taken prior to initiation of the blend campaign, as well as those taken during processing the first several batches were only analyzed for a limited number of analytes. Since the purpose of these samples was to ensure compatibility between the solution chemistry and the process equipment, only those analytes that might challenge the envelopes of associated safety bases were targeted.

The results of the inorganic analyses of the feed samples are contained in Table 13, those results for the concentrated bottoms are contained in Table 14, and those for the condensed overheads are contained in Table 15. Organic compound analyses were only performed on one sample from each of the three streams; therefore, the organic analysis results for all three streams are contained in Tables 16 and 17. The INTEC ALD analytical reports for these samples are contained in Appendix C. 


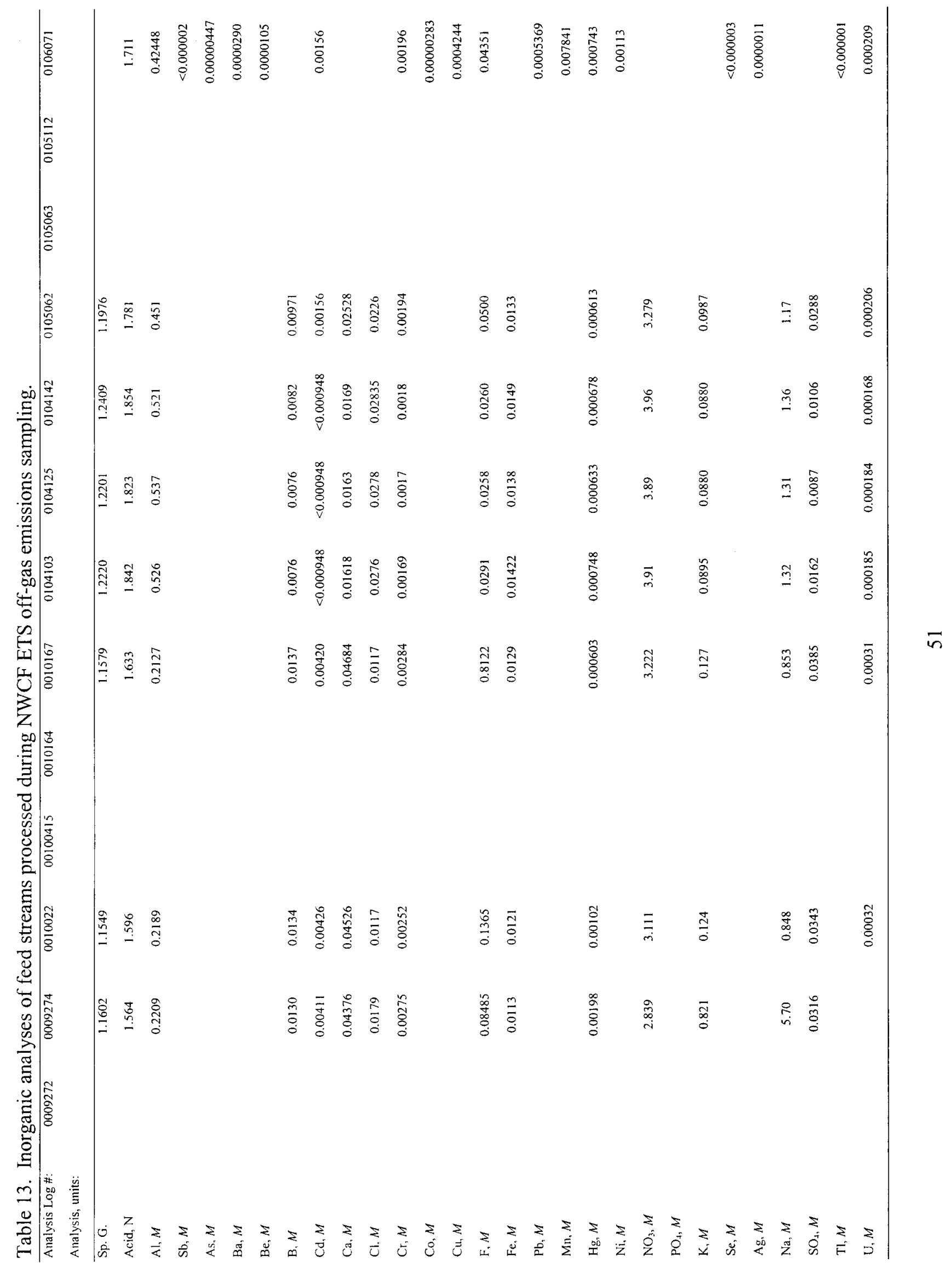




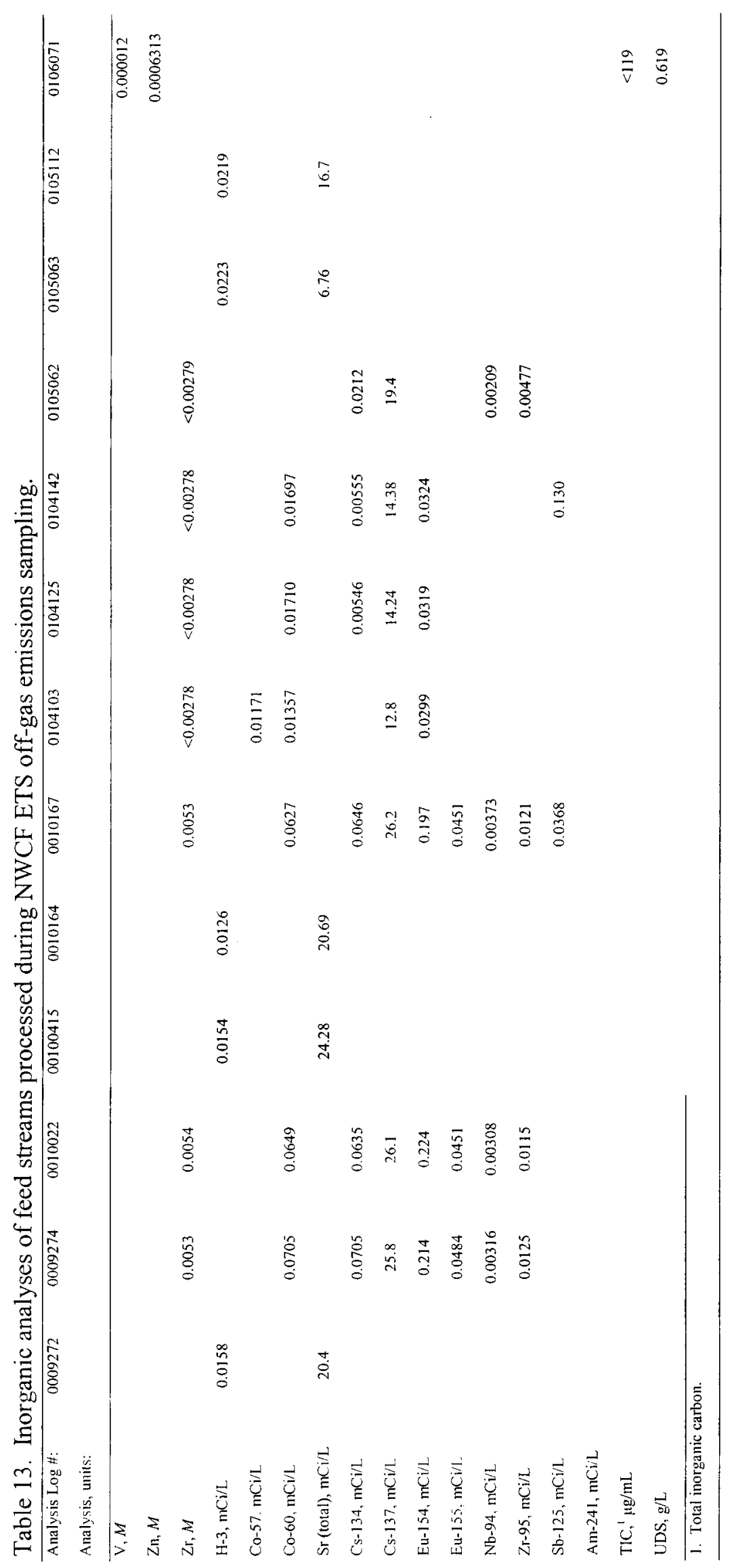

n 
Table 14. Inorganic analyses of bottoms streams during NWCF ETS off-gas emissions

\begin{tabular}{|c|c|c|c|c|}
\hline $\begin{array}{l}\text { Analysis Log \#: } \\
\text { Analysis, units: }\end{array}$ & 0105106 & 0106146 & 0106214 & 0106233 \\
\hline Sp. G. & 1.0747 & 1.3564 & & 1.3036 \\
\hline Acid, $N$ & 2.559 & 2.868 & 1.741 & 2.830 \\
\hline $\mathrm{Al}, M$ & 0.518 & 0.859 & 0.31256 & 0.711 \\
\hline $\mathrm{Sb}, M$ & & & 0.0000128 & \\
\hline As, $M$ & & & $<0.000008$ & \\
\hline $\mathrm{Ba}, M$ & & & 0.0000216 & \\
\hline $\mathrm{Be}, M$ & & & 0.0000067 & \\
\hline \multicolumn{5}{|l|}{$\mathrm{B}, M$} \\
\hline $\mathrm{Cd}, M$ & & & 0.00131 & \\
\hline \multicolumn{5}{|l|}{$\mathrm{Ca}, M$} \\
\hline $\mathrm{Cl}, M$ & 0.0226 & 0.02866 & & 0.03732 \\
\hline $\mathrm{Cr}, M$ & & & 0.001674 & \\
\hline $\mathrm{Co}, M$ & & & 0.0000241 & \\
\hline $\mathrm{Cu}, M$ & & & 0.0003003 & \\
\hline $\mathrm{F}, M$ & 0.0466 & 0.0900 & 0.052775 & 0.0684 \\
\hline \multicolumn{5}{|l|}{$\mathrm{Fe}, M$} \\
\hline $\mathrm{Pb}, M$ & & & 0.0004164 & \\
\hline $\mathrm{Mn}, M$ & & & 0.006174 & \\
\hline $\mathrm{Hg}, M$ & & & 0.000568 & \\
\hline $\mathrm{Ni}, M$ & & & 0.0009426 & \\
\hline $\mathrm{NO}_{3}, M$ & 4.03 & 5.27 & & 7.22 \\
\hline $\mathrm{PO}_{4}, M$ & 0.003871 & 0.01399 & & 0.01382 \\
\hline $\mathrm{K}, M$ & 0.0159 & 0.129 & & 0.154 \\
\hline $\mathrm{Se}, M$ & & & $<0.000012$ & \\
\hline $\mathrm{Ag}, M$ & & & $<0.000004$ & \\
\hline $\mathrm{Na}, M$ & 0.505 & 1.56 & & 1.91 \\
\hline \multicolumn{5}{|l|}{$\mathrm{SO}_{4}, M$} \\
\hline $\mathrm{Tl}, M$ & & & $<0.000004$ & \\
\hline$\cup, M$ & 0.000247 & 0.000311 & 0.000261 & 0.0004357 \\
\hline$\vee, M$ & & & 0.000011 & \\
\hline $\mathrm{Zn}, M$ & & & 0.000494 & \\
\hline \multicolumn{5}{|l|}{$\mathrm{Zr}, M$} \\
\hline $\mathrm{H}-3, \mathrm{mCi} / \mathrm{L}$ & 0.0108 & 0.0151 & & \\
\hline \multicolumn{5}{|l|}{$\mathrm{Co}-57, \mathrm{mCi} / \mathrm{L}$} \\
\hline $\mathrm{Co}-60, \mathrm{mCi} / \mathrm{L}$ & 0.0352 & & & \\
\hline \multicolumn{5}{|l|}{$\mathrm{Sr}$ (total), $\mathrm{mCi} / \mathrm{L}$} \\
\hline $\mathrm{Cs}-134, \mathrm{mCi} / \mathrm{L}$ & 0.0236 & & & \\
\hline $\mathrm{Cs}-137, \mathrm{mCi} / \mathrm{L}$ & 21.2 & & & \\
\hline Eu-154, mCi/L & 0.0904 & & & \\
\hline \multicolumn{5}{|l|}{$\mathrm{Eu}-155, \mathrm{mCi} / \mathrm{L}$} \\
\hline \multicolumn{5}{|l|}{$\mathrm{Nb}-94, \mathrm{mCi} / \mathrm{L}$} \\
\hline $\mathrm{Zr}-95, \mathrm{mCi} / \mathrm{L}$ & 0.00528 & & & \\
\hline \multicolumn{5}{|l|}{$\mathrm{Sb}-125, \mathrm{mCi} / \mathrm{L}$} \\
\hline \multicolumn{5}{|l|}{$\mathrm{Am}-241, \mathrm{mCi} / \mathrm{L}$} \\
\hline TIC, ${ }^{\prime} \mu \mathrm{g} / \mathrm{mL}$ & & & $<119$ & \\
\hline UDS, $\mathrm{g} / \mathrm{L}$ & 5.3 & 1.187 & 1.288 & 0.725 \\
\hline
\end{tabular}


Table 15. Inorganic analyses of condensate streams during NWCF ETS off-gas emissions

\begin{tabular}{|c|c|c|c|c|c|c|c|}
\hline $\begin{array}{l}\text { Analysis Log \#: } \\
\text { Analysis, units: }\end{array}$ & 0105061 & 0105073 & 0105087 & 0105092 & 0106145 & 0106221 & 0106241 \\
\hline Sp. G. & 1.0127 & 1.0121 & 1.0131 & 1.0131 & 1.0138 & & 1.0130 \\
\hline Acid, $N$ & 0.467 & 0.450 & 0.476 & 0.480 & 0.498 & 0.514 & 0.471 \\
\hline $\mathrm{Al}, M$ & 0.00108 & $<0.0008$ & $<0.00042$ & $<0.00075$ & $<0.00042$ & 0.00006312 & $<0.00042$ \\
\hline $\mathrm{Sb}, M$ & & & & & & $<0.0000004$ & \\
\hline As, $M$ & & & & & & $<0.0000004$ & \\
\hline $\mathrm{Ba}, M$ & & & & & & 0.000000066 & \\
\hline $\mathrm{Be}, M$ & & & & & & $<0.0000001$ & \\
\hline \multicolumn{8}{|l|}{$\mathrm{B}, M$} \\
\hline $\mathrm{Cd}, M$ & & & & & & $<0.00000004$ & \\
\hline \multicolumn{8}{|l|}{$\mathrm{Ca}, M$} \\
\hline $\mathrm{Cl}, M$ & 0.004928 & 0.00485 & 0.005156 & 0.00525 & 0.005602 & & 0.00539 \\
\hline $\mathrm{Cr}, M$ & & & & & & 0.00000052 & \\
\hline $\mathrm{Co}, M$ & & & & & & $<0.0000002$ & \\
\hline $\mathrm{Cu}, M$ & & & & & & $<0.0000003$ & \\
\hline $\mathrm{F}, M$ & $<0.00041$ & $<0.00037$ & $<0.00037$ & $<0.00037$ & $<0.00018$ & 0.0001791 & $<0.00018$ \\
\hline \multicolumn{8}{|l|}{$\mathrm{Fe}, M$} \\
\hline $\mathrm{Pb}, M$ & & & & & & $<0.0000004$ & \\
\hline $\mathrm{Mn}, M$ & & & & & & 0.00000024 & \\
\hline $\mathrm{Hg}, M$ & 0.0000229 & 0.0000189 & 0.0000186 & 0.0000115 & 0.0000274 & 0.00001969 & 0.0000163 \\
\hline $\mathrm{Ni}, M$ & & & & & & 0.00000055 & \\
\hline $\mathrm{NO}_{3}, M$ & 0.4011 & 0.4198 & 0.02049 & 0.4233 & 0.4393 & & 0.4007 \\
\hline \multicolumn{8}{|l|}{$\mathrm{PO}_{4}, M$} \\
\hline \multicolumn{8}{|l|}{$\mathrm{K}, M$} \\
\hline $\begin{array}{l}\mathrm{Se}, M \\
\mathrm{Ag}, M\end{array}$ & & & & & & $\begin{array}{l}<0.0000007 \\
<0.0000002\end{array}$ & \\
\hline \multicolumn{8}{|l|}{$\mathrm{Na}, M$} \\
\hline $\mathrm{SO}_{4}, M$ & 0.000153 & 0.000120 & $<0.00003$ & 0.000112 & 0.000083 & & $<0.000059$ \\
\hline $\begin{array}{l}\mathrm{Tl}, M \\
\mathrm{U}, M\end{array}$ & & & & & & $<0.0000002$ & \\
\hline $\begin{array}{l}\mathrm{U}, M \\
\mathrm{~V}, M\end{array}$ & $<0.0000014$ & $<0.0000014$ & $<0.0000014$ & $<0.0000014$ & $<0.0000014$ & $<0.0000014$ & $<0.0000014$ \\
\hline $\begin{array}{l}\mathrm{V}, M \\
\mathrm{Zn}, M\end{array}$ & & & & & & $<0.0000002$ & \\
\hline $\begin{array}{l}\operatorname{Zn}, M \\
\operatorname{Zr}, M\end{array}$ & & & & & & 0.00000081 & \\
\hline \multicolumn{8}{|l|}{$\mathrm{Zr}, M$} \\
\hline \multicolumn{8}{|l|}{$\begin{array}{l}\mathrm{H}-3, \mathrm{mCi} / \mathrm{L} \\
\mathrm{Co}-57 \mathrm{mCi} / \mathrm{L}\end{array}$} \\
\hline \multirow{2}{*}{\multicolumn{8}{|c|}{$\begin{array}{l}\mathrm{Co}-57, \mathrm{mCi} / \mathrm{L} \\
\mathrm{Co}-60, \mathrm{mCi} / \mathrm{L}\end{array}$}} \\
\hline \multirow{2}{*}{\multicolumn{7}{|c|}{$\mathrm{Sr}$ (total), $\mathrm{mCi} / \mathrm{L}$}} & \\
\hline & & & & & & & \\
\hline \multicolumn{8}{|l|}{$\mathrm{Cs}-134, \mathrm{mCi} / \mathrm{L}$} \\
\hline \multicolumn{8}{|l|}{$\mathrm{Cs}-137, \mathrm{mCi} / \mathrm{L}$} \\
\hline \multicolumn{8}{|l|}{$\mathrm{Eu}-154, \mathrm{mCi} / \mathrm{L}$} \\
\hline \multicolumn{8}{|l|}{ Eu- $155, \mathrm{mCi} / \mathrm{L}$} \\
\hline \multicolumn{8}{|l|}{$\mathrm{Nb}-94, \mathrm{mCi} / \mathrm{L}$} \\
\hline \multicolumn{8}{|l|}{$\mathrm{Zr}-95, \mathrm{mCi} / \mathrm{L}$} \\
\hline \multirow{2}{*}{\multicolumn{8}{|c|}{$\begin{array}{l}\mathrm{Sb}-125, \mathrm{mCi} / \mathrm{L} \\
\mathrm{Am}-241, \mathrm{mCi} / \mathrm{L}\end{array}$}} \\
\hline $\mathrm{Am}-241, \mathrm{mCi} / \mathrm{L}$ & & & & & & & \\
\hline $\mathrm{TIC},{ }^{\prime} \mu \mathrm{g} / \mathrm{mL}$ & & & & & & $<23.8$ & \\
\hline UDS, g/L & none visible & none visible & none visible & none visible & none visible & 0.0 & none visible \\
\hline 1. Total inorganic & arbon. & & & & & & \\
\hline
\end{tabular}


Table 16. VOC analyses of NWCF ETS streams during NWCF ETS off-gas emissions sampling.

\begin{tabular}{|c|c|c|c|c|c|c|}
\hline \multirow{2}{*}{ Stream } & \multicolumn{2}{|c|}{ Blended Feed } & \multicolumn{2}{|c|}{ Bottoms } & \multicolumn{2}{|c|}{ Condensate } \\
\hline & Result & LQ & Result & LQ & Result & $\mathrm{LQ}$ \\
\hline Analyte, units: & & & & & & \\
\hline $\mathrm{TOC}, \mu \mathrm{g} / \mathrm{mL}$ & 608.462 & & 754.99 & & 147.853 & \\
\hline Chloromethane, $\mu \mathrm{g} / \mathrm{L}$ & $<10$ & $\mathrm{UM}$ & $<10$ & $\mathrm{U}$ & $<10$ & $\mathrm{U}$ \\
\hline Vinyl Chloride, $\mu \mathrm{g} / \mathrm{L}$ & $<10$ & $\mathrm{U}$ & $<10$ & $\mathrm{U}$ & $<10$ & $\mathrm{U}$ \\
\hline Bromomethane, $\mu \mathrm{g} / \mathrm{L}$ & 4 & $\mathrm{~J}$ & 37 & B M & 160 & E B M \\
\hline Chloroethane, $\mu \mathrm{g} / \mathrm{L}$ & $<10$ & $\mathrm{U}$ & $<10$ & U & $<10$ & $\mathrm{U}$ \\
\hline Trichlorofluoromethane, $\mu \mathrm{g} / \mathrm{L}$ & $<10$ & $\mathrm{U}$ & $<10$ & $\mathrm{U}$ & $<10$ & $\mathrm{U}$ \\
\hline 1,1-Dichloroethene, $\mu \mathrm{g} / \mathrm{L}$ & $<10$ & $\mathrm{U}$ & $<10$ & U & $<10$ & $\mathrm{U}$ \\
\hline 1,1,2-Trichloro-1,2,2- & $<10$ & $\mathrm{U}$ & $<10$ & $\mathrm{U}$ & $<10$ & $\mathrm{U}$ \\
\hline Carbon disulfide, $\mu \mathrm{g} / \mathrm{L}$ & $<10$ & $\mathrm{U}$ & $<10$ & $\mathrm{U}$ & $<10$ & $\mathrm{U}$ \\
\hline Acetone, $\mu \mathrm{g} / \mathrm{L}$ & 9 & $\mathrm{~J}$ & $<20$ & UZ & 32 & $\mathrm{Y}$ \\
\hline Methylene chloride & $<10$ & $\mathrm{U}$ & $<20$ & $\mathrm{U} Z$ & $<20$ & $\mathrm{UZ}$ \\
\hline Trans-1,2-dichloroethene, $\mu \mathrm{g} / \mathrm{L}$ & $<10$ & $\mathrm{U}$ & $<10$ & $\mathrm{U}$ & $<10$ & U \\
\hline 1,1-Dichloroethane, $\mu \mathrm{g} / \mathrm{L}$ & $<10$ & $\mathrm{U}$ & $<10$ & $\mathrm{U}$ & $<10$ & $\mathrm{U}$ \\
\hline Cis-1,2-dichloroethene, $\mu \mathrm{g} / \mathrm{L}$ & $<10$ & $\mathrm{U}$ & $<10$ & U & $<10$ & $U$ \\
\hline 2-Butanone, $\mu \mathrm{g} / \mathrm{L}$ & $<10$ & $\mathrm{U}$ & $<10$ & $\mathrm{U}$ & $<10$ & U \\
\hline Chloroform, $\mu \mathrm{g} / \mathrm{L}$ & $<10$ & $\mathrm{U}$ & $<10$ & $\mathrm{U}$ & $<10$ & $\mathrm{U}$ \\
\hline $1,1,1$-Trichloroethane, $\mu \mathrm{g} / \mathrm{L}$ & $<10$ & $\mathrm{U}$ & $<10$ & $U$ & $<10$ & $\mathrm{U}$ \\
\hline Carbon tetrachloride, $\mu \mathrm{g} / \mathrm{L}$ & $<10$ & $\mathrm{U}$ & $<10$ & $\mathrm{U}$ & $<10$ & $\mathrm{U}$ \\
\hline Benzene, $\mu \mathrm{g} / \mathrm{L}$ & $<10$ & $\mathrm{U}$ & $<10$ & $\mathrm{U}$ & $<10$ & $\mathrm{U}$ \\
\hline 1,2-Dichloroethane, $\mu \mathrm{g} / \mathrm{L}$ & $<10$ & $\mathrm{UM}$ & $<10$ & $\mathrm{U}$ & $<10$ & $\mathrm{U}$ \\
\hline Trichloroethene, $\mu \mathrm{g} / \mathrm{L}$ & $<10$ & $\mathrm{U}$ & $<10$ & $\mathrm{U}$ & $<10$ & $\mathrm{U}$ \\
\hline 1,2-Dichloropropane, $\mu \mathrm{g} / \mathrm{L}$ & $<10$ & $\mathrm{U}$ & $<10$ & $\mathrm{U}$ & $<10$ & $\mathrm{U}$ \\
\hline Bromodichloromethane, $\mu \mathrm{g} / \mathrm{L}$ & $<10$ & $\mathrm{U}$ & $<10$ & $\mathrm{U}$ & $<10$ & U \\
\hline Cis-1,3-dichloropropene, $\mu \mathrm{g} / \mathrm{L}$ & $<10$ & $\mathrm{U}$ & $<10$ & $\mathrm{U}$ & $<10$ & $\mathrm{U}$ \\
\hline 4-Methyl-2-pentanone, $\mu \mathrm{g} / \mathrm{L}$ & $<10$ & $\mathrm{U}$ & $<10$ & U & $<10$ & $\mathrm{U}$ \\
\hline Toluene, $\mu \mathrm{g} / \mathrm{L}$ & $<10$ & $\mathrm{U}$ & $<10$ & $\mathrm{U}$ & $<10$ & $U$ \\
\hline Trans-1,3-dichloropropene, $\mu \mathrm{g} / \mathrm{L}$ & $<10$ & $\mathrm{U}$ & $<10$ & $\mathrm{U}$ & $<10$ & $U$ \\
\hline 1,1,2-Trichloroethane, $\mu \mathrm{g} / \mathrm{L}$ & $<10$ & $\mathrm{U}$ & $<10$ & $\mathrm{U}$ & $<10$ & $\mathrm{U}$ \\
\hline Tetrachloroethene, $\mu \mathrm{g} / \mathrm{L}$ & $<10$ & $\mathrm{U}$ & $<10$ & $\mathrm{U}$ & $<10$ & $\mathrm{U}$ \\
\hline 2-Hexanone, $\mu \mathrm{g} / \mathrm{L}$ & $<10$ & $\mathrm{U}$ & $<10$ & $\mathrm{U}$ & $<10$ & $\mathrm{U}$ \\
\hline Dibromochloromethane, $\mu \mathrm{g} / \mathrm{L}$ & $<10$ & $\mathrm{U}$ & $<10$ & $\mathrm{U}$ & $<10$ & U \\
\hline Chlorobenzene, $\mu \mathrm{g} / \mathrm{L}$ & $<10$ & $\mathrm{U}$ & $<10$ & $\mathrm{U}$ & $<10$ & $\mathrm{U}$ \\
\hline Ethylbenzene, $\mu \mathrm{g} / \mathrm{L}$ & $<10$ & $\mathrm{U}$ & $<10$ & $\mathrm{U}$ & $<10$ & $\mathrm{U}$ \\
\hline M-xylene and p-xylene, $\mu \mathrm{g} / \mathrm{L}$ & $<20$ & $\mathrm{U}$ & $<20$ & $\mathrm{U}$ & $<20$ & $\mathrm{U}$ \\
\hline O-xylene, $\mu \mathrm{g} / \mathrm{L}$ & $<10$ & $\mathrm{U}$ & $<10$ & $\mathrm{U}$ & $<10$ & $\mathrm{U}$ \\
\hline Styrene, $\mu \mathrm{g} / \mathrm{L}$ & $<10$ & $\mathrm{U}$ & $<10$ & $\mathrm{U}$ & $<10$ & $\mathrm{U}$ \\
\hline Bromoform. $\mu \mathrm{g} / \mathrm{L}$ & $<10$ & $\mathrm{U}$ & $<10$ & $\mathrm{U}$ & $<10$ & $\mathrm{U}$ \\
\hline 1,1,2,2-Tetrachloroethane, $\mu \mathrm{g} / \mathrm{L}$ & $<10$ & $\mathrm{U}$ & $<10$ & $\mathrm{U}$ & $<10$ & $\mathrm{U}$ \\
\hline Tentatively Identified Compounds: & & & & & & \\
\hline Unknowns, number & $1 / 10$ & $\mathrm{~J}$ & & & & \\
\hline
\end{tabular}


Table 17. SVOC analyses of NWCF ETS streams during NWCF ETS off-gas emissions

\begin{tabular}{|c|c|c|c|c|c|c|}
\hline \multirow{3}{*}{$\begin{array}{l}\text { Stream } \\
\text { Analysis Log \#: }\end{array}$} & \multirow{2}{*}{\multicolumn{2}{|c|}{$\begin{array}{c}\text { Blended Feed } \\
0106071\end{array}$}} & \multirow{2}{*}{\multicolumn{2}{|c|}{$\begin{array}{l}\text { Bottoms } \\
0106221\end{array}$}} & \multirow{2}{*}{\multicolumn{2}{|c|}{$\begin{array}{c}\text { Condensate } \\
0106214\end{array}$}} \\
\hline & & & & & & \\
\hline & Result & LQ & Result & LQ & Result & LQ \\
\hline \multicolumn{7}{|l|}{ Analyte, units: } \\
\hline $\mathrm{TOC}, \mu \mathrm{g} / \mathrm{mL}$ & 608.462 & & 754.99 & & 147.853 & \\
\hline N-Nitrosodimethylamine & $<20$ & $\mathrm{UM}$ & 36 & & 42 & \\
\hline Pyridine & $<20$ & $\mathrm{U}$ & $<20$ & $\mathrm{U}$ & $<20$ & $\mathrm{U}$ \\
\hline Phenol & $<20$ & U & $<20$ & U & $<20$ & U \\
\hline bis(2-Chloroethyl)ether & $<20$ & $\mathrm{U}$ & $<20$ & $\mathrm{U}$ & $<20$ & $\mathrm{U}$ \\
\hline 2-Chlorophenol & $<20$ & $U$ & $<20$ & $\mathrm{U}$ & $<20$ & $\mathrm{U}$ \\
\hline 1,3-Dichlorobenzene & $<20$ & $U$ & $<20$ & $\mathrm{U}$ & $<20$ & $\mathrm{U}$ \\
\hline 1,4-Dichlorobenzene & $<20$ & $U$ & $<20$ & $\mathrm{U}$ & $<20$ & $\mathrm{U}$ \\
\hline 1,2-Dichlorobenzene & $<20$ & U & $<20$ & $\mathrm{U}$ & $<20$ & $\mathrm{U}$ \\
\hline 2-Methylphenol & $<20$ & $U$ & $<20$ & $\mathrm{U}$ & $<20$ & $\mathrm{U}$ \\
\hline bis(2-Chloroisopropyl)ether & $<20$ & $\mathrm{U}$ & $<20$ & $\mathrm{U}$ & $<20$ & $\mathrm{U}$ \\
\hline 3 \& 4-Methylphenol & $<20$ & U & $<20$ & $\mathrm{U}$ & $<20$ & $\mathrm{U}$ \\
\hline N-Nitroso-di-n-propylamine & $<20$ & $U$ & $<20$ & $\mathrm{U}$ & $<20$ & $\mathrm{U}$ \\
\hline Hexachloroethane & $<20$ & $\mathrm{U}$ & $<20$ & $\mathrm{U}$ & $<20$ & $\mathrm{U}$ \\
\hline Nitrobenzene & $<20$ & $U \mathrm{M}$ & $<20$ & $\mathrm{U}$ & $<20$ & $U$ \\
\hline Isophorone & $<20$ & $U$ & $<20$ & $\mathrm{U}$ & $<20$ & $\mathrm{U}$ \\
\hline 2-Nitrophenol & $<20$ & $\mathrm{U}$ & $<20$ & $\mathrm{U}$ & $<20$ & $\mathrm{U}$ \\
\hline 2,4-Dimethylphenol & $<20$ & $\mathrm{U}$ & $<20$ & $\mathrm{U}$ & $<20$ & $\mathrm{U}$ \\
\hline bis(2-Chloroethoxy)methane) & $<20$ & $\mathrm{U}$ & $<20$ & U & $<20$ & $\mathrm{U}$ \\
\hline 2,4-Dichlorophenol & $<20$ & $\mathrm{U}$ & $<20$ & $\mathrm{U}$ & $<20$ & $\mathrm{U}$ \\
\hline 1,2,4-Trichlorobenzene & $<20$ & $\mathrm{U}$ & $<20$ & U & $<20$ & $U$ \\
\hline Naphthalene & $<20$ & $\mathrm{U}$ & $<20$ & $\mathrm{U}$ & $<20$ & $\mathrm{U}$ \\
\hline 4-Chloroaniline & $<20$ & U & $<20$ & $\mathrm{U}$ & $<20$ & $U$ \\
\hline Hexachlorobutadiene & $<20$ & $\mathrm{U}$ & $<20$ & $\mathrm{U}$ & $<20$ & $\mathrm{U}$ \\
\hline 4-Chloro-3-methylphenol & $<20$ & $\mathrm{U}$ & $<20$ & $\mathrm{U}$ & $<20$ & $\mathrm{U}$ \\
\hline 2-Methylnaphthalene & $<20$ & $\mathrm{U}$ & $<20$ & $\mathrm{U}$ & $<20$ & $\mathrm{U}$ \\
\hline Hexachlorocyclopentadiene & $<20$ & $U$ & $<20$ & $\mathrm{U}$ & $<20$ & $\mathrm{U}$ \\
\hline 2,4,6-Trichlorophenol & $<20$ & $\mathrm{U}$ & $<20$ & $\mathrm{U}$ & $<20$ & $\mathrm{U}$ \\
\hline 2,4,5-Trichlorophenol & $<20$ & $\mathrm{U}$ & $<20$ & $\mathrm{U}$ & $<20$ & $\mathrm{U}$ \\
\hline 2-Chloronaphthalene & $<20$ & $\mathrm{U}$ & $<20$ & $\mathrm{U}$ & $<20$ & $\mathrm{U}$ \\
\hline 2-Nitroaniline & $<20$ & $\mathrm{U}$ & $<20$ & $\mathrm{U}$ & $<20$ & $\mathrm{U}$ \\
\hline Dimethylphthalate & $<20$ & U & $<20$ & $\mathrm{U}$ & $<20$ & $\mathrm{U}$ \\
\hline 2,6-Dinitrotoluene & $<20$ & $\mathrm{U}$ & $<20$ & $U$ & $<20$ & $\mathrm{U}$ \\
\hline Acenaphthylene & $<20$ & $\mathrm{U}$ & $<20$ & $\mathrm{U}$ & $<20$ & $\mathrm{U}$ \\
\hline 3-Nitroaniline & $<20$ & $\mathrm{U}$ & $<20$ & U & $<20$ & $\mathrm{U}$ \\
\hline Acenaphthene & $<20$ & $\mathrm{U}$ & $<20$ & $\mathrm{U}$ & $<20$ & $\mathrm{U}$ \\
\hline
\end{tabular}


Table 17. SVOC analyses of NWCF ETS streams during NWCF ETS off-gas emissions

\begin{tabular}{|c|c|c|c|c|c|c|}
\hline \multirow{3}{*}{$\begin{array}{l}\text { Stream } \\
\text { Analysis Log \#: }\end{array}$} & \multirow{2}{*}{\multicolumn{2}{|c|}{$\begin{array}{c}\text { Blended Feed } \\
0106071\end{array}$}} & \multirow{2}{*}{\multicolumn{2}{|c|}{$\begin{array}{l}\text { Bottoms } \\
0106221\end{array}$}} & \multirow{2}{*}{\multicolumn{2}{|c|}{$\begin{array}{c}\text { Condensate } \\
0106214\end{array}$}} \\
\hline & & & & & & \\
\hline & Result & LQ & Result & LQ & Result & LQ \\
\hline \multicolumn{7}{|l|}{ Analyte, units: } \\
\hline 2,4-Dinitrophenol & 420 & $\mathrm{DMH}$ & 44 & $\mathrm{M}$ & 110 & $\mathrm{M}$ \\
\hline 4-Nitrophenol & $<20$ & $\mathrm{U}$ & $<20$ & UM & $<20$ & $\mathrm{UM}$ \\
\hline Dibenzofuran & $<20$ & $\mathrm{U}$ & $<20$ & $\mathrm{U}$ & $<20$ & $\mathrm{U}$ \\
\hline 2,4-Dinitrotoluene & $<20$ & $\mathrm{U}$ & $<20$ & $\mathrm{U}$ & $<20$ & $\bar{U}$ \\
\hline Diethylphthalate & $<20$ & U M & $<20$ & $U \mathrm{M}$ & $<20$ & $\mathrm{UM}$ \\
\hline 4-Chlorophenyl-phenylether & $<20$ & U & $<20$ & U & $<20$ & $\mathrm{U}$ \\
\hline Fluorene & $<20$ & $\mathrm{U}$ & $<20$ & U & $<20$ & $\mathrm{U}$ \\
\hline 4-Nitroaniline & $<20$ & $\mathrm{U}$ & $<20$ & $\mathrm{U}$ & $<20$ & $\mathrm{U}$ \\
\hline 4,6-Dinitro-2-methylphenol & $<20$ & UM & $<20$ & $\mathrm{UM}$ & $<20$ & $\mathrm{UM}$ \\
\hline N-Nitrosodiphenylamine & $<20$ & $\mathrm{U}$ & $<20$ & $\mathrm{U}$ & $<20$ & $\mathrm{U}$ \\
\hline Tri-n-butyl phosphate & & & & U & & $\mathrm{U}$ \\
\hline Azobenzene & & & & U & & $\mathrm{U}$ \\
\hline 4-Bromophenyl-phenylether & $<20$ & U & $<20$ & U & $<20$ & U \\
\hline Hexachlorobenzene & $<20$ & u & $<20$ & U & $<20$ & U \\
\hline Pentachlorophenol & $<40$ & $\mathrm{U}$ & $<40$ & $u$ & $<40$ & $\mathrm{U}$ \\
\hline Phenanthrene & $<20$ & U & $<20$ & U & $<20$ & $\mathrm{U}$ \\
\hline Anthracene & $<20$ & U & $<20$ & $\mathrm{U}$ & $<20$ & U \\
\hline Carbazole & $<20$ & U & $<20$ & $\mathrm{U}$ & $<20$ & $\mathrm{u}$ \\
\hline Di-n-butylphthalate & $<20$ & U & $<20$ & UM & $<20$ & $\mathrm{UM}$ \\
\hline Fluoranthene & $<20$ & U & $<20$ & $\mathrm{U}$ & $<20$ & $\mathrm{U}$ \\
\hline Pyrene & $<20$ & $\mathrm{U}$ & $<20$ & $\mathrm{U}$ & $<20$ & $\mathrm{U}$ \\
\hline Butylbenzylphthalate & $<20$ & $\mathrm{u}$ & $<20$ & $\mathrm{U}$ & $<20$ & U \\
\hline 3,3'-Dichlorobenzidine & $<20$ & $\mathrm{U}$ & $<20$ & $\mathrm{UM}$ & $<20$ & $\mathrm{UM}$ \\
\hline Chrysene & $<20$ & u & $<20$ & $\mathrm{U}$ & $<20$ & $\mathrm{U}$ \\
\hline Benzo(a)anthracene & $<20$ & $\mathrm{U}$ & $<20$ & $\mathrm{U}$ & $<20$ & $\mathrm{U}$ \\
\hline bis(2-Ethylhexyl)phthalate & $<20$ & U & $<140$ & $\mathrm{U}$ & $<20$ & U \\
\hline Di-n-octylphthalate & $<20$ & U & $<20$ & $\mathrm{U}$ & $<20$ & $u$ \\
\hline Benzo(b)fluoranthene & $<20$ & $\mathrm{U}$ & $<20$ & $\mathrm{U}$ & $<20$ & $\mathrm{U}$ \\
\hline Benzo(k)fluoranthene & $<20$ & $\mathrm{U}$ & $<20$ & $\mathrm{U}$ & $<20$ & U \\
\hline Benzo(a)pyrene & $<20$ & U & $<20$ & $\mathrm{U}$ & $<20$ & $\mathrm{U}$ \\
\hline Indeno(1,2,3-cd)pyrene & $<20$ & $\mathrm{U}$ & $<20$ & $\mathrm{u}$ & $<20$ & U \\
\hline Dibenzo(a,h)anthracene & $<20$ & U & $<20$ & $\mathrm{U}$ & $<20$ & $\mathrm{U}$ \\
\hline Benzo $(\mathrm{g}, \mathrm{h}, \mathrm{I})$ perylene & $<20$ & U & $<20$ & $\mathrm{U}$ & $<20$ & $\mathrm{U}$ \\
\hline \multicolumn{7}{|l|}{ Tentatively Identified Compounds: } \\
\hline Unknowns, number identified/conc. & $7 / 501$ & $J$ & $19 / 509$ & $\mathrm{~J}$ & $5 / 130$ & $J$ \\
\hline Tri-n-butyl phosphate & & & & & 17 & $J$ \\
\hline
\end{tabular}




\section{PROCESS OPERATING CONDITIONS}

The NWCF ETS is operated as described in Section 2. The evaporator is initially filled with fresh waste solution. The temperature in the evaporator flash column, prior to initiation of stream is usually between 50 and $60^{\circ} \mathrm{C}$. Steam flow is ramped from 0 to $1730 \mathrm{lb} / \mathrm{hr}$ in 15 to 45 minutes and then maintained for eight to ten hours until the desired solution density is obtained. Approximately 2 to 3 hours is required for the column to reach a full boil at around $100^{\circ} \mathrm{C}$. Fresh feed solution is added to the evaporator until near the end of the batch. The temperature increases $3-4^{\circ}$ throughout the batch as the concentration increases to the target density.

Offgas sampling at the beginning of the evaporator run was synchronized with the initiation of steam to the evaporator. Approximately 3 hours was needed to collect the offgas samples, including the SMVOC runs. This provided a representative average of the emissions during the startup period. SMVOC samples were actually started 15-20 minutes prior to steam initiation in order to capture any burst of volatile organic emissions on the onset of solution heating. Sample collection at the end of the evaporator batches was coordinated with the ETS operators in order to sample during the final 3-4 hours of the evaporator batch.

Process parameter data were collected during the NWCF ETS emissions sampling by the NWCF Distributive Control System (DCS). A history of key process variables was collected using fifteen-minute average data. The data were then tabulated for the times when the sampling was taking pläce. Appendix D compiles the process parameters for the sixteen sample collection runs.

The evaporator operated within normal operating parameters throughout the period of sample collection. The feed batches had very similar densities and temperatures, indicating very similar compositions. The total flow from the NWCF (containing the NWCF ETS emissions) was essentially constant limiting variation due to deposition or re-entrainment.

During the collection of sample train 0060-STRT-02, sampling was interrupted for an emergency drill while the evaporator batch continued. Sampling was interrupted from 0850 to 0922 on June 6, 2001 until sampling personnel were notified that they could continue. During this time, the average temperature in the evaporator increased from 76.4 to 97.4 degrees. 


\section{DQO ASSESSMENT AND PROJECT SURVEILLANCE}

Data quality objectives (DQOs) for the NWCF ETS offgas emissions project are defined in the quality assessment project plan (QAPjP, company document PLN-879). Sample collection in the field was coordinated by the Project Technical Leads (PTL) with independent surveillance performed by the Project Quality Assurance Officer (PQAO). The sample collection activities were monitored by the PTL and PQAO, thus ensuring that the sample collection activities were completed in accordance with the test plan and QAPJP and that the samples were maintained under proper custody and conditions at all times. All changes to the test plan required advance approval from the PTL and PQAO prior to being implemented during sample collection. A standard field change form was used to document the approvals for these changes.

The services of the INEEL Sample Management Office (SMO) were not enlisted to review the analytical data. This was previously completed on the NWCF Calciner offgas emissions inventory, but was not within the budget constraints of the current project. Therefore, a cursory review of the analytical data QA/QC requirements was completed by the PQAO and is provided in lieu of the Limitations and Validation (L\&V) reports that are provided by the SMO on previous projects.

\subsection{Documented Field Changes}

The QAPjP allowed for in-field changes to requirements of the QAPjP and sampling protocols as long as such changes were approved per Section 13.1 of the QAPJP. Also allowed by the QAPJP were properly approved changes to the sampling checklists. Seven field change forms capturing 11 different requested variances from the QAPJP or standard protocol were approved.

\subsubsection{VOC Sample Collection}

Method 0031 for VOCs still requires the storage of Tenax ${ }^{\circledR}$ and Anasorb- $747^{\circledR}$ tubes at less than $10^{\circ} \mathrm{C}$ after tube conditioning, during transport, and up to the time of tube usage in sampling. This is a typical protocol deviation among current laboratory service providers, since it is deemed unnecessary in conjunction with the common practice of sealing the resin tubes in air-tight containers and using a trip blank to identify potential fugitive contamination that may occur prior to use of the tubes in the field and during their return to the laboratory. Conditioned sample media availability, schedule slippage, and laboratory technical guidance were critical factors in the project field decision to waive this requirement via a field change once it was determined that the laboratory had not complied.

The method requirement that conditioned media tubes not be exposed to severe pressure variations during transport is satisfied amply by the multiple layers of containment used by the contract laboratory providing the sample tubes. Therefore, in this regard, no field change was applicable. It should also be noted that all of the Tenax ${ }^{\circledR}$ and Anasorb- $747^{\circledR}$ tubes were cooled to $0-10^{\circ} \mathrm{C}$ immediately following sample collection.

The initial intent of the contract sampling team was to not preserve aqueous samples collected for VOCs (Method 0031 ) by acidification with $8 \mathrm{~N} \mathrm{HCl}$ to a measured $\mathrm{pH}$ of $<2$. The BBWI Project Technical Lead and the PQAO pointed out that it would be preferable to perform this standard EPA preservation protocol on such aqueous samples and on associated reagent water blanks. A field change to the sampling checklist reflecting that guidance was approved and sampling team was so instructed. 
Another field change was proposed and approved to require another pair of Tenax $\circledast /$ Anasorb$747 \AA$ tubes be collected as a field blank for the second day of Method 0031 sampling. The project had already varied in the QAPjP from the standard method requirement for a field blank every two hours of sampling based on the expected sampling period and reviews of cost /benefit and technical applicability of this QC-related method requirement to sample train operations, as scheduled.

The last field change related to the 0031 sample trains and protocols was one to keep the flowrates at or below $0.5 \mathrm{Lpm}$ rather than going with the standard EPA Method 0031 upper limit of 1 $\mathrm{Lpm}$. This allowed four sets of tubes to be ran in the 0031 sampling trains over a typical sampling interval meeting or exceeding the method target sampling time of 2 hours. This is compliant with the intent of Section 1.8 of the standard EPA SMVOC method.

\subsubsection{SVOC Sample Collection}

One field change form was approved specific to the Method 010 trains for SVOCs. This change incorporated a final field dilution of the condensate fractions from these trains from just under a total volume of $500 \mathrm{~mL}$ to a total volume of 1 liter. This reflects a dilution normally performed on the samples after receipt at the laboratory. By performing this dilution in the field under close supervision of the project tech lead and sample team leads, the project was readily able to comply with the $70 \mathrm{~Bq} /$ gram ceiling limit for shipping the associated SVOC samples in a non-radiological classification per DOT regulations.

\subsubsection{SCS Trains and Screening}

A field change was approved to allow for cancellation of a scheduled third radiological contamination evaluation train (SCS-EVAP-3), based on consistent and low radioactivity results obtained for all previous sample contamination trains and routine gamma counting screening that was performed in accordance with MCP-1173, Package and Ship NWCF Offgas Emissions Samples Offsite for Analysis, Revision 2.

\subsubsection{Metals and Anions Sample Collection}

No field changes were required for the metals and anions sample collection runs. All runs proceeded in accordance with the sample collection checklists and test plans.

\subsubsection{Miscellaneous Decisions}

A final field change capturing five different field decisions was processed and approved. Per QAPjP language, not all of the decisions under this field change were required by project planning documents to be documented by a field change. However, the PQAO and the PTL decided it would be a best management practice to well document these decisions, and this process was the most accommodating and represented a configuration management control mechanism totally internal to the project. The following change elements were approved on this change form.

\section{Element1:}

It was determined that the $\mathrm{pH}$ meters supplied by the sampling contractor and in the field for this project could not meet reasonable time to stabilization criteria. Based on this fact, a field decision was made by PQAO and PTL to allow the samplers to do all $\mathrm{pH}$ measurements with $\mathrm{pH}$ paper versus requiring a meter. This was facilitated by the fact that in-field neutralizations of liquid sample fractions was not necessary as in the emissions inventory for 
the NWCF Calciner. Approval was given at beginning of sample collections for the use of indicator paper for $\mathrm{pH}$ measurements in $\mathrm{NaOH}$ impinger sample fractions due to the harsh effects of the high $\mathrm{pH}$.

\section{Element 2:}

This documents and certifies the assumption made early in project that the oxygen monitor readings of the sampled gas stream basically reflected the concentration of oxygen in ambient air. This assumption was used to relax standard EPA method calibration protocols and was already allowed by the QAPjP.

\section{Element 3:}

In conjunction with Element 2 above, it was decided to use oxygen cylinders already in inventory and marked as $20.8 \% \mathrm{O}_{2}$ rather than secure standards as cited in 40 CFR 60 . In an associated decision, it was determined that for this project, the percent drift determinations would be made over time lines comparable to the actual train run times versus the 24-hour comparison baselines found in the 40 CFR 60 regulations which were inferentially tied to continuous monitoring.

\section{Element 4:}

Similar to Element 3 above, two other decisions related to the operation of the "continuous emissions monitoring system (CEMS)" monitors were made before inception of sample collections. First, use of a non-heated Perma-Pure $\AA$ dryer to condition sample stream was authorized. Second, a rotameter with a $0-0.8 \mathrm{Lpm}$ range was selected for use with the $\mathrm{O}_{2}$ monitor. This resulted in a target flowrate of $0.75 \mathrm{Lpm}$ rather than the $1.0 \mathrm{Lpm}$ cited in the standard method.

\section{Element 5:}

Typical condensate recoveries from Method 0050 and Method 0060 trains were much less than $1.0 \mathrm{ml}$. The condensate and knockout $(\mathrm{KO})$ rinse was added to impinger $\mathrm{K} 1-\mathrm{K} 2$ contents and rinses. This was per approved checklists. Additionally, for VOCs, the condensate fraction was topped to fill a $20-\mathrm{ml}$ standard VOA vial and acidified with $8 \mathrm{~N} \mathrm{HCl}$ to a $\mathrm{pH}$ of $<2$. Reagent water blanks for VOCs analyses were processed in same fashion as 0031 condensates. In retrospect this decision element is redundant to other field changes already processed.

\subsection{Data Quality Indicators}

As of this point, all post-laboratory data evaluations for achievement of qualitative objectives and for quantitative data quality indicator acceptance criteria have been performed by the PTL and the PQAO. With the exception of a final evaluation of inter-train precision based on calculations of RSDs associated with results of surrogates from all trains for VOCs and all trains for SVOCs, the data quality indicators presented in the QAPjP have all been evaluated.

Reviews of analytical reports indicated that method performance and associated QC, as depicted in the lab reports, met analytical method and project planning document requirements, with a few instances of failures to meet individual QC criteria. The results for the vast majority of 
associated QC data meet QAPjP criteria and support current designated project uses of this data. As stated, an independent analytical data validation to the cited Statement of Work (SOW) requirements found inER-SOW-156, INEL Sample Management Office (SMO) Statement of Work for Inorganic \& Miscellaneous Classical Analyses, Revision 1, ER-SOW-169, Statement of Work for Organic Analyses Performed for INEL Sample Management Office, Revision 0, or in associated standard analytical methods has not been performed. A full data validation process could potentially lead to additional data qualifications based on standard methods or cited SOWs.

\subsection{Sampling Documentation Reviews}

As in past emissions tests conducted for the NWCF Calciner, all field sheets related to sample collections were reviewed in the field as the sampling proceeded. Reviews were conducted by both the PTL and by the PQAO, and were completed to schedule per the QAPjP. Minor omissions or errors in field-level paperwork were therefore immediately caught and corrections were implemented while the sampling team was still in the field, and/or, if deemed necessary, so that re-sampling could occur with minimal delays or additional costs. Most observations that required corrective actions involved the accuracy or completeness of field data forms, and these instances were actually infrequent with respect to the sheer volume of field data entries required for the project.

All data were manually recorded in the field into the associated logbooks, sample data sheets and sampling checklists associated for each sample train type used during project sample collections. The data on sample data sheets were then uploaded into laptop computer templates of these same respective forms. There were some observed anomalies between some of the field definitions on the paper copies versus those on the electronic templates. These were noted in the individual internal PQAO reports to the PTL during each train type $(0031,0030,0050,0060)$ testing period. The sampling contractor (SAIC) made accommodations in each case by either giving more specific instructions to the sample collectors regarding the required entries on the paper forms, or by changing appropriate entry fields in the electronic templates. All changes were reviewed and approved in the field as required by the QAPjP.

As a result of reviews of the field-level documentation, it was discovered that the sample collectors had noted that sample \#3411, Set\#1 Anasorb-747 ${ }^{\circledR}$ tube for Train 0031-END-1 had a crack at one end. Subsequent reviews of the laboratory entries into the related chain-of-custody forms did not indicate that this condition was noted by lab personnel on receipt of the samples. The laboratory analytical results appear consistent with other corresponding Anasorb- $747^{\circledR}$ results and meet the necessary surrogate and internal standard recoveries.

Reviews of calibration documentation for associated sampling equipment identified no deficiencies with respect to requirements. Reviews of chain-of-custody and request for analysis forms identified no deficiencies in those documents.

\subsection{Records Management}

Records associated with this project have been retained and filed in an approved secure central file location (CFL) per company records management requirements. Project records have been categorized and dispositioned as environmental records and are currently assigned a permanent retention period. Per the project QAPjP and related company requirements and procedures for the designation and management of quality records, the following records have been further characterized as quality records: 
- Quality Assurance Project Plan (QAPjP)

- Test Plans

- Logbooks

- Certificates of Analysis

- Calibrations

- Field Data Sheets

- Field Changes

- $\mathrm{COC} / \mathrm{RFA}$

- Analytical Data and Emissions Calculations Spreadsheets

- QA Reports

- Limitation and Validation Reports (not applicable yet for this project phase)

- Final Reports

Some of the above records are in paper form, some in electronic format, and some in dual media. All are stored appropriately in locked cabinets and controlled key access within the designated CFL. A file index has been prepared for these project records, and is available at the CFL. All records in CFL have been assigned appropriate INEEL uniform file codes in compliance with associated company procedures for management of files and records.

\subsection{Review of Spreadsheet Calculations}

As part of their contracted services, the sampling contractor provided spreadsheets which captured all appropriate field sampling data, analytical data, and project-required plant operations data, and which calculated from this data estimated total emissions rates from the NWCF ETS. As referred to elsewhere in this report, these estimates are conservative contributors to the emissions related to the operation of the ETS itself because other sources of miscellaneous plant tank operations continuously emit purge and/or vessel sparging gases to this same NWCF offgas line.

The first line of independent review and QC of these contractor spreadsheets occurred internal to the contractor. A second $100 \%$ review was conducted by the PTL, with any required corrections being implemented in conjunction the contractor for sake of configuration management and documenting general agreement with changes. Lastly, the PQAO reviewed in excess of $20 \%$ of the resultant pertinent entries after each iteration of changes. This comprehensive and iterated approach exceeded the basic requirement of the QAPjP that the PQAO verify only $20 \%$ of all entries and resultant calculation values. Once the sampling contractor lead, PTL, and PQAO were in agreement with the acceptability of the spreadsheets, the spreadsheets were noted as verified and released to be used on final report compilations. 


\subsection{Analytical Results}

\subsubsection{Data Reporting and Flagging}

The QAPjP, associated task order statements of work, and technical lead guidance to the analytical laboratory defined project-specific requirements for data flagging, assignment of " $<$ " symbols, and selection of most conservative and technically defensible result values for purposes of inserting conservative (high-biased) estimators of emissions rates for each identified compound into the emissions calculations. These requirements were in addition to the standard method-related data qualification flagging, and derive from the following EPA guidance documents:

- EPA 1998a, Guidance on Collection of Emissions Data to Support Site-Specific Risk Assessments at Hazardous Waste Combustion Facilities, EPA530-D-98-002, August.

- EPA 1998b, Human Health Risk Assessment Protocol for Hazardous Waste Combustion Facilities, EPA530-D-98-001 A, July, Appendix A-1, Table A-1.

Rule 1. When there was a non-detect below the MDL in a train fraction for a given compound, then the lesser of the RDL or the RL was used.

Rule 2. When the laboratory RL is greater than the RDL, and a compound was detected above the MDL in a train fraction, but the result was less than the RDL, then the RDL was used.

Rule 3. When the RL is less than the RDL, and a compound was detected in a given train fraction above both the MDL and RL values, but less than the RDL, then the RDL was used.

Rule 4. When the RL is less than the RDL, and a compound was detected above the MDL, but result was less than the RL, then the RL was used (not the RDL).

Rule 5. Any compound that was detected above the RDL was used for the risk calculation applications and no "<" ("less than") sign was assigned for that result in the respective train fraction.

Rule 6. When a result for a SVOC or VOC target compound in a given train fraction was greater than MDL, but less than the RL, and result was assigned an estimated flag, the " $<$ " flag was also assigned.

Rule 7. Whenever a "<" flag was assigned to any given train fraction result, then the "<" flag propagated to the train total for that compound, unless it was dropped due to protocol of rounding to significant figures in the train total summation process. (ASTM E29-93a 1999).

Rules 2 and 5 default to the RL, not the RDL, when the RL is less than the RDL, and the "hit" was less than the RL. This was justified since the RL is a statistically established conservative RL, established by the analytical laboratory. Therefore, team assessed uses of RL value for risk calculations as a justified approach. 
Most of the lab-assigned data qualifier flags are based on lab methods and procedures, and are standard to a large majority of environmental services laboratories. For metals analyses, the QAPjP required " $\mathrm{B}$ " flags to be assigned to metals results greater than MDL but less than the Reporting Limit.

Additionally, a system of assigning project flags "P", "N," and "A" to train total results was developed to evaluate the significance of each given target analyte result for the complete sampling train based on the relative occurrence of real hits for the various train fractions that comprised each sample train. The "P" indicated that related compound was detected in some train fractions, but not all. The "N" indicated that there was no positive detection in any train fraction for that compound. Lastly, the "A" flag on the train total result indicated that the compound was detected in all of the train sample fractions.

\subsubsection{Blank Corrections}

Blank corrections for metals trains are allowed by the cited EPA guidance documents and standard air sampling methods. Reagent blank corrections were only made where actual hits occurred in the reagent blanks.

The performance of the SMVOC blank sample indicated that residual acetone and methylene chloride was present in the sample collection line connecting the probe to the train setup. The resulted from failure to adequately purge the sample collection line following collection of the SVOC samples which involves rinsing the line with a mixture of acetone and methylene chloride. The VOC results showed a rapid decay of acetone and methylene chloride in successive sample fractions. In spite of the fugitive contamination (which was included in the emissions inventory), volatile species emissions were very low; therefore, this problem is not considered a significant limitation of the data set. In order to correct this anomaly, it is recommended that a separate VOST probe always be used to collect the SMVOC trains. In the present inventory, the Project Technical Lead authorized the SVOC Method 5 probe for the SMVOC runs to reduce the risks associated with insertion and removal of a second probe into the NWCF offgas duct.

\subsubsection{Data Reporting}

Data reporting from laboratory was comprised of Certificates of Analysis, EDDs, and actual final reports which included complete data packages constructed to the lab's data package level equivalent to the INEEL Tier 1 reporting requirements. The INEEL internal documents that prescribe these data package expectations are ER SOWs -156 and -169 . These reporting requirements were meant to allow for later validation of data should project management decide to subject this data to either a Level A or a Level B data validation per existing INEEL Sample Management Office procedures. Such a future validation decision might be made based on further identified uses of data or other criteria such as permit support, operational changes, planned facility modifications, etc.

\subsubsection{Analytical QC}

\subsubsection{MS/MSD and LCS/LCSD Samples}

Although analytical performance is treated in Section 5 of this report, there are some laboratory related QC aspects of results which need to be discussed in this section. The first QC topic area deriving from QAPjP relates to the selection and analysis of matrix spike samples and matrix spike duplicate samples, as well as the alternative approach of analyzing laboratory control sample spikes and duplicates. 
The structural configuration of these EPA standard methods air sampling trains and nonhomogeneity of matrices present in the various train sample fractions collected from the trains make traditional application of matrix spikes, matrix spike duplicates, and even sample splits very difficult. Splitting of sample fractions for analyses to calculate precision estimators obviously raises associated detection limits for those trains. Simultaneous or sequential operation and sample collections from two or three complete trains in order to derive estimates of precision and accuracy can quickly become very expensive and time consuming.

For this project, the PTL and contract laboratory project manager determined that either MS/MSDs or LCS/LCSDs with surrogate spikes would be utilized by analytical lab for various analyses. These associated surrogate spikes and acceptance criteria are listed in Table 3-1 of QAPjP. The RPD and percent recovery of these spikes were determined and provided by the contract laboratory. Evaluation of these data quality indicators was then accomplished later by the project team, using the criteria found in QAPjP Table 3-1.

The approach taken by laboratory for SVOCs was to analyze a front-half Composite LCS/LCSD and a back-half composite LCS/LCSD. For the aqueous matrices associated with 0010 trains, not only was a LCS/LCSD pair analyzed, but the lab also performed a 3-way split of the condensate and impinger contents for Run 2, using two of the split fractions to develop a MS/MSD pair. This allowed for generation of important matrix-specific information, but also tripled the detection limits for the non-QC sample aliquot. This is also discussed to a degree in Section 5.

Additionally, the XAD-2® tubes utilized in SVOC sampling were pre-spiked with $200 \mu \mathrm{g}$ of a ${ }^{13} \mathrm{C}_{3}$-Naphthalene sampling surrogate. The determined recoveries of this surrogate were evaluated later in the project, and are discussed in Section 5 of this report.. Application of this labeled surrogate occurs prior to sample train operation and is a comprehensive estimator for the overall accuracy of surrogate application, collection method, laboratory sample prep, and analytical method. Additionally, recovery result is an indicator of potential losses of surrogate (or other SVOCs) or sample media cross-contamination occurring during shipments of same media to field or during shipment of collected samples back to the lab. All of the recovery results for this labeled sampling surrogate were in control.

With regard to VOC analyses of 0031 train samples, the same approach was taken, except that the QAPjP listed the recovery surrogates typical to Method 0031 and the following matrix spike compounds: 1,1-dichloroethane, trichloroethane, benzene, toulene, and chlorobenzene. These were selected with technical guidance from the INEEL SMO and reflect a subset of the standard surrogates used in the laboratory method which implements SW-846 Method 8260B. No spikes of the Tenax $\otimes$ media prior to sample collection were required. Again, acceptance criteria for these data quality indicators related to precision in accuracy were presented in QAPjP Table 3-1. The analytical report from the lab does not discuss results for such a LCS/LCSD. This may be a potential project-specific deficiency in the VOCs analyses unless additional data are located in the raw data packages.

For the Method 0060 metals trains, performance of post digestion spikes in accordance with EPA Method 6010B was an authorized approach. Additionally, for mercury, the QAPjP specified a MS/MSD pair. All associated acceptance criteria in terms of RPDs and percent recoveries were given in QAPjP Table 3-1. Again, for the metals train configuration there is no technically representative way to pre-spike train fractions before sample collection. As of time of this report, PQOA has not confirmed that lab reported associated LCS/LCSD results required by QAPjP.

Given the complexities of these matrices, the project team chose not to define sample fraction selection criteria or required frequencies of MS/MSDs to the lab in the QAPJP. Ongoing technical 
consultations between the analytical lab and PTL determined the exact approaches which were taken in this regard for MS/MSD and LCS/LCSD analyses. They are included in the Requests-for-Analysis that were included in the Task Order Specific (TOS) Statement of Work (SOW) for sample analysis.

\subsubsection{Performance for Internal Standards and Surrogates}

Results for internal standards and surrogates for the VOCs analyses appeared to be acceptable based on QAPjP criteria. One outlier was Anasorb tube sample \#3364 where results were non-usable. Some difficulties were encountered in recovery of the internal standard perylene- $\mathrm{d}_{12}$, one of the six internal standards for the $8270 \mathrm{C}$ analysis of the SVOC samples. In order to obtain acceptable recovery of this standard, it was necessary for the analytical laboratory to dilute the samples. This increased the detection limits for the reference target analyte species. A more complete discussion on this anomaly is provided in Section 5 and the STL Final Analytical Report (STL 2001).

In summary, all QA/QC criteria meet the data quality objectives with only two notable exceptions. First, there was some difficulty in recovering perylene- $\mathrm{d}_{12}$, one of the six internal standards used by the contract analytical laboratory for the Method $8270 \mathrm{C} \mathrm{SVOC}$ analysis. The project used a trial XAD- $2^{\mathbb{B}}$ to determine that the performance based QA/QC indicators would likely be achieved without modifications to the sample collection or analytical procedures. Thus, the poor performance of perylene- $\mathrm{d}_{12}$ was not expected. It was necessary to dilute the final sample volume using methylene chloride (the same organic solvent that is used to extract the samples from the sample collection media) and then to "re-shoot" the sample with the GC/MS instrument. Dilutions of 10-100 times were needed to achieve acceptable recoveries of the perylene- $d_{12}$ standard. The implication of this result is that a few of target SVOC analyte data can only be considered an estimate, although the data are still usable for the emission inventory. This should not be considered a serious limitation of the data since all of the SVOC target analytes were typically less than the laboratory RL. In order to avoid this problem in future evaporator emissions testing, it is recommended that successive $1 \mathrm{~mL}$ samples be withdrawn from the sample solution during concentration (i.e., "blowdown') of the methylene chloride extraction solvent. This should be completed for the first run to determine the maximum concentration that is possible without failing to meet the specified internal standards recovery efficiencies.

Second, surrogate compound recoveries for one Anasorb ${ }^{\circledR}$ tube (A-3364 in Run 0031-STRT-1) failed to meet the acceptable recovery range. The results of the three corresponding Anasorb ${ }^{\circledR}$ tubes for this run were averaged and substituted for this tube. Surrogate performance and internal standard performance for all other Tenax $^{\circledR}$ and Anasorb ${ }^{\circledR}$ tubes was generally excellent; therefore, the quality of the four SMVOC runs provide an accurate measurement of the target VOC analytes. Thus, this limitation did not significantly impact the run results. The run total was comparable to the results for the other 3 SMVOC runs.

\subsection{Request for Analysis and Chain-of-Custody Forms}

The analytical services laboratory used for this project utilized a system of pre-printed labels for samples based on a predetermined master sample list, in conjunction with "Request-for-Analysis" (RFA) and COC forms. This system greatly minimized chances for sample identification errors during the sample collection process. This is critical when there are multiple sample fractions for each train, many of which must be accurately combined either in the field or at the laboratory after sample receipt and log-in. 
Reviews of closed out COC forms indicated only one instance of receipt of a shipping container without all container seals being intact. That occurrence was for the final probes rinses collected on June 25, 2001. There were otherwise no indications of any sample abnormalities observed for any sample receipts by the lab. All shipments of samples for analysis for organics arrived within acceptable temperature ranges per EPA criteria for sample preservation. Per the standard EPA methods, samples from 0050 and 0060 trains were not cooled during or prior to shipment.

\subsection{Field Assessments by PQAO}

In adherence with the QAPJP requirements for field assessments of sampling activities, surveillances/assessments were conducted for a single complete train run from each type of sample train used during the testing period for the NWCF ETS emissions. As a result some field changes processed, and corrective actions were taken in the field whenever necessary. There was no necessity per company procedures to initiate any forms for potential discrepancies or nonconformance reporting. Corrective actions were facilitated in the field during the respective sampling period.

Multiple types of checklists were utilized. Some criteria were based on the sampling protocol checklists themselves, while others dealt with good lab practice and work-site housekeeping. Housekeeping and lab practices ranged between acceptable to exemplary. These aspects were very important to this project given the spatial constraints of the sample collections area, rapidity of work schedule, and the multiple tasks occurring within the associated work hoods. Importance ranged from quality to safety, spill prevention, waste management, and effective radiological controls.

Field observations by the PQAO of the sampling team use of $\mathrm{pH}$ meters supplied by their company to perform $\mathrm{pH}$ measurements on project samples exhibited a failure of these meters to perform adequately with regard to stabilized readings in the buffered calibration standards or project samples. The PQAO, PTL, and SAIC sampling team lead agreed that substitution of $\mathrm{pH}$ indicator paper for all field $\mathrm{pH}$ measurements had adequate accuracy. This was facilitated by the fact that no sample neutralizations were required for this project phase. The section of this report dealing with field changes also discuses these points.

For this project, it was technically determined prior to inception of field activities that CEMS was not required to monitor the same miscellaneous gaseous emissions components that were a concern when the NWCF Calciner was running and being tested for emissions. In the past these emissions parameters included $\mathrm{O}_{2}, \mathrm{CO}_{2}, \mathrm{CO}, \mathrm{NO}, \mathrm{NO}_{2}, \mathrm{HCl}, \mathrm{CH}_{4}, \mathrm{SO}_{2}$ and total hydrocarbons (THC) and these derived from the aspects of feed to the Calciner and the physical presence of combustion products in the NWCF Calciner process. $\mathrm{HCl}$ emissions of ETS were evaluated using the results of the samples collected from the scheduled Method 0050 trains. For this project, dynamic monitoring was considered to be of technical value only for $\mathrm{O}_{2}$ emissions. Additionally, it was predicted that the oxygen levels would be extremely close to those of ambient air. The monitored results from $\mathrm{O}_{2}$ monitor throughout sampling campaign did actually reflect that oxygen concentration levels in the sampled offgas were essentially the same as ambient air concentrations of $\mathrm{O}_{2}$.

Latitude was built into the QAPJP regarding the operation and the calibration of the $\mathrm{O}_{2}$ monitor. Relief was given from the regulatory (40 CFR 60) requirements for the associated calibration gases, \% Drift measurements, calibration frequency, etc. Additionally, the technical applications to associating the $\mathrm{O}_{2}$ levels with fairly short interval sample train runs, versus the usual regulatory application of 24-hr continuous monitoring, justified the relaxed protocol. It was determined that an appropriate calibration frequency for this monitor was that the calibrations must 
occur prior to and after each test period, but not to exceed 24 hours between calibrations.

Requirements were adequately implemented in the field. As part of the assessment of the operation and calibrations of $\mathrm{O}_{2}$ monitor it was documented that samplers were misinterpreting one entry associated with the documenting of \% Drifts for this monitor. They were appropriately instructed in correct completion of the calibration sheets, and no further problems were observed. 


\section{OFFGAS EMISSIONS AND HEALTH RISK}

Species emissions rates were calculated for all target analytes and tentatively identified compounds emitted from the NWCF Evaporator Tank System. Data for the train totals listed in Appendix A and the field sample collection data listed in Appendix B were compiled in an Microsoft Excel Program spreadsheet to compute both emissions rates [g/s basis] and offgas concentrations $[\mu \mathrm{g} / \mathrm{dscm}$ basis], relative to conditions in the NWCF offgas duct where sampling was performed. Both the emissions rates and offgas concentrations summary sheets are included in Appendix B. Only a limited interpretation of emissions trends and potential risk to public health has been made at this time.

\subsection{Emissions Rates and Trends}

It was postulated that the release of organic compounds and volatile mercury present in the tank wastes would be higher at the start of the batch when the evaporator is filled with fresh feed. Conversely, it was hypothesized that metals emissions rates would increase with the density of the evaporator contents since the mechanism for non-volatile metals is primarily attributed to aerosol entrainment. Evolution of the organics, however, especially the semi-volatile organics, is a function of the evaporator temperature.

Figure 6 shows the average evaporator vessel temperature during a typical Method 5 sample collection period at the start and the end of an evaporator batch. The temperature was initially lower at the start of the run when steam to the heating coils was initiated. The temperature gradually increased, reaching the desired operating temperature of approximately $100^{\circ} \mathrm{C}$ at around 130 minutesalmost $3 / 4$ through the first sampling period. Fresh feed to the evaporator was not increased significantly until the evaporator temperature reached the boiling temperature. The sample collected at the end of the evaporator run was performed when the evaporator vessel was at the peak temperature. At this time, feed was continually being supplied to maintain a constant volume in the evaporator. The volume of waste solution fed to the evaporator during the final three hours of the batch varied, but was approximately equal to $1 / 2$ of the evaporator batch. 


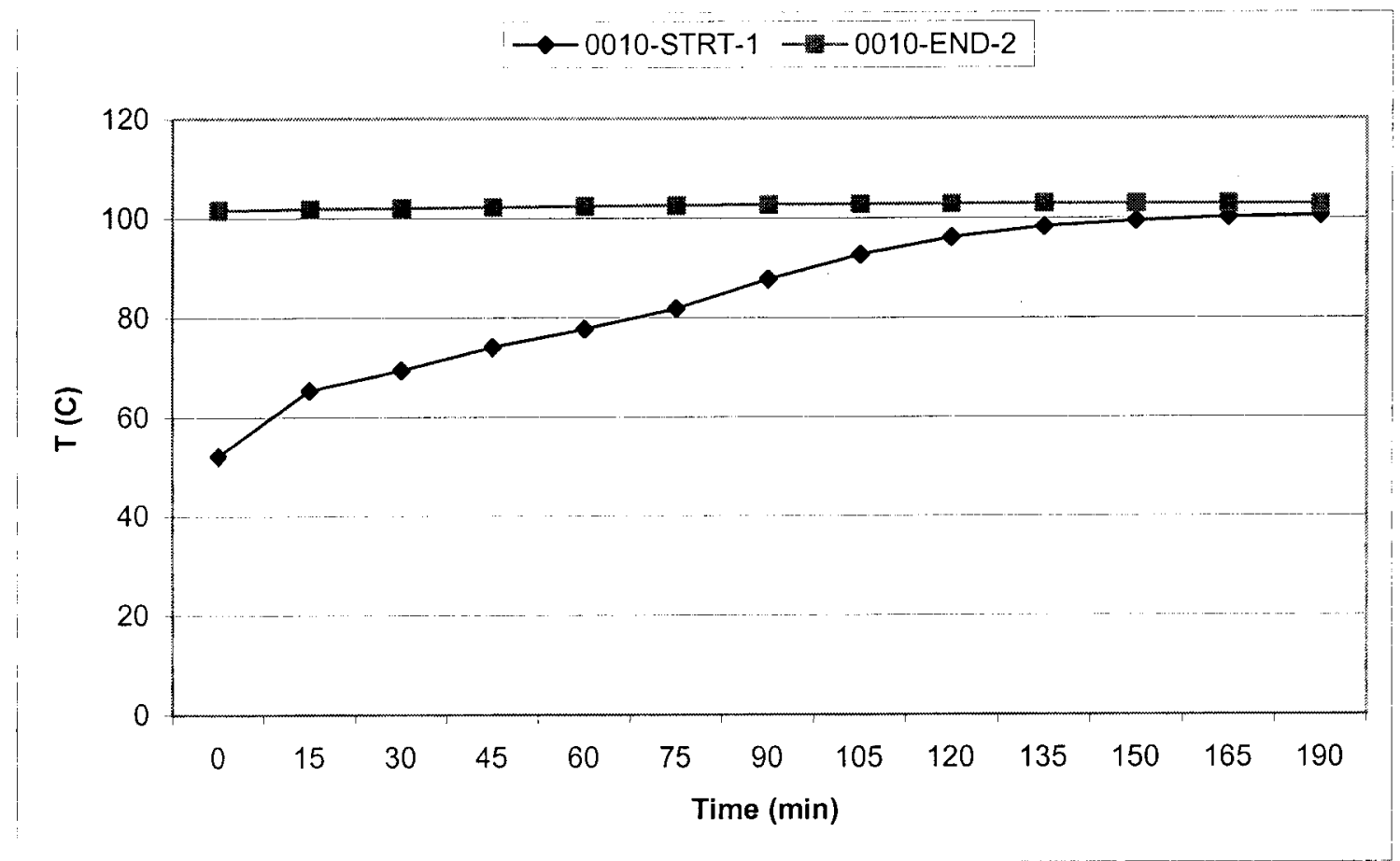

Figure 6. Average evaporator vessel temperature comparison for SVOC runs at the start and end on the evaporator batch.

\subsubsection{Organic Compounds}

Figure 7 plots the concentration of the 20 highest VOC compounds emitted from the evaporator. There are surprisingly small differences in the emissions rates at the beginning and end of the evaporator batch. The single highest volatile organic detected was dodecane, which was not a target analyte, but was reported as a tentatively identified compound. On a volumetric basis, the concentration of dodecane was only $54 \mathrm{ppbv}$. Acetone emissions were also relatively high, but still were in the low parts-per-billion range (i.e., $30 \mathrm{ppb}$ maximum). Some of the other volatile organic measurements were actually higher at the end of the batch, although the results were near the method detection limits and were susceptible to some process variations and sampling uncertainty at these lower levels. 


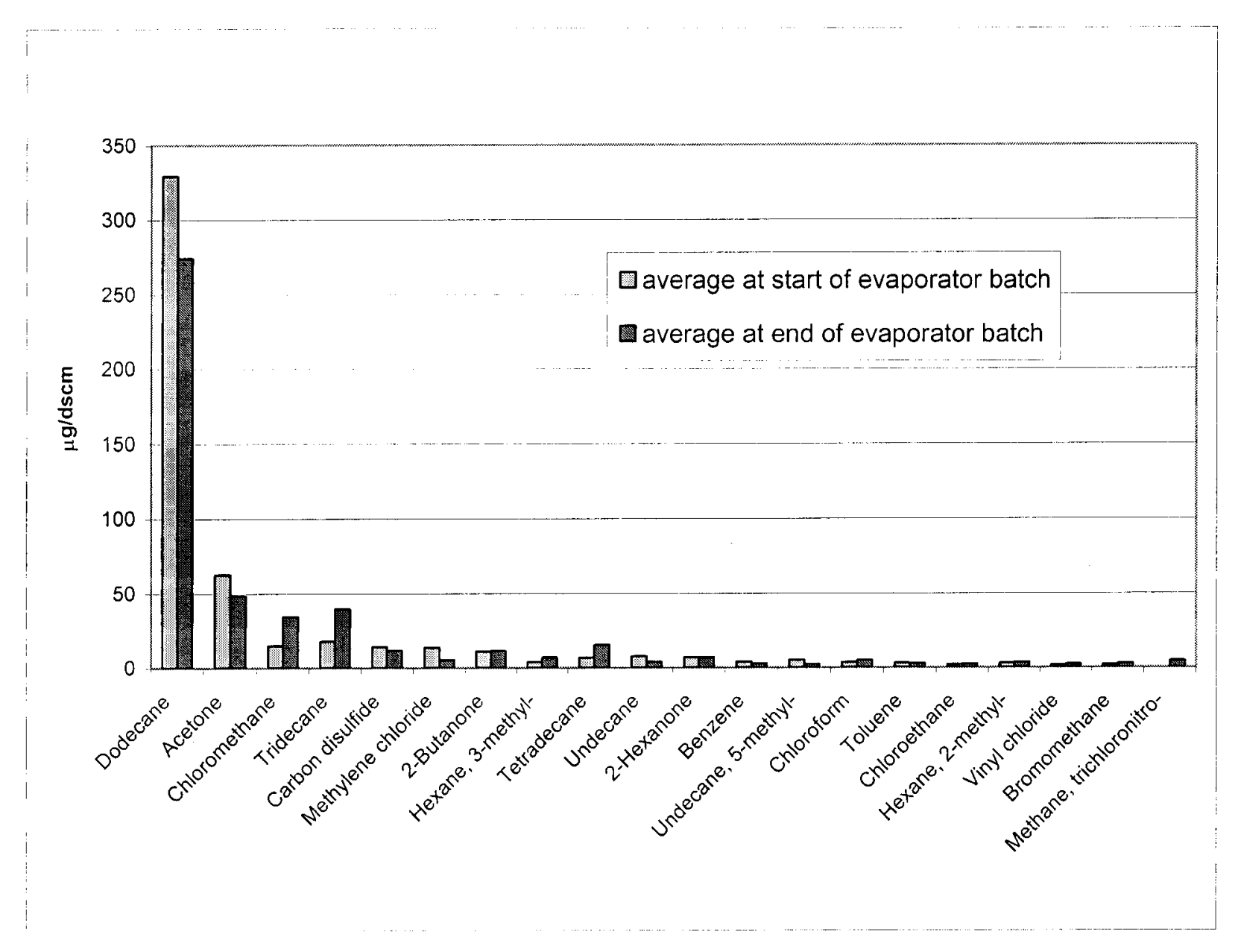

Figure 7. Comparison of volatile organic emissions at the beginning and end of evaporator batches.

The top 20 SVOCs measured in the offgas stream are plotted in Figure 8. Semivolatile organic compounds also appear to be only slightly higher at the start of the evaporator batch. Benzoic acid (a target analyte) and benzaldehyde (a tentatively identified compound) were the two most prevalent semivolatile organics emitted during operation of the NWCF ETS. The maximum emissions concentrations for benzoic acid and benzaldehyde were $310 \mathrm{ppb}$ and $80 \mathrm{ppb}$, respectively. On a volumetric basis, the sum of all volatile and semivolatile organics is less than $1 \mathrm{ppm}$. All other SVOCs measurements are near the method detection limits for the respective species.

With the exception of benzoic acid, all of the SVOC species emitted from the evaporator were also detected during the NWCF Calciner offgas emissions inventory (Boardman 2001). Nearly all of the compounds are derivatives of benzene or other cyclic compounds and are possibly the products of incomplete combustion of the kerosene used to heat the Calciner. These compounds probably entered the tank system when Calciner scrub was recycled to the tank farm. It is further postulated that benzoic acid and benaldehyde were either formed during combustion of the kerosene or they were formed by oxidation of benzene and toluene in the acidic waste solutions. Relatively higher emissions of benzoic acid can be explained by noting that it is readily stripped from waste solutions by steam. 
In summary, the rate of organic emissions at the start and end of the evaporator batches were not significantly different. This phenomena is attributed to the trade off between evaporator temperature and the volume of fresh waste solution fed to the evaporator during the respective sampling periods. The hourly total emissions rate for all volatile and semivolatile organic emissions was less than $0.02 \mathrm{lbs} / \mathrm{hr}$. This is significantly less than the $3 \mathrm{lbs} / \mathrm{hr}$ limit that is generally considered significant for RCRA waste treatment units permitting decisions.

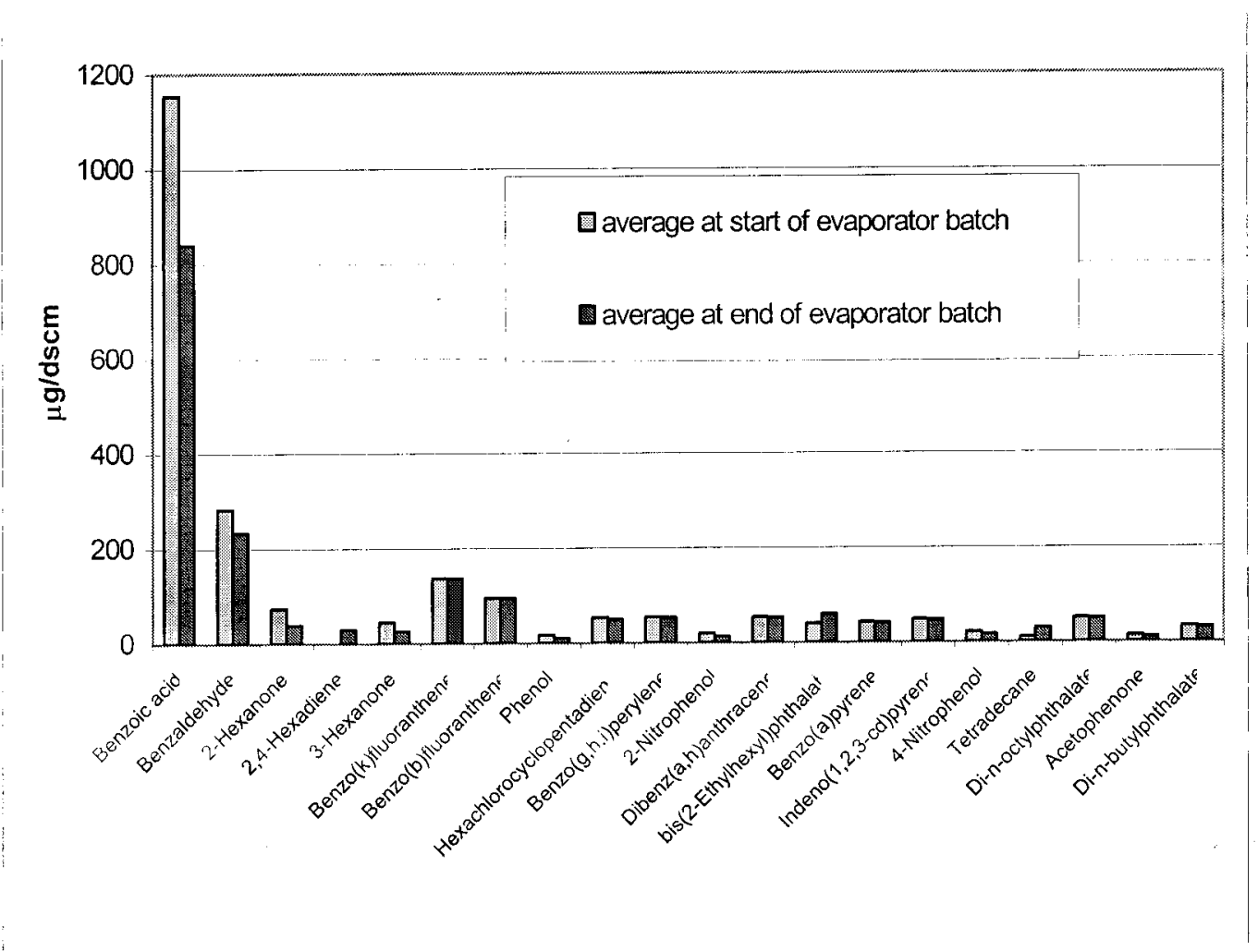

Figure 8. Comparison of semi-volatile organic emissions at the beginning and end of evaporator batches

\subsubsection{Inorganic Compounds}

The average metals emissions rates at the beginning and end of evaporator batches are plotted in Figure 9. As anticipated, metals emissions, including mercury, were typically higher at the end of an evaporator batch when the evaporator solution reached its maximum density. Assuming aerosol droplet entrainment with the overhead gas was constant throughout the run, then the emissions of all nonvolatile species should correlate with the solution density. The exception is mercury. If volatile elemental mercury exists as a dissolved gas in the waste solutions, then it would tend to be volatilized at the beginning of the evaporator runs. Otherwise, if the mercury is complexed or speciated in the wastes, then it would tend to be emitted as a non-volatile entrained species. These data indicate that mercury was mainly emitted as non-volatile particulate, although no speciation was attempted to distinguish elemental versus oxidized forms of mercury in the effluent gas stream. Aluminum, manganese, and zinc emissions appear to correlate with their relative abundance in the evaporator feed and bottoms. The emissions of all other metals were relatively low, as were their concentrations in the feed. 


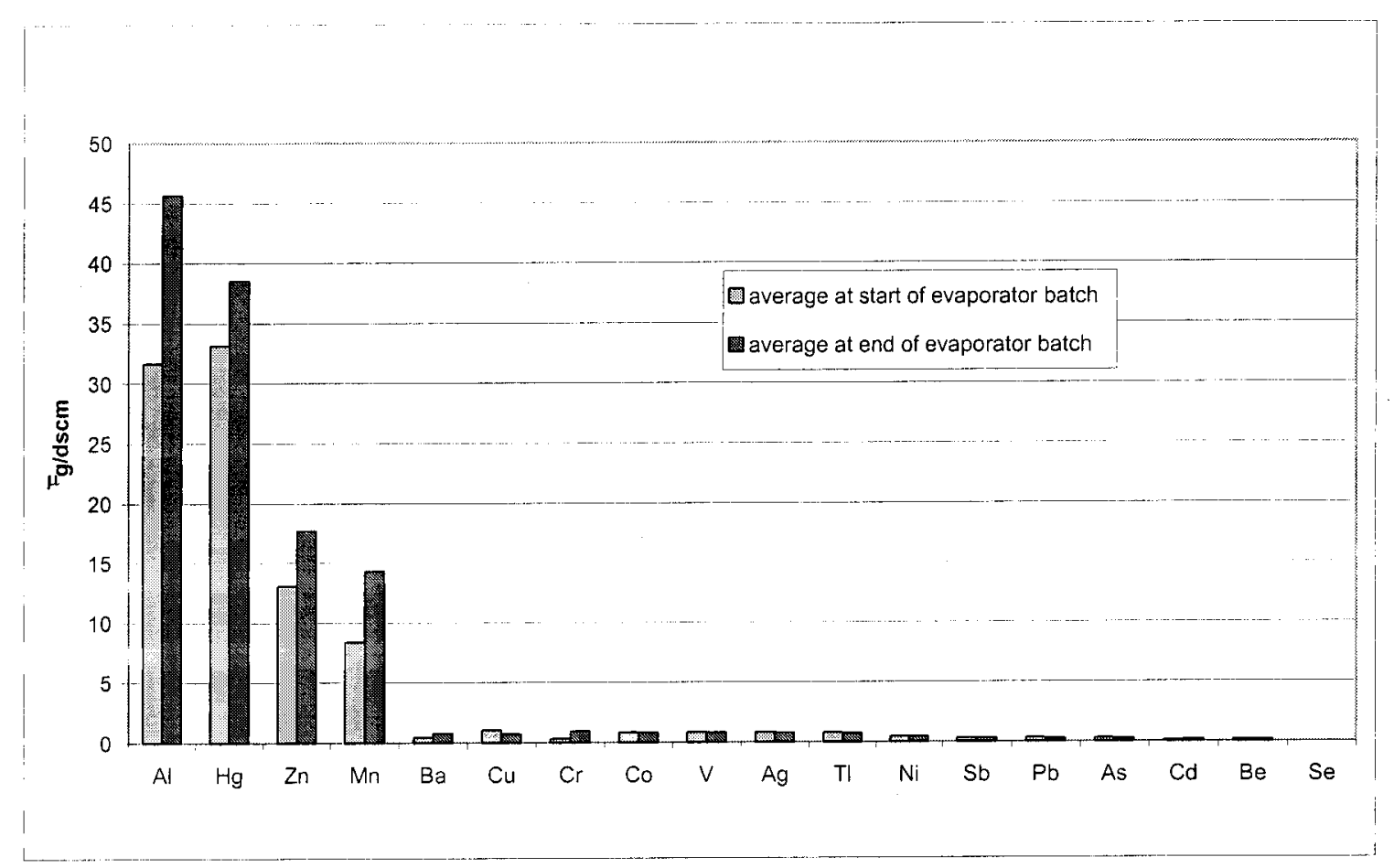

Figure 9. Comparison of metals emissions at the beginning and end of evaporator batches (not including final probe rinse species apportionment).

Throughout the sampling period, it was necessary to leave the 12-ft Method 5 sample collection probe at a fixed position in the offgas duct to reduce the potential for contamination spread and personnel radiation exposures. The probe was removed at the end of the 3 -week sampling period and rinsed with acetone, followed by nitric acid, to obtain a final probe rinse measurement of particulate and metals absorbed on the probe's glass liner. Approximately $93 \mathrm{dscm}$ of offgas sample was drawn through the probe over the duration of the offgas emissions inventory. The apportioned amount of particulate and metals for a single run is thus roughly $3 / 93[\mathrm{dscm} / \mathrm{dscm}]$ or $3.2 \%$ of the total particulate and metals mass measured in the final probe rinse. Thus, $3.2 \%$ of the particulate and metals detected in the final probe rinses should be apportioned to the run averages.

The difference between the four most abundant metals detected in the offgas and the apportioned amount in the final probe rinse, relative to the average emissions was $<10 \%$ for $\mathrm{Zn},<6 \%$ for $\mathrm{Al},<0.5 \%$ for $\mathrm{Mn}$, and $<0.1 \%$ for $\mathrm{Hg}$. The percent of apportioned probe mass for the minor species was also typically low, although a comparison of the results is skewed by the fact that the measurements are near or below the analytical method detection limits. In conclusion, these results indicate that the metals uptaken on the probe liner were insignificant with respect to obtaining an accurate emissions inventory for the target metals species.

Total particulate and chloride emissions rate averages at the start and end two evaporator batches are illustrated in Figures 10 and 11 . There were relatively small differences in the chloride emissions at the start and end of the batch. Hydrochloric acid levels are significantly higher as expected. Still, the sum of chloride emission contributions from $\mathrm{HCl}$ and $\mathrm{Cl}_{2}$ is less than 1 ppmv. 
Particulate emissions follow the trend of the semi-volatile organic species which were slightly higher at the beginning of the batch. Figure 11 also compares the apportioned particulate measurement for the final probe rinse (viz., $3.2 \%$ of the final probe particulate measurement as discussed above). The relative amount of particulate absorbed on the probe was $20-25 \%$ of the train total. It can be inferred that some SVOCs were also deposited on the probe liner, with the maximum being similar to the particulate. This fact should be taken into consideration when using the SVOC emissions rates.

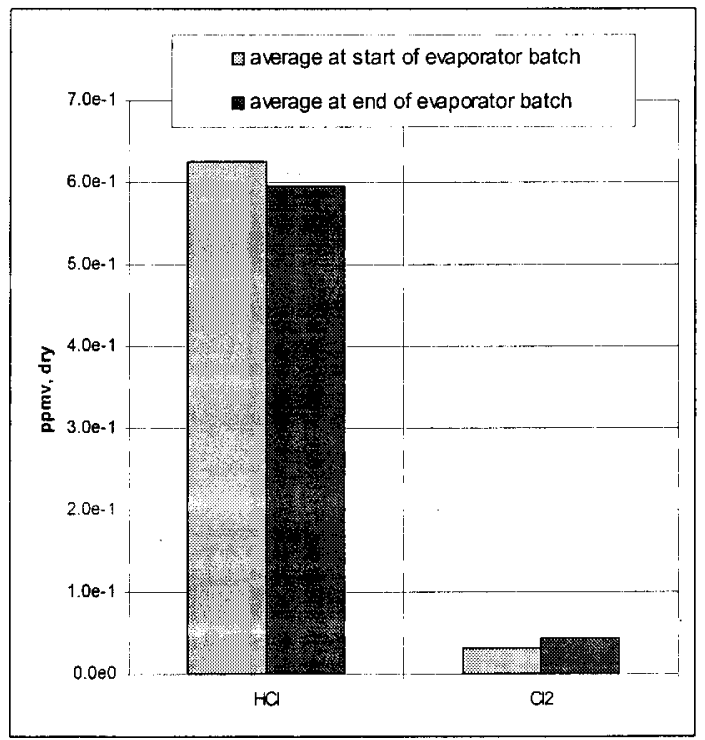

Figure 10. Comparison of chloride emissions at the beginning and end of evaporator batches.

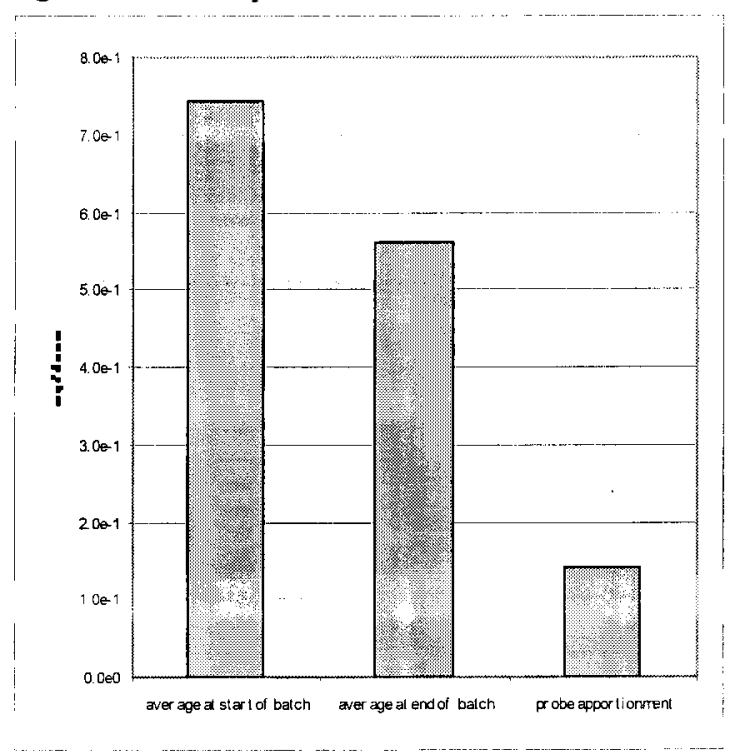

Figure 11. Comparison of particulate emissions at the beginning and end of the evaporator run with probe particulate apportionment. 


\subsection{Emissions Health Risk}

The emission rates of hazardous air pollutants from the NWCF ETS were used to calculate risk to human health. Pollutants from the NWCF ETS are released from the same point and under the same conditions as NWCF Calciner emissions. Therefore, to a close approximation, the NWCF ETS can be scaled to the risk terms previously determined for the NWCF Calciner. The NWCF Calciner results are described in NWCF Calciner Emissions Inventory -Final Report for Phase IV Testing (2001).

Compounds with an EPA hazards quotient (HQ) or a cancer risk (Risk) present in the NWCF ETS samples, were ratioed to the NWCF Calciner emissions rate to determine an estimated NWCF ETS risk. Maximum values were used to bound risks. The NWCF Calciner emission rates were normalized to an annual basis. To compare the NWCF ETS results, measured NWCF ETS emissions were multiplied by a factor of 0.274 to normalize them to an annual basis. This is based on the NWCF ETS operating twelve hours a day, 200 days a year.

It was observed that the emissions rates were much lower for the NWCF ETS than from the NWCF Calciner with the exception of benzoic acid. The semi-volatiles were the largest contributor to the HQ and the Risk. The largest contributor was a phthalate (bis (2 ethylhexyl)phthalate) which is a common sampling or laboratory contaminate from plastics such as tubing, bottles, etc. Most of the materials "detected" were present at levels below the RL and in only a few samples. Benzoic acid, the single organic found in high concentrations than the NWCF Calciner, has a relatively low cancer risk and hazard quotient compound. The total HQ for the NWCF ETS was $6.2 \mathrm{e}-6$ as compared to 3.3 e-03 for the NWCF Calciner and 0.25 for the EPA target criteria. The cancer risk was $1.3 \mathrm{e}-10$ compared to $1.9 \mathrm{e}-07$ for the NWCF Calciner and an EPA target of 1e-5.

A risk summary is given in Figures 12 and 13. Tables 18, 19, 20, and 21 give the concentration ratios and scaled risk factors.

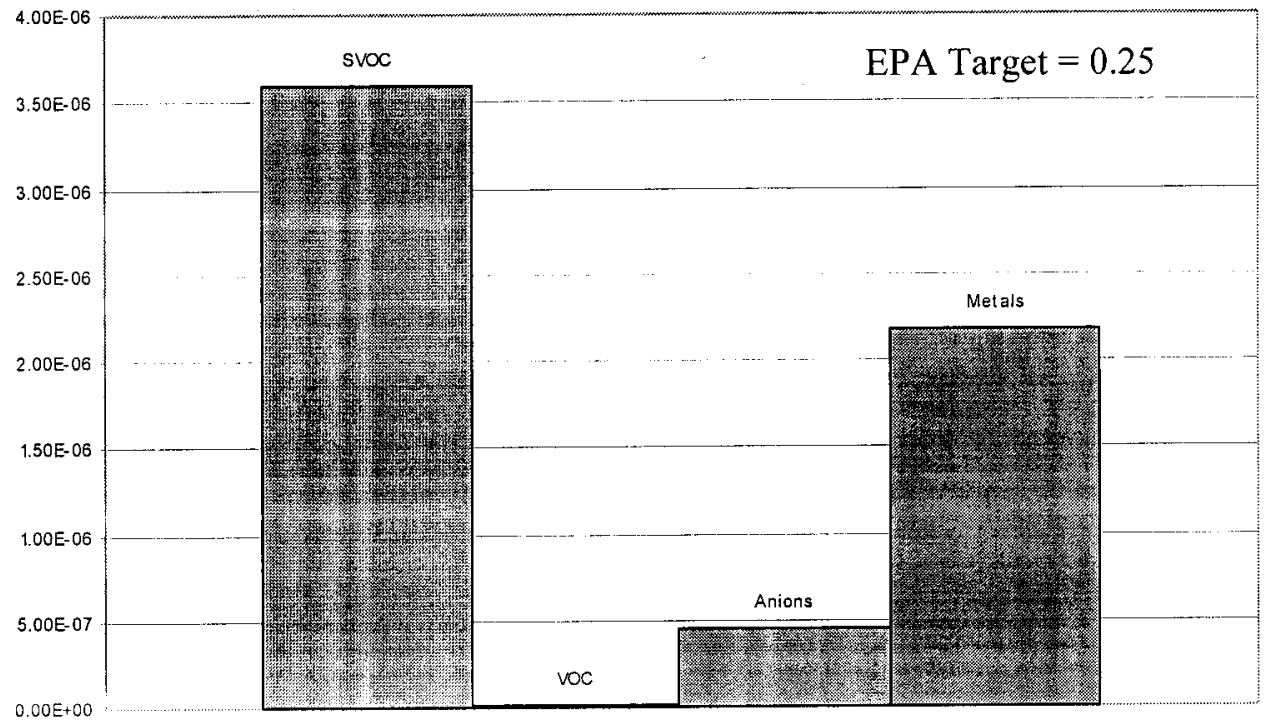

Figure 12. NWCF ETS EPA hazards quotient. 


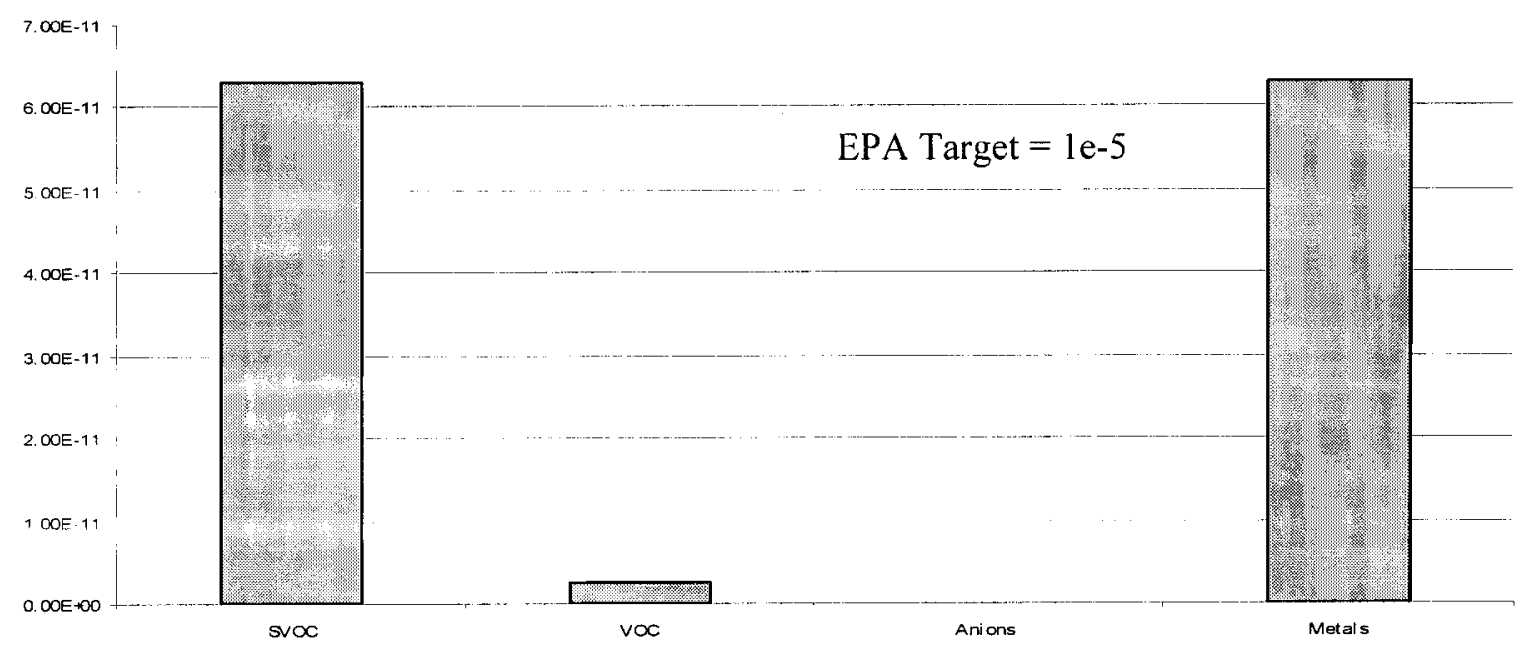

Figure 13. NWCF ETS cancer risk by pollutant category. 
Table 18. Risk scaling of Method 0010 analytes.

\begin{tabular}{|c|c|c|c|c|}
\hline Analyte & $\begin{array}{l}\text { NWCF } \\
\text { Hazard }\end{array}$ & $\begin{array}{c}\text { Concentration } \\
\text { Ratio }\end{array}$ & $\begin{array}{c}\text { NWCF ETS } \\
\text { Hazard }\end{array}$ & Risk \\
\hline Acenaphthene & $3.70 \mathrm{E}-12$ & $2.6^{1}$ & $9.60 \mathrm{E}-12$ & \\
\hline \multicolumn{5}{|l|}{ Acenaphthylene } \\
\hline Acetophenone & $5.60 \mathrm{E}-09$ & $0.048^{1}$ & $2.69 \mathrm{E}-10$ & \\
\hline Anthracene & $6.60 \mathrm{E}-12$ & $35^{1}$ & $2.31 \mathrm{E}-10$ & \\
\hline Benzoic acid & 4.50E-09 & 0.15 & $6.75 \mathrm{E}-10$ & \\
\hline \multicolumn{5}{|l|}{ Benzo(a)anthracene } \\
\hline \multicolumn{5}{|l|}{ Benzo(a)pyrene } \\
\hline \multicolumn{5}{|l|}{ Benzo(b)fluoranthene } \\
\hline \multicolumn{5}{|l|}{ Benzo(g,h,i)perylene } \\
\hline \multicolumn{5}{|l|}{ Benzo(k)fluoranthene } \\
\hline bis(2-Chloroethyl)ether & $9.90 \mathrm{E}-10$ & 0.02 & $1.98 \mathrm{E}-11$ & $2.00 \mathrm{E}-11$ \\
\hline bis(2-Ethylhexyl)phthalate & $1.70 \mathrm{E}-04$ & 0.013 & $2.21 \mathrm{E}-06$ & $2.00 \mathrm{E}-11$ \\
\hline 4-Bromophenyl-phenylether & $4.60 \mathrm{E}-07$ & 0.03 & $1.38 \mathrm{E}-08$ & \\
\hline Butylbenzylphthalate & 7.50E-09 & 0.02 & $1.50 \mathrm{E}-10$ & \\
\hline \multicolumn{5}{|l|}{ Carbazole } \\
\hline 2-Chloronaphthalene & $4.20 \mathrm{E}-09$ & 0.02 & $8.40 \mathrm{E}-11$ & \\
\hline 2-Chlorophenol & $2.20 \mathrm{E}-06$ & $8.00 \mathrm{E}-04$ & $1.76 \mathrm{E}-09$ & \\
\hline \multicolumn{5}{|l|}{ 4-Chlorophenyl phenyl ether } \\
\hline \multicolumn{5}{|l|}{ Chrysene } \\
\hline Di-n-butylphthalate & $4.50 \mathrm{E}-07$ & 0.025 & $1.13 \mathrm{E}-08$ & \\
\hline Di-n-octylphthalate & $3.50 \mathrm{E}-09$ & 0.1 & $3.50 \mathrm{E}-10$ & \\
\hline \multicolumn{5}{|l|}{ Dibenz(a,h)anthracene } \\
\hline \multicolumn{5}{|l|}{ Dibenzofuran } \\
\hline 1,2-Dichlorobenzene & $1.60 \mathrm{E}-09$ & 0.09 & $1.44 \mathrm{E}-10$ & \\
\hline 1,3-Dichlorobenzene & $9.90 \mathrm{E}-10$ & 0.1 & $9.90 \mathrm{E}-11$ & \\
\hline 1,4-Dichlorobenzene & $3.90 \mathrm{E}-10$ & 0.12 & $4.68 \mathrm{E}-11$ & \\
\hline Diethylphthalate & $3.20 \mathrm{E}-09$ & 0.02 & $6.40 \mathrm{E}-11$ & \\
\hline Dimethyl phthalate & $1.40 \mathrm{E}-10$ & $2.00 \mathrm{E}-02$ & $2.80 \mathrm{E}-12$ & \\
\hline 2,4-Dinitrotoluene & $3.90 \mathrm{E}-08$ & 0.014 & $5.46 \mathrm{E}-10$ & \\
\hline 2,6-Dinitrotoluene & $2.10 \mathrm{E}-06$ & 0.02 & 4.20E-08 & \\
\hline 1,2-Diphenylhydrazine & $3.50 \mathrm{E}-09$ & 0.02 & $7.00 \mathrm{E}-11$ & $8.00 \mathrm{E}-12$ \\
\hline Fluoranthene & $9.70 \mathrm{E}-10$ & $21.5^{1}$ & $2.09 \mathrm{E}-08$ & \\
\hline \multicolumn{5}{|l|}{ Fluorene } \\
\hline Hexachlorocyclopentadiene & $2.40 \mathrm{E}-04$ & $2.00 \mathrm{E}-03$ & $4.80 \mathrm{E}-07$ & \\
\hline Hexachlorobenzene & $6.10 \mathrm{E}-07$ & 0.02 & $1.22 \mathrm{E}-08$ & $1.50 \mathrm{E}-11$ \\
\hline \multicolumn{5}{|l|}{ Hexachlorobutadiene } \\
\hline Hexachloroethane & $8.20 \mathrm{E}-07$ & 0.014 & $1.15 \mathrm{E}-08$ & $1.50 \mathrm{E}-13$ \\
\hline \multicolumn{5}{|l|}{ Indeno(1,2,3-cd)pyrene } \\
\hline Isophorone & $3.90 \mathrm{E}-09$ & 0.02 & $7.80 \mathrm{E}-11$ & $1.00 \mathrm{E}-14$ \\
\hline 2-Methylnaphthalene & & & & \\
\hline
\end{tabular}


Table 18. Risk scaling of Method 0010 analytes.

\begin{tabular}{|c|c|c|c|c|}
\hline Analyte & $\begin{array}{l}\text { NWCF } \\
\text { Hazard }\end{array}$ & $\begin{array}{c}\text { Concentration } \\
\text { Ratio }\end{array}$ & $\begin{array}{l}\text { NWCF ETS } \\
\text { Hazard }\end{array}$ & Risk \\
\hline \multicolumn{5}{|l|}{$\overline{\text { N-Nitrosodimethylamine }}$} \\
\hline N-Nitrosodiphenylamine & $2.40 \mathrm{E}-12$ & 0.03 & $7.20 \mathrm{E}-14$ & \\
\hline Naphthalene & $3.60 \mathrm{E}-07$ & 0.014 & $5.04 \mathrm{E}-09$ & \\
\hline Nitrobenzene & $3.40 \mathrm{E}-09$ & $3.00 \mathrm{E}-03$ & $1.02 \mathrm{E}-11$ & \\
\hline \multicolumn{5}{|l|}{ 2-Nitrophenol } \\
\hline 4-Nitrophenol & $1.40 \mathrm{E}-06$ & $8.00 \mathrm{E}-04$ & $1.12 \mathrm{E}-09$ & \\
\hline \multicolumn{5}{|c|}{$\begin{array}{l}2,2 \text { '-Oxybis( } 1 \text {-chloropropane) } \\
\text { Phenanthrene }\end{array}$} \\
\hline Phenol & $2.90 \mathrm{E}-08$ & 4.00E-03 & $1.16 \mathrm{E}-10$ & \\
\hline Pyrene & $3.20 \mathrm{E}-08$ & $34^{\prime}$ & $1.09 \mathrm{E}-06$ & \\
\hline 1,2,4-Trichlorobenzene & 1.60E-09 & 0.1 & $1.60 \mathrm{E}-10$ & \\
\hline Total & & & $3.90 \mathrm{E}-06$ & $6.32 \mathrm{E}-11$ \\
\hline
\end{tabular}

Table 19. Risk scaling for Method 0031 analytes.

\begin{tabular}{|c|c|c|c|c|}
\hline Analyte & $\begin{array}{l}\text { NWCF } \\
\text { Hazard }\end{array}$ & $\begin{array}{c}\text { Concentration } \\
\text { Ratio }\end{array}$ & $\begin{array}{c}\text { NWCF ETS } \\
\text { Hazard }\end{array}$ & Risk \\
\hline Acetone & $6.30 \mathrm{E}-08$ & 0.023 & $1.45 \mathrm{E}-09$ & \\
\hline Benzene & 4.10E-06 & $7.70 \mathrm{E}-04$ & $3.16 \mathrm{E}-09$ & \\
\hline \multicolumn{5}{|l|}{ Bromomethane } \\
\hline 2-Butanone & $1.50 \mathrm{E}-09$ & 0.046 & $6.90 \mathrm{E}-11$ & \\
\hline Carbon disulfide & $9.00 \mathrm{E}-10$ & 0.147 & $1.32 \mathrm{E}-10$ & \\
\hline Carbon tetrachloride & $1.30 \mathrm{E}-08$ & 0.0351 & $4.56 \mathrm{E}-10$ & $1.70 \mathrm{E}-13$ \\
\hline Chlorobenzene & 4.80E-08 & 0.0076 & $3.65 \mathrm{E}-10$ & \\
\hline Chloroethane & $3.20 \mathrm{E}-11$ & 0.044 & $1.41 \mathrm{E}-12$ & \\
\hline Chloroform & $1.90 \mathrm{E}-08$ & 0.044 & $8.36 \mathrm{E}-10$ & $6.60 \mathrm{E}-13$ \\
\hline Chloromethane & $1.50 \mathrm{E}-09$ & 0.466 & $6.99 \mathrm{E}-10$ & $3.70 \mathrm{E}-13$ \\
\hline Dichlorodifluoromethane & $2.00 \mathrm{E}-09$ & 0.0466 & $9.32 \mathrm{E}-11$ & \\
\hline 1,2-Dichloroethane & $3.20 \mathrm{E}-08$ & 0.0356 & $1.14 \mathrm{E}-09$ & $3.00 \mathrm{E}-13$ \\
\hline 1,1-Dichloroethene & $1.00 \mathrm{E}-08$ & 0.041 & 4.10E-10 & $3.40 \mathrm{E}-13$ \\
\hline 1,2-Dichloropropane & $8.10 \mathrm{E}-08$ & 0.03 & $2.43 \mathrm{E}-09$ & $1.90 \mathrm{E}-13$ \\
\hline Methylene chloride & $5.50 \mathrm{E}-10$ & 0.877 & $4.82 \mathrm{E}-10$ & $4.80 \mathrm{E}-13$ \\
\hline Toluene & $7.20 \mathrm{E}-10$ & 0.055 & $3.96 \mathrm{E}-11$ & \\
\hline Trichlorofluoromethane & $8.30 \mathrm{E}-10$ & 0.02 & $1.66 \mathrm{E}-11$ & \\
\hline Vinyl chloride & $1.80 \mathrm{E}-12$ & 0.0356 & $6.41 \mathrm{E}-14$ & $6.40 \mathrm{E}-14$ \\
\hline o-Xylene & $4.80 \mathrm{E}-11$ & 0.018 & $8.64 \mathrm{E}-13$ & \\
\hline Totals & & & $1.18 \mathrm{E}-08$ & $2.574 \mathrm{E}-12$ \\
\hline
\end{tabular}


Table 20. Risk scaling for Method 0050 analytes.

\begin{tabular}{|c|c|c|c|c|}
\hline Analyte & NWCF Hazard & $\begin{array}{c}\text { Concentration } \\
\text { Ratio }\end{array}$ & $\begin{array}{c}\text { NWCF ETS } \\
\text { Hazard }\end{array}$ & Risk \\
\hline Chloride (as $\mathrm{HCl}$ ) & $1.50 \mathrm{E}-05$ & 0.02 & $3.00 \mathrm{E}-07$ & \\
\hline Chloride (as $\mathrm{Cl} 2$ ) & $1.70 \mathrm{E}-07$ & 0.0047 & $7.99 \mathrm{E}-10$ & \\
\hline Fluoride (as HF) & $3.50 \mathrm{E}-05$ & 0.00082 & $2.87 \mathrm{E}-08$ & \\
\hline Nitrate (as $\mathrm{HNO} 3$ ) & $9.50 \mathrm{E}-04$ & 0.00008 & $7.60 \mathrm{E}-08$ & \\
\hline \multicolumn{5}{|l|}{ Nitrite (as HNO2) } \\
\hline \multicolumn{5}{|l|}{ Particulate } \\
\hline Total & & & $4.05 \mathrm{E}-07$ & --- \\
\hline
\end{tabular}

Table 21. Risk scaling for Method 0060 analytes.

\begin{tabular}{|c|c|c|c|c|}
\hline Analyte & NWCF Hazard & $\begin{array}{c}\text { Concentration } \\
\text { Ratio }\end{array}$ & HLLWE Hazard & Risk \\
\hline Aluminum (Al) & $5.7 \mathrm{E}-09$ & 0.036 & $2.1 \mathrm{E}-10$ & \\
\hline Antimony (Sb) & $1.50 \mathrm{E}-09$ & 0.056 & $8.48 \mathrm{E}-11$ & \\
\hline Arsenic (As) & $1.30 \mathrm{E}-14$ & $2.2 \mathrm{E}-7$ & $7.8 \mathrm{E}-22$ & \\
\hline Barium $(\mathrm{Ba})$ & $7.50 \mathrm{E}-06$ & 0.0025 & $1.88 \mathrm{E}-08$ & \\
\hline \multicolumn{5}{|l|}{ Beryllium (Be) } \\
\hline Cadmium (Cd) & $1.70 \mathrm{E}-07$ & 0.026 & $4.5 \mathrm{E}-09$ & $1.70 \mathrm{E}-12$ \\
\hline Chromium (Cr) & $9.00 \mathrm{E}-07$ & 0.047 & 4.23E-08 & $6.0 \mathrm{E}-11$ \\
\hline \multicolumn{5}{|l|}{ Cobalt (Co) } \\
\hline Copper $(\mathrm{Cu})$ & $4.50 \mathrm{E}-09$ & 0.017 & $7.5 \mathrm{E}-11$ & \\
\hline Lead $(\mathrm{Pb})$ & $2.90 \mathrm{E}-08$ & 0.008 & $2.4 \mathrm{E}-10$ & \\
\hline Manganese (Mn) & $1.60 \mathrm{E}-05$ & 0.085 & $1.36 \mathrm{E}-06$ & \\
\hline Mercury (Hg) & $2.10 \mathrm{E}-04$ & 0.0036 & $7.56 \mathrm{E}-07$ & \\
\hline Nickel (Ni) & $2.30 \mathrm{E}-11$ & 0.07 & $1.61 \mathrm{E}-12$ & $1.30 \mathrm{E}-12$ \\
\hline Selenium (Se) & $8.20 \mathrm{E}-10$ & $2.00 \mathrm{E}-11$ & 1.64E-20 & \\
\hline \multicolumn{5}{|l|}{ Silver (Ag) } \\
\hline Thallium (Tl) & $2.7 \mathrm{E}-8$ & 0.14 & 4.0E-9 & \\
\hline \multicolumn{5}{|l|}{ Vanadium (V) } \\
\hline \multirow[t]{2}{*}{ Zinc $(\mathrm{Zn})$} & $2.00 \mathrm{E}-08$ & $5.00 \mathrm{E}-05$ & $1.00 \mathrm{E}-12$ & \\
\hline & & & Total & \\
\hline Total & & & 2.19E-06 & $6.3 \mathrm{E}-11$ \\
\hline
\end{tabular}




\section{CONCLUSIONS}

Characterization samples for the NWCF ETS were collected with only minor deviations from EPA protocols. Due to ALARA concerns, the samples were collected at a single point in the duct and the probe was not removed between sample trains. The NWCF ETS emissions rates for all species were relatively low in terms of regulatory emissions limits and health risk considerations. It was observed that organic compound emissions are slightly higher at the beginning of the batch while metals emissions, including mercury, are slightly higher at the end of the evaporator batch. Mercury emissions were less than $5 \mathrm{ppbv}(<40 \mu \mathrm{g} / \mathrm{dscm})$, while the sum of $\mathrm{HCl}$ and $\mathrm{Cl} 2$ emissions was less than 1 ppmv. The sum of all organic emissions also was less than 1 ppmv. Particulate emissions (included the apportioned particulate recovered in the final probe rinse) are less than $0.9 \mathrm{mg} / \mathrm{dscm}$ and less than $0.7 \mathrm{mg} / \mathrm{dscm}$ at the beginning and end of the evaporator batch, respectively.

The estimated HQ for the evaporator was $6.2 \mathrm{e}-6$ as compared to 0.25 for the EPA target criteria. The estimated cancer risk was $1.3 \mathrm{e}-10$ compared to an EPA target of $1.0 \mathrm{e}-5$. The NWCF ETS offgas emissions inventory was completed in accordance with the QAPjP developed and approved for this activity. Conventional EPA sampling and analytical methods were used to characterize volatile and semivolatile organic compounds, multiple metals, $\mathrm{HCl} / \mathrm{Cl}_{2}$, and particulate emissions.

Diligence in following sample checklists, continuous monitoring by either the Project Technical Leads and Quality Assurance Office, use of a master sample collection list, pre-defined sample labels, and RFA/COC documentation provided for the best possible sample collection accuracy and consistency. The data are believed to be accurate and representative of the NWCF ETS for the feed and system operating conditions during the offgas sampling period. A compilation of the process operating parameters, the offgas sample analytical data summaries, and the calculated emissions rates and liquid composition data for the evaporator feed, overhead condensate, and bottoms are included in the report appendices for permit applications purposes.

NWCF ETS operations were normal and consistent throughout the three-week sample collection period. Feed batches were consistent, as were the evaporator operating parameters and offgas system conditions. Radiation levels in the offgas samples were extremely low. Extended gamma scanning did not identify any gamma emitters in either the sample contamination trains or ongoing screening samples. Gross beta and gross alpha levels were only detected in the pico-curies range, easily meeting all of the analytical laboratory sample acceptance criteria. Tritium levels were low and proportionate to the low levels of moisture that were present in the NWCF offgas stream. Oxygen levels in the offgas duct were comparable to ambient air conditions.

Species absorption in the probe liner was minor. The exception may be organic particulate. The apportioned amount of organic particulate contained in the final probe rinse was approximately $25 \%$ of the average Method 0050 run total particulate measurements. This suggests that some semi-volatile organic matter could be potentially deposited on the probe liner. Even when the SVOC results are conservatively escalated by $25 \%$, to account for the maximum potential portion of semivolatile material adsorbed on the probe liner, the outcome of the emissions rates are risk calculations are not significant.

Metal adsorption on the probe was low for all metals. Less than $0.1 \%$ of the mercury was deposited on the probe liner. Therefore, apportionment of the final probe rinse to the Method 0060 Metals trains is not significant. 


\section{REFERENCES}

ASTM Standard E29-93a , 1999, "Standard Practice for Using Significant Digits in Test Data to Determine Conformance with Specifications."

Bechtel BWXT Idaho, LLC, 2000, Balance of Plant Sampling and Analysis Plan (Input to PEWE and LET\&D), PLN-613, Rev. 1, February 2000.

Bechtel BWXT Idaho, LLC, 2001, INTEC Quality Assurance Project Plan for HLLWE Offgas Emissions Inventory Project, PLN-880, Rev. 0, May 2001.

Bechtel BWXT Idaho, LLC, 2001, Test Plan for the HLLWE Gas Emission Inventory, PLN-879, Rev. 0, May 2001.

Bechtel BWXT Idaho, LLC, 2001, Package and Ship NWCF Offgas Emissions Samples Offsite for Analysis, MCP-1173, Rev. 2, June 2001.

Bechtel BWXT Idaho, LLC, Sample NWCF Offgas for Emissions Characterization, TPR-5496, Rev. 6, June 2001.

Boardman, R. D., L. J. Young, N. R. Soelberg, and L. A. Matejka, 2001, NWCF Calciner Emissions Inventory - Final Report for Phase IV Testing, EXT-01-00260, February 2001.

Severn Trent Services, 2001, Analytical Report for the Idaho Nuclear Technology and Engineering Center (INTEC) High Level Waste Evaporator (HLLWE) Effluent Gas Emissions Inventory, September 2001. 


\section{APPENDIX A}

ANALYTICAL DATA SUMMARIES 



\section{CONTENTS}

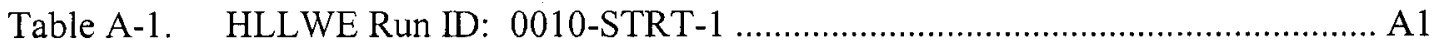

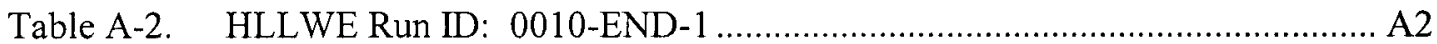

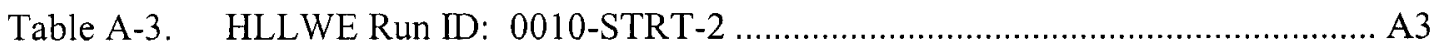

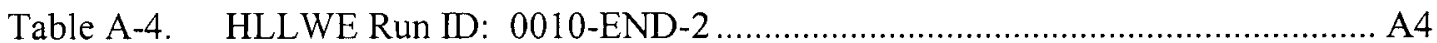

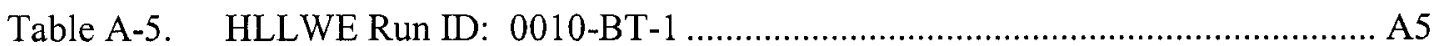

Table A-6. Run 2, XAD-2 Resin Tube Trip Blank/Reagent Blank................................. A6

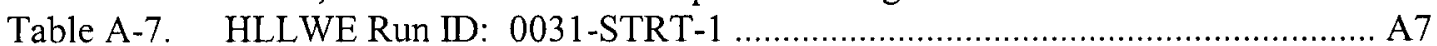

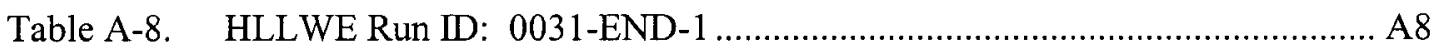

Table A-9. HLLWE Run ID: 0031-STRT-2 ...................................................... A9

Table A-10. HLLWE Run ID: 0031-END-2 …..................................................... A10

Table A-11. Run 2, VOST Tenax Tube Pair Field Blank ............................................ A11

Table A-12. Run 2, VOST Anasorb 747 Tube Field Blank ............................................. A12

Table A-13. HLLWE Run ID: 0050-STRT-1 ....................................................... A13

Table A-14. HLLWE Run ID: 0050-END-1 ......................................................... A14

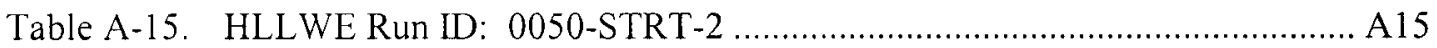

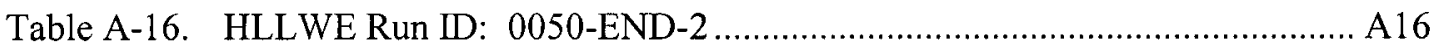

Table A-17. M5 Particulate and Anion Train Run 2 Reagent Blanks ............................. A17

Table A-18. M5 Particulate and Anion Train Run 2 INTEC

Deionized Water Reagent Blank ...................................................... A18

Table A-19. M5 Particulate and Anion Train Final Acetone Probe Rinse ...................... A19

Table A-20. HLLWE Run ID: 0060-STRT-1 ......................................................... A20

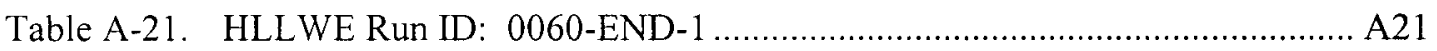

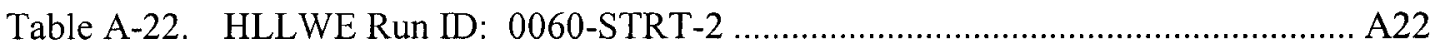

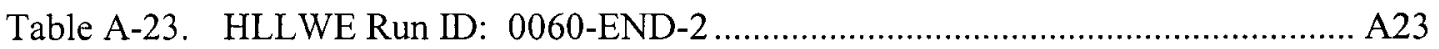

Table A-24. HLLWE Run ID: 0060-BT-1 ........................................................... A24

Table A-25. Method 0060 Multi-Metals Train (MMT) Analytical Results

Summary Run 2 Front Half Composite Reagent Blank ............................ A25

Table A-26. Method 0060 Multi-Metals Train (MMT) Analytical Results

Summary Run 2 Back Half Composite Reagent Blank ............................. A26

Table A-27. Method 0060 Multi-Metals Train (MMT) Analytical Results

Summary Run 2 Mercury Impinger Composite Reagent Blank ................ A27

Table A-28. Method 0060 Multi-Metals Train (MMT) Analytical Results

Summary Run 2 HCl Impinger Rinse Solution Reagent Blank ................. A28

Table A-29. Method 0060 Multi-Metals Train (MMT) Analytical Results

Summary Run 2 INTEC Deionized Water Reagent Blank

Table A-30. Final (Post-Test) Acetone Probe Rinse and Nitric Acid

Probe Rinse Composite Metallic Analyte Analytical Results Summary .... A-30 

BECHTEL BWXT IDAHO, LLC (BBWI)

INTEC HLLWE Effluent Gas Emissions Inventory

Idaho National Engineering and Environmental Laboratory (INEEL)

STL Knoxville Project Number: 142503.40

\section{MM-5 Train Summary - Run 1 Train Totals Semivolatile Organic Compounds Analytical Results Summary Table A-1. HLLWE Run ID: 0010-STRT-1}

Field Sample Name: Sample Description:
MM-5 Train

MM-5 Train Totals for Semivolatile Organic Compounds Analysis

\begin{tabular}{|c|c|c|c|c|c|c|c|c|c|c|}
\hline \multirow[b]{2}{*}{ Analyte } & \multirow{2}{*}{$\begin{array}{c}\text { CAS } \\
\text { Registry } \\
\text { Number }\end{array}$} & \multicolumn{2}{|c|}{$\begin{array}{c}\text { MM-5 Train } \\
\text { Front Half } \\
\text { Composite } \\
(\mu \mathrm{g})\end{array}$} & \multicolumn{2}{|c|}{$\begin{array}{c}\text { MM-5 Train } \\
\text { Back Half } \\
\text { Composite }^{2} \\
(\mu g)\end{array}$} & \multicolumn{2}{|c|}{$\begin{array}{c}\text { MM-5 Train } \\
\text { Condensate } \\
\text { Composite } \\
(\mu g) \\
\end{array}$} & \multicolumn{2}{|c|}{$\begin{array}{c}\text { MM-5 Train } \\
\text { Totals } \\
\text { (Total } \mu g \text { ) }\end{array}$} & \multirow{2}{*}{$\begin{array}{c}\text { Project } \\
\text { Specific } \\
\text { Flag }^{7}\end{array}$} \\
\hline & & Risk Result & Flag $^{5}$ & Risk Result I & Flag $^{5}$ & Risk Result & Flag $^{5}$ & Total $^{6}$ & Flag & \\
\hline \multicolumn{11}{|l|}{ Target Compound List } \\
\hline Acenaphthene & $83-32-9$ & 1.3 & U & 6.6 & $\mathrm{U}$ & 1.9 & $\mathrm{U}$ & $<9.8$ & & $N$ \\
\hline Acenaphthylene & $208-96-8$ & 1.3 & $\mathrm{U}$ & 6.6 & $\mathrm{U}$ & 1.6 & $\mathrm{U}$ & $<9.5$ & & $N$ \\
\hline Acetophenone & $9[8-86-2$ & 2.0 & $\mathrm{U}$ & 44 & $\mathrm{~J}$ & 2.6 & $\mathrm{U}$ & $<49$ & $\mathrm{~J}$ & $\mathrm{P}$ \\
\hline Aniline & $62-53-3$ & 2.5 & $\mathrm{U}$ & 94 & $\mathrm{U}$ & 18 & $\mathrm{U}$ & $<110$ & & $N$ \\
\hline Anthracene & $120-12-7$ & 1.3 & U & 6.6 & $\mathrm{U}$ & 1.6 & U & $<9.5$ & & $\mathrm{~N}$ \\
\hline Benzidine & $92-87-5$ & 100 & $\mathrm{U}$ & 500 & U & 66 & U & $<670$ & & $N$ \\
\hline Benzoic acid & $65-85-0$ & 100 & $\mathrm{U}$ & 4,800 & $E$ & 9.4 & $\mathrm{U}$ & $<4,900$ & $E$ & $P$ \\
\hline Benzo(a)anthracene & $56-55-3$ & 2.2 & $\mathrm{U}$ & 7.6 & $\mathrm{U}$ & 1.7 & $\mathrm{U}$ & $<12$ & & $\mathrm{~N}$ \\
\hline Benzo(a)pyrene & $50-32-8$ & 2.6 & $\mathrm{U}$ & 130 & $\mathrm{U}$ & 1.8 & U & $<130$ & & $\mathrm{~N}$ \\
\hline Benzo(b)fluoranthene & $205-99-2$ & 3.7 & $\mathrm{U}$ & 290 & $\mathrm{U}$ & 4.2 & $\mathrm{U}$ & $<300$ & & $\mathrm{~N}$ \\
\hline Benzo $(g, h, i)$ perylene & $191-24-2$ & 7.3 & $U$ & 160 & $\mathrm{U}$ & 2.1 & $\mathrm{U}$ & $<170$ & & $N$ \\
\hline Benzo(k)fluoranthene & $207-08-9$ & 5.5 & $\mathrm{U}$ & 420 & $\mathrm{U}$ & 2.9 & $\mathrm{U}$ & $<430$ & & $\mathrm{~N}$ \\
\hline Benzyl alcohol & $100-51-6$ & 92 & $\mathrm{U}$ & 470 & $\mathrm{U}$ & 4.2 & $\mathrm{U}$ & $<570$ & & $\mathrm{~N}$ \\
\hline bis(2-Chloroethoxy)methane & $111-91-1$ & 1.5 & $\mathrm{U}$ & 6.6 & $\mathrm{U}$ & 2.0 & $\mathrm{U}$ & $<10$ & & $\mathrm{~N}$ \\
\hline bis(2-Chloroethyl)ether & $111-44-4$ & 2.0 & U & 7.3 & U & 1.7 & $\mathrm{U}$ & $<11$ & & $N$ \\
\hline bis(2-Ethylhexyl)phthalate & $117-81-7$ & 14 & & 100 & $\mathrm{~J}$ & 11 & & $<120$ & $\mathrm{~J}$ & A \\
\hline 4-Bromophenyl-phenylether & $101-55-3$ & 1.4 & $\mathrm{U}$ & 6.6 & $\mathrm{U}$ & 1.4 & $\mathrm{U}$ & $<9.4$ & & $N$ \\
\hline Butylbenzylphthalate & $85-68-7$ & 2.9 & $\mathrm{U}$ & 7.9 & $\mathrm{U}$ & 2.3 & U & $<13$ & & $\mathrm{~N}$ \\
\hline Carbazole & $86-74-8$ & 2.0 & $\mathrm{U}$ & 8.4 & U & 2.2 & U & $<13$ & & $\mathrm{~N}$ \\
\hline 4-Chloro-3-methylphenol & $59-50-7$ & 2.6 & $\mathrm{U}$ & 8.1 & $\mathrm{U}$ & 6.6 & $\mathrm{U}$ & $<17$ & & $\mathrm{~N}$ \\
\hline 4-Chloroaniline & $106-47-8$ & 3.1 & $\mathrm{U}$ & 79 & $\mathrm{U}$ & 7.9 & $\mathrm{U}$ & $<90$ & & $\mathrm{~N}$ \\
\hline 2-Chioronaphthalene & $91-58-7$ & 1.3 & $\mathrm{U}$ & 6.6 & $\mathrm{U}$ & 1.4 & $\mathrm{U}$ & $<9.3$ & & $\mathrm{~N}$ \\
\hline 2-Chlorophenol & $95-57-8$ & 2.6 & $\mathrm{U}$ & 6.6 & $\mathrm{U}$ & 1.7 & $\mathrm{U}$ & $<11$ & & $\mathrm{~N}$ \\
\hline 4-Chlorophenyl phenyl ether & $7005-72-36$ & 1.3 & $\mathrm{U}$ & 6.6 & $\mathrm{U}$ & 3.1 & $\mathrm{U}$ & $<11$ & & $N$ \\
\hline Chrysene & $218-01-9$ & 2.3 & $\mathrm{U}$ & 8.4 & $\mathrm{U}$ & 1.3 & $\mathrm{U}$ & $<12$ & & $\mathrm{~N}$ \\
\hline
\end{tabular}

Last saved by Robin Gifford on 02/04/02 at 4:28 PM

E: My Documents $\backslash$ HLLWE SamplinglFinal Report \Appendix A $\backslash$ Appendix A-1. 0010-STRT-1.doc

Created on 2/4/2002 4:05 PM 
BECHTEL BWXT IDAHO, LLC (BBWI)

INTEC HLLWE Effluent Gas Emissions Inventory

Idaho National Engineering and Environmental Laboratory (INEEL)

STL Knoxville Project Number: 142503.40

\section{MM-5 Train Summary - Run 1 Train Totals (Continued) Semivolatile Organic Compounds Analytical Results Summary Table A-1. HLLWE Run ID: 0010-STRT-1}

\begin{tabular}{|c|c|c|c|c|c|c|c|c|c|c|}
\hline \multirow[b]{2}{*}{ Analyte } & \multirow{2}{*}{$\begin{array}{c}\text { CAS } \\
\text { Registry } \\
\text { Number }\end{array}$} & \multicolumn{2}{|c|}{$\begin{array}{c}\text { MM-5 Train } \\
\text { Front Half } \\
\text { Composite } \\
(\mu \mathrm{g})\end{array}$} & \multicolumn{2}{|c|}{$\begin{array}{c}\text { MM-5 Train } \\
\text { Back Half } \\
\text { Composite }^{2} \\
(\mu \mathrm{g})\end{array}$} & \multicolumn{2}{|c|}{$\begin{array}{c}\text { MM-5 Train } \\
\text { Condensate } \\
\text { Composite }^{3} \\
(\mu g) \\
\end{array}$} & \multicolumn{2}{|c|}{$\begin{array}{c}\text { MM-5 Train } \\
\text { Totals }^{4} \\
\text { (Total } \mu \mathrm{g}) \\
\end{array}$} & \multirow{2}{*}{$\begin{array}{c}\text { Project } \\
\text { Specific } \\
\text { Flag }^{7}\end{array}$} \\
\hline & & Risk Result & Flag 5 & Risk Result & Flag $^{5}$ & Risk Result & Flag 5 & Total $^{6}$ & Flag & \\
\hline Di-n-butylphthalate & $84-74-2$ & 1.9 & J & 100 & $\mathrm{U}$ & 2.3 & $\mathrm{U}$ & $<100$ & $\mathrm{~J}$ & $\mathrm{P}$ \\
\hline Di-n-octylphthalate & $117-84-0$ & 11 & & 150 & $\mathrm{U}$ & 2.5 & $\mathrm{~J}$ & $<160$ & J & $\mathrm{P}$ \\
\hline Dibenz(a,h)anthracene & $53-70-3$ & 5.2 & $\mathrm{U}$ & 160 & $\mathrm{U}$ & 2.9 & U & $<170$ & & $\mathrm{~N}$ \\
\hline Dibenzofuran & $132-64-9$ & 1.4 & U & 6.6 & $\mathrm{U}$ & 2.9 & $\mathrm{U}$ & $<11$ & & $N$ \\
\hline 1,2-Dichlorobenzene & $95-50-1$ & 2.2 & $\mathrm{U}$ & 6.8 & $\mathrm{U}$ & 1.7 & $\mathrm{U}$ & $<11$ & & $\mathrm{~N}$ \\
\hline 1,3-Dichlorobenzene & $541-73-1$ & 3.1 & $\mathrm{U}$ & 7.3 & $\mathrm{U}$ & 1.4 & $\mathrm{U}$ & $<12$ & & $\mathrm{~N}$ \\
\hline 1,4-Dichlorobenzene & $106-46-7$ & 2.9 & $\mathrm{U}$ & 11 & $\mathrm{~J}$ & 2.0 & $\mathrm{U}$ & $<16$ & $\mathrm{~J}$ & $\mathrm{P}$ \\
\hline 3,3'-Dichlorobenzidine & $91-94-1$ & 7.1 & $\mathrm{U}$ & 97 & U & 7.9 & U & $<110$ & & $\mathrm{~N}$ \\
\hline 2,4-Dichlorophenol & $120-83-2$ & 3.9 & $\mathrm{U}$ & 6.6 & $\mathrm{U}$ & 2.3 & $\mathrm{U}$ & $<13$ & & $\mathrm{~N}$ \\
\hline Diethylphthalate & $84-66-2$ & 5.4 & $J$ & 9.4 & U & 1.4 & $\mathrm{U}$ & $<16$ & $\mathrm{~J}$ & $\mathrm{P}$ \\
\hline Dimethyl phthalate & $131-11-3$ & 1.7 & $\mathrm{U}$ & 6.6 & $\mathrm{U}$ & 1.3 & $\mathrm{U}$ & $<9.6$ & & N \\
\hline 2,4-Dimethylphenol & $105-67-9$ & 7.6 & $\mathrm{U}$ & 50 & $\mathrm{U}$ & 1.5 & $\mathrm{U}$ & $<59$ & & $\mathrm{~N}$ \\
\hline 4,6-Dinitro-2-methylphenol & $534-52-1$ & 13 & $\mathrm{U}$ & 120 & $\mathrm{U}$ & 1.5 & $\mathrm{U}$ & $<130$ & & $\mathrm{~N}$ \\
\hline 2,4-Dinitrophenol & $51-28-5$ & 15 & $\mathrm{U}$ & 250 & $\mathrm{U}$ & 3.9 & $\mathrm{U}$ & $<270$ & & $\mathrm{~N}$ \\
\hline 2,4-Dinitrotoluene & $121-14-2$ & 4.2 & $\mathrm{U}$ & 6.6 & $\mathrm{U}$ & 2.6 & $\mathrm{U}$ & $<13$ & & $\mathrm{~N}$ \\
\hline 2,6-Dinitrotoluene & $606-20-2$ & 3.4 & $\mathrm{U}$ & 6.6 & $\mathrm{U}$ & 2.1 & $\mathrm{U}$ & $<12$ & & $\mathrm{~N}$ \\
\hline 1,2-Diphenylhydrazine & $122-66-7$ & 1.7 & $\mathrm{U}$ & 6.6 & $\mathrm{U}$ & 1.5 & $\mathrm{U}$ & $<9.8$ & & $\mathrm{~N}$ \\
\hline Fluoranthene & $206-44-0$ & 1.3 & $\mathrm{U}$ & 7.1 & U & 1.8 & U & $<10$ & & $\mathrm{~N}$ \\
\hline Fluorene & $86-73-7$ & 1.3 & $\mathrm{U}$ & 6.6 & $\mathrm{U}$ & 2.6 & $\mathrm{U}$ & $<10$ & & $N$ \\
\hline Hexachlorocyclopentadiene & $77-47-4$ & 26 & $\mathrm{U}$ & 130 & $\mathrm{U}$ & 6.6 & $\mathrm{U}$ & $<160$ & & $\mathrm{~N}$ \\
\hline Hexachlorobenzene & $118-74-1$ & 1.5 & $\mathrm{U}$ & 6.6 & $\mathrm{U}$ & 2.6 & $\mathrm{U}$ & $<11$ & & $\mathrm{~N}$ \\
\hline Hexachlorobutadiene & $87-68-3$ & 3.7 & U & 9.7 & $\mathrm{U}$ & 1.9 & $\mathrm{U}$ & $<15$ & & $\mathrm{~N}$ \\
\hline Hexachloroethane & $67-72-1$ & 6.6 & $\mathrm{U}$ & 7.1 & $\mathrm{U}$ & 1.9 & $\mathrm{U}$ & $<16$ & & $\mathrm{~N}$ \\
\hline Indeno $(1,2,3-\mathrm{cd})$ pyrene & $193-39-5$ & 5.5 & $\mathrm{U}$ & 140 & $\mathrm{U}$ & 2.3 & U & $<150$ & & $\mathrm{~N}$ \\
\hline Isophorone & $78-59-1$ & 1.7 & $\mathrm{U}$ & 6.6 & $\mathrm{U}$ & 1.8 & U & $<10$ & & $\mathrm{~N}$ \\
\hline 2-Methylnaphthalene & $91-57-6$ & 1.5 & U & 6.6 & $\mathrm{U}$ & 2.3 & $\mathrm{U}$ & $<10$ & & $\mathrm{~N}$ \\
\hline 2-Methylphenol & $95-48-7$ & 6.0 & $\mathrm{U}$ & 39 & $\mathrm{U}$ & 2.1 & $\mathrm{U}$ & $<47$ & & $\mathrm{~N}$ \\
\hline 3-Methylphenol \& 4-Methylphenol & $65794-96-9$ & 6.0 & $\mathrm{U}$ & 26 & $\mathrm{U}$ & 2.2 & $\mathrm{U}$ & $<34$ & & $\mathrm{~N}$ \\
\hline
\end{tabular}

Last saved by Robin Gifford on 02/04/02 at 4:28 PM

E:My Documents $Y$ HLLWE SamplingIFinal Report Appendix ALAppendix A-1. 0010-STRT-1.doc

Created on 2/4/2002 4:05 PM 
BECHTEL BWXT IDAHO, LLC (BBWI)

INTEC HLLWE Effluent Gas Emissions Inventory

Idaho National Engineering and Environmental Laboratory (INEEL)

STL Knoxville Project Number: 142503.40

\section{MM-5 Train Summary - Run 1 Train Totals (Continued) Semivolatile Organic Compounds Analytical Results Summary Table A-1. HLLWE Run ID: 0010-STRT-1}

\begin{tabular}{|c|c|c|c|c|c|c|c|c|c|c|}
\hline \multirow[b]{2}{*}{ Analyte } & \multirow{2}{*}{$\begin{array}{c}\text { CAS } \\
\text { Registry } \\
\text { Number } \\
\end{array}$} & \multicolumn{2}{|c|}{$\begin{array}{l}\text { MM-5 Train } \\
\text { Front Half } \\
\text { Composite } \\
(\mu \mathrm{g})\end{array}$} & \multicolumn{2}{|c|}{$\begin{array}{c}\text { MM-5 Train } \\
\text { Back Half } \\
\text { Composite }^{2} \\
(\mu g)\end{array}$} & \multicolumn{2}{|c|}{$\begin{array}{c}\text { MM-5 Train } \\
\text { Condensate } \\
\text { Composite }^{3} \\
(\mu g)\end{array}$} & \multicolumn{2}{|c|}{$\begin{array}{c}\text { MM-5 Train } \\
\text { Totals }^{4} \\
\text { (Total } \mu \mathrm{g}) \\
\end{array}$} & \multirow{2}{*}{$\begin{array}{c}\text { Project } \\
\text { Specific } \\
\text { Flag }^{7} \\
\end{array}$} \\
\hline & & Risk Result & Flag $^{5}$ & Risk Result & Flag $^{5}$ & Risk Result & Flag 5 & Total $^{6}$ & Flag & \\
\hline N-Nitroso-di-n-propylamine & $621-64-7$ & 1.9 & $\mathrm{U}$ & 6.6 & $\mathrm{U}$ & 2.3 & U & $<11$ & & $N$ \\
\hline N-Nitrosodimethylamine & $62-75-9$ & 1.9 & $\mathrm{U}$ & 6.6 & $\mathrm{U}$ & 2.2 & $U$ & $<11$ & & $N$ \\
\hline N-Nitrosodiphenylamine & $86-30-6$ & 1.6 & $\mathrm{U}$ & 12 & U & 1.4 & $\mathrm{U}$ & $<15$ & & $N$ \\
\hline Naphthalene & $91-20-3$ & 1.3 & $\mathrm{U}$ & 7.9 & $\mathrm{U}$ & 1.8 & $\mathrm{U}$ & $<11$ & & $N$ \\
\hline 2-Nitroaniline & $88-74-4$ & 1.5 & $\mathrm{U}$ & 6.6 & $\mathrm{U}$ & 2.9 & $\mathrm{U}$ & $<11$ & & $\mathrm{~N}$ \\
\hline 3-Nitroaniline & $99-09-2$ & 10 & U & 26 & $\mathrm{U}$ & 4.7 & $\mathrm{U}$ & $<41$ & & $\mathrm{~N}$ \\
\hline 4-Nitroaniline & $100-01-6$ & 6.0 & $\mathrm{U}$ & 26 & U & 3.9 & $\mathrm{U}$ & $<36$ & & $\mathrm{~N}$ \\
\hline Nitrobenzene & $98-95-1$ & 1.9 & U & 12 & $\mathrm{~J}$ & 1.7 & $U$ & $<16$ & $J$ & $\mathrm{P}$ \\
\hline 2-Nitrophenol & $88-75-5$ & 8.4 & $\mathrm{U}$ & 80 & & 2.6 & $\mathrm{U}$ & $<91$ & & $\mathrm{P}$ \\
\hline 4-Nitrophenol & $100-02-7$ & 8.7 & $\mathrm{U}$ & 63 & $\mathrm{~J}$ & 3.9 & $\mathrm{U}$ & $<76$ & $\mathrm{~J}$ & $\mathrm{P}$ \\
\hline 2,2 -Oxybis(1-chloropropane) ${ }^{8}$ & $108-60-1$ & 2.6 & $\mathrm{U}$ & 10 & $\mathrm{U}$ & 1.8 & $U$ & $<14$ & & $\mathrm{~N}$ \\
\hline Pentachlorobenzene & $608-93-5$ & 1.4 & $\mathrm{U}$ & 6.6 & $\mathrm{U}$ & 2.3 & $\mathrm{U}$ & $<10$ & & $\mathrm{~N}$ \\
\hline Pentachloronitrobenzene & $82-68-8$ & 2.0 & $\mathrm{U}$ & 6.6 & $\mathrm{U}$ & 2.6 & $\mathrm{U}$ & $<11$ & & $\mathrm{~N}$ \\
\hline Pentachlorophenol & $87-86-5$ & 50 & $\mathrm{U}$ & 250 & $\mathrm{U}$ & 3.4 & $\mathrm{U}$ & $<300$ & & $\mathrm{~N}$ \\
\hline Phenanthrene & $85-01-8$ & 1.3 & $\mathrm{U}$ & 6.6 & $\mathrm{U}$ & 1.9 & $\mathrm{U}$ & $<9.8$ & & $\mathrm{~N}$ \\
\hline Phenol & $108-95-2$ & 2.9 & $\mathrm{U}$ & 72 & & 2.2 & $\mathrm{U}$ & $<77$ & & $\mathrm{P}$ \\
\hline Pyrene & $129-00-0$ & 1.9 & $\mathrm{U}$ & 6.8 & U & 1.4 & $\mathrm{U}$ & $<10$ & & $\mathrm{~N}$ \\
\hline Pyridine & $110-86-1$ & 2.3 & U & 9.7 & $\mathrm{U}$ & 5.2 & $\mathrm{U}$ & $<17$ & & $\mathrm{~N}$ \\
\hline 1,2,4,5-Tetrachlorobenzene & $95-94-3$ & 2.3 & $\mathrm{U}$ & 6.6 & $\mathrm{U}$ & 2.2 & $\mathrm{U}$ & $<11$ & & $\mathrm{~N}$ \\
\hline 1,2,4-Trichlorobenzene & $120-82-1$ & 1.9 & $\mathrm{U}$ & 7.9 & $\mathrm{U}$ & 2.2 & $\mathrm{U}$ & $<12$ & & $\mathrm{~N}$ \\
\hline 2,4,5-Trichlorophenol & $95-95-4$ & 6.0 & $\mathrm{U}$ & 17 & $\mathrm{U}$ & 2.1 & $\mathrm{U}$ & $<25$ & & $N$ \\
\hline 2,4,6-Trichlorophenol & $88-06-2$ & 3.7 & $\mathrm{U}$ & 10 & $\mathrm{U}$ & 2.5 & $\mathrm{U}$ & $<16$ & & $\mathrm{~N}$ \\
\hline
\end{tabular}


BECHTEL BWXT IDAHO, LLC (BBWI)

INTEC HLLWE Effluent Gas Emissions Inventory

Idaho National Engineering and Environmental Laboratory (INEEL)

STL Knoxville Project Number: 142503.40

MM-5 Train Summary - Run 1 Train Totals (Continued) Semivolatile Organic Compounds Analytical Results Summary

Table A-1. HLLWE Run ID: 0010-STRT-1

\begin{tabular}{|c|c|c|c|c|c|c|c|}
\hline \multirow[b]{2}{*}{ Analyte } & \multirow{2}{*}{$\begin{array}{c}\text { CAS } \\
\text { Registry } \\
\text { Number } \\
\end{array}$} & $\begin{array}{l}\text { MM-5 Train } \\
\text { Front Half } \\
\text { Composite } \\
(\mu \mathrm{g})\end{array}$ & $\begin{array}{c}\text { MM-5 Train } \\
\text { Back Half } \\
\text { Composite }^{2} \\
(\mu \mathrm{g}) \\
\end{array}$ & $\begin{array}{c}\text { MM-5 Train } \\
\text { Condensate } \\
\text { Composite }^{3} \\
(\mu \mathrm{g}) \\
\end{array}$ & \multicolumn{2}{|c|}{$\begin{array}{c}\text { MM-5 Train } \\
\text { Totals }^{4} \\
\text { (Total } \mu \mathrm{g}) \\
\end{array}$} & \multirow{2}{*}{$\begin{array}{c}\text { Project } \\
\text { Specific } \\
\text { Flag }^{7} \\
\end{array}$} \\
\hline & & Risk Result Flag ${ }^{5}$ & Risk Result Flag ${ }^{5}$ & 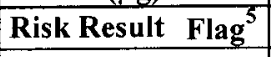 & Total $^{6}$ & Flag & \\
\hline \multicolumn{8}{|l|}{$T I C s^{9}$} \\
\hline Furan, 2,5-dimethyl- & $625-86-5$ & 4.1 & --- & 9.5 & 14 & $\mathrm{~N}, \mathrm{~J}, \mathrm{M}$ & $P$ \\
\hline 3-Hexanone & $589-38-8$ & --- & 190 & -- & 190 & $\mathrm{~N}, \mathrm{~J}, \mathrm{M}$ & $P$ \\
\hline 2-Hexanone & $591-78-6$ & -- & 230 & $\cdots$ & 230 & $\mathrm{~N}, \mathrm{~J}, \mathrm{M}$ & $\mathrm{P}$ \\
\hline Octane, 3-methyl- & $2216-33-3$ & 7.9 & --- & $\cdots$ & 7.9 & $\mathrm{~N}, \mathrm{~J}, \mathrm{M}$ & $\mathrm{P}$ \\
\hline Benzaldehyde & $100-52-7$ & --- & 1,100 & -- & 1,100 & $\mathrm{~N}, \mathrm{~J}, \mathrm{M}$ & $\mathrm{P}$ \\
\hline Dodecane & $112-40-3$ & $\ldots$ & 67 & --- & 67 & $\mathrm{~N}, \mathrm{~J}, \mathrm{M}$ & $\mathrm{P}$ \\
\hline Tridecane & $629-50-5$ & --- & 20 & -- & 20 & $\mathrm{~N}, \mathrm{~J}, \mathrm{M}$ & $\mathrm{P}$ \\
\hline Tetradecane & $629-59-4$ & 8.6 & -- & -- & 8.6 & $\mathrm{~N}, \mathrm{~J}, \mathrm{M}$ & $\mathrm{P}$ \\
\hline Pentadecane & $629-62-9$ & 15 & $\cdots$ & -- & 15 & $\mathrm{~N}, \mathrm{~J}, \mathrm{M}$ & $\mathrm{P}$ \\
\hline Phosphoric acid tributyl ester & $126-73-8$ & 23 & --- & $\cdots$ & 23 & $\mathrm{~N}, \mathrm{~J}, \mathrm{M}$ & $\mathrm{P}$ \\
\hline Cyclododecane & $294-62-2$ & 20 & -- & 11 & 31 & $\mathrm{~N}, \mathrm{~J}, \mathrm{M}$ & $\mathbf{P}$ \\
\hline Heptadecane & $629-78-7$ & 5.5 & --- & 3.4 & 8.9 & $\mathrm{~N}, \mathrm{~J}, \mathrm{M}$ & $\mathrm{P}$ \\
\hline Eicosane & $112-95-8$ & --- & --- & 2.0 & 2.0 & $\mathrm{~N}, \mathrm{~J}, \mathrm{M}$ & $P$ \\
\hline Hexadecanoic acid & $57-10-3$ & 5.4 & --- & --- & 5.4 & $\mathrm{~N}, J, M$ & $P$ \\
\hline Octadecanoic acid & $57-11-4$ & 3.6 & --- & --- & 3.6 & $\mathrm{~N}, \mathrm{~J}, \mathrm{M}$ & $P$ \\
\hline Phosphine oxide, triphenyl- & $791-28-6$ & --- & --- & 8.7 & 8.7 & $\mathrm{~N}, \mathrm{~J}, \mathrm{M}$ & $\mathrm{P}$ \\
\hline Heneicosane & $629-94-7$ & $\cdots$ & -- & 2.0 & 2.0 & $\mathrm{~N}, \mathrm{~J}, \mathrm{M}$ & $\mathrm{P}$ \\
\hline Tetratetracontane & $7098-22-8$ & -- & $-\cdots$ & 6.6 & 6.6 & $\mathrm{~N}, \mathrm{~J}, \mathrm{M}$ & $P$ \\
\hline Eicosane & $112-95-8$ & --- & --- & 4.1 & 4.1 & $\mathrm{~N}, J, M$ & $P$ \\
\hline
\end{tabular}

Last saved by Robin Gifford on 02/04/02 at 4:28 PM

E:My Documents\HLLWE Sampling\Final ReportłAppendix A\Appendix A-1. 0010-STRT-I.doc

Created on 2/4/2002 4:05 PM 


\section{BECHTEL BWXT IDAHO, LLC (BBWI) INTEC HLLWE Effluent Gas Emissions Inventory Idaho National Engineering and Environmental Laboratory (INEEL) STL Knoxville Project Number: 142503.40}

Footnotes:

1 The MM-5 Train Front Half Composite consists of the Particulate Filter and the Front Half of the Filter Holder and Probe Solvent Rinses.

2 The MM-5 Train Back Half Composite consists of the XAD-2 Resin Tube and the Back Half of the Filter Holder and Coil Condenser Solvent Rinses.

3 The MM-5 Train Condensate Composite consists of the Condensate and Impinger Contents and the Glassware Solvent Rinses.

4 The total mass for each semivolatile compound found in the MM-5 sampling train consists of the sum of the MM-5 train's Front Half Composite contents, the train's Back Half Composite contents, and the Condensate Composite. The calculation is as follows:

$($ Total $\mu \mathrm{g}$ in the Front Half $)+($ Total $\mu \mathrm{g}$ in the Back Half $)+($ Concentration in the Condensate Composite $\times$ Condensate Composite Volume) $=$ Total $\mu \mathrm{g}$ in the MM-5 Sampling Train.

Therefore: $\quad(\mu \mathrm{g})+(\mu \mathrm{g})+(\mu \mathrm{g} /$ Liter $\times$ Liter $)=$ Total $\mu \mathrm{g}$

The MM-5 Train Run Total (in Total $\mu \mathrm{g}$ ) is the sum of results for the three (3) MM-5 train sample fractions using the following guidelines:

- When the train component analytical result is greater than the laboratory reporting limit (RL), the result included in the train total is the actual analytical result or "hit" determined by the laboratory.

- When the train component analytical result is greater than the reliable detection level (RDL), but less than the laboratory reporting limit $(\mathrm{RL})$, the result included in the train total is actual analytical result or "hit" determined by the laboratory and the corresponding " $\mathrm{J}$ " flag is carried through the calculation to the train total.

- When the train analytical component result is less than the RDL, but greater than the method detection limit (MDL), the result included in the train total is the RDL and the corresponding "J" flag is carried through the calculation to the train total.

- When the train component analytical result is not detected down to the MDL, the result included in the train total is the RDL and the corresponding " $U$ " flag is carried through the calculation to the train total.

- It should be noted that when the RDL is selected as the default value using the guidelines above, but the RDL is greater than the $\mathrm{RL}$, the $\mathrm{RL}$ is included in the train total.

The data flags attached to the MM-5 Train Total are the cumulative set of flags for each train component included as part of the MM5 train total. A flag attached to an MM-5 train component is carried through to the "MM-5 Train Total" column when the associated component analytical result is a significant number in comparison to the MM-5 Train Total. That is, if the MM-5 Train Total is affected by an MM-5 train component analytical result, the flag is carried through to the MM- 5 Train Total, but if the MM-5 Train Total is not affected by an MM-5 train component, the flag is not carried through to the MM-5 Train Total. The combinations of train fractions are conducted following the standard practice of using significant figures found in ASTM E29-93a(1999), "Standard Practice for Using Significant Digits in Test Data to Determine Conformance with Specifications" and Severn Trent Laboratories standard operating procedure number QA-004, "Rounding and Significant Figures". 


\section{BECHTEL BWXT IDAHO, LLC (BBWI) \\ INTEC HLLWE Effluent Gas Emissions Inventory \\ Idaho National Engineering and Environmental Laboratory (INEEL) \\ STL Knoxville Project Number: 142503.40}

5 This flag is the laboratory data flag that corresponds to EPA guidelines. The data flags for these samples are as follows:

- A "U" qualifier indicates that this analyte was analyzed for, but was not detected down to the MDL.

- A " $\mathrm{J}$ " qualifier indicates that this compound was detected, but at a concentration below the laboratory RL. The analytical result is therefore an estimated value.

- A "B" qualifier indicates that this compound was found in the associated laboratory method blank. Under these conditions these values are regarded as estimated values.

- A "D" qualifier indicates that this result was obtained through dilution of the sample. This original analysis yielded a result that exceeded the calibration range.

- An "N" qualifier indicates that this compound is a tentatively identified compound (TIC). Therefore the value is estimated.

- An "E" qualifier indicates that this compound exceeded the calibration range of the instrument.

- An "A" qualifier indicates that this result is an Aldol-condensation product.

- An "M" qualifier indicates that this result was measured against the nearest internal standard and assumed a response factor of one (1).

6 When listed, the less than $(<)$ sign indicates that at least one sample fraction result is either a "non-detect" value down to the MDL of the measurement that carries, or an estimated "hit" value that is below the RDL. In either case, the final value for the fraction that is included in the data set total is the default RDL value and the actual value of the total is known to be less than $(<)$ the displayed result.

7 Entries in this column are project-specific train total flags that are applied to the run total values and are not standard EPA data flags. These project-specific flags are utilized for the INEEL NWCF HLLWE Effluent Gas Emissions Inventory project and are defined as follows:

- An " $N$ " flag in this column indicates that the compound was not measured (detected) in any of the sampling train components, or fractions.

- A "P" flag in this column indicates that the compound was measured (detected) in one or more of the train components, or fractions, but not in all of the sampling train fractions.

- An "A" flag in this column indicates that the compound was measured (detected) in all of the sampling train components, or fractions.

8 Bis(2-chloroisopropyl)ether and 2,2'-Oxybis(1-chloropropane) are synonyms.

9 The tentatively identified compounds (TICs) were identified by conducting a mass spectral library search using the NBS library of data. It should be noted that TICs that give the same mass spectral match for GC peaks at different retention times are listed separately with the same compound identity. Under these conditions the compounds are likely indistinguishable isomers of the same compound. However, insufficient evidence is available to determine unequivocal identities.

Last saved by Robin Gifford on 02/04/02 at 4:28 PM

E:IMy Documents UHLLWE Sampling $\backslash$ Final Report Appendix A\Appendix A-1. 0010-STRT-1.doc

Created on 2/4/2002 4:05 PM 
BECHTEL BWXT IDAHO, LLC (BBWI)

INTEC HLLWE Effluent Gas Emissions Inventory

Idaho National Engineering and Environmental Laboratory (INEEL)

STL Knoxville Project Number: 142503.40

\section{MM-5 Train Summary - Run 3 Train Totals \\ Semivolatile Organic Compounds Analytical Results Summary \\ Table A-2. HLLWE Run ID: 0010-END-1}

Field Sample Name:

Sample Description:
MM-5 Train

MM-5 Train Totals for Semivolatile Organic Compounds Analysis

\begin{tabular}{|c|c|c|c|c|c|c|c|c|c|c|}
\hline \multirow[b]{2}{*}{ Analyte } & \multirow{2}{*}{$\begin{array}{c}\text { CAS } \\
\text { Registry } \\
\text { Number } \\
\end{array}$} & \multicolumn{2}{|c|}{$\begin{array}{l}\text { MM-5 Train } \\
\text { Front Half } \\
\text { Composite } \\
(\mu \mathrm{g})\end{array}$} & \multicolumn{2}{|c|}{$\begin{array}{c}\text { MM-5 Train } \\
\text { Back Half } \\
\text { Composite }^{2} \\
(\mu \mathrm{g}) \\
\end{array}$} & \multicolumn{2}{|c|}{$\begin{array}{c}\text { MM-5 Train } \\
\text { Condensate } \\
\text { Composite }^{3} \\
(\mu g) \\
\end{array}$} & \multicolumn{2}{|c|}{$\begin{array}{c}\text { MM-5 Train } \\
\text { Totals } \\
{ }^{4} \\
\text { Total } \mu \mathrm{g}) \\
\end{array}$} & \multirow{2}{*}{$\begin{array}{l}\text { Project } \\
\text { Specific } \\
\text { Flag }^{7}\end{array}$} \\
\hline & & Risk Result & Flag $^{5}$ & Risk Result & Flag 5 & Risk Result & Flag $^{5}$ & Total $^{6}$ & Flag & \\
\hline \multicolumn{11}{|l|}{ Target Compound List } \\
\hline Acenaphthene & $83-32-9$ & 1.3 & $\mathrm{U}$ & 6.6 & $\mathrm{U}$ & 1.9 & $\mathrm{U}$ & $<9.8$ & & $\mathrm{~N}$ \\
\hline Acenaphthylene & $208-96-8$ & 1.3 & $\mathrm{U}$ & 6.6 & U & 1.6 & $\mathrm{U}$ & $<9.5$ & & $N$ \\
\hline Acetophenone & $9[8-86-2$ & 2.0 & U & 31 & J & 2.6 & U & $<36$ & $J$ & $\mathrm{P}$ \\
\hline Aniline & $62-53-3$ & 2.5 & $\mathrm{U}$ & 94 & $\mathrm{U}$ & 18 & $\mathrm{U}$ & $<110$ & & $\mathrm{~N}$ \\
\hline Anthracene & $120-12-7$ & 1.3 & $\mathrm{U}$ & 6.6 & $\mathrm{U}$ & 1.6 & $\mathrm{U}$ & $<9.5$ & & $\mathrm{~N}$ \\
\hline Benzidine & $92-87-5$ & 100 & $\mathrm{U}$ & 500 & $\mathrm{U}$ & 66 & $\mathrm{U}$ & $<670$ & & $N$ \\
\hline Benzoic acid & $65-85-0$ & 100 & $\mathrm{U}$ & 2,500 & $\mathrm{E}$ & 9.4 & $\mathrm{U}$ & $<2,600$ & $\mathrm{E}$ & $\mathrm{P}$ \\
\hline Benzo(a)anthracene & $56-55-3$ & 2.2 & $\mathrm{U}$ & 7.6 & U & 1.7 & $\mathrm{U}$ & $<12$ & & $\mathrm{~N}$ \\
\hline Benzo(a)pyrene & $50-32-8$ & 2.6 & $\mathrm{U}$ & 130 & $\mathrm{U}$ & 1.8 & U & $<130$ & & $\mathrm{~N}$ \\
\hline Benzo(b)fluoranthene & $205-99-2$ & 3.7 & U & 290 & $\mathrm{U}$ & 4.2 & $\mathrm{U}$ & $<300$ & & $\mathrm{~N}$ \\
\hline Benzo(g,h,i)perylene & $191-24-2$ & 7.3 & U & 160 & $U$ & 2.1 & $\mathrm{U}$ & $<170$ & & $N$ \\
\hline Benzo(k)fluoranthene & $207-08-9$ & 5.5 & $\mathrm{U}$ & 420 & U & 2.9 & $\mathrm{U}$ & $<430$ & & $\mathrm{~N}$ \\
\hline Benzyl alcohol & $100-51-6$ & 92 & $\mathrm{U}$ & 470 & $U$ & 4.2 & $\mathrm{U}$ & $<570$ & & $\mathrm{~N}$ \\
\hline bis(2-Chloroethoxy)methane & $111-91-1$ & 1.5 & $\mathrm{U}$ & 6.6 & $\mathrm{U}$ & 2.0 & U & $<10$ & & $\mathrm{~N}$ \\
\hline bis(2-Chloroethyl)ether & $111-44-4$ & 2.0 & U & 7.3 & $\mathrm{U}$ & 1.7 & $\mathrm{U}$ & $<11$ & & $\mathrm{~N}$ \\
\hline bis(2-Ethylhexyl)phthalate & $117-81-7$ & 49 & & 100 & $\mathrm{U}$ & 68 & & $<220$ & & $P$ \\
\hline 4-Bromophenyl-phenylether & $101-55-3$ & 1.4 & $\mathrm{U}$ & 6.6 & $\mathrm{U}$ & 1.4 & $\mathrm{U}$ & $<9.4$ & & N \\
\hline Butylbenzylphthalate & $85-68-7$ & 2.9 & $\mathrm{U}$ & 7.9 & $\mathrm{U}$ & 2.3 & $\mathrm{U}$ & $<13$ & & $N$ \\
\hline Carbazole & $86-74-8$ & 2.0 & $\mathrm{U}$ & 8.4 & $\mathrm{U}$ & 2.2 & $\mathrm{U}$ & $<13$ & & $N$ \\
\hline 4-Chloro-3-methylphenol & $59-50-7$ & 2.6 & $\mathrm{U}$ & 8.1 & $\mathrm{U}$ & 6.6 & $\mathrm{U}$ & $<17$ & & $\mathrm{~N}$ \\
\hline 4-Chloroaniline & $106-47-8$ & 3.1 & $\mathrm{U}$ & 79 & $\mathrm{U}$ & 7.9 & $\mathrm{U}$ & $<90$ & & $N$ \\
\hline 2-Chloronaphthalene & $91-58-7$ & 1.3 & $\mathrm{U}$ & 6.6 & $\mathrm{U}$ & 1.4 & $\mathrm{U}$ & $<9.3$ & & $\mathrm{~N}$ \\
\hline 2-Chlorophenol & $95-57-8$ & 2.6 & $\mathrm{U}$ & 6.6 & $\mathrm{U}$ & 1.7 & $\mathrm{U}$ & $<11$ & & $\mathrm{~N}$ \\
\hline 4-Chlorophenyl phenyl ether & $7005-72-36$ & 1.3 & $\mathrm{U}$ & 6.6 & $\mathrm{U}$ & 3.1 & $\mathrm{U}$ & $<11$ & & $\mathrm{~N}$ \\
\hline Chrysene & $218-01-9$ & 2.3 & $\mathrm{U}$ & 8.4 & $\mathrm{U}$ & 1.3 & $\mathrm{U}$ & $<12$ & & $\mathrm{~N}$ \\
\hline
\end{tabular}

Last saved by Robin Gifford on 02/04/02 at 4:29 PM

E:My Documents $\backslash$ HLLWE Sampling $\backslash F i n a l$ Report Appendix A AAppendix A-2. 0010-END-1.doc

Created on 2/4/2002 4:18 PM 
MM-5 Train Summary - Run 3 Train Totals (Continued) Semivolatile Organic Compounds Analytical Results Summary Table A-2. HLLWE Run ID: 0010-END-1

\begin{tabular}{|c|c|c|c|c|c|c|c|c|c|c|}
\hline \multirow[b]{2}{*}{ Analyte } & \multirow{2}{*}{$\begin{array}{c}\text { CAS } \\
\text { Registry } \\
\text { Number }\end{array}$} & \multicolumn{2}{|c|}{$\begin{array}{c}\text { MM-5 Train } \\
\text { Front Half } \\
\text { Composite } \\
(\mu \mathrm{g})\end{array}$} & \multicolumn{2}{|c|}{$\begin{array}{l}\text { MM-5 Train } \\
\text { Back Half } \\
\text { Composite }{ }^{2} \\
(\mu g)\end{array}$} & \multicolumn{2}{|c|}{$\begin{array}{c}\text { MM-5 Train } \\
\text { Condensate } \\
\text { Composite } \\
(\mu \mathrm{g}) \\
\end{array}$} & \multicolumn{2}{|c|}{$\begin{array}{l}\text { MM-5 Train } \\
\text { Totals }^{4} \\
(\text { Total } \mu g) \\
\end{array}$} & \multirow{2}{*}{$\begin{array}{l}\text { Project } \\
\text { Specific } \\
\text { Flag }^{7}\end{array}$} \\
\hline & & Risk Result & Flag 5 & Risk Result & Flag $^{5}$ & Risk Result & Flag 5 & Total $^{6}$ & Flag & \\
\hline Di-n-butylphthalate & $84-74-2$ & 1.9 & J & 100 & $\mathrm{U}$ & 2.3 & $\mathrm{U}$ & $<100$ & J & $\mathrm{P}$ \\
\hline Di-n-octylphthalate & $117-84-0$ & 8.4 & $\mathrm{~J}$ & 150 & $\mathrm{U}$ & 2.5 & $J$ & $<160$ & $J$ & $P$ \\
\hline Dibenz $(\mathrm{a}, \mathrm{h})$ anthracene & $53-70-3$ & 5.2 & $\mathrm{U}$ & 160 & $\mathrm{U}$ & 2.9 & $\mathrm{U}$ & $<170$ & & N \\
\hline Dibenzofuran & $132-64-9$ & 1.4 & U & 6.6 & $\mathrm{U}$ & 2.9 & $\mathrm{U}$ & $<11$ & & $\mathrm{~N}$ \\
\hline 1,2-Dichlorobenzene & $95-50-1$ & 2.2 & $\mathrm{U}$ & 6.8 & $\mathrm{U}$ & 1.7 & $U$ & $<11$ & & $N$ \\
\hline 1,3-Dichlorobenzene & $541-73-1$ & 3.1 & $\mathrm{U}$ & 7.3 & U & 1.4 & $\mathrm{U}$ & $<12$ & & $\mathrm{~N}$ \\
\hline 1,4-Dichlorobenzene & $106-46-7$ & 2.9 & $\mathrm{U}$ & 6.8 & $\mathrm{~J}$ & 2.0 & $\mathrm{U}$ & $<12$ & $J$ & $\mathrm{P}$ \\
\hline 3,3'-Dichlorobenzidine & $91-94-1$ & 7.1 & $\mathrm{U}$ & 97 & $\mathrm{U}$ & 7.9 & $\mathrm{U}$ & $<110$ & & $\mathrm{~N}$ \\
\hline 2,4-Dichlorophenol & $120-83-2$ & 3.9 & $\mathrm{U}$ & 6.6 & $\mathrm{U}$ & 2.3 & $\mathrm{U}$ & $<13$ & & $\mathrm{~N}$ \\
\hline Diethylphthalate & $84-66-2$ & 3.9 & $\mathrm{U}$ & 9.4 & $\mathrm{U}$ & 1.4 & $\mathrm{U}$ & $<15$ & & $\mathrm{~N}$ \\
\hline Dimethyl phthalate & $131-11-3$ & 1.7 & $\mathrm{U}$ & 6.6 & $\mathrm{U}$ & 1.3 & $\mathrm{U}$ & $<9.6$ & & $\mathrm{~N}$ \\
\hline 2,4-Dimethylphenol & $105-67-9$ & 7.6 & $\mathrm{U}$ & 50 & $\mathrm{U}$ & 1.5 & $\mathrm{U}$ & $<59$ & & $N$ \\
\hline 4,6-Dinitro-2-methylphenol & $534-52-1$ & 13 & $\mathrm{U}$ & 120 & $\mathrm{U}$ & 1.5 & $\mathrm{U}$ & $<130$ & & $\mathrm{~N}$ \\
\hline 2,4-Dinitrophenol & $51-28-5$ & 16 & $\mathrm{U}$ & 250 & U & 3.9 & $\mathrm{U}$ & $<270$ & & $\mathrm{~N}$ \\
\hline 2,4-Dinitrotoluene & $121-14-2$ & 4.2 & $\mathrm{U}$ & 6.6 & $\mathrm{U}$ & 2.6 & $\mathrm{U}$ & $<13$ & & $N$ \\
\hline 2,6-Dinitrotoluene & $606-20-2$ & 3.4 & $\mathrm{U}$ & 6.6 & $U$ & 2.1 & $U$ & $<12$ & & $N$ \\
\hline 1,2-Diphenylhydrazine & $122-66-7$ & 1.7 & $U$ & 6.6 & $\mathrm{U}$ & 1.5 & $\mathrm{U}$ & $<9.8$ & & $\mathrm{~N}$ \\
\hline Fluoranthene & $206-44-0$ & 1.3 & $\mathrm{U}$ & 7.1 & $\mathrm{U}$ & 1.8 & U & $<10$ & & $N$ \\
\hline Fluorene & $86-73-7$ & 1.3 & $\mathrm{U}$ & 6.6 & $\mathrm{U}$ & 2.6 & $\mathrm{U}$ & $<10$ & & $N$ \\
\hline Hexachlorocyclopentadiene & $77-47-4$ & 26 & $\mathrm{U}$ & 130 & U & 6.6 & $\mathrm{U}$ & $<160$ & & $\mathrm{~N}$ \\
\hline Hexachlorobenzene & $118-74-1$ & 1.5 & $\mathrm{U}$ & 6.6 & $\mathrm{U}$ & 2.6 & $\mathrm{U}$ & $<11$ & & $N$ \\
\hline Hexachlorobutadiene & $87-68-3$ & 3.7 & $\mathrm{U}$ & 9.7 & $\mathrm{U}$ & 1.9 & $\mathrm{U}$ & $<15$ & & $\mathrm{~N}$ \\
\hline Hexachloroethane & $67-72-1$ & 6.6 & $\mathrm{U}$ & 7.1 & $\mathrm{U}$ & 1.9 & $\mathrm{U}$ & $<16$ & & $\mathrm{~N}$ \\
\hline Indeno(1,2,3-cd)pyrene & $193-39-5$ & 5.5 & U & 140 & U & 2.3 & $\mathrm{U}$ & $<150$ & & $\mathrm{~N}$ \\
\hline Isophorone & $78-59-1$ & 1.7 & U & 6.6 & $\mathrm{U}$ & 1.8 & $\mathrm{U}$ & $<10$ & & $\mathrm{~N}$ \\
\hline 2-Methylnaphthalene & $91-57-6$ & 1.5 & $\mathrm{U}$ & 6.6 & $\mathrm{U}$ & 2.3 & $\mathrm{U}$ & $<10$ & & $N$ \\
\hline 2-Methylphenol & $95-48-7$ & 6.0 & $\mathrm{U}$ & 39 & $\mathrm{U}$ & 2.1 & $\mathrm{U}$ & $<47$ & & $\mathrm{~N}$ \\
\hline 3-Methylphenol \& 4-Methylphenol & $65794-96-9$ & 6.0 & $\mathrm{U}$ & 26 & $\mathrm{U}$ & 2.2 & U & $<34$ & & $\mathrm{~N}$ \\
\hline
\end{tabular}


BECHTEL BWXT IDAHO, LLC (BBWI)

INTEC HLLWE Effluent Gas Emissions Inventory

Idaho National Engineering and Environmental Laboratory (INEEL)

STL Knoxville Project Number: 142503.40

\section{MM-5 Train Summary - Run 3 Train Totals (Continued) Semivolatile Organic Compounds Analytical Results Summary Table A-2. HLLWE Run ID: 0010-END-1}

\begin{tabular}{|c|c|c|c|c|c|c|c|c|c|c|}
\hline \multirow[b]{2}{*}{ Analyte } & \multirow{2}{*}{$\begin{array}{c}\text { CAS } \\
\text { Registry } \\
\text { Number } \\
\end{array}$} & \multicolumn{2}{|c|}{$\begin{array}{c}\text { MM-5 Train } \\
\text { Front Half } \\
\text { Composite } \\
(\mu \mathrm{g})\end{array}$} & \multicolumn{2}{|c|}{$\begin{array}{c}\text { MM-5 Train } \\
\text { Back Half } \\
\text { Composite }^{2} \\
(\mu g)\end{array}$} & \multicolumn{2}{|c|}{$\begin{array}{c}\text { MM-5 Train } \\
\text { Condensate } \\
\text { Composite }^{3} \\
(\mu \mathrm{g}) \\
\end{array}$} & \multicolumn{2}{|c|}{$\begin{array}{c}\text { MM-5 Train } \\
\text { Totals }^{4} \\
\text { (Total } \mu \mathrm{g}) \\
\end{array}$} & \multirow{2}{*}{$\begin{array}{c}\text { Project } \\
\text { Specific } \\
\text { Flag }^{7}\end{array}$} \\
\hline & & Risk Result & Flag 5 & Risk Result & Flag $^{5}$ & Risk Result & Flag $^{5}$ & Total $^{6}$ & Flag & \\
\hline N-Nitroso-di-n-propylamine & $621-64-7$ & 1.9 & $\mathrm{U}$ & 6.6 & $\mathrm{U}$ & 2.3 & $\mathrm{U}$ & $<11$ & & $\mathrm{~N}$ \\
\hline N-Nitrosodimethylamine & $62-75-9$ & 1.9 & $\underline{U}$ & 6.6 & $\mathrm{U}$ & 2.2 & $\mathrm{U}$ & $<11$ & & $\mathrm{~N}$ \\
\hline N-Nitrosodiphenylamine & $86-30-6$ & 1.6 & $\mathrm{U}$ & 12 & $\mathrm{U}$ & 1.4 & $\mathrm{U}$ & $<15$ & & $N$ \\
\hline Naphthalene & $91-20-3$ & 1.3 & $\mathrm{U}$ & 7.9 & $\mathrm{U}$ & 1.8 & $\mathrm{U}$ & $<11$ & & $\mathrm{~N}$ \\
\hline 2-Nitroaniline & $88-74-4$ & 1.5 & $\mathrm{U}$ & 6.6 & $\mathrm{U}$ & 2.9 & $\mathrm{U}$ & $<11$ & & $\mathrm{~N}$ \\
\hline 3-Nitroaniline & $99-09-2$ & 10 & $\mathrm{U}$ & 26 & U & 4.7 & $\mathrm{U}$ & $<41$ & & $N$ \\
\hline 4-Nitroaniline & $100-01-6$ & 6.0 & $\mathrm{U}$ & 26 & $\mathrm{U}$ & 3.9 & $\mathrm{U}$ & $<36$ & & $\mathrm{~N}$ \\
\hline Nitrobenzene & $98-95-1$ & 1.9 & $\mathrm{U}$ & 11 & J & 1.7 & U & $<15$ & $\mathrm{~J}$ & $\mathrm{P}$ \\
\hline 2-Nitrophenol & $88-75-5$ & 8.4 & $\mathrm{U}$ & 30 & $J$ & 2.6 & $\mathrm{U}$ & $<41$ & $\mathbf{J}$ & $\mathrm{P}$ \\
\hline 4-Nitrophenol & $100-02-7$ & 8.7 & $\mathrm{U}$ & 42 & $\mathrm{U}$ & 3.9 & $\mathrm{U}$ & $<55$ & & $\mathrm{~N}$ \\
\hline $2,2^{\prime}$-Oxybis(1-chloropropane) ${ }^{8}$ & $108-60-1$ & 2.6 & $\mathrm{U}$ & 10 & $\mathrm{U}$ & 1.8 & $\mathrm{U}$ & $<14$ & & $N$ \\
\hline Pentachlorobenzene & $608-93-5$ & 1.4 & $\mathrm{U}$ & 6.6 & $\mathrm{U}$ & 2.3 & $\mathrm{U}$ & $<10$ & & $\mathrm{~N}$ \\
\hline Pentachloronitrobenzene & $82-68-8$ & 2.0 & $\mathrm{U}$ & 6.6 & $\mathrm{U}$ & 2.6 & $\mathrm{U}$ & $<11$ & & $\mathrm{~N}$ \\
\hline Pentachlorophenol & $87-86-5$ & 50 & $\mathrm{U}$ & 250 & U & 3.4 & $\mathrm{U}$ & $<300$ & & $\mathrm{~N}$ \\
\hline Phenanthrene & $85-01-8$ & 1.3 & $\mathrm{U}$ & 6.6 & $\mathrm{U}$ & 1.9 & U & $<9.8$ & & $N$ \\
\hline Phenol & $108-95-2$ & 2.9 & $\mathrm{U}$ & 32 & J & 2.2 & $\mathbf{J}$ & $<37$ & $\mathbf{J}$ & $\mathrm{P}$ \\
\hline Pyrene & $129-00-0$ & 1.9 & $\mathrm{U}$ & 6.8 & $\mathrm{U}$ & 1.4 & $\mathrm{U}$ & $<10$ & & N \\
\hline Pyridine & $110-86-1$ & 2.3 & U & 9.7 & $\mathrm{U}$ & 5.2 & $\mathrm{U}$ & $<17$ & & $\mathrm{~N}$ \\
\hline 1,2,4,5-Tetrachlorobenzene & $95-94-3$ & 2.3 & $\mathrm{U}$ & 6.6 & $\mathrm{U}$ & 2.2 & $\mathrm{U}$ & $<11$ & & $\mathrm{~N}$ \\
\hline 1,2,4-Trichlorobenzene & $120-82-1$ & 1.9 & $\mathrm{U}$ & 7.9 & $\mathrm{U}$ & 2.2 & $\mathrm{U}$ & $<12$ & & $\mathrm{~N}$ \\
\hline 2,4,5-Trichlorophenol & $95-95-4$ & 6.0 & $\mathrm{U}$ & 17 & $\mathrm{U}$ & 2.1 & $\mathrm{U}$ & $<25$ & & $\mathrm{~N}$ \\
\hline 2,4,6-Trichlorophenol & $88-06-2$ & 3.7 & $\mathrm{U}$ & 10 & $U$ & 2.5 & $\mathrm{U}$ & $<16$ & & $\mathrm{~N}$ \\
\hline
\end{tabular}




\section{MM-5 Train Summary - Run 3 Train Totals (Continued) Semivolatile Organic Compounds Analytical Results Summary Table A-2. HLLWE Run ID: 0010-END-1}

\begin{tabular}{|c|c|c|c|c|c|c|c|}
\hline \multirow[b]{2}{*}{ Analyte } & \multirow{2}{*}{$\begin{array}{c}\text { CAS } \\
\text { Registry } \\
\text { Number } \\
\end{array}$} & $\begin{array}{c}\text { MM-5 Train } \\
\text { Front Half } \\
\text { Composite } \\
(\mu \mathrm{g})\end{array}$ & $\begin{array}{c}\text { MM-5 Train } \\
\text { Back Half } \\
\text { Composite }^{2} \\
(\mu g)\end{array}$ & $\begin{array}{c}\text { MM-5 Train } \\
\text { Condensate } \\
\text { Composite }^{3} \\
(\mu g)\end{array}$ & \multicolumn{2}{|c|}{$\begin{array}{c}\text { MM-5 Train } \\
\text { Totals }^{4} \\
\text { (Total } \mu g) \\
\end{array}$} & \multirow{2}{*}{$\begin{array}{c}\text { Project } \\
\text { Specific } \\
\text { Flag }^{7} \\
\end{array}$} \\
\hline & & Risk Result Flag ${ }^{5}$ & Risk Result Flag ${ }^{5}$ & Risk Result Flag $^{5}$ & Total $^{6}$ & Flag & \\
\hline \multicolumn{8}{|l|}{$T I C s^{9}$} \\
\hline Furan, 2,5-dimethyl- & $625-86-5$ & 4.7 & $\ldots$ & 3.1 & 7.8 & $\mathrm{~N}, \mathrm{~J}, \mathrm{M}$ & $\mathrm{P}$ \\
\hline 3-Hexanone & $589-38-8$ & $-\cdots$ & 96 & $-\cdots$ & 96 & $\mathrm{~N}, \mathrm{~J}, \mathrm{M}$ & $\mathrm{P}$ \\
\hline 2-Hexanone & $591-78-6$ & --- & 120 & -- & 120 & $\mathrm{~N}, \mathrm{~J}, \mathrm{M}$ & $\mathrm{P}$ \\
\hline Heptane, 2,3-dimethyl- & $3074-71-3$ & 9.9 & --- & $\cdots$ & 9.9 & $\mathrm{~N}, \mathrm{~J}, \mathrm{M}$ & $\mathbf{P}$ \\
\hline Benzaldehyde & $100-52-7$ & $--\cdot$ & 740 & --- & 740 & $\mathrm{~N}, \mathrm{~J}, \mathrm{M}$ & $\mathrm{P}$ \\
\hline Formic acid, phenylmethyl ester & $104-57-4$ & --- & 72 & $-\ldots$ & 72 & $\mathrm{~N}, \mathrm{~J}, \mathrm{M}$ & $\mathrm{P}$ \\
\hline Benzaldehyde, 4-ethyl- & $4748-78-1$ & $\cdots$ & 57 & $-\cdots$ & 57 & $\mathrm{~N}, \mathrm{~J}, \mathrm{M}$ & $\mathrm{P}$ \\
\hline Dodecane & $112-40-3$ & $-\cdots$ & 48 & --- & 48 & $\mathrm{~N}, J, M$ & $P$ \\
\hline Tridecane & $629-50-5$ & -- & 21 & --- & 21 & $\mathrm{~N}, \mathrm{~J}, \mathrm{M}$ & $\mathrm{P}$ \\
\hline 2,4-Hexadiene & $592-46-1$ & $\cdots$ & 45 & -- & 45 & $\mathrm{~N}, \mathrm{~J}, \mathrm{M}$ & $\mathrm{P}$ \\
\hline 2,4-Hexadiene & $592-46-1$ & $-\cdots$ & 110 & $\cdots$ & 110 & $\mathrm{~N}, \mathrm{~J}, \mathrm{M}$ & $\mathrm{P}$ \\
\hline Tetradecane & $629-59-4$ & $-\cdots$ & 96 & $-\cdots$ & 96 & $\mathrm{~N}, \mathrm{~J}, \mathrm{M}$ & $P$ \\
\hline Phosphoric acid tributyl ester & $126-73-8$ & 16 & --- & $\ldots$ & 16 & $\mathrm{~N}, \mathrm{~J}, \mathrm{M}$ & $\mathrm{P}$ \\
\hline Cyclododecane & $294-62-2$ & 24 & --- & 6.5 & 30 & $\mathrm{~N}, \mathrm{~J}, \mathrm{M}$ & $\mathrm{P}$ \\
\hline Heptadecane & $629-78-7$ & 4.0 & $\cdots$ & --- & 4.0 & $\mathrm{~N}, \mathrm{~J}, \mathrm{M}$ & $\mathrm{P}$ \\
\hline Octadecanoic acid & $57-11-4$ & 2.0 & $-\cdots$ & -- & 2.0 & $\mathrm{~N}, \mathrm{~J}, \mathrm{M}$ & $\mathrm{P}$ \\
\hline
\end{tabular}




\section{BECHTEL BWXT IDAHO, LLC (BBWI) \\ INTEC HLLWE Effluent Gas Emissions Inventory \\ Idaho National Engineering and Environmental Laboratory (INEEL) \\ STL Knoxville Project Number: 142503.40}

\section{Footnotes:}

1 The MM-5 Train Front Half Composite consists of the Particulate Filter and the Front Half of the Filter Holder and Probe Solvent Rinses.

2 The MM-5 Train Back Half Composite consists of the XAD-2 Resin Tube and the Back Half of the Filter Holder and Coil Condenser Solvent Rinses.

3 The MM-5 Train Condensate Composite consists of the Condensate and Impinger Contents and the Glassware Solvent Rinses.

4 The total mass for each semivolatile compound found in the MM-5 sampling train consists of the sum of the MM-5 train's Front Half Composite contents, the train's Back Half Composite contents, and the Condensate Composite. The calculation is as follows:

(Total $\mu \mathrm{g}$ in the Front Half) $+($ Total $\mu \mathrm{g}$ in the Back Half $)+($ Concentration in the Condensate Composite $\times$ Condensate Composite Volume)

$=$ Total $\mu \mathrm{g}$ in the MM-5 Sampling Train.

Therefore: $\quad(\mu \mathrm{g})+(\mu \mathrm{g})+(\mu \mathrm{g} /$ Liter $\times$ Liter $)=$ Total $\mu \mathrm{g}$

The MM-5 Train Run Total (in Total $\mu \mathrm{g}$ ) is the sum of results for the three (3) MM-5 train sample fractions using the following guidelines:

- When the train component analytical result is greater than the laboratory reporting limit (RL), the result included in the train total is the actual analytical result or "hit" determined by the laboratory.

- When the train component analytical result is greater than the reliable detection level (RDL), but less than the laboratory reporting limit (RL), the result included in the train total is actual analytical result or "hit" determined by the laboratory and the corresponding " $\mathrm{J}$ " flag is carried through the calculation to the train total.

- When the train analytical component result is less than the RDL, but greater than the method detection limit (MDL), the result included in the train total is the RDL and the corresponding "J" flag is carried through the calculation to the train total.

- When the train component analytical result is not detected down to the MDL, the result included in the train total is the RDL and the corresponding " $U$ " flag is carried through the calculation to the train total.

- It should be noted that when the RDL is selected as the default value using the guidelines above, but the RDL is greater than the $\mathrm{RL}$, the RL is included in the train total.

The data flags attached to the MM-5 Train Total are the cumulative set of flags for each train component included as part of the MM5 train total. A flag attached to an MM-5 train component is carried through to the "MM-5 Train Total" column when the associated component analytical result is a significant number in comparison to the MM- 5 Train Total. That is, if the MM- 5 Train Total is affected by an MM-5 train component analytical result, the flag is carried through to the MM-5 Train Total, but if the MM-5 Train Total is not affected by an MM-5 train component, the flag is not carried through to the MM-5 Train Total. The combinations of train fractions are conducted following the standard practice of using significant figures found in ASTM E29-93a(1999), "Standard Practice for Using Significant Digits in Test Data to Determine Conformance with Specifications" and Severn Trent Laboratories standard operating procedure number QA-004, "Rounding and Significant Figures". 


\section{BECHTEL BWXT IDAHO, LLC (BBWI) INTEC HLLWE Effluent Gas Emissions Inventory Idaho National Engineering and Environmental Laboratory (INEEL) STL Knoxville Project Number: 142503.40}

5 This flag is the laboratory data flag that corresponds to EPA guidelines. The data flags for these samples are as follows:

- A "U" qualifier indicates that this analyte was analyzed for, but was not detected down to the MDL.

- A "J" qualifier indicates that this compound was detected, but at a concentration below the laboratory RL. The analytical result is therefore an estimated value.

- A "B" qualifier indicates that this compound was found in the associated laboratory method blank. Under these conditions these values are regarded as estimated values.

- A "D" qualifier indicates that this result was obtained through dilution of the sample. This original analysis yielded a result that exceeded the calibration range.

- An "N" qualifier indicates that this compound is a tentatively identified compound (TIC). Therefore the value is estimated.

- An "E" qualifier indicates that this compound exceeded the calibration range of the instrument.

- An " $A$ " qualifier indicates that this result is an Aldol-condensation product.

- An "M" qualifier indicates that this result was measured against the nearest internal standard and assumed a response factor of one (1).

6 When listed, the less than $(<)$ sign indicates that at least one sample fraction result is either a "non-detect" value down to the MDL of the measurement that carries, or an estimated "hit" value that is below the RDL. In either case, the final value for the fraction that is included in the data set total is the default RDL value and the actual value of the total is known to be less than $(<)$ the displayed result.

7 Entries in this column are project-specific train total flags that are applied to the run total values and are not standard EPA data flags. These project-specific flags are utilized for the INEEL NWCF HLLWE Effluent Gas Emissions Inventory project and are defined as follows:

- An "N" flag in this column indicates that the compound was not measured (detected) in any of the sampling train components, or fractions.

- A "P" flag in this column indicates that the compound was measured (detected) in one or more of the train components, or fractions, but not in all of the sampling train fractions.

- An " $\mathrm{A}$ " flag in this column indicates that the compound was measured (detected) in all of the sampling train components, or fractions.

Bis(2-chloroisopropyl)ether and 2,2'-Oxybis(1-chloropropane) are synonyms.

9 The tentatively identified compounds (TICs) were identified by conducting a mass spectral library search using the NBS library of data. It should be noted that TICs that give the same mass spectral match for GC peaks at different retention times are listed separately with the same compound identity. Under these conditions the compounds are likely indistinguishable isomers of the same compound. However, insufficient evidence is available to determine unequivocal identities.

Last saved by Robin Gifford on 02/04/02 at 4:29 PM

E:LMy Documents $\backslash$ HLLWE SamplingไFinal Report Appendix AlAppendix A-2. 0010-END-1.doe

Created on $2 / 4 / 20024: 18 \mathrm{PM}$ 
BECHTEL BWXT IDAHO, LLC (BBWI)

INTEC HLLWE Effluent Gas Emissions Inventory

Idaho National Engineering and Environmental Laboratory (INEEL)

STL Knoxville Project Number: 142503.40

\section{MM-5 Train Summary - Run 2 Train Totals Semivolatile Organic Compounds Analytical Results Summary Table A-3. HLLWE Run ID: 0010-STRT-2}

Field Sample Name: Sample Description
MM-5 Train

MM-5 Train Totals for Semivolatile Organic Compounds Analysis

\begin{tabular}{|c|c|c|c|c|c|c|c|c|c|c|}
\hline \multirow[b]{2}{*}{ Analyte } & \multirow{2}{*}{$\begin{array}{c}\text { CAS } \\
\text { Registry } \\
\text { Number } \\
\end{array}$} & \multicolumn{2}{|c|}{$\begin{array}{c}\text { MM-5 Train } \\
\text { Front Half } \\
\text { Composite } \\
(\mu g)\end{array}$} & \multicolumn{2}{|c|}{$\begin{array}{c}\text { MM-5 Train } \\
\text { Back Haif } \\
\text { Composite }{ }^{2} \\
(\mu \mathrm{g})\end{array}$} & \multicolumn{2}{|c|}{$\begin{array}{c}\text { MM-5 Train } \\
\text { Condensate } \\
\text { Composite } \\
(\mu \mathrm{g}) \\
\end{array}$} & \multicolumn{2}{|c|}{$\begin{array}{c}\text { MM-5 Train } \\
\text { Totals } \\
{ }^{4} \\
\text { (Total } \mu \mathrm{g}) \\
\end{array}$} & \multirow{2}{*}{$\begin{array}{l}\text { Project } \\
\text { Specific } \\
\text { Flag }^{7} \\
\end{array}$} \\
\hline & & Risk Result & Flag $^{5}$ & Risk Result & Flag 5 & Risk Result & Flag $^{5}$ & Total $^{6}$ & Flag & \\
\hline \multicolumn{11}{|l|}{ Target Compound List } \\
\hline Acenaphthene & $83-32-9$ & 1.3 & $\mathrm{U}$ & 6.6 & U & 8.0 & J & $<16$ & $\mathrm{~J}$ & $P$ \\
\hline Acenaphthylene & $208-96-8$ & 1.3 & $\mathrm{U}$ & 6.6 & $\mathrm{U}$ & 7.6 & J & $<16$ & $\mathrm{~J}$ & $\mathrm{P}$ \\
\hline Acetophenone & $9[8-86-2$ & 2.0 & $\mathrm{U}$ & 32 & $\mathrm{~J}$ & 7.9 & $\mathrm{U}$ & $<42$ & $J$ & $\mathrm{P}$ \\
\hline Aniline & $62-53-3$ & 2.5 & $\mathrm{U}$ & 94 & $\mathrm{U}$ & 55 & $\mathrm{U}$ & $<150$ & & $\mathrm{~N}$ \\
\hline Anthracene & $120-12-7$ & 1.3 & $\mathrm{U}$ & 6.6 & U & 8.3 & J & $<16$ & $\mathrm{~J}$ & $P$ \\
\hline Benzidine & $92-87-5$ & 100 & $\mathrm{U}$ & 500 & $\mathrm{U}$ & 200 & U & $<800$ & & $N$ \\
\hline Benzoic acid & $65-85-0$ & 100 & U & 2,200 & $\mathrm{E}$ & 29 & $\mathrm{U}$ & $<2,300$ & $\mathrm{E}$ & $\mathrm{P}$ \\
\hline Benzo(a)anthracene & $56-55-3$ & 2.2 & $\mathrm{U}$ & 7.6 & $\mathrm{U}$ & 9.0 & $\mathrm{~J}$ & $<19$ & $\mathrm{~J}$ & $\mathrm{P}$ \\
\hline Benzo(a)pyrene & $50-32-8$ & 2.6 & $\mathrm{U}$ & 130 & $\mathrm{U}$ & 9.3 & $J$ & $<140$ & $\mathrm{~J}$ & $\mathrm{P}$ \\
\hline Benzo(b)fluoranthene & $205-99-2$ & 3.7 & $\mathrm{U}$ & 290 & $\mathrm{U}$ & 13 & $\mathrm{~J}$ & $<300$ & $\mathrm{~J}$ & $\mathrm{P}$ \\
\hline Benzo $(g, h, i)$ perylene & $191-24-2$ & 7.3 & $\mathrm{U}$ & 160 & $U$ & 9.5 & $\mathrm{~J}$ & $<180$ & $\mathrm{~J}$ & $\mathrm{P}$ \\
\hline Benzo(k)fluoranthene & $207-08-9$ & 5.5 & $\mathrm{U}$ & 420 & $\mathrm{U}$ & 9.0 & J & $<430$ & $\mathrm{~J}$ & $\mathrm{P}$ \\
\hline Benzyl alcohol & $100-51-6$ & 92 & $\mathrm{U}$ & 470 & $\mathrm{U}$ & 12 & U & $<570$ & & $\mathrm{~N}$ \\
\hline bis(2-Chloroethoxy)methane & $111-91-1$ & 1.5 & U & 6.6 & $\mathrm{U}$ & 6.0 & U & $<14$ & & $N$ \\
\hline bis(2-Chloroethyl)ether & $111-44-4$ & 2.0 & $\mathrm{U}$ & 7.3 & $\mathrm{U}$ & 6.4 & $J$ & $<16$ & $\mathrm{~J}$ & $\mathrm{P}$ \\
\hline bis(2-Ethylhexyl)phthalate & $117-81-7$ & 16 & & 100 & $\mathrm{~J}$ & 16 & J & $<130$ & $\mathrm{~J}$ & A \\
\hline 4-Bromophenyl-phenylether & $101-55-3$ & 1.4 & $\mathrm{U}$ & 6.6 & $\mathrm{U}$ & 10 & $\mathrm{~J}$ & $<18$ & $J$ & $\mathrm{P}$ \\
\hline Butylbenzylphthalate & $85-68-7$ & 2.9 & $\mathrm{U}$ & 7.9 & $\mathrm{U}$ & 8.2 & J & $<19$ & $\mathbf{J}$ & $\mathrm{P}$ \\
\hline Carbazole & $86-74-8$ & 2.0 & $U$ & 8.4 & $\mathrm{U}$ & 7.2 & $\mathrm{~J}$ & $<18$ & J & $\mathrm{P}$ \\
\hline 4-Chloro-3-methylphenol & $59-50-7$ & 2.6 & $\mathrm{U}$ & 8.1 & $\mathrm{U}$ & 20 & $\mathrm{U}$ & $<31$ & & $\mathrm{~N}$ \\
\hline 4-Chloroaniline & $106-47-8$ & 3.1 & $\mathrm{U}$ & 79 & $\mathrm{U}$ & 24 & $\mathrm{U}$ & $<110$ & & $\mathrm{~N}$ \\
\hline 2-Chloronaphthalene & $91-58-7$ & 1.3 & $\mathrm{U}$ & 6.6 & $\mathrm{U}$ & 6.8 & $\mathrm{~J}$ & $<15$ & $\mathrm{~J}$ & $\mathrm{P}$ \\
\hline 2-Chlorophenol & $95-57-8$ & 2.6 & $\mathrm{U}$ & 6.6 & $\mathrm{U}$ & 5.4 & $\mathrm{~J}$ & $<15$ & $J$ & $P$ \\
\hline 4-Chlorophenyl phenyl ether & $7005-72-36$ & 1.3 & $\mathrm{U}$ & 6.6 & $\mathrm{U}$ & 9.4 & $\mathrm{~J}$ & $<17$ & $\mathrm{~J}$ & $\mathrm{P}$ \\
\hline Chrysene & $218-01-9$ & 2.3 & $\mathrm{U}$ & 8.4 & $\mathrm{U}$ & 9.9 & $\mathrm{~J}$ & $<21$ & $\mathrm{~J}$ & $P$ \\
\hline
\end{tabular}

Last saved by Robin Gifford on 02/04/02 at 4:30 PM

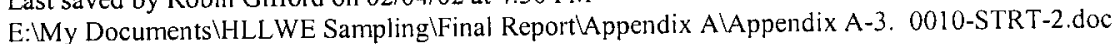

Created on 2/4/2002 4:23 PM 
BECHTEL BWXT IDAHO, LLC (BBWI)

INTEC HLLWE Effluent Gas Emissions Inventory

Idaho National Engineering and Environmental Laboratory (INEEL)

STL Knoxville Project Number: 142503.40

\section{MM-5 Train Summary - Run 2 Train Totals (Continued) Semivolatile Organic Compounds Analytical Results Summary Table A-3. HLLWE Run ID: 0010-STRT-2}

\begin{tabular}{|c|c|c|c|c|c|c|c|c|c|c|}
\hline \multirow[b]{2}{*}{ Analyte } & \multirow{2}{*}{$\begin{array}{c}\text { CAS } \\
\text { Registry } \\
\text { Number } \\
\end{array}$} & \multicolumn{2}{|c|}{$\begin{array}{c}\text { MM-5 Train } \\
\text { Front Half } \\
\text { Composite } \\
(\mu \mathrm{g}) \\
\end{array}$} & \multicolumn{2}{|c|}{$\begin{array}{c}\text { MM-5 Train } \\
\text { Back Half } \\
\text { Composite }^{2} \\
(\mu \mathrm{g}) \\
\end{array}$} & \multicolumn{2}{|c|}{$\begin{array}{c}\text { MM-5 Train } \\
\text { Condensate } \\
\text { Composite } \\
(\mu g)\end{array}$} & \multicolumn{2}{|c|}{$\begin{array}{c}\text { MM-5 Train } \\
\text { Totals }^{4} \\
\text { (Total } \mu \mathrm{g})\end{array}$} & \multirow{2}{*}{$\begin{array}{c}\text { Project } \\
\text { Specific } \\
\text { Flag }^{7}\end{array}$} \\
\hline & & Risk Result & Flag $^{5}$ & Risk Result & Flag $^{5}$ & Risk Result & Flag $^{5}$ & Total $^{6}$ & Flag & \\
\hline Di-n-butylphthalate & $84-74-2$ & 1.9 & $\mathrm{U}$ & 100 & $\mathrm{U}$ & 8.4 & $J$ & $<110$ & $\mathrm{~J}$ & $P$ \\
\hline Di-n-octylphthalate & $117-84-0$ & 5.5 & $\mathrm{~J}$ & 150 & $\mathrm{U}$ & 7.6 & $\mathrm{U}$ & $<160$ & $\mathrm{~J}$ & $\mathrm{P}$ \\
\hline Dibenz(a,h)anthracene & $53-70-3$ & 5.2 & $\mathrm{U}$ & 160 & $\mathrm{U}$ & 8.4 & $\mathrm{~J}$ & $<170$ & $\mathrm{~J}$ & $\mathrm{P}$ \\
\hline Dibenzofuran & $132-64-9$ & 1.4 & U & 6.6 & U & 8.7 & $J$ & $<17$ & $\mathbf{J}$ & $\mathrm{P}$ \\
\hline 1,2-Dichlorobenzene & $95-50-1$ & 2.2 & U & 6.8 & $\mathrm{U}$ & 6.6 & $J$ & $<16$ & $J$ & $P$ \\
\hline 1,3-Dichlorobenzene & $541-73-1$ & 3.1 & $\mathrm{U}$ & 7.3 & U & 6.5 & $J$ & $<17$ & J & $\mathrm{P}$ \\
\hline 1,4-Dichlorobenzene & $106-46-7$ & 2.9 & U & 6.8 & $\mathrm{~J}$ & 6.2 & $J$ & $<16$ & $\mathrm{~J}$ & $\mathrm{P}$ \\
\hline 3,3'-Dichlorobenzidine & $91-94-1$ & 7.1 & $\mathrm{U}$ & 97 & U & 23 & $\mathrm{U}$ & $<130$ & & $\mathrm{~N}$ \\
\hline 2,4-Dichlorophenol & $120-83-2$ & 3.9 & $\mathrm{U}$ & 6.6 & $\mathrm{U}$ & 7.1 & $\mathrm{U}$ & $<18$ & & $N$ \\
\hline Diethylphthalate & $84-66-2$ & 3.9 & $\mathrm{U}$ & 9.4 & $\mathrm{U}$ & 8.9 & $\mathrm{~J}$ & $<22$ & $J$ & $\mathrm{P}$ \\
\hline Dimethyl phthalate & $131-11-3$ & 1.7 & $U$ & 6.6 & $\mathrm{U}$ & 8.1 & $\mathrm{~J}$ & $<16$ & $\mathrm{~J}$ & $\mathrm{P}$ \\
\hline 2,4-Dimethylphenol & $105-67-9$ & 7.6 & $\mathrm{U}$ & 50 & $\mathrm{U}$ & 4.7 & $\mathrm{U}$ & $<62$ & & $N$ \\
\hline 4,6-Dinitro-2-methylphenol & $534-52-1$ & 13 & $\mathrm{U}$ & 120 & $\mathrm{U}$ & 4.5 & $\mathrm{U}$ & $<140$ & & N \\
\hline 2,4-Dinitrophenol & $51-28-5$ & 15 & $\mathrm{U}$ & 250 & U & 12 & $\mathrm{U}$ & $<280$ & & $N$ \\
\hline 2,4-Dinitrotoluene & $121-14-2$ & 4.2 & $\mathrm{U}$ & 6.6 & $\mathrm{U}$ & 8.1 & $J$ & $<19$ & $\mathrm{~J}$ & $\mathrm{P}$ \\
\hline 2,6-Dinitrotoluene & $606-20-2$ & 3.4 & $\mathrm{U}$ & 6.6 & $\mathrm{U}$ & 6.3 & $J$ & $<16$ & $\mathbf{J}$ & $\mathrm{P}$ \\
\hline 1,2-Diphenylhydrazine & $122-66-7$ & 1.7 & $\mathrm{U}$ & 6.6 & $U$ & 7.5 & $\mathbf{J}$ & $<16$ & $\mathbf{J}$ & $\mathrm{P}$ \\
\hline Fluoranthene & $206-44-0$ & 1.3 & U & 7.1 & U & 8.5 & $J$ & $<17$ & $\mathrm{~J}$ & $\mathrm{P}$ \\
\hline Fluorene & $86-73-7$ & 1.3 & $U$ & 6.6 & $\mathrm{U}$ & 8.4 & $\mathrm{~J}$ & $<16$ & $\mathrm{~J}$ & $P$ \\
\hline Hexachlorocyclopentadiene & $77-47-4$ & 26 & $\mathrm{U}$ & 130 & U & 20 & $\mathrm{U}$ & $<180$ & & $\mathrm{P}$ \\
\hline Hexachlorobenzene & $118-74-1$ & 1.5 & $\mathrm{U}$ & 6.6 & $\mathrm{U}$ & 8.3 & $J$ & $<16$ & $\mathrm{~J}$ & $\mathrm{P}$ \\
\hline Hexachlorobutadiene & $87-68-3$ & 3.7 & $\mathrm{U}$ & 9.7 & $\mathrm{U}$ & 6.5 & J & $<20$ & $\mathrm{~J}$ & $P$ \\
\hline Hexachloroethane & $67-72-1$ & 6.6 & $\mathrm{U}$ & 7.1 & $\mathrm{U}$ & 6.4 & $\mathrm{~J}$ & $<20$ & $\mathrm{~J}$ & $P$ \\
\hline Indeno(1,2,3-cd)pyrene & $193-39-5$ & 5.5 & $\mathrm{U}$ & 140 & U & 7.0 & $\mathrm{~J}$ & $<150$ & $J$ & $P$ \\
\hline Isophorone & $78-59-1$ & 1.7 & $\mathrm{U}$ & 6.6 & $\mathrm{U}$ & 7.4 & $\mathrm{~J}$ & $<16$ & $\mathrm{~J}$ & $\mathrm{P}$ \\
\hline 2-Methylnaphthalene & $91-57-6$ & 1.5 & $\mathrm{U}$ & 6.6 & $\mathrm{U}$ & 6.8 & $\mathrm{~J}$ & $<15$ & $J$ & $\mathrm{P}$ \\
\hline 2-Methylphenol & $95-48-7$ & 6.0 & $\mathrm{U}$ & 39 & $\mathrm{U}$ & 6.3 & $\mathrm{U}$ & $<51$ & & $\mathrm{~N}$ \\
\hline 3-Methylphenol \& 4-Methylphenol & $65794-96-9$ & 6.0 & $\mathrm{U}$ & 26 & $\mathrm{U}$ & 6.8 & $U$ & $<39$ & & $\mathrm{~N}$ \\
\hline
\end{tabular}


BECHTEL BWXT IDAHO, LLC (BBWI)

INTEC HLLWE Effluent Gas Emissions Inventory

Idaho National Engineering and Environmental Laboratory (INEEL)

STL Knoxville Project Number: 142503.40

\section{MM-5 Train Summary - Run 2 Train Totals (Continued) Semivolatile Organic Compounds Analytical Results Summary Table A-3. HLLWE Run ID: 0010-STRT-2}

\begin{tabular}{|c|c|c|c|c|c|c|c|c|c|c|}
\hline \multirow[b]{2}{*}{ Analyte } & \multirow{2}{*}{$\begin{array}{c}\text { CAS } \\
\text { Registry } \\
\text { Number } \\
\end{array}$} & \multicolumn{2}{|c|}{$\begin{array}{c}\text { MM-5 Train } \\
\text { Front Half } \\
\text { Composite }{ }^{1} \\
(\mu g)\end{array}$} & \multicolumn{2}{|c|}{$\begin{array}{c}\text { MM-5 Train } \\
\text { Back Half } \\
\text { Composite }^{2} \\
(\mu g)\end{array}$} & \multicolumn{2}{|c|}{$\begin{array}{c}\text { MM-5 Train } \\
\text { Condensate } \\
\text { Composite } \\
(\mu \mathrm{g}) \\
\end{array}$} & \multicolumn{2}{|c|}{$\begin{array}{c}\text { MM-5 Train } \\
\text { Totals }^{4} \\
\text { (Total } \mu \mathrm{g}) \\
\end{array}$} & \multirow{2}{*}{$\begin{array}{c}\text { Project } \\
\text { Specific } \\
\text { Flag }^{7} \\
\end{array}$} \\
\hline & & Risk Result & Flag $^{5}$ & \begin{tabular}{|l|} 
Risk Result \\
\end{tabular} & Flag 5 & \begin{tabular}{|l|} 
Risk Result \\
\end{tabular} & Flag $^{5}$ & Total $^{6}$ & Flag & \\
\hline N-Nitroso-di-n-propylamine & $621-64-7$ & 1.9 & $\mathrm{U}$ & 6.6 & $\mathrm{U}$ & 6.8 & $\mathrm{U}$ & $<15$ & & $N$ \\
\hline N-Nitrosodimethylamine & $62-75-9$ & 1.9 & $\mathrm{U}$ & 6.6 & $\mathrm{U}$ & 6.6 & J & $<15$ & $\mathbf{J}$ & $\mathrm{P}$ \\
\hline N-Nitrosodiphenylamine & $86-30-6$ & 1.6 & $\mathrm{U}$ & 12 & $\mathrm{U}$ & 7.6 & $\mathrm{~J}$ & $<21$ & $\mathbf{J}$ & $\mathrm{P}$ \\
\hline Naphthalene & $91-20-3$ & 1.3 & $\mathrm{U}$ & 7.9 & U & 7.4 & $J$ & $<17$ & $\mathrm{~J}$ & $\mathrm{P}$ \\
\hline 2-Nitroaniline & $88-74-4$ & 1.5 & $\mathrm{U}$ & 6.6 & $\mathrm{U}$ & 8.4 & $\mathrm{U}$ & $<17$ & & $\mathrm{~N}$ \\
\hline 3-Nitroaniline & $99-09-2$ & 10 & $\mathrm{U}$ & 26 & U & 14 & $\mathrm{U}$ & $<50$ & & $\mathrm{~N}$ \\
\hline 4-Nitroaniline & $100-01-6$ & 6.0 & $\mathrm{U}$ & 26 & $\mathrm{U}$ & 12 & $\mathrm{U}$ & $<44$ & & $N$ \\
\hline Nitrobenzene & $98-95-1$ & 1.9 & $\mathrm{U}$ & 9.2 & $J$ & 8.8 & $\mathrm{~J}$ & $<20$ & J & $\mathbf{P}$ \\
\hline 2-Nitrophenol & $88-75-5$ & 8.4 & $\mathrm{U}$ & 21 & $\mathrm{~J}$ & 7.9 & $\mathrm{~J}$ & $<37$ & J & $\mathrm{P}$ \\
\hline 4-Nitrophenol & $100-02-7$ & 8.7 & $\mathrm{U}$ & 42 & $\mathrm{U}$ & 12 & $\mathrm{U}$ & $<63$ & & $N$ \\
\hline $2,2^{\prime}$-Oxybis (1-chloropropane $)^{8}$ & $108-60-1$ & 2.6 & $\mathrm{U}$ & 10 & $\mathrm{U}$ & 9.7 & $J$ & $<22$ & $\mathrm{~J}$ & $\mathrm{P}$ \\
\hline Pentachlorobenzene & $608-93-5$ & 1.4 & $\mathrm{U}$ & 6.6 & $\mathrm{U}$ & 6.8 & $\mathrm{U}$ & $<15$ & & $N$ \\
\hline Pentachloronitrobenzene & $82-68-8$ & 2.0 & U & 6.6 & $\mathrm{U}$ & 7.9 & $\mathrm{U}$ & $<16$ & & $\mathrm{~N}$ \\
\hline Pentachlorophenol & $87-86-5$ & 50 & U & 250 & $\mathrm{U}$ & 10 & $\mathrm{U}$ & $<310$ & & $\mathrm{~N}$ \\
\hline Phenanthrene & $85-01-8$ & 1.3 & $\mathrm{U}$ & 6.6 & $\mathrm{U}$ & 8.6 & $\mathbf{J}$ & $<17$ & $\mathrm{~J}$ & $\mathrm{P}$ \\
\hline Phenol & $108-95-2$ & 2.9 & $\mathrm{U}$ & 23 & $\mathbf{J}$ & 7.4 & $\mathrm{~J}$ & $<33$ & $\mathrm{~J}$ & $\mathrm{P}$ \\
\hline Pyrene & $129-00-0$ & 1.9 & U & 6.8 & $\mathrm{U}$ & 9.4 & $\mathrm{~J}$ & $<18$ & J & $\mathrm{P}$ \\
\hline Pyridine & $110-86-1$ & 2.3 & $\mathrm{U}$ & 9.7 & $\mathrm{U}$ & 16 & $\mathrm{U}$ & $<28$ & & $N$ \\
\hline 1,2,4,5-Tetrachlorobenzene & $95-94-3$ & 2.3 & $\mathrm{U}$ & 6.6 & $\mathrm{U}$ & 6.6 & $\mathrm{U}$ & $<16$ & & $N$ \\
\hline 1,2,4-Trichlorobenzene & $120-82-1$ & 1.9 & $\mathrm{U}$ & 7.9 & $\mathrm{U}$ & 7.4 & $\mathrm{~J}$ & $<17$ & J & $\mathrm{P}$ \\
\hline $2,4,5-$ Trichlorophenol & $95-95-4$ & 6.0 & $\mathrm{U}$ & 17 & $\mathrm{U}$ & 6.3 & $\mathrm{U}$ & $<29$ & & $\mathrm{~N}$ \\
\hline 2,4,6-Trichlorophenol & $88-06-2$ & 3.7 & $\mathrm{U}$ & 10 & $\mathrm{U}$ & 7.6 & $\mathrm{U}$ & $<21$ & & $\mathrm{~N}$ \\
\hline
\end{tabular}


BECHTEL BWXT IDAHO, LLC (BBWI)

INTEC HLLWE Effluent Gas Emissions Inventory

Idaho National Engineering and Environmental Laboratory (INEEL)

STL Knoxville Project Number: 142503.40

\section{MM-5 Train Summary - Run 2 Train Totals (Continued) Semivolatile Organic Compounds Analytical Results Summary Table A-3. HLLWE Run ID: 0010-STRT-2}

\begin{tabular}{|c|c|c|c|c|c|c|c|}
\hline \multirow[b]{2}{*}{ Analyte } & \multirow{2}{*}{$\begin{array}{c}\text { CAS } \\
\text { Registry } \\
\text { Number }\end{array}$} & $\begin{array}{c}\text { MM-5 Train } \\
\text { Front Half } \\
\text { Composite } \\
(\mu g)\end{array}$ & $\begin{array}{c}\text { MM-5 Train } \\
\text { Back Half } \\
\text { Composite }^{2} \\
(\mu g)\end{array}$ & $\begin{array}{c}\text { MM-5 Train } \\
\text { Condensate } \\
\text { Composite }^{3} \\
(\mu \mathrm{g})\end{array}$ & \multicolumn{2}{|c|}{$\begin{array}{c}\text { MM-5 Train } \\
\text { Totals }^{4} \\
(\text { Total } \mu \mathrm{g}) \\
\end{array}$} & \multirow{2}{*}{$\begin{array}{c}\text { Project } \\
\text { Specific } \\
\text { Flag }^{7}\end{array}$} \\
\hline & & ${\text { Risk Result } \text { Flag }^{5}}$ & Risk Result Flag ${ }^{5}$ & Risk Result Flag $^{5}$ & Total $^{6}$ & Flag & \\
\hline \multicolumn{8}{|l|}{$T I C s^{2}$} \\
\hline Furan, 2,5-dimethyl- & $625-86-5$ & 9.8 & --- & 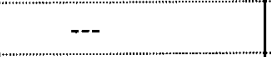 & 9.8 & $\mathrm{~N}, \mathrm{~J}, \mathrm{M}$ & $\mathrm{P}$ \\
\hline 3-Hexanone & $589-38-8$ & -- & 96 & -- & 96 & $\mathrm{~N}, \mathrm{~J}, \mathrm{M}$ & $\mathrm{P}$ \\
\hline Heptane, 2,5-dimethyl- & $2216-30-0$ & 7.1 & --- & -- & 7.1 & $\mathrm{~N}, \mathrm{~J}, \mathrm{M}$ & $\mathrm{P}$ \\
\hline Benzaldehyde & $100-52-7$ & $\cdots$ & 670 & $-\cdots$ & 670 & $\mathrm{~N}, \mathrm{~J}, \mathrm{M}$ & $\mathrm{P}$ \\
\hline Formic acid, phenylmethyl este & $104-57-4$ & -- & 52 & --- & 52 & $\mathrm{~N}, \mathrm{~J}, \mathrm{M}$ & $\mathrm{P}$ \\
\hline Dodecane & $112-40-3$ & $+\cdots$ & 55 & --- & 55 & $\mathrm{~N}, \mathrm{~J}, \mathrm{M}$ & $\mathbf{P}$ \\
\hline Tridecane & $629-50-5$ & -- & 18 & $\cdots$ & 18 & $\mathrm{~N}, \mathrm{~J}, \mathrm{M}$ & $\mathrm{P}$ \\
\hline Naphthalene, I-methyl- & $90-12-0$ & --- & -- & 7.8 & 7.8 & $\mathrm{~N}, \mathrm{~J}, \mathrm{M}$ & $\mathrm{P}$ \\
\hline Tetradecane & $629-59-4$ & --- & 56 & $-\cdots$ & 56 & $\mathrm{~N}, \mathrm{~J}, \mathrm{M}$ & $\mathrm{P}$ \\
\hline Cyclododecane & $294-62-2$ & 5.6 & $\cdots$ & --- & 5.6 & $\mathrm{~N}, \mathrm{~J}, \mathrm{M}$ & $\mathrm{P}$ \\
\hline Hexanedioic acid, bis(2-ethylh) & $103-23-1$ & -- & --- & 10 & 10 & $\mathrm{~N}, \mathrm{~J}, \mathrm{M}$ & $\mathrm{P}$ \\
\hline 1,2-Benzenedicarboxylic acid, & $1330-96-7$ & $-\cdots$ & $--\cdot$ & 8.4 & 8.4 & $\mathrm{~N}, \mathrm{~J}, \mathrm{M}$ & $\mathrm{P}$ \\
\hline Benzo(e)pyrene & $192-97-2$ & $\cdots$ & -- & 14 & 14 & $\mathrm{~N}, \mathrm{~J}, \mathrm{M}$ & $\mathrm{P}$ \\
\hline
\end{tabular}




\section{BECHTEL BWXT IDAHO, LLC (BBWI) INTEC HLLWE Effluent Gas Emissions Inventory Idaho National Engineering and Environmental Laboratory (INEEL) STL Knoxville Project Number: 142503.40}

\section{Footnotes:}

1 The MM-5 Train Front Half Composite consists of the Particulate Filter and the Front Half of the Filter Holder and Probe Solvent Rinses.

2 The MM-5 Train Back Half Composite consists of the XAD-2 Resin Tube and the Back Half of the Filter Holder and Coil Condenser Solvent Rinses.

3 The MM-5 Train Condensate Composite consists of the Condensate and Impinger Contents and the Glassware Solvent Rinses.

4 The total mass for each semivolatile compound found in the MM-5 sampling train consists of the sum of the MM-5 train's Front Half Composite contents, the train's Back Half Composite contents, and the Condensate Composite. The calculation is as follows:

$($ Total $\mu \mathrm{g}$ in the Front Half $)+($ Total $\mu \mathrm{g}$ in the Back Half $)+($ Concentration in the Condensate Composite $x$ Condensate Composite Volume)

$=$ Total $\mu \mathrm{g}$ in the MM-5 Sampling Train.

Therefore: $(\mu \mathrm{g})+(\mu \mathrm{g})+(\mu \mathrm{g} /$ Liter $\times$ Liter $)=$ Total $\mu \mathrm{g}$

The MM-5 Train Run Total (in Total $\mu \mathrm{g}$ ) is the sum of results for the three (3) MM-5 train sample fractions using the following guidelines:

- When the train component analytical result is greater than the laboratory reporting limit (RL), the result included in the train total is the actual analytical result or "hit" determined by the laboratory.

- When the train component analytical result is greater than the reliable detection level (RDL), but less than the laboratory reporting limit $(\mathrm{RL})$, the result included in the train total is actual analytical result or "hit" determined by the laboratory and the corresponding " $\mathrm{J}$ " flag is carried through the calculation to the train total.

- When the train analytical component result is less than the RDL, but greater than the method detection limit (MDL), the result included in the train total is the RDL and the corresponding " $\mathrm{J}$ " flag is carried through the calculation to the train total.

- When the train component analytical result is not detected down to the MDL, the result included in the train total is the RDL and the corresponding " $U$ " flag is carried through the calculation to the train total.

- It should be noted that when the RDL is selected as the default value using the guidelines above, but the RDL is greater than the $\mathrm{RL}$, the RL is included in the train total.

The data flags attached to the MM-5 Train Total are the cumulative set of flags for each train component included as part of the MM5 train total. A flag attached to an MM-5 train component is carried through to the "MM-5 Train Total" column when the associated component analytical result is a significant number in comparison to the MM-5 Train Total. That is, if the MM-5 Train Total is affected by an MM-5 train component analytical result, the flag is carried through to the MM- 5 Train Total, but if the MM- 5 Train Total is not affected by an MM-5 train component, the flag is not carried through to the MM-5 Train Total. The combinations of train fractions are conducted following the standard practice of using significant figures found in ASTM E29-93a(1999), "Standard Practice for Using Significant Digits in Test Data to Determine Conformance with Specifications" and Severn Trent Laboratories standard operating procedure number QA-004, "Rounding and Significant Figures". 


\section{BECHTEL BWXT IDAHO, LLC (BBWI) \\ INTEC HLLWE Effluent Gas Emissions Inventory \\ Idaho National Engineering and Environmental Laboratory (INEEL) \\ STL Knoxville Project Number: 142503.40}

5 This flag is the laboratory data flag that corresponds to EPA guidelines. The data flags for these samples are as follows:

- A "U" qualifier indicates that this analyte was analyzed for, but was not detected down to the MDL.

- A "J" qualifier indicates that this compound was detected, but at a concentration below the laboratory RL. The analytical result is therefore an estimated value.

- A "B" qualifier indicates that this compound was found in the associated laboratory method blank. Under these conditions these values are regarded as estimated values.

- A " $D$ " qualifier indicates that this result was obtained through dilution of the sample. This original analysis yielded a result that exceeded the calibration range.

- An "N" qualifier indicates that this compound is a tentatively identified compound (TIC). Therefore the value is estimated.

- An "E" qualifier indicates that this compound exceeded the calibration range of the instrument.

- An " $\mathrm{A}$ " qualifier indicates that this result is an Aldol-condensation product.

- An "M" qualifier indicates that this result was measured against the nearest internal standard and assumed a response factor of one (1).

- A "Q" qualifier indicates that this result was quantitated against the response factor of a calibration standard.

6 When listed, the less than $(<)$ sign indicates that at least one sample fraction result is either a "non-detect" value down to the MDL of the measurement that carries, or an estimated "hit" value that is below the RDL. In either case, the final value for the fraction that is included in the data set total is the default RDL value and the actual value of the total is known to be less than $(<)$ the displayed result.

7 Entries in this column are project-specific train total flags that are applied to the run total values and are not standard EPA data flags. These project-specific flags are utilized for the INEEL NWCF HLLWE Effluent Gas Emissions Inventory project and are defined as follows:

- An "N" flag in this column indicates that the compound was not measured (detected) in any of the sampling train components, or fractions.

- A "P" flag in this column indicates that the compound was measured (detected) in one or more of the train components, or fractions, but not in all of the sampling train fractions.

- An "A" flag in this column indicates that the compound was measured (detected) in all of the sampling train components, or fractions.

8 Bis(2-chloroisopropyl)ether and 2,2'-Oxybis(1-chloropropane) are synonyms.

9 The tentatively identified compounds (TICs) were identified by conducting a mass spectral library search using the NBS library of data. It should be noted that TICs that give the same mass spectral match for GC peaks at different retention times are listed separately with the same compound identity. Under these conditions the compounds are likely indistinguishable isomers of the same compound. However, insufficient evidence is available to determine unequivocal identities. 
BECHTEL BWXT IDAHO, LLC (BBWI)

INTEC HLLWE Effluent Gas Emissions Inventory

Idaho National Engineering and Environmental Laboratory (INEEL)

STL Knoxville Project Number: 142503.40

\section{MM-5 Train Summary - Run 4 Train Totals \\ Semivolatile Organic Compounds Analytical Results Summary \\ Table A-4. HLLWE Run ID: 0010-END-2}

Field Sample Name

Sample Description:

MM-5 Train

MM-5 Train Totals for Semivolatile Organic Compounds Analysis

\begin{tabular}{|c|c|c|c|c|c|c|c|c|c|c|}
\hline \multirow[b]{2}{*}{ Analyte } & \multirow{2}{*}{$\begin{array}{c}\text { CAS } \\
\text { Registry } \\
\text { Number }\end{array}$} & \multicolumn{2}{|c|}{$\begin{array}{c}\text { MM-5 Train } \\
\text { Front Half } \\
\text { Composite } \\
(\mu \mathrm{g})\end{array}$} & \multicolumn{2}{|c|}{$\begin{array}{c}\text { MM-5 Train } \\
\text { Back Half } \\
\text { Composite }^{2} \\
(\mu \mathrm{g}) \\
\end{array}$} & \multicolumn{2}{|c|}{$\begin{array}{c}\text { MM-5 Train } \\
\text { Condensate } \\
\text { Composite }^{3} \\
(\mu \mathrm{g}) \\
\end{array}$} & \multicolumn{2}{|c|}{$\begin{array}{c}\text { MM-5 Train } \\
\text { Totals }^{4} \\
\text { (Total } \mu \mathrm{g})\end{array}$} & \multirow{2}{*}{$\begin{array}{l}\text { Project } \\
\text { Specific } \\
\text { Flag }^{7}\end{array}$} \\
\hline & & Risk Result & Flag $^{5}$ & Risk Result & Flag $^{5}$ & Risk Result & Flag 5 & Total $^{6}$ & Flag & \\
\hline \multicolumn{11}{|l|}{ Target Compound List } \\
\hline Acenaphthene & $83-32-9$ & 1.3 & $\mathrm{U}$ & 6.6 & $\mathrm{U}$ & 1.9 & $\mathrm{U}$ & $<9.8$ & & $\mathrm{~N}$ \\
\hline Acenaphthylene & $208-96-8$ & 1.3 & $\mathrm{U}$ & 6.6 & $\mathrm{U}$ & 1.6 & $\mathrm{U}$ & $<9.5$ & & $N$ \\
\hline Acetophenone & $9[8-86-2$ & 2.0 & $\mathrm{U}$ & 32 & $J$ & 2.6 & $\mathrm{U}$ & $<37$ & $J$ & $\mathrm{P}$ \\
\hline Aniline & $62-53-3$ & 2.5 & $\mathrm{U}$ & 94 & $\mathrm{U}$ & 18 & $\mathrm{U}$ & $<110$ & & $\mathrm{~N}$ \\
\hline Anthracene & $120-12-7$ & 1.3 & U & 6.6 & $\mathrm{U}$ & 1.6 & $\mathrm{U}$ & $<9.5$ & & $N$ \\
\hline Benzidine & $92-87-5$ & 100 & $\mathrm{U}$ & 500 & $\mathrm{U}$ & 66 & $\mathrm{U}$ & $<670$ & & $\mathrm{~N}$ \\
\hline Benzoic acid & $65-85-0$ & 100 & $\mathrm{U}$ & 2,600 & $\mathrm{E}$ & 9.4 & $\mathrm{U}$ & $<2,700$ & $E$ & $P$ \\
\hline Benzo(a)anthracene & $56-55-3$ & 2.2 & $\mathrm{U}$ & 7.6 & $\mathrm{U}$ & 1.7 & $\mathrm{U}$ & $<12$ & & $\mathrm{~N}$ \\
\hline Benzo(a)pyrene & $50-32-8$ & 2.6 & U & 130 & $\mathrm{U}$ & 1.8 & $\mathrm{U}$ & $<130$ & & $N$ \\
\hline Benzo(b)fluoranthene & $205-99-2$ & 3.7 & U & 290 & U & 4.2 & $\mathrm{U}$ & $<300$ & & $\mathrm{~N}$ \\
\hline Benzo(g,h,i)perylene & $191-24-2$ & 7.3 & U & 160 & U & 2.1 & U & $<170$ & & $N$ \\
\hline Benzo(k)fluoranthene & $207-08-9$ & 5.5 & $\mathrm{U}$ & 420 & $\mathrm{U}$ & 2.9 & $\mathrm{U}$ & $<430$ & & $\mathrm{~N}$ \\
\hline Benzyl alcohol & $100-51-6$ & 92 & $\mathrm{U}$ & 470 & U & 4.2 & U & $<570$ & & $\mathrm{~N}$ \\
\hline bis(2-Chloroethoxy)methane & $111-91-1$ & 1.5 & $\mathrm{U}$ & 6.6 & $\mathrm{U}$ & 2.0 & $\mathrm{U}$ & $<10$ & & $\mathrm{~N}$ \\
\hline bis(2-Chloroethyl)ether & $111-44-4$ & 2.0 & $\mathrm{U}$ & 7.3 & U & 1.7 & $\mathrm{U}$ & $<11$ & & $\mathrm{~N}$ \\
\hline bis(2-Ethylhexyl)phthalate & $117-81-7$ & 57 & & 100 & $\mathrm{~J}$ & 6.1 & $\mathrm{~J}$ & $<160$ & $J$ & A \\
\hline 4-Bromophenyl-phenylether & $10 !-55-3$ & 1.4 & $\mathrm{U}$ & 6.6 & $\mathrm{U}$ & 1.4 & $\mathrm{U}$ & $<9.4$ & & $N$ \\
\hline Butylbenzylphthalate & $85-68-7$ & 2.9 & $\mathrm{U}$ & 7.9 & $\mathrm{U}$ & 2.3 & $\mathrm{U}$ & $<13$ & & $N$ \\
\hline Carbazole & $86-74-8$ & 2.0 & $U$ & 8.4 & $\mathrm{U}$ & 2.2 & $\mathrm{U}$ & $<13$ & & $N$ \\
\hline 4-Chloro-3-methylphenol & $59-50-7$ & 2.6 & $\mathrm{U}$ & 8.1 & $\mathrm{U}$ & 6.6 & $\mathrm{U}$ & $<17$ & & $\mathrm{~N}$ \\
\hline 4-Chloroaniline & $106-47-8$ & 3.1 & $\mathrm{U}$ & 79 & $\mathrm{U}$ & 7.9 & $\mathrm{U}$ & $<90$ & & $N$ \\
\hline 2-Chloronaphthalene & $91-58-7$ & 1.3 & $\mathrm{U}$ & 6.6 & $\mathrm{U}$ & 1.4 & $\mathrm{U}$ & $<9.3$ & & $\mathrm{~N}$ \\
\hline 2-Chlorophenol & $95-57-8$ & 2.6 & $\mathrm{U}$ & 6.6 & U & 1.7 & $\mathrm{U}$ & $<11$ & & $N$ \\
\hline 4-Chlorophenyl phenyl ether & $7005-72-36$ & 1.3 & $\mathrm{U}$ & 6.6 & $\mathrm{U}$ & 3.1 & $\mathrm{U}$ & $<11$ & & $\mathrm{~N}$ \\
\hline Chrysene & $218-01-9$ & 2.3 & $\mathrm{U}$ & 8.4 & $\mathrm{U}$ & 1.3 & U & $<12$ & & $\mathrm{~N}$ \\
\hline
\end{tabular}

Last saved by Patti Carswell on 02/04/02 at 4:40 PM

E:My Documents $\backslash H L L W E$ SamplinglFinal Report\Appendix AlAppendix A-4. 0010-END-2.doc

Created on 2/4/2002 4:40 PM 
BECHTEL BWXT IDAHO, LLC (BBWI)

INTEC HLLWE Effluent Gas Emissions Inventory

Idaho National Engineering and Environmental Laboratory (INEEL)

STL Knoxville Project Number: 142503.40

\section{MM-5 Train Summary - Run 4 Train Totals (Continued) Semivolatile Organic Compounds Analytical Results Summary Table A-4. HLLWE Run ID: 0010-END-2}

\begin{tabular}{|c|c|c|c|c|c|c|c|c|c|c|}
\hline \multirow[b]{2}{*}{ Analyte } & \multirow{2}{*}{$\begin{array}{c}\text { CAS } \\
\text { Registry } \\
\text { Number } \\
\end{array}$} & \multicolumn{2}{|c|}{$\begin{array}{c}\text { MM-5 Train } \\
\text { Front Half } \\
\text { Composite } \\
(\mu g)\end{array}$} & \multicolumn{2}{|c|}{$\begin{array}{c}\text { MM-5 Train } \\
\text { Back Half } \\
\text { Composite } \\
(\mu \mathrm{g})\end{array}$} & \multicolumn{2}{|c|}{$\begin{array}{c}\text { MM-5 Train } \\
\text { Condensate } \\
\text { Composite } \\
(\mu \mathrm{g})\end{array}$} & \multicolumn{2}{|c|}{$\begin{array}{c}\text { MM-5 Train } \\
\text { Totals } \\
\text { (Total } \mu \mathrm{g})\end{array}$} & \multirow{2}{*}{$\begin{array}{c}\text { Project } \\
\text { Specific } \\
\text { Flag }^{7} \\
\end{array}$} \\
\hline & & Risk Result & Flag 5 & Risk Result & Flag 5 & Risk Result & Flag 5 & Total $^{6}$ & Flag & \\
\hline Di-n-butylphthalate & $84-74-2$ & 1.9 & $\mathrm{~J}$ & 100 & $\mathrm{U}$ & 2.3 & $\mathrm{U}$ & $<100$ & $\mathrm{~J}$ & $\mathrm{P}$ \\
\hline Di-n-octylphthalate & $117-84-0$ & 5.5 & $\mathrm{~J}$ & 150 & $\mathrm{U}$ & 2.5 & J & $<160$ & J & $\mathrm{P}$ \\
\hline Dibenz(a,h)anthracene & $53-70-3$ & 5.2 & $\mathrm{U}$ & 160 & $\mathrm{U}$ & 2.9 & $\mathrm{U}$ & $<170$ & & $\mathrm{~N}$ \\
\hline Dibenzofuran & $132-64-9$ & 1.4 & $\mathrm{U}$ & 6.6 & U & 2.9 & $\mathrm{U}$ & $<11$ & & $N$ \\
\hline 1,2-Dichlorobenzene & $95-50-1$ & 2.2 & $\mathrm{U}$ & 6.8 & $\mathrm{U}$ & 1.7 & $\mathrm{U}$ & $<11$ & & $N$ \\
\hline 1,3-Dichlorobenzene & $541-73-1$ & 3.1 & $\mathrm{U}$ & 7.3 & $\mathrm{U}$ & 1.4 & $\mathrm{U}$ & $<12$ & & $\mathrm{~N}$ \\
\hline 1,4-Dichlorobenzene & $106-46-7$ & 2.9 & $\mathrm{U}$ & 16 & $\mathrm{~J}$ & 2.0 & $\mathrm{U}$ & $<21$ & $\mathrm{~J}$ & $\mathrm{P}$ \\
\hline 3,3'-Dichlorobenzidine & $91-94-1$ & 7.1 & $\mathrm{U}$ & 97 & $\mathrm{U}$ & 7.9 & $\mathrm{U}$ & $<110$ & & $N$ \\
\hline 2,4-Dichlorophenol & $120-83-2$ & 3.9 & $\mathrm{U}$ & 6.6 & $\mathrm{U}$ & 2.3 & $\mathrm{U}$ & $<13$ & & $\mathrm{~N}$ \\
\hline Diethylphthalate & $84-66-2$ & 3.9 & $\mathrm{U}$ & 9.4 & $\mathrm{U}$ & 1.4 & $\mathrm{U}$ & $<15$ & & $\mathrm{~N}$ \\
\hline Dimethyl phthalate & $131-11-3$ & 1.7 & $\mathrm{U}$ & 6.6 & $\mathrm{U}$ & 1.3 & $\mathrm{U}$ & $<9.6$ & & $\mathrm{~N}$ \\
\hline 2,4-Dimethylphenol & $105-67-9$ & 7.6 & $\mathrm{U}$ & 50 & $\mathrm{U}$ & 1.5 & $\mathrm{U}$ & $<59$ & & $N$ \\
\hline 4,6-Dinitro-2-methylphenol & $534-52-1$ & 13 & $\mathrm{U}$ & 120 & $\mathrm{U}$ & 1.5 & $\mathrm{U}$ & $<130$ & & $\mathrm{~N}$ \\
\hline 2,4-Dinitrophenol & $51-28-5$ & 16 & $\mathrm{U}$ & 250 & U & 3.9 & $\mathrm{U}$ & $<270$ & & $N$ \\
\hline 2,4-Dinitrotoluene & $121-14-2$ & 4.2 & $\mathrm{U}$ & 6.6 & $\mathrm{U}$ & 2.6 & $\mathrm{U}$ & $<13$ & & $\mathrm{~N}$ \\
\hline 2,6-Dinitrotoluene & $606-20-2$ & 3.4 & $\mathrm{U}$ & 6.6 & $\mathrm{U}$ & 2.1 & $\mathrm{U}$ & $<12$ & & $N$ \\
\hline 1,2-Diphenylhydrazine & $122-66-7$ & 1.7 & $\mathrm{U}$ & 6.6 & $\mathrm{U}$ & 1.5 & $\mathrm{U}$ & $<9.8$ & & $\mathrm{~N}$ \\
\hline Fluoranthene & $206-44-0$ & 1.3 & $\mathrm{U}$ & 7.1 & $\mathrm{U}$ & 1.8 & $\mathrm{U}$ & $<10$ & & $N$ \\
\hline Fluorene & $86-73-7$ & 1.3 & $\mathrm{U}$ & 6.6 & $\mathrm{U}$ & 2.6 & $\mathrm{U}$ & $<10$ & & $N$ \\
\hline Hexachlorocyclopentadiene & $77-47-4$ & 26 & $\mathrm{U}$ & 130 & U & 6.6 & $\mathrm{U}$ & $<160$ & & $N$ \\
\hline Hexachlorobenzene & $118-74-1$ & 1.5 & $\mathrm{U}$ & 6.6 & $\mathrm{U}$ & 2.6 & $\mathrm{U}$ & $<11$ & & $\mathrm{~N}$ \\
\hline Hexachlorobutadiene & $87-68-3$ & 3.7 & $\mathrm{U}$ & 9.7 & $\mathrm{U}$ & 1.9 & $\mathrm{U}$ & $<15$ & & $N$ \\
\hline Hexachloroethane & $67-72-1$ & 6.6 & $\mathrm{U}$ & 7.1 & $\mathrm{U}$ & 1.9 & $\mathrm{U}$ & $<16$ & & $\mathrm{~N}$ \\
\hline Indeno(1,2,3-cd)pyrene & $193-39-5$ & 5.5 & $\mathrm{U}$ & 140 & $\mathrm{U}$ & 2.3 & $\mathrm{U}$ & $<150$ & & $N$ \\
\hline Isophorone & $78-59-1$ & 1.7 & $\mathrm{U}$ & 6.6 & $\mathrm{U}$ & 1.8 & $\mathrm{U}$ & $<10$ & & $\mathrm{~N}$ \\
\hline 2-Methylnaphthalene & $91-57-6$ & 1.5 & $\mathrm{U}$ & 6.6 & $\mathrm{U}$ & 2.3 & $\mathrm{U}$ & $<10$ & & $\mathrm{~N}$ \\
\hline 2-Methylphenol & $95-48-7$ & 6.0 & $\mathrm{U}$ & 39 & $\mathrm{U}$ & 2.1 & $\mathrm{U}$ & $<47$ & & $\mathrm{~N}$ \\
\hline 3-Methylphenol \& 4-Methylphenol & $65794-96-9$ & 6.0 & $\mathrm{U}$ & 26 & $\mathrm{U}$ & 2.2 & $\mathrm{U}$ & $<34$ & & $\mathrm{~N}$ \\
\hline
\end{tabular}

Last saved by Patti Carswell on 02/04/02 at 4:40 PM

E:My Documents $\backslash H L L W E$ Sampling $\backslash$ Final ReportlAppendix AlAppendix A-4. 0010-END-2.doc

Created on 2/4/2002 4:40 PM 
BECHTEL BWXT IDAHO, LLC (BBWI)

INTEC HLLWE Effluent Gas Emissions Inventory

Idaho National Engineering and Environmental Laboratory (INEEL)

STL Knoxville Project Number: 142503.40

\section{MM-5 Train Summary - Run 4 Train Totals (Continued) Semivolatile Organic Compounds Analytical Results Summary Table A-4. HLLWE Run ID: 0010-END-2}

\begin{tabular}{|c|c|c|c|c|c|c|c|c|c|c|}
\hline \multirow[b]{2}{*}{ Analyte } & \multirow{2}{*}{$\begin{array}{c}\text { CAS } \\
\text { Registry } \\
\text { Number } \\
\end{array}$} & \multicolumn{2}{|c|}{$\begin{array}{c}\text { MM-5 Train } \\
\text { Front Half } \\
\text { Composite } \\
(\mu \mathrm{g})\end{array}$} & \multicolumn{2}{|c|}{$\begin{array}{c}\text { MM-5 Train } \\
\text { Back Half } \\
\text { Composite }^{2} \\
(\mu g)\end{array}$} & \multicolumn{2}{|c|}{$\begin{array}{c}\text { MM-5 Train } \\
\text { Condensate } \\
\text { Composite } \\
(\mu g)\end{array}$} & \multicolumn{2}{|c|}{$\begin{array}{c}\text { MM-5 Train } \\
\text { Totals }^{4} \\
\text { (Total } \mu \mathrm{g})\end{array}$} & \multirow{2}{*}{$\begin{array}{c}\text { Project } \\
\text { Specific } \\
\text { Flag }^{7}\end{array}$} \\
\hline & & Risk Result & Flag $^{5}$ & Risk Result & Flag $^{5}$ & \begin{tabular}{|l|} 
Risk Result \\
\end{tabular} & Flag $^{5}$ & Total $^{6}$ & Flag & \\
\hline N-Nitroso-di-n-propylamine & $621-64-7$ & 1.9 & $\mathrm{U}$ & 6.6 & $\mathrm{U}$ & 2.3 & $\mathrm{U}$ & $<11$ & & $N$ \\
\hline N-Nitrosodimethylamine & $62-75-9$ & 1.9 & $\mathrm{U}$ & 6.6 & $\mathrm{U}$ & 2.2 & $\mathrm{U}$ & $<11$ & & $\mathrm{~N}$ \\
\hline N-Nitrosodiphenylamine & $86-30-6$ & 1.6 & $\mathrm{U}$ & 12 & $\mathrm{U}$ & 1.4 & $\mathrm{U}$ & $<15$ & & $\mathrm{~N}$ \\
\hline Naphthalene & $91-20-3$ & 1.3 & $\mathrm{U}$ & 7.9 & U & 1.8 & $\mathrm{U}$ & $<11$ & & $\mathrm{~N}$ \\
\hline 2-Nitroaniline & $88-74-4$ & 1.5 & $\mathrm{U}$ & 6.6 & $\mathrm{U}$ & 2.9 & $\mathrm{U}$ & $<11$ & & $\mathrm{~N}$ \\
\hline 3-Nitroaniline & $99-09-2$ & 10 & $\mathrm{U}$ & 26 & $\mathrm{U}$ & 4.7 & $\mathrm{U}$ & $<41$ & & $N$ \\
\hline 4-Nitroaniline & $100-01-6$ & 6.0 & U & 26 & $\mathrm{U}$ & 3.9 & $\mathrm{U}$ & $<36$ & & $N$ \\
\hline Nitrobenzene & $98-95-1$ & 1.9 & $\mathrm{U}$ & 8.5 & $\mathrm{~J}$ & 1.7 & $\mathrm{U}$ & $<12$ & $J$ & $\mathrm{P}$ \\
\hline 2-Nitrophenol & $88-75-5$ & 8.4 & $\mathrm{U}$ & 40 & $\mathrm{~J}$ & 2.6 & $\mathrm{U}$ & $<51$ & $J$ & $\mathrm{P}$ \\
\hline 4-Nitrophenol & $100-02-7$ & 8.7 & $\mathrm{U}$ & 42 & $\mathbf{J}$ & 3.9 & $U$ & $<55$ & $\mathrm{~J}$ & $\mathrm{P}$ \\
\hline $2,2^{\prime}$-Oxybis(1-chloropropane $)^{8}$ & $108-60-1$ & 2.6 & $\mathrm{U}$ & 10 & $\mathrm{U}$ & 1.8 & $\mathrm{U}$ & $<14$ & & $\mathrm{~N}$ \\
\hline Pentachlorobenzene & $608-93-5$ & 1.4 & $\mathrm{U}$ & 6.6 & $\mathrm{U}$ & 2.3 & $\mathrm{U}$ & $<10$ & & $\mathrm{~N}$ \\
\hline Pentachloronitrobenzene & $82-68-8$ & 2.0 & $\mathrm{U}$ & 6.6 & U & 2.6 & $\mathrm{U}$ & $<11$ & & $\mathrm{~N}$ \\
\hline Pentachlorophenol & $87-86-5$ & 50 & U & 320 & $\mathrm{U}$ & 3.4 & $\mathrm{U}$ & $<370$ & & $\mathrm{~N}$ \\
\hline Phenanthrene & $85-01-8$ & 1.3 & $\mathrm{U}$ & 6.6 & $\mathrm{U}$ & 1.9 & $\mathrm{U}$ & $<9.8$ & & $\mathrm{~N}$ \\
\hline Phenol & $108-95-2$ & 2.9 & $\mathrm{U}$ & 34 & $J$ & 2.2 & $J$ & $<39$ & $J$ & $\mathrm{P}$ \\
\hline Pyrene & $129-00-0$ & 1.9 & $\mathrm{U}$ & 6.8 & $\mathrm{U}$ & 1.4 & $\mathrm{U}$ & $<10$ & & $\mathrm{~N}$ \\
\hline Pyridine & $110-86-1$ & 2.3 & $U$ & 9.7 & U & 5.2 & $\mathrm{U}$ & $<17$ & & $\mathrm{~N}$ \\
\hline 1,2,4,5-Tetrachlorobenzene & $95-94-3$ & 2.3 & $\mathrm{U}$ & 6.6 & $\mathrm{U}$ & 2.2 & $\mathrm{U}$ & $<11$ & & $\mathrm{~N}$ \\
\hline 1,2,4-Trichlorobenzene & $120-82-1$ & 1.9 & $\mathrm{U}$ & 7.9 & $\mathrm{U}$ & 2.2 & $\mathrm{U}$ & $<12$ & & $\mathrm{~N}$ \\
\hline 2,4,5-Trichlorophenol & $95-95-4$ & 6.0 & U & 17 & $\mathrm{U}$ & 2.1 & $\mathrm{U}$ & $<25$ & & $\mathrm{~N}$ \\
\hline 2,4,6-Trichlorophenol & $88-06-2$ & 3.7 & $\mathrm{U}$ & 10 & $\mathrm{U}$ & 2.5 & $\mathrm{U}$ & $<16$ & & $\mathrm{~N}$ \\
\hline
\end{tabular}

Last saved by Patti Carswell on 02/04/02 at 4:40 PM 
BECHTEL BWXT IDAHO, LLC (BBWI)

INTEC HLLWE Effluent Gas Emissions Inventory

Idaho National Engineering and Environmental Laboratory (INEEL)

STL Knoxville Project Number: 142503.40

\section{MM-5 Train Summary - Run 4 Train Totals (Continued) Semivolatile Organic Compounds Analytical Results Summary Table A-4. HLLWE Run ID: 0010-END-2}

\begin{tabular}{|c|c|c|c|c|c|c|c|}
\hline \multirow[b]{2}{*}{ Analyte } & \multirow{2}{*}{$\begin{array}{c}\text { CAS } \\
\text { Registry } \\
\text { Number }\end{array}$} & $\begin{array}{c}\text { MM-5 Train } \\
\text { Front Half } \\
\text { Composite } \\
(\mu \mathrm{g})\end{array}$ & $\begin{array}{c}\text { MM-5 Train } \\
\text { Back Half } \\
\text { Composite }^{2} \\
(\mu \mathrm{g})\end{array}$ & $\begin{array}{c}\text { MM-5 Train } \\
\text { Condensate } \\
\text { Composite } \\
(\mu \mathrm{g}) \\
\end{array}$ & \multicolumn{2}{|c|}{$\begin{array}{c}\text { MM-5 Train } \\
\text { Totals } \\
\text { (Total } \mu \mathrm{g})\end{array}$} & \multirow{2}{*}{$\begin{array}{c}\text { Project } \\
\text { Specific } \\
\text { Flag }^{7} \\
\end{array}$} \\
\hline & & 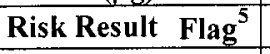 & Risk Result Flag ${ }^{5}$ & Risk Result Flag $^{5}$ & Total $^{6}$ & Flag & \\
\hline \multicolumn{8}{|l|}{$\mathrm{TICs}^{9}$} \\
\hline 3-Hexanone & $589-38-8$ & $-\cdots$ & 70 & $-\cdots$ & 70 & $\mathrm{~N}, \mathrm{~J}, \mathrm{M}$ & $P$ \\
\hline Benzaldehyde & $100-52-7$ & $-\cdots$ & 730 & --- & 730 & $\mathrm{~N}, \mathrm{~J}, \mathrm{M}$ & $P$ \\
\hline 2-Cyclohexene-1-one, 3-methyl- & $1193-18-6$ & $-\cdots$ & --- & 3.2 & 3.2 & $\mathrm{~N}, \mathrm{~J}, \mathrm{M}$ & $P$ \\
\hline Formic acid, phenylmethyl ester & $104-57-4$ & $\cdots$ & 95 & --- & 95 & $\mathrm{~N}, \mathrm{~J}, \mathrm{M}$ & $\mathrm{P}$ \\
\hline Benzaldehyde, ethyl- & $53951-50-1$ & $\cdots$ & 61 & --- & 61 & $\mathrm{~N}, \mathrm{~J}, \mathrm{M}$ & $P$ \\
\hline Dodecane & $112-40-3$ & $-\cdots$ & 37 & --- & 37 & $\mathrm{~N}, \mathrm{~J}, \mathrm{M}$ & $P$ \\
\hline Tridecane & $629-50-5$ & -- & 21 & $-\cdots$ & 21 & $\mathrm{~N}, J, M$ & $P$ \\
\hline 2,4-Hexadiene & $592-46-1$ & --- & 28 & --- & 28 & $\mathrm{~N}, \mathrm{~J}, \mathrm{M}$ & $\mathrm{P}$ \\
\hline 2,5-Diethylphenol & $876-20-0$ & $-\cdots$ & 100 & --- & 100 & $\mathrm{~N}, \mathrm{~J}, \mathrm{M}$ & $\mathrm{P}$ \\
\hline Tetradecane & $629-59-4$ & -- & 99 & -- & 99 & $\mathrm{~N}, \mathrm{~J}, \mathrm{M}$ & $\mathrm{P}$ \\
\hline Hexatriacontane & $630-06-8$ & 5.7 & -- & $\cdots$ & 5.7 & $\mathrm{~N}, \mathrm{~J}, \mathrm{M}$ & $\mathrm{P}$ \\
\hline Phosphoric acid tributyl ester & $126-73-8$ & 24 & $-\cdots$ & --- & 24 & $\mathrm{~N}, \mathrm{~J}, \mathrm{M}$ & $\mathrm{P}$ \\
\hline Cyclododecane & $294-62-2$ & 13 & $\cdots$ & -- & 13 & $\mathrm{~N}, \mathrm{~J}, \mathrm{M}$ & $\mathrm{P}$ \\
\hline Pentadecane & $629-62-9$ & 4.5 & $-\cdots$ & --- & 4.5 & $\mathrm{~N}, J, M$ & $\mathrm{P}$ \\
\hline Heneicosane & $629-94-7$ & 9.1 & --- & $\cdots$ & 9.1 & $\mathrm{~N}, \mathrm{~J}, \mathrm{M}$ & $\mathrm{P}$ \\
\hline Tetracosane & $646-31-1$ & 19 & -- & $-\cdots$ & 19 & $\mathrm{~N}, \mathrm{~J}, \mathrm{M}$ & $\mathrm{P}$ \\
\hline Pentacosane & $629-99-2$ & 35 & -- & -- & 35 & $\mathrm{~N}, \mathrm{~J}, \mathrm{M}$ & $\mathrm{P}$ \\
\hline Hexacosane & $630-01-3$ & 64 & $-\cdots$ & --- & 64 & $\mathrm{~N}, \mathrm{~J}, \mathrm{M}$ & $\mathrm{P}$ \\
\hline Heptacosane & $593-49-7$ & 83 & --- & --- & 83 & $\mathrm{~N}, \mathrm{~J}, \mathrm{M}$ & $\mathrm{P}$ \\
\hline Pentacosane & $629-99-2$ & -- & $\cdots$ & 2.2 & 2.2 & $\mathrm{~N}, \mathrm{~J}, \mathrm{M}$ & $P$ \\
\hline Hexatriacontane & $630-06-8$ & 100 & --- & $\cdots$ & 100 & $\mathrm{~N}, J, M$ & $\mathrm{P}$ \\
\hline Hexatriacontane & $630-06-8$ & 67 & --- & -- & 67 & $\mathrm{~N}, \mathrm{~J}, \mathrm{M}$ & $\mathrm{P}$ \\
\hline Hexatriacontane & $630-06-8$ & 32 & --- & -- & 32 & $\mathrm{~N}, \mathrm{~J}, \mathrm{M}$ & $\mathrm{P}$ \\
\hline Eicosane & $112-95-8$ & 19 & $\ldots$ & -- & 19 & $\mathrm{~N}, \mathrm{~J}, \mathrm{M}$ & $\mathrm{P}$ \\
\hline Tetracosane & $646-31-1$ & 7.4 & -- & -- & 7.4 & $\mathrm{~N}, \mathrm{~J}, \mathrm{M}$ & $\mathrm{P}$ \\
\hline
\end{tabular}




\section{BECHTEL BWXT IDAHO, LLC (BBWI) \\ INTEC HLLWE Effluent Gas Emissions Inventory \\ Idaho National Engineering and Environmental Laboratory (INEEL) \\ STL Knoxville Project Number: 142503.40}

\section{Footnotes:}

1 The MM-5 Train Front Half Composite consists of the Particulate Filter and the Front Half of the Filter Holder and Probe Solvent Rinses.

2 The MM-5 Train Back Half Composite consists of the XAD-2 Resin Tube and the Back Half of the Filter Holder and Coil Condenser Solvent Rinses.

3 The MM-5 Train Condensate Composite consists of the Condensate and Impinger Contents and the Glassware Solvent Rinses.

4 The total mass for each semivolatile compound found in the MM-5 sampling train consists of the sum of the MM-5 train's Front Half Composite contents, the train's Back Half Composite contents, and the Condensate Composite. The calculation is as follows:

(Total $\mu \mathrm{g}$ in the Front Half $)+($ Total $\mu \mathrm{g}$ in the Back Half $)+($ Concentration in the Condensate Composite $\times$ Condensate Composite Volume)

$=$ Total $\mu \mathrm{g}$ in the MM-5 Sampling Train.

Therefore: $\quad(\mu g)+(\mu g)+(\mu g /$ Liter $x$ Liter $)=$ Total $\mu g$

The MM-5 Train Run Total (in Total $\mu \mathrm{g}$ ) is the sum of results for the three (3) MM-5 train sample fractions using the following guidelines:

- When the train component analytical result is greater than the laboratory reporting limit (RL), the result included in the train total is the actual analytical result or "hit" determined by the laboratory.

- When the train component analytical result is greater than the reliable detection level (RDL), but less than the laboratory reporting limit (RL), the result included in the train total is actual analytical result or "hit" determined by the laboratory and the corresponding " $\mathrm{J}$ " flag is carried through the calculation to the train total.

- When the train analytical component result is less than the RDL, but greater than the method detection limit (MDL), the result included in the train total is the RDL and the corresponding " $J$ " flag is carried through the calculation to the train total.

- When the train component analytical result is not detected down to the MDL, the result included in the train total is the RDL and the corresponding " $U$ " flag is carried through the calculation to the train total.

- It should be noted that when the RDL is selected as the default value using the guidelines above, but the RDL is greater than the $\mathrm{RL}$, the $\mathrm{RL}$ is included in the train total.

The data flags attached to the MM-5 Train Total are the cumulative set of flags for each train component included as part of the MM5 train total. A flag attached to an MM-5 train component is carried through to the "MM-5 Train Total" column when the associated component analytical result is a significant number in comparison to the MM-5 Train Total. That is, if the MM-5 Train Total is affected by an MM-5 train component analytical result, the flag is carried through to the MM-5 Train Total, but if the MM-5 Train Total is not affected by an MM-5 train component, the flag is not carried through to the MM-5 Train Total. The combinations of train fractions are conducted following the standard practice of using significant figures found in ASTM E29-93a(1999), "Standard Practice for Using Significant Digits in Test Data to Determine Conformance with Specifications" and Severn Trent Laboratories standard operating procedure number QA-004, "Rounding and Significant Figures". 
BECHTEL BWXT IDAHO, LLC (BBWI)

INTEC HLLWE Effluent Gas Emissions Inventory

Idaho National Engineering and Environmental Laboratory (INEEL)

STL Knoxville Project Number: 142503.40

5 This flag is the laboratory data flag that corresponds to EPA guidelines. The data flags for these samples are as follows:

- A "U" qualifier indicates that this analyte was analyzed for, but was not detected down to the MDL.

- A "J" qualifier indicates that this compound was detected, but at a concentration below the laboratory RL. The analytical result is therefore an estimated value.

- $A$ " $B$ " qualifier indicates that this compound was found in the associated laboratory method blank. Under these conditions these values are regarded as estimated values.

- A "D" qualifier indicates that this result was obtained through dilution of the sample. This original analysis yielded a result that exceeded the calibration range.

- An "N" qualifier indicates that this compound is a tentatively identified compound (TIC). Therefore the value is estimated.

- An "E" qualifier indicates that this compound exceeded the calibration range of the instrument.

- An "A" qualifier indicates that this result is an Aldol-condensation product.

- An " $M$ " qualifier indicates that this result was measured against the nearest internal standard and assumed a response factor of one (1).

6 When listed, the less than $(<)$ sign indicates that at least one sample fraction result is either a "non-detect" value down to the MDL of the measurement that carries, or an estimated "hit" value that is below the RDL. In either case, the final value for the fraction that is included in the data set total is the default RDL value and the actual value of the total is known to be less than $(<)$ the displayed result.

7 Entries in this column are project-specific train total flags that are applied to the run total values and are not standard EPA data flags. These project-specific flags are utilized for the INEEL NWCF HLLWE Effluent Gas Emissions Inventory project and are defined as follows:

- An "N" flag in this column indicates that the compound was not measured (detected) in any of the sampling train components, or fractions.

- A "P" flag in this column indicates that the compound was measured (detected) in one or more of the train components, or fractions, but not in all of the sampling train fractions.

- An "A" flag in this column indicates that the compound was measured (detected) in all of the sampling train components, or fractions.

Bis(2-chloroisopropyl)ether and 2,2'-Oxybis(1-chloropropane) are synonyms.

9 The tentatively identified compounds (TICs) were identified by conducting a mass spectral library search using the NBS library of data. It should be noted that TICs that give the same mass spectral match for GC peaks at different retention times are listed separately with the same compound identity. Under these conditions the compounds are likely indistinguishable isomers of the same compound. However, insufficient evidence is available to determine unequivocal identities.

Last saved by Patti Carswell on 02/04/02 at 4:40 PM

E:My Documents $\backslash H L L W E$ Sampling Final Report\Appendix A\Appendix A-4. 0010-END-2.doc

Created on 2/4/2002 4:40 PM 
BECHTEL BWXT IDAHO, LLC (BBWI)

INTEC HLLWE Effluent Gas Emissions Inventory

Idaho National Engineering and Environmental Laboratory (INEEL)

STL Knoxville Project Number: 142503.40

\section{MM-5 Blank Train Summary - Run 2 Train Totals Semivolatile Organic Compounds Analytical Results Summary Table A-5. HLLWE Run ID: 0010-BT-1}

Field Sample Name: Sample Description:
MM-5 Blank Train

MM-5 Blank Train Totals for Semivolatile Organic Compounds Analysis

\begin{tabular}{|c|c|c|c|c|c|c|c|c|c|c|}
\hline \multirow[b]{2}{*}{ Analyte } & \multirow{2}{*}{$\begin{array}{c}\text { CAS } \\
\text { Registry } \\
\text { Number } \\
\end{array}$} & \multicolumn{2}{|c|}{$\begin{array}{c}\text { MM-5 Train } \\
\text { Front Half } \\
\text { Composite }{ }^{1} \\
(\mu g)\end{array}$} & \multicolumn{2}{|c|}{$\begin{array}{c}\text { MM-5 Train } \\
\text { Back Half } \\
\text { Composite } \\
(\mu \mathrm{g})\end{array}$} & \multicolumn{2}{|c|}{$\begin{array}{c}\text { MM-5 Train } \\
\text { Condensate } \\
\text { Composite }^{3} \\
(\mu \mathrm{g})\end{array}$} & \multicolumn{2}{|c|}{$\begin{array}{c}\text { MM-5 Train } \\
\text { Totals } \\
{ }^{4} \\
\text { Total } \mu g) \\
\end{array}$} & \multirow{2}{*}{$\begin{array}{c}\text { Project } \\
\text { Specific } \\
\text { Flag }^{7}\end{array}$} \\
\hline & & Risk Result & Flag $^{5}$ & Risk Result & Flag $^{5}$ & \begin{tabular}{|l|} 
Risk Result \\
\end{tabular} & Flag ${ }^{5}$ & Total $^{6}$ & Flag & \\
\hline \multicolumn{11}{|l|}{ Target Compound List } \\
\hline Acenaphthene & $83-32-9$ & 1.3 & U & 1.3 & $U$ & 1.9 & $\mathrm{U}$ & $<4.5$ & & $\mathrm{~N}$ \\
\hline Acenaphthylene & $208-96-8$ & 1.3 & $\mathrm{U}$ & 1.3 & $\mathrm{U}$ & 1.6 & $\mathrm{U}$ & $<4.2$ & & $\mathrm{~N}$ \\
\hline Acetophenone & $9[8-86-2$ & 2.0 & $\mathrm{U}$ & 6.3 & $\mathrm{~J}$ & 2.6 & $\mathrm{U}$ & $<11$ & $J$ & $\mathrm{P}$ \\
\hline Aniline & $62-53-3$ & 2.5 & $\mathrm{U}$ & 19 & $\mathrm{U}$ & 18 & U & $<40$ & & $\mathrm{~N}$ \\
\hline Anthracene & $120-12-7$ & 1.3 & $\mathrm{U}$ & 1.3 & U & 1.6 & $\mathrm{U}$ & $<4.2$ & & $\mathrm{~N}$ \\
\hline Benzidine & $92-87-5$ & 100 & $\mathrm{U}$ & 100 & $\mathrm{U}$ & 66 & $\mathrm{U}$ & $<270$ & & $N$ \\
\hline Benzoic acid & $65-85-0$ & 100 & $\mathrm{U}$ & 100 & $\mathrm{U}$ & 9.4 & $\mathrm{U}$ & $<210$ & & $\mathrm{~N}$ \\
\hline Benzo(a)anthracene & $56-55-3$ & 2.2 & U & 1.5 & U & 1.7 & $\mathrm{U}$ & $<5.4$ & & $\mathrm{~N}$ \\
\hline Benzo(a)pyrene & $50-32-8$ & 2.6 & $\mathrm{U}$ & 1.3 & $\mathrm{U}$ & 1.8 & $\mathrm{U}$ & $<5.7$ & & $N$ \\
\hline Benzo(b)fluoranthene & $205-99-2$ & 3.7 & $\mathrm{U}$ & 2.9 & $\mathrm{U}$ & 4.2 & $\mathrm{U}$ & $<11$ & & $N$ \\
\hline Benzo(g,h,i)perylene & $191-24-2$ & 7.3 & $\mathrm{U}$ & 1.6 & $\mathrm{U}$ & 2.1 & U & $<11$ & & $\mathrm{~N}$ \\
\hline Benzo(k)fluoranthene & $207-08-9$ & 5.5 & $\mathrm{U}$ & 4.2 & U & 2.9 & $\mathrm{U}$ & $<13$ & & $N$ \\
\hline Benzyl alcohol & $100-51-6$ & 92 & $\mathrm{U}$ & 92 & $\mathrm{U}$ & 4.2 & $\mathrm{U}$ & $<190$ & & $N$ \\
\hline bis(2-Chloroethoxy)methane & $111-91-1$ & 1.5 & $\mathrm{U}$ & 1.3 & $\mathrm{U}$ & 2.0 & $\mathrm{U}$ & $<4.8$ & & $\mathrm{~N}$ \\
\hline bis(2-Chloroethyl)ether & $111-44-4$ & 2.0 & $U$ & 1.5 & $\mathrm{U}$ & 1.7 & $\mathrm{U}$ & $<5.2$ & & $\mathrm{~N}$ \\
\hline bis(2-Ethylhexyl)phthalate & $117-81-7$ & 15 & & 99 & & 15 & & 130 & & A \\
\hline 4-Bromophenyl-phenylether & $101-55-3$ & 1.4 & $\mathrm{U}$ & 1.3 & $U$ & 1.4 & $U$ & $<4.1$ & & $\mathrm{~N}$ \\
\hline Butylbenzylphthalate & $85-68-7$ & 2.9 & $\mathrm{U}$ & 1.6 & $\mathrm{U}$ & 2.3 & U & $<6.8$ & & $\mathrm{~N}$ \\
\hline Carbazole & $86-74-8$ & 2.0 & $\mathrm{U}$ & 1.7 & U & 2.2 & U & $<5.9$ & & $\mathrm{~N}$ \\
\hline 4-Chloro-3-methylphenol & $59-50-7$ & 2.6 & $\mathrm{U}$ & 1.6 & $\mathrm{U}$ & 6.6 & $\mathrm{U}$ & $<11$ & & $\mathrm{~N}$ \\
\hline 4-Chloroaniline & $106-47-8$ & 3.1 & $\mathrm{U}$ & 16 & $\mathrm{U}$ & 7.9 & $\mathrm{U}$ & $<27$ & & $\mathrm{~N}$ \\
\hline 2-Chloronaphthalene & $91-58-7$ & 1.3 & $\mathrm{U}$ & 1.3 & $\mathrm{U}$ & 1.4 & $\mathrm{U}$ & $<4.0$ & & $\mathrm{~N}$ \\
\hline 2-Chlorophenol & $95-57-8$ & 2.6 & $\mathrm{U}$ & 1.3 & $\mathrm{U}$ & 1.7 & $U$ & $<5.6$ & & $\mathrm{~N}$ \\
\hline 4-Chlorophenyl phenyl ether & $7005-72-36$ & 1.3 & $\mathrm{U}$ & 1.3 & $\mathrm{U}$ & 3.1 & $\mathrm{U}$ & $<5.7$ & & $\mathrm{~N}$ \\
\hline Chrysene & $218-01-9$ & 2.3 & U & 1.7 & $\mathrm{U}$ & 1.3 & U & $<5.3$ & & $\mathrm{~N}$ \\
\hline
\end{tabular}

Last saved by Patti Carswell on 02/04/02 at 4:45 PM

G:INEEL HLLWE Report 2001 10010 Train Reports\MM5 0010-BT-1.doc

Created on 8/2/2001 12:46 PM 
BECHTEL BWXT IDAHO, LLC (BBWI)

INTEC HLLWE Effluent Gas Emissions Inventory

Idaho National Engineering and Environmental Laboratory (INEEL)

STL Knoxville Project Number: 142503.40

\section{MM-5 Blank Train Summary - Run 2 Train Totals (Continued) Semivolatile Organic Compounds Analytical Results Summary Table A-5. HLLWE Run ID: 0010-BT-1}

\begin{tabular}{|c|c|c|c|c|c|c|c|c|c|c|}
\hline \multirow[b]{2}{*}{ Analyte } & \multirow{2}{*}{$\begin{array}{c}\text { CAS } \\
\text { Registry } \\
\text { Number } \\
\end{array}$} & \multicolumn{2}{|c|}{$\begin{array}{c}\text { MM-5 Train } \\
\text { Front Half } \\
\text { Composite }^{1} \\
(\mu \mathrm{g})\end{array}$} & \multicolumn{2}{|c|}{$\begin{array}{c}\text { MM-5 Train } \\
\text { Back Half } \\
\text { Composite }^{2} \\
(\mu \mathrm{g}) \\
\end{array}$} & \multicolumn{2}{|c|}{$\begin{array}{c}\text { MM-5 Train } \\
\text { Condensate } \\
\text { Composite }^{3} \\
(\mu \mathrm{g}) \\
\end{array}$} & \multicolumn{2}{|c|}{$\begin{array}{c}\text { MM-5 Train } \\
\text { Totals }^{4} \\
\text { (Total } \mu \mathrm{g}) \\
\end{array}$} & \multirow{2}{*}{$\begin{array}{c}\text { Project } \\
\text { Specific } \\
\text { Flag }^{7}\end{array}$} \\
\hline & & Risk Result & Flag 5 & Risk Result & Flag 5 & Risk Result & Flag 5 & Total $^{6}$ & Flag & \\
\hline Di-n-butylphthalate & $84-74-2$ & 1.9 & $\mathrm{~J}$ & 20 & $\mathrm{U}$ & 2.3 & $\mathrm{~J}$ & $<24$ & $\mathrm{~J}$ & $P$ \\
\hline Di-n-octylphthalate & $117-84-0$ & 5.5 & J & 10 & & 3.3 & $J$ & $<19$ & $J$ & $\mathrm{~A}$ \\
\hline Dibenz(a,h)anthracene & $53-70-3$ & 5.2 & U & 1.6 & $\mathrm{U}$ & 2.9 & $\mathrm{U}$ & $<9.7$ & & $\mathrm{~N}$ \\
\hline Dibenzofuran & $132-64-9$ & 1.4 & $\mathrm{U}$ & 1.3 & $\mathrm{U}$ & 2.9 & $\mathrm{U}$ & $<5.6$ & & $N$ \\
\hline 1,2-Dichlorobenzene & $95-50-1$ & 2.2 & $\mathrm{U}$ & 1.3 & U & 1.7 & $\mathrm{U}$ & $<5.2$ & & $\mathrm{~N}$ \\
\hline 1,3-Dichlorobenzene & $541-73-1$ & 3.1 & $\mathrm{U}$ & 1.5 & $\mathrm{U}$ & 1.4 & $\mathrm{U}$ & $<6.0$ & & $\mathrm{~N}$ \\
\hline 1,4-Dichlorobenzene & $106-46-7$ & 2.9 & U & 12 & & 2.0 & U & $<17$ & & $\mathrm{P}$ \\
\hline 3,3'-Dichlorobenzidine & $91-94-1$ & 7.1 & $\mathrm{U}$ & 19 & U & 7.9 & $\mathrm{U}$ & $<34$ & & $N$ \\
\hline 2,4-Dichlorophenol & $120-83-2$ & 3.9 & $\mathrm{U}$ & 1.3 & $\mathrm{U}$ & 2.3 & $\mathrm{U}$ & $<7.5$ & & N \\
\hline Diethylphthalate & $84-66-2$ & 3.9 & U & 1.9 & $\mathrm{U}$ & 1.4 & $\mathrm{U}$ & $<7.2$ & & $\mathrm{~N}$ \\
\hline Dimethyl phthalate & $131-11-3$ & 1.7 & U & 1.3 & $\mathrm{U}$ & 1.3 & $\mathrm{U}$ & $<4.3$ & & $\mathrm{~N}$ \\
\hline 2,4-Dimethylphenol & $105-67-9$ & 7.6 & $\mathrm{U}$ & 10 & $\mathrm{U}$ & 1.5 & $\mathrm{U}$ & $<19$ & & $\mathrm{~N}$ \\
\hline 4,6-Dinitro-2-methylphenol & $534-52-1$ & 13 & $\mathrm{U}$ & 23 & $\mathrm{U}$ & 1.5 & $\mathrm{U}$ & $<38$ & & $\mathrm{~N}$ \\
\hline 2,4-Dinitrophenol & $51-28-5$ & 15 & U & 50 & U & 3.9 & U & $<69$ & & $\mathrm{~N}$ \\
\hline 2,4-Dinitrotoluene & $121-14-2$ & 4.2 & $\mathrm{U}$ & 1.3 & $\mathrm{U}$ & 2.6 & $\mathrm{U}$ & $<8.1$ & & $\mathrm{~N}$ \\
\hline 2,6-Dinitrotoluene & $606-20-2$ & 3.4 & $\mathrm{U}$ & 1.3 & $\mathrm{U}$ & 2.1 & $U$ & $<6.8$ & & $\mathrm{~N}$ \\
\hline 1,2-Diphenylhydrazine & $122-66-7$ & 1.7 & $\mathrm{U}$ & 1.3 & $\mathrm{U}$ & 1.5 & $\mathrm{U}$ & $<4.5$ & & $\mathrm{~N}$ \\
\hline Fluoranthene & $206-44-0$ & 1.3 & $\mathrm{U}$ & 1.4 & U & 1.8 & $\mathrm{U}$ & $<4.5$ & & $\mathrm{~N}$ \\
\hline Fluorene & $86-73-7$ & 1.3 & U & 1.3 & $\mathrm{U}$ & 2.6 & $\mathrm{U}$ & $<5.2$ & & $\mathrm{~N}$ \\
\hline Hexachlorocyclopentadiene & $77-47-4$ & 26 & U & 26 & $\mathrm{U}$ & 6.6 & U & $<59$ & & $\mathrm{~N}$ \\
\hline Hexachlorobenzene & $118-74-1$ & 1.5 & $\mathrm{U}$ & 1.3 & U & 2.6 & $\mathrm{U}$ & $<5.4$ & & $\mathrm{~N}$ \\
\hline Hexachlorobutadiene & $87-68-3$ & 3.7 & $\mathrm{U}$ & 1.9 & $\mathrm{U}$ & 1.9 & $U$ & $<7.5$ & & $\mathrm{~N}$ \\
\hline Hexachloroethane & $67-72-1$ & 6.6 & $\mathrm{U}$ & 1.4 & $\mathrm{U}$ & 1.9 & $\mathrm{U}$ & $<9.9$ & & $\mathrm{~N}$ \\
\hline Indeno( $1,2,3-\mathrm{cd})$ pyrene & $193-39-5$ & 5.5 & $\mathrm{U}$ & 1.4 & $\mathrm{U}$ & 2.3 & $\mathrm{U}$ & $<9.2$ & & $\mathrm{~N}$ \\
\hline Isophorone & $78-59-1$ & 1.7 & U & 1.3 & $\mathrm{U}$ & 1.8 & U & $<4.8$ & & $\mathrm{~N}$ \\
\hline 2-Methylnaphthalene & $91-57-6$ & 1.5 & $\mathrm{U}$ & 1.3 & $\mathrm{U}$ & 2.3 & $\mathrm{U}$ & $<5.1$ & & $\mathrm{~N}$ \\
\hline 2-Methylphenol & $95-48-7$ & 6.0 & $\mathrm{U}$ & 7.9 & $\mathrm{U}$ & 2.1 & $U$ & $<16$ & & $\mathrm{~N}$ \\
\hline 3-Methylphenol \& 4-Methylphenol & $65794-96-9$ & 6.0 & $\mathrm{U}$ & 5.2 & $U$ & 2.2 & $\mathrm{U}$ & $<13$ & & $N$ \\
\hline
\end{tabular}

Last saved by Patti Carswell on 02/04/02 at 4:45 PM

G:INEEL HLLWE. Report 200110010 Train ReportsIMM5_0010-BT-1.doc

Created on 8/2/2001 12:46 PM 
BECHTEL BWXT IDAHO, LLC (BBWI)

INTEC HLLWE Effluent Gas Emissions Inventory

Idaho National Engineering and Environmental Laboratory (INEEL)

STL Knoxville Project Number: 142503.40

\section{MM-5 Blank Train Summary - Run 2 Train Totals (Continued) \\ Semivolatile Organic Compounds Analytical Results Summary \\ Table A-5. HLLWE Run ID: 0010-BT-1}

\begin{tabular}{|c|c|c|c|c|c|c|c|c|c|c|}
\hline \multirow[b]{2}{*}{ Analyte } & \multirow{2}{*}{$\begin{array}{c}\text { CAS } \\
\text { Registry } \\
\text { Number }\end{array}$} & \multicolumn{2}{|c|}{$\begin{array}{c}\text { MM-5 Train } \\
\text { Front Half } \\
\text { Composite }^{1} \\
(\mu g)\end{array}$} & \multicolumn{2}{|c|}{$\begin{array}{c}\text { MM-5 Train } \\
\text { Back Half } \\
\text { Composite }{ }^{2} \\
(\mu g) \\
\end{array}$} & \multicolumn{2}{|c|}{$\begin{array}{c}\text { MM-5 Train } \\
\text { Condensate } \\
\text { Composite }^{3} \\
(\mu \mathrm{g}) \\
\end{array}$} & \multicolumn{2}{|c|}{$\begin{array}{c}\text { MM-5 Train } \\
\text { Totals }^{4} \\
\text { (Total } \mu \mathrm{g}) \\
\end{array}$} & \multirow{2}{*}{$\begin{array}{c}\text { Project } \\
\text { Specific } \\
\text { Flag }^{7}\end{array}$} \\
\hline & & \begin{tabular}{|l|} 
Risk Result \\
\end{tabular} & Flag $^{5}$ & Risk Result & Flag $^{5}$ & Risk Result & Flag $^{5}$ & Total $^{6}$ & Flag & \\
\hline N-Nitroso-di-n-propylamine & $621-64-7$ & 1.9 & $\mathrm{U}$ & 1.3 & $\mathrm{U}$ & 2.3 & $U$ & $<5.5$ & & $\mathrm{~N}$ \\
\hline N-Nitrosodimethylamine & $62-75-9$ & 1.9 & $\mathrm{U}$ & 1.3 & $\mathrm{U}$ & 2.2 & $\mathrm{U}$ & $<5.4$ & & $\mathrm{~N}$ \\
\hline N-Nitrosodiphenylamine & $86-30-6$ & 1.6 & $\mathrm{U}$ & 2.3 & U & 1.4 & U & $<5.3$ & & $N$ \\
\hline Naphthalene & $91-20-3$ & 1.3 & U & 1.6 & $J$ & 1.8 & $\mathrm{U}$ & $<4.7$ & $J$ & $\mathrm{P}$ \\
\hline 2-Nitroaniline & $88-74-4$ & 1.5 & $\mathrm{U}$ & 1.3 & $\mathrm{U}$ & 2.9 & $\mathrm{U}$ & $<5.7$ & & $\mathrm{~N}$ \\
\hline 3-Nitroaniline & $99-09-2$ & 10 & $\mathrm{U}$ & 5.2 & $\mathrm{U}$ & 4.7 & $\mathrm{U}$ & $<20$ & & $\mathrm{~N}$ \\
\hline 4-Nitroaniline & $100-01-6$ & 6.0 & $\mathrm{U}$ & 5.2 & $\mathrm{U}$ & 3.9 & $\mathrm{U}$ & $<15$ & & $\mathrm{~N}$ \\
\hline Nitrobenzene & $98-95-1$ & 1.9 & $\mathrm{U}$ & 1.5 & $\mathrm{U}$ & 1.7 & $\mathrm{U}$ & $<5.1$ & & $\mathrm{~N}$ \\
\hline 2-Nitrophenol & $88-75-5$ & 8.4 & $\mathrm{U}$ & 1.3 & $\mathrm{U}$ & 2.6 & $\mathrm{U}$ & $<12$ & & $\mathrm{~N}$ \\
\hline 4-Nitrophenol & $100-02-7$ & 8.7 & $\mathrm{U}$ & 8.7 & $U$ & 3.9 & U & $<21$ & & $\mathrm{~N}$ \\
\hline $2,2^{\prime}-$ Oxybis(1-chloropropane $)^{8}$ & $108-60-1$ & 2.6 & U & 2.0 & $\mathrm{U}$ & 1.8 & U & $<6.4$ & & $N$ \\
\hline Pentachlorobenzene & $608-93-5$ & 1.4 & U & 1.3 & $\mathrm{U}$ & 2.3 & $\mathrm{U}$ & $<5.0$ & & $N$ \\
\hline Pentachloronitrobenzene & $82-68-8$ & 2.0 & $\mathrm{U}$ & 1.3 & U & 2.6 & $\mathrm{U}$ & $<5.9$ & & $N$ \\
\hline Pentachlorophenol & $87-86-5$ & 50 & U & 50 & $\mathrm{U}$ & 3.4 & $\mathrm{U}$ & $<100$ & & $N$ \\
\hline Phenanthrene & $85-01-8$ & 1.3 & $\mathrm{U}$ & 1.3 & $\mathrm{U}$ & 1.9 & $\mathrm{U}$ & $<4.5$ & & $\mathrm{~N}$ \\
\hline Phenol & $108-95-2$ & 2.9 & U & 2.4 & $\mathrm{U}$ & 2.2 & $\mathrm{U}$ & $<7.5$ & & $\mathrm{~N}$ \\
\hline Pyrene & $129-00-0$ & 1.9 & $\mathrm{U}$ & 1.4 & $\mathrm{U}$ & 1.4 & $\mathrm{U}$ & $<4.7$ & & $\mathrm{~N}$ \\
\hline Pyridine & $110-86-1$ & 2.3 & $\mathrm{U}$ & 1.9 & U & 5.2 & $\mathrm{U}$ & $<9.4$ & & $\mathrm{~N}$ \\
\hline 1,2,4,5-Tetrachlorobenzene & $95-94-3$ & 2.3 & $\mathrm{U}$ & 1.3 & $\mathrm{U}$ & 2.2 & $\mathrm{U}$ & $<5.8$ & & $\mathrm{~N}$ \\
\hline $1,2,4$-Trichlorobenzene & $120-82-1$ & 1.9 & $\mathrm{U}$ & 1.5 & U & 2.2 & $\mathrm{U}$ & $<5.6$ & & $N$ \\
\hline 2,4,5-Trichlorophenol & $95-95-4$ & 6.0 & $\mathrm{U}$ & 3.4 & $\mathrm{U}$ & 2.1 & $U$ & $<12$ & & $N$ \\
\hline 2,4,6-Trichlorophenol & $88-06-2$ & 3.7 & $\mathrm{U}$ & 2.0 & U & 2.5 & $\mathrm{U}$ & $<8.2$ & & $\mathrm{~N}$ \\
\hline
\end{tabular}

Last saved by Patti Carswelt on 02/04/02 at 4:45 PM

G:INEEL HLLWE Report 200110010 Train Reports\MM5_0010-BT-1.doc

Created on 8/2/2001 12:46 PM 
BECHTEL BWXT IDAHO, LLC (BBWI)

INTEC HLLWE Effluent Gas Emissions Inventory

Idaho National Engineering and Environmental Laboratory (INEEL)

STL Knoxville Project Number: 142503.40

\section{MM-5 Blank Train Summary - Run 2 Train Totals (Continued) Semivolatile Organic Compounds Analytical Results Summary Table A-5. HLLWE Run ID: 0010-BT-1}

\begin{tabular}{|c|c|c|c|c|c|c|c|}
\hline \multirow[b]{2}{*}{ Analyte } & \multirow{2}{*}{$\begin{array}{c}\text { CAS } \\
\text { Registry } \\
\text { Number }\end{array}$} & $\begin{array}{c}\text { MM-5 Train } \\
\text { Front Half } \\
\text { Composite } \\
(\mu g)\end{array}$ & $\begin{array}{c}\text { MM-5 Train } \\
\text { Back Half } \\
\text { Composite }^{2} \\
(\mu g)\end{array}$ & $\begin{array}{c}\text { MM-5 Train } \\
\text { Condensate } \\
\text { Composite }^{3} \\
(\mu g)\end{array}$ & \multicolumn{2}{|c|}{$\begin{array}{c}\text { MM-5 Train } \\
\text { Totals }^{4} \\
\text { (Total } \mu g) \\
\end{array}$} & \multirow{2}{*}{$\begin{array}{c}\text { Project } \\
\text { Specific } \\
\text { Flag }^{7} \\
\end{array}$} \\
\hline & & Risk Result Flag ${ }^{5}$ & Risk Result Flag ${ }^{5}$ & Risk Result Flag $^{5}$ & Total $^{6}$ & Flag & \\
\hline \multicolumn{8}{|l|}{$T I C s^{9}$} \\
\hline Furan, 2,5-dimethyl- & $625-86-5$ & 12 & --- & --- & 12 & $\mathrm{~N}, \mathrm{~J}, \mathrm{M}$ & $\mathrm{P}$ \\
\hline Heptane, 2,5-dimethyl- & $2216-30-0$ & 9.0 & -- & -- & 9.0 & $\mathrm{~N}, \mathrm{~J}, \mathrm{M}$ & $\mathrm{P}$ \\
\hline Heptane, 2,3-dimethyl- & $3074-71-3$ & 11 & -- & $\cdots$ & 11 & $\mathrm{~N}, J, M$ & $\mathrm{P}$ \\
\hline Benzaldehyde & $100-52-7$ & --- & 7.3 & $\cdots$ & 7.3 & $\mathrm{~N}, \mathrm{~J}, \mathrm{M}$ & $\mathrm{P}$ \\
\hline Benzoic acid, methyl ester & $93-58-3$ & -- & 8.4 & --- & 8.4 & $\mathrm{~N}, \mathrm{~J}, \mathrm{M}$ & $\mathrm{P}$ \\
\hline Benzaldehyde, ethyl- & $53951-50-1$ & -- & 14 & $--\cdot$ & 14 & $\mathrm{~N}, \mathrm{~J}, \mathrm{M}$ & $\mathrm{P}$ \\
\hline Pentadecane & $629-62-9$ & 4.4 & $-\cdots$ & $-\cdots$ & 4.4 & $\mathrm{~N}, \mathrm{~J}, \mathrm{M}$ & $\mathrm{P}$ \\
\hline Cyclododecane & $294-62-2$ & 11 & 8.7 & 10 & 30 & $\mathrm{~N}, \mathrm{~J}, \mathrm{M}$ & $\mathrm{P}$ \\
\hline Heptadecane & $629-78-7$ & 3.0 & $\cdots$ & -- & 3.0 & $\mathrm{~N}, \mathrm{~J}, \mathrm{M}$ & $\mathrm{P}$ \\
\hline Eicosane & $112-95-8$ & $\cdots$ & -- & 3.7 & 3.7 & $\mathrm{~N}, J, M$ & $\mathrm{P}$ \\
\hline Heneicosane & $629-94-7$ & $\cdots$ & 4.5 & $\cdots$ & 4.5 & $\mathrm{~N}, \mathrm{~J}, \mathrm{M}$ & $\mathrm{P}$ \\
\hline Octodecane & $593-45-3$ & -- & 10 & $\cdots$ & 10 & $\mathrm{~N}, \mathrm{~J}, \mathrm{M}$ & $\mathrm{P}$ \\
\hline Phosphine oxide, triphenyl- & $791-28-6$ & $\cdots$ & -- & 26 & 26 & $\mathrm{~N}, \mathrm{~J}, \mathrm{M}$ & $P$ \\
\hline Nonacosane & $630-03-5$ & -- & 10 & --- & 10 & N,J,M & $\mathrm{P}$ \\
\hline Eicosane & $112-95-8$ & -- & 14 & -- & 14 & $\mathrm{~N}, J, M$ & $\mathrm{P}$ \\
\hline Hexatriacontane & $630-06-8$ & --- & 23 & $\cdots$ & 23 & $\mathrm{~N}, \mathrm{~J}, \mathrm{M}$ & $\mathrm{P}$ \\
\hline Tetracosane & $646-31-1$ & -- & 18 & -- & 18 & $\mathrm{~N}, \mathrm{~J}, \mathrm{M}$ & $\mathrm{P}$ \\
\hline Heneicosane & $629-94-7$ & --- & $\cdots$ & 3.6 & 3.6 & $\mathrm{~N}, \mathrm{~J}, \mathrm{M}$ & $\mathrm{P}$ \\
\hline Tetratriacontane & $14167-59-0$ & $\cdots$ & 11 & --- & 11 & $\mathrm{~N}, \mathrm{~J}, \mathrm{M}$ & $\mathrm{P}$ \\
\hline Eicosane & $112-95-8$ & --- & 9.2 & $\ldots$ & 9.2 & $\mathrm{~N}, \mathrm{~J}, \mathrm{M}$ & $\mathrm{P}$ \\
\hline
\end{tabular}




\section{BECHTEL BWXT IDAHO, LLC (BBWI) \\ INTEC HLLWE Effluent Gas Emissions Inventory \\ Idaho National Engineering and Environmental Laboratory (INEEL) \\ STL Knoxville Project Number: 142503.40}

\section{Footnotes:}

1 The MM-5 Train Front Half Composite consists of the Particulate Filter and the Front Half of the Filter Holder and Probe Solvent Rinses.

2 The MM-5 Train Back Half Composite consists of the XAD-2 Resin Tube and the Back Half of the Filter Holder and Coil Condenser Solvent Rinses.

3 The MM-5 Train Condensate Composite consists of the Condensate and Impinger Contents and the Glassware Solvent Rinses.

4 The total mass for each semivolatile compound found in the MM-5 sampling train consists of the sum of the MM-5 train's Front Half Composite contents, the train's Back Half Composite contents, and the Condensate Composite. The calculation is as follows:

(Total $\mu \mathrm{g}$ in the Front Half $)+($ Total $\mu \mathrm{g}$ in the Back Half $)+($ Concentration in the Condensate Composite $\times$ Condensate Composite Volume)

$=$ Total $\mu g$ in the MM-5 Sampling Train.

Therefore: $\quad(\mu \mathrm{g})+(\mu \mathrm{g})+(\mu \mathrm{g} /$ Liter $\times$ Liter $)=$ Total $\mu \mathrm{g}$

The MM-5 Train Run Total (in Total $\mu \mathrm{g}$ ) is the sum of results for the three (3) MM-5 train sample fractions using the following guidelines:

- When the train component analytical result is greater than the laboratory reporting limit (RL), the result included in the train total is the actual analytical result or "hit" determined by the laboratory.

- When the train component analytical result is greater than the reliable detection level (RDL), but less than the laboratory reporting limit (RL), the result included in the train total is actual analytical result or "hit" determined by the laboratory and the corresponding "J" flag is carried through the calculation to the train total.

- When the train analytical component result is less than the RDL, but greater than the method detection limit (MDL), the result included in the train total is the RDL and the corresponding " $\mathrm{J}$ " flag is carried through the calculation to the train total.

- When the train component analytical result is not detected down to the MDL, the result included in the train total is the RDL and the corresponding "U" flag is carried through the calculation to the train total.

- It should be noted that when the RDL is selected as the default value using the guidelines above, but the RDL is greater than the $R L$, the RL is included in the train total.

The data flags attached to the MM-5 Train Total are the cumulative set of flags for each train component included as part of the MM5 train total. A flag attached to an MM-5 train component is carried through to the "MM-5 Train Total" column when the associated component analytical result is a significant number in comparison to the MM-5 Train Total. That is, if the MM-5 Train Total is affected by an MM-5 train component analytical result, the flag is carried through to the MM-5 Train Total, but if the MM-5 Train Total is not affected by an MM-5 train component, the flag is not carried through to the MM-5 Train Total. The combinations of train fractions are conducted following the standard practice of using significant figures found in ASTM E29-93a(1999), "Standard Practice for Using Significant Digits in Test Data to Determine Conformance with Specifications" and Severn Trent Laboratories standard operating procedure number QA-004, "Rounding and Significant Figures".

Last saved by Patti Carswell on 02/04/02 at 4:45 PM 


\section{BECHTEL BWXT IDAHO, LLC (BBWI) \\ INTEC HLLWE Effluent Gas Emissions Inventory \\ Idaho National Engineering and Environmental Laboratory (INEEL) \\ STL Knoxville Project Number: 142503.40}

5 This flag is the laboratory data flag that corresponds to EPA guidelines. The data flags for these samples are as follows:

- A "U" qualifier indicates that this analyte was analyzed for, but was not detected down to the MDL.

- A "J" qualifier indicates that this compound was detected, but at a concentration below the laboratory RL. The analytical result is therefore an estimated value.

- A "B" qualifier indicates that this compound was found in the associated laboratory method blank. Under these conditions these values are regarded as estimated values.

- A "D" qualifier indicates that this result was obtained through dilution of the sample. This original analysis yielded a result that exceeded the calibration range.

- An "N" qualifier indicates that this compound is a tentatively identified compound (TIC). Therefore the value is estimated.

- An "E" qualifier indicates that this compound exceeded the calibration range of the instrument.

- An "A" qualifier indicates that this result is an Aldol-condensation product.

- An "M" qualifier indicates that this result was measured against the nearest internal standard and assumed a response factor of one (1).

6 When listed, the less than $(<)$ sign indicates that at least one sample fraction result is either a "non-detect" value down to the MDL of the measurement that carries, or an estimated "hit" value that is below the RDL. In either case, the final value for the fraction that is included in the data set total is the default RDL value and the actual value of the total is known to be less than $(<)$ the displayed result.

7 Entries in this column are project-specific train total flags that are applied to the run total values and are not standard EPA data flags. These project-specific flags are utilized for the INEEL NWCF HLLWE Effluent Gas Emissions Inventory project and are defined as follows:

- An " $N$ " flag in this column indicates that the compound was not measured (detected) in any of the sampling train components, or fractions.

- A "P" flag in this column indicates that the compound was measured (detected) in one or more of the train components, or fractions, but not in all of the sampling train fractions.

- An "A" flag in this column indicates that the compound was measured (detected) in all of the sampling train components, or fractions.

Bis(2-chloroisopropyl)ether and 2,2'-Oxybis( 1-chloropropane) are synonyms.

9 The tentatively identified compounds (TICs) were identified by conducting a mass spectral library search using the NBS library of data. It should be noted that TICs that give the same mass spectral match for GC peaks at different retention times are listed separately with the same compound identity. Under these conditions the compounds are likely indistinguishable isomers of the same compound. However, insufficient evidence is available to determine unequivocal identities.

Last saved by Patti Carswell on 02/04/02 at 4:45 PM

G:VINEEL HLLWE Report 200110010 Train Reports\MM5_0010-BT-1.doc

Created on 8/2/2001 12:46 PM 
BECHTEL BWXT IDAHO, LLC (BBWI)

INTEC HLLWE Effluent Gas Emissions Inventory

Idaho National Engineering and Environmental Laboratory (INEEL)

STL Knoxville Project Number: 142503.40

\section{MM-5 Train Analytical Results Summary \\ Table A-6. Run 2, XAD-2 Resin Tube Trip Blank/Reagent Blank}

Field Sample Name: Sample Description: Field Sample ID:

STL Sample No.
MM-5 Train XAD-2 Resin Tube Trip Blank/Reagent Blank

MM-5 Train XAD-2 Resin Tube Trip Blank/Reagent Blank for Semivolatile Organic Compounds Analysis A-3378

H1F250162-007

\begin{tabular}{|c|c|c|c|c|c|c|c|}
\hline \multirow[b]{2}{*}{ Analyte } & \multirow{2}{*}{$\begin{array}{l}\text { CAS } \\
\text { Registry } \\
\text { Number }\end{array}$} & \multicolumn{6}{|c|}{$\begin{array}{c}\text { MM-5 Train } \\
\text { XAD-2 Resin Tube Trip Blank/Reagent Blank } \\
(\mu \mathrm{g})\end{array}$} \\
\hline & & Lab Result ${ }^{2}$ & $\mathrm{MDL}^{3}$ & $\mathrm{RDL}^{4}$ & $\mathbf{R L}^{5}$ & Risk Result & Flag $^{6}$ \\
\hline Acenaphthene & $83-32-9$ & ND & 0.50 & 1.3 & 10 & $<1.3$ & \\
\hline Acenaphthylene & $208-96-8$ & ND & 0.50 & 1.3 & 10 & $<1.3$ & \\
\hline Acetophenone & $98-86-2$ & 2.7 & 2.4 & 6.3 & 10 & 6.3 & J \\
\hline Aniline & $62-53-3$ & $\mathrm{ND}$ & 7.3 & 19 & 20 & $<19$ & \\
\hline Anthracene & $120-12-7$ & ND & 0.50 & 1.3 & 10 & $<1.3$ & \\
\hline Benzidine & $92-87-5$ & ND & 51 & 130 & 100 & $<100$ & \\
\hline Benzoic acid & $65-85-0$ & ND & 46 & 120 & 100 & $<100$ & \\
\hline Benzo(a)anthracene & $56-55-3$ & ND & 0.58 & 1.5 & 10 & $<1.5$ & \\
\hline Benzo(a)pyrene & $50-32-8$ & ND & 0.50 & 1.3 & 10 & $<1.3$ & \\
\hline Benzo(b)fluoranthene & $205-99-2$ & ND & 1.1 & 2.9 & 10 & $<2.9$ & \\
\hline Benzo(g,h,i)perylene & $191-24-2$ & ND & 0.62 & 1.6 & 10 & $<1.6$ & \\
\hline Benzo(k)fluoranthene & $207-08-9$ & ND & 1.6 & 4.2 & 10 & $<4.2$ & \\
\hline Benzyl alcohol & $100-51-6$ & ND & 35 & 92 & 100 & $<92$ & \\
\hline bis(2-Chloroethoxy)methane & $111-91-1$ & ND & 0.50 & 1.3 & 10 & $<1.3$ & \\
\hline bis(2-Chloroethyl)ether & $111-44-4$ & ND & 0.56 & 1.5 & 10 & $<1.5$ & \\
\hline bis(2-Ethylhexyl)phthalate & $117-81-7$ & ND & 10 & 26 & 20 & $<20$ & \\
\hline 4-Bromophenyl-phenylether & $101-55-3$ & ND & 0.50 & 1.3 & 10 & $<1.3$ & \\
\hline Butylbenzylphthalate & $85-68-7$ & ND & 0.61 & 1.6 & 10 & $<1.6$ & \\
\hline Carbazole & $86-74-8$ & ND & 0.64 & 1.7 & 10 & $<1.7$ & \\
\hline 4-Chloro-3-methylphenol & $59-50-7$ & ND & 0.62 & 1.6 & 10 & $<1.6$ & \\
\hline 4-Chloroaniline & $106-47-8$ & ND & 6.0 & 16 & 20 & $<16$ & \\
\hline 2-Chloronaphthalene & $91-58-7$ & ND & 0.50 & 1.3 & 10 & $<1.3$ & \\
\hline 2-Chlorophenol & $95-57-8$ & ND & 0.50 & 1.3 & 10 & $<1.3$ & \\
\hline 4-Chlorophenyl phenyl ether & $7005-72-36$ & $\mathrm{ND}$ & 0.50 & 1.3 & 10 & $<1.3$ & \\
\hline Chrysene & $218-01-9$ & ND & 0.64 & 1.7 & 10 & $<1.7$ & \\
\hline
\end{tabular}


BECHTEL BWXT IDAHO, LLC (BBWI)

INTEC HLLWE Effluent Gas Emissions Inventory

Idaho National Engineering and Environmental Laboratory (INEEL)

STL Knoxville Project Number: 142503.40

MM-5 Train Analytical Results Summary (Continued)

Table A-6. Run 2, XAD-2 Resin Tube Trip Blank/Reagent Blank

\begin{tabular}{|c|c|c|c|c|c|c|c|}
\hline \multirow[b]{2}{*}{ Analyte } & \multirow{2}{*}{$\begin{array}{c}\text { CAS } \\
\text { Registry } \\
\text { Number }\end{array}$} & \multicolumn{6}{|c|}{$\begin{array}{c}\text { MM-5 Train } \\
\text { XAD-2 Resin Tube Trip Blank/Reagent Blank } \\
(\mu \mathrm{g})^{\prime}\end{array}$} \\
\hline & & Lab Result ${ }^{2}$ & MDL $^{3}$ & $\mathrm{RDL}^{4}$ & $\mathbf{R L}^{5}$ & Risk Result & Flag $^{6}$ \\
\hline Di-n-butylphthalate & $84-74-2$ & ND & 10 & 26 & 20 & $<20$ & \\
\hline Di-n-octylphthalate & $117-84-0$ & $\mathrm{ND}$ & 0.56 & 1.5 & 10 & $<1.5$ & \\
\hline Dibenz(a,h)anthracene & $53-70-3$ & ND & 0.60 & 1.6 & 10 & $<1.6$ & \\
\hline Dibenzofuran & $132-64-9$ & ND & 0.50 & 1.3 & 10 & $<1.3$ & \\
\hline 1,2-Dichlorobenzene & $95-50-1$ & ND & 0.51 & 1.3 & 10 & $<1.3$ & \\
\hline 1,3-Dichlorobenzene & $541-73-1$ & ND & 0.57 & 1.5 & 10 & $<1.5$ & \\
\hline 1,4-Dichlorobenzene & $106-46-7$ & 9.0 & 0.53 & 1.4 & 10 & 9.0 & $\mathrm{~J}$ \\
\hline 3,3'-Dichlorobenzidine & 91-94-1 & ND & 7.4 & 19 & 50 & $<19$ & \\
\hline 2,4-Dichlorophenol & $120-83-2$ & ND & 0.50 & 1.3 & 10 & $<1.3$ & \\
\hline Diethylphthalate & $84-66-2$ & ND & 0.73 & 1.9 & 10 & $<1.9$ & \\
\hline Dimethyl phthalate & $131-11-3$ & ND & 0.50 & 1.3 & 10 & $<1.3$ & \\
\hline 2,4-Dimethylphenol & $105-67-9$ & ND & 6.3 & 16 & 10 & $<10$ & \\
\hline 4,6-Dinitro-2-methylphenol & $534-52-1$ & $\mathrm{ND}$ & 8.7 & 23 & 50 & $<23$ & \\
\hline 2,4-Dinitrophenol & $51-28-5$ & ND & 22 & 58 & 50 & $<50$ & \\
\hline 2,4-Dinitrotoluene & $121-14-2$ & ND & 0.50 & 1.3 & 10 & $<1.3$ & \\
\hline 2,6-Dinitrotoluene & $606-20-2$ & ND & 0.50 & 1.3 & 10 & $<1.3$ & \\
\hline 1,2-Diphenylhydrazine & $122-66-7$ & ND & 0.50 & 1.3 & 10 & $<1.3$ & \\
\hline Fluoranthene & $206-44-0$ & ND & 0.54 & 1.4 & 10 & $<1.4$ & \\
\hline Fluorene & $86-73-7$ & ND & 0.50 & 1.3 & 10 & $<1.3$ & \\
\hline Hexachlorocyclopentadiene & $77-47-4$ & ND & 10 & 26 & 50 & $<26$ & \\
\hline Hexachlorobenzene & $118-74-1$ & ND & 0.50 & 1.3 & 10 & $<1.3$ & \\
\hline Hexachlorobutadiene & $87-68-3$ & ND & 0.74 & 1.9 & 10 & $<1.9$ & \\
\hline Hexachloroethane & $67-72-1$ & ND & 0.54 & 1.4 & 10 & $<1.4$ & \\
\hline Indeno(1,2,3-cd)pyrene & $193-39-5$ & ND & 0.54 & 1.4 & 10 & $<1.4$ & \\
\hline Isophorone & $78-59-1$ & ND & 0.50 & 1.3 & 10 & $<1.3$ & \\
\hline 2-Methylnaphthalene & $91-57-6$ & ND & 0.50 & 1.3 & 10 & $<1.3$ & \\
\hline 2-Methylphenol & $95-48-7$ & ND & 3.0 & 7.9 & 10 & $<7.9$ & \\
\hline 3-Methylphenol \& 4-Methylphenol & $65794-96-9$ & ND & 2.0 & 5.2 & 10 & $<5.2$ & \\
\hline
\end{tabular}

Last saved by Robin Gifford on 02/04/02 at 4:56 PM

E: My Documents\HLLWE Sampling $\backslash F i n a l$ ReportlAppendix A\Table A-6. 0010-TB.doc

Created on 2/4/2002 4:53 PM 
BECHTEL BWXT IDAHO, LLC (BBWI)

INTEC HLLWE Effluent Gas Emissions Inventory

Idaho National Engineering and Environmental Laboratory (INEEL)

STL Knoxville Project Number: 142503.40

\section{MM-5 Train Analytical Results Summary (Continued) Table A-6. Run 2, XAD-2 Resin Tube Trip Blank/Reagent Blank}

\begin{tabular}{|c|c|c|c|c|c|c|c|}
\hline \multirow[b]{2}{*}{ Analyte } & \multirow{2}{*}{$\begin{array}{c}\text { CAS } \\
\text { Registry } \\
\text { Number }\end{array}$} & \multicolumn{6}{|c|}{$\begin{array}{c}\text { MM-5 Train } \\
\text { XAD-2 Resin Tube Trip Blank/Reagent Blank } \\
(\mu \mathrm{g}){ }^{1}\end{array}$} \\
\hline & & Lab Result ${ }^{2}$ & $\mathrm{MDL}^{3}$ & $\mathrm{RDL}^{4}$ & $\mathbf{R L}^{5}$ & Risk Result & Flag $^{6}$ \\
\hline N-Nitroso-di-n-propylamine & $621-64-7$ & ND & 0.50 & 1.3 & 10 & $<1.3$ & \\
\hline N-Nitrosodimethylamine & $62-75-9$ & ND & 0.50 & 1.3 & 10 & $<1.3$ & \\
\hline N-Nitrosodiphenylamine & $86-30-6$ & ND & 0.87 & 2.3 & 10 & $<2.3$ & \\
\hline Naphthalene & $91-20-3$ & ND & 0.60 & 1.6 & 10 & $<1.6$ & \\
\hline 2-Nitroaniline & $88-74-4$ & ND & 0.50 & 1.3 & 50 & $<1.3$ & \\
\hline 3-Nitroaniline & $99-09-2$ & ND & 2.0 & 5.2 & 50 & $<5.2$ & \\
\hline 4-Nitroaniline & $100-01-6$ & ND & 2.0 & 5.2 & 50 & $<5.2$ & \\
\hline Nitrobenzene & $98-95-1$ & ND & 0.57 & 1.5 & 10 & $<1.5$ & \\
\hline 2-Nitrophenol & $88-75-5$ & ND & 0.50 & 1.3 & 10 & $<1.3$ & \\
\hline 4-Nitrophenol & $100-02-7$ & ND & 3.3 & 8.7 & 50 & $<8.7$ & \\
\hline 2,2'-Oxybis(1-chloropropane) ${ }^{7}$ & $108-60-1$ & ND & 0.76 & 2.0 & 10 & $<2.0$ & \\
\hline Pentachlorobenzene & $608-93-5$ & ND & 0.50 & 1.3 & 10 & $<1.3$ & \\
\hline Pentachloronitrobenzene & $82-68-8$ & ND & 0.50 & 1.3 & 50 & $<1.3$ & \\
\hline Pentachlorophenol & $87-86-5$ & ND & 25 & 66 & 50 & $<50$ & \\
\hline Phenanthrene & $85-01-8$ & ND & 0.50 & 1.3 & 10 & $<1.3$ & \\
\hline Phenol & $108-95-2$ & ND & 0.90 & 2.4 & 10 & $<2.4$ & \\
\hline Pyrene & $129-00-0$ & ND & 0.53 & 1.4 & 10 & $<1.4$ & \\
\hline Pyridine & $110-86-1$ & ND & 0.74 & 1.9 & 20 & $<1.9$ & \\
\hline 1,2,4,5-Tetrachlorobenzene & $95-94-3$ & ND & 0.50 & 1.3 & 10 & $<1.3$ & \\
\hline 1,2,4-Trichlorobenzene & $120-82-1$ & ND & 0.59 & 1.5 & 10 & $<1.5$ & \\
\hline 2,4,5-Trichlorophenol & $95-95-4$ & ND & 1.3 & 3.4 & 10 & $<3.4$ & \\
\hline 2,4,6-Trichlorophenol & $88-06-2$ & ND & 0.75 & 2.0 & 10 & $<2.0$ & \\
\hline
\end{tabular}


BECHTEL BWXT IDAHO, LLC (BBWI)

INTEC HLLWE Effluent Gas Emissions Inventory

Idaho National Engineering and Environmental Laboratory (INEEL)

STL Knoxville Project Number: 142503.40

\section{MM-5 Train Analytical Results Summary (Continued) Table A-6. Run 2, XAD-2 Resin Tube Trip Blank/Reagent Blank}

\section{Sampling Surrogate Recoveries:}

\begin{tabular}{|c|c|c|}
\hline Sampling Surrogate Compound ${ }^{8}$ & $\begin{array}{c}\text { Percent Recovery } \\
(\%)\end{array}$ & $\begin{array}{c}\text { Project Target } \\
\text { Recovery Limits } \\
(\%)\end{array}$ \\
\hline${ }^{13} \mathrm{C}_{6}$-Naphthalene & $66 \%$ & $50-150 \%$ \\
\hline
\end{tabular}

Surrogate Standard Recoveries:

\begin{tabular}{|c|c|c|}
\hline Surrogate Standard Compound & $\begin{array}{c}\text { Percent Recovery } \\
(\%)\end{array}$ & $\begin{array}{c}\text { Laboratory } \\
\text { Recovery } \\
\text { Limits } \\
(\%) \\
\end{array}$ \\
\hline 2-Fluorophenol & $55 \%$ & $19-100 \%$ \\
\hline Phenol- $d_{5}$ & $61 \%$ & $15-124 \%$ \\
\hline Nitrobenzene- $\mathrm{d}_{5}$ & $63 \%$ & $35-122 \%$ \\
\hline 2-Fluorobiphenyl & $67 \%$ & $34-115 \%$ \\
\hline $2,4,6$-Tribromophenol & $0.0 \%{ }^{9}$ & $33-130 \%$ \\
\hline Terphenyl- $\mathrm{d}_{14}$ & $84 \%$ & $28-132 \%$ \\
\hline
\end{tabular}

Sample Collection and Analysis Dates:

$\begin{array}{ll}\text { Date(s) Collected: } & \text { June 21, 2001 } \\ \text { Date(s) of Extraction: } & \text { June 26, 2001 } \\ \text { Date(s) of Analysis: } & \text { July 02, 2001 }\end{array}$

\section{Preparation and Analysis Methods:}

SW-846 Method 0010: "Modified Method 5 Sampling Train"

SW-846 Method 3542: "Extraction of Semivolatile Analytes Collected Using Method 0010 (Modified Method 5 Sampling Train)"

SW-846 Method 8270C: "Semivolatile Organic Compounds by Gas Chromatography/Mass Spectrometry (GC/MS)

Capillary Column Technique" 
BECHTEL BWXT IDAHO, LLC (BBWI)

INTEC HLLWE Effluent Gas Emissions Inventory

Idaho National Engineering and Environmental Laboratory (INEEL)

STL Knoxville Project Number: 142503.40

\section{MM-5 Train \\ Table A-6. Run 2, XAD-2 Resin Tube Trip Blank/Reagent Blank \\ Tentatively Identified Compound (TIC) Summary}

\begin{tabular}{|l|c|c|c|c|}
\hline TICs ${ }^{10}$ & $\begin{array}{c}\text { CAS } \\
\text { Registry } \\
\text { Number }\end{array}$ & $\begin{array}{c}\text { Approximate } \\
\text { Retention } \\
\text { Time } \\
(\mathbf{m i n})\end{array}$ & $\begin{array}{c}\text { Sample } \\
\text { Result } \\
(\mu \mathrm{g})\end{array}$ & $\begin{array}{c}\text { TIC } \\
\text { Flag }\end{array}$ \\
\hline Benzaldehyde & & & 5.3 & N,J,M \\
\hline Benzoic acid, methyl ester & $100-52-7$ & 3.60 & 6.5 & N,J,M \\
\hline Benzaldehyde, ethyl- & $93-58-3$ & 4.72 & 4.6 & N,J,M \\
\hline Heptacosane & $53951-50-1$ & 5.40 & 3.1 & N,J,M \\
\hline Heneicosane & $593-49-7$ & 11.48 & 5.8 & N,J,M \\
\hline Tetratracontane & $629-94-7$ & 11.73 & 8.5 & N,J,M \\
\hline Hexatriacontane & $7098-22-8$ & 11.96 & 8.9 & N,J,M \\
\hline Hexatriacontane & $630-06-8$ & 12.19 & 8.2 & N,J,M \\
\hline Heneicosane & $630-06-8$ & 12.41 & 14 & N,J,M \\
\hline Heptacosane & $629-94-7$ & 12.64 & 9.1 & N,J,M \\
\hline
\end{tabular}


BECHTEL BWXT IDAHO, LLC (BBWI)

INTEC HLLWE Effluent Gas Emissions Inventory

Idaho National Engineering and Environmental Laboratory (INEEL)

STL Knoxville Project Number: 142503.40

\section{MM-5 Train Analytical Results Summary \\ Table A-6. Run 2, XAD-2 Resin Tube Trip Blank/Reagent Blank (Continued)}

\section{Footnotes:}

1 Based on the selection rules, the bolded value is the value or default value assigned to the analyte using the following guidelines:

- When the analytical result is greater than the laboratory reporting limit (RL), the result selected by boldface type is the actual analytical result or "hit" determined by the laboratory.

- When the analytical result is greater than the reliable detection level (RDL), but less than the laboratory reporting limit (RL), the result selected by boldface type is the actual analytical result or "hit" determined by the laboratory.

- When the analytical result is less than the RDL, but greater than the method detection limit (MDL), the result selected by boldface type is the RDL.

- When the analytical result is not detected down to the MDL, the result selected by boldface type is the RDL.

- It should be noted that when the RDL is selected using the guidelines above, but the RL is less than the RDL, the RL is included as the "Risk Result".

2 This value is the laboratory sample result. When the analytical result is "ND" or not detected, the laboratory analysis did not detect the analyte down to the MDL.

3 This value is the laboratory Method Detection Limit (MDL) derived according to requirements outlined in 40 CFR Part 136 , Appendix B.

4 The RDL is the Reliable Detection Limit. The RDL is the detection level recommended by EPA's National Research Laboratory in Cincinnati, Ohio, Environmental Monitoring Systems Laboratory (EMSL) in Cincinnati, Ohio, American Chemical Society (ACS) Committee on Environmental Improvement and the Drinking Water Standards Division (DWSD). It is defined as 2.623 times the MDL (2.623 X MDL).

5 The RL is the laboratory Reporting Limit (RL).

6 This flag is the laboratory data flag that corresponds to EPA guidelines. The data flags for these samples are as follows:

- A "U" qualifier indicates that this analyte was analyzed for, but was not detected down to the MDL.

- A " $\mathrm{J}$ " qualifier indicates that this compound was detected, but at a concentration below the laboratory RL. The analytical result is therefore an estimated value.

- A "B" qualifier indicates that this compound was found in the associated laboratory method blank. Under these conditions these values are regarded as estimated values.

- A "D" qualifier indicates that this result was obtained through dilution of the sample. This original analysis yielded a result that exceeded the calibration range.

- An "N" qualifier indicates that this compound is a tentatively identified compound (TIC). Therefore the value is estimated.

- An "E" qualifier indicates that this compound exceeded the calibration range of the instrument.

Last saved by Robin Gifford on 02/04/02 at 4:56 PM

E:IMy DocumentsiHLLWE Sampling\Final Reporthappendix AlTable A-6. 0010-TB.doc

Created on 2/4/2002 4:53 PM 


\section{MM-5 Train Analytical Results Summary Table A-6. Run 2, XAD-2 Resin Tube Trip Blank/Reagent Blank (Continued)}

- An " $\mathrm{A}$ " qualifier indicates that this result is an Aldol-condensation product.

- An " $M$ " qualifier indicates that this result was measured against the nearest internal standard and assumed a response factor of one (1).

7 Bis(2-chloroisopropyl)ether and 2,2'-Oxybis(1-chloropropane) are synonyms.

8 This material is a sampling surrogate and is an isotopically-labeled compound spiked on the XAD-2 Resin Tube prior to the collection of sample on the MM-5 sampling train.

9 This percent recovery is outside of the laboratory target recovery range.

10 The tentatively identified compounds (TICs) were identified by conducting a mass spectral library search using the NBS library of data. It should be noted that TICs that give the same mass spectral match for GC peaks at different retention times are listed separately with the same compound identity. Under these conditions the compounds are likely indistinguishable isomers of the same compound. However, insufficient evidence is available to determine unequivocal identities. 

BECHTEL BWXT IDAHO, LLC (BBWI)

INTEC HLLWE Effluent Gas Emissions Inventory

Idaho National Engineering and Environmental Laboratory (INEEL)

STL Knoxville Project Number: 142503.40

\section{VOST Summary - Run 1 Train Totals Method 0031 Volatile Organic Compounds Analytical Results Summary Table A-7. HLLWE Run ID: 0031-STRT-1}

Field Sample Name: Volatile Organic Sampling Train (VOST) Totals

Sample Description: Tenax ${ }^{\oplus}$ and Anasorb $747^{\oplus}$ Tube Sets (Sets \#1, \#2, \#3, and \#4) and the VOST Condensate for Volatile Organic Compounds (VOC) Analysis

\begin{tabular}{|c|c|c|c|c|c|c|c|c|c|c|c|c|c|c|}
\hline \multirow[b]{2}{*}{ Analyte } & \multirow[b]{2}{*}{$\begin{array}{c}\text { CAS } \\
\text { Registry } \\
\text { Number } \\
\end{array}$} & \multicolumn{2}{|c|}{$\begin{array}{c}\text { VOST } \\
\text { Tube Set \#1 } \\
\text { (Total } \mu \text { g/Set) } \\
\end{array}$} & \multicolumn{2}{|c|}{$\begin{array}{c}\text { VOST } \\
\text { Tube Set \#2 } \\
\text { (Total } \mu \mathrm{g} / \mathrm{Set}) \\
\end{array}$} & \multicolumn{2}{|c|}{$\begin{array}{c}\text { VOST } \\
\text { Tube Set \#3 } \\
\text { (Total } \mu \mathrm{g} / \text { Set) }\end{array}$} & \multicolumn{2}{|c|}{$\begin{array}{c}\text { VOST } \\
\text { Tube Set \#4 } \\
\text { (Total } \mu \mathrm{g} / \mathrm{Set}) \\
\end{array}$} & \multicolumn{2}{|c|}{$\begin{array}{c}\text { VOST } \\
\text { Condensate } \\
\text { (Total } \mu \mathrm{g})\end{array}$} & \multicolumn{2}{|c|}{$\begin{array}{c}\text { VOST } \\
\text { Total }^{1} \\
\text { (Total } \mu \mathrm{g})\end{array}$} & \multirow[b]{2}{*}{$\begin{array}{c}\text { Project } \\
\text { Specific } \\
\text { Flag } \\
\end{array}$} \\
\hline & & $\begin{array}{c}\text { Risk } \\
\text { Result }^{2}\end{array}$ & $\mathrm{Flag}^{3}$ & $\begin{array}{c}\text { Risk } \\
\text { Result }^{2} \\
\end{array}$ & Flag $^{3}$ & $\begin{array}{c}\text { Risk } \\
\text { Result }^{2}\end{array}$ & Flag $^{3}$ & $\begin{array}{c}\text { Risk } \\
\text { Result }^{2}\end{array}$ & Flag $^{3}$ & $\begin{array}{c}\text { Risk } \\
\text { Result }^{4} \\
\end{array}$ & Flags & Total & Flag & \\
\hline \multicolumn{15}{|l|}{ Target Compound List } \\
\hline Acetone & $67-64-1$ & 2.4 & $\mathrm{~B}$ & 1.1 & $\mathrm{~B}$ & 1.0 & $\mathrm{~B}$ & 1.3 & $\mathrm{~B}$ & 1.4 & B & 7.2 & $\mathrm{~B}$ & $A$ \\
\hline Acrylonitrile & $107-13-1$ & $<0.58$ & & $<0.58$ & & $<0.58$ & & $<0.58$ & & $<0.51$ & & $<2.8$ & & $\mathrm{~N}$ \\
\hline Benzene & $71-43-2$ & $<0.17$ & & $<0.11$ & & $<0.046$ & & $<0.043$ & & $<0.027$ & & $<0.40$ & & $P$ \\
\hline Bromobenzene & $108-86-1$ & $<0.020$ & & $<0.020$ & & $<0.020$ & & $<0.020$ & & $<0.036$ & & $<0.12$ & & $\mathrm{~N}$ \\
\hline Bromochloromethane & $74-97-5$ & $<0.030$ & & $<0.030$ & & $<0.030$ & & $<0.030$ & & $<0.028$ & & $<0.15$ & & $N$ \\
\hline Bromodichloromethane & $75-27-4$ & $<0.022$ & & $<0.022$ & & $<0.022$ & & $<0.022$ & & $<0.033$ & & $<0.12$ & & $N$ \\
\hline Bromoform & $75-25-2$ & $<0.038$ & & $<0.038$ & & $<0.038$ & & $<0.038$ & & $<0.025$ & & $<0.18$ & & $N$ \\
\hline Bromomethane & $74-83-9$ & $<0.030$ & $J$ & $<0.038$ & J & $<0.030$ & J & $<0.054$ & $\mathrm{~J}$ & $<0.020$ & & $<0.17$ & $J$ & $\mathrm{P}$ \\
\hline 2-Butanone & $78-93-3$ & $<0.20$ & $\mathrm{~J}$ & $<0.20$ & & $<0.20$ & & $<0.20$ & & $<0.093$ & & $<0.89$ & $J$ & $\mathrm{P}$ \\
\hline n-Butylbenzene & $104-51-8$ & $<0.032$ & & $<0.032$ & & $<0.032$ & & $<0.032$ & & $<0.025$ & & $<0.15$ & & $\mathrm{~N}$ \\
\hline sec-Butylbenzene & $135-98-8$ & $<0.017$ & & $<0.017$ & & $<0.017$ & & $<0.017$ & & $<0.017$ & & $<0.085$ & & $\mathrm{~N}$ \\
\hline tert-Butylbenzene & $98-06-6$ & $<0.032$ & & $<0.032$ & & $<0.032$ & & $<0.032$ & & $<0.014$ & & $<0.14$ & & $\mathrm{~N}$ \\
\hline Carbon disulfide & $75-15-0$ & $<0.37$ & & $<0.24$ & & $<0.16$ & & $<0.19$ & & $<0.011$ & & $<0.97$ & & $\mathrm{P}$ \\
\hline Carbon tetrachloride & $56-23-5$ & $<0.036$ & & $<0.036$ & & $<0.036$ & & $<0.036$ & & $<0.020$ & & $<0.16$ & & $\mathrm{~N}$ \\
\hline Chlorobenzene & $108-90-7$ & $<0.017$ & & $<0.017$ & & $<0.017$ & & $<0.017$ & & $<0.027$ & & $<0.095$ & & $\mathrm{~N}$ \\
\hline Chlorodibromomethane & $124-48-1$ & $<0.030$ & & $<0.030$ & & $<0.030$ & & $<0.030$ & & $<0.029$ & & $<0.15$ & & $\mathrm{~N}$ \\
\hline Chloroethane & $75-00-3$ & $<0.036$ & & $<0.036$ & J & $<0.036$ & $\mathrm{~J}$ & $<0.043$ & $J$ & $<0.014$ & & $<0.16$ & $J$ & $\mathrm{P}$ \\
\hline Chloroform & $67-66-3$ & $<0.036$ & & $<0.036$ & & $<0.036$ & $\mathrm{~J}$ & $<0.10$ & & $<0.031$ & & $<0.24$ & $J$ & $\mathrm{P}$ \\
\hline Chloromethane & $74-87-3$ & 0.15 & $J$ & 0.28 & J & 0.14 & $\mathrm{~J}$ & 0.61 & & $<0.011$ & & 1.2 & J & $\mathrm{P}$ \\
\hline 2-Chlorotoluene & $95-49-8$ & $<0.0094$ & & $<0.0094$ & & $<0.0094$ & & $<0.0094$ & & $<0.021$ & & $<0.059$ & & $\mathrm{~N}$ \\
\hline 4-Chlorotoluene & $106-43-4$ & $<0.0094$ & & $<0.0094$ & & $<0.0094$ & & $<0.0094$ & & $<0.021$ & & $<0.059$ & & $\mathrm{~N}$ \\
\hline 1,2-Dibromo-3-chloropropane & $96-12-8$ & $<0.058$ & & $<0.058$ & & $<0.058$ & & $<0.058$ & & $<0.042$ & & $<0.27$ & & $\mathrm{~N}$ \\
\hline 1,2-Dibromoethane & $106-93-4$ & $<0.040$ & & $<0.040$ & & $<0.040$ & & $<0.040$ & & $<0.042$ & & $<0.20$ & & $\mathrm{~N}$ \\
\hline Dibromomethane & $74-95-3$ & $<0.034$ & & $<0.034$ & & $<0.034$ & & $<0.034$ & & $<0.030$ & & $<0.17$ & & $N$ \\
\hline 1,2-Dichlorobenzene & $95-50-1$ & $<0.040$ & & $<0.040$ & & $<0.040$ & & $<0.040$ & & $<0.019$ & & $<0.18$ & & $\mathrm{~N}$ \\
\hline 1,3-Dichlorobenzene & $541-73-1$ & $<0.020$ & & $<0.020$ & & $<0.020$ & & $<0.020$ & & $<0.022$ & & $<0.10$ & & $\mathrm{~N}$ \\
\hline 1,4-Dichlorobenzene & $106-46-7$ & $<0.028$ & & $<0.028$ & & $<0.028$ & & $<0.028$ & & $<0.023$ & & $<0.14$ & & $N$ \\
\hline
\end{tabular}

Last saved by Patti Carswell on 02/05/02 at 7:24 AM

E:My Documents HHLLWE SamplingtFinal Reporthppendix AlTable A-7. 0031-STRT-1.doc

Created on 2/5/2002 7:13 AM 
BECHTEL BWXT IDAHO, LLC (BBWI)

INTEC HLLWE Effluent Gas Emissions Inventory

Idaho National Engineering and Environmental Laboratory (INEEL)

STL Knoxville Project Number: 142503.40

\section{VOST Summary - Run 1 Train Totals (Continued) Method 0031 Volatile Organic Compounds Analytical Results Summary \\ Table A-7. HLLWE Run ID: 0031-STRT-1}

\begin{tabular}{|c|c|c|c|c|c|c|c|c|c|c|c|c|c|c|}
\hline \multirow[b]{2}{*}{ Analyte } & \multirow{2}{*}{$\begin{array}{c}\text { CAS } \\
\text { Registry } \\
\text { Number } \\
\end{array}$} & \multicolumn{2}{|c|}{$\begin{array}{c}\text { VOST } \\
\text { Tube Set \#1 } \\
\text { (Total } \mu \mathrm{g} / \text { Set) } \\
\end{array}$} & \multicolumn{2}{|c|}{$\begin{array}{c}\text { VOST } \\
\text { Tube Set \#2 } \\
\text { (Total } \mu \text { g/Set) }\end{array}$} & \multicolumn{2}{|c|}{$\begin{array}{c}\text { VOST } \\
\text { Tube Set } \# 3 \\
\text { (Total } \mu g / \text { Set) }\end{array}$} & \multicolumn{2}{|c|}{$\begin{array}{c}\text { VOST } \\
\text { Tube Set \#4 } \\
\text { (Total } \mu g / \text { Set) } \\
\end{array}$} & \multicolumn{2}{|c|}{\begin{tabular}{|c|c|}
$\begin{array}{c}\text { VOST } \\
\text { Condensate } \\
(\text { Total } \mu \mathrm{g})\end{array}$ \\
\end{tabular}} & \multicolumn{2}{|c|}{$\begin{array}{c}\text { VOST } \\
\text { Total' } \\
\text { (Total } \mu \mathrm{g})\end{array}$} & \multirow{2}{*}{$\begin{array}{c}\text { Project } \\
\text { Specific } \\
\text { Flag }^{6} \\
\end{array}$} \\
\hline & & Result ${ }^{2}$ & Flag $^{3}$ & Result $^{2}$ & Flag $^{3}$ & Result $^{2}$ & $\mathrm{Flag}^{3}$ & Result & Flag $^{3}$ & Result $^{4}$ & Flags & Total & Flag & \\
\hline Dichlorodifluoromethane & $75-71-8$ & $<0.064$ & & $<0.061$ & & $<0.057$ & & $<0.062$ & & $<0.011$ & & $<0.26$ & & $\mathrm{P}$ \\
\hline 1,1-Dichloroethane & $75-34-3$ & $<0.034$ & & $<0.034$ & & $<0.034$ & & $<0.034$ & & $<0.017$ & & $<0.15$ & & $\mathrm{~N}$ \\
\hline 1,2-Dichloroethane & $107-06-2$ & $<0.034$ & J & $<0.034$ & & $<0.034$ & & $<0.034$ & & $<0.020$ & & $<0.16$ & $\mathrm{~J}$ & $\mathrm{P}$ \\
\hline 1,1-Dichloroethene & $75-35-4$ & $<0.036$ & & $<0.036$ & $\mathrm{~J}$ & $<0.036$ & $\mathrm{~J}$ & $<0.036$ & $\mathrm{~J}$ & $<0.016$ & & $<0.16$ & $\mathrm{~J}$ & $\mathrm{P}$ \\
\hline cis-1,2-Dichloroethene & $156-59-2$ & $<0.032$ & & $<0.032$ & & $<0.032$ & & $<0.032$ & & $<0.021$ & & $<0.15$ & & N \\
\hline trans-1,2-Dichloroethene & $156-60-5$ & $<0.038$ & & $<0.038$ & & $<0.038$ & & $<0.038$ & & $<0.014$ & & $<0.17$ & & $N$ \\
\hline 1,2-Dichloropropane & $78-87-5$ & $<0.026$ & & $<0.026$ & & $<0.026$ & & $<0.026$ & & $<0.023$ & & $<0.13$ & & $\mathrm{~N}$ \\
\hline 1,3-Dichloropropane & $142-28-9$ & $<0.038$ & & $<0.038$ & & $<0.038$ & & $<0.038$ & & $<0.022$ & & $<0.17$ & & $\mathrm{~N}$ \\
\hline 2.2-Dichloropropanc & $594-20-7$ & $<0.036$ & & $<0.036$ & & $<0.036$ & & $<0.036$ & & $<0.011$ & & $<0.16$ & & $\mathrm{~N}$ \\
\hline 1,1-Dichloropropene & $563-58-6$ & $<0.040$ & & $<0.040$ & & $<0.040$ & & $<0.040$ & & $<0.016$ & & $<0.18$ & & $\mathrm{~N}$ \\
\hline cis-1,3-Dichloropropene & $10061-01-5$ & $<0.024$ & & $<0.024$ & & $<0.024$ & & $<0.024$ & & $<0.030$ & & $<0.13$ & & $N$ \\
\hline trans-1,3-Dichloropropene & $10061-02-6$ & $<0.030$ & & $<0.030$ & & $<0.030$ & & $<0.030$ & & $<0.028$ & & $<0.15$ & & $\mathrm{~N}$ \\
\hline Ethylbenzene & $100-41-4$ & $<0.018$ & & $<0.018$ & & $<0.018$ & & $<0.018$ & & $<0.021$ & & $<0.093$ & & $N$ \\
\hline Hexachlorobutadiene & $87-68-3$ & $<0.050$ & & $<0.050$ & & $<0.050$ & & $<0.050$ & & $<0.025$ & & $<0.22$ & & $\mathrm{~N}$ \\
\hline 2-Hexanone & $591-78-6$ & $<0.13$ & & $<0.13$ & & $<0.13$ & & $<0.13$ & & $<0.036$ & & $<0.56$ & & $\mathrm{~N}$ \\
\hline Isopropylbenzene & $98-82-8$ & $<0.013$ & & $<0.013$ & & $<0.013$ & & $<0.013$ & & $<0.018$ & & $<0.070$ & & $N$ \\
\hline p-Isopropyltoluene & $99-87-6$ & $<0.024$ & & $<0.024$ & & $<0.024$ & & $<0.024$ & & $<0.018$ & & $<0.11$ & & $\mathrm{~N}$ \\
\hline Methylene chloride & $75-09-2$ & 13 & $E$ & 3.0 & E,B & 1.1 & B & 0.96 & $\mathrm{~B}$ & 0.12 & B & 18 & $E, B$ & A \\
\hline 4-Methyl-2-pentanone & $108-10-1$ & $<0.14$ & & $<0.14$ & & $<0.14$ & & $<0.14$ & & $<0.030$ & & $<0.59$ & & $\mathrm{~N}$ \\
\hline Naphthalene & $91-20-3$ & $<0.050$ & & $<0.050$ & & $<0.050$ & & $<0.050$ & & $<0.011$ & & $<0.21$ & & $N$ \\
\hline n-Propylbenzene & $103-65-1$ & $<0.011$ & & $<0.011$ & & $<0.011$ & & $<0.012$ & & $<0.022$ & & $<0.067$ & & $\mathrm{~N}$ \\
\hline Styrene & $100-42-5$ & $<0.014$ & & $<0.014$ & & $<0.014$ & & $<0.014$ & & $<0.022$ & & $<0.078$ & & $\mathrm{~N}$ \\
\hline $1,1,1,2$-Tetrachloroethane & $630-20-6$ & $<0.019$ & & $<0.019$ & & $<0.019$ & & $<0.019$ & & $<0.023$ & & $<0.099$ & & $\mathrm{~N}$ \\
\hline 1,1,2,2-Tetrachloroethane & $79-34-5$ & $<0.050$ & & $<0.050$ & & $<0.050$ & & $<0.050$ & & $<0.025$ & & $<0.22$ & & $\mathrm{~N}$ \\
\hline Tetrachloroethene & $127-18-4$ & $<0.032$ & & $<0.032$ & & $<0.032$ & & $<0.032$ & & $<0.021$ & & $<0.15$ & & $N$ \\
\hline Toluene & $108-88-3$ & $<0.032$ & $\mathrm{~J}$ & 0.041 & J & $<0.023$ & J & 0.064 & $\mathrm{~J}$ & $<0.028$ & & $<0.19$ & $\mathrm{~J}$ & $\mathrm{P}$ \\
\hline 1.2,3-Trichlorobenzene & $87-61-6$ & $<0.050$ & & $<0.050$ & & $<0.050$ & & $<0.050$ & & $<0.011$ & & $<0.21$ & & $N$ \\
\hline 1,2,4-Trichlorobenzene & $120-82-1$ & $<0.050$ & & $<0.050$ & & $<0.050$ & & $<0.050$ & & $<0.025$ & & $<0.22$ & & $\mathrm{~N}$ \\
\hline 1,1,1-Trichloroethane & $71-55-6$ & $<0.044$ & & $<0.044$ & & $<0.044$ & & $<0.044$ & & $<0.018$ & & $<0.19$ & & $\mathrm{~N}$ \\
\hline 1.1,2-Trichloroethane & $79-00-5$ & $<0.036$ & & $<0.036$ & & $<0.036$ & & $<0.036$ & & $<0.022$ & & $<0.17$ & & $\mathrm{~N}$ \\
\hline
\end{tabular}

Created by Patti Carswell Created on 2/5/2002 7:13 AM Last saved by Patti Carswell on 02/05/02 at 7:24 AM Last printed 2/5/2002 7:24 AM

Filename and Path: E:My Documents\HLLWE SamplinglFinal Report LAppendix AiTable A-7. 0031-STRT-1.doc 
BECHTEL BWXT IDAHO, LLC (BBWI)

INTEC HLLWE Effluent Gas Emissions Inventory

Idaho National Engineering and Environmental Laboratory (INEEL)

STL Knoxville Project Number: 142503.40

\section{VOST Summary - Run 1 Train Totals (Continued) Method 0031 Volatile Organic Compounds Analytical Results Summary \\ Table A-7. HLLWE Run ID: 0031-STRT-1}

\begin{tabular}{|c|c|c|c|c|c|c|c|c|c|}
\hline \multirow[b]{2}{*}{ Analyte } & \multirow[b]{2}{*}{$\begin{array}{c}\text { CAS } \\
\text { Registry } \\
\text { Number }\end{array}$} & $\begin{array}{c}\text { VOST } \\
\text { Tube Set } \# 1 \\
\text { (Total } \mu \mathrm{g} / \mathrm{Set} \text { ) } \\
\end{array}$ & $\begin{array}{c}\text { VOST } \\
\text { Tube Set \#2 } \\
\text { (Total } \mu g / \text { Set) }\end{array}$ & $\begin{array}{c}\text { VOST } \\
\text { Tube Set \#3 } \\
\text { (Total } \mu g / \text { Set) }\end{array}$ & $\begin{array}{c}\text { VOST } \\
\text { Tube Set \#4 } \\
\text { (Total } \mu \mathrm{g} / \mathrm{Set}) \\
\end{array}$ & $\begin{array}{c}\begin{array}{c}\text { VOST } \\
\text { Condensate } \\
\text { (Total } \mu \mathrm{g})\end{array} \\
\end{array}$ & \multicolumn{2}{|c|}{$\begin{array}{c}\text { VOST } \\
\text { Total }^{1} \\
(\text { Total } \mu \mathrm{g}) \\
\end{array}$} & \multirow[b]{2}{*}{$\begin{array}{c}\text { Project } \\
\text { Specific } \\
\text { Flag }^{6} \\
\end{array}$} \\
\hline & & $\begin{array}{cc}\text { Risk } & \\
\text { Result }^{2} & \text { Flag }^{3} \\
\end{array}$ & 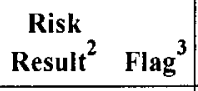 & $\begin{array}{cc}\text { Risk } & \\
\text { Result }^{2} & \text { Flag }^{3} \\
\end{array}$ & $\begin{array}{cc}\begin{array}{c}\text { Risk } \\
\text { Result }^{2}\end{array} \text { Flag }^{3} \\
\end{array}$ & $\begin{array}{c}\text { Risk } \\
\text { Result }^{4} \quad \text { Flag }^{5} \\
\end{array}$ & Total & Flag & \\
\hline Trichloroethene & $79-01-6$ & $<0.034$ & $<0.034$ & $<0.034$ & $<0.034$ & $<0.020$ & $<0.16$ & & $\mathrm{~N}$ \\
\hline Trichlorofluoromethane & $75-69-4$ & $<0.036$ & $<0.036$ & $<0.036$ & $<0.036$ & $<0.011$ & $<0.16$ & $J$ & $\mathrm{P}$ \\
\hline 1,2,3-Trichloropropane & $96-18-4$ & $<0.050$ & $<0.050$ & $<0.050$ & $<0.050$ & $<0.036$ & $<0.24$ & & $\mathrm{~N}$ \\
\hline 1,2,4-Trimethylbenzene & $95-63-6$ & $<0.015$ & $<0.015$ & $<0.015$ & $<0.015$ & $<0.042$ & $<0.10$ & & $\mathrm{~N}$ \\
\hline 1,3,5-Trimethylbenzene & $108-67-8$ & $<0.010$ & $<0.010$ & $<0.010$ & $<0.010$ & $<0.019$ & $<0.059$ & & $\mathrm{~N}$ \\
\hline Vinyl chloride & $75-01-4$ & $<0.013$ & $<0.016$ & $<0.013$ & $<0.022$ & $<0.068$ & $<0.13$ & J & $\mathrm{P}$ \\
\hline$m$-Xylene \& p-Xylene & $136777-61-2$ & $<0.10$ & $<0.10$ & $<0.10$ & $<0.10$ & $<0.042$ & $<0.44$ & & $\mathrm{~N}$ \\
\hline o-Xylene & $95-47-6$ & $<0.013$ & $<0.013$ & $<0.013$ & $<0.013$ & $<0.025$ & $<0.077$ & & $\mathrm{~N}$ \\
\hline \multicolumn{10}{|l|}{$T I C s^{7}$} \\
\hline Hexane, 2-methyl- & $591-76-4$ & $-\cdots$ & -- & --- & 0.17 & $-\cdots$ & 0.17 & $\mathrm{~N}, \mathrm{~J}, \mathrm{M}$ & P \\
\hline Pentane, 2,3-dimethyl- & $565-59-3$ & $\cdots$ & --- & --- & 0.18 & $-\cdots$ & 0.18 & $\mathrm{~N}, \mathrm{~J}, \mathrm{M}$ & P \\
\hline Butane, 1-chloro- & $109-69-3$ & -- & --- & -- & 0.057 & $\cdots$ & 0.057 & $\mathrm{~N}, \mathrm{~J}, \mathrm{M}$ & P \\
\hline Hexane, 3-methyl- & $589-34-4$ & -- & -- & --- & 0.38 & --- & 0.38 & $\mathrm{~N}, \mathrm{~J}, \mathrm{M}$ & P \\
\hline Cyclohexene & $110-83-8$ & $\cdots$ & 0.044 & 0.027 & 0.033 & $\cdots$ & 0.10 & $\mathrm{~N}, \mathrm{~J}, \mathrm{M}$ & P \\
\hline 1-Heptene & $592-76-7$ & -- & --- & --- & 0.054 & -- & 0.054 & $\mathrm{~N}, \mathrm{~J}, \mathrm{M}$ & $\mathrm{P}$ \\
\hline Cyclohexane, methyl- & $108-87-2$ & -- & $\cdots$ & $\ldots$ & 0.11 & $\cdots$ & 0.11 & $\mathrm{~N}, \mathrm{~J}, \mathrm{M}$ & P \\
\hline Hexane, 2,4-dimethyl- & $589-43-5$ & $-\cdots$ & - & -- & 0.11 & $\cdots$ & 0.11 & $\mathrm{~N}, J, M$ & P \\
\hline Cyclopentane, ethyl- & $1640-89-7$ & --- & $\cdots$ & $-\cdots$ & 0.028 & $--\cdot$ & 0.028 & $\mathrm{~N}, \mathrm{~J}, \mathrm{M}$ & P \\
\hline Octane & $111-65-9$ & 0.027 & $\cdots$ & $\cdots$ & $\cdots$ & --- & 0.027 & $\mathrm{~N}, \mathrm{~J}, \mathrm{M}$ & P \\
\hline Decane & $124-18-5$ & -- & 0.055 & 0.060 & $\cdots$ & $\cdots$ & 0.12 & $\mathrm{~N}, \mathrm{~J}, \mathrm{M}$ & $P$ \\
\hline Undecane & $1120-21-4$ & 0.44 & 0.23 & 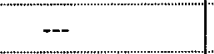 & 0.37 & $-\cdots$ & 1.0 & $\mathrm{~N}, \mathrm{~J}, \mathrm{M}$ & $P$ \\
\hline Undecane, 5-methyl- & $1632-70-8$ & 0.13 & 0.091 & 0.14 & 0.28 & --- & 0.64 & $\mathrm{~N}, \mathrm{~J}, \mathrm{M}$ & P \\
\hline Decane, 2,9-dimethyl- & $1002-17-1$ & 0.064 & $-\cdots$ & --- & $-\cdots$ & --- & 0.064 & $\mathrm{~N}, \mathrm{~J}, \mathrm{M}$ & $\mathrm{P}$ \\
\hline Dodecane & $112-40-3$ & 5.3 & 4.6 & 7.2 & 14 & $-\cdots$ & 31 & $\mathrm{~N}, \mathrm{~J}, \mathrm{M}$ & $\mathrm{P}$ \\
\hline Undecane, 2,6-dimethyl- & $17301-23-4$ & $\cdots$ & - & $\cdots$ & 0.11 & $\cdots$ & 0.11 & $\mathrm{~N}, \mathrm{~J}, \mathrm{M}$ & $\mathrm{P}$ \\
\hline Cyclohexane, hexyl- & $4292-75-5$ & $\cdots$ & $\cdots$ & 0.059 & $\cdots$ & $\cdots$ & 0.059 & $\mathrm{~N}, \mathrm{~J}, \mathrm{M}$ & $\mathrm{P}$ \\
\hline Tridecane & $629-50-5$ & 0.39 & 0.32 & $0.5 !$ & 1.6 & $-\cdots$ & 2.8 & $\mathrm{~N}, \mathrm{~J}, \mathrm{M}$ & $\mathrm{P}$ \\
\hline Tetradecane & $629-59-4$ & 0.15 & 0.19 & 0.22 & 0.36 & $\cdots$ & 0.92 & $\mathrm{~N}, \mathrm{~J}, \mathrm{M}$ & $\mathrm{P}$ \\
\hline
\end{tabular}




\section{BECHTEL BWXT IDAHO, LLC (BBWI) \\ INTEC HLLWE Effluent Gas Emissions Inventory Idaho National Engineering and Environmental Laboratory (INEEL) STL Knoxville Project Number: 142503.40}

\section{Footnotes:}

1 The Method 0031 VOST Run Total (in Total $\mu \mathrm{g}$ ) is the sum of results for the four (4) VOST tube sets and the condensate sample collected during the same sampling run using the following guidelines:

- When the train component analytical result is greater than the laboratory reporting limit (RL), the result included in the train total is the actual analytical result or "hit" determined by the laboratory.

- When the train component analytical result is greater than the reliable detection level (RDL), but less than the laboratory reporting limit $(\mathrm{RL})$, the result included in the train total is the actual analytical result or "hit" determined by the laboratory and the corresponding " $\mathrm{J}$ " flag is carried through the calculation to the train total.

- When the train analytical component result is less than the RDL, but greater than the method detection limit (MDL), the result included in the train total is the RDL and the corresponding "J" flag is carried through the calculation to the train total.

- When the train component analytical result is not detected down to the MDL, the result included in the train total is the RDL and the corresponding "U" flag is carried through the calculation to the train total

- It should be noted that when the RDL is selected as the default value using the guidelines above, but the RDL is greater than the RL, the RL is included in the train total.

The data flags attached to the VOST Total are the cumulative set of flags contributed by each train tube set included as part of the VOST total. A flag attached to a VOST component is carried through to the "VOST Total" column when the associated component analytical result is a significant number in comparison to the VOST Total. That is, if the VOST Total is affected by a VOST component analytical result, the associated flag is carried through to the VOST Total, but if the VOST Total is not affected by a VOST component, the associated flag is not carried through to the VOST Total. The combinations of train fractions are conducted following the standard practice of using significant figures found in ASTM E29-93a(1999), "Standard Practice for Using Significant Digits in Test Data to Determine Conformance with Specifications" and Scvern Trent Laboratories standard operating procedure number QA-004, "Rounding and Significant Figures".

2 The Method 0031 VOST Tube Set (Total $\mu \mathrm{g} / \mathrm{Set}$ ) result consists of the sum of the analytical results for the two (2) Tenax ${ }^{\circledR}$ resin tube contents (analyzed together) and the analytical result for the Anasorb $747^{\circledR}$ Tube contents. The calculation is as follows:

(Total $\mu \mathrm{g}$ on the Tenax ${ }^{\circledR}$ Tubes $\# 1$ and \#2) $+\left(\right.$ Total $\mu \mathrm{g}$ on the Anasorb $747^{\circledR}$ Tube) $=$ Total $\mu \mathrm{g}$ on the Method 0031 VOST tube set. Therefore: $(\mu \mathrm{g})+(\mu \mathrm{g})=$ Total $\mu \mathrm{g} / \mathrm{set}$

When listed, the less than $(<)$ sign indicates that at least one sample fraction result is either a "non-detect" value down to the MDL of the measurement that carries, or an estimated "hit" value that is below the RDL. In either case, the final value included in the tube set total is the default RDL value and the actual value is known to be less than $(<)$ the displayed result.

3 The data flags in this column for the VOST Tube Set are the cumulative set of flags contributed by each individual train component included as part of the VOST total. A flag attached to a VOST component is carried through to the "VOST Tube Set" column when the associated component analytical result is a significant number in comparison to the VOST tube set total. That is, if the VOST Tube Set Total is affected by a VOST component analytical result, the associated flag is carried through to the VOST Tube Set Total, but if the VOST Tube Set Total is not affected by a VOST component analytical result, the associated flag is not carried through to the VOST Tube Set Total.

4 The VOST Condensate result was obtained by multiplying the sample's corresponding RDL or "hit" by the VOST condensate volume.

5. This flag is the laboratory data flag that corresponds to EPA guidelines. The data flags for these samples are as follows:

- A "U" qualifier indicates that this analyte was analyzed for, but was not detected down to the MDL.

- An "E" flag indicates that the result exceeded the upper calibration range. The analytical result is therefore an estimated value. 
- A "J" flag indicates that this compound was detected, but at a concentration below the laboratory RL. The analytical result is therefore an estimated value.

- A "B" flag indicates that this compound was found in the associated laboratory method blank. Under these conditions this value is regarded as an estimated value.

- A "Y" flag indicates that this compound is an indistinguishable isomer as a tentatively identified compound (TIC).

- An "N" flag indicates that there is presumptive evidence that this compound is present in the sample based on spectral evidence.

- An "M" flag indicates that this result was measured against the nearest internal standard and assumed a response factor of one (1).

- A "D" flag indicates that this result was obtained by a dilution of the sample. The original analysis yielded an analytical result that exceeded the calibration range.

6. Entries in this column are project-specific train total flags that are applied to the run total values and are not standard EPA data flags. These project-specific flags are utilized for the INEEL NWCF HLLWE Effluent Gas Emissions Inventory project and are defined as follows:

- An "N" flag in this column indicates that the compound was not measured (detected) in any of the sampling train components, or fractions.

- A "P" flag in this column indicates that the compound was measured (detected) in one or more of the train components, or fractions, but not in all of the sampling train fractions.

- An "A" flag in this column indicates that the compound was measured (detected) in all of the sampling train components, or fractions.

7. The tentatively identified compounds (TICs) were identified by conducting a mass spectral library search using the NBS library of data. 



\section{BECHTEL BWXT IDAHO, LLC (BBWI) \\ INTEC HLLWE Effluent Gas Emissions Inventory \\ Idaho National Engineering and Environmental Laboratory (INEEL) \\ STL Knoxville Project Number: 142503.40}

\section{VOST Summary - Run 3 Train Totals Method 0031 Volatile Organic Compounds Analytical Results Summary Table A-8. HLLWE Run ID: 0031-END-1}

Field Sample Name: Volatile Organic Sampling Train (VOST) Totals

Sample Description: Tenax ${ }^{\oplus}$ and Anasorb $747^{\circ}$ Tube Sets (Sets \#1, \#2,\#3, and \#4) and the VOST Condensate for Volatile Organic Compounds (VOC) Analysis

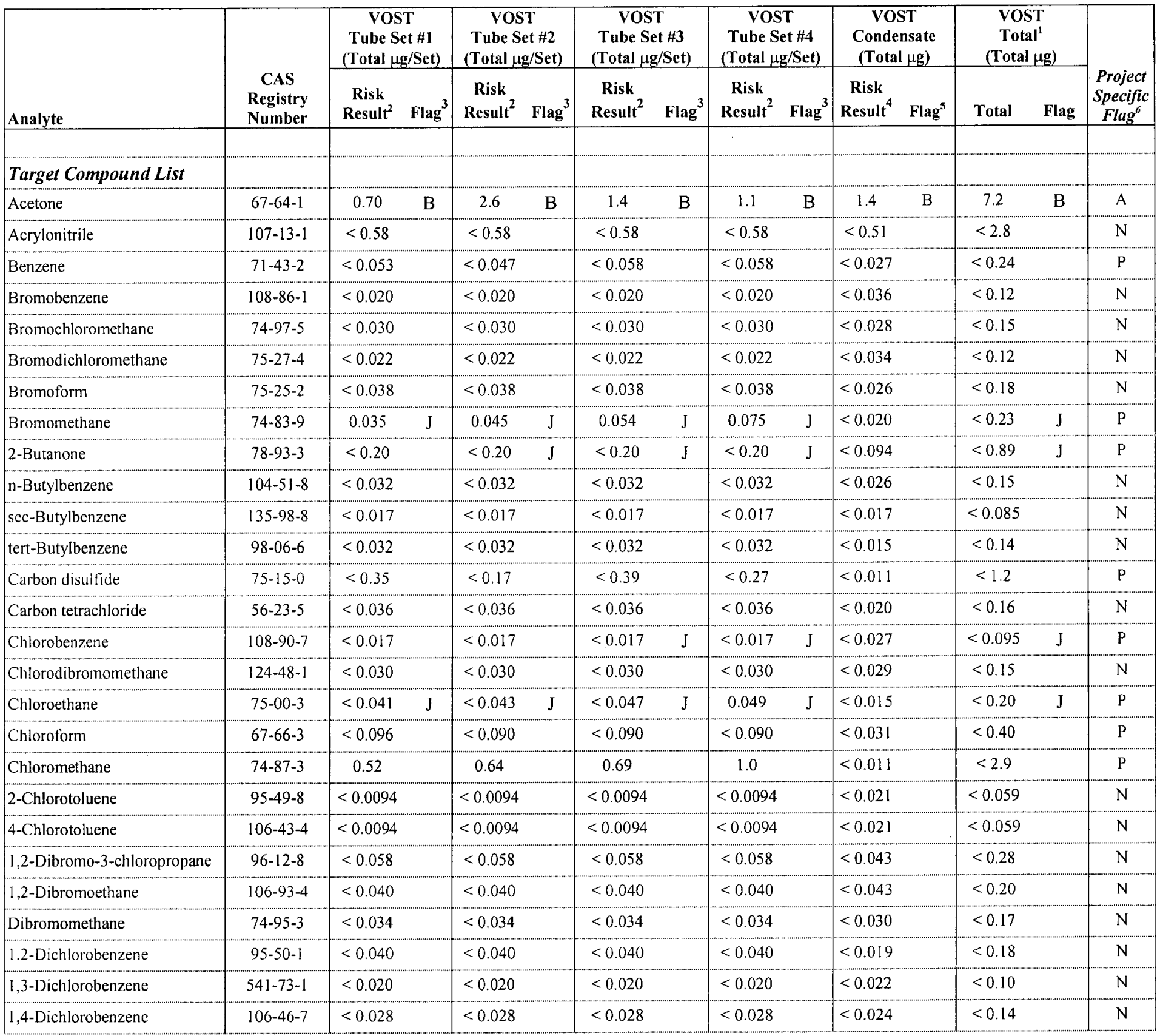


BECHTEL BWXT IDAHO, LLC (BBWI)

INTEC HLLWE Effluent Gas Emissions Inventory

Idaho National Engineering and Environmental Laboratory (INEEL)

STL Knoxville Project Number: 142503.40

\section{VOST Summary - Run 3 Train Totals (Continued) Method 0031 Volatile Organic Compounds Analytical Results Summary \\ Table A-8. HLLWE Run ID: 0031-END-1}

\begin{tabular}{|c|c|c|c|c|c|c|c|c|c|}
\hline \multirow[b]{2}{*}{ Analyte } & \multirow{2}{*}{$\begin{array}{c}\text { CAS } \\
\text { Registry } \\
\text { Number }\end{array}$} & $\begin{array}{c}\text { VOST } \\
\text { Tube Set } \# 1 \\
\text { (Total } \mu \mathrm{g} / \mathrm{Set}) \\
\end{array}$ & $\begin{array}{c}\text { VOST } \\
\text { Tube Set } \# 2 \\
\text { (Total } \mu g / \text { Set) }\end{array}$ & $\begin{array}{c}\text { VOST } \\
\text { Tube Set \#3 } \\
(\text { Total } \mu g / \text { Set) }\end{array}$ & $\begin{array}{c}\text { VOST } \\
\text { Tube Set \#4 } \\
\text { (Total } \mu \mathrm{g} / \mathrm{Set} \text { ) }\end{array}$ & \begin{tabular}{|c|c|}
$\begin{array}{c}\text { VOST } \\
\text { Condensate } \\
\text { (Total } \mu \mathrm{g})\end{array}$ \\
\end{tabular} & \multicolumn{2}{|c|}{$\begin{array}{c}\text { VOST } \\
\text { Total }^{1} \\
\text { (Total } \mu \mathrm{g}) \\
\end{array}$} & \multirow{2}{*}{$\begin{array}{r}\text { Projec } \\
\text { Specifi } \\
\text { Flag }^{6}\end{array}$} \\
\hline & & Result $^{2}$ Flag $^{3}$ & Result $^{2}$ Flag $^{3}$ & Result $^{2} \quad$ Flag $^{3}$ & Result $^{2}$ Flag $^{3}$ & Result $^{4}$ Flag $^{5}$ & Total & Flag & \\
\hline Dichlorodifluoromethane & $75-71-8$ & $<0.035$ & $<0.037$ & $<0.034$ & $<0.046$ & $<0.011$ & $<0.16$ & $\mathrm{~J}$ & $\mathrm{P}$ \\
\hline 1,1-Dichloroethane & $75-34-3$ & $<0.034$ & $<0.034$ & $<0.034$ & $<0.034$ & $<0.017$ & $<0.15$ & & $\mathrm{~N}$ \\
\hline 1,2-Dichloroethane & $107-06-2$ & $<0.034$ & $<0.034$ & $<0.034$ & $<0.034$ & $<0.020$ & $<0.16$ & $\mathrm{~J}$ & $\mathrm{P}$ \\
\hline 1,1-Dichloroethene & $75-35-4$ & $<0.036$ & $<0.041$ & $<0.039$ & $<0.044$ & $<0.016$ & $<0.18$ & $J$ & $\mathrm{P}$ \\
\hline cis-1,2-Dichloroethene & $156-59-2$ & $<0.032$ & $<0.032$ & $<0.032$ & $<0.032$ & $<0.021$ & $<0.15$ & & $\mathrm{~N}$ \\
\hline trans-1,2-Dichloroethene & $156-60-5$ & $<0.038$ & $<0.038$ & $<0.038$ & $<0.038$ & $<0.013$ & $<0.16$ & & $N$ \\
\hline 1,2-Dichloropropane & $78-87-5$ & $<0.026$ & $<0.026$ & $<0.026$ & $<0.026$ & $<0.024$ & $<0.13$ & $\mathrm{~J}$ & $\mathrm{P}$ \\
\hline 1,3-Dichloropropane & $142-28-9$ & $<0.038$ & $<0.038$ & $<0.038$ & $<0.038$ & $<0.022$ & $<0.17$ & & $\mathrm{~N}$ \\
\hline 2,2-Dichloropropane & $594-20-7$ & $<0.036$ & $<0.036$ & $<0.036$ & $<0.036$ & $<0.011$ & $<0.16$ & & $\mathrm{~N}$ \\
\hline 1,1-Dichloropropene & $563-58-6$ & $<0.040$ & $<0.040$ & $<0.040$ & $<0.040$ & $<0.016$ & $<0.18$ & & $\mathrm{~N}$ \\
\hline cis-1,3-Dichloropropene & $10061-01-5$ & $<0.024$ & $<0.024$ & $<0.024$ & $<0.024$ & $<0.030$ & $<0.13$ & & $\mathrm{~N}$ \\
\hline trans-1,3-Dichloropropene & $10061-02-6$ & $<0.030$ & $<0.030$ & $<0.030$ & $<0.030$ & $<0.028$ & $<0.15$ & & $\mathrm{~N}$ \\
\hline Ethylbenzene & $100-41-4$ & $<0.018$ & $<0.018$ & $<0.018$ & $<0.018$ & $<0.021$ & $<0.093$ & & $\mathrm{~N}$ \\
\hline Hexachlorobutadiene & $87-68-3$ & $<0.050$ & $<0.050$ & $<0.050$ & $<0.050$ & $<0.025$ & $<0.22$ & & $\mathrm{~N}$ \\
\hline 2-Hexanone & $591-78-6$ & $<0.13$ & $<0.13$ & $<0.13$ & $<0.13$ & $<0.036$ & $<0.56$ & & $\mathrm{~N}$ \\
\hline Isopropylbenzene & $98-82-8$ & $<0.013$ & $<0.013$ & $<0.013$ & $<0.013$ & $<0.018$ & $<0.070$ & & $\mathrm{~N}$ \\
\hline p-Isopropyltoluene & $99-87-6$ & $<0.024$ & $<0.024$ & $<0.024$ & $<0.024$ & $<0.018$ & $<0.11$ & & $\mathrm{~N}$ \\
\hline Methylene chloride & $75-09-2$ & 0.59 & 0.26 & 0.42 & 0.28 & 0.13 & 1.7 & B & A \\
\hline 4-Methyl-2-pentanone & $108-10-1$ & $<0.14$ & $<0.14$ & $<0.14$ & $<0.14$ & $<0.030$ & $<0.59$ & & $\mathrm{~N}$ \\
\hline Naphthalene & $91-20-3$ & $<0.050$ & $<0.050$ & $<0.050$ & $<0.050$ & $<0.011$ & $<0.21$ & & $\mathrm{~N}$ \\
\hline n-Propylbenzene & $103-65-1$ & $<0.011$ & $<0.011$ & $<0.011$ & $<0.011$ & $<0.022$ & $<0.066$ & & $N$ \\
\hline Styrene & $100-42-5$ & $<0.014$ & $<0.014$ & $<0.014$ & $<0.014$ & $<0.022$ & $<0.078$ & & N \\
\hline 1,1,1,2-Tetrachloroethane & $630-20-6$ & $<0.019$ & $<0.019$ & $<0.019$ & $<0.019$ & $<0.024$ & $<0.10$ & & $\mathrm{~N}$ \\
\hline 1,1,2,2-Tetrachloroethane & $79-34-5$ & $<0.050$ & $<0.050$ & $<0.050$ & $<0.050$ & $<0.025$ & $<0.22$ & & $\mathrm{~N}$ \\
\hline Tetrachloroethene & $127-18-4$ & $<0.032$ & $<0.032$ & $<0.032$ & $<0.032$ & $<0.021$ & $<0.15$ & & $\mathrm{~N}$ \\
\hline Toluene & $108-88-3$ & 0.14 & 0.051 & $<0.032$ & $<0.031$ & $<0.028$ & $<0.28$ & $J$ & $\mathrm{P}$ \\
\hline 1,2,3-Trichlorobenzene & $87-61-6$ & $<0.050$ & $<0.050$ & $<0.050$ & $<0.050$ & $<0.011$ & $<0.21$ & & $\mathrm{~N}$ \\
\hline 1,2,4-Trichlorobenzene & $120-82-1$ & $<0.050$ & $<0.050$ & $<0.050$ & $<0.050$ & $<0.025$ & $<0.22$ & & $\mathrm{~N}$ \\
\hline 1,1,1-Trichloroethane & $71-55-6$ & $<0.044$ & $<0.044$ & $<0.044$ & $<0.044$ & $<0.018$ & $<0.19$ & & $\mathrm{~N}$ \\
\hline
\end{tabular}


BECHTEL BWXT IDAHO, LLC (BBWI)

INTEC HLLWE Effluent Gas Emissions Inventory

Idaho National Engineering and Environmental Laboratory (INEEL)

STL Knoxville Project Number: 142503.40

\section{VOST Summary - Run 3 Train Totals (Continued) Method 0031 Volatile Organic Compounds Analytical Results Summary Table A-8. HLLWE Run ID: 0031-END-1}

\begin{tabular}{|c|c|c|c|c|c|c|c|c|c|}
\hline \multirow[b]{2}{*}{ Analyte } & \multirow{2}{*}{$\begin{array}{c}\text { CAS } \\
\text { Registry } \\
\text { Number }\end{array}$} & $\begin{array}{c}\text { VOST } \\
\text { Tube Set \#1 } \\
\text { (Total } \mu \mathrm{g} / \mathrm{Set}) \\
\end{array}$ & $\begin{array}{c}\text { VOST } \\
\text { Tube Set } \# 2 \\
\text { (Total } \mu \mathrm{g} / \text { Set) } \\
\end{array}$ & $\begin{array}{c}\text { VOST } \\
\text { Tube Set \#3 } \\
\text { (Total } \mu \mathrm{g} / \text { Set) }\end{array}$ & $\begin{array}{c}\text { VOST } \\
\text { Tube Set \#4 } \\
\text { (Total } \mu g / \text { Set) } \\
\end{array}$ & \multirow{2}{*}{\begin{tabular}{|c|}
$\begin{array}{c}\text { VOST } \\
\text { Condensate } \\
\text { (Total } \mu \mathrm{g})\end{array}$ \\
$\begin{array}{c}\text { Risk } \\
\text { Result }^{4} \quad \text { Flag }^{5}\end{array}$ \\
\end{tabular}} & \multicolumn{2}{|c|}{$\begin{array}{c}\text { VOST } \\
\text { Total }^{1} \\
\text { (Total } \mu \mathrm{g}) \\
\end{array}$} & \multirow[b]{2}{*}{$\begin{array}{c}\text { Project } \\
\text { Specific } \\
\text { Flag }^{6}\end{array}$} \\
\hline & & $\begin{array}{c}\text { Risk } \\
\text { Result }^{2} \quad \text { Flag }^{3} \\
\end{array}$ & 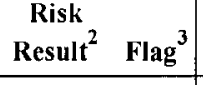 & $\begin{array}{c}\text { Risk } \\
\text { Result }^{2} \quad \text { Flag }^{3} \\
\end{array}$ & \begin{tabular}{cc}
\multicolumn{2}{c}{ Risk } \\
Result $^{2}$ & Flag $^{3}$ \\
\end{tabular} & & Total & Flag & \\
\hline 1,1,2-Trichloroethane & $79-00-5$ & $<0.036$ & $<0.036$ & $<0.036$ & $<0.036$ & $<0.022$ & $<0.17$ & & $\mathrm{~N}$ \\
\hline Trichloroethene & $79-01-6$ & $<0.034$ & $<0.034$ & $<0.034$ & $<0.034$ & $<0.020$ & $<0.16$ & & $N$ \\
\hline Trichlorofluoromethane & $75-69-4$ & $<0.036$ & $<0.036$ & $<0.036$ & $<0.036$ & $<0.011$ & $<0.16$ & $J$ & $\mathrm{P}$ \\
\hline 1,2,3-Trichloropropane & $96-18-4$ & $<0.050$ & $<0.050$ & $<0.050$ & $<0.050$ & $<0.036$ & $<0.24$ & & $\mathrm{~N}$ \\
\hline 1,2,4-Trimethylbenzene & $95-63-6$ & $<0.015$ & $<0.015$ & $<0.015$ & $<0.015$ & $<0.043$ & $<0.10$ & & $\mathrm{~N}$ \\
\hline 1,3,5-Trimethylbenzene & $108-67-8$ & $<0.010$ & $<0.010$ & $<0.010$ & $<0.010$ & $<0.019$ & $<0.059$ & & $N$ \\
\hline Vinyl chloride & $75-01-4$ & $<0.020$ & $<0.025$ & 0.023 & 0.039 & $<0.068$ & $<0.18$ & $J$ & $P$ \\
\hline m-Xylene \& p-Xylene & $136777-61-2$ & $<0.10$ & $<0.10$ & $<0.10$ & $<0.10$ & $<0.043$ & $<0.44$ & & $\mathrm{~N}$ \\
\hline o-Xylene & $95-47-6$ & $<0.013$ & $<0.013$ & $<0.013$ & $<0.013$ & $<0.026$ & $<0.078$ & $\mathrm{~J}$ & P \\
\hline \multicolumn{10}{|l|}{$T / C^{7}$} \\
\hline Pentane, 3,3-dimethyl- & $562-49-2$ & 0.059 & $\cdots$ & $\ldots$ & $--\cdot$ & $\cdots$ & 0.059 & $\mathrm{~N}, \mathrm{~J}, \mathrm{M}$ & $\mathrm{P}$ \\
\hline Hexane, 2-methyl- & $591-76-4$ & 0.27 & 0.037 & $\ldots$ & --- & --- & 0.31 & $\mathrm{~N}, \mathrm{~J}, \mathrm{M}$ & $\mathrm{P}$ \\
\hline Pentane, 2,3-dimethyl- & $565-59-3$ & $\cdots$ & 0.031 & --- & $\cdots$ & 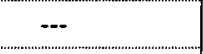 & 0.031 & $\mathrm{~N}, \mathrm{~J}, \mathrm{M}$ & $\mathrm{P}$ \\
\hline Hexane, 3-methyl- & $589-34-4$ & 0.65 & 0.076 & 0.031 & $\cdots$ & $\ldots$ & 0.76 & $\mathrm{~N}, J, \mathrm{M}$ & $P$ \\
\hline Cyclohexene & $110-83-8$ & 0.036 & $\cdots$ & $\ldots$ & $\cdots$ & $\cdots$ & 0.036 & $\mathrm{~N}, \mathrm{~J}, \mathrm{M}$ & $\mathrm{P}$ \\
\hline Cyclobutane, ethenyl- & $2597-49-1$ & --- & 0.029 & 0.044 & 0.047 & --- & 0.12 & $\mathrm{~N}, \mathrm{~J}, \mathrm{M}$ & $\mathrm{P}$ \\
\hline Cyclopentane, 1,2-dimethyl-, t & $822-50-4$ & 0.079 & $-\cdots$ & --- & --- & $\cdots$ & 0.079 & $\mathrm{~N}, \mathrm{~J}, \mathrm{M}$ & $P$ \\
\hline Cyclohexane, methyl- & $108-87-2$ & 0.20 & --- & --- & --- & --- & 0.20 & $\mathrm{~N}, \mathrm{~J}, \mathrm{M}$ & $P$ \\
\hline Hexane, 2,4-dimethyl- & $589-43-5$ & 0.16 & 0.025 & --- & --- & $\ldots$ & 0.18 & $\mathrm{~N}, \mathrm{~J}, \mathrm{M}$ & $\mathrm{P}$ \\
\hline Cyclopentane, ethyl- & $1640-89-7$ & 0.041 & $\cdots$ & --- & $\cdots$ & -- & 0.041 & $\mathrm{~N}, \mathrm{~J}, \mathrm{M}$ & $\mathrm{P}$ \\
\hline Methane, trichloronitro- & $76-06-2$ & $\cdots$ & -- & --- & 0.36 & $\cdots$ & 0.36 & $\mathrm{~N}, \mathrm{~J}, \mathrm{M}$ & $\mathrm{P}$ \\
\hline Benzonitrile & $100-47-0$ & --- & 0.074 & 0.058 & 0.059 & -- & 0.19 & $\mathrm{~N}, \mathrm{~J}, \mathrm{M}$ & $P$ \\
\hline Undecane & $1120-21-4$ & 0.21 & -- & 0.14 & 0.15 & $\cdots$ & 0.50 & $\mathrm{~N}, J, M$ & $\mathrm{P}$ \\
\hline Undecane, 5-methyl- & $1632-70-8$ & $\cdots$ & --- & 0.076 & 0.10 & $\cdots$ & 0.18 & $\mathrm{~N}, \mathrm{~J}, \mathrm{M}$ & $\mathrm{P}$ \\
\hline Dodecane & $112-40-3$ & 8.4 & 5.7 & 5.2 & 6.8 & $\cdots$ & 26 & $\mathrm{~N}, \mathrm{~J}, \mathrm{M}$ & $P$ \\
\hline Indecant & $629-50-5$ & 0.48 & 0.64 & 0.58 & 0.89 & --- & 3.1 & $\mathrm{~N}, \mathrm{~J}, \mathrm{M}$ & $\mathrm{P}$ \\
\hline Tetradecane & $629-59-4$ & 0.40 & 0.24 & --- & 0.37 & -- & 1.0 & $\mathrm{~N}, \mathrm{~J}, \mathrm{M}$ & $\mathrm{P}$ \\
\hline Hexadecane & $544-76-3$ & --- & --- & 0.21 & $-\cdots$ & --- & 0.21 & $\mathrm{~N}, \mathrm{~J}, \mathrm{M}$ & $P$ \\
\hline
\end{tabular}




\section{BECHTEL BWXT IDAHO, LLC (BBWI) \\ INTEC HLLWE Effluent Gas Emissions Inventory \\ Idaho National Engineering and Environmental Laboratory (INEEL) \\ STL Knoxville Project Number: 142503.40}

\section{Footnotes:}

1 The Method 0031 VOST Run Total (in Total $\mu \mathrm{g}$ ) is the sum of results for the four (4) VOST tube sets and the condensate sample collected during the same sampling run using the following guidelines:

- When the train component analytical result is greater than the laboratory reporting limit (RL), the result included in the train total is the actual analytical result or "hit" determined by the laboratory.

- When the train component analytical result is greater than the reliable detection level (RDL), but less than the laboratory reporting limit (RL), the result included in the train total is the actual analytical result or "hit" determined by the laboratory and the corresponding "J" flag is carried through the calculation to the train total.

- When the train analytical component result is less than the RDL, but greater than the method detection limit (MDL), the result included in the train total is the RDL and the corresponding "J" flag is carried through the calculation to the train total.

- When the train component analytical result is not detected down to the MDL, the result included in the train total is the RDL and the corresponding " $U$ " flag is carried through the calculation to the train total.

- It should be noted that when the RDL is selected as the default value using the guidelines above, but the RDL is greater than the RL, the RL is included in the train total.

The data flags attached to the VOST Total are the cumulative set of flags contributed by each train tube set included as part of the VOST total. A flag attached to a VOST component is carried through to the "VOST Total" column when the associated component analytical result is a significant number in comparison to the VOST Total. That is, if the VOST Total is affected by a VOST component analytical result, the associated flag is carried through to the VOST Total, but if the VOST Total is not affected by a VOST component, the associated flag is not carried through to the VOST Total. The combinations of train fractions are conducted following the standard practice of using significant figures found in ASTM E29-93a(1999), "Standard Practice for Using Significant Digits in Test Data to Determine Conformance with Specifications" and Severn Trent Laboratories standard operating procedure number QA-004, "Rounding and Significant Figures".

2 The Method 0031 VOST Tube Set (Total $\mu \mathrm{g} / \mathrm{Set}$ ) result consists of the sum of the analytical results for the two (2) Tenax ${ }^{\text {(B) }}$ resin tube contents (analyzed together) and the analytical result for the Anasorb $747^{\circledR}$ Tube contents. The calculation is as follows:

(Total $\mu \mathrm{g}$ on the Tenax ${ }^{(8)}$ Tubes $\# 1$ and $\# 2$ ) $+\left(\right.$ Total $\mu \mathrm{g}$ on the Anasorb $\left.747^{(}\right)$Tube) $=$Total $\mu \mathrm{g}$ on the Method 0031 VOST tube set. Therefore: $(\mu \mathrm{g})+(\mu \mathrm{g})=$ Total $\mu \mathrm{g} / \mathrm{set}$

When listed, the less than $(<)$ sign indicates that at least one sample fraction result is either a "non-detect" value down to the MDL of the measurement that carries, or an estimated "hit" value that is below the RDL. In either case, the final value included in the tube set total is the default RDL value and the actual value is known to be less than $(<)$ the displayed result.

3 The data flags in this column for the VOST Tube Set are the cumulative set of flags contributed by each individual train component included as part of the VOST total. A flag attached to a VOST component is carried through to the "VOST Tube Set" column when the associated component analytical result is a significant number in comparison to the VOST tube set total. That is, if the VOST Tube Set Total is affected by a VOST component analytical result, the associated flag is carried through to the VOST Tube Set Total, but if the VOST Tube Set Total is not affected by a VOST component analytical result, the associated flag is not carried through to the VOST Tube Set Total.

4 The VOST Condensate result was obtained by multiplying the sample's corresponding RDL or "hit" by the VOST condensate volume.

5 This flag is the laboratory data flag that corresponds to EPA guidelines. The data flags for these samples are as follows:

- A " $U$ " qualifier indicates that this analyte was analyzed for, but was not detected down to the MDL.

- An "E" flag indicates that the result exceeded the upper calibration range. The analytical result is therefore an estimated value. 
- A "J" flag indicates that this compound was detected, but at a concentration below the laboratory RL. The analytical result is therefore an estimated value.

- A "B" flag indicates that this compound was found in the associated laboratory method blank. Under these conditions this value is regarded as an estimated value.

- A "Y" flag indicates that this compound is an indistinguishable isomer as a tentatively identified compound (TIC).

- An " $N$ " flag indicates that there is presumptive evidence that this compound is present in the sample based on spectral evidence.

- An "M" flag indicates that this result was measured against the nearest internal standard and assumed a response factor of one (1).

- A "D" flag indicates that this result was obtained by a dilution of the sample. The original analysis yielded an analytical result that exceeded the calibration range.

- Entries in this column are project-specific train total flags that are applied to the run total values and are not standard EPA data flags. These project-specific flags are utilized for the INEEL NWCF HLLWE Effluent Gas Emissions Inventory project and are defined as follows:

- An "N" flag in this column indicates that the compound was not measured (detected) in any of the sampling train components, or fractions.

- A "P" flag in this column indicates that the compound was measured (detected) in one or more of the train components, or fractions, but not in all of the sampling train fractions.

- An "A" flag in this column indicates that the compound was measured (detected) in all of the sampling train components, or fractions.

7. The tentatively identified compounds (TICs) were identified by conducting a mass spectral library search using the NBS library of data. 



\section{VOST Summary - Run 2 Train Totals Method 0031 Volatile Organic Compounds Analytical Results Summary Table A-9. HLLWE Run ID: 0031-STRT-2}

Field Sample Name: Volatile Organic Sampling Train (VOST) Totals

Sample Description: Tenax $x^{\circledast}$ and Anasorb $747^{\circledast}$ Tube Sets (Sets \#1, \#2,\#3, and \#4) and the VOST Condensate for Volatile Organic Compounds (VOC) Analysis

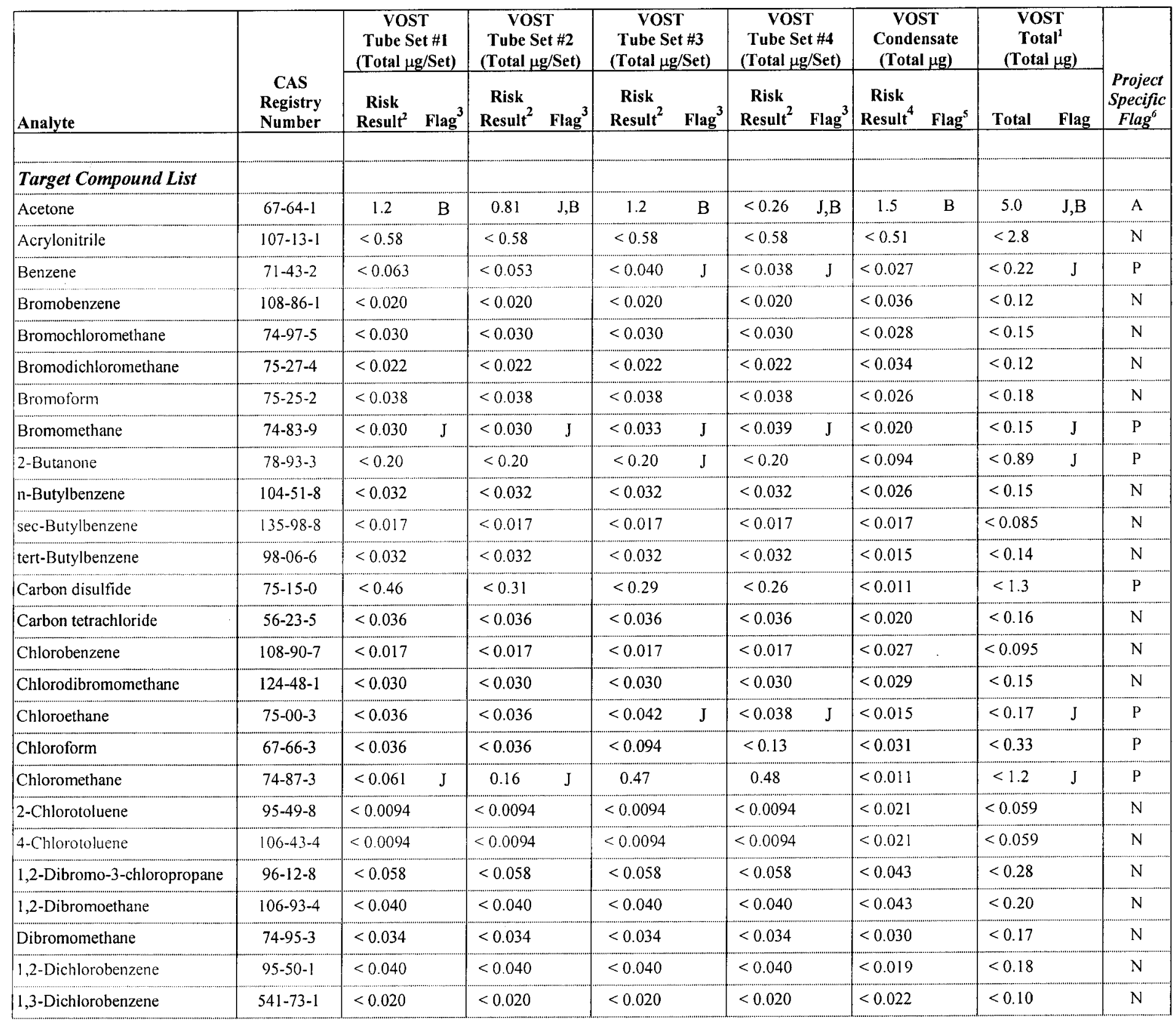




\section{BECHTEL BWXT IDAHO, LLC (BBWI) \\ INTEC HLLWE Effluent Gas Emissions Inventory Idaho National Engineering and Environmental Laboratory (INEEL) STL Knoxville Project Number: 142503.40}

\section{VOST Summary - Run 2 Train Totals (Continued) Method 0031 Volatile Organic Compounds Analytical Results Summary Table A-9. HLLWE Run ID: 0031-STRT-2}

\begin{tabular}{|c|c|c|c|c|c|c|c|c|c|c|c|c|}
\hline \multirow[b]{2}{*}{ Analyte } & \multirow{2}{*}{$\begin{array}{c}\text { CAS } \\
\text { Registry } \\
\text { Number }\end{array}$} & $\begin{array}{c}\text { VOST } \\
\text { Tube Set \#1 } \\
\text { (Total } \mu g / \text { Set) }\end{array}$ & $\begin{array}{c}\text { VOST } \\
\text { Tube Set \#2 } \\
\text { (Total } \mu \mathrm{g} / \text { Set) }\end{array}$ & \multicolumn{2}{|c|}{$\begin{array}{c}\text { VOST } \\
\text { Tube Set \#3 } \\
\text { (Total } \mu \mathrm{g} / \mathrm{Set} \text { ) }\end{array}$} & \multicolumn{2}{|c|}{$\begin{array}{c}\text { VOST } \\
\text { Tube Set \#4 } \\
\text { (Total } \mu \mathrm{g} / \text { Set) }\end{array}$} & \multicolumn{2}{|c|}{$\begin{array}{c}\text { VOST } \\
\text { Condensate } \\
(\text { Total } \mu \mathrm{g}) \\
\end{array}$} & \multicolumn{2}{|c|}{$\begin{array}{c}\text { VOST } \\
\text { Total }^{1} \\
(\text { Total } \mu \mathrm{g}) \\
\end{array}$} & \multirow{2}{*}{$\begin{array}{c}\text { Project } \\
\text { Specific } \\
\text { Flag }^{6} \\
\end{array}$} \\
\hline & & $\begin{array}{c}\begin{array}{c}\text { Risk } \\
\text { Result }^{2}\end{array} \text { Flag }^{3} \\
\end{array}$ & Result $^{2} \quad$ Flag $^{3}$ & Result $^{2}$ & Flag $^{3}$ & Result $^{2}$ & Flag $^{3}$ & Result $^{4}$ & Flag $^{5}$ & Total & Flag & \\
\hline 1,4-Dichlorobenzene & $106-46-7$ & $<0.028$ & $<0.028$ & $<0.028$ & & $<0.028$ & & $<0.024$ & & $<0.14$ & & $\mathrm{~N}$ \\
\hline Dichlorodifluoromethane & $75-71-8$ & $<0.047$ & $<0.054$ & $<0.048$ & & $<0.039$ & & $<0.011$ & & $<0.20$ & & $\mathrm{P}$ \\
\hline 1,1-Dichloroethane & $75-34-3$ & $<0.034$ & $<0.034$ & $<0.034$ & & $<0.034$ & & $<0.017$ & & $<0.15$ & & $\mathrm{~N}$ \\
\hline 1,2-Dichloroethane & $107-06-2$ & $<0.034$ & $<0.034$ & $<0.034$ & & $<0.034$ & & $<0.020$ & & $<0.16$ & & $\mathrm{~N}$ \\
\hline 1,1-Dichloroethene & $75-35-4$ & $<0.036$ & $<0.036$ & $<0.036$ & $\mathrm{~J}$ & $<0.036$ & $\mathrm{~J}$ & $<0.016$ & & $<0.16$ & $\mathrm{~J}$ & $\mathrm{P}$ \\
\hline cis-1,2-Dichloroethene & $156-59-2$ & $<0.032$ & $<0.032$ & $<0.032$ & & $<0.032$ & & $<0.021$ & & $<0.15$ & & $\mathrm{~N}$ \\
\hline trans-1,2-Dichloroethene & $156-60-5$ & $<0.038$ & $<0.038$ & $<0.038$ & & $<0.038$ & & $<0.013$ & & $<0.16$ & & $\mathrm{~N}$ \\
\hline 1,2-Dichloropropane & $78-87-5$ & $<0.026$ & $<0.026$ & $<0.026$ & & $<0.026$ & & $<0.024$ & & $<0.13$ & & N \\
\hline 1,3-Dichloropropane & $142-28-9$ & $<0.038$ & $<0.038$ & $<0.038$ & & $<0.038$ & & $<0.022$ & & $<0.17$ & & $N$ \\
\hline 2,2-Dichloropropane & $594-20-7$ & $<0.036$ & $<0.036$ & $<0.036$ & & $<0.036$ & & $<0.011$ & & $<0.16$ & & $\mathrm{~N}$ \\
\hline 1,1-Dichloropropene & $563-58-6$ & $<0.040$ & $<0.040$ & $<0.040$ & & $<0.040$ & & $<0.016$ & & $<0.18$ & & $\mathrm{~N}$ \\
\hline cis-1,3-Dichloropropene & $10061-01-5$ & $<0.024$ & $<0.024$ & $<0.024$ & & $<0.024$ & & $<0.030$ & & $<0.13$ & & $N$ \\
\hline trans-1,3-Dichloropropene & $10061-02-6$ & $<0.030$ & $<0.030$ & $<0.030$ & & $<0.030$ & & $<0.028$ & & $<0.15$ & & $\mathrm{~N}$ \\
\hline Ethylbenzene & $100-41-4$ & $<0.018$ & $<0.018$ & $<0.018$ & & $<0.018$ & & $<0.021$ & & $<0.093$ & & $N$ \\
\hline Hexachlorobutadiene & $87-68-3$ & $<0.050$ & $<0.050$ & $<0.050$ & & $<0.050$ & & $<0.025$ & & $<0.22$ & & $\mathrm{~N}$ \\
\hline 2-Hexanone & $591-78-6$ & $<0.13$ & $<0.13$ & $<0.13$ & & $<0.13$ & & $<0.036$ & & $<0.56$ & & $N$ \\
\hline Isopropylbenzene & $98-82-8$ & $<0.013$ & $<0.013$ & $<0.013$ & & $<0.013$ & & $<0.018$ & & $<0.070$ & & $N$ \\
\hline p-Isopropyltoluene & $99-87-6$ & $<0.024$ & $<0.024$ & $<0.024$ & & $<0.024$ & & $<0.018$ & & $<0.11$ & & $N$ \\
\hline Methylene chloride & $75-09-2$ & 0.55 & 0.15 & 0.13 & $\mathrm{~B}$ & 0.12 & $\mathrm{~B}$ & 0.13 & B & 1.1 & B & A \\
\hline 4-Methyl-2-pentanone & $108-10-1$ & $<0.14$ & $<0.14$ & $<0.14$ & & $<0.14$ & & $<0.030$ & & $<0.59$ & & $\mathrm{~N}$ \\
\hline Naphthalene & $91-20-3$ & $<0.050$ & $<0.050$ & $<0.050$ & & $<0.050$ & & $<0.011$ & & $<0.21$ & & $\mathrm{~N}$ \\
\hline n-Propylbenzene & $103-65-1$ & $<0.011$ & $<0.011$ & $<0.011$ & & $<0.011$ & & $<0.022$ & & $<0.066$ & & $\mathrm{~N}$ \\
\hline Styrene & $100-42-5$ & $<0.014$ & $<0.014$ & $<0.014$ & & $<0.014$ & & $<0.022$ & & $<0.078$ & & $\mathrm{~N}$ \\
\hline 1,1,1,2-Tetrachloroethane & $630-20-6$ & $<0.019$ & $<0.019$ & $<0.019$ & & $<0.019$ & & $<0.024$ & & $<0.10$ & & $\mathrm{~N}$ \\
\hline 1,1,2,2-Tetrachloroethane & $79-34-5$ & $<0.050$ & $<0.050$ & $<0.050$ & & $<0.050$ & & $<0.025$ & & $<0.22$ & & N \\
\hline Tetrachloroethene & $127-18-4$ & $<0.032$ & $<0.032$ & $<0.032$ & & $<0.032$ & & $<0.021$ & & $<0.15$ & & $\mathrm{~N}$ \\
\hline Toluene & $108-88-3$ & $<0.027$ & 0.17 & 0.031 & J & 0.045 & $J$ & $<0.028$ & & $<0.30$ & $\mathrm{~J}$ & P \\
\hline 1,2,3-Trichlorobenzene & $87-61-6$ & $<0.050$ & $<0.050$ & $<0.050$ & & $<0.050$ & & $<0.011$ & & $<0.21$ & & $N$ \\
\hline 1,2,4-Trichlorobenzene & $120-82-1$ & $<0.050$ & $<0.050$ & $<0.050$ & & $<0.050$ & & $<0.025$ & & $<0.22$ & & $N$ \\
\hline
\end{tabular}


BECHTEL BWXT IDAHO, LLC (BBWI)

INTEC HLLWE Effluent Gas Emissions Inventory

Idaho National Engineering and Environmental Laboratory (INEEL)

STL Knoxville Project Number: 142503.40

\section{VOST Summary - Run 2 Train Totals (Continued) Method 0031 Volatile Organic Compounds Analytical Results Summary \\ Table A-9. HLLWE Run ID: 0031-STRT-2}

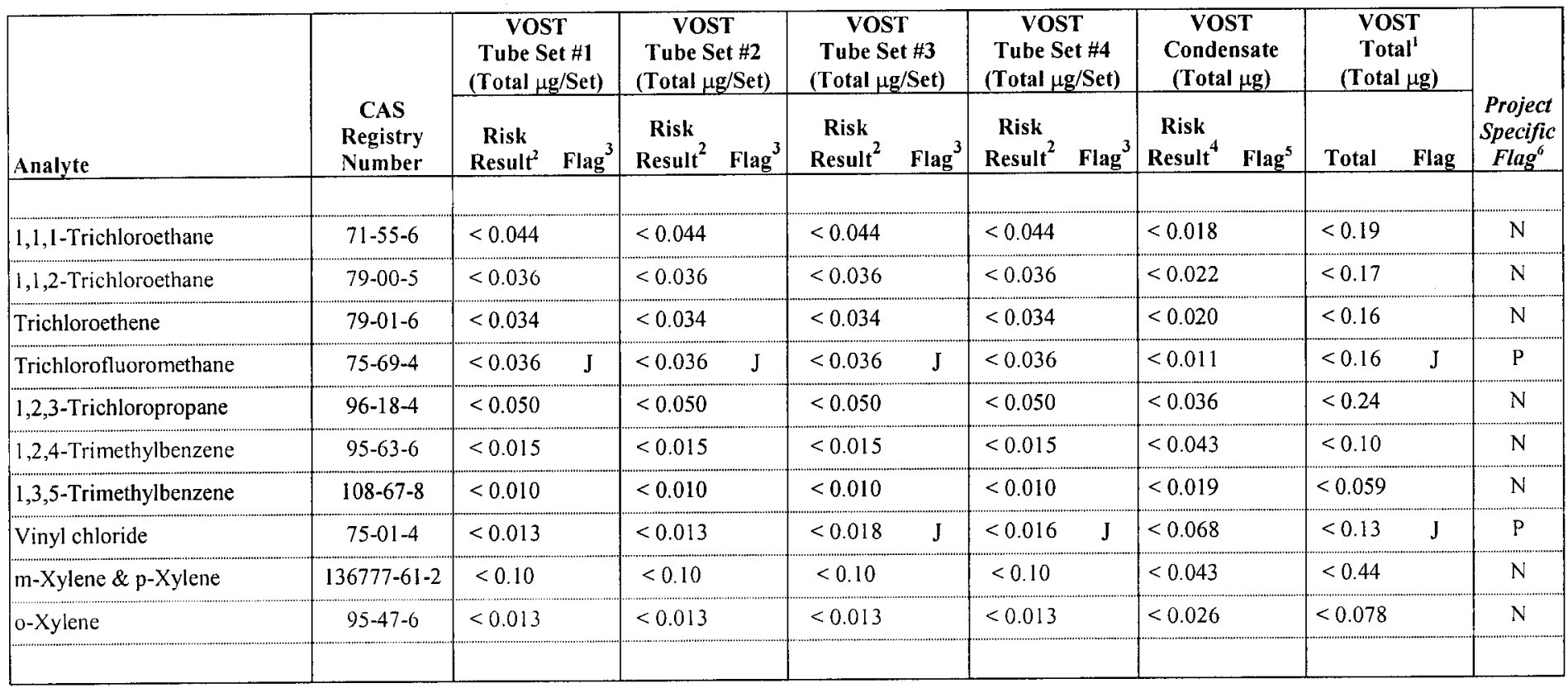


BECHTEL BWXT IDAHO, LLC (BBWI)

INTEC HLLWE Effluent Gas Emissions Inventory

Idaho National Engineering and Environmental Laboratory (INEEL)

STL Knoxville Project Number: 142503.40

\section{VOST Summary - Run 2 Train Totals (Continued) Method 0031 Volatile Organic Compounds Analytical Results Summary \\ Table A-9. HLLWE Run ID: 0031-STRT-2}

\begin{tabular}{|c|c|c|c|c|c|c|c|c|c|}
\hline \multirow[b]{2}{*}{ Analyte } & \multirow{2}{*}{$\begin{array}{c}\text { CAS } \\
\text { Registry } \\
\text { Number }\end{array}$} & \multirow{2}{*}{$\begin{array}{c}\text { VOST } \\
\text { Tube Set \#I } \\
\text { (Total Hg/Set) } \\
\begin{array}{c}\text { Risk } \\
\text { Result }{ }^{2} \quad \text { Flag }^{3}\end{array} \\
\end{array}$} & \multirow{2}{*}{$\begin{array}{c}\begin{array}{c}\text { VOST } \\
\text { Tube Set } \# 2 \\
\text { (Total } \mu \mathrm{g} / \mathrm{Set} \text { ) }\end{array} \\
\begin{array}{c}\text { Risk } \\
\text { Result }^{2} \quad \text { Flag }^{3}\end{array} \\
\end{array}$} & \multirow{2}{*}{$\begin{array}{c}\begin{array}{c}\text { VOST } \\
\text { Tube Set } \# 3 \\
(\text { Total } \mu \text { g/Set) }\end{array} \\
\begin{array}{c}\text { Risk } \\
\text { Result }^{2} \quad \text { Flag }^{3}\end{array} \\
\end{array}$} & \multirow{2}{*}{ 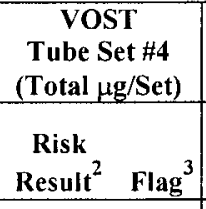 } & \multirow{2}{*}{\begin{tabular}{|c|}
$\begin{array}{c}\text { VOST } \\
\text { Condensate } \\
\text { (Total } \mu \mathrm{g})\end{array}$ \\
Risk $^{4} \quad$ Flag $^{5}$ \\
Result $^{4} \quad{ }^{2}$
\end{tabular}} & \multicolumn{2}{|c|}{$\begin{array}{c}\text { VOST } \\
\text { Total }^{1} \\
(\text { Total } \mu \mathrm{g}) \\
\end{array}$} & \multirow{2}{*}{$\begin{array}{c}\text { Project } \\
\text { Specific } \\
\text { Flag }^{6} \\
\end{array}$} \\
\hline & & & & & & & Total & Flag & \\
\hline \multicolumn{10}{|l|}{$T I C s^{7}$} \\
\hline Hexane, 2-methyl- & $591-76-4$ & --- & 0.17 & 0.059 & 0.072 & --- & 0.30 & $\mathrm{~N}, \mathrm{~J}, \mathrm{M}$ & $\mathrm{P}$ \\
\hline Pentane, 2,3-dimethyl- & $565-59-3$ & --- & $\cdots$ & 0.057 & 0.056 & --- & 0.11 & $\mathrm{~N}, \mathrm{~J}, \mathrm{M}$ & $\mathrm{P}$ \\
\hline Hexane, 3-methyl- & $589-34-4$ & $\cdots$ & --- & 0.12 & 0.15 & --- & 0.27 & $\mathrm{~N}, \mathrm{~J}, \mathrm{M}$ & $\mathrm{P}$ \\
\hline Pentane, 3-ethyl- & $617-78-7$ & --- & -- & 0.034 & $-\cdots$ & --- & 0.034 & $\mathrm{~N}, \mathrm{~J}, \mathrm{M}$ & $\mathrm{P}$ \\
\hline Cyclohexene & $110-83-8$ & 0.030 & 0.042 & 0.036 & 0.073 & -- & 0.18 & $\mathrm{~N}, \mathrm{~J}, \mathrm{M}$ & $\mathrm{P}$ \\
\hline Cyclopentane, 1,2-dimethyl- & $2452-99-5$ & --- & 0.053 & -- & -- & $\cdots$ & 0.053 & $\mathrm{~N}, J, M$ & $\mathrm{P}$ \\
\hline Cyclohexane, methyl- & $108-87-2$ & $\cdots$ & 0.13 & 0.032 & 0.037 & $\cdots$ & 0.20 & $\mathrm{~N}, \mathrm{~J}, \mathrm{M}$ & P \\
\hline Hexane, 2,4-dimethyl- & $589-43-5$ & -- & 0.13 & 0.037 & 0.037 & $\cdots$ & 0.20 & $\mathrm{~N}, \mathrm{~J}, \mathrm{M}$ & $\mathrm{P}$ \\
\hline Cyclopentane, ethyl- & $1640-89-7$ & --- & 0.036 & $\ldots$ & --- & --- & 0.036 & $\mathrm{~N}, \mathrm{~J}, \mathrm{M}$ & P \\
\hline Benzonitrile & $100-47-0$ & 0.038 & 0.034 & $\cdots$ & --- & -- & 0.072 & $\mathrm{~N}, J, \mathrm{M}$ & P \\
\hline Tridecane & $629-50-5$ & 0.082 & $-\cdots$ & $-\cdots$ & $\cdots$ & --- & 0.082 & $\mathrm{~N}, J, M$ & ${ }^{P}$ \\
\hline Undecane & $1120-21-4$ & --- & 0.065 & 0.10 & 0.080 & --- & 0.24 & $\mathrm{~N}, J, M$ & $P$ \\
\hline Decane, 2,2,5-trimethyl- & $62237-96-1$ & -- & $\cdots$ & $-\cdots$ & 0.060 & --- & 0.060 & $\mathrm{~N}, J, M$ & $\mathrm{P}$ \\
\hline Undecane, 5-methyl- & $1632-70-8$ & 0.052 & 0.051 & 0.11 & $--\cdot$ & -- & 0.21 & $\mathrm{~N}, J, M$ & $P$ \\
\hline Dodecane & $112-40-3$ & 3.4 & 3.4 & 8.1 & 6.8 & --- & 22 & $\mathrm{~N}, \mathrm{~J}, \mathrm{M}$ & P \\
\hline Dodecane, 6-methyl- & $6044-71-9$ & --- & $\cdots$ & 0.052 & $\cdots$ & $\cdots$ & 0.052 & $\mathrm{~N}, \mathrm{~J}, \mathrm{M}$ & $\mathrm{P}$ \\
\hline Undecane, 2,6-dimethyl- & $17301-23-4$ & -.- & $\ldots$ & $\ldots$ & 0.046 & --- & 0.046 & $\mathrm{~N}, \mathrm{~J}, \mathrm{M}$ & $P$ \\
\hline Tridecane & $629-50-5$ & 0.34 & 0.29 & 0.84 & 0.93 & --- & 2.4 & $\mathrm{~N}, J, M$ & $\mathrm{P}$ \\
\hline Tetradecane & $629-59-4$ & 0.17 & 0.16 & 0.28 & 0.37 & --- & 0.98 & $\mathrm{~N}, \mathrm{~J}, \mathrm{M}$ & $\mathrm{P}$ \\
\hline
\end{tabular}




\section{BECHTEL BWXT IDAHO, LLC (BBWI) \\ INTEC HLLWE Effluent Gas Emissions Inventory \\ Idaho National Engineering and Environmental Laboratory (INEEL) \\ STL Knoxville Project Number: 142503.40}

Footnotes:

1 The Method 0031 VOST Run Total (in Total $\mu \mathrm{g}$ ) is the sum of results for the four (4) VOST tube sets and the condensate sample collected during the same sampling run using the following guidelines:

- When the train component analytical result is greater than the laboratory reporting limit (RL), the result included in the train total is the actual analytical result or "hit" determined by the laboratory.

- When the train component analytical result is greater than the reliable detection level (RDL), but less than the laboratory reporting limit $(\mathrm{RL})$, the result included in the train total is the actual analytical result or "hit" determined by the laboratory and the corresponding "J" flag is carried through the calculation to the train total.

- When the train analytical component result is less than the RDL, but greater than the method detection limit (MDL), the result included in the train total is the RDL and the corresponding "J" flag is carried through the calculation to the train total.

- When the train component analytical result is not detected down to the MDL, the result included in the train total is the RDL and the corresponding " $U$ " flag is carried through the calculation to the train total.

- It should be noted that when the RDL is selected as the default value using the guidelines above, but the RDL is greater than the RL, the RL is included in the train total.

The data flags attached to the VOST Total are the cumulative set of flags contributed by each train tube set included as part of the VOST total. A flag attached to a VOST component is carried through to the "VOST Total" column when the associated component analytical result is a significant number in comparison to the VOST Total. That is, if the VOST Total is affected by a VOST component analytical result, the associated flag is carried through to the VOST Total, but if the VOST Total is not affected by a VOST component, the associated flag is not carried through to the VOST Total. The combinations of train fractions are conducted following the standard practice of using significant figures found in ASTM E29-93a(1999), "Standard Practice for Using Significant Digits in Test Data to Determine Conformance with Specifications" and Severn Trent Laboratories standard operating procedure number QA-004, "Rounding and Significant Figures".

2 The Method 0031 VOST Tube Set (Total $\mu \mathrm{g} / \mathrm{Set}$ ) result consists of the sum of the analytical results for the two (2) Tenax ${ }^{(\bullet)}$ resin tube contents (analyzed together) and the analytical result for the Anasorb $747^{\circledR}$ Tube contents. The calculation is as follows:

(Total $\mu$ g on the Tenax ${ }^{\circledR}$ Tubes \#1 and \#2) $+\left(\right.$ Total $\mu g$ on the Anasorb $747^{\circledR}$ Tube) $=$ Total $\mu$ g on the Method 0031 VOST tube set. Therefore: $(\mu \mathrm{g})+(\mu \mathrm{g})=$ Total $\mu \mathrm{g} / \mathrm{set}$

When listed, the less than $(<)$ sign indicates that at least one sample fraction result is either a "non-detect" value down to the MDL of the measurement that carries, or an estimated "hit" value that is below the RDL. In either case, the final value included in the tube set total is the default RDL value and the actual value is known to be less than $(<)$ the displayed result.

3 The data flags in this column for the VOST Tube Set are the cumulative set of flags contributed by each individual train component included as part of the VOST total. A flag attached to a VOST component is carried through to the "VOST Tube Set" column when the associated component analytical result is a significant number in comparison to the VOST tube set total. That is, if the VOST Tube Set Total is affected by a VOST component analytical result, the associated flag is carried through to the VOST Tube Set Total, but if the VOST Tube Set Total is not affected by a VOST component analytical result, the associated flag is not carried through to the VOST Tube Set Total.

4 The VOST Condensate result was obtained by multiplying the sample's corresponding RDL or "hit" by the VOST condensate volume.

5 This flag is the laboratory data flag that corresponds to EPA guidelines. The data flags for these samples are as follows:

- A "U" qualifier indicates that this analyte was analyzed for, but was not detected down to the MDL.

- An "E" flag indicates that the result exceeded the upper calibration range. The analytical result is therefore an estimated value. 


\section{BECHTEL BWXT IDAHO, LLC (BBWI) \\ INTEC HLLWE Effluent Gas Emissions Inventory \\ Idaho National Engineering and Environmental Laboratory (INEEL) \\ STL Knoxville Project Number: 142503.40}

- A "J" flag indicates that this compound was detected, but at a concentration below the laboratory RL. The analytical result is therefore an estimated value.

- A "B" flag indicates that this compound was found in the associated laboratory method blank. Under these conditions this value is regarded as an estimated value.

- A "Y" flag indicates that this compound is an indistinguishable isomer as a tentatively identified compound (TIC).

- An "N" flag indicates that there is presumptive evidence that this compound is present in the sample based on spectral evidence.

- An " $\mathrm{M}$ " flag indicates that this result was measured against the nearest internal standard and assumed a response factor of one (1).

- A "D" flag indicates that this result was obtained by a dilution of the sample. The original analysis yielded an analytical result that exceeded the calibration range.

6. Entries in this column are project-specific train total flags that are applied to the run total values and are not standard EPA data flags. These project-specific flags are utilized for the INEEL NWCF HLLWE Effluent Gas Emissions Inventory project and are defined as follows:

- An "N" flag in this column indicates that the compound was not measured (detected) in any of the sampling train components, or fractions.

- A "P" flag in this column indicates that the compound was measured (detected) in one or more of the train components, or fractions, but not in all of the sampling train fractions.

- An "A" flag in this column indicates that the compound was measured (detected) in all of the sampling train components, or fractions.

7. The tentatively identified compounds (TICs) were identified by conducting a mass spectral library search using the NBS library of data. 
BECHTEL BWXT IDAHO, LLC (BBWI)

INTEC HLLWE Effluent Gas Emissions Inventory

Idaho National Engineering and Environmental Laboratory (INEEL)

STL Knoxville Project Number: 142503.40

\section{VOST Summary - Run 4 Train Totals Method 0031 Volatile Organic Compounds Analytical Results Summary Table A-10. HLLWE Run ID: 0031-END-2}

Field Sample Name: Volatile Organic Sampling Train (VOST) Totals

Sample Description: Tenax ${ }^{\circledR}$ and Anasorb $747^{\oplus}$ Tube Sets (Sets \#1, \#2, \#3, and \#4) and the VOST Condensate for Volatile Organic Compounds (VOC) Analysis

\begin{tabular}{|c|c|c|c|c|c|c|c|c|c|c|c|c|c|c|}
\hline \multirow[b]{2}{*}{ Analyte } & \multirow{2}{*}{$\begin{array}{c}\text { CAS } \\
\text { Registry } \\
\text { Number } \\
\end{array}$} & \multicolumn{2}{|c|}{$\begin{array}{c}\text { VOST } \\
\text { Tube Set \#1 } \\
(\text { Total } \mu \mathrm{g} / \mathrm{Set}) \\
\end{array}$} & \multicolumn{2}{|c|}{$\begin{array}{c}\text { VOST } \\
\text { Tube Set \#2 } \\
(\text { Total } \mu \mathrm{g} / \text { Set }) \\
\end{array}$} & \multicolumn{2}{|c|}{$\begin{array}{c}\text { VOST } \\
\text { Tube Set \#3 } \\
(\text { Total } \mu \mathrm{g} / \text { Set) } \\
\end{array}$} & \multicolumn{2}{|c|}{$\begin{array}{c}\text { VOST } \\
\text { Tube Set \#4 } \\
\text { (Total } \mu \mathrm{g} / \mathrm{Set})\end{array}$} & \multicolumn{2}{|c|}{$\begin{array}{c}\text { VOST } \\
\text { Condensate } \\
\text { (Total } \mu \mathrm{g} \text { ) }\end{array}$} & \multicolumn{2}{|c|}{$\begin{array}{c}\text { VOST } \\
\text { Total }^{1} \\
\text { (Total } \mu \mathrm{g})\end{array}$} & \multirow{2}{*}{$\begin{array}{c}\text { Project } \\
\text { Specific } \\
\text { Flag }^{6}\end{array}$} \\
\hline & & Result & Flag $^{3}$ & Result $^{2}$ & Flag $^{3}$ & Result $^{2}$ & Flag $^{3}$ & Result $^{2}$ & Flag $^{3}$ & Result $^{4}$ & Flag ${ }^{5}$ & Total & Flag & \\
\hline \multicolumn{15}{|l|}{ Target Compound List } \\
\hline Acetone & $67-64-1$ & $<0.46$ & $\mathrm{~J}, \mathrm{~B}$ & 0.59 & $\mathrm{~B}$ & 1.3 & $\mathrm{~B}$ & 1.4 & $\mathrm{~B}$ & 0.081 & $\mathrm{~J}, \mathrm{~B}$ & $<3.8$ & $\mathrm{~J}, \mathrm{~B}$ & A \\
\hline Acrylonitrile & $107-13-1$ & $<0.58$ & & $<0.58$ & & $<0.58$ & & $<0.58$ & & $<0.51$ & & $<2.8$ & & $\mathrm{~N}$ \\
\hline Benzene & $71-43-2$ & $<0.052$ & & $<0.034$ & & $<0.044$ & & $<0.047$ & & $<0.027$ & & $<0.20$ & & $\mathrm{P}$ \\
\hline Bromobenzene & $108-86-1$ & $<0.020$ & & $<0.020$ & & $<0.020$ & & $<0.020$ & & $<0.036$ & & $<0.12$ & & $\mathrm{~N}$ \\
\hline Bromochloromethane & $74-97-5$ & $<0.030$ & & $<0.030$ & & $<0.030$ & & $<0.030$ & & $<0.028$ & & $<0.15$ & & $\mathrm{~N}$ \\
\hline Bromodichloromethane & $75-27-4$ & $<0.022$ & & $<0.022$ & & $<0.022$ & & $<0.022$ & & $<0.033$ & & $<0.12$ & & $\mathrm{~N}$ \\
\hline Bromoform & $75-25-2$ & $<0.038$ & & $<0.038$ & & $<0.038$ & & $<0.038$ & & $<0.025$ & & $<0.18$ & & $N$ \\
\hline Bromomethane & $74-83-9$ & $<0.030$ & $J$ & $<0.055$ & $J$ & $<0.041$ & J & $<0.049$ & J & $<0.020$ & & $<0.20$ & $\mathrm{~J}$ & $P$ \\
\hline 2-Butanone & $78-93-3$ & $<0.20$ & & $<0.20$ & & $<0.20$ & & $<0.20$ & & $<0.093$ & & $<0.89$ & & $\mathrm{~N}$ \\
\hline n-Butylbenzene & $104-51-8$ & $<0.032$ & & $<0.032$ & & $<0.032$ & & $<0.032$ & & $<0.025$ & & $<0.15$ & & $\mathrm{~N}$ \\
\hline sec-Butylbenzene & $135-98-8$ & $<0.017$ & & $<0.017$ & & $<0.017$ & & $<0.017$ & & $<0.017$ & & $<0.085$ & & $\mathrm{~N}$ \\
\hline tert-Butylbenzene & $98-06-6$ & $<0.032$ & & $<0.032$ & & $<0.032$ & & $<0.032$ & & $<0.014$ & & $<0.14$ & & N \\
\hline Carbon disulfide & $75-15-0$ & 0.22 & & $<0.036$ & & $<0.18$ & & $<0.19$ & & $<0.011$ & & $<0.64$ & & $\mathrm{P}$ \\
\hline Carbon tetrachloride & $56-23-5$ & $<0.036$ & $J$ & $<0.036$ & & $<0.036$ & & $<0.036$ & J & $<0.020$ & & $<0.16$ & $J$ & $P$ \\
\hline Chlorobenzene & $108-90-7$ & $<0.017$ & $J$ & $<0.017$ & & $<0.017$ & J & $<0.017$ & $\mathrm{~J}$ & $<0.027$ & & $<0.095$ & $\mathrm{~J}$ & $P$ \\
\hline Chlorodibromomethane & $124-48-1$ & $<0.030$ & & $<0.030$ & & $<0.030$ & & $<0.030$ & & $<0.029$ & & $<0.15$ & & $\mathrm{~N}$ \\
\hline Chloroethane & $75 \cdot 00-3$ & $<0.036$ & $J$ & $<0.036$ & $J$ & $<0.036$ & $\mathrm{~J}$ & $<0.036$ & $J$ & $<0.014$ & & $<0.16$ & $\mathrm{~J}$ & $P$ \\
\hline Chloroform & $67-66-3$ & $<0.15$ & & $<0.036$ & & $<0.087$ & & $<0.087$ & & $<0.031$ & & $<0.39$ & & $P$ \\
\hline Chloromethane & $74-87-3$ & 0.46 & & $<0.70$ & & 0.65 & J & 0.70 & & $<0.011$ & & $<2.5$ & J & $P$ \\
\hline 2-Chlorotoluene & $95-49-8$ & $<0.0094$ & & $<0.0094$ & & $<0.0094$ & & $<0.0094$ & & $<0.021$ & & $<0.059$ & & $\mathrm{~N}$ \\
\hline 4-Chlorotoluene & $106-43-4$ & $<0.0094$ & & $<0.0094$ & & $<0.0094$ & & $<0.0094$ & & $<0.021$ & & $<0.059$ & & $\mathrm{~N}$ \\
\hline 1,2-Dibromo-3-chloropropane & $96-12-8$ & $<0.058$ & & $<0.058$ & & $<0.058$ & & $<0.058$ & & $<0.042$ & & $<0.27$ & & $\mathrm{~N}$ \\
\hline 1,2-Dibromoethane & $106-93-4$ & $<0.040$ & & $<0.040$ & & $<0.040$ & & $<0.040$ & & $<0.042$ & & $<0.20$ & & $N$ \\
\hline Dibromomethane & $74-95-3$ & $<0.034$ & & $<0.034$ & & $<0.034$ & & $<0.034$ & & $<0.030$ & & $<0.17$ & & N \\
\hline 1,2-Dichlorobenzene & $95-50-1$ & $<0.040$ & & $<0.040$ & & $<0.040$ & & $<0.040$ & & $<0.019$ & & $<0.18$ & & $\mathrm{~N}$ \\
\hline 1,3-Dichlorobenzene & $541-73-1$ & $<0.020$ & & $<0.020$ & & $<0.020$ & & $<0.020$ & & $<0.022$ & & $<0.10$ & & $N$ \\
\hline 1,4-Dichlorobenzene & $106-46-7$ & $<0.028$ & & $<0.028$ & & $<0.028$ & & $<0.028$ & & $<0.023$ & & $<0.14$ & & $\mathrm{~N}$ \\
\hline
\end{tabular}

Last saved by Patti Carswell on 02/05/02 at 7:54 AM

E:IMy Documents\HLLWE Sampling\Final Report\Appendix AlTable A-10. 0031-END-2.doc

Created on 2/5/2002 7:51 AM 


\section{BECHTEL BWXT IDAHO, LLC (BBWI) \\ INTEC HLLWE Effluent Gas Emissions Inventory \\ Idaho National Engineering and Environmental Laboratory (INEEL) \\ STL Knoxville Project Number: 142503.40}

\section{VOST Summary - Run 4 Train Totals (Continued) Method 0031 Volatile Organic Compounds Analytical Results Summary Table A-10. HLLWE Run ID: 0031-END-2}

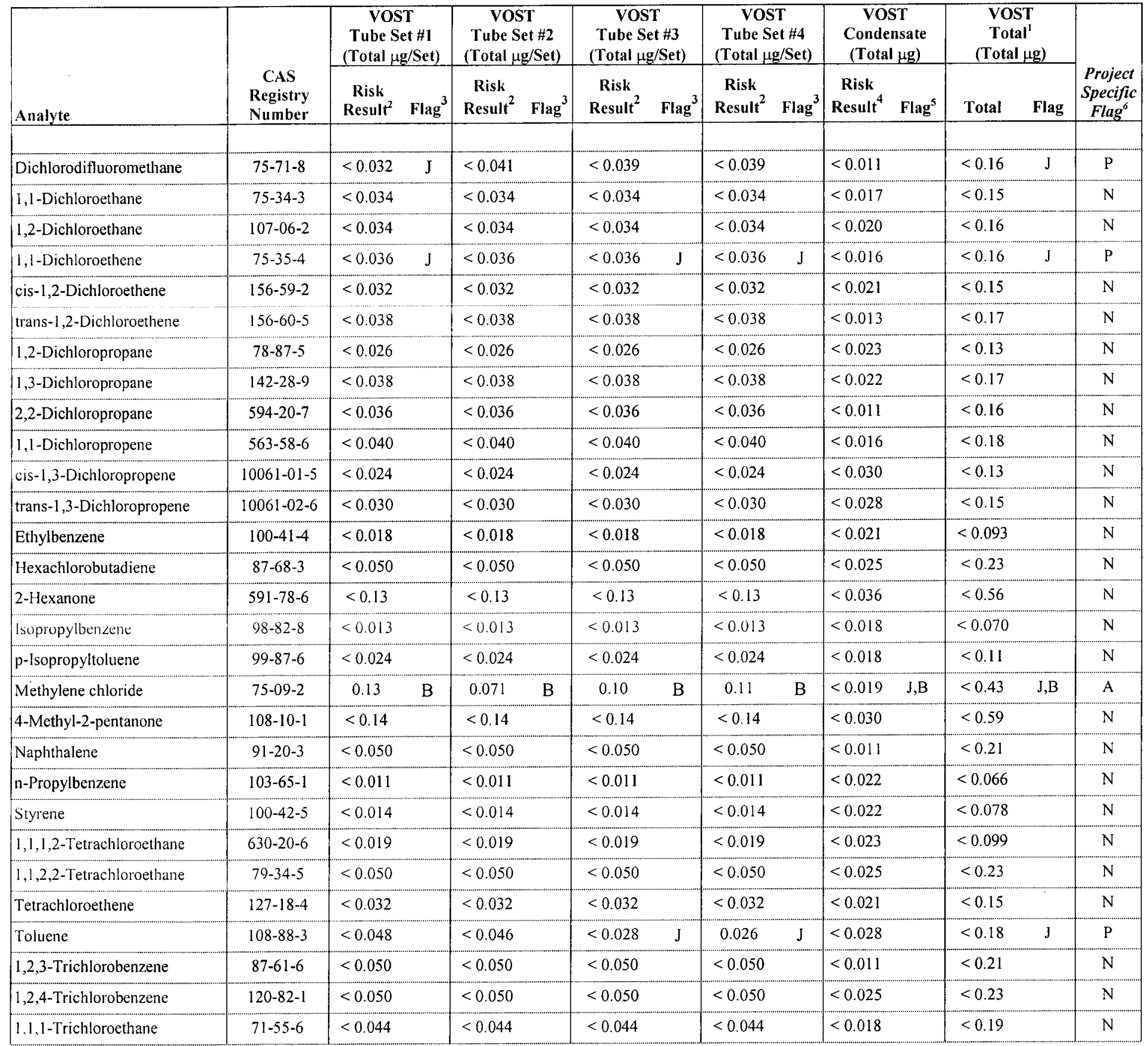


BECHTEL BWXT IDAHO, LLC (BBWI)

INTEC HLLWE Effluent Gas Emissions Inventory

Idaho National Engineering and Environmental Laboratory (INEEL)

STL Knoxville Project Number: 142503.40

\section{VOST Summary - Run 4 Train Totals (Continued) Method 0031 Volatile Organic Compounds Analytical Results Summary Table A-10. HLLWE Run ID: 0031-END-2}

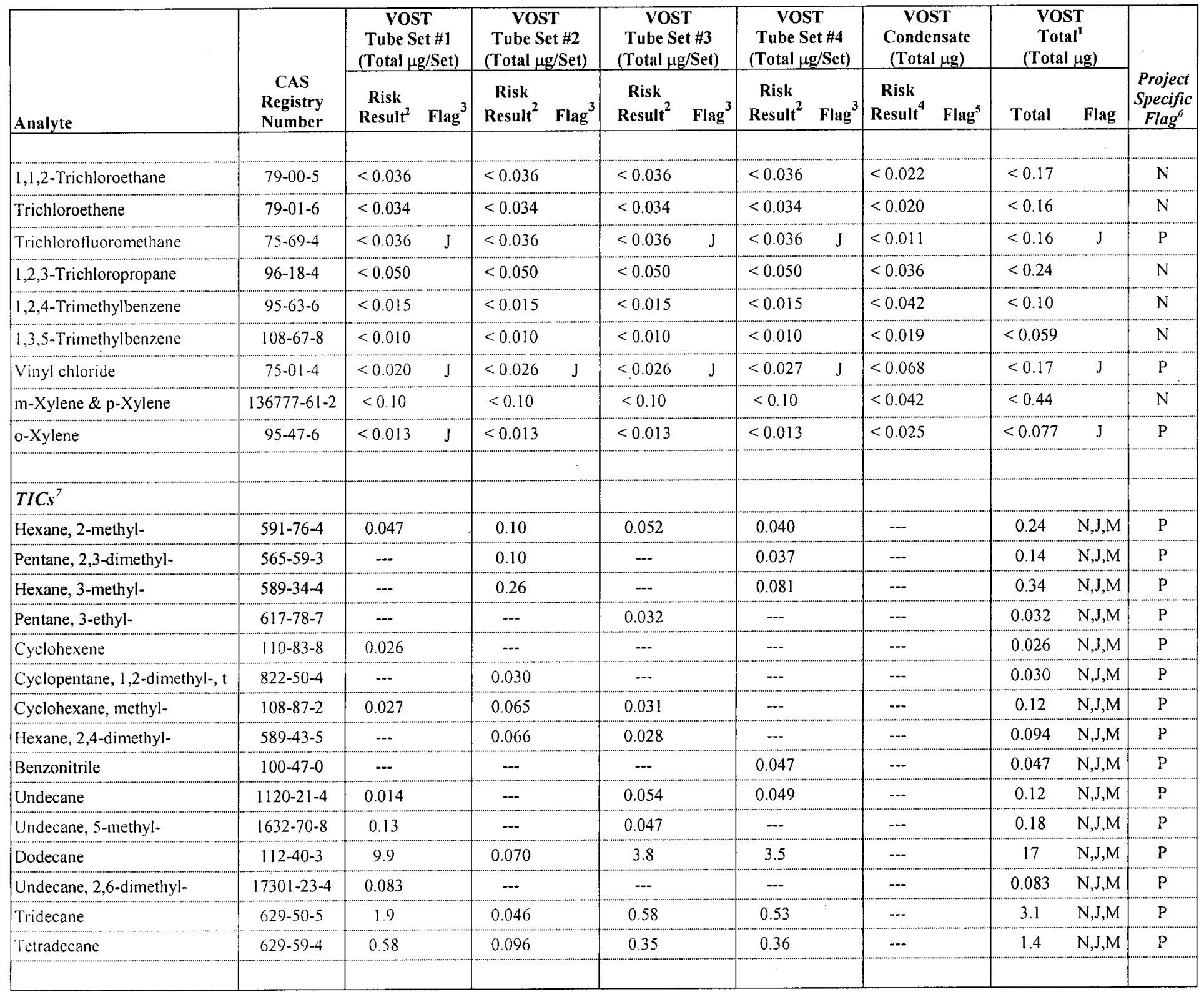




\section{BECHTEL BWXT IDAHO, LLC (BBWI) \\ INTEC HLLWE Effluent Gas Emissions Inventory \\ Idaho National Engineering and Environmental Laboratory (INEEL) \\ STL Knoxville Project Number: 142503.40}

\section{Footnotes:}

1 The Method 0031 VOST Run Total (in Total $\mu \mathrm{g}$ ) is the sum of results for the four (4) VOST tube sets and the condensate sample collected during the same sampling run using the following guidelines:

- When the train component analytical result is greater than the laboratory reporting limit (RL), the result included in the train total is the actual analytical result or "hit" determined by the laboratory.

- When the train component analytical result is greater than the reliable detection level (RDL), but less than the laboratory reporting limit (RL), the result included in the train total is the actual analytical result or "hit" determined by the laboratory and the corresponding " $J$ " flag is carried through the calculation to the train total.

- When the train analytical component result is less than the RDL, but greater than the method detection limit (MDL), the result included in the train total is the RDL and the corresponding " $\mathrm{J}$ " flag is carried through the calculation to the train total.

- When the train component analytical result is not detected down to the MDL, the result included in the train total is the RDL and the corresponding " $U$ " flag is carried through the calculation to the train total.

- It should be noted that when the RDL is selected as the default value using the guidelines above, but the RDL is greater than the RL, the RL is included in the train total.

The data flags attached to the VOST Total are the cumulative set of flags contributed by each train tube set included as part of the VOST total. A flag attached to a VOST component is carried through to the "VOST Total" column when the associated component analytical result is a significant number in comparison to the VOST Total. That is, if the VOST Total is affected by a VOST component analytical result, the associated flag is carried through to the VOST Total, but if the VOST Total is not affected by a VOST component, the associated flag is not carried through to the VOST Total. The combinations of train fractions are conducted following the standard practice of using significant figures found in ASTM E29-93a(1999), "Standard Practice for Using Significant Digits in Test Data to Determine Conformance with Specifications" and Severn Trent Laboratories standard operating procedure number QA-004, "Rounding and Significant Figures".

2 The Method 0031 VOST Tube Set (Total $\mu \mathrm{g} / \mathrm{Set}$ ) result consists of the sum of the analytical results for the two (2) Tenax ${ }^{\circledR}$ resin tube contents (analyzed together) and the analytical result for the Anasorb $747^{\circledR}$ Tube contents. The calculation is as follows:

$\left(\right.$ Total $\mu \mathrm{g}$ on the Tenax ${ }^{\circledR}$ Tubes \#1 and \#2) $+\left(\right.$ Total $\mu \mathrm{g}$ on the Anasorb $747^{\circledR}$ Tube $)=$ Total $\mu \mathrm{g}$ on the Method 0031 VOST tube set. Therefore: $(\mu \mathrm{g})+(\mu \mathrm{g})=$ Total $\mu \mathrm{g} / \mathrm{set}$

When listed, the less than $(<)$ sign indicates that at least one sample fraction result is either a "non-detect" value down to the MDL of the measurement that carries, or an estimated "hit" value that is below the RDL. In either case, the final value included in the tube set total is the default RDL value and the actual value is known to be less than $(<)$ the displayed result.

3 The data flags in this column for the VOST Tube Set are the cumulative set of flags contributed by each individual train component included as part of the VOST total. A flag attached to a VOST component is carried through to the "VOST Tube Set" column when the associated component analytical result is a significant number in comparison to the VOST tube set total. That is, if the VOST Tube Set Total is affected by a VOST component analytical result, the associated flag is carried through to the VOST Tube Set Total, but if the VOST Tube Set Total is not affected by a VOST component analytical result, the associated flag is not carried through to the VOST Tube Set Total.

4 The VOST Condensate result was obtained by multiplying the sample's corresponding RDL or "hit" by the VOST condensate volume.

5 This flag is the laboratory data flag that corresponds to EPA guidelines. The data flags for these samples are as follows:

- A "U" qualifier indicates that this analyte was analyzed for, but was not detected down to the MDL.

- An "E" flag indicates that the result exceeded the upper calibration range. The analytical result is therefore an estimated value. 


\section{BECHTEL BWXT IDAHO, LLC (BBWI)}

INTEC HLLWE Effluent Gas Emissions Inventory

\section{Idaho National Engineering and Environmental Laboratory (INEEL)}

STL Knoxville Project Number: 142503.40

- A "J" flag indicates that this compound was detected, but at a concentration below the laboratory RL. The analytical result is therefore an estimated value.

- A "B" flag indicates that this compound was found in the associated laboratory method blank. Under these conditions this value is regarded as an estimated value.

- A "Y" flag indicates that this compound is an indistinguishable isomer as a tentatively identified compound (TIC).

- An "N" flag indicates that there is presumptive evidence that this compound is present in the sample based on spectral evidence.

- An " $\mathrm{M}$ " flag indicates that this result was measured against the nearest internal standard and assumed a response factor of one (1).

- $\mathrm{A}$ " $\mathrm{D}$ " flag indicates that this result was obtained by a dilution of the sample. The original analysis yielded an analytical result that exceeded the calibration range.

6 Entries in this column are project-specific train total flags that are applied to the run total values and are not standard EPA data flags. These project-specific flags are utilized for the INEEL NWCF HLLWE Effluent Gas Emissions Inventory project and are defined as follows:

- An "N" flag in this column indicates that the compound was not measured (detected) in any of the sampling train components, or fractions.

- A "P" flag in this column indicates that the compound was measured (detected) in one or more of the train components, or fractions, but not in all of the sampling train fractions.

- An "A" flag in this column indicates that the compound was measured (detected) in all of the sampling train components, or fractions.

7. The tentatively identified compounds (TICs) were identified by conducting a mass spectral library search using the NBS library of data. 

BECHTEL BWXT IDAHO, LLC (BBWI)

INTEC HLLWE Effluent Gas Emissions Inventory

Idaho National Engineering and Environmental Laboratory (INEEL)

STL Knoxville Project Number: 142503.40

\section{VOST Analytical Results Summary Table A-11. Run 2, VOST Tenax ${ }^{\circledR}$ Tube Pair Field Blank}

Field Sample Name: Sample Description: Field Sample Number(s): STL Sample Number(s).
Volatile Organic Sampling Train (VOST)

VOST Tenax ${ }^{\oplus}$ Tube Pair Field Blank for Volatile Organic Compounds Analysis A-3392

H1F250144-019

\begin{tabular}{|c|c|c|c|c|c|c|c|}
\hline \multirow[b]{2}{*}{ Analyte } & \multirow{2}{*}{$\begin{array}{c}\text { CAS } \\
\text { Registry } \\
\text { Number }\end{array}$} & \multicolumn{6}{|c|}{$\begin{array}{c}\text { Tenax }{ }^{(3)} \text { Tube Pair } \\
\text { Field Blank } \\
\text { ( } \mu \text { g/Sample) }\end{array}$} \\
\hline & & Lab Result $^{1}$ & $\mathrm{MDL}^{2}$ & $\mathbf{R D L}^{3}$ & $\mathbf{R L}^{4}$ & Risk Result $^{5}$ & Flag $^{6}$ \\
\hline Acetone & $67-64-1$ & 0.027 & 0.024 & 0.063 & 0.10 & $<0.063$ & $\mathrm{~J}, \mathrm{~B}$ \\
\hline Acrylonitrile & $107-13-1$ & ND & 0.11 & 0.29 & 0.50 & $<0.29$ & \\
\hline Benzene & $71-43-2$ & ND & 0.0064 & 0.017 & 0.025 & $<0.017$ & \\
\hline Bromobenzene & $108-86-1$ & ND & 0.0039 & 0.010 & 0.025 & $<0.010$ & \\
\hline Bromochloromethane & $74-97-5$ & ND & 0.0056 & 0.015 & 0.025 & $<0.015$ & \\
\hline Bromodichloromethane & $75-27-4$ & ND & 0.0042 & 0.011 & 0.025 & $<0.011$ & \\
\hline Bromoform & $75-25-2$ & ND & 0.0074 & 0.019 & 0.025 & $<0.019$ & \\
\hline Bromomethane & $74-83-9$ & ND & 0.0059 & 0.015 & 0.050 & $<0.015$ & \\
\hline 2-Butanone & $78-93-3$ & ND & 0.038 & 0.10 & 0.10 & $<0.10$ & \\
\hline n-Butylbenzene & $104-51-8$ & ND & 0.0061 & 0.016 & 0.025 & $<0.016$ & \\
\hline sec-Butylbenzene & $135-98-8$ & ND & 0.0032 & 0.0084 & 0.025 & $<0.0084$ & \\
\hline tert-Butylbenzene & $98-06-6$ & ND & 0.0062 & 0.016 & 0.025 & $<0.016$ & \\
\hline Carbon disulfide & $75-15-0$ & ND & 0.0070 & 0.018 & 0.025 & $<0.018$ & \\
\hline Carbon tetrachloride & $56-23-5$ & ND & 0.0069 & 0.018 & 0.025 & $<0.018$ & \\
\hline Chlorobenzene & $108-90-7$ & ND & 0.0032 & 0.0084 & 0.025 & $<0.0084$ & \\
\hline Chlorodibromomethane & $124-48-1$ & ND & 0.0056 & 0.015 & 0.025 & $<0.015$ & \\
\hline Chloroethane & $75-00-3$ & ND & 0.0068 & 0.018 & 0.050 & $<0.018$ & \\
\hline Chloroform & $67-66-3$ & ND & 0.0070 & 0.018 & 0.025 & $<0.018$ & \\
\hline Chloromethane & $74-87-3$ & ND & 0.0048 & 0.013 & 0.050 & $<0.013$ & \\
\hline 2-Chlorotoluene & $95-49-8$ & ND & 0.0018 & 0.0047 & 0.025 & $<0.0047$ & \\
\hline 4-Chlorotoluene & $106-43-4$ & ND & 0.0018 & 0.0047 & 0.025 & $<0.0047$ & \\
\hline 1,2-Dibromo-3-chloropropane & $96-12-8$ & ND & 0.011 & 0.029 & 0.050 & $<0.029$ & \\
\hline 1,2-Dibromoethane & $106-93-4$ & ND & 0.0075 & 0.020 & 0.025 & $<0.020$ & \\
\hline Dibromomethane & $74-95-3$ & ND & 0.0064 & 0.017 & 0.025 & $<0.017$ & \\
\hline 1,2-Dichlorobenzene & $95-50-1$ & ND & 0.0077 & 0.020 & 0.025 & $<0.020$ & \\
\hline 1,3-Dichlorobenzene & $541-73-1$ & ND & 0.0038 & 0.010 & 0.025 & $<0.010$ & \\
\hline 1,4-Dichlorobenzene & $106-46-7$ & ND & 0.0055 & 0.014 & 0.025 & $<0.014$ & \\
\hline Dichlorodifluoromethane & $75-71-8$ & ND & 0.0051 & 0.013 & 0.025 & $<0.013$ & \\
\hline
\end{tabular}


BECHTEL BWXT IDAHO, LLC (BBWI)

INTEC HLLWE Effluent Gas Emissions Inventory

Idaho National Engineering and Environmental Laboratory (INEEL)

STL Knoxville Project Number: 142503.40

VOST Analytical Results Summary (Continued)

Table A-11. Run 2, VOST Tenax ${ }^{\circledR}$ Tube Pair Field Blank

\begin{tabular}{|c|c|c|c|c|c|c|c|}
\hline \multirow[b]{2}{*}{ Analyte } & \multirow{2}{*}{$\begin{array}{c}\text { CAS } \\
\text { Registry } \\
\text { Number }\end{array}$} & \multicolumn{6}{|c|}{ 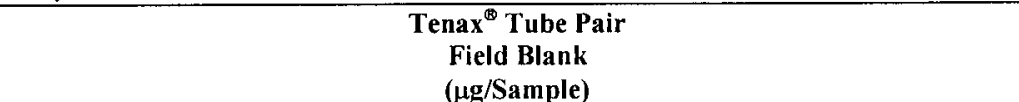 } \\
\hline & & Lab Result ${ }^{1}$ & $\mathrm{MDL}^{2}$ & $\mathrm{RDL}^{3}$ & $\mathbf{R L}^{4}$ & Risk Result ${ }^{5}$ & Flag $^{6}$ \\
\hline 1,1-Dichloroethane & $75-34-3$ & ND & 0.0064 & 0.017 & 0.025 & $<0.017$ & \\
\hline 1,2-Dichloroethane & $107-06-2$ & ND & 0.0066 & 0.017 & 0.025 & $<0.017$ & \\
\hline 1,1-Dichloroethene & $75-35-4$ & ND & 0.0067 & 0.018 & 0.025 & $<0.018$ & \\
\hline cis-1,2-Dichloroethene & $156-59-2$ & ND & 0.0062 & 0.016 & 0.025 & $<0.016$ & \\
\hline trans-1,2-Dichloroethene & $156-60-5$ & ND & 0.0074 & 0.019 & 0.025 & $<0.019$ & \\
\hline 1,2-Dichloropropane & $78-87-5$ & ND & 0.0049 & 0.013 & 0.025 & $<0.013$ & \\
\hline 1,3-Dichloropropane & $142-28-9$ & ND & 0.0073 & 0.019 & 0.025 & $<0.019$ & \\
\hline 2,2-Dichloropropane & $594-20-7$ & ND & 0.0070 & 0.018 & 0.025 & $<0.018$ & \\
\hline 1,1-Dichloropropene & $563-58-6$ & ND & 0.0077 & 0.020 & 0.025 & $<0.020$ & \\
\hline cis-1,3-Dichloropropene & $10061-01-5$ & ND & 0.0046 & 0.012 & 0.025 & $<0.012$ & \\
\hline trans-1,3-Dichloropropene & $10061-02-6$ & ND & 0.0059 & 0.015 & 0.025 & $<0.015$ & \\
\hline Ethylbenzene & $100-41-4$ & ND & 0.0035 & 0.0092 & 0.025 & $<0.0092$ & \\
\hline Hexachlorobutadiene & $87-68-3$ & ND & 0.012 & 0.031 & 0.025 & $<0.025$ & \\
\hline 2-Hexanone & $591-78-6$ & ND & 0.024 & 0.063 & 0.10 & $<0.063$ & \\
\hline Isopropylbenzene & $98-82-8$ & ND & 0.0024 & 0.0063 & 0.025 & $<0.0063$ & \\
\hline p-lsopropyltoluene & $99-87-6$ & ND & 0.0044 & 0.012 & 0.025 & $<0.012$ & \\
\hline Methylene chloride & $75-09-2$ & 0.025 & 0.016 & 0.042 & 0.025 & 0.025 & $\mathrm{~B}$ \\
\hline 4-Methyl-2-pentanone & $108-10-1$ & ND & 0.027 & 0.071 & 0.10 & $<0.071$ & \\
\hline Naphthalene & $91-20-3$ & ND & 0.014 & 0.037 & 0.025 & $<0.025$ & \\
\hline n-Propylbenzene & $103-65-1$ & ND & 0.0021 & 0.0055 & 0.025 & $<0.0055$ & \\
\hline Styrene & $100-42-5$ & ND & 0.0026 & 0.0068 & 0.025 & $<0.0068$ & \\
\hline 1,1,1,2-Tetrachloroethane & $630-20-6$ & ND & 0.0037 & 0.0097 & 0.025 & $<0.0097$ & \\
\hline 1,1,2,2-Tetrachloroethane & $79-34-5$ & ND & 0.0097 & 0.025 & 0.025 & $<0.025$ & \\
\hline Tetrachloroethene & $127-18-4$ & ND & 0.0062 & 0.016 & 0.025 & $<0.016$ & \\
\hline Toluene & $108-88-3$ & ND & 0.0025 & 0.0066 & 0.025 & $<0.0066$ & \\
\hline 1,2,3-Trichlorobenzene & $87-61-6$ & ND & 0.014 & 0.037 & 0.025 & $<0.025$ & \\
\hline 1,2,4-Trichlorobenzene & $120-82-1$ & ND & 0.013 & 0.034 & 0.025 & $<0.025$ & \\
\hline 1,1,1-Trichloroethane & $71-55-6$ & ND & 0.0082 & 0.022 & 0.025 & $<0.022$ & \\
\hline 1,1,2-Trichloroethane & $79-00-5$ & ND & 0.0070 & 0.018 & 0.025 & $<0.018$ & \\
\hline Trichloroethene & $79-01-6$ & ND & 0.0065 & 0.017 & 0.025 & $<0.017$ & \\
\hline Trichlorofluoromethane & $75-69-4$ & ND & 0.0068 & 0.018 & 0.050 & $<0.018$ & \\
\hline 1,2,3-Trichloropropane & $96-18-4$ & ND & 0.010 & 0.026 & 0.025 & $<0.025$ & \\
\hline 1,2,4-Trimethylbenzene & $95-63-6$ & ND & 0.0029 & 0.0076 & 0.025 & $<0.0076$ & \\
\hline
\end{tabular}

Last saved by Patti Carswell on 02/05/02 at 8:07 AM

E:My Documents\HLLWE SamplinglFinal Report\Appendix AlTable A-11.0031_FB_3392.doc

Created on 2/5/2002 8:07 AM 
BECHTEL BWXT IDAHO, LLC (BBWI)

INTEC HLLWE Effluent Gas Emissions Inventory

Idaho National Engineering and Environmental Laboratory (INEEL)

STL Knoxville Project Number: 142503.40

VOST Analytical Results Summary (Continued)

Table A-11. Run 2, VOST Tenax ${ }^{\circledR}$ Tube Pair Field Blank

\begin{tabular}{|c|c|c|c|c|c|c|c|}
\hline \multirow[b]{2}{*}{ Analyte } & \multirow{2}{*}{$\begin{array}{c}\text { CAS } \\
\text { Registry } \\
\text { Number }\end{array}$} & \multicolumn{6}{|c|}{$\begin{array}{c}\text { Tenax }{ }^{\circledast} \text { Tube Pair } \\
\text { Field Blank } \\
(\mu g / \text { Sample })\end{array}$} \\
\hline & & Lab Result ${ }^{1}$ & $\mathrm{MDL}^{2}$ & $\mathbf{R D L}^{3}$ & $\mathbf{R L}^{4}$ & Risk Result $^{5}$ & Flag $^{6}$ \\
\hline 1,3,5-Trimethylbenzene & $108-67-8$ & ND & 0.0019 & 0.0050 & 0.025 & $<0.0050$ & \\
\hline Vinyl chloride & $75-01-4$ & ND & 0.0025 & 0.0066 & 0.025 & $<0.0066$ & \\
\hline m-Xylene \& p-Xylene & $136777-61-2$ & ND & 0.025 & 0.066 & 0.050 & $<0.050$ & \\
\hline o-Xylene & $95-47-6$ & ND & 0.0025 & 0.0066 & 0.025 & $<0.0066$ & \\
\hline
\end{tabular}

Surrogate Recoveries:

\begin{tabular}{|lcc|}
\hline Surrogate Compound & $\begin{array}{c}\text { Percent Recovery } \\
(\%)\end{array}$ & $\begin{array}{c}\text { Laboratory Recovery } \\
\text { Limits } \\
(\%)\end{array}$ \\
\hline Dibromofluoromethane & & $50-150 \%$ \\
$1,2-$ Dichloroethane- $\mathrm{d}_{4}$ & $76 \%$ & $50-150 \%$ \\
Toluene- $\mathrm{d}_{8}$ & $70 \%$ & $50-150 \%$ \\
Bromofluorobenzene & $102 \%$ & $50-150 \%$ \\
\end{tabular}

Last saved by Patti Carswell on 02/05/02 at 8:07 AM 
BECHTEL BWXT IDAHO, LLC (BBWI)

INTEC HLLWE Effluent Gas Emissions Inventory

Idaho National Engineering and Environmental Laboratory (INEEL)

STL Knoxville Project Number: 142503.40

VOST Analytical Results Summary (Continued)

Table A-11. Run 2, VOST Tenax ${ }^{\circledR}$ Tube Pair Field Blank

Sample Collection and Analysis Dates:

\begin{tabular}{|l|c|}
\cline { 2 - 2 } \multicolumn{1}{c|}{} & Date \\
\hline Date(s) Collected: & June 21,2001 \\
\hline Date(s) of Extraction: & July 02, 2001 \\
\hline Date(s) of Analysis: & July 02, 2001 \\
\hline
\end{tabular}

Sample Collection. Preparation. and Analysis Dates:

SW-846 Method 0031: SW-846 Method 5041A

SW-846 Method 8260B
"Sampling Method for Volatile Organic Compounds (SMVOC)"

"Analysis for Desorption of Sorbent Cartridges from Volatile Organic Sampling Train (VOST)"

"Volatile Organic Compounds by Gas Chromatography/Mass Spectrometry (GC/MS)"

vOST Tentatively Identified Compound (TIC) Summary

\begin{tabular}{|llccc|}
\hline TIC $^{7}$ & $\begin{array}{c}\text { CAS } \\
\text { Number }\end{array}$ & $\begin{array}{c}\text { Approximate } \\
\text { Retention Time } \\
(\text { min.) }\end{array}$ & $\begin{array}{c}\text { Sample } \\
\text { Result } \\
(\mu \mathrm{g})\end{array}$ & $\begin{array}{c}\text { TIC } \\
\text { Flag }\end{array}$ \\
\hline Hexane, 3-methyl- & $589-34-4$ & 4.46 & 0.028 & $\mathrm{~N}, \mathrm{~J}, \mathrm{M}$ \\
\hline
\end{tabular}




\section{BECHTEL BWXT IDAHO, LLC (BBWI) \\ INTEC HLLWE Effluent Gas Emissions Inventory Idaho National Engineering and Environmental Laboratory (INEEL) STL Knoxville Project Number: 142503.40}

\section{Footnotes:}

1 This value is the laboratory sample result. When the analytical result is "ND" or not detected, the laboratory analysis did not detect the analyte down to the MDL.

2 This value is the laboratory Method Detection Limit (MDL) derived according to requirements outlined in 40 CFR Part 136 , Appendix B.

3 The RDL is the Reliable Detection Limit. The RDL is the detection level recommended by EPA's National Research Laboratory in Cincinnati, Ohio, Environmental Monitoring Systems Laboratory (EMSL) in Cincinnati, Ohio, American Chemical Society (ACS) Committee on Environmental Improvement and the Drinking Water Standards Division (DWSD). It is defined as 2.623 times the MDL (2.623 X MDL).

4 The RL is the laboratory Reporting Limit (RL).

5 Based on the selection rules, the bolded value is the value or default value assigned to the analyte using the following guidelines:

- When the analytical result is greater than the laboratory reporting limit (RL), the result selected by boldface type is the actual analytical result or "hit" determined by the laboratory.

- When the analytical result is greater than the reliable detection level (RDL), but less than the laboratory reporting limit (RL), the result selected by boldface type is the actual analytical result or "hit" determined by the laboratory.

- When the analytical result is less than the RDL, but greater than the method detection limit (MDL), the result selected by boldface type is the RDL.

- When the analytical result is not detected down to the MDL, the result selected by boldface type is the RDL.

- It should be noted that when the RDL is selected using the guidelines above, but the RL is less than the RDL, the RL is included as the "Risk Result".

o This flag is the laboratory data flag that corresponds to EPA guidelines. The data flags for these samples are as follows:

- A "U" qualifier indicates that this analyte was analyzed for, but was not detected down to the MDL.

- An "E" flag indicates that the result exceeded the upper calibration range. The analytical result is therefore an estimated value.

- A " J" flag indicates that this compound was detected, but at a concentration below the laboratory RL. The analytical result is therefore an estimated value.

- A "B" flag indicates that this compound was found in the associated laboratory method blank. Under these conditions this value is regarded as an estimated value.

- A "Y" flag indicates that this compound is an indistinguishable isomer as a tentatively identified compound (TIC).

- An "N" flag indicates that there is presumptive evidence that this compound is present in the sample based on spectral evidence.

- An " $\mathrm{M}$ " flag indicates that this result was measured against the nearest internal standard and assumed a response factor of one (1).

- A "D" flag indicates that this result was obtained by a dilution of the sample. The original analysis yielded an analytical result that exceeded the calibration range.

7 The tentatively identified compounds (TICs) were identified by conducting a mass spectral library search using the NBS library of data. 



\section{BECHTEL BWXT IDAHO, LLC (BBWI) \\ INTEC HLLWE Effluent Gas Emissions Inventory \\ Idaho National Engineering and Environmental Laboratory (INEEL) \\ STL Knoxville Project Number: 142503.40}

\section{VOST Analytical Results Summary Table A-12. Run 2, VOST Anasorb 747 Tube Field Blank}

Field Sample Name:

Sample Description:

Field Sample Number(s):

STL Sample Number(s)
Volatile Organic Sampling Train (VOST)

VOST Anasorb 747 Tube Field Blank for Volatile Organic Compounds Analysis A-3393

H1F250144-020

\begin{tabular}{|c|c|c|c|c|c|c|c|}
\hline \multirow[b]{2}{*}{ Analyte } & \multirow{2}{*}{$\begin{array}{c}\text { CAS } \\
\text { Registry } \\
\text { Number }\end{array}$} & \multicolumn{6}{|c|}{$\begin{array}{c}\text { Anasorb } 747 \text { Tube } \\
\text { Field Blank } \\
\text { ( } \mu \mathrm{g} / \text { Sample })\end{array}$} \\
\hline & & Lab Result $^{1}$ & $\mathrm{MDL}^{2}$ & $\mathbf{R D L}^{3}$ & $\mathbf{R L}^{4}$ & Risk Result & Flag $^{6}$ \\
\hline Acetone & $67-64-1$ & 0.034 & 0.024 & 0.063 & 0.10 & $<0.063$ & $\mathrm{~J}, \mathrm{~B}$ \\
\hline Acrylonitrile & $107-13-1$ & ND & 0.11 & 0.29 & 0.50 & $<0.29$ & \\
\hline Benzene & $71-43-2$ & ND & 0.0064 & 0.017 & 0.025 & $<0.017$ & \\
\hline Bromobenzene & $108-86-1$ & ND & 0.0039 & 0.010 & 0.025 & $<0.010$ & \\
\hline Bromochloromethane & $74-97-5$ & ND & 0.0056 & 0.015 & 0.025 & $<0.015$ & \\
\hline Bromodichloromethane & $75-27-4$ & ND & 0.0042 & 0.011 & 0.025 & $<0.011$ & \\
\hline Bromoform & $75-25-2$ & ND & 0.0074 & 0.019 & 0.025 & $<0.019$ & \\
\hline Bromomethane & $74-83-9$ & ND & 0.0059 & 0.015 & 0.050 & $<0.015$ & \\
\hline 2-Butanone & $78-93-3$ & ND & 0.038 & 0.10 & 0.10 & $<0.10$ & \\
\hline n-Butylbenzene & $104-51-8$ & $\mathrm{ND}$ & 0.0061 & 0.016 & 0.025 & $<0.016$ & \\
\hline sec-Butylbenzene & $135-98-8$ & ND & 0.0032 & 0.0084 & 0.025 & $<0.0084$ & \\
\hline tert-Butylbenzene & $98-06-6$ & ND & 0.0062 & 0.016 & 0.025 & $<0.016$ & \\
\hline Carbon disulfide & $75-15-0$ & ND & 0.0070 & 0.018 & 0.025 & $<0.018$ & \\
\hline Carbon tetrachloride & $56-23-5$ & ND & 0.0069 & 0.018 & 0.025 & $<0.018$ & \\
\hline Chlorobenzene & $108-90-7$ & ND & 0.0032 & 0.0084 & 0.025 & $<0.0084$ & \\
\hline Chlorodibromomethane & $124-48-1$ & ND & 0.0056 & 0.015 & 0.025 & $<0.015$ & \\
\hline Chloroethane & $75-00-3$ & ND & 0.0068 & 0.018 & 0.050 & $<0.018$ & \\
\hline Chloroform & $67-66-3$ & ND & 0.0070 & 0.018 & 0.025 & $<0.018$ & \\
\hline Chloromethane & $74-87-3$ & ND & 0.0048 & 0.013 & 0.050 & $<0.013$ & \\
\hline 2-Chlorotoluene & $95-49-8$ & ND & 0.0018 & 0.0047 & 0.025 & $<0.0047$ & \\
\hline 4-Chlorotoluene & $106-43-4$ & ND & 0.0018 & 0.0047 & 0.025 & $<0.0047$ & \\
\hline 1,2-Dibromo-3-chloropropane & $96-12-8$ & ND & 0.011 & 0.029 & 0.050 & $<0.029$ & \\
\hline 1,2-Dibromoethane & $106-93-4$ & ND & 0.0075 & 0.020 & 0.025 & $<0.020$ & \\
\hline Dibromomethane & $74-95-3$ & ND & 0.0064 & 0.017 & 0.025 & $<0.017$ & \\
\hline 1,2-Dichlorobenzene & $95-50-1$ & ND & 0.0077 & 0.020 & 0.025 & $<0.020$ & \\
\hline 1,3-Dichlorobenzene & $541-73-1$ & ND & 0.0038 & 0.010 & 0.025 & $<0.010$ & \\
\hline 1,4-Dichlorobenzene & $106-46-7$ & ND & 0.0055 & 0.014 & 0.025 & $<0.014$ & \\
\hline Dichlorodifluoromethane & $75-71-8$ & ND & 0.0051 & 0.013 & 0.025 & $<0.013$ & \\
\hline
\end{tabular}

Last saved by Patti Carswell on 02/05/02 at 8:11 AM 


\section{BECHTEL BWXT IDAHO, LLC (BBWI) \\ INTEC HLLWE Effluent Gas Emissions Inventory \\ Idaho National Engineering and Environmental Laboratory (INEEL) \\ STL Knoxville Project Number: 142503.40}

\section{VOST Analytical Results Summary (Continued)}

Table A-12. Run 2, VOST Anasorb 747 Tube Field Blank

\begin{tabular}{|c|c|c|c|c|c|c|c|}
\hline \multirow[b]{2}{*}{ Analyte } & \multirow{2}{*}{$\begin{array}{c}\text { CAS } \\
\text { Registry } \\
\text { Number }\end{array}$} & \multicolumn{6}{|c|}{$\begin{array}{c}\text { Anasorb 747 Tube } \\
\text { Field Blank } \\
(\mu \mathrm{g} / \text { Sample })\end{array}$} \\
\hline & & Lab Result ${ }^{1}$ & $\mathrm{MDL}^{2}$ & $\mathbf{R D L}^{3}$ & $\mathbf{R L}^{+}$ & Risk Result $^{5}$ & Flag $^{6}$ \\
\hline 1,1-Dichloroethane & $75-34-3$ & ND & 0.0064 & 0.017 & 0.025 & $<0.017$ & \\
\hline 1,2-Dichloroethane & $107-06-2$ & ND & 0.0066 & 0.017 & 0.025 & $<0.017$ & \\
\hline 1,1-Dichloroethene & $75-35-4$ & ND & 0.0067 & 0.018 & 0.025 & $<0.018$ & \\
\hline cis-1,2-Dichloroethene & $156-59-2$ & ND & 0.0062 & 0.016 & 0.025 & $<0.016$ & \\
\hline trans-1,2-Dichloroethene & $156-60-5$ & ND & 0.0074 & 0.019 & 0.025 & $<0.019$ & \\
\hline 1,2-Dichloropropane & $78-87-5$ & ND & 0.0049 & 0.013 & 0.025 & $<0.013$ & \\
\hline 1,3-Dichloropropane & $142-28-9$ & ND & 0.0073 & 0.019 & 0.025 & $<0.019$ & \\
\hline 2,2-Dichloropropane & $594-20-7$ & ND & 0.0070 & 0.018 & 0.025 & $<0.018$ & \\
\hline 1,1-Dichloropropene & $563-58-6$ & ND & 0.0077 & 0.020 & 0.025 & $<0.020$ & \\
\hline cis-1,3-Dichloropropene & $10061-01-5$ & $\mathrm{ND}$ & 0.0046 & 0.012 & 0.025 & $<0.012$ & \\
\hline trans-1,3-Dichloropropene & $10061-02-6$ & ND & 0.0059 & 0.015 & 0.025 & $<0.015$ & \\
\hline Ethylbenzene & $100-4 !-4$ & ND & 0.0035 & 0.0092 & 0.025 & $<0.0092$ & \\
\hline Hexachlorobutadiene & $87-68-3$ & ND & 0.012 & 0.031 & 0.025 & $<0.025$ & \\
\hline 2-Hexanone & $591-78-6$ & ND & 0.024 & 0.063 & 0.10 & $<0.063$ & \\
\hline Isopropylbenzene & $98-82-8$ & ND & 0.0024 & 0.0063 & 0.025 & $<0.0063$ & \\
\hline p-Isopropyltoluene & $99-87-6$ & ND & 0.0044 & 0.012 & 0.025 & $<0.012$ & \\
\hline Methylene chloride & $75-09-2$ & 0.027 & 0.016 & 0.042 & 0.025 & 0.027 & $\mathrm{~B}$ \\
\hline 4-Methyl-2-pentanone & $108-10-1$ & ND & 0.027 & 0.071 & 0.10 & $<0.071$ & \\
\hline Naphthalene & $91-20-3$ & ND & 0.014 & 0.037 & 0.025 & $<0.025$ & \\
\hline n-Propylbenzene & $103-65-1$ & ND & 0.0021 & 0.0055 & 0.025 & $<0.0055$ & \\
\hline Styrene & $100-42-5$ & ND & 0.0026 & 0.0068 & 0.025 & $<0.0068$ & \\
\hline 1,1,1,2-Tetrachloroethane & $630-20-6$ & $\mathrm{ND}$ & 0.0037 & 0.0097 & 0.025 & $<0.0097$ & \\
\hline 1,1,2,2-Tetrachloroethane & $79-34-5$ & ND & 0.0097 & 0.025 & 0.025 & $<0.025$ & \\
\hline Tetrachloroethene & $127-18-4$ & ND & 0.0062 & 0.016 & 0.025 & $<0.016$ & \\
\hline Toluene & $108-88-3$ & 0.12 & 0.0025 & 0.0066 & 0.025 & 0.12 & \\
\hline 1,2,3-Trichlorobenzene & $87-61-6$ & ND & 0.014 & 0.037 & 0.025 & $<0.025$ & \\
\hline 1,2,4-Trichlorobenzene & $120-82-1$ & ND & 0.013 & 0.034 & 0.025 & $<0.025$ & \\
\hline 1,1,1-Trichloroethane & $71-55-6$ & ND & 0.0082 & 0.022 & 0.025 & $<0.022$ & \\
\hline 1,1,2-Trichloroethane & $79-00-5$ & ND & 0.0070 & 0.018 & 0.025 & $<0.018$ & \\
\hline Trichloroethene & $79-01-6$ & ND & 0.0065 & 0.017 & 0.025 & $<0.017$ & \\
\hline Trichlorofluoromethane & $75-69-4$ & ND & 0.0068 & 0.018 & 0.050 & $<0.018$ & \\
\hline 1,2,3-Trichloropropane & $96-18-4$ & ND & 0.010 & 0.026 & 0.025 & $<0.025$ & \\
\hline 1,2,4-Trimethylbenzene & $95-63-6$ & ND & 0.0029 & 0.0076 & 0.025 & $<0.0076$ & \\
\hline
\end{tabular}

Last saved by Patti Carswell on 02/05/02 at 8:11 AM

G:INNEEL HLLWE Report 2001 0031 Train Reports $\backslash V O S T$ 0031_FB_3393.doc

Created on 8/2/2001 3:55 PM 
BECHTEL BWXT IDAHO, LLC (BBWI)

INTEC HLLWE Effluent Gas Emissions Inventory

Idaho National Engineering and Environmental Laboratory (INEEL)

STL Knoxville Project Number: 142503.40

VOST Analytical Results Summary (Continued)

Table A-12. Run 2, VOST Anasorb 747 Tube Field Blank

\begin{tabular}{|c|c|c|c|c|c|c|c|}
\hline \multirow[b]{2}{*}{ Analyte } & \multirow{2}{*}{$\begin{array}{c}\text { CAS } \\
\text { Registry } \\
\text { Number }\end{array}$} & \multicolumn{6}{|c|}{$\begin{array}{c}\text { Anasorb } 747 \text { Tube } \\
\text { Field Blank } \\
(\mu \mathrm{g} / \text { Sample }) \\
\end{array}$} \\
\hline & & Lab Result & $\mathrm{MDL}^{2}$ & $\mathrm{RDL}^{3}$ & $\mathbf{R L ^ { 4 }}$ & Risk Result $^{5}$ & Flag $^{6}$ \\
\hline 1,3,5-Trimethylbenzene & $108-67-8$ & ND & 0.0019 & 0.0050 & 0.025 & $<0.0050$ & \\
\hline Vinyl chloride & $75-01-4$ & ND & 0.0025 & 0.0066 & 0.025 & $<0.0066$ & \\
\hline m-Xylene \& p-Xylene & $136777-61-2$ & ND & 0.025 & 0.066 & 0.050 & $<0.050$ & \\
\hline o-Xylene & $95-47-6$ & ND & 0.0025 & 0.0066 & 0.025 & $<0.0066$ & \\
\hline
\end{tabular}

Surrogate Recoveries:

\begin{tabular}{|lcc|}
\hline Surrogate Compound & $\begin{array}{c}\text { Percent Recovery } \\
(\%)\end{array}$ & $\begin{array}{c}\text { Limits } \\
(\%)\end{array}$ \\
\hline Dibromofluoromethane & & $50-150 \%$ \\
$1,2-$ Dichloroethane- $\mathrm{d}_{4}$ & $77 \%$ & $50-150 \%$ \\
Toluene- $\mathrm{d}_{8}$ & $68 \%$ & $50-150 \%$ \\
Bromofluorobenzene & $99 \%$ & $50-150 \%$ \\
\end{tabular}


BECHTEL BWXT IDAHO, LLC (BBWI)

INTEC HLLWE Effluent Gas Emissions Inventory

Idaho National Engineering and Environmental Laboratory (INEEL)

STL Knoxville Project Number: 142503.40

\section{VOST Analytical Results Summary (Continued)}

\section{Table A-12. Run 2, VOST Anasorb 747 Tube Field Blank}

Sample Collection and Analusis Dates:

\begin{tabular}{|l|c|}
\cline { 2 - 2 } \multicolumn{1}{c|}{} & Date \\
\hline Date(s) Collected: & June 21, 2001 \\
\hline Date(s) of Extraction: & July 02, 2001 \\
\hline Date(s) of Analysis: & July 02, 2001 \\
\hline
\end{tabular}

Sample Collection. Preparation. and Analysis Dates:

SW-846 Method 0031: SW-846 Method 5041A: SW-846 Method 8260B:
"Sampling Method for Volatile Organic Compounds (SMVOC)"

"Analysis for Desorption of Sorbent Cartridges from Volatile Organic Sampling Train (VOST)"

"Volatile Organic Compounds by Gas Chromatography/Mass Spectrometry (GC/MS)"

VOST Tentatively Identified Compound (TIC) Summary

\begin{tabular}{|lcccc|}
\hline TIC $^{7}$ & $\begin{array}{c}\text { CAS } \\
\text { Number }\end{array}$ & $\begin{array}{c}\text { Approximate } \\
\text { Retention Time } \\
(\text { min.) }\end{array}$ & $\begin{array}{c}\text { Sample } \\
\text { Result } \\
(\mu)^{\prime}\end{array}$ & $\begin{array}{c}\text { TIC } \\
\text { Flag }\end{array}$ \\
\hline Cyclohexane, methyl- & & & 0.17 & $\mathrm{~N}, \mathrm{~J}, \mathrm{M}$ \\
Hexane, 2,4-dimethyl- & $108-87-2$ & 5.32 & 0.16 & $\mathrm{~N}, \mathrm{~J}, \mathrm{M}$ \\
Cyclopentane, ethyl- & $589-43-5$ & 5.40 & 0.044 & $\mathrm{~N}, \mathrm{~J}, \mathrm{M}$ \\
Pentane, 2,3-dimethyl- & $1640-89-7$ & 5.49 & 0.30 & $\mathrm{~N}, \mathrm{~J}, \mathrm{M}$ \\
Pentane, 3,3-dimethyl- & $565-59-3$ & 4.36 & 0.064 & $\mathrm{~N}, \mathrm{~J}, \mathrm{M}$ \\
Hexane, 2-methyl- & $562-49-2$ & 4.13 & 0.26 & $\mathrm{~N}, \mathrm{~J}, \mathrm{M}$ \\
\hline
\end{tabular}




\section{BECHTEL BWXT IDAHO, LLC (BBWI) INTEC HLLWE Effluent Gas Emissions Inventory Idaho National Engineering and Environmental Laboratory (INEEL) STL Knoxville Project Number: 142503.40}

\section{Footnotes:}

1 This value is the laboratory sample result. When the analytical result is "ND" or not detected, the laboratory analysis did not detect the analyte down to the MDL.

2 This value is the laboratory Method Detection Limit (MDL) derived according to requirements outlined in 40 CFR Part 136 , Appendix B.

3 The RDL is the Reliable Detection Limit. The RDL is the detection level recommended by EPA's National Research Laboratory in Cincinnati, Ohio, Environmental Monitoring Systems Laboratory (EMSL) in Cincinnati, Ohio, American Chemical Society (ACS) Committee on Environmental Improvement and the Drinking Water Standards Division (DWSD). It is defined as 2.623 times the MDL (2.623 X MDL).

4 The RL is the laboratory Reporting Limit (RL).

5 Based on the selection rules, the bolded value is the value or default value assigned to the analyte using the following guidelines:

- When the analytical result is greater than the laboratory reporting limit (RL), the result selected by boldface type is the actual analytical result or "hit" determined by the laboratory.

- When the analytical result is greater than the reliable detection level (RDL), but less than the laboratory reporting limit (RL), the result selected by boldface type is the actual analytical result or "hit" determined by the laboratory.

- When the analytical result is less than the RDL, but greater than the method detection limit (MDL), the result selected by boldface type is the RDL.

- When the analytical result is not detected down to the MDL, the result selected by boldface type is the RDL.

- It should be noted that when the RDL is selected using the guidelines above, but the RL is less than the RDL, the RL is included as the "Risk Result".

6 This flag is the laboratory data flag that corresponds to EPA guidelines. The data flags for these samples are as follows:

- A "U" qualifier indicates that this analyte was analyzed for, but was not detected down to the MDL.

- An "E" flag indicates that the result exceeded the upper calibration range. The analytical result is therefore an estimated value.

- A "J" flag indicates that this compound was detected, but at a concentration below the laboratory RL. The analytical result is therefore an estimated value.

- A "B" flag indicates that this compound was found in the associated laboratory method blank. Under these conditions this value is regarded as an estimated value.

- A "Y" flag indicates that this compound is an indistinguishable isomer as a tentatively identified compound (TIC).

- An "N" flag indicates that there is presumptive evidence that this compound is present in the sample based on spectral evidence.

- An "M" flag indicates that this result was measured against the nearest internal standard and assumed a response factor of one (1).

- A "D" flag indicates that this result was obtained by a dilution of the sample. The original analysis yielded an analytical result that exceeded the calibration range.

7 The tentatively identified compounds (TICs) were identified by conducting a mass spectral library search using the NBS library of data. 



\section{M5 Particulate and Anion Train - Run 1 Analytical Results Summary Table A-13. HLLWE Run ID: 0050-STRT-1}

Field Sample Name: Sample Description: Field Sample Number(s): STL Sample No.:
M5 $\mathrm{HCl} / \mathrm{Cl}_{2}$ and Particulate Train

Particulate Filter and Acetone Probe Rinse Samples for Particulate Determination A-3308 and A-3309

H1F210104-001 and H1F210104-002

\begin{tabular}{|lccc|}
\hline Analyte & $\begin{array}{c}\text { Particulate Filter } \\
\text { Particulate Weight } \\
(\mu \mathrm{g})\end{array}$ & $\begin{array}{c}\text { Acetone Probe Rinse } \\
\text { Particulate Weight } \\
(\mu \mathrm{g})\end{array}$ & $\begin{array}{c}\text { Particulate } \\
\text { Total } \\
\text { Particulate }\end{array}$ \\
\hline & $100 \mathrm{~B}$ & 3,400 & $3,500 \mathrm{~B}$ \\
\hline
\end{tabular}

Field Sample Name: Sample Description: Field Sample Number(s): STL Sample No:
$\mathrm{M} 5 \mathrm{HCl} / \mathrm{Cl}_{2}$ and Particulate Train

$0.1 \mathrm{~N} \mathrm{H}_{2} \mathrm{SO}_{4}$ Impinger Solution for Chloride, Fluoride, Nitrate, and Nitrite Analysis A-3310

H1F210104-003

\begin{tabular}{|c|c|c|c|c|c|c|}
\hline Analyte & $\begin{array}{l}\text { Laboratory } \\
\text { Result }^{2} \\
\text { (Total mg) }\end{array}$ & $\mathrm{MDL}^{3}$ & $\mathbf{R D L}^{4}$ & $\mathbf{R L}^{5}$ & $\begin{array}{c}\text { Risk } \\
\text { Result } \\
\text { (Total mg) }^{6}\end{array}$ & Flag $^{7}$ \\
\hline Chloride (as $\mathrm{HCl})^{8}$ & 1.9 & 1.7 & 4.5 & 2.9 & $<2.9$ & B \\
\hline Fluoride (as HF) ${ }^{9}$ & ND & 0.075 & 0.20 & 1.5 & $<0.20$ & \\
\hline Nitrate (as $\left.\mathrm{HNO}_{3}\right)^{10}$ & 11 & 0.072 & 0.19 & 0.72 & 11 & \\
\hline Nitrite (as $\left.\mathrm{HNO}_{2}\right)^{11}$ & ND & 0.073 & 0.19 & 0.73 & $<0.19$ & \\
\hline
\end{tabular}




\section{M5 Particulate and Anion Train - Run 1 \\ Analytical Results Summary (Continued) \\ Table A-13. HLLWE Run ID: 0050-STRT-1}

Field Sample Name:

Sample Description:

M5 $\mathrm{HCl} / \mathrm{Cl}_{2}$ and Particulate Train

Field Sample Number(s):

$0.1 \mathrm{~N} \mathrm{NaOH}$ Impinger Solution for Chlorine, Fluoride, Nitrate, and Nitrite Analysis

STL Sample No.: A-3311

HIF210104-004

\begin{tabular}{|c|c|c|c|c|c|c|}
\hline Analyte & $\begin{array}{c}\text { Laboratory } \\
\text { Result }^{2} \\
\text { (Total mg) }\end{array}$ & MDL $^{3}$ & $\mathrm{RDL}^{4}$ & $\mathbf{R L}^{5}$ & $\begin{array}{c}\text { Risk } \\
\text { Result } \\
\text { (Total mg) }^{6}\end{array}$ & Flag $^{7}$ \\
\hline Chlorine (as $\left.\mathrm{Cl}_{2}\right)^{12}$ & ND & 0.16 & 0.42 & 0.29 & $<0.29$ & \\
\hline Fluoride (as HF) ${ }^{9}$ & ND & 0.075 & 0.20 & 1.5 & $<0.20$ & \\
\hline Nitrate (as $\left.\mathrm{HNO}_{3}\right)^{10}$ & 0.85 & 0.014 & 0.037 & 0.14 & 0.85 & \\
\hline Nitrite (as $\left.\mathrm{HNO}_{2}\right)^{11}$ & 1.4 & 0.073 & 0.19 & 0.73 & 1.4 & \\
\hline
\end{tabular}

Sample Collection and Analysis Dates:

\begin{tabular}{|l|c|c|c|c|}
\cline { 2 - 5 } \multicolumn{1}{c|}{} & \multicolumn{1}{c|}{$\begin{array}{c}\text { Particulate } \\
\text { Filter }\end{array}$} & $\begin{array}{c}\text { Acetone } \\
\text { Probe Rinse }\end{array}$ & $\begin{array}{c}\mathbf{0 . 1 N} \mathbf{H}_{2} \text { SO }_{4} \text { Impinger } \\
\text { Composite }\end{array}$ & $\begin{array}{c}\mathbf{0 . 1 N} \text { NaOH Impinger } \\
\text { Composite }\end{array}$ \\
\hline $\begin{array}{l}\text { Date(s) Collected: } \\
\text { Date(s) of Preparation- }\end{array}$ & June 07, 2001 & June 07, 2001 & June 07, 2001 & June 07, 2001 \\
Analysis: & June 22-26, 2001 & June 25-26, 2001 & June 29, 2001 & July 02, 2001 \\
& & & & \\
\hline
\end{tabular}

Preparation and Analysis Methods:

EPA Method 5: "Particulate Emissions from Stationary Sources"

SW-846 Method 9056: "Determination of Inorganic Anions by Ion Chromatography"

Last saved by Robin Gifford on E:MMY DOCUMENTS $\backslash$ HLLWE SAMPLINGIFINAL REPORTVAPPENDIX AITABLE A-13. 0050-STRT-1.DOC Last printed on 2/5/2002 10:50 AM2/5/2002 at 10:50 AM 
BECHTEL BWXT IDAHO, LLC (BBWI)

INTEC HLLWE Effluent Gas Emissions Inventory

Idaho National Engineering and Environmental Laboratory (INEEL)

STL Knoxville Project Number: 142503.40

\section{M5 Particulate and Anion Train - Run 1 \\ Analytical Results Summary (Continued) \\ Table A-13. HLLWE Run ID: 0050-STRT-1}

\section{Footnotes:}

1 The Total M-5 Particulate result is the sum of the acetone probe rinse particulate weight and the particulate filter particulate weight.

2 This value is the laboratory sample result. When the analytical result is "ND" or not detected, the laboratory analysis did not detect the analyte down to the method detection limit (MDL).

3 This value is the laboratory MDL derived according to requirements outlined in 40 CFR Part 136, Appendix B. The MDL has been calculated as $\mathrm{Cl}_{2}, \mathrm{HCl}, \mathrm{HF}, \mathrm{HNO}_{3}$, or $\mathrm{HNO}_{2}$, as appropriate.

4 The RDL is the Reliable Detection Limit. The RDL is the detection level recommended by EPA's National Research Laboratory in Cincinnati, Ohio, Environmental Monitoring Systems Laboratory (EMSL) in Cincinnati, Ohio, American Chemical Society (ACS) Committee on Environmental Improvement and the Drinking Water Standards Division (DWSD). It is defined as 2.623 times the MDL (2.623 X MDL). The RDL has been calculated as $\mathrm{Cl}_{2}, \mathrm{HCl}, \mathrm{HF}, \mathrm{HNO}_{3}$, or $\mathrm{HNO}_{2}$, as appropriate

5 The RL is the laboratory Reporting Limit (RL). The RL has been calculated as $\mathrm{Cl}_{2}, \mathrm{HCl}, \mathrm{HF}, \mathrm{HNO}_{3}$, or $\mathrm{HNO}_{2}$, as appropriate.

6 The bolded value for each analyte is the value or default value assigned to the analyte. This value was determined using the following guidelines:

- When the analytical result is greater than the laboratory reporting limit (RL), the "Risk Result" is the actual analytical result or "hit" determined by the laboratory.

- When the analytical result is greater than the reliable detection level (RDL), but less than the laboratory reporting limit (RL), the "Risk Result" is the actual analytical result or "hit" determined by the laboratory.

- When the analytical result is less than the RDL, but greater than the method detection limit (MDL), the default value is the RDL.

- When the analytical result is not detected down to the MDL, the "Risk Result" is the RDL.

- It should be noted that when the RDL is selected as the default value using the guidelines above, but the RDL is greater than the $\mathrm{RL}$, the $\mathrm{RL}$ is used as the risk result.

7 This flag is the laboratory data flag that corresponds to EPA guidelines. The data flags for these samples are as follows:

- A "U" flag indicates that this analyte was analyzed for, but was not detected down to the MDL.

$A$ " $B$ " flag indicates that the result for this analyte was below the RL and is therefore considered to be an estimated value.

8 The calculation of the total milligrams ( $\mathrm{mg}$ ) of $\mathrm{HCl}$ in the Impinger Composite is as follows:

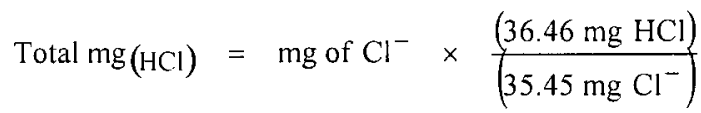

Where: $\quad 36.46=$ the molecular weight of $\mathrm{HCl}$ in $\mathrm{mg} / \mathrm{mg}$-mole and

$35.45=$ the atomic weight of $\mathrm{Cl}^{-}$in $\mathrm{mg} / \mathrm{mg}$-mole.

Last saved by Robin Gifford on E:LMY DOCUMENTS\HLLWE SAMPLINGYFINAL REPORTAPPENDIX AITABLE A-13. 0050-STRT-1.DOC Last printed on 2/5/2002 10:50 AM2/5/2002 at 10:50 AM 
BECHTEL BWXT IDAHO, LLC (BBWI)

INTEC HLLWE Effluent Gas Emissions Inventory

Idaho National Engineering and Environmental Laboratory (INEEL)

STL Knoxville Project Number: 142503.40

\section{M5 Particulate and Anion Train - Run 1 \\ Analytical Results Summary (Continued) \\ Table A-13. HLLWE Run ID: 0050-STRT-1}

9 The calculation of the total milligrams ( $\mathrm{mg}$ ) of HF in Impinger Composite is as follows:

$$
\text { Total } \mathrm{mg}(\mathrm{HF})=\mathrm{mg} \text { of } \mathrm{F}^{-} \times \frac{(20.01 \mathrm{mgHF})}{\left(19.00 \mathrm{mgF}^{-}\right)}
$$

Where: $\quad 20.01=$ the molecular weight of $\mathrm{HF}$ in $\mathrm{mg} / \mathrm{mg}$-mole and

$19.00=$ the atomic weight of $\mathrm{F}^{-}$in $\mathrm{mg} / \mathrm{mg}$-mole.

10 The calculation of the total milligrams (mg) of $\mathrm{HNO}_{3}$ in the Impinger Composite is as follows:

$$
\text { Total } \mathrm{mg}\left(\mathrm{HNO}_{3}\right)=\mathrm{mg} \mathrm{of} \mathrm{NO}_{3}^{-} \times \frac{(63.01 \mathrm{mg} \mathrm{HNO} 3)}{\left.62.00 \mathrm{mg} \mathrm{NO}_{3}^{-}\right)}
$$

Where: $\quad 63.01=$ the molecular weight of $\mathrm{HNO}_{3}$ in $\mathrm{mg} / \mathrm{mg}$-mole and $62.00=$ the molecular weight of $\mathrm{NO}_{3}^{-}{ }^{-}$in $\mathrm{mg} / \mathrm{mg}$-mole.

11 The calculation of the total milligrams ( $\mathrm{mg}$ ) of $\mathrm{HNO}_{2}$ in the Impinger Composite is as follows:

$$
\text { Total } \mathrm{mg}\left(\mathrm{HNO}_{2}\right)=\mathrm{mg} \text { of } \mathrm{NO}_{2}{ }^{-} \times \frac{(47.01 \mathrm{mg} \mathrm{HNO} 2)}{\left(46.01 \mathrm{mg} \mathrm{NO}_{2}^{-}\right)}
$$

Where: $\quad 47.01=$ the molecular weight of $\mathrm{HNO}_{2}$ in $\mathrm{mg} / \mathrm{mg}$-mole and

$46.01=$ the molecular weight of $\mathrm{NO}_{2}^{-}$in $\mathrm{mg} / \mathrm{mg}-\mathrm{mole}$.

12 No additional calculation was required to be performed to obtain the total milligrams (mg) of $\mathrm{Cl}_{2}$ in the $\mathrm{NaOH}$ Impinger Composite. Note that the $\mathrm{NaOH}$ Impingers were treated with $\mathrm{NaS}_{2} \mathrm{O}_{3}$ prior to the analysis of chlorine. 


\section{M5 Particulate and Anion Train - Run 3 Analytical Results Summary Table A-14. HLLWE Run ID: 0050-END-1}

Field Sample Name: Sample Description: Field Sample Number(s): STL Sample No:
M5 $\mathrm{HCl} / \mathrm{Cl}_{2}$ and Particulate Train

Particulate Filter and Acetone Probe Rinse Samples for Particulate Determination A-3338 and A-3339

H1F210104-018 and H1F210104-019

\begin{tabular}{|c|c|c|c|}
\hline Analyte & $\begin{array}{c}\text { Particulate Filter } \\
\text { Particulate Weight } \\
(\mu \mathrm{g})\end{array}$ & $\begin{array}{c}\text { Acetone Probe Rinse } \\
\text { Particulate Weight } \\
(\mu \mathrm{g})\end{array}$ & $\begin{array}{c}\text { Particulate } \\
\text { Total } \\
\text { (Total } \mu \text { g of Particulate) }\end{array}$ \\
\hline Particulate & $300 \mathrm{~B}$ & 2,600 & $2,900 \mathrm{~B}$ \\
\hline
\end{tabular}

Field Sample Name: Sample Description: Field Sample Number(s): STL Sample No.:
M5 $\mathrm{HCl} / \mathrm{Cl}_{2}$ and Particulate Train

$0.1 \mathrm{~N} \mathrm{H}_{2} \mathrm{SO}_{4}$ Impinger Solution for Chloride, Fluoride, Nitrate, and Nitrite Analysis A-3340

H1F210104-020

\begin{tabular}{|c|c|c|c|c|c|c|}
\hline Analyte & $\begin{array}{c}\text { Laboratory } \\
\text { Result }^{2} \\
\text { (Total mg) }\end{array}$ & MDL $^{3}$ & $\mathrm{RDL}^{4}$ & RL $^{5}$ & $\begin{array}{c}\text { Risk } \\
\text { Result } \\
\text { (Total mg) }\end{array}$ & Flag $^{7}$ \\
\hline Chloride (as $\mathrm{HCl})^{8}$ & 2.1 & 1.7 & 4.4 & 2.9 & $<2.9$ & B \\
\hline Fluoride (as $\mathrm{HF})^{9}$ & ND & 0.075 & 0.20 & 1.5 & $<0.20$ & \\
\hline Nitrate (as $\left.\mathrm{HNO}_{3}\right)^{10}$ & 9.8 & 0.072 & 0.19 & 0.72 & 9.8 & \\
\hline Nitrite (as $\left.\mathrm{HNO}_{2}\right)^{\natural}$ & ND & 0.073 & 0.19 & 0.73 & $<0.19$ & \\
\hline
\end{tabular}

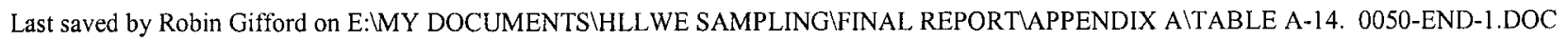
Last printed on 2/5/2002 11:11 AM2/5/2002 at 11:11 AM 
BECHTEL BWXT IDAHO, LLC (BBWI)

INTEC HLLWE Effluent Gas Emissions Inventory

Idaho National Engineering and Environmental Laboratory (INEEL)

STL Knoxville Project Number: 142503.40

\section{M5 Particulate and Anion Train - Run 3 \\ Analytical Results Summary (Continued) \\ Table A-14. HLLWE Run ID: 0050-END-1}

Field Sample Name:

Sample Description:

M5 $\mathrm{HCl} / \mathrm{Cl}_{2}$ and Particulate Train

Field Sample Number(s):

$0.1 \mathrm{~N} \mathrm{NaOH}$ Impinger Solution for Chlorine, Fluoride, Nitrate, and Nitrite Analysis

STL Sample No.: A-3341

H1F210104-021

\begin{tabular}{|c|c|c|c|c|c|c|}
\hline Analyte & $\begin{array}{c}\text { Laboratory } \\
\text { Result }^{2} \\
\text { (Total mg) }^{\text {Tot }}\end{array}$ & $\mathrm{MDL}^{3}$ & RDL $^{4}$ & $\mathbf{R L}^{5}$ & $\begin{array}{c}\text { Risk } \\
\text { Result } \\
\text { (Total mg) }\end{array}$ & Flag $^{7}$ \\
\hline Chlorine (as $\left.\mathrm{Cl}_{2}\right)^{12}$ & 0.42 & 0.32 & 0.85 & 0.57 & $<0.57$ & B \\
\hline Fluoride (as $\mathrm{HF})^{9}$ & ND & 0.075 & 0.20 & 1.5 & $<0.20$ & \\
\hline Nitrate $\left(\text { as } \mathrm{HNO}_{3}\right)^{10}$ & 0.61 & 0.014 & 0.038 & 0.14 & 0.61 & \\
\hline Nitrite (as $\left.\mathrm{HNO}_{2}\right)^{\prime \prime}$ & 3.6 & 0.029 & 0.076 & 0.29 & 3.6 & \\
\hline
\end{tabular}

Sample Collection and Analysis Dates:

\begin{tabular}{|l|c|c|c|c|}
\cline { 2 - 5 } \multicolumn{1}{c|}{} & \multicolumn{1}{c|}{$\begin{array}{c}\text { Particulate } \\
\text { Filter }\end{array}$} & $\begin{array}{c}\text { Acetone } \\
\text { Probe Rinse }\end{array}$ & $\begin{array}{c}\text { 0.1N H } \text { SO }_{4} \text { Impinger } \\
\text { Composite }\end{array}$ & $\begin{array}{c}\text { 0.1N NaOH Impinger } \\
\text { Composite }\end{array}$ \\
\hline $\begin{array}{l}\text { Date(s) Collected: } \\
\begin{array}{l}\text { Date(s) of Preparation- } \\
\text { Analysis: }\end{array}\end{array}$ & June 07, 2001 & June 07, 2001 & June 07, 2001 & June 07, 2001 \\
& June 22-26, 2001 & June 25-26, 2001 & June 29, 2001 & July 02, 2001 \\
\hline
\end{tabular}

Preparation and Analysis Methods:

EPA Method 5: "Particulate Emissions from Stationary Sources"

SW-846 Method 9056: "Determination of Inorganic Anions by Ion Chromatography"

Last saved by Robin Gifford on E:IMY DOCUMENTS\HLLWE SAMPLINGFINAL REPORTAAPPENDIX AITABLE A-14. 0050-END-1.DOC Last printed on 2/5/2002 11:11 AM2/5/2002 at 11:11 AM 
BECHTEL BWXT IDAHO, LLC (BBWI)

INTEC HLLWE Effluent Gas Emissions Inventory

Idaho National Engineering and Environmental Laboratory (INEEL)

STL Knoxville Project Number: 142503.40

\section{M5 Particulate and Anion Train - Run 3 \\ Analytical Results Summary (Continued) Table A-14. HLLWE Run ID: 0050-END-1}

Footnotes:

1 The Total M-5 Particulate result is the sum of the acetone probe rinse particulate weight and the particulate filter particulate weight.

2 This value is the laboratory sample result. When the analytical result is "ND" or not detected, the laboratory analysis did not detect the analyte down to the method detection limit (MDL).

3 This value is the laboratory MDL derived according to requirements outlined in 40 CFR Part 136, Appendix B. The MDL has been calculated as $\mathrm{Cl}_{2}, \mathrm{HCl}, \mathrm{HF}, \mathrm{HNO}_{3}$, or $\mathrm{HNO}_{2}$, as appropriate.

4 The RDL is the Reliable Detection Limit. The RDL is the detection level recommended by EPA's National Research Laboratory in Cincinnati, Ohio, Environmental Monitoring Systems Laboratory (EMSL) in Cincinnati, Ohio, American Chemical Society (ACS) Committee on Environmental Improvement and the Drinking Water Standards Division (DWSD). It is defined as 2.623 times the MDL (2.623 X MDL). The RDL has been calculated as $\mathrm{Cl}_{2}, \mathrm{HCl}, \mathrm{HF}, \mathrm{HNO}_{3}$, or $\mathrm{HNO}_{2}$, as appropriate.

5 The RL is the laboratory Reporting Limit (RL). The RL has been calculated as $\mathrm{Cl}_{2}, \mathrm{HCl}, \mathrm{HF}, \mathrm{HNO}_{3}$, or $\mathrm{HNO}_{2}$, as appropriate.

6 The bolded value for each analyte is the value or default value assigned to the analyte. This value was determined using the following guidelines:

- When the analytical result is greater than the laboratory reporting limit (RL), the "Risk Result" is the actual analytical result or "hit" determined by the laboratory.

- When the analytical result is greater than the reliable detection level (RDL), but less than the laboratory reporting limit (RL), the "Risk Result" is the actual analytical result or "hit" determined by the laboratory.

- When the analytical result is less than the RDL, but greater than the method detection limit (MDL), the default value is the RDL.

- When the analytical result is not detected down to the MDL, the "Risk Result" is the RDL.

- It should be noted that when the RDL is selected as the default value using the guidelines above, but the RDL is greater than the $R L$, the $R L$ is used as the risk result.

7 This flag is the laboratory data flag that corresponds to EPA guidelines. The data flags for these samples are as follows:

- A " $U$ " flag indicates that this analyte was analyzed for, but was not detected down to the MDL.

$A$ " $\mathrm{B}$ " flag indicates that the result for this analyte was below the RL and is therefore considered to be an estimated value.

8 The calculation of the total milligrams (mg) of $\mathrm{HCl}$ in the Impinger Composite is as follows:

$$
\text { Total mg }(\mathrm{HCl})=\mathrm{mg} \mathrm{of} \mathrm{Cl}^{-} \times \frac{(36.46 \mathrm{mg} \mathrm{HCl})}{\left(35.45 \mathrm{mg} \mathrm{Cl}^{-}\right)}
$$

Where: $\quad 36.46=$ the molecular weight of $\mathrm{HCl}$ in $\mathrm{mg} / \mathrm{mg}$-mole and

$35.45=$ the atomic weight of $\mathrm{Cl}^{-}$in $\mathrm{mg} / \mathrm{mg}$-mole.

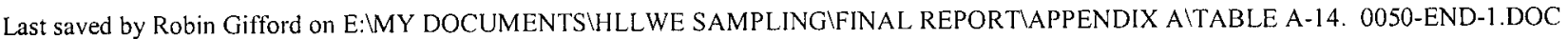
Last printed on 2/5/2002 11:11 AM2/5/2002 at 11:11 AM 
BECHTEL BWXT IDAHO, LLC (BBWI)

INTEC HLLWE Effluent Gas Emissions Inventory

Idaho National Engineering and Environmental Laboratory (INEEL)

STL Knoxville Project Number: 142503.40

\section{M5 Particulate and Anion Train - Run 3 \\ Analytical Results Summary (Continued) \\ Table A-14. HLLWE Run ID: 0050-END-1}

9 The calculation of the total milligrams (mg) of HF in Impinger Composite is as follows:

$$
\text { Total } \mathrm{mg}(\mathrm{HF})=\mathrm{mg} \mathrm{of} \mathrm{F}^{-} \times \frac{(20.01 \mathrm{mgHF})}{\left(19.00 \mathrm{mgF}^{-}\right)}
$$

Where: $\quad 20.01=$ the molecular weight of $\mathrm{HF}$ in $\mathrm{mg} / \mathrm{mg}-$ mole and

$19.00=$ the atomic weight of $F^{-}$in $\mathrm{mg} / \mathrm{mg}^{-}$mole.

10 The calculation of the total milligrams $(\mathrm{mg})$ of $\mathrm{HNO}_{3}$ in the Impinger Composite is as follows:

$$
\text { Total mg }\left(\mathrm{HNO}_{3}\right)=\operatorname{mg} \text { of } \mathrm{NO}_{3}{ }^{-} \times \frac{\left(63.01 \mathrm{mg} \mathrm{HNO}_{3}\right)}{\left(62.00 \mathrm{mg} \mathrm{NO}_{3}^{-}\right)}
$$

Where: $\quad 63.01=$ the molecular weight of $\mathrm{HNO}_{3}$ in $\mathrm{mg} / \mathrm{mg}$-mole and

$62.00=$ the molecular weight of $\mathrm{NO}_{3}{ }^{-}$in $\mathrm{mg} / \mathrm{mg}$-mole.

11 The calculation of the total milligrams ( $\mathrm{mg}$ ) of $\mathrm{HNO}_{2}$ in the Impinger Composite is as follows:

$$
\text { Total } \mathrm{mg}\left(\mathrm{HNO}_{2}\right)=\mathrm{mg} \text { of } \mathrm{NO}_{2}{ }^{-} \times \frac{\left.(47.01 \mathrm{mg} \mathrm{HNO})_{2}\right)}{\left(46.01 \mathrm{mg} \mathrm{NO}{ }^{-}\right)}
$$

Where: $\quad 47.01=$ the molecular weight of $\mathrm{HNO}_{2}$ in $\mathrm{mg} / \mathrm{mg}$-mole and

$46.01=$ the molecular weight of $\mathrm{NO}_{2}{ }^{-}$in $\mathrm{mg} / \mathrm{mg}$-mole.

12 No additional calculation was required to be performed to obtain the total milligrams ( $\mathrm{mg}$ ) of $\mathrm{Cl}_{2}$ in the $\mathrm{NaOH}$ Impinger Composite. Note that the $\mathrm{NaOH}$ Impingers were treated with $\mathrm{NaS}_{2} \mathrm{O}_{3}$ prior to the analysis of chlorine. 


\section{M5 Particulate and Anion Train - Run 2 Analytical Results Summary \\ Table A-15. HLLWE Run ID: 0050-STRT-2}

Field Sample Name:

Sample Description:

Field Sample Number(s):

STL Sample No.:
$\mathrm{M} 5 \mathrm{HCl} / \mathrm{Cl}_{2}$ and Particulate Train

Particulate Filter and Acetone Probe Rinse Samples for Particulate Determination A-3312 and A-3313

H1F210104-005 and H1F210104-006

\begin{tabular}{|lccc|}
\hline Analyte & $\begin{array}{c}\text { Particulate Filter } \\
\text { Particulate Weight } \\
(\mu \mathrm{g})\end{array}$ & $\begin{array}{c}\text { Acetone Probe Rinse } \\
\text { Particulate Weight } \\
(\mu \mathrm{g})\end{array}$ & $\begin{array}{c}\text { Particulate } \\
\text { Total } \\
\text { Particulate }\end{array}$ \\
\hline & 600 & 500 & 1,100 \\
\hline
\end{tabular}

Field Sample Name: Sample Description: Field Sample Number(s): STL Sample No.:

\begin{tabular}{|c|c|c|c|c|c|c|}
\hline Analyte & $\begin{array}{c}\text { Laboratory } \\
\text { Result }^{2} \\
\text { (Total mg) }\end{array}$ & MDL $^{3}$ & $\mathrm{RDL}^{4}$ & $\mathbf{R L}^{5}$ & $\begin{array}{c}\text { Risk } \\
\text { Result } \\
{\text { (Total } \mathrm{mg})^{6}}^{6}\end{array}$ & Flag $^{7}$ \\
\hline Chloride (as $\mathrm{HCl})^{8}$ & 1.7 & 1.6 & 4.3 & 2.9 & $<2.9$ & B \\
\hline Fluoride (as $\mathrm{HF})^{9}$ & ND & 0.074 & 0.19 & 1.5 & $<0.19$ & \\
\hline Nitrate $\left(\text { as } \mathrm{HNO}_{3}\right)^{10}$ & 8.4 & 0.071 & 0.19 & 0.71 & 8.4 & \\
\hline Nitrite $\left(\text { as } \mathrm{HNO}_{2}\right)^{11}$ & ND & 0.14 & 0.38 & 1.4 & $<0.38$ & \\
\hline
\end{tabular}


BECHTEL BWXT IDAHO, LLC (BBWI)

INTEC HLLWE Effluent Gas Emissions Inventory

Idaho National Engineering and Environmental Laboratory (INEEL)

STL Knoxville Project Number: 142503.40

\section{M5 Particulate and Anion Train - Run 2 \\ Analytical Results Summary (Continued) \\ Table A-15. HLLWE Run ID: 0050-STRT-2}

Field Sample Name:

Sample Description:

Field Sample Number(s):

M5 $\mathrm{HCl} / \mathrm{Cl}_{2}$ and Particulate Train

STL Sample No.

$0.1 \mathrm{~N} \mathrm{NaOH}$ Impinger Solution for Chlorine, Fluoride, Nitrate, and Nitrite Analysis

A-3315

HIF210104-008

\begin{tabular}{|c|c|c|c|c|c|c|}
\hline Analyte & $\begin{array}{c}\text { Laboratory } \\
\text { Result }^{2} \\
\text { (Total mg) }\end{array}$ & $\mathrm{MDL}^{3}$ & RDL $^{4}$ & $\mathbf{R L}^{5}$ & $\begin{array}{c}\text { Risk } \\
\text { Result } \\
\text { (Total mg) }^{6}\end{array}$ & Flag $^{7}$ \\
\hline Chlorine $\left(\text { as } \mathrm{Cl}_{2}\right)^{12}$ & ND & 0.16 & 0.42 & 0.28 & $<0.28$ & \\
\hline Fluoride (as $\mathrm{HF})^{9}$ & ND & 0.074 & 0.19 & 1.5 & $<0.19$ & \\
\hline Nitrate $\left(\text { as } \mathrm{HNO}_{3}\right)^{10}$ & 0.22 & 0.014 & 0.037 & 0.14 & 0.22 & \\
\hline Nitrite (as $\left.\mathrm{HNO}_{2}\right)^{11}$ & 1.2 & 0.029 & 0.075 & 0.29 & 1.2 & \\
\hline
\end{tabular}

Sample Collection and Analysis Dates:

\begin{tabular}{|l|c|c|c|c|}
\cline { 2 - 5 } \multicolumn{1}{c|}{} & $\begin{array}{c}\text { Particulate } \\
\text { Filter }\end{array}$ & $\begin{array}{c}\text { Acetone } \\
\text { Probe Rinse }\end{array}$ & $\begin{array}{c}\mathbf{0 . 1 N} \mathbf{H}_{2} \mathrm{SO}_{\mathbf{4}} \text { Impinger } \\
\text { Composite }\end{array}$ & $\begin{array}{c}\text { 0.1N NaOH Impinger } \\
\text { Composite }\end{array}$ \\
\hline $\begin{array}{l}\text { Date(s) Collected: } \\
\begin{array}{l}\text { Date(s) of Preparation- } \\
\text { Analysis: }\end{array}\end{array}$ & June 11,2001 & June 11, 2001 & June 11,2001 & June 11, 2001 \\
& June 22-26,2001 & June 25-26, 2001 & June 29, 2001 & July 05, 2001 \\
\hline
\end{tabular}

\section{Preparation and Analysis Methods:}

EPA Method 5: "Particulate Emissions from Stationary Sources"

SW-846 Method 9056: "Determination of Inorganic Anions by Ion Chromatography"

Last saved by Robin Gifford on E:MMY DOCUMENTS IHLLWE SAMPLINGIFINAL REPORTAAPPENDIX AITABLE A-15. 0050-STRT-2.DOC Last printed on 2/5/2002 11:14 AM2/5/2002 at 11:14 AM 
BECHTEL BWXT IDAHO, LLC (BBWI)

INTEC HLLWE Effluent Gas Emissions Inventory

Idaho National Engineering and Environmental Laboratory (INEEL)

STL Knoxville Project Number: 142503.40

\section{M5 Particulate and Anion Train - Run 2 \\ Analytical Results Summary (Continued) \\ Table A-15. HLLWE Run ID: 0050-STRT-2}

\section{Footnotes:}

1 The Total M-5 Particulate result is the sum of the acetone probe rinse particulate weight and the particulate filter particulate weight.

2 This value is the laboratory sample result. When the analytical result is "ND" or not detected, the laboratory analysis did not detect the analyte down to the method detection limit (MDL).

3 This value is the laboratory MDL derived according to requirements outlined in 40 CFR Part 136, Appendix B. The MDL has been calculated as $\mathrm{Cl}_{2}, \mathrm{HCl}, \mathrm{HF}, \mathrm{HNO}_{3}$, or $\mathrm{HNO}_{2}$, as appropriate.

4 The RDL is the Reliable Detection Limit. The RDL is the detection level recommended by EPA's National Research Laboratory in Cincinnati, Ohio, Environmental Monitoring Systems Laboratory (EMSL) in Cincinnati, Ohio, American Chemical Society (ACS) Committee on Environmental Improvement and the Drinking Water Standards Division (DWSD). It is defined as 2.623 times the MDL (2.623 X MDL). The RDL has been calculated as $\mathrm{Cl}_{2}, \mathrm{HCl}, \mathrm{HF}, \mathrm{HNO}_{3}$, or $\mathrm{HNO}_{2}$, as appropriate.

5 The RL is the laboratory Reporting Limit (RL). The RL has been calculated as $\mathrm{Cl}_{2}, \mathrm{HCl}, \mathrm{HF}, \mathrm{HNO}_{3}$, or $\mathrm{HNO}_{2}$, as appropriate.

6 The bolded value for each analyte is the value or default value assigned to the analyte. This value was determined using the following guidelines:

- When the analytical result is greater than the laboratory reporting limit (RL), the "Risk Result" is the actual analytical result or "hit" determined by the laboratory.

- When the analytical result is greater than the reliable detection level (RDL), but less than the laboratory reporting limit (RL), the "Risk Result" is the actual analytical result or "hit" determined by the laboratory.

- When the analytical result is less than the RDL, but greater than the method detection limit (MDL), the default value is the RDL.

- When the analytical result is not detected down to the MDL, the "Risk Result" is the RDL.

- It should be noted that when the RDL is selected as the default value using the guidelines above, but the RDL is greater than the $\mathrm{RL}$, the RL is used as the risk result.

7 This flag is the laboratory data flag that corresponds to EPA guidelines. The data flags for these samples are as follows:

- A " $U$ " flag indicates that this analyte was analyzed for, but was not detected down to the MDL.

$A$ " $B$ " flag indicates that the result for this analyte was below the RL and is therefore considered to be an estimated value.

8 The calculation of the total milligrams $(\mathrm{mg})$ of $\mathrm{HCl}$ in the Impinger Composite is as follows:

$$
\text { Total mg }(\mathrm{HCl})=\operatorname{mg~of~Cl}{ }^{-} \times \frac{(36.46 \mathrm{mg} \mathrm{HCl})}{\left(35.45 \mathrm{mg} \mathrm{Cl}^{-}\right)}
$$

Where: $\quad 36.46=$ the molecular weight of $\mathrm{HCl}$ in $\mathrm{mg} / \mathrm{mg}$-mole and

$35.45=$ the atomic weight of $\mathrm{Cl}^{-}$in $\mathrm{mg} / \mathrm{mg}$-mole.

Last saved by Robin Gifford on E:MY DOCUMENTS\HLLWE SAMPLINGIFINAL REPORT TAPPENDIX AITABLE A-15. 0050-STRT-2.DOC Last printed on $2 / 5 / 200211: 14$ AM2/5/2002 at 11:14 AM 
BECHTEL BWXT IDAHO, LLC (BBWI)

INTEC HLLWE Effluent Gas Emissions Inventory

Idaho National Engineering and Environmental Laboratory (INEEL)

STL Knoxville Project Number: 142503.40

\section{M5 Particulate and Anion Train - Run 2 Analytical Results Summary (Continued) Table A-15. HLLWE Run ID: 0050-STRT-2}

9 The calculation of the total milligrams (mg) of HF in Impinger Composite is as follows:

$$
\text { Total } \mathrm{mg}(\mathrm{HF})=\mathrm{mg} \mathrm{of} \mathrm{F}^{-} \times \frac{(20.01 \mathrm{mgHF})}{\left(19.00 \mathrm{mgF}^{-}\right)}
$$

Where: $\quad 20.01=$ the molecular weight of $\mathrm{HF}$ in $\mathrm{mg} / \mathrm{mg}$-mole and

$19.00=$ the atomic weight of $\mathrm{F}^{-}$in $\mathrm{mg} / \mathrm{mg}$-mole.

10 The calculation of the total milligrams (mg) of $\mathrm{HNO}_{3}$ in the Impinger Composite is as follows:

$$
\text { Total } \mathrm{mg}\left(\mathrm{HNO}_{3}\right)=\text { mg of } \mathrm{NO}_{3}^{-} \times \frac{\left(63.01 \mathrm{mg} \mathrm{\textrm {HNO } _ { 3 }}\right)}{\left(62.00 \mathrm{mg} \mathrm{NO}_{3}^{-}\right)}
$$

Where: $\quad 63.01=$ the molecular weight of $\mathrm{HNO}_{3}$ in $\mathrm{mg} / \mathrm{mg}$-mole and $62.00=$ the molecular weight of $\mathrm{NO}_{3}{ }^{-}$in $\mathrm{mg} / \mathrm{mg}$-mole.

11 The calculation of the total milligrams $(\mathrm{mg})$ of $\mathrm{HNO}_{2}$ in the Impinger Composite is as follows:

$$
\text { Total } m g\left(\mathrm{HNO}_{2}\right)=\operatorname{mg} \text { of } \mathrm{NO}_{2}^{-} \times \frac{(47.01 \mathrm{mg} \mathrm{HNO} 2)}{\left(46.01 \mathrm{mg} \mathrm{NO}{ }_{2}^{-}\right)}
$$

Where: $\quad 47.01=$ the molecular weight of $\mathrm{HNO}_{2}$ in $\mathrm{mg} / \mathrm{mg}$-mole and

$46.01=$ the molecular weight of $\mathrm{NO}_{2}^{-}$in $\mathrm{mg} / \mathrm{mg}-$ mole.

12 No additional calculation was required to be performed to obtain the total milligrams $(\mathrm{mg})$ of $\mathrm{Cl}_{2}$ in the $\mathrm{NaOH}$ Impinger Composite Note that the $\mathrm{NaOH}$ Impingers were treated with $\mathrm{NaS}_{2} \mathrm{O}_{3}$ prior to the analysis of chlorine. 


\section{M5 Particulate and Anion Train - Run 4 Analytical Results Summary Table A-16. HLLWE Run ID: 0050-END-2}

Field Sample Name: Sample Description: Field Sample Number(s): STL Sample No:
$\mathrm{M} 5 \mathrm{HCl} / \mathrm{Cl}_{2}$ and Particulate Train

Particulate Filter and Acetone Probe Rinse Samples for Particulate Determination A-3342 and A-3343

H1F210104-022 and H1F210104-023

\begin{tabular}{|c|c|c|c|}
\hline Analyte & $\begin{array}{c}\text { Particulate Filter } \\
\text { Particulate Weight } \\
(\mu \mathrm{g})\end{array}$ & $\begin{array}{c}\text { Acetone Probe Rinse } \\
\text { Particulate Weight } \\
(\mu \mathrm{g})\end{array}$ & $\begin{array}{c}\text { Particulate }^{1} \\
\text { Total } \\
\text { (Total } \mu \text { g of Particulate) }\end{array}$ \\
\hline Particulate & 700 & $100 \mathrm{~B}$ & $800 \mathrm{~B}$ \\
\hline
\end{tabular}

Field Sample Name: Sample Description: Field Sample Number(s) STL Sample No.:

\begin{tabular}{|c|c|c|c|c|c|c|}
\hline Analyte & $\begin{array}{c}\text { Laboratory } \\
\text { Result } \\
\text { (Total mg) }\end{array}$ & $\mathrm{MDL}^{3}$ & $\mathrm{RDL}^{4}$ & $\mathbf{R L}^{5}$ & $\begin{array}{c}\text { Risk } \\
\text { Result } \\
{\text { (Total } \mathbf{m g})^{6}}^{6}\end{array}$ & Flag $^{7}$ \\
\hline Chloride (as $\mathrm{HCl})^{8}$ & 1.8 & 1.6 & 4.3 & 2.9 & $<2.9$ & B \\
\hline Fluoride (as HF) ${ }^{9}$ & ND & 0.074 & 0.19 & 1.5 & $<0.19$ & \\
\hline Nitrate (as $\left.\mathrm{HNO}_{3}\right)^{10}$ & 6.6 & 0.071 & 0.19 & 0.71 & 6.6 & \\
\hline Nitrite (as $\left.\mathrm{HNO}_{2}\right)^{11}$ & ND & 0.14 & 0.38 & 1.4 & $<0.38$ & \\
\hline
\end{tabular}

M5 $\mathrm{HCl} / \mathrm{Cl}_{2}$ and Particulate Train

$0.1 \mathrm{~N} \mathrm{H}_{2} \mathrm{SO}_{4}$ Impinger Solution for Chloride, Fluoride, Nitrate, and Nitrite Analysis A-3344 A-3344 
BECHTEL BWXT IDAHO, LLC (BBWI)

INTEC HLLWE Effluent Gas Emissions Inventory

Idaho National Engineering and Environmental Laboratory (INEEL)

STL Knoxville Project Number: 142503.40

\section{M5 Particulate and Anion Train - Run 4 Analytical Results Summary (Continued) Table A-16. HLLWE Run ID: 0050-END-2}

Field Sample Name: Sample Description: Field Sample Number(s): STL Sample No.:

$\mathrm{M} 5 \mathrm{HCl} / \mathrm{Cl}_{2}$ and Particulate Train $0.1 \mathrm{~N} \mathrm{NaOH}$ Impinger Solution for Chlorine, Fluoride, Nitrate, and Nitrite Analysis A-3345

H1F210104-025

\begin{tabular}{|c|c|c|c|c|c|c|}
\hline Analyte & $\begin{array}{c}\text { Laboratory } \\
\text { Result }^{2} \\
\text { (Total mg) }\end{array}$ & $\mathrm{MDL}^{3}$ & $\mathrm{RDL}^{4}$ & $\mathrm{RL}^{5}$ & $\begin{array}{c}\text { Risk } \\
\text { Result } \\
\text { (Total mg) }^{6}\end{array}$ & Flag $^{7}$ \\
\hline Chlorine (as $\left.\mathrm{Cl}_{2}\right)^{12}$ & ND & 0.16 & 0.42 & 0.29 & $<0.29$ & \\
\hline Fluoride (as HF) ${ }^{9}$ & ND & 0.075 & 0.20 & 1.5 & $<0.20$ & \\
\hline Nitrate (as $\left.\mathrm{HNO}_{3}\right)^{10}$ & 0.51 & 0.014 & 0.038 & 0.14 & 0.51 & \\
\hline Nitrite (as $\left.\mathrm{HNO}_{2}\right)^{11}$ & 2.7 & 0.015 & 0.038 & 0.15 & 2.7 & \\
\hline
\end{tabular}

Sample Collection and Analysis Dates:

\begin{tabular}{|l|c|c|c|c|}
\cline { 2 - 5 } \multicolumn{1}{c|}{} & \multicolumn{1}{c|}{$\begin{array}{c}\text { Particulate } \\
\text { Filter }\end{array}$} & $\begin{array}{c}\text { Acetone } \\
\text { Probe Rinse }\end{array}$ & $\begin{array}{c}\mathbf{0 . 1 N} \mathbf{H}_{2} \text { SO }_{4} \text { Impinger } \\
\text { Composite }\end{array}$ & $\begin{array}{c}\mathbf{0 . 1 N} \text { NaOH Impinger } \\
\text { Composite }\end{array}$ \\
\hline $\begin{array}{l}\text { Date(s) Collected: } \\
\text { Date(s) of Preparation- }\end{array}$ & June 11,2001 & June 11, 2001 & June 11, 2001 & June 11, 2001 \\
Analysis: & June 22-26,2001 & June 25-26, 2001 & June 29, 2001 & July 05, 2001 \\
& & & & \\
\hline
\end{tabular}

Preparation and Analvsis Methods:

EPA Method 5: "Particulate Emissions from Stationary Sources"

SW-846 Method 9056: "Determination of Inorganic Anions by Ion Chromatography" 
BECHTEL BWXT IDAHO, LLC (BBWI)

INTEC HLLWE Effluent Gas Emissions Inventory

Idaho National Engineering and Environmental Laboratory (INEEL)

STL Knoxville Project Number: 142503.40

\section{M5 Particulate and Anion Train - Run 4 \\ Analytical Results Summary (Continued) \\ Table A-16. HLLWE Run ID: 0050-END-2}

Footnotes:

1 The Total M-5 Particulate result is the sum of the acetone probe rinse particulate weight and the particulate filter particulate weight.

2 This value is the laboratory sample result. When the analytical result is "ND" or not detected, the laboratory analysis did not detect the analyte down to the method detection limit (MDL).

3 This value is the laboratory MDL derived according to requirements outlined in 40 CFR Part 136, Appendix B. The MDL has been calculated as $\mathrm{Cl}_{2}, \mathrm{HCl}, \mathrm{HF}, \mathrm{HNO}_{3}$, or $\mathrm{HNO}_{2}$, as appropriate.

4 The RDL is the Reliable Detection Limit. The RDL is the detection level recommended by EPA's National Research Laboratory in Cincinnati, Ohio, Environmental Monitoring Systems Laboratory (EMSL) in Cincinnati, Ohio, American Chemical Society (ACS) Committee on Environmental Improvement and the Drinking Water Standards Division (DWSD). It is defined as 2.623 times the MDL (2.623 X MDL). The RDL has been calculated as $\mathrm{Cl}_{2}, \mathrm{HCl}, \mathrm{HF}, \mathrm{HNO}_{3}$, or $\mathrm{HNO}_{2}$, as appropriate.

5 The RL is the laboratory Reporting Limit (RL). The RL has been calculated as $\mathrm{Cl}_{2}, \mathrm{HCl}, \mathrm{HF}, \mathrm{HNO}_{3}$, or $\mathrm{HNO}_{2}$, as appropriate.

6 The bolded value for each analyte is the value or default value assigned to the analyte. This value was determined using the following guidelines:

- When the analytical result is greater than the laboratory reporting limit (RL), the "Risk Result" is the actual analytical result or "hit" determined by the laboratory.

- When the analytical result is greater than the reliable detection level (RDL), but less than the laboratory reporting limit (RL), the "Risk Result" is the actual analytical result or "hit" determined by the laboratory.

- When the analytical result is less than the RDL, but greater than the method detection limit (MDL), the default value is the RDL.

- When the analytical result is not detected down to the MDL, the "Risk Result" is the RDL.

- It should be noted that when the RDL is selected as the default value using the guidelines above, but the RDL is greater than the $\mathrm{RL}$, the RL is used as the risk result.

7 This flag is the laboratory data flag that corresponds to EPA guidelines. The data flags for these samples are as follows:

- A "U" flag indicates that this analyte was analyzed for, but was not detected down to the MDL.

A "B" flag indicates that the result for this analyte was below the RL and is therefore considered to be an estimated value.

8 The calculation of the total milligrams ( $\mathrm{mg}$ ) of $\mathrm{HCl}$ in the Impinger Composite is as follows:

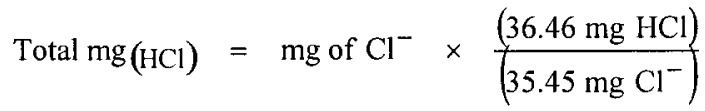

Where: $\quad 36.46=$ the molecular weight of $\mathrm{HCl}$ in $\mathrm{mg} / \mathrm{mg}$-mole and

$35.45=$ the atomic weight of $\mathrm{Cl}^{-}$in $\mathrm{mg} / \mathrm{mg}$-mole.

Last saved by Robin Gifford on EIMY DOCUMENTS HI.L.WE SAMPLINGIFINAL REPORT \APPENDIX AITABLE A-16. 0050-END-2.DOC Last printed on 2/5/2002 11:16 AM2/5/2002 at 11:16 AM 
BECHTEL BWXT IDAHO, LLC (BBWI)

INTEC HLLWE Effluent Gas Emissions Inventory

Idaho National Engineering and Environmental Laboratory (INEEL)

STL Knoxville Project Number: 142503.40

\section{M5 Particulate and Anion Train - Run 4 Analytical Results Summary (Continued) Table A-16. HLLWE Run ID: 0050-END-2}

9 The calculation of the total milligrams ( $\mathrm{mg}$ ) of HF in Impinger Composite is as follows:

$$
\text { Total mg(HF) } \mathrm{mg} \mathrm{of}^{-} \times \frac{(20.01 \mathrm{mgHF})}{\left(19.00 \mathrm{mgF}^{-}\right)}
$$

Where: $\quad 20.0 \mathrm{l}=$ the molecular weight of $\mathrm{HF}$ in $\mathrm{mg} / \mathrm{mg}$-mole and

$19.00=$ the atomic weight of $\mathrm{F}^{-}$in $\mathrm{mg} / \mathrm{mg}$-mole.

10 The calculation of the total milligrams ( $\mathrm{mg}$ ) of $\mathrm{HNO}_{3}$ in the Impinger Composite is as follows:

$$
\text { Total } \mathrm{mg}\left(\mathrm{HNO}_{3}\right)=\mathrm{mg} \text { of } \mathrm{NO}_{3}{ }^{-} \times \frac{\left.(63.01 \mathrm{mg} \mathrm{HNO})_{3}\right)}{62.00 \mathrm{mg} \mathrm{NO}{ }^{-}}
$$

Where: $\quad 63.01=$ the molecular weight of $\mathrm{HNO}_{3}$ in $\mathrm{mg} / \mathrm{mg}$-mole and $62.00=$ the molecular weight of $\mathrm{NO}_{3}{ }^{-}$in $\mathrm{mg} / \mathrm{mg}$-mole.

11 The calculation of the total milligrams $(\mathrm{mg})$ of $\mathrm{HNO}_{2}$ in the Impinger Composite is as follows:

$$
\text { Total } \mathrm{mg}\left(\mathrm{HNO}_{2}\right)=\mathrm{mg} \text { of } \mathrm{NO}_{2}{ }^{-} \times \frac{(47.01 \mathrm{mg} \mathrm{HNO} 2)}{\left(46.01 \mathrm{mg} \mathrm{NO}_{2}^{-}\right)}
$$

Where: $\quad 47.01=$ the molecular weight of $\mathrm{HNO}_{2}$ in $\mathrm{mg} / \mathrm{mg}$-mole and $46.01=$ the molecular weight of $\mathrm{NO}_{2}^{-}$in $\mathrm{mg} / \mathrm{mg}$-mole.

12 No additional calculation was required to be performed to obtain the total milligrams ( $\mathrm{mg}$ ) of $\mathrm{Cl}_{2}$ in the $\mathrm{NaOH}$ Impinger Composite. Note that the $\mathrm{NaOH}$ Impingers were treated with $\mathrm{NaS}_{2} \mathrm{O}_{3}$ prior to the analysis of chlorine. 


\section{Table A-17. M5 Particulate and Anion Train - Run 2 Reagent Blanks Analytical Results Summary}

Field Sample Name:

Sample Description:

Field Sample Number(s):

STL Sample No.:
$\mathrm{M} 5 \mathrm{HCl} / \mathrm{Cl}_{2}$ and Particulate Train Reagent Blanks

Particulate Filter and Acetone Probe Rinse Reagent Blanks for Particulate Determination A-3316 and A-3317

H1F210104-009 and H1F210104-010

\begin{tabular}{|lccc|}
\hline Analyte & $\begin{array}{c}\text { Particulate Filter } \\
\text { Particulate Weight } \\
(\mu \mathrm{g})\end{array}$ & $\begin{array}{c}\text { Acetone Probe Rinse } \\
\text { Particulate Weight } \\
(\mu g)\end{array}$ & $\begin{array}{c}\text { Particulate }^{1} \\
\text { Total } \\
\text { Particulate }\end{array}$ \\
\hline & $500 \mathrm{U}$ & $500 \mathrm{U}$ & $1,000 \mathrm{U}$ \\
\hline
\end{tabular}

Field Sample Name: Sample Description: Field Sample Number(s): STL Sample No.:
M5 $\mathrm{HCl} / \mathrm{Cl}_{2}$ and Particulate Train Reagent Blanks

$0.1 \mathrm{~N} \mathrm{H}_{2} \mathrm{SO}_{4}$ Impinger Solution Reagent Blanks for Chloride, Fluoride, Nitrate, and Nitrite Analysis A-3318

H1F210104-011

\begin{tabular}{|c|c|c|c|c|c|c|}
\hline Analyte & $\begin{array}{c}\text { Laboratory } \\
\text { Result }^{2} \\
\text { (Total mg) }\end{array}$ & $\mathrm{MDL}^{3}$ & $\mathrm{RDL}^{4}$ & $\mathbf{R} \mathbf{L}^{5}$ & $\begin{array}{c}\text { Risk } \\
\text { Result } \\
\text { (Total mg) }^{6}\end{array}$ & Flag $^{7}$ \\
\hline Chloride (as $\mathrm{HCl})^{8}$ & 1.6 & 1.2 & 3.0 & 2.0 & $<2.0$ & B \\
\hline Fluoride (as HF) ${ }^{9}$ & ND & 0.052 & 0.14 & 1.0 & $<0.14$ & \\
\hline Nitrate $\left(\text { as } \mathrm{HNO}_{3}\right)^{10}$ & ND & 0.020 & 0.053 & 0.20 & $<0.053$ & \\
\hline Nitrite (as $\left.\mathrm{HNO}_{2}\right)^{11}$ & ND & 0.10 & 0.27 & 1.0 & $<0.27$ & \\
\hline
\end{tabular}

Last saved by Robin Gifford on E:MMY DOCUMENTS\HLLWE SAMPLINGIFINAL REPORTIAPPENDIX AITABLE A-17. 0050-STRT-2 RBS.DOC

Last printed on 2/5/2002 12:07 PM2/5/2002 at 12:07 PM 
BECHTEL BWXT IDAHO, LLC (BBWI)

INTEC HLLWE Effluent Gas Emissions Inventory

Idaho National Engineering and Environmental Laboratory (INEEL)

STL Knoxville Project Number: 142503.40

\section{Table A-17. M5 Particulate and Anion Train - Run 2 Reagent Blanks Analytical Results Summary (Continued)}

Field Sample Name: Sample Description: Field Sample Number(s): STL Sample No:
$\mathrm{M} 5 \mathrm{HCl} / \mathrm{Cl}_{2}$ and Particulate Train Reagent Blanks $0.1 \mathrm{~N} \mathrm{NaOH}$ Impinger Solution Reagent Blanks for Chlorine, Fluoride, Nitrate, and Nitrite Analysis A-3319

H1F210104-012

\begin{tabular}{|c|c|c|c|c|c|c|}
\hline Analyte & $\begin{array}{c}\text { Laboratory } \\
\text { Result }^{2} \\
\text { (Total mg) }\end{array}$ & $\mathrm{MDL}^{3}$ & RDL $^{4}$ & $\mathbf{R L}^{5}$ & $\begin{array}{c}\text { Risk } \\
\text { Result } \\
\text { (Total mg) }^{6} \\
\end{array}$ & Flag $^{7}$ \\
\hline Chlorine $\left(\text { as } \mathrm{Cl}_{2}\right)^{12}$ & ND & 0.11 & 0.30 & 0.20 & $<0.20$ & \\
\hline Fluoride (as $\mathrm{HF})^{9}$ & ND & 0.11 & 0.28 & 2.1 & $<0.28$ & \\
\hline Nitrate (as $\left.\mathrm{HNO}_{3}\right)^{10}$ & 0.012 & 0.010 & 0.027 & 0.10 & $<0.027$ & B \\
\hline Nitrite (as $\left.\mathrm{HNO}_{2}\right)^{11}$ & ND & 0.010 & 0.027 & 0.10 & $<0.027$ & \\
\hline
\end{tabular}

Sample Collection and Analysis Dates:

\begin{tabular}{|l|c|c|c|c|}
\cline { 2 - 5 } \multicolumn{1}{c|}{} & $\begin{array}{c}\text { Particulate } \\
\text { Filter }\end{array}$ & $\begin{array}{c}\text { Acetone } \\
\text { Probe Rinse }\end{array}$ & $\begin{array}{c}\mathbf{0 . 1 N} \text { H}_{2} \text { SO Impinger } \\
\text { Composite }\end{array}$ & $\begin{array}{c}\text { 0.1N NaOH Impinger } \\
\text { Composite }\end{array}$ \\
\hline $\begin{array}{l}\text { Date(s) Collected: } \\
\begin{array}{l}\text { Date(s) of Preparation- } \\
\text { Analysis: }\end{array}\end{array}$ & June 11,2001 & June 11,2001 & June 11, 2001 & June 11, 2001 \\
\hline
\end{tabular}

\section{Preparation and Analysis Methods:}

EPA Method 5: "Particulate Emissions from Stationary Sources"

SW-846 Method 9056: "Determination of Inorganic Anions by Ion Chromatography" 
BECHTEL BWXT IDAHO, LLC (BBWI)

INTEC HLLWE Effluent Gas Emissions Inventory

Idaho National Engineering and Environmental Laboratory (INEEL)

STL Knoxville Project Number: 142503.40

\section{Table A-17. M5 Particulate and Anion Train - Run 2 Reagent Blanks Analytical Results Summary (Continued)}

\section{Footnotes:}

1 The Total M-5 Particulate result is the sum of the acetone probe rinse particulate weight and the particulate filter particulate weight.

2 This value is the laboratory sample result. When the analytical result is "ND" or not detected, the laboratory analysis did not detect the analyte down to the method detection limit (MDL).

3 This value is the laboratory MDL derived according to requirements outlined in 40 CFR Part 136, Appendix B. The MDL has been calculated as $\mathrm{Cl}_{2}, \mathrm{HCl}, \mathrm{HF}, \mathrm{HNO}_{3}$, or $\mathrm{HNO}_{2}$, as appropriate.

4 The RDL is the Reliable Detection Limit. The RDL is the detection level recommended by EPA's National Research Laboratory in Cincinnati, Ohio, Environmental Monitoring Systems Laboratory (EMSL) in Cincinnati, Ohio, American Chemical Society (ACS) Committee on Environmental Improvement and the Drinking Water Standards Division (DWSD). It is defined as 2.623 times the $\operatorname{MDL}\left(2.623 \times \mathrm{MDL}\right.$ ). The RDL has been calculated as $\mathrm{Cl}_{2}, \mathrm{HCl}, \mathrm{HF}, \mathrm{HNO}_{3}$, or $\mathrm{HNO}_{2}$, as appropriate.

5 The RL is the laboratory Reporting Limit (RL). The RL has been calculated as $\mathrm{Cl}_{2}, \mathrm{HCl}, \mathrm{HF}, \mathrm{HNO}_{3}$, or $\mathrm{HNO}_{2}$, as appropriate.

6 The bolded value for each analyte is the value or default value assigned to the analyte. This value was determined using the following guidelines:

- When the analytical result is greater than the laboratory reporting limit (RL), the "Risk Result" is the actual analytical result or "hit" determined by the laboratory.

- When the analytical result is greater than the reliable detection level (RDL), but less than the laboratory reporting limit (RL), the "Risk Result" is the actual analytical result or "hit" determined by the laboratory.

- When the analytical result is less than the RDL, but greater than the method detection limit (MDL), the default value is the RDL.

- When the analytical result is not detected down to the MDL, the "Risk Result" is the RDL.

- It should be noted that when the RDL is selected as the default value using the guidelines above, but the RDL is greater than the $\mathrm{RL}$, the $\mathrm{RL}$ is used as the risk result.

7 This flag is the laboratory data flag that corresponds to EPA guidelines. The data flags for these samples are as follows:

- A "U" flag indicates that this analyte was analyzed for, but was not detected down to the MDL.

$A$ " $B$ " flag indicates that the result for this analyte was below the RL and is therefore considered to be an estimated value.

8 The calculation of the total milligrams (mg) of $\mathrm{HCl}$ in the Impinger Composite is as follows:

$$
\text { Total mg }(\mathrm{HCl})=m g \text { of } \mathrm{Cl}^{-} \times \frac{(36.46 \mathrm{mg} \mathrm{HCl})}{\left(35.45 \mathrm{mg} \mathrm{Cl}^{-}\right)}
$$

Where: $\quad 36.46=$ the molecular weight of $\mathrm{HCl}$ in $\mathrm{mg} / \mathrm{mg}$-mole and

$35.45=$ the atomic weight of $\mathrm{Cl}^{-}$in $\mathrm{mg} / \mathrm{mg}$-mole.

Last saved by Robin Gifford on E:IMY DOCUMENTS

RBS.DOC

Last printed on 2/5/2002 12:07 PM2/5/2002 at 12:07 PM 
BECHTEL BWXT IDAHO, LLC (BBWI)

INTEC HLLWE Effluent Gas Emissions Inventory

Idaho National Engineering and Environmental Laboratory (INEEL)

STL Knoxville Project Number: 142503.40

\section{Table A-17. M5 Particulate and Anion Train - Run 2 Reagent Blanks Analytical Results Summary (Continued)}

9 The calculation of the total milligrams $(\mathrm{mg})$ of $\mathrm{HF}$ in Impinger Composite is as follows:

$$
\text { Total } \mathrm{mg}(\mathrm{HF})=\mathrm{mg} \text { of } \mathrm{F}^{-} \quad \times \frac{(20.01 \mathrm{mgHF})}{\left(19.00 \mathrm{mgF}^{-}\right)}
$$

Where: $\quad 20.01=$ the molecular weight of $\mathrm{HF}$ in $\mathrm{mg} / \mathrm{mg}$-mole and

$19.00=$ the atomic weight of $\mathrm{F}^{-}$in $\mathrm{mg} / \mathrm{mg}$-mole.

10 The calculation of the total milligrams (mg) of $\mathrm{HNO}_{3}$ in the Impinger Composite is as follows:

$$
\text { Total mg }\left(\mathrm{HNO}_{3}\right)=\mathrm{mg} \text { of } \mathrm{NO}_{3}^{-} \times \frac{(63.01 \mathrm{mg} \mathrm{HNO})_{3}}{\left(62.00 \mathrm{mg} \mathrm{NO}{ }^{-}\right.}
$$

Where: $\quad 63.01=$ the molecular weight of $\mathrm{HNO}_{3}$ in $\mathrm{mg} / \mathrm{mg}$-mole and

$62.00=$ the molecular weight of $\mathrm{NO}_{3}^{-}{ }^{-}$in $\mathrm{mg} / \mathrm{mg}$-mole.

11 The calculation of the total milligrams (mg) of $\mathrm{HNO}_{2}$ in the Impinger Composite is as follows:

$$
\text { Total } m g\left(\mathrm{HNO}_{2}\right)=\operatorname{mg} \text { of } \mathrm{NO}_{2}{ }^{-} \times \frac{(47.01 \mathrm{mg} \mathrm{HNO} 2)}{\left(46.01 \mathrm{mg} \mathrm{NO}_{2}^{-}\right)}
$$

Where: $\quad 47.01=$ the molecular weight of $\mathrm{HNO}_{2}$ in $\mathrm{mg} / \mathrm{mg}$-mole and

$46.01=$ the molecular weight of $\mathrm{NO}_{2}^{-}$in $\mathrm{mg} / \mathrm{mg}-\mathrm{mole}$.

12 No additional calculation was required to be performed to obtain the total milligrams $(\mathrm{mg})$ of $\mathrm{Cl}_{2}$ in the $\mathrm{NaOH}$ Impinger Composite. Note that the $\mathrm{NaOH}$ Impingers were treated with $\mathrm{NaS}_{2} \mathrm{O}_{3}$ prior to the analysis of chlorine. 


\section{Table A-18. M5 Particulate and Anion Train Run 2 INTEC Deionized Water Reagent Blank Analytical Results Summary}

Field Sample Name: Sample Description: Field Sample Number(s): STL Sample No.:
M5 HCl/Cl 2 and Particulate Train INTEC D.1. Water Reagent Blank

INTEC D.I. Water Reagent Blank for Chloride, Fluoride, Nitrate, and Nitrite Analysis A-3349

H1F210104-013

\begin{tabular}{|c|c|c|c|c|c|c|}
\hline Analyte & $\begin{array}{c}\text { Laboratory } \\
\text { Result }^{1} \\
\text { (Total } \mathrm{mg} \text { ) }\end{array}$ & $\mathrm{MDL}^{2}$ & $\mathrm{RDL}^{3}$ & $\mathbf{R L}^{4}$ & $\begin{array}{c}\text { Risk } \\
\text { Result } \\
\text { (Total mg) }\end{array}$ & Flag $^{6}$ \\
\hline Chloride (as $\mathrm{HCl})^{7}$ & ND & 0.060 & 0.16 & 0.10 & $<0.10$ & \\
\hline Fluoride (as HF) ${ }^{8}$ & ND & 0.0054 & 0.014 & 0.11 & $<0.014$ & \\
\hline Nitrate (as $\left.\mathrm{HNO}_{3}\right)^{9}$ & 0.0058 & 0.0052 & 0.014 & 0.052 & $<0.014$ & B \\
\hline Nitrite (as $\left.\mathrm{HNO}_{2}\right)^{10}$ & ND & 0.01 & 0.014 & 0.052 & $<0.014$ & \\
\hline
\end{tabular}

Sample Collection and Analysis Dates:

Date(s) Collected:

Date(s) of Preparation-Analysis:
June 11, 2001

June 29, 2001

\section{Preparation and Analysis Methods:}

EPA Method 5: "Particulate Emissions from Stationary Sources"

SW-846 Method 9056: "Determination of Inorganic Anions by Ion Chromatography" 
BECHTEL BWXT IDAHO, LLC (BBWI)

INTEC HLLWE Effluent Gas Emissions Inventory

Idaho National Engineering and Environmental Laboratory (INEEL)

STL Knoxville Project Number: 142503.40

\section{Table A-18. M5 Particulate and Anion Train \\ Run 2 INTEC Deionized Water Reagent Blank Analytical Results Summary (Continued)}

Footnotes:

1 This value is the laboratory sample result. When the analytical result is "ND" or not detected, the laboratory analysis did not detect the analyte down to the method detection limit (MDL).

2 This value is the laboratory MDL derived according to requirements outlined in 40 CFR Part 136, Appendix B. The MDL has been calculated as $\mathrm{Cl}_{2}, \mathrm{HCl}, \mathrm{HF}, \mathrm{HNO}_{3}$, or $\mathrm{HNO}_{2}$, as appropriate.

3 The RDL is the Reliable Detection Limit. The RDL is the detection level recommended by EPA's National Research Laboratory in Cincinnati, Ohio, Environmental Monitoring Systems Laboratory (EMSL) in Cincinnati, Ohio, American Chemical Society (ACS) Committee on Environmental Improvement and the Drinking Water Standards Division (DWSD). It is defined as 2.623 times the MDL (2.623 X MDL). The RDL has been calculated as $\mathrm{Cl}_{2}, \mathrm{HCl}, \mathrm{HF}, \mathrm{HNO}_{3}$, or $\mathrm{HNO}_{2}$, as appropriate.

4 The RL is the laboratory Reporting Limit (RL). The RL has been calculated as $\mathrm{Cl}_{2}, \mathrm{HCl}, \mathrm{HF}, \mathrm{HNO}_{3}$, or $\mathrm{HNO}_{2}$, as appropriate.

5 The bolded value for each analyte is the value or default value assigned to the analyte. This value was determined using the following guidelines:

- When the analytical result is greater than the laboratory reporting limit (RL), the "Risk Result" is the actual analytical result or "hit" determined by the laboratory.

- When the analytical result is greater than the reliable detection level (RDL), but less than the laboratory reporting limit (RL), the "Risk Result" is the actual analytical result or "hit" determined by the laboratory.

- When the analytical result is less than the RDL, but greater than the method detection limit (MDL), the default value is the RDL.

- When the analytical result is not detected down to the MDL, the "Risk Result" is the RDL.

- It should be noted that when the RDL is selected as the default value using the guidelines above, but the RDL is greater than the $R L$, the $R L$ is used as the risk result.

6 This flag is the laboratory data flag that corresponds to EPA guidelines. The data flags for these samples are as follows:

- A "U" flag indicates that this analyte was analyzed for, but was not detected down to the MDL.

$A$ " $B$ " flag indicates that the result for this analyte was below the RL and is therefore considered to be an estimated value.

${ }^{7}$ The calculation of the total milligrams $(\mathrm{mg})$ of $\mathrm{HCl}$ in the Impinger Composite is as follows:

$$
\text { Total mg(HCl })=m g \text { of } \mathrm{Cl}^{-} \times \frac{(36.46 \mathrm{mg} \mathrm{HCl})}{\left(35.45 \mathrm{mg} \mathrm{Cl}^{-}\right)}
$$

Where: $\quad 36.46=$ the molecular weight of $\mathrm{HCl}$ in $\mathrm{mg} / \mathrm{mg}$-mole and

$35.45=$ the atomic weight of $\mathrm{Cl}^{-}$in $\mathrm{mg} / \mathrm{mg}$-mole.

Last saved by Robin Gifford on E:MY DOCUMENTSIHLLWE SAMPLINGIFINAL REPORT\APPENDIX A TTABLE A-18. 0050-STRT- DI H20 RB.DOC

Last printed on 2/5/2002 12:12 PM2/5/2002 at 12:12 PM 


\section{Table A-18. M5 Particulate and Anion Train Run 2 INTEC Deionized Water Reagent Blank Analytical Results Summary (Continued)}

${ }^{8}$ The calculation of the total milligrams (mg) of HF in Impinger Composite is as follows:

$$
\text { Total } \mathrm{mg}(\mathrm{HF})=\mathrm{mg} \text { of } \mathrm{F}^{-} \times \frac{(20.01 \mathrm{mgHF})}{\left(19.00 \mathrm{mgF}^{-}\right)}
$$

Where: $\quad 20.01=$ the molecular weight of $\mathrm{HF}$ in $\mathrm{mg} / \mathrm{mg}$-mole and

$19.00=$ the atomic weight of $\mathrm{F}^{-}$in $\mathrm{mg} / \mathrm{mg}$-mole

${ }^{9}$ The calculation of the total milligrams ( $\mathrm{mg}$ ) of $\mathrm{HNO}_{3}$ in the Impinger Composite is as follows:

$$
\text { Total mg } \left.\left(\mathrm{HNO}_{3}\right)=\operatorname{mg} \text { of } \mathrm{NO}_{3}^{-} \times \frac{(63.01 \mathrm{mg} \mathrm{HNO}}{3}\right)
$$

Where: $\quad 63.01=$ the molecular weight of $\mathrm{HNO}_{3}$ in $\mathrm{mg} / \mathrm{mg}$-mole and $62.00=$ the molecular weight of $\mathrm{NO}_{3}{ }^{-}$in $\mathrm{mg} / \mathrm{mg}-$ mole.

${ }^{10}$ The calculation of the total milligrams $(\mathrm{mg})$ of $\mathrm{HNO}_{2}$ in the Impinger Composite is as follows:

$$
\text { Total mg }\left(\mathrm{HNO}_{2}\right)=\text { mg of } \mathrm{NO}_{2}^{-} \times \frac{(47.01 \mathrm{mg} \mathrm{HNO} 2)}{\left(46.01 \mathrm{mg} \mathrm{NO}_{2}^{-}\right)}
$$

Where: $\quad 47.01=$ the molecular weight of $\mathrm{HNO}_{2}$ in $\mathrm{mg} / \mathrm{mg}$-mole and $46.01=$ the molecular weight of $\mathrm{NO}_{2}^{-}$in $\mathrm{mg} / \mathrm{mg}$-mole. 

BECHTEL BWXT IDAHO, LLC (BBWI)

INTEC HLLWE Effluent Gas Emissions Inventory

Idaho National Engineering and Environmental Laboratory (INEEL)

STL Knoxville Project Number: 142503.40

\section{Table A-19. M5 Particulate and Anion Train \\ Final Acetone Probe Rinse \\ Analytical Results Summary}

Field Sample Name:

Sample Description:

Field Sample Number(s):

STL Sample No.:
M5 $\mathrm{HCl} / \mathrm{Cl}_{2}$ and Particulate Train Final Acetone Probe Rinse

Final Acetone Probe Rinse for Particulate Determination

A-3346

H1 G030222-001

\begin{tabular}{|lc|}
\hline Analyte & $\begin{array}{c}\text { Final Acetone Probe Rinse } \\
\text { Particulate Weight } \\
(\mu \mathrm{g})^{1}\end{array}$ \\
\hline Particulate & 6,200 \\
\hline
\end{tabular}

\begin{tabular}{|l|c|} 
Sample Collection and Analysis Dates: & Final Acetone Probe Rinse \\
\hline & \\
Date(s) Collected: & June 25, 2001 \\
Date(s) of Preparation-Analysis: & July 03-06, 2001 \\
\hline
\end{tabular}

\section{Preparation and Analysis Methods:}

EPA Method 5: $\quad$ "Particulate Emissions from Stationary Sources"

SW-846 Method 9056: "Determination of Inorganic Anions by Ion Chromatography"

\section{Footnotes:}

1 This flag is the laboratory data flag that corresponds to EPA guidelines. The data flags for these samples are as follows:

- A "U" flag indicates that this analyte was analyzed for, but was not detected down to the MDL.

$A$ " $B$ " flag indicates that the result for this analyte was below the RL and is therefore considered to be an estimated value. 

BECHTEL BWXT IDAHO, LLC (BBWI)

INTEC HLLWE Effluent Gas Emissions Inventory

Idaho National Engineering and Environmental Laboratory (INEEL)

STL Knoxville Project Number: 142503.40

\section{Method 0060 Multi-Metals Train (MMT) Train Total Summary - Run 1 Train Totals Metallic Analyte Analytical Results Summary Table A-20. HLLWE Run ID: 0060-STRT-1}

Field Sample Name: Sample Description:
Method 0060 Multi-Metals Train (MMT)

Method 0060 Multi-Metals Train (MMT) Totals for Metals Analysis

\begin{tabular}{|c|c|c|c|c|c|c|c|c|}
\hline \multirow[b]{2}{*}{ Analyte } & \multirow{2}{*}{$\begin{array}{c}\text { CAS } \\
\text { Registry } \\
\text { Number }\end{array}$} & \multicolumn{2}{|c|}{$\begin{array}{c}\text { MMT } \\
\text { Front Half } \\
\text { Composite }^{1} \\
(\mu \mathrm{g})\end{array}$} & \multicolumn{2}{|c|}{$\begin{array}{c}\text { MMT } \\
\text { Back Half }^{2} \\
\text { Composite }^{2} \\
(\mu \mathrm{g})\end{array}$} & \multicolumn{2}{|c|}{$\begin{array}{c}\text { MMT } \\
\text { Sampling Train } \\
\text { Totals }^{3} \\
(\text { Total } \mu \mathrm{g})\end{array}$} & \multirow{2}{*}{$\begin{array}{c}\text { Project } \\
\text { Specific } \\
\text { Flag }^{6}\end{array}$} \\
\hline & & Risk Result & Flag $^{4}$ & Risk Result & Flag ${ }^{4}$ & Total $^{5}$ & Flag & \\
\hline Aluminum (Al) & $7429-90-5$ & 95 & & 42 & & 140 & & A \\
\hline Antimony (Sb) & $7440-36-0$ & 2.9 & B & 1.7 & B & $<4.6$ & B & A \\
\hline Arsenic (As) & $7440-38-2$ & 0.92 & $\mathrm{U}$ & 0.66 & B & $<1.6$ & B & $\mathrm{P}$ \\
\hline Barium (Ba) & $7440-39-3$ & 4.8 & B & 1.9 & B & 6.7 & B & A \\
\hline Beryllium (Be) & $7440-41-7$ & 0.23 & B & 0.42 & $\mathrm{U}$ & $<0.65$ & B & $\mathrm{P}$ \\
\hline Cadmium (Cd) & $7440-43-9$ & 0.32 & B & 0.14 & B & $<0.46$ & B & A \\
\hline Chromium $(\mathrm{Cr})$ & $7440-47-3$ & 1.7 & & 1.3 & & 3.0 & & A \\
\hline Cobalt (Co) & $7440-48-4$ & 1.3 & B & 1.4 & B & $<2.7$ & B & A \\
\hline Copper $(\mathrm{Cu})$ & $7440-50-8$ & 0.94 & B & 3.4 & & 4.3 & B & A \\
\hline Lead $(\mathrm{Pb})$ & $7439-92-1$ & 0.52 & $\mathrm{U}$ & 0.78 & B & $<1.3$ & B & $\mathrm{P}$ \\
\hline Manganese (Mn) & $7439-96-5$ & 2.8 & & 16 & & 19 & & A \\
\hline Mercury (Hg) & $7439-97-0$ & 0.37 & B & 100 & & 100 & & A \\
\hline Nickel (Ni) & $7440-02-0$ & 3.8 & B & 1.2 & B & $<5.0$ & B & A \\
\hline Selenium (Se) & $7782-49-2$ & 2.4 & & 0.78 & B & 3.2 & B & A \\
\hline Silver (Ag) & $7440-22-4$ & 1.9 & U & 0.71 & $\mathrm{U}$ & $<2.6$ & & $\mathrm{~N}$ \\
\hline Thallium ( $\mathrm{Tl}$ ) & $7440-28-0$ & 1.0 & $\mathrm{U}$ & 1.5 & U & $<2.5$ & & $\mathrm{~N}$ \\
\hline Vanadium (V) & $7440-62-2$ & 1.3 & $\mathrm{U}$ & 1.4 & U & $<2.7$ & & $\mathrm{~N}$ \\
\hline Zinc (Zn) & $7440-66-6$ & 18 & & 43 & & 61 & & A \\
\hline
\end{tabular}




\section{BECHTEL BWXT IDAHO, LLC (BBWI) \\ INTEC HLLWE Effluent Gas Emissions Inventory \\ Idaho National Engineering and Environmental Laboratory (INEEL) \\ STL Knoxville Project Number: 142503.40}

\section{Footnotes:}

1 The MMT Front Half Composite consists of the Quartz Fiber Particulate Filter and the 0.1 N Nitric Acid Probe Rinse Solution.

2 The MMT Back Half configuration includes seven (7) impingers. The first impinger remains empty prior to sampling and serves as a moisture knockout impinger. The second and third impingers are charged with $5 \% \mathrm{HNO}_{3} / 10 \% \mathrm{H}_{2} \mathrm{O}_{2}$ and are analyzed with the first impinger contents for all of the metallic analytes including mercury. The fourth impinger is left empty and serves to protect the mercury trapping solution from carryover. Its contents and glassware rinses are analyzed separately for mercury, only. The fifth and sixth impingers contain $4 \% \mathrm{KMnO}_{4} / 10 \% \mathrm{H}_{2} \mathrm{SO}_{4}$ and are also analyzed for mercury, only. The seventh impinger contains silica gel for a final scrubbing of residual moisture. The MMT back half mercury $(\mathrm{Hg})$ result is the sum of the mercury in the $5 \% \mathrm{HNO}_{3} / 10 \% \mathrm{H}_{2} \mathrm{O}_{2}$ fraction, the fourth empty impinger fraction, the $4 \% \mathrm{KMnO}_{4} / 10 \% \mathrm{H}_{2} \mathrm{SO}_{4}$ fraction, and the $8 \mathrm{~N} \mathrm{HCl}$ impinger rinse fraction. The total micrograms (ug) of mercury in these fractions is included in the MMT back half result.

3 The total mass for each metal found in the MMT sampling train consists of the sum of the MMT train's Front Half metals content plus the train's Back Half metals content. The calculation is as follows:

(Total $\mu g$ in the Front Half) $+($ Total $\mu g$ in the Back Half $)=$ Total $\mu g$ in the Multi-Metals Sampling Train. Therefore: $(\mu \mathrm{g})+(\mu \mathrm{g})=$ Total $\mu \mathrm{g}$

The MMT Sampling Train Total value was obtained by summing the MMT Front Half results and the MMT Back Half results using the following guidelines:

- When the train component analytical result is greater than the laboratory reporting limit (RL), the result included in the train total is the actual analytical result or "hit" determined by the laboratory.

- When the train component analytical result is greater than the reliable detection level (RDL), but less than the laboratory reporting limit (RL), the result included in the train total is actual analytical result or "hit" determined by the laboratory and the corresponding "B" flag is carried through the calculation to the train total.

- When the train analytical component result is less than the RDL, but greater than the method detection limit (MDL), the result included in the train total is the RDL and the corresponding " $\mathrm{B}$ " flag is carried through the calculation to the train total.

- When the train component analytical result is not detected down to the MDL, the result included in the train total is the RDL and the corresponding " $U$ " flag is carried through the calculation to the train total.

- It should be noted that when the RDL is selected as the default value using the guidelines above, but the RDL is greater than the RL, the RL is included in the train total.

The data flags attached to the MMT Totals are the cumulative set of flags for each train component included as part of the MMT total. A flag attached to a MMT component is carried through to the "MMT Sampling Train Total" column when the associated component analytical result is a significant number in comparison to the MMT Total. That is, if the MMT Total is affected by a MMT component analytical result, the associated flag is carried to the MMT Train Total, but if the MMT Train Total is not affected by a MMT component, the associated flag is not carried through to the MMT Train Total. The combinations of train fractions are conducted following the standard practice of using significant figures found in ASTM E29-93a(1999), "Standard Practice for Using Significant Digits in Test Data to Determine Conformance with Specifications" and Severn Trent Laboratories standard operating procedure number QA-004, "Rounding and Significant Figures".

4 This flag is the laboratory data flag that corresponds to EPA guidelines. The data flags for these samples are as follows:

- A "U" qualifier indicates that this analyte was analyzed for, but was not detected down to the MDL.

- A "B" flag indicates that this analyte was detected, but at a concentration below the laboratory RL. The analytical result is therefore an estimated value. 
BECHTEL BWXT IDAHO, LLC (BBWI)

INTEC HLLWE Effluent Gas Emissions Inventory

Idaho National Engineering and Environmental Laboratory (INEEL)

STL Knoxville Project Number: 142503.40

5 When listed, the less than $(<)$ sign indicates that at least one sample fraction result is either a "non-detect" value down to the MDL of the measurement that carries, or an estimated "hit" value that is below the RDL. In either case, the final value for the fraction that is included in the data set total is the default RDL value and the actual value of the total is known to be less than $(<)$ the displayed result.

- Entries in this column are project-specific train total flags that are applied to the run total values and are not standard EPA data flags. These project-specific flags are utilized for the INEEL NWCF HLLWE Effluent Gas Emissions Inventory project and are defined as follows:

- An "N" flag in this column indicates that the compound was not measured (detected) in any of the sampling train components, or fractions.

- A "P" flag in this column indicates that the compound was measured (detected) in one or more of the train components, or fractions, but not in all of the sampling train fractions.

- An "A" flag in this column indicates that the compound was measured (detected) in all of the sampling train components, or fractions. 

BECHTEL BWXT IDAHO, LLC (BBWI)

INTEC HLLWE Effluent Gas Emissions Inventory

Idaho National Engineering and Environmental Laboratory (INEEL)

STL Knoxville Project Number: 142503.40

\section{Method 0060 Multi-Metals Train (MMT) Train Total Summary - Run 3 Train Totals Metallic Analyte Analytical Results Summary \\ Table A-21. HLLWE Run ID: 0060-END-1}

Field Sample Name: Sample Description:
Method 0060 Multi-Metals Train (MMT)

Method 0060 Multi-Metals Train (MMT) Totals for Metals Analysis

\begin{tabular}{|c|c|c|c|c|c|c|c|c|}
\hline \multirow[b]{2}{*}{ Analyte } & \multirow{2}{*}{$\begin{array}{c}\text { CAS } \\
\text { Registry } \\
\text { Number }\end{array}$} & \multicolumn{2}{|c|}{$\begin{array}{c}\text { MMT } \\
\text { Front Half } \\
\text { Composite } \\
(\mu \mathrm{g})\end{array}$} & \multicolumn{2}{|c|}{$\begin{array}{c}\text { MMT } \\
\text { Back Half }^{2} \\
\text { Composite }^{2} \\
(\mu g)\end{array}$} & \multicolumn{2}{|c|}{$\begin{array}{c}\text { MMT } \\
\text { Sampling Train } \\
\text { Totals }{ }^{3} \\
(\text { Total } \mu \mathrm{g}) \\
\end{array}$} & \multirow{2}{*}{$\begin{array}{c}\text { Project } \\
\text { Specific } \\
\text { Flag }^{6}\end{array}$} \\
\hline & & Risk Result & Flag $^{4}$ & Risk Result & Flag $^{4}$ & Total $^{5}$ & Flag & \\
\hline Aluminum (AI) & $7429-90-5$ & 230 & & 38 & & 270 & & A \\
\hline Antimony (Sb) & $7440-36-0$ & 3.4 & B & 1.7 & B & $<5.1$ & B & A \\
\hline Arsenic (As) & $7440-38-2$ & 0.92 & $\mathrm{U}$ & 0.66 & $\mathrm{U}$ & $<1.6$ & & $\mathrm{~N}$ \\
\hline Barium (Ba) & $7440-39-3$ & 8.5 & $\mathrm{~B}$ & 1.6 & B & 10 & B & A \\
\hline Beryllium (Be) & $7440-41-7$ & 0.23 & $\mathrm{U}$ & 0.42 & $\mathrm{~B}$ & $<0.65$ & $\mathrm{~B}$ & $\mathrm{P}$ \\
\hline Cadmium $(\mathrm{Cd})$ & $7440-43-9$ & 0.68 & & 0.14 & $\mathrm{~B}$ & $<0.82$ & $\mathrm{~B}$ & $A$ \\
\hline Chromium (Cr) & $7440-47-3$ & 3.4 & & 1.3 & & 4.7 & & A \\
\hline Cobalt $(\mathrm{Co})$ & $7440-48-4$ & 1.3 & $\mathrm{U}$ & 1.4 & $\mathrm{U}$ & $<2.7$ & & $\mathrm{~N}$ \\
\hline Copper $(\mathrm{Cu})$ & $7440-50-8$ & 2.2 & $\mathrm{~B}$ & 1.4 & $\mathrm{~B}$ & 3.6 & B & A \\
\hline Lead $(\mathrm{Pb})$ & $7439-92-1$ & 0.52 & $\mathrm{~B}$ & 0.78 & $\mathrm{~B}$ & $<1.3$ & $\mathrm{~B}$ & A \\
\hline Manganese (Mn) & $7439-96-5$ & 5.4 & & 18 & & 23 & & $A$ \\
\hline Mercury (Hg) & $7439-97-6$ & 0.37 & B & 150 & & 150 & & $A$ \\
\hline Nickel (Ni) & $7440-02-0$ & 4.9 & & 1.1 & $\mathrm{~B}$ & $<6.0$ & B & A \\
\hline Selenium (Se) & $7782-49-2$ & 1.5 & & 0.63 & U & $<2.1$ & & $\mathrm{P}$ \\
\hline Silver (Ag) & $7440-22-4$ & 1.9 & $\mathrm{U}$ & 0.71 & $\mathrm{U}$ & $<2.6$ & & $\mathrm{~N}$ \\
\hline Thallium (Tl) & $7440-28-0$ & 1.0 & $\mathrm{U}$ & 1.4 & $\mathrm{U}$ & $<2.4$ & & $\mathrm{~N}$ \\
\hline Vanadium (V) & $7440-62-2$ & 1.3 & $\mathrm{U}$ & 1.4 & $\mathrm{U}$ & $<2.7$ & & $\mathrm{~N}$ \\
\hline Zinc $(\mathrm{Zn})$ & $7440-66-6$ & 89 & & 30 & & 120 & & A \\
\hline
\end{tabular}




\section{Footnotes:}

1 The MMT Front Half Composite consists of the Quartz Fiber Particulate Filter and the 0.1 N Nitric Acid Probe Rinse Solution.

2 The MMT Back Half configuration includes seven (7) impingers. The first impinger remains empty prior to sampling and serves as a moisture knockout impinger. The second and third impingers are charged with $5 \% \mathrm{HNO}_{3} / 10 \% \mathrm{H}_{2} \mathrm{O}_{2}$ and are analyzed with the first impinger contents for all of the metallic analytes including mercury. The fourth impinger is left empty and serves to protect the mercury trapping solution from carryover. Its contents and glassware rinses are analyzed separately for mercury, only. The fifth and sixth impingers contain $4 \% \mathrm{KMnO}_{4} / 10 \% \mathrm{H}_{2} \mathrm{SO}_{4}$ and are also analyzed for mercury, only. The seventh impinger contains silica gel for a final scrubbing of residual moisture. The MMT back half mercury $(\mathrm{Hg})$ result is the sum of the mercury in the $5 \% \mathrm{HNO}_{3} / 10 \% \mathrm{H}_{2} \mathrm{O}_{2}$ fraction, the fourth empty impinger fraction, the $4 \% \mathrm{KMnO}_{4} / 10 \% \mathrm{H}_{2} \mathrm{SO}_{4}$ fraction, and the $8 \mathrm{~N} \mathrm{HCl}$ impinger rinse fraction. The total micrograms (ug) of mercury in these fractions is included in the MMT back half result.

3 The total mass for each metal found in the MMT sampling train consists of the sum of the MMT train's Front Half metals content plus the train's Back Half metals content. The calculation is as follows:

(Total $\mu \mathrm{g}$ in the Front Half $)+($ Total $\mu \mathrm{g}$ in the Back Half $)=$ Total $\mu \mathrm{g}$ in the Multi-Metals Sampling Train. Therefore: $(\mu \mathrm{g})+(\mu \mathrm{g})=$ Total $\mu \mathrm{g}$

The MMT Sampling Train Total value was obtained by summing the MMT Front Half results and the MMT Back Half results using the following guidelines:

- When the train component analytical result is greater than the laboratory reporting limit (RL), the result included in the train total is the actual analytical result or "hit" determined by the laboratory.

- When the train component analytical result is greater than the reliable detection level (RDL), but less than the laboratory reporting limit (RL), the result included in the train total is actual analytical result or "hit" determined by the laboratory and the corresponding " $B$ " flag is carried through the calculation to the train total.

- When the train analytical component result is less than the RDL, but greater than the method detection limit (MDL), the result included in the train total is the RDL and the corresponding " $\mathrm{B}$ " flag is carried through the calculation to the train total.

- When the train component analytical result is not detected down to the MDL, the result included in the train total is the RDL and the corresponding " $\mathrm{U}$ " flag is carried through the calculation to the train total.

- It should be noted that when the RDL is selected as the default value using the guidelines above, but the RDL is greater than the RL, the $\mathrm{RL}$ is included in the train total.

The data flags attached to the MMT Totals are the cumulative set of flags for each train component included as part of the MMT total. A flag attached to a MMT component is carried through to the "MMT Sampling Train Total" column when the associated component analytical result is a significant number in comparison to the MMT Total. That is, if the MMT Total is affected by a MMT component analytical result, the associated flag is carried to the MMT Train Total, but if the MMT Train Total is not affected by a MMT component, the associated flag is not carried through to the MMT Train Total. The combinations of train fractions are conducted following the standard practice of using significant figures found in ASTM E29-93a(1999), "Standard Practice for Using Significant Digits in Test Data to Determine Conformance with Specifications" and Severn Trent Laboratories standard operating procedure number QA-004, "Rounding and Significant Figures".

4 This flag is the laboratory data flag that corresponds to EPA guidelines. The data flags for these samples are as follows:

- A "U" qualifier indicates that this analyte was analyzed for, but was not detected down to the MDL. 
BECHTEL BWXT IDAHO, LLC (BBWI)

INTEC HLLWE Effluent Gas Emissions Inventory

Idaho National Engineering and Environmental Laboratory (INEEL)

STL Knoxville Project Number: 142503.40

- A "B" flag indicates that this analyte was detected, but at a concentration below the laboratory RL. The analytical result is therefore an estimated value.

5 When listed, the less than $(<)$ sign indicates that at least one sample fraction result is either a "non-detect" value down to the MDL of the measurement that carries, or an estimated "hit" value that is below the RDL. In either case, the final value for the fraction that is included in the data set total is the default RDL value and the actual value of the total is known to be less than $(<)$ the displayed result.

6 Entries in this column are project-specific train total flags that are applied to the run total values and are not standard EPA data flags. These project-specific flags are utilized for the INEEL NWCF HLLWE Effluent Gas Emissions Inventory project and are defined as follows:

- An "N" flag in this column indicates that the compound was not measured (detected) in any of the sampling train components, or fractions.

- A "P" flag in this column indicates that the compound was measured (detected) in one or more of the train components, or fractions, but not in all of the sampling train fractions.

- An "A" flag in this column indicates that the compound was measured (detected) in all of the sampling train components, or fractions. 

BECHTEL BWXT IDAHO, LLC (BBWI)

INTEC HLLWE Effluent Gas Emissions Inventory

Idaho National Engineering and Environmental Laboratory (INEEL)

STL Knoxville Project Number: 142503.40

\section{Method 0060 Multi-Metals Train (MMT) Train Total Summary - Run 2 Train Totals Metallic Analyte Analytical Results Summary Table A-22. HLLWE Run ID: 0060-STRT-2}

Field Sample Name Sample Description:
Method 0060 Multi-Metals Train (MMT)

Method 0060 Multi-Metals Train (MMT) Totals for Metals Analysis

\begin{tabular}{|c|c|c|c|c|c|c|c|c|}
\hline \multirow[b]{2}{*}{ Analyte } & \multirow{2}{*}{$\begin{array}{c}\text { CAS } \\
\text { Registry } \\
\text { Number }\end{array}$} & \multicolumn{2}{|c|}{$\begin{array}{c}\text { MMT } \\
\text { Front Half } \\
\text { Composite }^{1} \\
(\mu \mathrm{g})\end{array}$} & \multicolumn{2}{|c|}{$\begin{array}{c}\text { MMT } \\
\text { Back Half }^{2} \\
\text { Composite }^{2} \\
(\mu \mathrm{g})\end{array}$} & \multicolumn{2}{|c|}{$\begin{array}{c}\text { MMT } \\
\text { Sampling Train } \\
\text { Totals }^{3} \\
(\text { Total } \mu \mathrm{g}) \\
\end{array}$} & \multirow{2}{*}{$\begin{array}{c}\text { Project } \\
\text { Specific } \\
\text { Flag }^{6} \\
\end{array}$} \\
\hline & & Risk Result & Flag ${ }^{4}$ & Risk Result & Flag $^{4}$ & Total $^{5}$ & Flag & \\
\hline Aluminum (Al) & $7429-90-5$ & 42 & & 34 & & 76 & & $A$ \\
\hline Antimony (Sb) & $7440-36-0$ & 2.8 & B & 1.7 & B & $<4.5$ & B & A \\
\hline Arsenic (As) & $7440-38-2$ & 0.92 & U & 0.66 & $\mathrm{U}$ & $<1.6$ & & $\mathrm{~N}$ \\
\hline Barium (Ba) & $7440-39-3$ & 3.5 & B & 1.6 & B & 5.1 & B & A \\
\hline Beryllium (Be) & $7440-41-7$ & 0.23 & B & 0.42 & $\mathrm{U}$ & $<0.65$ & B & $P$ \\
\hline Cadmium (Cd) & $7440-43-9$ & 0.13 & $\mathrm{U}$ & 0.14 & B & $<0.27$ & B & $\mathrm{P}$ \\
\hline Chromium (Cr) & $7440-47-3$ & 1.3 & & 1.2 & & 2.5 & & A \\
\hline Cobalt (Co) & $7440-48-4$ & 1.3 & $\mathrm{U}$ & 1.4 & U & $<2.7$ & & $\mathrm{~N}$ \\
\hline Copper (Cu) & $7440-50-8$ & 0.66 & B & 1.6 & B & $<2.3$ & B & A \\
\hline Lead $(\mathrm{Pb})$ & $7439-92-1$ & 0.52 & $\mathrm{U}$ & 0.89 & B & $<1.4$ & B & $\mathrm{P}$ \\
\hline Manganese (Mn) & $7439-96-5$ & 16 & & 22 & & 38 & & A \\
\hline Mercury (Hg) & 7439-97-6 & 0.37 & B & 110 & & 110 & & A \\
\hline Nickel (Ni) & $7440-02-0$ & 3.3 & B & 1.2 & B & $<4.5$ & B & A \\
\hline Selenium (Se) & $7782-49-2$ & 2.0 & & 0.63 & B & $<2.6$ & B & A \\
\hline Silver (Ag) & $7440-22-4$ & 1.9 & $\mathrm{U}$ & 0.71 & U & $<2.6$ & & $\mathrm{~N}$ \\
\hline Thallium (Tl) & $7440-28-0$ & 1.0 & $\mathrm{U}$ & 1.5 & U & $<2.5$ & & $\mathrm{~N}$ \\
\hline Vanadium (V) & $7440-62-2$ & 1.3 & U & 1.4 & $\mathrm{U}$ & $<2.7$ & & $\mathrm{~N}$ \\
\hline $\operatorname{Zinc}(Z n)$ & $7440-66-6$ & 10 & & 20 & & 30 & & $\mathrm{~A}$ \\
\hline
\end{tabular}




\section{BECHTEL BWXT IDAHO, LLC (BBWI) INTEC HLLWE Effluent Gas Emissions Inventory Idaho National Engineering and Environmental Laboratory (INEEL) STL Knoxville Project Number: 142503.40}

\section{Footnotes:}

1 The MMT Front Half Composite consists of the Quartz Fiber Particulate Filter and the 0.1 N Nitric Acid Probe Rinse Solution.

2 The MMT Back Half configuration includes seven (7) impingers. The first impinger remains empty prior to sampling and serves as a moisture knockout impinger. The second and third impingers are charged with $5 \% \mathrm{HNO}_{3} / 10 \% \mathrm{H}_{2} \mathrm{O}_{2}$ and are analyzed with the first impinger contents for all of the metallic analytes including mercury. The fourth impinger is left empty and serves to protect the mercury trapping solution from carryover. Its contents and glassware rinses are analyzed separately for mercury, only. The fifth and sixth impingers contain $4 \% \mathrm{KMnO}_{4} / 10 \% \mathrm{H}_{2} \mathrm{SO}_{4}$ and are also analyzed for mercury, only. The seventh impinger contains silica gel for a final scrubbing of residual moisture. The MMT back half mercury $(\mathrm{Hg})$ result is the sum of the mercury in the $5 \% \mathrm{HNO}_{3} / 10 \% \mathrm{H}_{2} \mathrm{O}_{2}$ fraction, the fourth empty impinger fraction, the $4 \% \mathrm{KMnO}_{4} / 10 \% \mathrm{H}_{2} \mathrm{SO}_{4}$ fraction, and the $8 \mathrm{~N} \mathrm{HCl}$ impinger rinse fraction. The total micrograms (ug) of mercury in these fractions is included in the MMT back half result.

3 The total mass for each metal found in the MMT sampling train consists of the sum of the MMT train's Front Half metals content plus the train's Back Half metals content. The calculation is as follows:

(Total $\mu \mathrm{g}$ in the Front Half) $+($ Total $\mu \mathrm{g}$ in the Back Half $)=$ Total $\mu \mathrm{g}$ in the Multi-Metals Sampling Train. Therefore: $(\mu \mathrm{g})+(\mu \mathrm{g})=$ Total $\mu \mathrm{g}$

The MMT Sampling Train Total value was obtained by summing the MMT Front Half results and the MMT Back Half results using the following guidelines:

- When the train component analytical result is greater than the laboratory reporting limit (RL), the result included in the train total is the actual analytical result or "hit" determined by the laboratory.

- When the train component analytical result is greater than the reliable detection level (RDL), but less than the laboratory reporting limit (RL), the result included in the train total is actual analytical result or "hit" determined by the laboratory and the corresponding "B" flag is carried through the calculation to the train total.

- When the train analytical component result is less than the RDL, but greater than the method detection limit (MDL), the result included in the train total is the RDL and the corresponding " $\mathrm{B}$ " flag is carried through the calculation to the train total.

- When the train component analytical result is not detected down to the MDL, the result included in the train total is the RDL and the corresponding " $U$ " flag is carried through the calculation to the train total.

- It should be noted that when the RDL is selected as the default value using the guidelines above, but the RDL is greater than the RL, the $\mathrm{RL}$ is included in the train total.

The data flags attached to the MMT Totals are the cumulative set of flags for each train component included as part of the MMT total. A flag attached to a MMT component is carried through to the "MMT Sampling Train Total" column when the associated component analytical result is a significant number in comparison to the MMT Total. That is, if the MMT Total is affected by a MMT component analytical result, the associated flag is carried to the MMT Train Total, but if the MMT Train Total is not affected by a MMT component, the associated flag is not carried through to the MMT Train Total. The combinations of train fractions are conducted following the standard practice of using significant figures found in ASTM E29-93a(1999), "Standard Practice for Using Significant Digits in Test Data to Determine Conformance with Specifications" and Severn Trent Laboratories standard operating procedure number QA-004, "Rounding and Significant Figures".

4 This flag is the laboratory data flag that corresponds to EPA guidelines. The data flags for these samples are as follows:

- A "U" qualifier indicates that this analyte was analyzed for, but was not detected down to the MDL.

- A "B" flag indicates that this analyte was detected, but at a concentration below the laboratory RL. The analytical result is therefore an estimated value. 
BECHTEL BWXT IDAHO, LLC (BBWI)

INTEC HLLWE Effluent Gas Emissions Inventory

Idaho National Engineering and Environmental Laboratory (INEEL)

STL Knoxville Project Number: 142503.40

$5 \quad$ When listed, the less than $(<)$ sign indicates that at least one sample fraction result is either a "non-detect" value down to the MDL of the measurement that carries, or an estimated "hit" value that is below the RDL. In either case, the final value for the fraction that is included in the data set total is the default RDL value and the actual value of the total is known to be less than $(<)$ the displayed result.

6 Entries in this column are project-specific train total flags that are applied to the run total values and are not standard EPA data flags. These project-specific flags are utilized for the INEEL NWCF HLLWE Effluent Gas Emissions Inventory project and are defined as follows:

- An "N" flag in this column indicates that the compound was not measured (detected) in any of the sampling train components, or fractions.

- A "P" flag in this column indicates that the compound was measured (detected) in one or more of the train components, or fractions, but not in all of the sampling train fractions.

- An "A" flag in this column indicates that the compound was measured (detected) in all of the sampling train components, or fractions. 

BECHTEL BWXT IDAHO, LLC (BBWI)

INTEC HLLWE Effluent Gas Emissions Inventory

Idaho National Engineering and Environmental Laboratory (INEEL)

STL Knoxville Project Number: 142503.40

\section{Method 0060 Multi-Metals Train (MMT) Train Total Summary - Run 4 Train Totals Metallic Analyte Analytical Results Summary \\ Table A-23. HLLWE Run ID: 0060-END-2}

Field Sample Name:

Sample Description:
Method 0060 Multi-Metals Train (MMT)

Method 0060 Multi-Metals Train (MMT) Totals for Metals Analysis

\begin{tabular}{|c|c|c|c|c|c|c|c|c|}
\hline \multirow[b]{2}{*}{ Analyte } & \multirow{2}{*}{$\begin{array}{c}\text { CAS } \\
\text { Registry } \\
\text { Number }\end{array}$} & \multicolumn{2}{|c|}{$\begin{array}{c}\text { MMT } \\
\text { Front Half } \\
\text { Composite }^{1} \\
(\mu \mathrm{g})\end{array}$} & \multicolumn{2}{|c|}{$\begin{array}{c}\text { MMT } \\
\text { Back Half } \\
\text { Composite }^{2} \\
(\mu \mathrm{g})\end{array}$} & \multicolumn{2}{|c|}{$\begin{array}{c}\text { MMT } \\
\text { Sampling Train } \\
\text { Totals }^{3} \\
(\text { Total } \mu \mathrm{g})\end{array}$} & \multirow{2}{*}{$\begin{array}{c}\text { Projec } \\
\text { Specifi } \\
\text { Flag }^{6} \\
\end{array}$} \\
\hline & & Risk Result & Flag $^{4}$ & Risk Result & Flag $^{4}$ & Total $^{5}$ & Flag & \\
\hline Aluminum (Al) & $7429-90-5$ & 44 & & 29 & & 73 & & A \\
\hline Antimony (Sb) & $7440-36-0$ & 2.4 & B & 1.7 & B & $<4.1$ & B & A \\
\hline Arsenic (As) & $7440-38-2$ & 0.92 & $\mathrm{U}$ & 0.66 & $\mathrm{U}$ & $<1.6$ & & $\mathrm{~N}$ \\
\hline Barium (Ba) & $7440-39-3$ & 3.6 & B & 1.1 & B & 4.7 & B & A \\
\hline Beryllium (Be) & $7440-41-7$ & 0.23 & U & 0.42 & B & $<0.65$ & B & $P$ \\
\hline Cadmium (Cd) & $7440-43-9$ & 0.13 & U & 0.14 & U & $<0.27$ & & $N$ \\
\hline Chromium $(\mathrm{Cr})$ & $7440-47-3$ & 1.2 & & 3.9 & & 5.1 & & A \\
\hline Cobalt $(\mathrm{Co})$ & $7440-48-4$ & 1.3 & $\mathrm{U}$ & 1.4 & $\mathrm{U}$ & $<2.7$ & & $\mathrm{~N}$ \\
\hline Copper $(\mathrm{Cu})$ & $7440-50-8$ & 0.66 & $\mathrm{U}$ & 0.71 & B & $<1.4$ & B & $\mathrm{P}$ \\
\hline Lead $(\mathrm{Pb})$ & $7439-92-1$ & 0.52 & $\mathrm{U}$ & 0.68 & B & $<1.2$ & B & $\mathrm{P}$ \\
\hline Manganese $(\mathrm{Mn})$ & $7439-96-5$ & 23 & & 49 & & 72 & & A \\
\hline Mercury $(\mathrm{Hg})$ & $7439-97-6$ & 0.37 & B & 110 & & 110 & & A \\
\hline Nickel (Ni) & $7440-02-0$ & 3.0 & B & 1.1 & B & $<4.1$ & B & A \\
\hline Selenium $(\mathrm{Se})$ & $7782-49-2$ & 1.7 & & 0.63 & B & $<2.3$ & B & A \\
\hline Silver (Ag) & $7440-22-4$ & 1.9 & $\mathrm{U}$ & 0.71 & U & $<2.6$ & & $N$ \\
\hline Thallium ( $\mathrm{Tl}$ ) & $7440-28-0$ & 1.0 & U & 1.4 & U & $<2.4$ & & $\mathrm{~N}$ \\
\hline Vanadium (V) & $7440-62-2$ & 1.3 & $\mathrm{U}$ & 1.4 & $\mathrm{U}$ & $<2.7$ & & $\mathrm{~N}$ \\
\hline Zinc $(Z n)$ & $7440-66-6$ & 6.6 & & 10 & & 17 & & A \\
\hline
\end{tabular}


BECHTEL BWXT IDAHO, LLC (BBWI)

INTEC HLLWE Effluent Gas Emissions Inventory

Idaho National Engineering and Environmental Laboratory (INEEL)

STL Knoxville Project Number: 142503.40

\section{Footnotes:}

1 The MMT Front Half Composite consists of the Quartz Fiber Particulate Filter and the 0.1 N Nitric Acid Probe Rinse Solution.

2 The MMT Back Half configuration includes seven (7) impingers. The first impinger remains empty prior to sampling and serves as a moisture knockout impinger. The second and third impingers are charged with $5 \% \mathrm{HNO}_{3} / 10 \% \mathrm{H}_{2} \mathrm{O}_{2}$ and are analyzed with the first impinger contents for all of the metallic analytes including mercury. The fourth impinger is left empty and serves to protect the mercury trapping solution from carryover. Its contents and glassware rinses are analyzed separately for mercury, only. The fifth and sixth impingers contain $4 \% \mathrm{KMnO}_{4} / 10 \% \mathrm{H}_{2} \mathrm{SO}_{4}$ and are also analyzed for mercury, only. The seventh impinger contains silica gel for a final scrubbing of residual moisture. The MMT back half mercury $(\mathrm{Hg})$ result is the sum of the mercury in the $5 \% \mathrm{HNO}_{3} / 10 \% \mathrm{H}_{2} \mathrm{O}_{2}$ fraction, the fourth empty impinger fraction, the $4 \% \mathrm{KMnO}_{4} / 10 \% \mathrm{H}_{2} \mathrm{SO}_{4}$ fraction, and the $8 \mathrm{~N} \mathrm{HCl}$ impinger rinse fraction. The total micrograms (ug) of mercury in these fractions is included in the MMT back half result.

3 The total mass for each metal found in the MMT sampling train consists of the sum of the MMT train's Front Half metals content plus the train's Back Half metals content. The calculation is as follows:

(Total $\mu \mathrm{g}$ in the Front Half) $+($ Total $\mu \mathrm{g}$ in the Back Half) $=$ Total $\mu \mathrm{g}$ in the Multi-Metals Sampling Train. Therefore: $(\mu \mathrm{g})+(\mu \mathrm{g})=$ Total $\mu \mathrm{g}$

The MMT Sampling Train Total value was obtained by summing the MMT Front Half results and the MMT Back Half results using the following guidelines:

- When the train component analytical result is greater than the laboratory reporting limit (RL), the result included in the train total is the actual analytical result or "hit" determined by the laboratory.

- When the train component analytical result is greater than the reliable detection level (RDL), but less than the laboratory reporting limit (RL), the result included in the train total is actual analytical result or "hit" determined by the laboratory and the corresponding " $\mathrm{B}$ " flag is carried through the calculation to the train total.

- When the train analytical component result is less than the RDL, but greater than the method detection limit (MDL), the result included in the train total is the RDL and the corresponding " $\mathrm{B}$ " flag is carried through the calculation to the train total.

- When the train component analytical result is not detected down to the MDL, the result included in the train total is the RDL and the corresponding " $U$ " flag is carried through the calculation to the train total.

- It should be noted that when the RDL is selected as the default value using the guidelines above, but the RDL is greater than the RL, the $\mathrm{RL}$ is included in the train total.

The data flags attached to the MMT Totals are the cumulative set of flags for each train component included as part of the MMT total. A flag attached to a MMT component is carried through to the "MMT Sampling Train Total" column when the associated component analytical result is a significant number in comparison to the MMT Total. That is, if the MMT Total is affected by a MMT component analytical result, the associated flag is carried to the MMT Train Total, but if the MMT Train Total is not affected by a MMT component, the associated flag is not carried through to the MMT Train Total. The combinations of train fractions are conducted following the standard practice of using significant figures found in ASTM E29-93a(1999), "Standard Practice for Using Significant Digits in Test Data to Determine Conformance with Specifications" and Severn Trent Laboratories standard operating procedure number QA-004, "Rounding and Significant Figures".

4 This flag is the laboratory data flag that corresponds to EPA guidelines. The data flags for these samples are as follows:

- A "U" qualifier indicates that this analyte was analyzed for, but was not detected down to the MDL. 
BECHTEL BWXT IDAHO, LLC (BBWI)

INTEC HLLWE Effluent Gas Emissions Inventory

Idaho National Engineering and Environmental Laboratory (INEEL)

STL Knoxville Project Number: 142503.40

- A "B" flag indicates that this analyte was detected, but at a concentration below the laboratory RL. The analytical result is therefore an estimated value.

5 When listed, the less than $(<)$ sign indicates that at least one sample fraction result is either a "non-detect" value down to the MDL of the measurement that carries, or an estimated "hit" value that is below the RDL. In either case, the final value for the fraction that is included in the data set total is the default RDL value and the actual value of the total is known to be less than $(<)$ the displayed result.

6 Entries in this column are project-specific train total flags that are applied to the run total values and are not standard EPA data flags. These project-specific flags are utilized for the INEEL NWCF HLLWE Effluent Gas Emissions Inventory project and are defined as follows:

- An "N" flag in this column indicates that the compound was not measured (detected) in any of the sampling train components, or fractions.

- A "P" flag in this column indicates that the compound was measured (detected) in one or more of the train components, or fractions, but not in all of the sampling train fractions.

- An "A" flag in this column indicates that the compound was measured (detected) in all of the sampling train components, or fractions. 

BECHTEL BWXT IDAHO, LLC (BBWI)

INTEC HLLWE Effluent Gas Emissions Inventory

Idaho National Engineering and Environmental Laboratory (INEEL)

STL Knoxville Project Number: 142503.40

\section{Method 0060 Multi-Metals Blank Train (MMT) Train Total Summary \\ Run 2 Blank Train Totals \\ Metallic Analyte Analytical Results Summary \\ Table A-24. HLLWE Run ID: 0060-BT-1}

Field Sample Name:

Sample Description:
Method 0060 Multi-Metals Blank Train (MMT)

Method 0060 Multi-Metals Blank Train (MMT) Totals for Metals Analysis

\begin{tabular}{|c|c|c|c|c|c|c|c|c|}
\hline \multirow[b]{2}{*}{ Analyte } & \multirow{2}{*}{$\begin{array}{c}\text { CAS } \\
\text { Registry } \\
\text { Number } \\
\end{array}$} & \multicolumn{2}{|c|}{$\begin{array}{c}\text { MMT } \\
\text { Front Half } \\
\text { Composite } \\
(\mu \mathrm{g})\end{array}$} & \multicolumn{2}{|c|}{$\begin{array}{c}\text { MMT } \\
\text { Back Half } \\
\text { Composite }^{2} \\
(\mu \mathrm{g})\end{array}$} & \multicolumn{2}{|c|}{$\begin{array}{c}\text { MMT } \\
\text { Sampling Train } \\
\text { Totals }^{3} \\
(\text { Total } \mu \mathrm{g}) \\
\end{array}$} & \multirow{2}{*}{$\begin{array}{c}\text { Project } \\
\text { Specific } \\
\text { Flag }^{6}\end{array}$} \\
\hline & & Risk Result & Flag $^{4}$ & Risk Result & Flag $^{4}$ & Total $^{5}$ & Flag & \\
\hline Aluminum (Al) & $7429-90-5$ & 46 & & 29 & & 75 & & A \\
\hline Antimony (Sb) & $7440-36-0$ & 2.5 & B & 1.7 & B & $<4.2$ & B & A \\
\hline Arsenic (As) & $7440-38-2$ & 0.92 & B & 0.66 & $\mathrm{U}$ & $<1.6$ & B & $\mathbf{P}$ \\
\hline Barium $(\mathrm{Ba})$ & $7440-39-3$ & 3.4 & B & 1.3 & B & 4.7 & B & A \\
\hline Beryllium (Be) & $7440-41-7$ & 0.23 & $\mathrm{U}$ & 0.42 & $\mathrm{U}$ & $<0.65$ & & $\mathrm{~N}$ \\
\hline Cadmium (Cd) & $7440-43-9$ & 0.13 & $\mathrm{U}$ & 0.14 & B & $<0.27$ & B & $\mathbf{P}$ \\
\hline Chromium (Cr) & $7440-47-3$ & 0.66 & U & 0.66 & U & $<1.3$ & & $\mathrm{~N}$ \\
\hline Cobalt (Co) & $7440-48-4$ & 1.3 & U & 1.4 & U & $<2.7$ & & $\mathrm{~N}$ \\
\hline Copper $(\mathrm{Cu})$ & $7440-50-8$ & 0.66 & $\mathrm{U}$ & 0.71 & B & $<1.4$ & B & $P$ \\
\hline Lead $(\mathrm{Pb})$ & $7439-92-1$ & 0.52 & U & 0.68 & B & $<1.2$ & B & $P$ \\
\hline Manganese (Mn) & $7439-96-5$ & 1.6 & & 3,700 & & 3,700 & & A \\
\hline Mercury (Hg) & $7439-97-6$ & 0.37 & U & 2.7 & $\mathrm{U}$ & $<3.1$ & & $\mathrm{~N}$ \\
\hline Nickel (Ni) & $7440-02-0$ & 2.8 & B & 1.2 & B & $<4.0$ & B & A \\
\hline Selenium (Se) & $7782-49-2$ & 1.7 & & 0.63 & U & $<2.3$ & & $\mathrm{P}$ \\
\hline Silver (Ag) & $7440-22-4$ & 1.9 & $\mathrm{U}$ & 0.71 & $\mathrm{U}$ & $<2.6$ & & $\mathrm{~N}$ \\
\hline Thallium (TI) & $7440-28-0$ & 1.0 & U & 1.5 & U & $<2.5$ & & $\mathrm{~N}$ \\
\hline Vanadium (V) & $7440-62-2$ & 1.3 & U & 1.4 & $\mathrm{U}$ & $<2.7$ & & $\mathrm{~N}$ \\
\hline $\operatorname{Zinc}(\mathrm{Zn})$ & $7440-66-6$ & 4.7 & & 34 & & 39 & & A \\
\hline
\end{tabular}




\section{BECHTEL BWXT IDAHO, LLC (BBWI) \\ INTEC HLLWE Effluent Gas Emissions Inventory \\ Idaho National Engineering and Environmental Laboratory (INEEL) STL Knoxville Project Number: 142503.40}

\section{Footnotes:}

1 The MMT Front Half Composite consists of the Quartz Fiber Particulate Filter and the 0.1 N Nitric Acid Probe Rinse Solution.

2 The MMT Back Half configuration includes seven (7) impingers. The first impinger remains empty prior to sampling and serves as a moisture knockout impinger. The second and third impingers are charged with $5 \% \mathrm{HNO}_{3} / 10 \% \mathrm{H}_{2} \mathrm{O}_{2}$ and are analyzed with the first impinger contents for all of the metallic analytes including mercury. The fourth impinger is left empty and serves to protect the mercury trapping solution from carryover. Its contents and glassware rinses are analyzed separately for mercury, only. The fifth and sixth impingers contain $4 \% \mathrm{KMnO}_{4} / 10 \% \mathrm{H}_{2} \mathrm{SO}_{4}$ and are also analyzed for mercury, only. The seventh impinger contains silica gel for a final scrubbing of residual moisture. The MMT back half mercury $(\mathrm{Hg})$ result is the sum of the mercury in the $5 \% \mathrm{HNO}_{3} / 10 \% \mathrm{H}_{2} \mathrm{O}_{2}$ fraction, the fourth empty impinger fraction, the $4 \% \mathrm{KMnO}_{4} / 10 \% \mathrm{H}_{2} \mathrm{SO}_{4}$ fraction, and the $8 \mathrm{~N} \mathrm{HCl}$ impinger rinse fraction. The total micrograms (ug) of mercury in these fractions is included in the MMT back half result.

3 The total mass for each metal found in the MMT sampling train consists of the sum of the MMT train's Front Half metals content plus the train's Back Half metals content. The calculation is as follows:

(Total $\mu \mathrm{g}$ in the Front Half $)+($ Total $\mu \mathrm{g}$ in the Back Half $)=$ Total $\mu \mathrm{g}$ in the Multi-Metals Sampling Train. Therefore: $(\mu \mathrm{g})+(\mu \mathrm{g})=$ Total $\mu \mathrm{g}$

The MMT Sampling Train Total value was obtained by summing the MMT Front Half results and the MMT Back Half results using the following guidelines:

- When the train component analytical result is greater than the laboratory reporting limit (RL), the result included in the train total is the actual analytical result or "hit" determined by the laboratory.

- When the train component analytical result is greater than the reliable detection level (RDL), but less than the laboratory reporting limit (RL), the result included in the train total is actual analytical result or "hit" determined by the laboratory and the corresponding " $\mathrm{B}$ " flag is carried through the calculation to the train total.

- When the train analytical component result is less than the RDL, but greater than the method detection limit (MDL), the result included in the train total is the RDL and the corresponding " $\mathrm{B}$ " flag is carried through the calculation to the train total.

- When the train component analytical result is not detected down to the MDL, the result included in the train total is the RDL and the corresponding "U" flag is carried through the calculation to the train total

- It should be noted that when the RDL is selected as the default value using the guidelines above, but the RDL is greater than the RL, the $\mathrm{RL}$ is included in the train total.

The data flags attached to the MMT Totals are the cumulative set of flags for each train component included as part of the MMT total. A flag attached to a MMT component is carried through to the "MMT Sampling Train Total" column when the associated component analytical result is a significant number in comparison to the MMT Total. That is, if the MMT Total is affected by a MMT component analytical result, the associated flag is carried to the MMT Train Total, but if the MMT Train Total is not affected by a MMT component, the associated flag is not carried through to the MMT Train Total. The combinations of train fractions are conducted following the standard practice of using significant figures found in ASTM E29-93a(1999), "Standard Practice for Using Significant Digits in Test Data to Determine Conformance with Specifications" and Severn Trent Laboratories standard operating procedure number QA-004, "Rounding and Significant Figures".

4 This flag is the laboratory data flag that corresponds to EPA guidelines. The data flags for these samples are as follows:

- A "U" qualifier indicates that this analyte was analyzed for, but was not detected down to the MDL.

- A "B" flag indicates that this analyte was detected, but at a concentration below the laboratory RL. The analytical result is therefore an estimated value. 
BECHTEL BWXT IDAHO, LLC (BBWI)

INTEC HLLWE Effluent Gas Emissions Inventory

Idaho National Engineering and Environmental Laboratory (INEEL)

STL Knoxville Project Number: 142503.40

$5 \quad$ When listed, the less than $(<)$ sign indicates that at least one sample fraction result is either a "non-detect" value down to the MDL of the measurement that carries, or an estimated "hit" value that is below the RDL. In either case, the final value for the fraction that is included in the data set total is the default RDL value and the actual value of the total is known to be less than $(<)$ the displayed result.

6 Entries in this column are project-specific train total flags that are applied to the run total values and are not standard EPA data flags. These project-specific flags are utilized for the INEEL NWCF HLLWE Effluent Gas Emissions Inventory project and are defined as follows:

- An " $N$ " flag in this column indicates that the compound was not measured (detected) in any of the sampling train components, or fractions.

- A "P" flag in this column indicates that the compound was measured (detected) in one or more of the train components, or fractions, but not in all of the sampling train fractions.

- An "A" flag in this column indicates that the compound was measured (detected) in all of the sampling train components, or fractions. 

BECHTEL BWXT IDAHO, LLC (BBWI)

INTEC HLLWE Effluent Gas Emissions Inventory

Idaho National Engineering and Environmental Laboratory (INEEL)

STL Knoxville Project Number: 142503.40

\section{Table A-25. Method 0060 Multi-Metals Train (MMT) Analytical Results Summary Run 2 Front Half Composite Reagent Blank}

Field Sample Name:

Sample Description:

Field Sample ID:

STL Sample No:
Method 0060 Multi-Metals Train (MMT) Front Half Composite Reagent Blanks

Quartz Fiber Particulate Filter and 0.1 N Nitric Acid Probe Rinse Solution Reagent Blanks for Metals (including Mercury) Analysis

A-3297 and A-3298

H1F200234-011

\begin{tabular}{|c|c|c|c|c|c|c|c|}
\hline \multirow[b]{2}{*}{ Analyte } & \multirow[b]{2}{*}{$\begin{array}{c}\text { CAS Registry } \\
\text { Number }\end{array}$} & \multicolumn{6}{|c|}{$\begin{array}{c}\text { MMT } \\
\text { Front Half Composite Reagent Blank } \\
\text { Total } \mu \mathrm{g}\end{array}$} \\
\hline & & Lab Result ${ }^{1}$ & $\mathrm{MDL}^{2}$ & $\mathrm{RDL}^{3}$ & $\mathbf{R L}^{4}$ & $\begin{array}{c}\text { Risk Result } \\
\text { for Blank } \\
\text { Correction } \\
\end{array}$ & Flag $^{6}$ \\
\hline Aluminum (Al) & $7429-90-5$ & 43 & 5.2 & 14 & 20 & 43 & \\
\hline Antimony (Sb) & $7440-36-0$ & 2.7 & 0.60 & 1.6 & 6.0 & 2.7 & B \\
\hline Arsenic (As) & $7440-38-2$ & 0.72 & 0.35 & 0.92 & 1.0 & 0.72 & B \\
\hline Barium (Ba) & $7440-39-3$ & 3.6 & 0.35 & 0.92 & 20 & 3.6 & B \\
\hline Beryllium (Be) & $7440-41-7$ & 0.20 & 0.089 & 0.23 & 0.50 & 0.20 & B \\
\hline Cadmium (Cd) & $7440-43-9$ & ND & 0.050 & 0.13 & 0.50 & 0 & \\
\hline Chromium (Cr) & $7440-47-3$ & 0.92 & 0.25 & 0.66 & 1.0 & 0.92 & B \\
\hline Cobalt (Co) & $7440-48-4$ & ND & 0.50 & 1.3 & 5.0 & 0 & \\
\hline Copper $(\mathrm{Cu})$ & $7440-50-8$ & ND & 0.25 & 0.66 & 2.5 & 0 & \\
\hline Lead $(\mathrm{Pb})$ & $7439-92-1$ & ND & 0.20 & 0.52 & 1.0 & 0 & \\
\hline Manganese (Mn) & $7439-96-5$ & 0.52 & 0.15 & 0.39 & 1.5 & 0.52 & B \\
\hline Mercury (Hg) & $7439-97-6$ & ND & 0.14 & 0.37 & 0.40 & 0 & \\
\hline Nickel (Ni) & $7440-02-0$ & 2.9 & 0.44 & 1.2 & 4.0 & 2.9 & B \\
\hline Selenium (Se) & $7782-49-2$ & 2.9 & 0.35 & 0.92 & 1.0 & 2.9 & \\
\hline Silver (Ag) & $7440-22-4$ & ND & 0.71 & 1.9 & 2.0 & 0 & \\
\hline Thallium (TI) & $7440-28-0$ & ND & 0.40 & 1.0 & 2.0 & 0 & \\
\hline Vanadium (V) & $7440-62-2$ & ND & 0.50 & 1.3 & 5.0 & 0 & \\
\hline $\operatorname{Zinc}(Z n)$ & $7440-66-6$ & 3.1 & 0.23 & 0.60 & 2.0 & 3.1 & \\
\hline
\end{tabular}


BECHTEL BWXT IDAHO, LLC (BBWI)

INTEC HLLWE Effluent Gas Emissions Inventory

Idaho National Engineering and Environmental Laboratory (INEEL)

STL Knoxville Project Number: 142503.40

\section{Table A-25. Method 0060 Multi-Metals Train (MMT) Analytical Results Summary (Continued) Run 2 Front Half Composite Reagent Blank}

Sample Collection and Analysis Dates:

$\begin{array}{ll}\text { Date(s) Collected: } & \text { June 07, 2001 } \\ \text { Date(s) of Digestion (Metals): } & \text { June 24, 2001 } \\ \text { Date(s) of Digestion (Mercury): } & \text { June 25, 2001 } \\ \text { Date(s) of Analysis (Metals): } & \text { June 28, 2001 } \\ \text { Date(s) of Analysis (Mercury): } & \text { June 26, 2001 }\end{array}$

\section{Preparation and Analvsis Methods:}

SW-846 Method 0060: "Determination of Metals in Stack Emissions"

SW-846 Method 7470A: "Mercury in Liquid Waste (Manual Cold Vapor Technique)"

SW-846 Method 6010B: "Inductively Coupled Plasma - Atomic Emission Spectroscopy"

\section{Footnotes:}

1 This value is the laboratory sample result. When the analytical result is "ND" or not detected, the laboratory analysis did not detect the analyte down to the MDL.

2 This value is the laboratory Method Detection Limit (MDL) derived according to requirements outlined in 40 CFR Part 136 , Appendix B.

3 The RDL is the Reliable Detection Limit. The RDL is the detection level recommended by EPA's National Research Laboratory in Cincinnati, Ohio, Environmental Monitoring Systems Laboratory (EMSL) in Cincinnati, Ohio, American Chemical Society (ACS) Committee on Environmental Improvement and the Drinking Water Standards Division (DWSD). It is defined as 2.623 times the MDL (2.623 X MDL).

4 The RL is the laboratory Reporting Limit (RL).

5 The column titled "Risk Result for Blank Correction" presents a " 0 " for elements that were analyzed for but not detected down to the method detection limit (MDL). Method 0060 specifically calls out the criteria that are to be applied to blank correct multi-metals train (MMT) data.

6 This flag is the laboratory data flag that corresponds to EPA guidelines. The data flags for these samples are as follows:

- A "B" flag indicates that this analyte was detected, but at a concentration below the laboratory RL. The analytical result is therefore an estimated value. 
BECHTEL BWXT IDAHO, LLC (BBWI)

INTEC HLLWE Effluent Gas Emissions Inventory

Idaho National Engineering and Environmental Laboratory (INEEL)

STL Knoxville Project Number: 142503.40

Table A-26. Method 0060 Multi-Metals Train (MMT) Analytical Results Summary Run 2 Back Half Composite Reagent Blank

Field Sample Name: Sample Description: Field Sample ID: STL Sample No:
Method 0060 Multi-Metals Train (MMT) Back Half Impingers Reagent Blank $5 \% \mathrm{HNO}_{3}$ and $10 \% \mathrm{H}_{2} \mathrm{O}_{2}$ Impingers Reagent Blank for Metals (including Mercury) Analysis A-3299 H1F200234-012

\begin{tabular}{|c|c|c|c|c|c|c|c|}
\hline \multirow[b]{2}{*}{ Analyte } & \multirow[b]{2}{*}{$\begin{array}{c}\text { CAS Registry } \\
\text { Number }\end{array}$} & \multicolumn{6}{|c|}{$\begin{array}{c}\text { MMT } \\
\text { Back Half Composite Reagent Blank } \\
\text { Total } \mu \mathrm{g} \\
\end{array}$} \\
\hline & & Lab Result $^{1}$ & $\mathbf{M D L}^{2}$ & $\mathbf{R D L}^{3}$ & $\mathbf{R L}^{4}$ & $\begin{array}{l}\text { Risk Result } \\
\text { for Blank } \\
\text { Correction } \\
\end{array}$ & Flag $^{6}$ \\
\hline Aluminum (Al) & $7429-90-5$ & 19 & 3.5 & 9.2 & 23 & 19 & B \\
\hline Antimony (Sb) & $7440-36-0$ & 0.86 & 0.69 & 1.8 & 6.9 & 0.86 & B \\
\hline Arsenic (As) & $7440-38-2$ & ND & 0.26 & 0.68 & 1.1 & 0 & \\
\hline Barium (Ba) & $7440-39-3$ & 0.96 & 0.34 & 0.89 & 23 & 0.96 & B \\
\hline Beryllium (Be) & $7440-41-7$ & ND & 0.17 & 0.45 & 0.57 & 0 & \\
\hline Cadmium (Cd) & $7440-43-9$ & 0.059 & 0.057 & 0.15 & 0.57 & 0.059 & B \\
\hline Chromium (Cr) & $7440-47-3$ & 0.91 & 0.26 & 0.68 & 1.1 & 0.91 & B \\
\hline Cobalt (Co) & $7440-48-4$ & ND & 0.57 & 1.5 & 5.7 & 0 & \\
\hline Copper $(\mathrm{Cu})$ & $7440-50-8$ & ND & 0.29 & 0.76 & 2.9 & 0 & \\
\hline Lead $(\mathrm{Pb})$ & $7439-92-1$ & 0.43 & 0.27 & 0.71 & 1.1 & 0.43 & B \\
\hline Manganese $(\mathrm{Mn})$ & $7439-96-5$ & 14 & 0.17 & 0.45 & 1.7 & 14 & \\
\hline Mercury $(\mathrm{Hg})$ & $7439-97-6$ & ND & 0.20 & 0.52 & 0.80 & 0 & \\
\hline Nickel (Ni) & $7440-02-0$ & 0.56 & 0.46 & 1.2 & 4.6 & 0.56 & B \\
\hline Selenium $(\mathrm{Se})$ & $7782-49-2$ & 0.28 & 0.25 & 0.66 & 1.1 & 0.28 & B \\
\hline Silver (Ag) & $7440-22-4$ & ND & 0.29 & 0.76 & 2.3 & 0 & \\
\hline Thallium (Tl) & $7440-28-0$ & ND & 0.58 & 1.5 & 2.3 & 0 & \\
\hline Vanadium (V) & $7440-62-2$ & ND & 0.57 & 1.5 & 5.7 & 0 & \\
\hline Zinc $(Z n)$ & $7440-66-6$ & 4.7 & 0.51 & 1.3 & 2.3 & 4.7 & \\
\hline
\end{tabular}


BECHTEL BWXT IDAHO, LLC (BBWI)

INTEC HLLWE Effluent Gas Emissions Inventory

Idaho National Engineering and Environmental Laboratory (INEEL)

STL Knoxville Project Number: 142503.40

\title{
Table A-26. Method 0060 Multi-Metals Train (MMT) Analytical Results Summary (Continued) Run 2 Back Half Composite Reagent Blank
}

\section{Sample Collection and Analvsis Dates:}

\author{
Date(s) Collected: June 07, 2001 \\ Date(s) of Digestion (Metals): June 24, 2001 \\ Date(s) of Digestion (Mercury): June 25, 2001 \\ Date(s) of Analysis (Metals): June 28, 2001 \\ Date(s) of Analysis (Mercury): June 26, 2001
}

\section{Preparation and Analysis Methods:}

SW-846 Method 0060: $\quad$ "Determination of Metals in Stack Emissions"

SW-846 Method 7470A: $\quad$ "Mercury in Liquid Waste (Manual Cold Vapor Technique)"

SW-846 Method 6010B: "Inductively Coupled Plasma - Atomic Emission Spectroscopy"

\section{Footnotes:}

1 This value is the laboratory sample result. When the analytical result is "ND" or not detected, the laboratory analysis did not detect the analyte down to the MDL.

2 This value is the laboratory Method Detection Limit (MDL) derived according to requirements outlined in 40 CFR Part 136, Appendix B.

3 The RDL is the Reliable Detection Limit. The RDL is the detection level recommended by EPA's National Research Laboratory in Cincinnati, Ohio, Environmental Monitoring Systems Laboratory (EMSL) in Cincinnati, Ohio, American Chemical Society (ACS) Committee on Environmental Improvement and the Drinking Water Standards Division (DWSD). It is defined as 2.623 times the MDL (2.623 X MDL).

4 The RL is the laboratory Reporting Limit (RL).

5 The column titled "Risk Result for Blank Correction" presents a " 0 " for elements that were analyzed for but not detected down to the method detection limit (MDL). Method 0060 specifically calls out the criteria that are to be applied to blank correct multi-metals train (MMT) data.

6 This flag is the laboratory data flag that corresponds to EPA guidelines. The data flags for these samples are as follows:

- A "B" flag indicates that this analyte was detected, but at a concentration below the laboratory RL. The analytical result is therefore an estimated value. 


\section{Table A-27. Method 0060 Multi-Metals Train (MMT) Analytical Results Summary Run 2 Mercury Impinger Composite Reagent Blank}

Field Sample Name: Sample Description: Field Sample ID: STL Sample No:
Method 0060 Multi-Metals Train (MMT) Mercury Impingers Reagent Blank $4 \% \mathrm{KMnO}_{4}$ and $10 \% \mathrm{H}_{2} \mathrm{SO}_{4}$ Impingers Reagent Blank for Mercury $(\mathrm{Hg})$ Analysis A- 3300

H1F200234-013

\begin{tabular}{|c|c|c|c|c|c|c|c|}
\hline \multirow[b]{2}{*}{ Analyte } & \multirow[b]{2}{*}{$\begin{array}{c}\text { CAS Registry } \\
\text { Number }\end{array}$} & \multicolumn{6}{|c|}{$\begin{array}{c}\text { MMT } \\
\text { Mercury Impinger Composite } \\
\text { Total } \mu \mathrm{g}\end{array}$} \\
\hline & & Lab Result ${ }^{1}$ & $\mathrm{MDL}^{2}$ & $\mathbf{R D L}^{3}$ & $\mathbf{R L}^{4}$ & $\begin{array}{c}\text { Risk Result } \\
\text { for Blank } \\
\text { Correction }\end{array}$ & Flag $^{6}$ \\
\hline Mercury (Hg) & $7439-97-6$ & ND & 0.10 & 0.26 & 0.42 & 0 & \\
\hline
\end{tabular}

\section{Sample Collection and Analysis Dates:}

Date(s) Collected:

Date(s) of Digestion:

Date(s) of Analysis:
June 07, 2001

June 25, 2001

June 26,2001

\section{Preparation and Analvsis Methods:}

SW-846 Method 0060: "Determination of Metals in Stack Emissions"

SW-846 Method 7470A: $\quad$ "Mercury in Liquid Waste (Manual Cold Vapor Technique)" 
BECHTEL BWXT IDAHO, LLC (BBWI)

INTEC HLLWE Effluent Gas Emissions Inventory

Idaho National Engineering and Environmental Laboratory (INEEL)

STL Knoxville Project Number: 142503.40

\section{Table A-27. Method 0060 Multi-Metals Train (MMT) Analytical Results Summary (Continued) Run 2 Mercury Impinger Composite Reagent Blank}

\section{Footnotes:}

1 This value is the laboratory sample result. When the analytical result is "ND" or not detected, the laboratory analysis did not detect the analyte down to the MDL.

2 This value is the laboratory Method Detection Limit (MDL) derived according to requirements outlined in 40 CFR Part 136, Appendix B.

3 The RDL is the Reliable Detection Limit. The RDL is the detection level recommended by EPA's National Research Laboratory in Cincinnati, Ohio, Environmental Monitoring Systems Laboratory (EMSL) in Cincinnati, Ohio, American Chemical Society (ACS) Committee on Environmental Improvement and the Drinking Water Standards Division (DWSD). It is defined as 2.623 times the MDL (2.623 X MDL).

4 The RL is the laboratory Reporting Limit (RL).

s The column titled "Risk Result for Blank Correction" presents a " 0 " for elements that were analyzed for but not detected down to the method detection limit (MDL). Method 0060 specifically calls out the criteria that are to be applied to blank correct multi-metals train (MMT) data.

6 This flag is the laboratory data flag that corresponds to EPA guidelines. The data flags for these samples are as follows:

- A "B" flag indicates that this analyte was detected, but at a concentration below the laboratory RL. The analytical result is therefore an estimated value. 


\section{Table A-28. Method 0060 Multi-Metals Train (MMT) Analytical Results Summary Run $2 \mathrm{HCl}$ Impinger Rinse Solution Reagent Blank}

Field Sample Name: Sample Description: Field Sample ID:

STL Sample No.:
Method 0060 Multi-Metals Train (MMT) Mercury Impinger HCl Rinse Solution Reagent Blank $8 \mathrm{~N} \mathrm{HCl}$ Impinger Rinse Solution Reagent Blank for Mercury $(\mathrm{Hg})$ Analysis A-3301

H1F200234-014

\begin{tabular}{|c|c|c|c|c|c|c|c|}
\hline \multirow[b]{2}{*}{ Analyte } & \multirow[b]{2}{*}{$\begin{array}{c}\text { CAS Registry } \\
\text { Number }\end{array}$} & \multicolumn{6}{|c|}{$\begin{array}{c}\text { MMT } \\
\text { 8N HCI Mercury Impinger Rinse } \\
\text { Total } \mu \mathrm{g} \\
\end{array}$} \\
\hline & & Lab Result $^{1}$ & $\mathrm{MDL}^{2}$ & $\mathrm{RDL}^{3}$ & $\mathbf{R L}^{4}$ & $\begin{array}{c}\text { Risk Result } \\
\text { for Blank } \\
\text { Correction }^{5}\end{array}$ & Flag $^{6}$ \\
\hline Mercury $(\mathrm{Hg})$ & $7439-97-6$ & ND & 0.23 & 0.60 & 0.92 & 0 & \\
\hline
\end{tabular}

\section{Sample Collection and Analysis Dates:}

Date(s) Collected:

Date(s) of Digestion:

Date(s) of Analysis:
June 07,2001

June 25, 2001

June 26, 2001

\section{Preparation and Analysis Methods:}

SW-846 Method 0060:

SW-846 Method 7470A:
"Determination of Metals in Stack Emissions"

"Mercury in Liquid Waste (Manual Cold Vapor Technique)" 


\section{Table A-28. Method 0060 Multi-Metals Train (MMT) Analytical Results Summary (Continued) Run $2 \mathrm{HCl}$ Impinger Rinse Solution Reagent Blank}

\section{Footnotes:}

1 This value is the laboratory sample result. When the analytical result is "ND" or not detected, the laboratory analysis did not detect the analyte down to the MDL.

2 This value is the laboratory Method Detection Limit (MDL) derived according to requirements outlined in 40 CFR Part 136, Appendix B.

3 The RDL is the Reliable Detection Limit. The RDL is the detection level recommended by EPA's National Research Laboratory in Cincinnati, Ohio, Environmental Monitoring Systems Laboratory (EMSL) in Cincinnati, Ohio, American Chemical Society (ACS) Committee on Environmental Improvement and the Drinking Water Standards Division (DWSD). It is defined as 2.623 times the MDL (2.623 X MDL).

4 The RL is the laboratory Reporting Limit (RL).

5 The column titled "Risk Result for Blank Correction" presents a " 0 " for elements that were analyzed for but not detected down to the method detection limit (MDL). Method 0060 specifically calls out the criteria that are to be applied to blank correct multi-metals train (MMT) data.

6 This flag is the laboratory data flag that corresponds to EPA guidelines. The data flags for these samples are as follows:

- A "B" flag indicates that this analyte was detected, but at a concentration below the laboratory RL. The analytical result is therefore an estimated value. 
BECHTEL BWXT IDAHO, LLC (BBWI)

INTEC HLLWE Effluent Gas Emissions Inventory

Idaho National Engineering and Environmental Laboratory (INEEL)

STL Knoxville Project Number: 142503.40

\section{Table A-29. Method 0060 Multi-Metals Train (MMT) Analytical Results Summary Run 2 INTEC Deionized Water Reagent Blank}

Field Sample Name: Sample Description: Field Sample ID: STL Sample No:
INTEC Deionized (D.I.) Water Reagent Blank

INTEC Deionized (D.I.) Water Reagent Blank for Metals (including Mercury) Analysis A-3348

H1F200234-015

\begin{tabular}{|c|c|c|c|c|c|c|c|}
\hline \multirow[b]{2}{*}{ Analyte } & \multirow[b]{2}{*}{$\begin{array}{c}\text { CAS Registry } \\
\text { Number }\end{array}$} & \multicolumn{6}{|c|}{$\begin{array}{c}\text { MMT } \\
\text { INTEC Deionized (D.I.) Water Reagent Blank } \\
\text { Total } \mu \mathrm{g}\end{array}$} \\
\hline & & Lab Result $^{1}$ & $\mathrm{MDL}^{2}$ & $\mathrm{RDL}^{3}$ & $\mathrm{RL}^{4}$ & $\begin{array}{c}\text { Risk Result } \\
\text { for Blank } \\
\text { Correction } \\
\end{array}$ & Flag $^{6}$ \\
\hline Aluminum (Al) & $7429-90-5$ & 5.2 & 5.2 & 14 & 20 & 5.2 & $\mathrm{~B}$ \\
\hline Antimony (Sb) & $7440-36-0$ & ND & 0.60 & 1.6 & 6.0 & 0 & \\
\hline Arsenic (As) & $7440-38-2$ & ND & 0.35 & 0.92 & 1.0 & 0 & \\
\hline Barium (Ba) & $7440-39-3$ & ND & 0.35 & 0.92 & 20 & 0 & \\
\hline Beryllium (Be) & $7440-41-7$ & 0.13 & 0.089 & 0.23 & 0.50 & 0.13 & B \\
\hline Cadmium (Cd) & $7440-43-9$ & ND & 0.050 & 0.13 & 0.50 & 0 & \\
\hline Chromium (Cr) & $7440-47-3$ & ND & 0.25 & 0.66 & 1.0 & 0 & \\
\hline Cobalt (Co) & $7440-48-4$ & ND & 0.50 & 1.3 & 5.0 & 0 & \\
\hline Copper $(\mathrm{Cu})$ & $7440-50-8$ & ND & 0.25 & 0.66 & 2.5 & 0 & \\
\hline Lead $(\mathrm{Pb})$ & $7439-92-1$ & ND & 0.20 & 0.52 & 1.0 & 0 & \\
\hline Manganese (Mn) & $7439-96-5$ & ND & 0.15 & 0.39 & 1.5 & 0 & \\
\hline Mercury $(\mathrm{Hg})$ & $7439-97-6$ & ND & 0.14 & 0.37 & 0.40 & 0 & \\
\hline Nickel (Ni) & $7440-02-0$ & ND & 0.44 & 1.2 & 4.0 & 0 & \\
\hline Selenium (Se) & $7782-49-2$ & ND & 0.35 & 0.92 & 1.0 & 0 & \\
\hline Silver (Ag) & $7440-22-4$ & ND & 0.71 & 1.9 & 2.0 & 0 & \\
\hline Thallium (Tl) & $7440-28-0$ & ND & 0.40 & 1.0 & 2.0 & 0 & \\
\hline Vanadium (V) & $7440-62-2$ & ND & 0.50 & 1.3 & 5.0 & 0 & \\
\hline Zinc $(Z n)$ & $7440-66-6$ & 0.93 & 0.23 & 0.60 & 2.0 & 0.93 & B \\
\hline
\end{tabular}


BECHTEL BWXT IDAHO, LLC (BBWI)

INTEC HLLWE Effluent Gas Emissions Inventory

Idaho National Engineering and Environmental Laboratory (INEEL)

STL Knoxville Project Number: 142503.40

\section{Table A-29. Method 0060 Multi-Metals Train (MMT) Analytical Results Summary (Continued) Run 2 INTEC Deionized Water Reagent Blank}

\section{Sample Collection and Analysis Dates:}

Date(s) Collected:

Date(s) of Digestion (Metals):

Date(s) of Digestion (Mercury):

Date(s) of Analysis (Metals):

Date(s) of Analysis (Mercury):
June 07,2001

June 24,2001

June 25,2001

June 28,2001

June 26, 2001

\section{Preparation and Analvsis Methods:}

SW-846 Method 0060: "Determination of Metals in Stack Emissions"

SW-846 Method 7470A: "Mercury in Liquid Waste (Manual Cold Vapor Technique)"

SW-846 Method 6010B: $\quad$ "Inductively Coupled Plasma - Atomic Emission Spectroscopy"

\section{Footnotes:}

1 This value is the laboratory sample result. When the analytical result is "ND" or not detected, the laboratory analysis did not detect the analyte down to the MDL.

2 This value is the laboratory Method Detection Limit (MDL) derived according to requirements outlined in 40 CFR Part 136, Appendix B.

The RDL is the Reliable Detection Limit. The RDL is the detection level recommended by EPA's National Research Laboratory in Cincinnati, Ohio, Environmental Monitoring Systems Laboratory (EMSL) in Cincinnati, Ohio, American Chemical Society (ACS) Committee on Environmental Improvement and the Drinking Water Standards Division (DWSD). It is defined as 2.623 times the MDL (2.623 X MDL).

$+\quad$ The RL is the laboratory Reporting Limit (RL).

5 The column titled "Risk Result for Blank Correction" presents a " 0 " for elements that were analyzed for but not detected down to the method detection limit (MDL). Method 0060 specifically calls out the criteria that are to be applied to blank correct multi-metals train (MMT) data.

6 This flag is the laboratory data flag that corresponds to EPA guidelines. The data flags for these samples are as follows:

- A "B" flag indicates that this analyte was detected, but at a concentration below the laboratory RL. The analytical result is therefore an estimated value. 


\section{Table A-30. Final (Post-Test) Acetone Probe Rinse and Nitric Acid Probe Rinse Composite Metallic Analyte Analytical Results Summary}

Field Sample Name:

Sample Description:

Field Sample ID:

STL Sample No:
Final (Post-Test) Acetone Probe Rinse and Nitric Acid Probe Rinse Composite

Final (Post-Test) Acetone Probe Rinse and Nitric Acid Probe Rinse Composite Sample for Metals (including Mercury) Analysis

A-3346 and A-3347

H1G030222-002

\begin{tabular}{|c|c|c|c|c|c|c|c|}
\hline \multirow[b]{2}{*}{ Analyte } & \multirow{2}{*}{$\begin{array}{c}\text { CAS } \\
\text { Registry } \\
\text { Number }\end{array}$} & \multicolumn{6}{|c|}{$\begin{array}{l}\text { Final (Post-Test) Acetone Probe Rinse and Nitric Acid Probe Rinse Composite Sample } \\
\text { Total } \mu \mathrm{g}^{1}\end{array}$} \\
\hline & & Lab Result $^{2}$ & MDL $^{3}$ & $\mathrm{RDL}^{4}$ & $\mathrm{RL}^{5}$ & Risk Result ${ }^{6,7}$ & Flag $^{8}$ \\
\hline Aluminum (Al) & $7429-90-5$ & 120 & 5.2 & 14 & 20.0 & 120 & \\
\hline Antimony $(\mathrm{Sb})$ & $7440-36-0$ & 1.3 & 0.60 & 1.6 & 6.0 & $<1.6$ & B \\
\hline Arsenic (As) & $7440-38-2$ & ND & 0.35 & 0.92 & 1.0 & $<0.92$ & \\
\hline Barium (Ba) & $7440-39-3$ & 3.2 & 0.35 & 0.92 & 20.0 & 3.2 & B \\
\hline Beryllium (Be) & $7440-41-7$ & ND & 0.089 & 0.23 & 0.50 & $<0.23$ & \\
\hline Cadmium $(\mathrm{Cd})$ & $7440-43-9$ & 4.1 & 0.050 & 0.13 & 0.50 & 4.1 & \\
\hline Chromium $(\mathrm{Cr})$ & $7440-47-3$ & 1.7 & 0.25 & 0.66 & 1.0 & 1.7 & \\
\hline Cobalt $(\mathrm{Co})$ & $7440-48-4$ & 0.85 & 0.50 & 1.3 & 5.0 & $<1.3$ & B \\
\hline Copper $(\mathrm{Cu})$ & $7440-50-8$ & 2.5 & 0.25 & 0.66 & 2.5 & 2.5 & \\
\hline Lead $(\mathrm{Pb})$ & $7439-92-1$ & 3.2 & 0.20 & 0.52 & 1.0 & 3.2 & \\
\hline Manganese (Mn) & $7439-96-5$ & 6.1 & 0.15 & 0.39 & 1.5 & 6.1 & \\
\hline Mercury (Hg) & $7439-97-6$ & 0.77 & 0.14 & 0.37 & 0.40 & 0.77 & \\
\hline Nickel (Ni) & $7440-02-0$ & 3.2 & 0.44 & 1.2 & 4.0 & 3.2 & B \\
\hline Selenium (Se) & $7782-49-2$ & ND & 0.35 & 0.92 & 1.0 & $<0.92$ & \\
\hline Silver (Ag) & $7440-22-4$ & 1.8 & 0.71 & 1.9 & 2.0 & $<1.9$ & B \\
\hline Thallium (Tl) & $7440-28-0$ & ND & 0.40 & 1.0 & 2.0 & $<1.0$ & \\
\hline Vanadium (V) & $7440-62-2$ & ND & 0.50 & 1.3 & 5.0 & $<1.3$ & \\
\hline Zinc $(\mathrm{Zn})$ & $7440-66-6$ & 50 & 0.23 & 0.60 & 2.0 & 50 & \\
\hline
\end{tabular}


BECHTEL BWXT IDAHO, LLC (BBWI)

INTEC HLLWE Effluent Gas Emissions Inventory

Idaho National Engineering and Environmental Laboratory (INEEL)

STL Knoxville Project Number: 142503.40

Table A-30. Final (Post-Test) Acetone Probe Rinse and Nitric Acid Probe Rinse Composite Metallic Analyte Analytical Results Summary

(Continued)

Sample Collection and Analysis Dates:

$\begin{array}{ll}\text { Date(s) Collected: } & \text { June 25, 2001 } \\ \text { Date(s) of Digestion (Metals): } & \text { July 08, 2001 } \\ \text { Date(s) of Digestion (Mercury): } & \text { July 08, 2001 } \\ \text { Date(s) of Analysis (Metals): } & \text { July 28, 2001 } \\ \text { Date(s) of Analysis (Mercury): } & \text { July 08, 2001 }\end{array}$

Preparation and Analysis Methods:

SW-846 Method 0060:

SW-846 Method 7470A:

SW-846 Method 6010B:
"Determination of Metals in Stack Emissions"

"Mercury in Liquid Waste (Manual Cold Vapor Technique)"

"Inductively Coupled Plasma - Atomic Emission Spectroscopy" 
BECHTEL BWXT IDAHO, LLC (BBWI)

INTEC HLLWE Effluent Gas Emissions Inventory

Idaho National Engineering and Environmental Laboratory (INEEL)

STL Knoxville Project Number: 142503.40

\section{Table A-30. Final (Post-Test) Acetone Probe Rinse and Nitric Acid Probe Rinse Composite Metallic Analyte Analytical Results Summary (Continued)}

\section{Footnotes:}

1 Based on the selection rules, the bolded value is the value or default value assigned to the analyte and is carried through to the sampling train total, if it is determined to be significant.

2 This value is the laboratory sample result. When the analytical result is "ND" or not detected, the laboratory analysis did not detect the analyte down to the MDL.

3 This value is the laboratory Method Detection Limit (MDL) derived according to requirements outlined in 40 CFR Part 136 , Appendix B

4 The RDL is the Reliable Detection Limit. The RDL is the detection level recommended by EPA's National Research Laboratory in Cincinnati, Ohio, Environmental Monitoring Systems Laboratory (EMSL) in Cincinnati, Ohio, American Chemical Society (ACS) Committee on Environmental Improvement and the Drinking Water Standards Division (DWSD). It is defined as 2.623 times the MDL $(2.623 \times \mathrm{MDL})$.

5 The RL is the laboratory Reporting Limit (RL).

6 Based on the selection rules, the bolded value is the value or default value assigned to the analyte using the following guidelines:

- When the analytical result is greater than the laboratory reporting limit (RL), the result selected by boldface type is the actual analytical result or "hit" determined by the laboratory.

- When the analytical result is greater than the reliable detection level (RDL), but less than the laboratory reporting limit (RL), the result selected by boldface type is the actual analytical result or "hit" determined by the laboratory.

- When the analytical result is less than the RDL, but greater than the method detection limit (MDL), the result selected by boldface type is the RDL.

- When the analytical result is not detected down to the MDL, the result selected by boldface type is the RDL.

- It should be noted that when the RDL is selected using the guidelines above, but the RL is less than the RDL, the RL is included as the "Risk Result".

7. When listed, the less than $(<)$ sign indicates that the sample result is either a "non-detect" value down to the MDL of the measurement that carries, or an estimated "hit" value that is below the RDL. In either case, the final value is the default RDL value and the actual value is known to be less than $(<)$ the displayed result.

8 This flag is the laboratory data flag that corresponds to EPA guidelines. The data flags for these samples are as follows:

- A "U" qualifier indicates that this analyte was analyzed for, but was not detected down to the MDL.

- A "B" flag indicates that this analyte was detected, but at a concentration below the laboratory RL. The analytical result is therefore an estimated value. 


\section{APPENDIX B}

\section{OFFGAS SAMPLING DATA}





\section{CONTENTS}

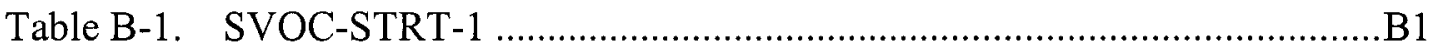

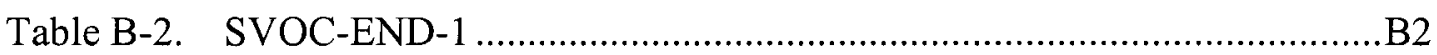

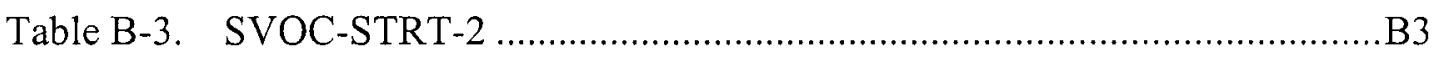

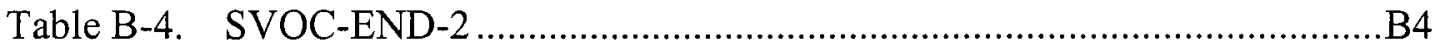

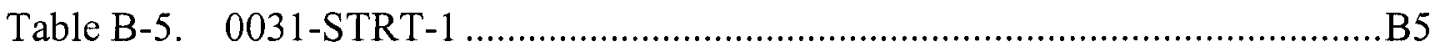

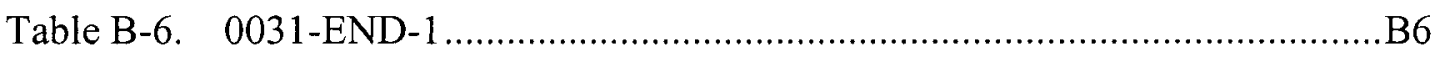

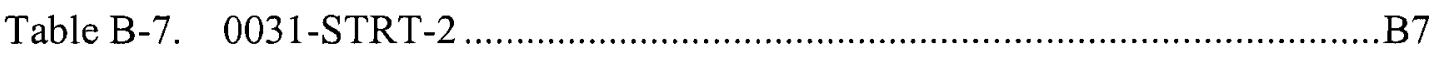

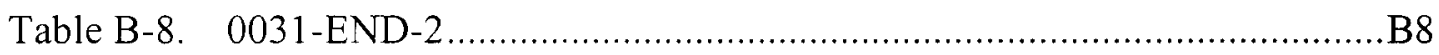

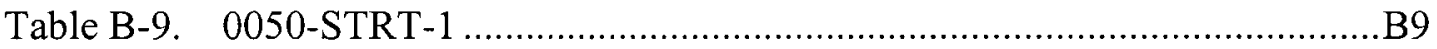

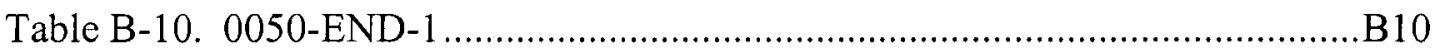

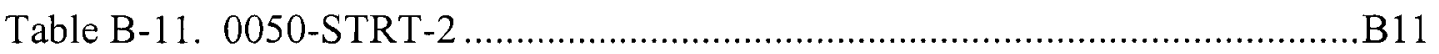

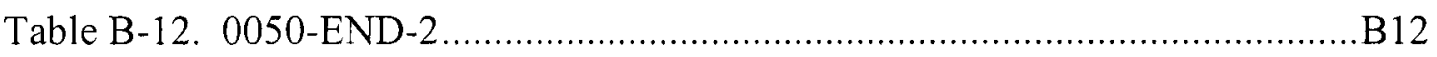

Table B-13. 0060-STRT-1 …............................................................................ B13

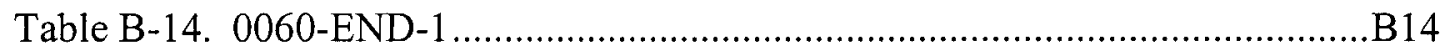

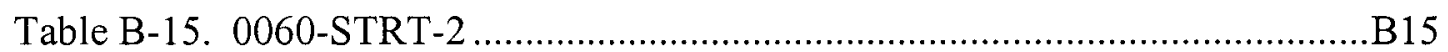

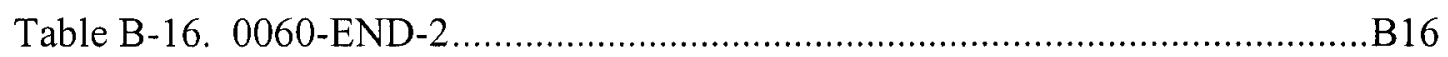

Table B-17. SVOC emission rates - grams per second comparisons ..................... 17

Table B-18. 0031 emission rates - grams per second comparisons......................... 18

Table B-19. 0050 emission rates - grams per second comparisons........................19

Table B-20. 0060 emission rates - grams per second comparisons........................ 20

Table B-21. 0060 blank corrected emission rates - grams per second

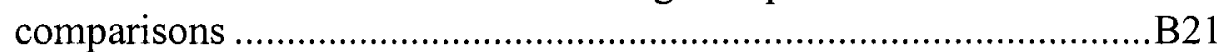

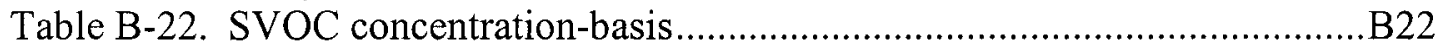

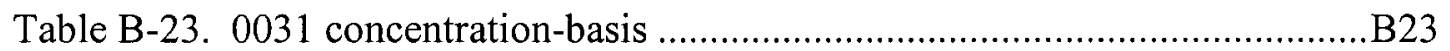

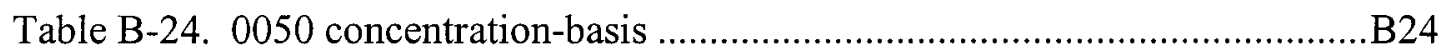

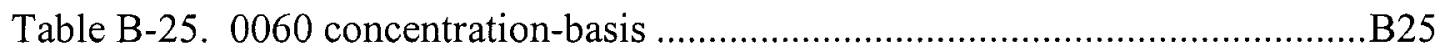

Table B-26. 0060 blank corrected concentration-basis.................................... 26 



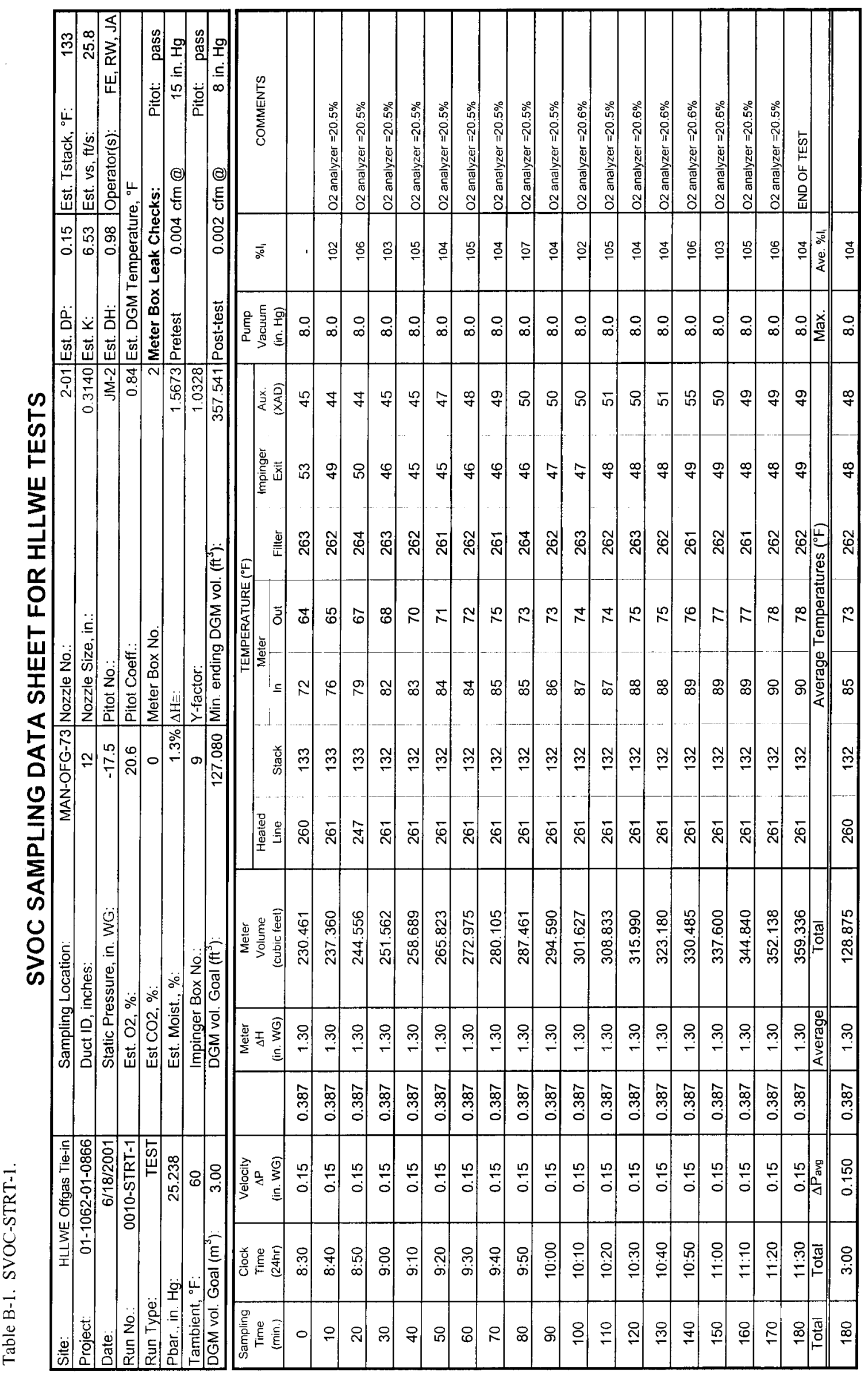




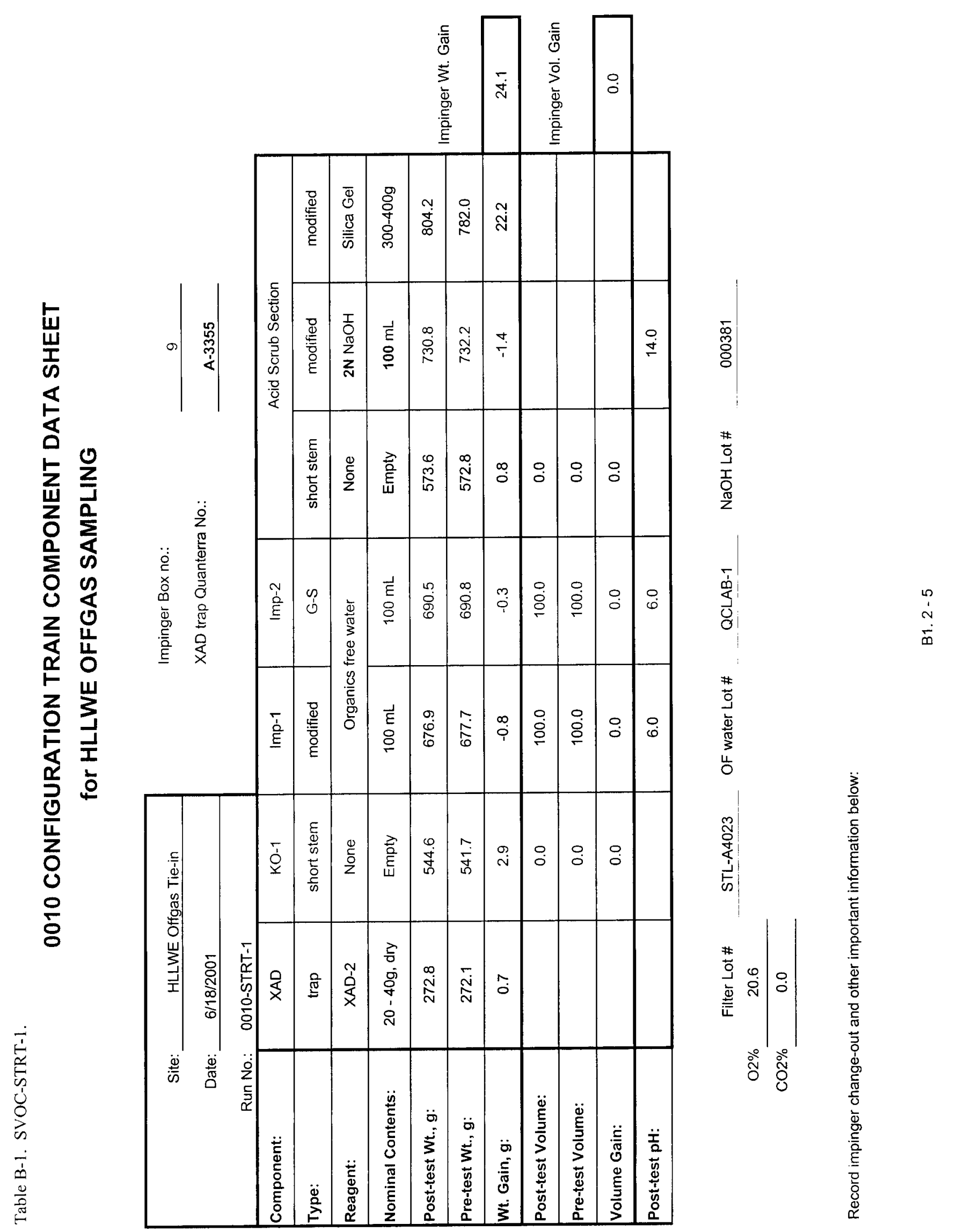


Table B-1. SVOC-STRT-1.

FIELD DATA CALCULATIONS

\begin{tabular}{|c|c|c|c|}
\hline $\begin{array}{r}\text { Project: } \\
\text { Run Date: } \\
\text { Run Identification: }\end{array}$ & $\begin{array}{r}01-1062 \\
6 / 18 \\
0010-5\end{array}$ & $\begin{array}{l}1-0866 \\
001 \\
\text { RT-1 }\end{array}$ & \\
\hline PARAMETER & SYMBOL & UNITS & \\
\hline Absolute Pressure in the Duct & Pabs & in. $\mathrm{Hg}$ & 23.951 \\
\hline Average Duct Gas Temperature & Ts & $\mathrm{R}$ & 592 \\
\hline Average Meter Temperature & $\mathrm{Tm}$ & $\mathrm{R}$ & 538 \\
\hline Average Gas Oxygen Content & $\mathrm{Co} 2, \mathrm{~m}$ & $\%$ & 20.6 \\
\hline Average Gas Carbon Dioxide Content & $\mathrm{Cco} 2, \mathrm{~m}$ & $\%$ & 0.0 \\
\hline Total Impinger Weight Gain (water) & Ww & grams & 24.1 \\
\hline Nozzle Area & An & $\mathrm{ft}^{2}$ & 0.000538 \\
\hline Duct Area & As & $\mathrm{ft}^{2}$ & 0.7854 \\
\hline Sample Volume & VmStd & dscf & 110.435 \\
\hline Sample Volume (SI) & VmStdm & dscm & 3.127 \\
\hline Average Sampling Rate & Qm & dscf/m & 0.614 \\
\hline Volume of Water Vapor & VwStd & scf & 1.136 \\
\hline Volume of Water Vapor (SI) & VwStdm & $\mathrm{scm}$ & 0.0322 \\
\hline Moisture Fraction & Bws & - & 0.010 \\
\hline Dry Gas Molecular Weight & Md & $\mathrm{g} / \mathrm{g}-\mathrm{mol}$ & 28.82 \\
\hline Wet Gas Molecular Weight & Ms & $\mathrm{g} / \mathrm{g}-\mathrm{mol}$ & 28.71 \\
\hline Gas Velocity at Nozzle & vn & $\mathrm{ft} / \mathrm{s}$ & 25.8 \\
\hline Gas Velocity at Nozzle (SI) & $\mathrm{vnm}$ & $\mathrm{m} / \mathrm{s}$ & 7.86 \\
\hline Average Gas Velocity & vncor & $\mathrm{ft} / \mathrm{s}$ & 21.77 \\
\hline Dry Offgas Flow Rate & Qsd & $\mathrm{dscf} / \mathrm{h}$ & 43,494 \\
\hline Dry Offgas Flow Rate (SI) & Qsdm & $\mathrm{dscm} / \mathrm{h}$ & $1,231.6$ \\
\hline Actual Offgas Flow Rate & $Q$ & $\operatorname{acf} / \mathrm{h}$ & 61,566 \\
\hline Intermediate Isokinetic Rate & li & $\%$ & 104.6 \\
\hline Final Isokinetic Rate & I & $\%$ & 104.3 \\
\hline
\end{tabular}


Table B-1. SVOC-STRT-1.

\begin{tabular}{|c|c|c|c|c|c|c|c|c|c|c|c|c|}
\hline \multirow[t]{3}{*}{$\begin{array}{r}\text { Project: } \\
\text { Run Date: } \\
\text { Run Identification: } \\
\text { Run Type: } \\
\text { Lab Report Date: } \\
\text { Lab Report Status: (preliminary or } \\
\text { final) } \\
\end{array}$} & \multicolumn{2}{|c|}{$\begin{array}{c}01-1062-01-0866 \\
6 / 18 / 2001 \\
\text { O010-STRT-1 } \\
\text { TEST } \\
\text { 8/28/2001 } \\
\text { Final }\end{array}$} & \multicolumn{10}{|c|}{$\begin{array}{l}\text { RESULTS } \\
\text { - without blank corrections } \\
\text { - final presentation should be rounded to two significant digits }\end{array}$} \\
\hline & \multicolumn{6}{|c|}{ CONCENTRATIONS } & \multicolumn{6}{|c|}{ MASS FLOW RATES } \\
\hline & & $\begin{array}{c}\text { Actual } \\
(\mu g / a c m)\end{array}$ & & & & $\begin{array}{l}\text { dard } \\
\text { m) }\end{array}$ & & & & s/sec & & $\mathrm{lb} / \mathrm{h}$ \\
\hline Acenaphthene & $<$ & $2.214 \mathrm{e} 0$ & $<$ & $3.1 \mathrm{e} 0$ & $<$ & $3.1 \mathrm{e} 0$ & $<$ & $6.4 \mathrm{e} 1$ & $\mid<$ & $1.1 \mathrm{e}-6$ & $<$ & $8.5 e-6$ \\
\hline Acenaphthylene & $<$ & $2.1 \in 0$ & $<$ & $3.0 \mathrm{e} 0$ & $<$ & $3.0 \mathrm{e} 0$ & $<$ & $6.2 e 1$ & $\mid<$ & $1.0 \mathrm{e}-6$ & $<$ & $8.2 e-6$ \\
\hline Acetophenone & $<, d$ & $1.1 \mathrm{e} 1$ & $<, J$ & $1.6 e 1$ & $<\rfloor$, & $1.6 \mathrm{e} 1$ & $<, J$ & $3.2 e 2$ & $2<, J$ & $5.4 e-6$ & $<, J$ & $4.3 e-5$ \\
\hline Aniline & $<$ & $2.5 e 1$ & $<$ & $3.5 \mathrm{e} 1$ & $<$ & $3.5 \mathrm{e} 1$ & $<$ & $7.2 \mathrm{e} 2$ & $<$. & $1.2 \mathrm{e}-5$ & $<$ & $9.6 e-5$ \\
\hline Anthracene & $<$ & 2.100 & $<$ & $3.0 e 0$ & $<$ & $3.0 \mathrm{e} 0$ & $<$ & $6.2 \mathrm{e} 1$ & $<$ & $1.0 \mathrm{e}-6$ & $<$ & $8.2 e-6$ \\
\hline Benzidine & $<$ & $1.5 \mathrm{e} 2$ & $<$ & $2.1 \mathrm{e} 2$ & $<$ & $2.1 \mathrm{e} 2$ & $<$ & $4.4 \mathrm{e} 3$ & $\mid<$ & $7.3 e-5$ & $<$ & $5.8 e-4$ \\
\hline Benzoic acid & $E$ & $1.1 \mathrm{e} 3$ & E & $1.6 \mathrm{e} 3$ & $E$ & $1.6 \mathrm{e} 3$ & E & $3.2 \mathrm{e} 4$ & $E$ & $5.4 e-4$ & E & $4.3 e-3$ \\
\hline Benzo(a)anthracene & $<$ & $2.7 e 0$ & $<$ & $3.8 \mathrm{eo}$ & $<$ & $3.8 \mathrm{e} 0$ & $<$ & $7.9 \mathrm{e} 1$ & $\leq$ & $1.3 e-6$ & $<$ & $1.0 \mathrm{e}-5$ \\
\hline Benzo(a)pyrene & $<$ & $2.9 e 1$ & $<$ & $4.1 \mathrm{e} 1$ & $<$ & $4.2 \mathrm{e} 1$ & $<$ & $8.5 \mathrm{e} 2$ & $<$ & $1.4 \mathrm{e}-5$ & $<$ & $1.1 e-4$ \\
\hline Benzo(b)fluoranthene & $<$ & $6.8 \mathrm{e} 1$ & $<$ & $9.5 \mathrm{e} 1$ & $<$ & $9.6 \mathrm{e} 1$ & $<$ & $2.0 \mathrm{e} 3$ & $<$ & $3.3 e-5$ & $<$ & $2.6 e-4$ \\
\hline Benzo(g,h,i)perylene & $<$ & $3.8 \mathrm{e} 1$ & $<$ & $5.4 \mathrm{e} 1$ & $<$ & $5.4 \mathrm{e} 1$ & $<$ & $1.1 \mathrm{e} 3$ & $<$ & $1.9 e-5$ & $<$ & $1.5 e-4$ \\
\hline Benzo(k)fluoranthene & $<$ & $9.7 \mathrm{e} 1$ & $<$ & $1.4 \mathrm{e} 2$ & $<$ & $1.4 \mathrm{e} 2$ & $<$ & $2.8 \mathrm{e} 3$ & $<$ & $4.7 e-5$ & $<$ & $3.7 e-4$ \\
\hline Benzyl alcohol & $<$ & $1.3 \mathrm{e} 2$ & $<$ & $1.8 \mathrm{e} 2$ & $<$ & $1.8 \mathrm{e} 2$ & $<$ & $3.7 \mathrm{e} 3$ & $<$ & $6.2 e-5$ & $<$ & $4.9 e-4$ \\
\hline bis(2-Chloroethoxy)methane & $<$ & $2.3 \mathrm{e} 0$ & $<$ & $3.2 \mathrm{e} 0$ & $<$ & $3.2 \mathrm{eo}$ & $<$ & $6.6 \mathrm{e} 1$ & $\mid<$ & $1.1 e-6$ & $<$ & 8.7e-6 \\
\hline bis(2-Chloroethyl)ether & k & $2.5 \mathrm{e} 0$ & $<$ & $3.5 \mathrm{e} 0$ & $<$ & $3.5 \mathrm{eo}$ & $<$ & $7.2 \mathrm{e1}$ & $\mid<$ & $1.2 e-6$ & $<$ & $9.6 \mathrm{e}-6$ \\
\hline bis (2-Ethylhexyl)phthalate & $<, \sqrt{ }$ & $2.7 \mathrm{e} 1$ & $<, J$ & $3.8 \mathrm{e} 1$ & $<, J$ & $3.8 \mathrm{e} 1$ & $<, j$ & $7.9 \mathrm{e} 2$ & $<, J$ & $1.3 e-5$ & $<, \mathrm{J}$ & $1.0 \mathrm{e}-4$ \\
\hline 4-Bromophenyl-phenylether & $<$ & 2.100 & $<$ & 3.000 & $<$ & $3.0 \mathrm{eo}$ & $<$ & $6.2 \mathrm{e} 1$ & $<$ & $1.0 e-6$ & $<$ & $8.2 e-6$ \\
\hline Butylbenzylph thalate & $<$ & $2.9 \mathrm{e} 0$ & $<$ & $4.1 \in 0$ & $<$ & $4.2 \mathrm{eo}$ & $<$ & $8.5 \mathrm{e} 1$ & $\mid<$ & $1.4 \mathrm{e}-6$ & $<$ & $1.1 e-5$ \\
\hline Carbazole & < & $2.9 \mathrm{e} 0$ & |< & $4.1 \mathrm{e} 0$ & $<$ & $4.2 \mathrm{e0}$ & $<$ & $8.5 \mathrm{e} 1$ & $\mid<$ & $1.4 e-6$ & $<$ & $1.1 e-5$ \\
\hline 4-Chloro-3-methylphenol & $<$ & $3.8 \mathrm{e} 0$ & $<$ & $5.4 \mathrm{e} 0$ & $\leq$ & $5.4 \mathrm{e} 0$ & $<$ & $1.1 \mathrm{e} 2$ & $<$ & $1.9 \mathrm{e}-6$ & $<$ & $1.5 \mathrm{e}-5$ \\
\hline 4-Chloroaniline & $<$ & $2.0 \mathrm{e} 1$ & $<$ & $2.8 \mathrm{e} 1$ & $<$ & $2.9 \mathrm{e} 1$ & $<$ & $5.9 \mathrm{e} 2$ & $<<$ & $9.8 \mathrm{e}-6$ & $<$ & $7.8 \mathrm{e}-5$ \\
\hline 2-Chloronaphthalene & k & $2.1 \mathrm{e} 0$ & $<$ & $2.9 \mathrm{e} 0$ & $<$ & $3.0 \mathrm{eo}$ & $<$ & $6.1 \mathrm{e} 1$ & $\mid<$ & $1.0 \mathrm{e}-6$ & $<$ & $8.1 e-6$ \\
\hline 2-Chlorophenol & $<$ & $2.5 \mathrm{e} 0$ & $<$ & $3.5 \mathrm{e} 0$ & $<$ & $3.5 \mathrm{eo}$ & $<$ & $7.2 \mathrm{e} 1$ & $<$ & $1.2 \mathrm{e}-6$ & $<$ & $9.6 \mathrm{e}-6$ \\
\hline 4-Chlorophenyl phenyl ether & $<$ & $2.5 e 0$ & $<$ & $3.5 \mathrm{e} 0$ & $<$ & $3.5 \mathrm{eo}$ & $<$ & $7.2 \mathrm{e} 1$ & $<$ & $1.2 \mathrm{e}-6$ & $\leq$ & $9.6 e-6$ \\
\hline Chrysene & $<$ & $2.7 \mathrm{e} 0$ & $<$ & $3.8 \mathrm{e0}$ & $<$ & $3.8 \mathrm{eo}$ & $<$ & $7.9 \mathrm{e} 1$ & $<$ & $1.3 e-6$ & $<$ & $1.0 e-5$ \\
\hline Di-n-butylphthalate & $<, J$ & $2.3 e 1$ & $<, J$ & $3.2 \mathrm{e} 1$ & $<, J$ & $3.2 \mathrm{e} 1$ & $<, J$ & $6.6 \mathrm{e} 2$ & $<, J$ & $1.1 \mathrm{e}-5$ & $<, J$ & $8.7 e-5$ \\
\hline Di-n-octylphthalate & $<, J$ & $3.6 \mathrm{e} 1$ & $<, J$ & $5.1 \mathrm{e} 1$ & $<, J$ & $5.1 e 1$ & $<, J$ & $1.1 \mathrm{e} 3$ & $<, J$ & $1.8 e-5$ & $<, J$ & $1.4 e-4$ \\
\hline Dibenz $(a, h)$ anthracene & $<$ & $3.8 \mathrm{e} 1$ & $\leq$ & $5.4 \mathrm{e} 1$ & $<$ & $5.4 \mathrm{e} 1$ & $<$ & $1.1 \mathrm{e} 3$ & $<$ & $1.9 e-5$ & $<$ & $1.5 \mathrm{e}-4$ \\
\hline Dibenzofuran & $<$ & $2.5 e 0$ & $<$ & $3.5 \mathrm{e} 0$ & $<$ & $3.5 \mathrm{e0}$ & $<$ & $7.2 \mathrm{e} 1$ & $<$ & $1.2 \mathrm{e}-6$ & $<$ & $9.6 e-6$ \\
\hline 1,2-Dichlorobenzene & $<$ & $2.5 \mathrm{e} 0$ & $<$ & $3.5 \mathrm{e} 0$ & $<$ & $3.5 e 0$ & $<$ & $7.2 \mathrm{e} 1$ & $\mid<$ & $1.2 e-6$ & $<$ & $9.6 \mathrm{e}-6$ \\
\hline 1,3-Dichlorobenzene & $<$ & $2.7 \mathrm{e} 0$ & $<$ & $3.8 \mathrm{e} 0$ & $<$ & $3.8 \mathrm{e} 0$ & $<$ & $7.9 \mathrm{e} 1$ & $<$ & $1.3 e-6$ & $<$ & $1.0 e-5$ \\
\hline 1,4-Dichlorobenzene & $<, \sqrt{ }$ & $3.6 \mathrm{e} 0$ & $<, J$ & $5.1 \mathrm{e} 0$ & $<, J$ & $5.1 \mathrm{e} 0$ & $<, J$ & $1.1 \mathrm{e} 2$ & $<, \mathrm{J}$ & $1.8 \mathrm{e}-6$ & $<, \mathrm{J}$ & $1.4 \mathrm{e}-5$ \\
\hline 3,3'-Dichlorobenzidine & $<$ & $2.5 \mathrm{e} 1$ & $<$ & $3.5 \mathrm{e} 1$ & $<$ & $3.5 \mathrm{e} 1$ & $<$ & $7.2 \mathrm{e} 2$ & $<$ & $1.2 \mathrm{e}-5$ & $<$ & $9.6 \mathrm{e}-5$ \\
\hline 2,4-Dichlorophenol & $<$ & $2.9 \mathrm{e} 0$ & $<$ & $4.1 \mathrm{e} 0$ & $<$ & $4.2 \mathrm{eo}$ & < & $8.5 \mathrm{e} 1$ & $\mid<$ & $1.4 e-6$ & < & $1.1 e-5$ \\
\hline Diethylphthaiate & $<, d$ & $3.6 \mathrm{e} 0$ & $<, J$ & $5.1 \mathrm{e} 0$ & $<, J$ & $5.1 \mathrm{eo}$ & $<, J$ & $1.1 \mathrm{e} 2$ & $<, J$ & $1.8 e-6$ & $<, J$ & $1.4 \mathrm{e}-5$ \\
\hline Dimethyl phthalate & $<$ & 2.200 & $<$ & $3.0 e 0$ & $<$ & $3.1 \mathrm{eo}$ & $<$ & $6.3 e 1$ & $\mid<$ & $1.1 \mathrm{e}-6$ & $<$ & $8.3 e-6$ \\
\hline 2,4-Dimethylphenol & $<$ & $1.3 \mathrm{e} 1$ & $<$ & $1.9 \mathrm{e} 1$ & $<$ & $1.9 \mathrm{e} 1$ & $<$ & $3.9 \mathrm{e} 2$ & $<$ & $6.5 e-6$ & $<$ & $5.1 e-5$ \\
\hline 4,6-Dinitro-2-methylphenol & $<$ & $2.9 \mathrm{e} 1$ & $<$ & $4.1 \mathrm{e} 1$ & $<$ & $4.2 \mathrm{e} 1$ & $<$ & $8.5 \mathrm{e} 2$ & $<$ & $1.4 \mathrm{e}-5$ & $<$ & 1. $1 \mathrm{e}-4$ \\
\hline 2,4-Dinitrophenol & $<$ & $6.1 \mathrm{e} 1$ & $<$ & $8.5 e 1$ & $<$ & $8.6 e 1$ & $<$ & $1.8 \mathrm{e} 3$ & $<$ & $3.0 e-5$ & $<$ & $2.3 e-4$ \\
\hline 2,4-Dinitrotoluene & $\leq$ & $2.9 \mathrm{e}$ & $<$ & $4.1 \mathrm{e} 0$ & $<$ & $4.2 \mathrm{eO}$ & $<$ & $8.5 e 1$ & $<$ & $1.4 \mathrm{e}-6$ & $<$ & $1.1 \mathrm{e}-5$ \\
\hline 2,6-Dinitrotoluene & $<$ & $2.7 e 0$ & $<$ & $3.8 \mathrm{e} 0$ & $<$ & $3.8 \mathrm{e} 0$ & $<$ & $7.9 \mathrm{e} 1$ & $\mid<$ & $1.3 e-6$ & $<$ & $1.0 \mathrm{e}-5$ \\
\hline 1,2-Diphenylhydrazine & $<$ & $2.2 \mathrm{e} 0$ & $<$ & $3.1 \mathrm{e} 0$ & $<$ & $3.1 \mathrm{eo}$ & $<$ & $6.4 \mathrm{e} 1$ & $\mid<$ & $1.1 e-6$ & $<$ & $8.5 e-6$ \\
\hline Fluoranthene & $<$ & $2.3 \mathrm{e} 0$ & $<$ & $3.2 \mathrm{e} 0$ & $<$ & $3.2 \mathrm{eo}$ & $<$ & $6.6 \mathrm{e} 1$ & $<$ & $1.1 e-6$ & $<$ & $8.7 e-6$ \\
\hline Fluorene & $<$ & $2.3 \mathrm{e} 0$ & $<$ & $3.2 \mathrm{e} 0$ & $<$ & $3.2 \mathrm{eO}$ & $<$ & $6.6 \mathrm{e} 1$ & $\leq$ & $1.1 \mathrm{e}-6$ & $<$ & $8.7 e-6$ \\
\hline Hexachlorocyclopentadiene & $<$ & $3.6 \mathrm{e} 1$ & $<$ & $5.1 \in 1$ & $<$ & $5.1 e 1$ & $<$ & $1.1 \mathrm{e} 3$ & $<$ & $1.8 \mathrm{e}-5$ & $<$ & $1.4 \mathrm{e}-4$ \\
\hline Hexachlorobenzene & $<$ & $2.5 \mathrm{e} 0$ & $<$ & $3.5 \mathrm{e} 0$ & $<$ & $3.5 \mathrm{e} 0$ & $<$ & $7.2 \mathrm{e} 1$ & $<$ & $1.2 \mathrm{e}-6$ & $<$ & $9.6 e-6$ \\
\hline Hexachlorobutadiene & $<$ & $3.4 \mathrm{e} 0$ & $<$ & $4.7 \mathrm{e} 0$ & $<$ & $4.8 \mathrm{eo}$ & $<$ & $9.8 \mathrm{e} 1$ & $<$ & $1.6 e-6$ & $<$ & $1.3 e-5$ \\
\hline Hexachloroethane & $\leq$ & $3.6 e 0$ & $\leq$ & $5.1 e 0$ & $<$ & $5.1 \mathrm{eo}$ & $<$ & $1.1 \mathrm{e} 2$ & $<$ & $1.8 \mathrm{e}-6$ & $<$ & $1.4 \mathrm{e}-5$ \\
\hline Indeno $(1,2,3$-cd)pyrene & $<$ & $3.4 \mathrm{e} 1$ & $<$ & $4.7 \mathrm{e} 1$ & $<$ & $4.8 \mathrm{e} 1$ & $<$ & $9.8 \mathrm{e} 2$ & $<$ & $1.6 e-5$ & $<$ & $1.3 e-4$ \\
\hline Isophorone & $<$ & $2.3 \mathrm{e} 0$ & $<$ & $3.2 \mathrm{e} 0$ & $<$ & $3.2 \mathrm{eo}$ & $<$ & $6.6 \mathrm{e} 1$ & $<$ & $1.1 \mathrm{e}-6$ & $<$ & 8.7e-6 \\
\hline 2-Methylnaphthalene & $<$ & $2.3 e 0$ & $<$ & $3.2 \mathrm{e} 0$ & $<$ & $3.2 \mathrm{e} 0$ & $<$ & $6.6 \mathrm{e1}$ & $<$ & $1.1 \mathrm{e}-6$ & $<$ & $8.7 e-6$ \\
\hline 2-Methylphenol & $<$ & $1.1 \mathrm{e} 1$ & $<$ & $1.5 \mathrm{e} 1$ & $<$ & $1.5 \mathrm{e} 1$ & $<$ & $3.1 \mathrm{e} 2$ & $<$ & $5.1 e-6$ & $<$ & $4.1 e-5$ \\
\hline 3-Methylphenol \& 4-Methylphenol & $<$ & $7.7 \mathrm{e} 0$ & $<$ & $1.1 \mathrm{e} 1$ & $<$ & $1.1 \mathrm{e} 1$ & $<$ & $2.2 \mathrm{e} 2$ & $<$ & $3.7 e-6$ & $<$ & $3.0 \mathrm{e}-5$ \\
\hline N-Nitroso-di-n-propylamine & $<$ & $2.5 e 0$ & $<$ & $3.5 \mathrm{e} 0$ & $<$ & $3.5 \mathrm{e} 0$ & $<$ & $7.2 \mathrm{e} 1$ & $<$ & $1.2 \mathrm{e}-6$ & $<$ & $9.6 e-6$ \\
\hline N-Nitrosodimethylamine & $<$ & $2.5 \mathrm{e} 0$ & $<$ & $3.5 \mathrm{e} 0$ & $<$ & $3.5 e 0$ & $<$ & $7.2 \mathrm{e} 1$ & $<$ & $1.2 \mathrm{e}-6$ & $<$ & $9.6 e-6$ \\
\hline N-Nitrosodiphenylamine & $<$ & $3.4 e 0$ & $\leq$ & $4.7 \mathrm{eo}$ & $<$ & 4.800 & $<$ & $9.8 \mathrm{e} 1$ & $<$ & $1.6 \mathrm{e}-6$ & $<$ & $1.3 e-5$ \\
\hline Naphthalene & $<$ & $2.5 \mathrm{e} 0$ & $\bar{\varepsilon}$ & $3.5 \mathrm{e} 0$ & $<$ & $3.5 \mathrm{eo}$ & $<$ & $7.2 \mathrm{e} 1$ & $<$ & $1.2 e-6$ & $<$ & $9.6 e-6$ \\
\hline 2-Nitroaniline & $<$ & $2.5 \mathrm{eO}$ & $<$ & $3.5 \mathrm{e} 0$ & $<$ & $3.5 \mathrm{eo}$ & $<$ & $7.2 \mathrm{e} 1$ & k & $1.2 \mathrm{e}-6$ & $<$ & $9.6 e-6$ \\
\hline 3-Nitroaniline & $<$ & $9.3 \mathrm{eo}$ & $<$ & $1.3 \mathrm{e} 1$ & $<$ & $1.3 e 1$ & $<$ & $2.7 \mathrm{e} 2$ & $<$ & $4.5 e-6$ & $<$ & $3.6 e-5$ \\
\hline 4-Nitroaniline & k & 8.100 & $k$ & $1.1 \mathrm{e} 1$ & $<$ & $1.2 \mathrm{e} 1$ & $<$ & $2.4 \mathrm{e} 2$ & $<$ & $3.9 e-6$ & $<$ & $3.1 \mathrm{e}-5$ \\
\hline
\end{tabular}


Table B-1. SVOC-STRT-1

\begin{tabular}{|c|c|c|c|c|c|c|c|c|c|c|c|c|}
\hline \multirow[t]{3}{*}{\begin{tabular}{|r|r|} 
Project: \\
Run Date: \\
Run identification: \\
Run Type: \\
Lab Report Date: \\
Lab Report Status: (preliminary or \\
final)
\end{tabular}} & \multicolumn{2}{|c|}{$\begin{array}{c}01-1062-01-0866 \\
6 / 18 / 2001 \\
0010-\text { STRT-1 } \\
\text { TEST } \\
8 / 28 / 2001 \\
\text { Final }\end{array}$} & \multicolumn{10}{|c|}{$\begin{array}{c}\text { RESULTS } \\
\text { - without blank corrections } \\
\text { - final presentation should be rounded to two significant digits }\end{array}$} \\
\hline & \multirow{2}{*}{\multicolumn{2}{|c|}{$\begin{array}{c}\text { Actual } \\
(\mu \mathrm{g} / \mathrm{acm})\end{array}$}} & $\mathrm{ONCE}$ & RATION & & & \multicolumn{6}{|c|}{ MASS FLOW RATES } \\
\hline & & & & & $\begin{array}{r}\text { Dry } \\
(\mu \mathrm{s}\end{array}$ & $\begin{array}{l}\text { ndard } \\
\mathrm{cm})\end{array}$ & & & & isec & & \\
\hline Nitrobenzene & $<, J$ & $3.6 \mathrm{e} 0$ & $<, J$ & $5.1 \mathrm{e0}$ & $<, J$ & 5.100 & $<, J$ & $1.1 \mathrm{e} 2$ & $<, J$ & $1.8 \mathrm{e}-6$ & $<, J$ & $1.4 \mathrm{e}-5$ \\
\hline 2-Nitrophenol & $<$ & $2.1 \mathrm{e} 1$ & $<$ & $2.9 \mathrm{e} 1$ & $<$ & $2.9 \mathrm{e} 1$ & $<$ & $6.0 \mathrm{e} 2$ & $<$ & $1.0 e-5$ & < & $7.9 \mathrm{e}-5$ \\
\hline 4-Nitrophenol & $<\mathrm{J}$ & $1.7 \mathrm{e} 1$ & $<, J$ & $2.4 \mathrm{e} 1$ & $<, J$ & $2.4 \mathrm{e} 1$ & $<, J$ & $5.0 \mathrm{e} 2$ & $<, J$ & $8.3 e-6$ & $<, J$ & $6.6 \mathrm{e}-5$ \\
\hline 2,2'-Oxybis(1-chloropropane) & $<$ & $3.2 \mathrm{eo}$ & $<$ & $4.4 \mathrm{e} 0$ & $<$ & 4.500 & $<$ & $9.2 e 1$ & $<$ & $1.5 e-6$ & $<$ & $1.2 \mathrm{e}-5$ \\
\hline Pentachiorobenzene & k & $2.3 \mathrm{e} 0$ & $<$ & $3.2 \mathrm{e} 0$ & $<$ & $3.2 \mathrm{e} 0$ & $<$ & $6.6 \mathrm{e} 1$ & $<$ & $1.1 \mathrm{e}-6$ & < & $8.7 \mathrm{e}-6$ \\
\hline Pentachloronitrobenzene & < & $2.5 \mathrm{e} 0$ & $<$ & $3.5 \mathrm{e} 0$ & < & $3.5 \mathrm{e} 0$ & < & $7.2 \mathrm{e} 1$ & < & $1.2 e-6$ & < & $9.6 \mathrm{e}-6$ \\
\hline Pentachlorophenol & $<$ & $6.8 \mathrm{e} 1$ & $<$ & $9.5 \mathrm{e} 1$ & |< & $9.6 \mathrm{e} 1$ & $<$ & $2.0 \mathrm{e} 3$ & |< & $3.3 e-5$ & $<$ & $2.6 e-4$ \\
\hline Phenanthrene & < & $2.2 \mathrm{e} 0$ & $\leq$ & $3.1 \mathrm{e} 0$ & $<$ & 3.100 & $<$ & $6.4 \mathrm{e} 1$ & $\leq$ & $1.1 e-6$ & $<$ & $8.5 e-6$ \\
\hline Phenol & k & $1.7 e 1$ & $<$ & $2.4 \mathrm{e} 1$ & $<$ & $2.5 \mathrm{e} 1$ & < & $5.1 \mathrm{e} 2$ & $<$ & $8.4 \mathrm{e}-6$ & $<$ & $6.7 e-5$ \\
\hline Pyrene & $<$ & $2.3 \mathrm{eo}$ & $<$ & $3.2 \mathrm{eo}$ & $<$ & $3.2 \mathrm{eo}$ & $<$ & $6.6 \mathrm{e} 1$ & $<$ & $1.1 e-6$ & $<$ & $8.7 e-6$ \\
\hline Pyridine & $<$ & $3.8 \mathrm{e} 0$ & $<$ & $5.4 \mathrm{e} 0$ & $<$ & $5.4 \mathrm{e} 0$ & $<$ & $1.1 \mathrm{e} 2$ & $<$ & $1.9 e-6$ & $<$ & $1.5 e-5$ \\
\hline 1,2,4,5-Tetrachlorobenzene & |< & $2.5 \mathrm{e} 0$ & $<$ & $3.5 \mathrm{e} 0$ & $<$ & $3.5 \mathrm{e} 0$ & $<$ & $7.2 \mathrm{e}$ & $\leq$ & $1.2 e-6$ & $<$ & $9.6 \mathrm{e}-6$ \\
\hline 1,2,4-Trichlorobenzene & k & 2.700 & $<$ & $3.8 \mathrm{e} 0$ & $<$ & $3.8 \mathrm{e} 0$ & $<$ & $7.9 \mathrm{e} 1$ & $<$ & $1.3 e-6$ & $<$ & $1.0 \mathrm{e}-5$ \\
\hline 2,4,5-Trichlorophenol & < & $5.6 \mathrm{e} 0$ & $<$ & $7.9 \mathrm{e}$ & $<$ & $8.0 \mathrm{e} 0$ & $<$ & $1.6 \mathrm{e} 2$ & $<$ & $2.7 e-6$ & $<$ & $2.2 \mathrm{e}-5$ \\
\hline $\begin{array}{r}\text { 2,4,6-Trichlorophenol } \\
\text { TICs }\end{array}$ & $<$ & $3.6 \mathrm{e} 0$ & $<$ & $5.1 \mathrm{e} 0$ & $<$ & $5.1 \mathrm{eo}$ & $<$ & $1.1 \mathrm{e} 2$ & $<$ & $1.8 \mathrm{e}-6$ & k & $1.4 \mathrm{e}-5$ \\
\hline Furan, 2,5-dimethyl- & $\mathrm{N}, \mathrm{J}, \mathrm{M}$ & $3.2 \mathrm{e} 0$ & $\mathrm{~N}, \mathrm{~J}, \mathrm{M}$ & $4.4 \mathrm{e} 0$ & $\mathrm{~N}, \mathrm{~J}, \mathrm{M}$ & $4.5 \mathrm{e} 0$ & $\mathrm{~N}, \mathrm{~J}, \mathrm{M}$ & $9.2 \mathrm{e} 1$ & $\mathrm{~N}, J, \mathrm{M}$ & $1.5 e-6$ & $N, J, M$ & $1.2 \mathrm{e}-5$ \\
\hline 3-Hexanone & $\mathrm{N}, \mathrm{J}, \mathrm{M}$ & $4.3 \mathrm{e} 1$ & $\mathrm{~N}, \mathrm{~J}, \mathrm{M}$ & $6.0 \mathrm{e} 1$ & $\mathrm{~N}, \mathrm{~J}, \mathrm{M}$ & $6.1 e 1$ & $\mathrm{~N}, \mathrm{~J}, \mathrm{M}$ & $1.2 \mathrm{e} 3$ & $\mathrm{~N}, J, M$ & $2.1 \mathrm{e}-5$ & $\mathrm{~N}, \mathrm{~J}, \mathrm{M}$ & $1.6 \mathrm{e}-4$ \\
\hline 2-Hexanone & N.J.M & $5.2 \mathrm{e} 1$ & $\mathrm{~N}, J, M$ & $7.3 \mathrm{e} 1$ & $\mathrm{~N}, \mathrm{~J}, \mathrm{M}$ & $7.4 \mathrm{e} 1$ & $N, J, M$ & $1.5 \mathrm{e} 3$ & $\mathrm{~N}, J, \mathrm{M}$ & $2.5 e-5$ & $\mathrm{~N}, \mathrm{~J}, \mathrm{M}$ & $2.0 \mathrm{e}-4$ \\
\hline Octan & $N, J, M$ & $1.8 \mathrm{e} 0$ & $\mathrm{~N}, J, M$ & $2.5 \mathrm{e} 0$ & $N, J, M$ & $2.5 \mathrm{e} 0$ & $N, J, M$ & $5.2 \mathrm{e} 1$ & $N, J, M$ & $8.6 e-7$ & $\mathrm{~N}, \mathrm{~J}, \mathrm{M}$ & $6.9 e-6$ \\
\hline Benzaldehyde & $\mathrm{N}, \mathrm{J}, \mathrm{M}$ & $2.5 \mathrm{e} 2$ & $\mathrm{~N}, \mathrm{~J}, \mathrm{M}$ & $3.5 \mathrm{e} 2$ & $\mathrm{~N}, \mathrm{~J}, \mathrm{M}$ & $3.5 \mathrm{e} 2$ & $\mathrm{~N}, \mathrm{~J}, \mathrm{M}$ & $7.2 \mathrm{e} 3$ & $\bar{N}, J, M$ & $1.2 e-4$ & $\mathrm{~N}, \mathrm{~J}, \mathrm{M}$ & $9.6 e-4$ \\
\hline Dodecane & $\mathrm{N}, J, M$ & $1.5 \mathrm{e} 1$ & $\mathrm{~N}, \mathrm{~J}, \mathrm{M}$ & $2.1 \mathrm{e} 1$ & $\mathrm{~N}, \mathrm{~J}, \mathrm{M}$ & $2.1 \mathrm{e} 1$ & $\mathrm{~N}, J, M$ & $4.4 \mathrm{e} 2$ & N,J,M & $7.3 e-6$ & $\mathrm{~N}, \mathrm{~J}, \mathrm{M}$ & $5.8 \mathrm{e}-5$ \\
\hline Tridecane & $\mathrm{N}, \mathrm{J}, \mathrm{M}$ & $4.5 e 0$ & $\mathrm{~N}, \mathrm{~J}, \mathrm{M}$ & $6.3 \mathrm{e} 0$ & $N, J, M$ & $6.4 \mathrm{e0}$ & $N, J, M$ & $1.3 \mathrm{e} 2$ & $\mathrm{~N}, \mathrm{~J}, \mathrm{M}$ & $2.2 e-6$ & $\mathrm{~N}, \mathrm{~J}, \mathrm{M}$ & $1.7 e-5$ \\
\hline Tetradecane & $N, J, M$ & $1.9 \mathrm{e} 0$ & $\mathrm{~N}, \mathrm{~J}, \mathrm{M}$ & $2.7 e 0$ & $N, J, M$ & 2.800 & $N, U, M$ & $5.6 \mathrm{e}$ & $N, J, M$ & $-9.4 \mathrm{e}-7$ & $\mathrm{~N}, \mathrm{~J}, \mathrm{M}$ & $7.5 \mathrm{e}-6$ \\
\hline Pentadecane & $\mathrm{N}, \mathrm{J}, \mathrm{M}$ & $3.4 \mathrm{e} 0$ & $\mathrm{~N}, \mathrm{~J}, \mathrm{M}$ & $4.7 \mathrm{e} 0$ & $N, J, M$ & $4.8 \mathrm{e} 0$ & $\mathrm{~N}, J, \mathrm{M}$ & $9.8 e 1$ & $\mathrm{~N}, J, \mathrm{M}$ & $1.6 e-6$ & & $1.3 e-5$ \\
\hline Phosphoric acid tributyl ester & $\mathrm{N}, \mathrm{J}, \mathrm{M}$ & $5.2 \mathrm{e} 0$ & $\mathrm{~N}, \mathrm{~J}, \mathrm{M}$ & $7.3 \mathrm{e} 0$ & $\mathrm{~N}, J, M$ & $7.4 \mathrm{e} 0$ & $\mathrm{~N}, J, \mathrm{M}$ & $1.5 \mathrm{e} 2$ & $\mathrm{~N}, \mathrm{~J}, \mathrm{M}$ & $2.5 e-6$ & $\mathrm{~N}, \mathrm{~J}, \mathrm{M}$ & $2.0 e-5$ \\
\hline Cyclododecane & $\mathrm{N}, \mathrm{J}, \mathrm{M}$ & $7.0 \mathrm{e} 0$ & $\mathrm{~N}, \mathrm{~J}, \mathrm{M}$ & $9.8 \mathrm{e} 0$ & $\mathrm{~N}, \mathrm{~J}, \mathrm{M}$ & $9.9 \mathrm{e0}$ & $\mathrm{N}, \mathrm{J}, \mathrm{M}$ & $2.0 \mathrm{e} 2$ & $\mathrm{~N}, \mathrm{~J}, \mathrm{M}$ & $3.4 \mathrm{e}-6$ & $\mathrm{~N}, \mathrm{~J}, \mathrm{M}$ & $2.7 \mathrm{e}-5$ \\
\hline Heptadecane & $\mathrm{N}, J, \mathrm{M}$ & 2.000 & N.J.M & $2.8 \mathrm{e} 0$ & $\mathrm{~N}, \mathrm{~J}, \mathrm{M}$ & $2,8 \mathrm{e} 0$ & $\mathrm{~N}, J, M$ & $5.8 \mathrm{e} 1$ & $\mathrm{~N}, J, \mathrm{M}$ & $9.7 \mathrm{e}-7$ & $\mathrm{~N}, J, M$ & $7.7 e-6$ \\
\hline Eicosan & $\mathrm{N}, \mathrm{J}, \mathrm{M}$ & $1.4 \mathrm{e} 0$ & $\mathrm{~N}, J, \mathrm{M}$ & $1.9 \mathrm{e} 0$ & $N, J, M$ & $2.0 \mathrm{e} 0$ & $\bar{N}, J, \bar{M}$ & $4.0 \mathrm{e} 1$ & $\bar{N}, U, M$ & $6.7 \mathrm{e}-7$ & $\mathrm{~N}, J, M$ & $5.3 e-6$ \\
\hline Hexade & $\mathrm{N}$ & $1.2 \mathrm{e} 0$ & $\mathrm{~N}, \mathrm{~J}, \mathrm{M}$ & $1.7 \mathrm{e}$ & $\mathrm{N}, J, M$ & $1.7 \mathrm{eo}$ & $\mathrm{N}, \mathrm{J}, \mathrm{M}$ & $3.5 \mathrm{e} 1$ & $\mathrm{~N}, \mathrm{~J}, \mathrm{M}$ & $5.9 e-7$ & $\mathrm{~N}, \mathrm{~J}, \mathrm{M}$ & $4.7 e-6$ \\
\hline Octadecanoic acid & $\mathrm{N}, \downarrow, \mathrm{M}$ & $8.1 e-1$ & $\mathrm{~N}, J, M$ & $1.1 \mathrm{e0}$ & $\mathrm{N}, J, \mathrm{M}$ & $1.2 \mathrm{e} 0$ & $\mathrm{~N}, J, M$ & $2.4 \mathrm{e} 1$ & $\mathrm{~N}, \mathrm{~J}, \mathrm{M}$ & $3.9 e-7$ & $\mathrm{~N}, J, \mathrm{M}$ & $3.1 e-6$ \\
\hline Phosphine oxide, triphenyl- & $\mathrm{N}, \mathrm{J}, \mathrm{M}$ & $2.0 \mathrm{e} 0$ & $\mathrm{~N}, J, M$ & $2.8 \mathrm{e} 0$ & $\mathrm{~N}, J, \mathrm{M}$ & $2.8 \mathrm{e} 0$ & $N, J, M$ & $5.7 \mathrm{e} 1$ & $N, J, M$ & $9.5 e-7$ & $\mathrm{~N}, \mathrm{~J}, \mathrm{M}$ & $7.6 e-6$ \\
\hline Heneicosane & $\mathrm{N}, J, \mathrm{M}$ & $4.5 \mathrm{e}-1$ & $\mathrm{~N}, \mathrm{~J}, \mathrm{M}$ & $6.3 \mathrm{e}-1$ & $\mathrm{~N}, J, \mathrm{M}$ & $6.4 \mathrm{e}-1$ & $\mathrm{~N}, \mathrm{~J}, \mathrm{M}$ & $1.3 \mathrm{e} 1$ & $\mathrm{~N}, \mathrm{~J}, \mathrm{M}$ & $2.2 \mathrm{e}-7$ & $\mathrm{~N}, \mathrm{~J}, \mathrm{M}$ & $1.7 \mathrm{e}-6$ \\
\hline $\begin{array}{l}\text { Tetratetracontane } \\
\text { Heptane, 2,5-dimethyl- } \\
\text { Heptane, 2,3-dimethyl- }\end{array}$ & $\mathrm{N}, J, \mathrm{M}$ & $1.5 \mathrm{e} 0$ & $\mathrm{~N}, \mathrm{~J}, \mathrm{M}$ & $2.1 \mathrm{eo}$ & $\mathrm{N}, \mathrm{J}, \mathrm{M}$ & $2.1 \mathrm{eo}$ & $N, J, M$ & $4.3 e 1$ & $\mathrm{~N}, \mathrm{~J}, \mathrm{M}$ & $7.2 \mathrm{e}-7$ & $\mathrm{~N}, \mathrm{~J}, \mathrm{M}$ & $5.7 e-6$ \\
\hline $\begin{array}{l}\text { Benzaldehyde, ethyl- } \\
\text { Octodecane } \\
\text { Nonacosane } \\
\text { Hexatriacontane }\end{array}$ & & & & & & & & & & & & \\
\hline $\begin{array}{l}\text { Tetracosane } \\
\text { Tetratriacontane }\end{array}$ & & & & & & & & & & & & \\
\hline
\end{tabular}





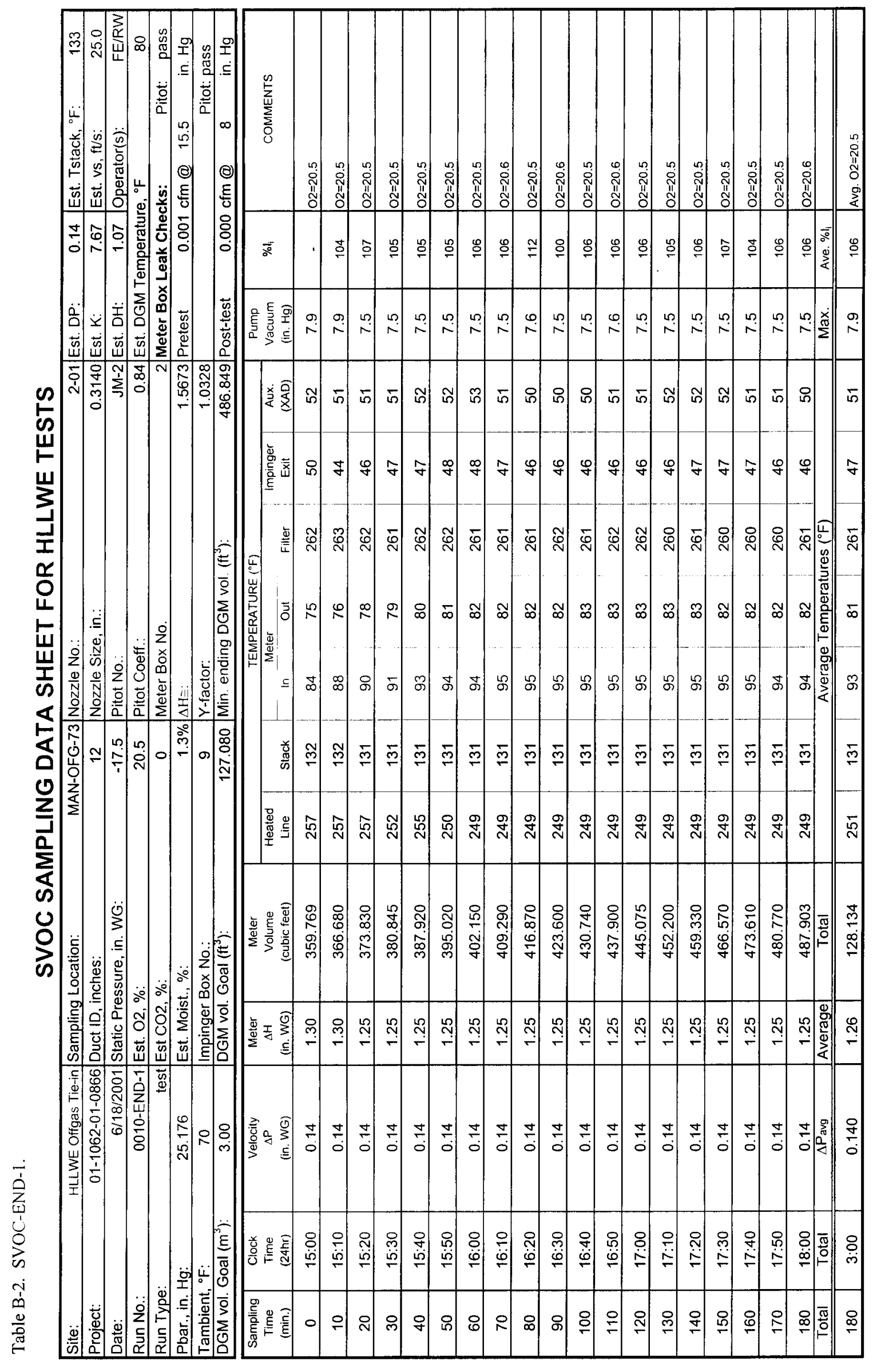




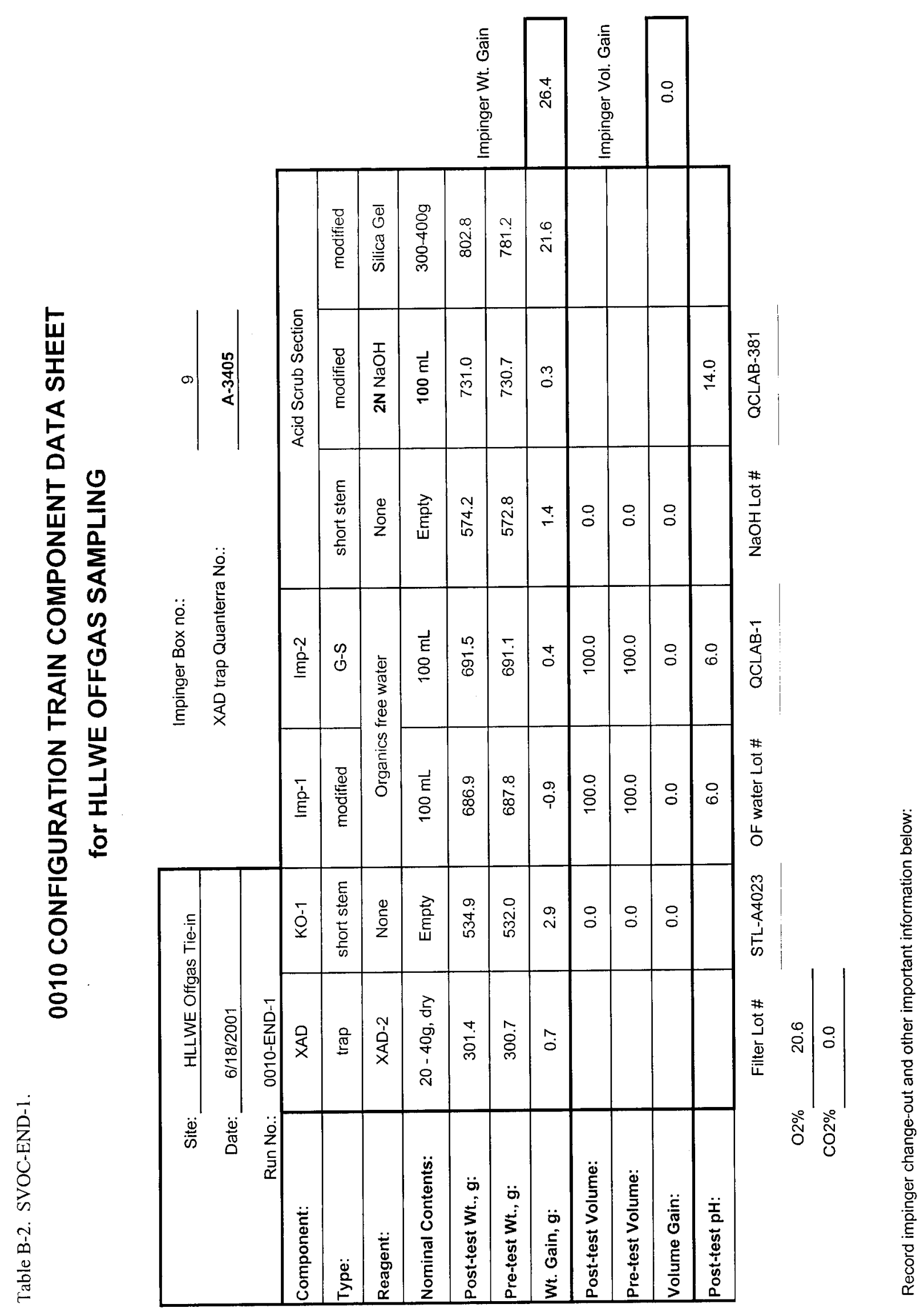


Table B-2. SVOC-END-1.

FIELD DATA CALCULATIONS

\begin{tabular}{|c|c|c|c|}
\hline $\begin{array}{r}\text { Project: } \\
\text { Run Date: } \\
\text { Run Identification: }\end{array}$ & $\begin{array}{r}01-1062-( \\
6 / 18 / 2 \\
0010-E\end{array}$ & $\begin{array}{l}1-0866 \\
001 \\
\text { ND-1 }\end{array}$ & \\
\hline PARAMETER & SYMBOL & UNITS & \\
\hline Absolute Pressure in the Duct & Pabs & in. $\mathrm{Hg}$ & 23.889 \\
\hline Average Duct Gas Temperature & Ts & $\mathrm{R}$ & 591 \\
\hline Average Meter Temperature & $\mathrm{Tm}$ & $\mathrm{R}$ & 547 \\
\hline Average Gas Oxygen Content & $\mathrm{Co} 2, \mathrm{~m}$ & $\%$ & 20.6 \\
\hline Average Gas Carbon Dioxide Content & $\mathrm{Cco} 2, \mathrm{~m}$ & $\%$ & 0.0 \\
\hline Total Impinger Weight Gain (water) & Ww & grams & 26.4 \\
\hline Nozzle Area & An & $\mathrm{ft}^{2}$ & 0.000538 \\
\hline Duct Area & As & $\mathrm{ft}^{2}$ & 0.7854 \\
\hline Sample Volume & VmStd & dscf & 107.847 \\
\hline Sample Volume (SI) & VmStdm & $\mathrm{dscm}$ & 3.054 \\
\hline Average Sampling Rate & Qm & $\mathrm{dscf} / \mathrm{m}$ & 0.599 \\
\hline Volume of Water Vapor & VwStd & scf & 1.245 \\
\hline Volume of Water Vapor (SI) & VwStdm & $\mathrm{scm}$ & 0.0352 \\
\hline Moisture Fraction & Bws & - & 0.011 \\
\hline Dry Gas Molecular Weight & Md & g/g-mol & 28.82 \\
\hline Wet Gas Molecular Weight & Ms & g/g-mol & 28.70 \\
\hline Gas Velocity at Nozzle & $\mathrm{vn}$ & $\mathrm{ft} / \mathrm{s}$ & 24.9 \\
\hline Gas Velocity at Nozzle (SI) & vnm & $\mathrm{m} / \mathrm{s}$ & 7.60 \\
\hline Average Gas Velocity & vncor & $\mathrm{ft} / \mathrm{s}$ & 21.05 \\
\hline Dry Offgas Flow Rate & Qsd & $d s c f / h$ & 41,960 \\
\hline Dry Offgas Flow Rate (SI) & Qsdm & $\mathrm{dscm} / \mathrm{h}$ & $1,188.2$ \\
\hline Actual Offgas Flow Rate & Q & acf $/ \mathrm{h}$ & 59,517 \\
\hline Intermediate Isokinetic Rate & li & $\%$ & 105.8 \\
\hline Final Isokinetic Rate & $\mathbf{I}$ & $\%$ & 105.6 \\
\hline
\end{tabular}

B2. 3 - 5 


\begin{tabular}{|c|c|c|c|c|c|c|c|c|c|c|c|c|}
\hline \multirow[t]{3}{*}{$\begin{array}{rr}\text { Project: } \\
\text { Run Date: } \\
\text { Run Identification: } \\
\text { Run Type: } \\
\text { Lab Report Date: } \\
\text { Lab Report Status: (preliminary or } \\
\text { final) } \\
\end{array}$} & \multicolumn{2}{|c|}{$\begin{array}{c}01-1062-01- \\
0866 \\
6 / 18 / 2001 \\
0010-E N D-1 \\
\text { test } \\
8 / 28 / 2001 \\
\text { Final }\end{array}$} & \multicolumn{10}{|c|}{$\begin{array}{c}\text { RESULTS } \\
\text { - without blank corrections } \\
\text { - final presentation should be rounded to two significant digits }\end{array}$} \\
\hline & \multicolumn{6}{|c|}{ CONCENTRATIONS } & \multicolumn{6}{|c|}{ MASS FLOW RATES } \\
\hline & & $\begin{array}{l}\mathrm{Jal} \\
\mathrm{cm} \text { ) }\end{array}$ & & & & $\begin{array}{l}\mathrm{idard} \\
\mathrm{cm} \text { ) }\end{array}$ & & & & $3 / \mathrm{sec}$ & & $b / h$ \\
\hline Acenaphthene & $<$ & $2.3 \mathrm{e} 0$ & $<$ & $3.2 e 0$ & $<$ & $3.2 \mathrm{e} 0$ & $<$ & $6.4 \mathrm{e} 1$ & $\mid<$ & $1.1 \mathrm{e}-6$ & $<$ & $8.4 e-6$ \\
\hline Acenaphthylene & $<$ & $2.2 \mathrm{e0}$ & $<$ & $3.1 e 0$ & $<$ & $3.1 \mathrm{e} 0$ & $<$ & $6.2 \mathrm{e} 1$ & $\mid<$ & $1.0 e-6$ & $<$ & 8.1e-6 \\
\hline Acetophenone & $<, \sqrt{ }$ & $8.3 \mathrm{e0}$ & $<. J$ & $1.2 e^{1}$ & $<, J$ & $1.2 \mathrm{e} 1$ & $<, J$ & $2.3 \mathrm{e} 2$ & $<<J$ & $3.9 e-6$ & $<, J$ & $3.1 \mathrm{e}-5$ \\
\hline Aniline & $<$ & $2.5 \mathrm{e} 1$ & $<$ & $3.6 \mathrm{e} 1$ & $<$ & $3.6 \mathrm{e} 1$ & $<$ & $7.1 \mathrm{e} 2$ & $<$ & $1.2 \mathrm{e}-5$ & $\leq$ & $9.4 e-5$ \\
\hline Anthracene & $<$ & $2.2 \mathrm{e} 0$ & $<$ & $3.1 \mathrm{e} 0$ & $<$ & $3.1 \mathrm{e} 0$ & $<$ & $6.2 \mathrm{e}^{1}$ & $<$ & $1.0 \mathrm{e}-6$ & $<$ & $8.1 e-6$ \\
\hline Benzidine & $<$ & $1.5 \mathrm{e} 2$ & $<$ & $2.2 \mathrm{e} 2$ & $<$ & $2.2 \mathrm{e} 2$ & $<$ & $4.3 \mathrm{e} 3$ & $\mid<$ & $7.2 \mathrm{e}-5$ & $<$ & $5.7 \mathrm{e}-4$ \\
\hline Benzoic acid & $<, E$ & $6.0 \mathrm{e} 2$ & $<, E$ & $8.4 \mathrm{e} 2$ & $<, E$ & $8.5 e 2$ & $<, E$ & $1.7 \mathrm{e} 4$ & $<, E$ & $2.8 \mathrm{e}-4$ & $<, E$ & $2.2 \mathrm{e}-3$ \\
\hline Benzo(a) anthracene & $\leq$ & $2.8 \mathrm{e} 0$ & $\leq$ & $3.9 e 0$ & $\leq \ldots$ & $3.9 \mathrm{e} 0$ & $\leq$ & $7.8 \mathrm{e} 1$ & $<$ & $-1.3 e-6$ & $<$ & $1.0 e-5$ \\
\hline Benzo(a)pyrene & k & $3.0 \mathrm{e} 1$ & k & $4.2 \mathrm{e} 1$ & $<$ & $4.3 \mathrm{e} 1$ & $\bar{k}$ & $8.4 \mathrm{e} 2$ & $<<$ & $1.4 \mathrm{e}-5$ & $<$ & $1.1 \mathrm{e}-4$ \\
\hline Benzo(b)fluoranthene & $<$ & $6.9 e 1$ & $<$ & $9.7 e^{1}$ & $<$ & $9.8 \mathrm{e} 1$ & $<$ & $1.9 \mathrm{e} 3$ & $\mid<$ & $3.2 \mathrm{e}-5$ & $<$ & $2.6 \mathrm{e}-4$ \\
\hline Benzo(g,h,i)perylene & $<$ & $3.9 \mathrm{e} 1$ & $<$ & $5.5 \mathrm{e} 1$ & $<$ & $5.6 e 1$ & $<$ & $1.1 \mathrm{e} 3$ & $\mid<$ & $1.8 e-5$ & $<$ & $1.5 \mathrm{e}-4$ \\
\hline Benzo(k)fluoranthene & $<$ & $9.9 \mathrm{e} 1$ & k & $1.4 \mathrm{e}^{2}$ & $<$ & $1.4 \mathrm{e} 2$ & $<$ & $2.8 \mathrm{e} 3$ & $\mid \leq$ & $4.6 e-5$ & $<$ & $3.7 \mathrm{e}-4$ \\
\hline Benzyl alcohol & < & $1.3 \mathrm{e} 2$ & $<$ & $1.8 \mathrm{e} 2$ & $<$ & $1.9 \mathrm{e} 2$ & $<$ & $3.7 \mathrm{e} 3$ & $<$ & $6.2 e-5$ & $<$ & $4.9 e-4$ \\
\hline bis(2-Chloroethoxy)methane & $<$ & $2.3 \mathrm{e} 0$ & $<$ & $3.2 e 0$ & $<$ & $3.3 \mathrm{e} 0$ & $<$ & $6.5 \mathrm{e} 1$ & $<$ & 1. $1 \mathrm{e}-6$ & $<$ & $8.6 e-6$ \\
\hline bis(2-Chloroethyl)ether & $<$ & $2.5 \mathrm{e0}$ & < & $3.6 e 0$ & $<$ & $3.6 \mathrm{e} 0$ & $<$ & $7.1 \mathrm{e} 1$ & $\mid<$ & 1.2e-6 & $<$ & $9.4 \mathrm{e}-6$ \\
\hline bis(2-Ethylhexyl)phthalate & $<$ & $5.1 \mathrm{e} 1$ & $\leq$ & $7.1 \mathrm{e} 1$ & $\leq$ & $7.2 \mathrm{e} 1$ & $\leq$ & $1.4 \mathrm{e} 3$ & $\leq$ & $2.4 \mathrm{e}-5$ & $\leq$ & $1.9 \mathrm{e}-4$ \\
\hline 4-Bromophenyl-phenylether & $<$ & $2.2 \mathrm{e0}$ & $\overline{<}$ & $3.0 e 0$ & $\overline{<}$ & $3.1 \mathrm{e} 0$ & $<$ & $6.1 \mathrm{e} 1$ & $<$ & $1.0 \mathrm{e}-6$ & $\bar{s}$ & $8.1 \mathrm{e}-6$ \\
\hline Butylbenzy|phthalate & $<$ & 3.000 & $<$ & $4.2 e 0$ & $<$ & $4.3 \mathrm{e} 0$ & $<$ & $8.4 \mathrm{e} t$ & $<<$ & $1.4 \mathrm{e}-6$ & $<$ & $1.1 e-5$ \\
\hline Carbazole & $<$ & 3.000 & $<$ & $4.2 \mathrm{e} 0$ & $<$ & $4.3 \mathrm{e} 0$ & $<$ & $8.4 \mathrm{e} 1$ & $\mid<$ & $1.4 \mathrm{e}-6$ & $<$ & $1.1 e-5$ \\
\hline 4-Chloro-3-methylphenol & $<$ & $3.9 \mathrm{e} 0$ & $\leq$ & $5.5 \mathrm{e} 0$ & $<$ & $5.6 \mathrm{e} 0$ & $\leq$ & $1.1 \mathrm{e} 2$ & $<$ & $1.8 \mathrm{e}-6$ & $<$ & $1.5 e-5$ \\
\hline 4-Chioroaniline & $<$ & $2.1 \mathrm{e} 1$ & < & $2.9 \mathrm{e} 1$ & $<$ & $2.9 \mathrm{e} 1$ & $<$ & $5.8 \mathrm{e} 2$ & $<$ & $9.7 e-6$ & $<$ & $7.7 e-5$ \\
\hline 2-Chloronaphthalene & $<$ & $2.1 \mathrm{eo}$ & $<$ & $3.0 \mathrm{e} 0$ & $<$ & $3.0 \mathrm{e} 0$ & $<$ & $6.0 \mathrm{e} 1$ & $<$ & $1.0 \mathrm{e}-6$ & $<$ & $8.0 e-6$ \\
\hline 2-Chtorophenol & $<$ & $2.5 \mathrm{eo}$ & < & $3.6 \mathrm{e} 0$ & $<$ & $3.6 \mathrm{e} 0$ & $<$ & $7.1 \mathrm{e} 1$ & $\mid<$ & 1. $2 \mathrm{e}-6$ & $<$ & $9.4 e-6$ \\
\hline 4-Chlorophenyl phenyl ether & $<$ & $2.5 e 0$ & $\leq$ & $3.6 \mathrm{e} 0$ & $<$ & $3.6 \mathrm{e} 0$ & $\leq$ & $7.1 \mathrm{e} 1$ & $1<$ & $12 \mathrm{e}-6$ & $\leq$ & $9.4 e-6$ \\
\hline Chrysene & $<$ & $2.8 \mathrm{e} 0$ & $<$ & $3.9 \mathrm{e} 0$ & $<$ & $3.9 \mathrm{e} 0$ & $\overline{<}$ & $7.8 \mathrm{e} 1$ & $<$ & $1.3 e-6$ & $<$ & $1.0 \mathrm{e}-5$ \\
\hline Di-n-butylphthalate & $<, j$ & $2.3 \mathrm{e} 1$ & $<, J$ & $3.2 \mathrm{e} 1$ & $<, J$ & $3.3 \mathrm{e} 1$ & $<, J$ & $6.5 \mathrm{e} 2$ & $<<J$ & 1. $1 \mathrm{e}-5$ & $<, J$ & $8.6 e-5$ \\
\hline Di-n-octylphthalate & $<, j$ & $3.7 \mathrm{e} 1$ & $<, J$ & $5.2 e^{1}$ & $<, J$ & $5.2 \mathrm{e} 1$ & $<, J$ & $7.0 \mathrm{e} 3$ & $<, J$ & $1.7 e-5$ & $<, J$ & $1.4 \mathrm{e}-4$ \\
\hline Oibenz(a,h)anthracene & $<$ & 3.901 & $\leq$ & $5.5 \mathrm{e} 1$ & $<$ & $5.6 \mathrm{e} 1$ & $\leq$ & $1.1 \mathrm{e} 3$ & $3 \mid<$ & $18 \mathrm{e}-5$ & $<$ & $1.5 \mathrm{e}-4$ \\
\hline Dibenzofuran & $<<$ & $2.5 \mathrm{eO}$ & k & $3.6 \mathrm{eo}$ & $<$ & $3.6 \mathrm{e} 0$ & e & $7.1 \mathrm{e} 1$ & $1<$ & $1.2 \mathrm{e}-6$ & $<$ & $9.4 \mathrm{e}-6$ \\
\hline 1,2-Dichlorobenzene & k & $2.5 \mathrm{e} 0$ & $<$ & $3.6 \mathrm{e} 0$ & $<$ & $3.6 \mathrm{e} 0$ & $<$ & $7.1 \mathrm{e} 1$ & $\mid<$ & $1.2 e-6$ & $<$ & $8.4 e-6$ \\
\hline i,3-Dichlorobenzene & $<$ & $2.8 \mathrm{e} 0$ & $<$ & $3.9 \mathrm{eo}$ & $<$ & $3.9 \mathrm{e} 0$ & $<$ & $7.8 \mathrm{e} 1$ & $\mid<$ & $1.3 e-6$ & $<$ & $1.0 \mathrm{e}-5$ \\
\hline 1,4-Dichlorobenzene & $\mathrm{J}$ & $4.4 \mathrm{e} 0$ & $J$ & $6.2 \mathrm{e} 0$ & $\mathrm{~J}$ & $6.2 \mathrm{e} 0$ & $\mathrm{~J}$ & $1.2 \mathrm{e} 2$ & $\mathrm{~J}$ & $2.1 \mathrm{e}-6$ & 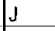 & $1.6 \mathrm{e}-5$ \\
\hline 3,3'-Dichlorobenzidine & $<$ & $2.5 \mathrm{e} 1$ & $<$ & $3.6 \mathrm{e} 1$ & $<$ & $3.6 \mathrm{e} 1$ & $<$ & $7.1 \mathrm{e} 2$ & $<<$ & $1.2 \mathrm{e}-5$ & $<$ & $9.4 \mathrm{e}-5$ \\
\hline 2,4-Dichlorophenol & $<$ & $3.0 \mathrm{e} 0$ & $<$ & $4.2 \mathrm{e} 0$ & $<$ & $4.3 \mathrm{e} 0$ & $<$ & $8.4 \mathrm{e} 1$ & $<$ & $1.4 \mathrm{e}-6$ & $<$ & $1.1 \mathrm{e}-5$ \\
\hline Diethylphthalate & < & $3.5 \mathrm{e} 0$ & $<$ & $4.9 \mathrm{e} 0$ & $<$ & $4.9 \mathrm{e} 0$ & $<$ & $9.7 \mathrm{e} 1$ & $1<$ & $1.6 e-6$ & $<$ & $1.3 e-5$ \\
\hline Dimethyl phthalate & $\leq$ & $2.2 \mathrm{eo}$ & $\leq$ & $3.1 \mathrm{e} 0$ & $<$ & $3.1 \mathrm{e} 0$ & $<$ & $6.2 \mathrm{e} 1$ & $\mid<$ & $1.0 \mathrm{e}-6$ & $<$ & $8.2 e-6$ \\
\hline 2,4-Dimethylphenol & $<$ & $1.4 e 1$ & $<$ & $1.9 \mathrm{e} 1$ & $<$ & $1.9 \mathrm{e} 1$ & $<$ & $3.8 \mathrm{e} 2$ & $<$ & $6.4 e-6$ & $<$ & $5.1 e-5$ \\
\hline 4,6-Dinitro-2-methyiphenol & $<$ & 3.001 & $<$ & $4.2 \mathrm{e} 1$ & $<$ & $4.3 \mathrm{e} 1$ & $<$ & $8.4 \mathrm{e} 2$ & $2<$ & $1.4 \mathrm{e}-5$ & $<$ & $1.1 e-4$ \\
\hline 2,4-Dinitrophenol & $<$ & $6.2 \mathrm{e} 1$ & $<$ & $8.7 \mathrm{e}^{1}$ & $<$ & $8.8 e 1$ & $<$ & $1.8 \mathrm{e} 3$ & $<$ & $2.9 e-5$ & $<$ & $2.3 \mathrm{e}-4$ \\
\hline 2,4-Dinitrotoluene & $<$ & $3.0 \mathrm{e} 0$ & $<\leq$ & $4.2 \mathrm{e0}$ & $<$ & $4.3 \mathrm{e} 0$ & $<$ & $8.4 \mathrm{e} 1$ & $<<$ & $1.4 \mathrm{e}-6$ & $\leq$ & $1.1 e-5$ \\
\hline 2,6-Dinitrotoluene & $<$ & $2.8 \mathrm{e0}$ & $<$ & $3.9 \mathrm{e} 0$ & $<$ & $3.9 \mathrm{e} 0$ & $<$ & $7.8 \mathrm{e} 1$ & $<$ & $1.3 e-6$ & $<$ & $1.0 \mathrm{e}-5$ \\
\hline 1,2-Diphenylhydrazine & $<$ & $2.3 \mathrm{e} 0$ & $<$ & $3.2 \mathrm{e} 0$ & $<$ & $3.2 \mathrm{e} 0$ & $<$ & $6.4 \mathrm{e} 1$ & $\mid<$ & $1.1 \mathrm{e}-6$ & $<$ & $8.4 e-6$ \\
\hline Fluoranthene & $<$ & $2.3 \mathrm{e} 0$ & $<$ & $3.2 \mathrm{e} 0$ & $<$ & $3.3 \mathrm{e} 0$ & $<$ & $6.5 \mathrm{e} 1$ & $\mid<$ & $1.1 \mathrm{e}-6$ & $<$ & $8.6 \mathrm{e}-6$ \\
\hline Fluorene & $<$ & $2.3 \mathrm{e} 0$ & $<$ & $3.2 e 0$ & $<$ & $3.3 \mathrm{e} 0$ & $<$ & $6.5 \mathrm{e} 1$ & $1<$ & $1.1 \mathrm{e}-6$ & $<$ & $8.6 \mathrm{e}-6$ \\
\hline Hexachlorocyclopentadiene & $<$ & $3.7 \mathrm{e} 1$ & $<$ & $5.2 \mathrm{e} 1$ & $<$ & $5.2 \mathrm{e} 1$ & k & $1.0 \mathrm{e} 3$ & $3<<$ & $1.7 e-5$ & $<$ & $1.4 \mathrm{e}-4$ \\
\hline Hexachlorobenzene & $<$ & $2.5 \mathrm{eo}$ & $<$ & $3.6 e 0$ & $<$ & $3.6 \mathrm{e} 0$ & $<$ & $7.1 \mathrm{e} 1$ & $1<$ & $1.2 \mathrm{e}-6$ & $<$ & $9.4 e-6$ \\
\hline Hexachlorobutadiene & $<$ & $3.5 \mathrm{eo}$ & $<$ & 4.900 & $<$ & $4.9 \mathrm{e} 0$ & $<$ & $9.7 \mathrm{e} 1$ & $<$ & $1.6 e-6$ & $<$ & $1.3 e-5$ \\
\hline Hexachloroethane & $<$ & $3.7 \mathrm{eo}$ & $1<$ & $5.2 \mathrm{e} 0$ & $\leq$ & $5.2 \mathrm{e} 0$ & $<$ & $1.0 \mathrm{e} 2$ & $<<$ & $1.7 \mathrm{e}-6$ & $<$ & $1.4 \theta-5$ \\
\hline Indeno(1,2,3-cd)pyrene & $<$ & $3.5 \mathrm{e} 1$ & $<$ & $4.9 \mathrm{e} 1$ & $<$ & $\overline{4} . \overline{\mathrm{e} 1}$ & k & $9.7 \mathrm{e} 2$ & $<<$ & $1.6 e-5$ & $<$ & $1.3 e-4$ \\
\hline Isophorone & $<$ & $2.3 \mathrm{eo}$ & $<$ & $3.2 \mathrm{e} 0$ & $<$ & $3.3 \mathrm{e} 0$ & $<$ & $6.5 \mathrm{e} 1$ & $\mid<$ & $1.1 \mathrm{e}-6$ & $<$ & $8.6 e-6$ \\
\hline 2-Methylnaphthalene & k & $2.3 \mathrm{eo}$ & < & $3.2 \mathrm{eO}$ & $<$ & $3.3 \mathrm{e} 0$ & $<$ & $6.5 \mathrm{et}$ & $<$ & $1.1 \mathrm{e}-6$ & $<$ & $8.6 \theta-6$ \\
\hline 2-Methylphenol & $<$ & $1.1 \mathrm{e} 1$ & $<$ & $1.5 \mathrm{e} 1$ & $<$ & $1.5 e 1$ & $<$ & $3.0 \mathrm{e} 2$ & $<<$ & $5.1 \mathrm{e}-6$ & $<$ & $4.00-5$ \\
\hline 3-Methylphenol \& 4-Methylphenol & $<$ & $7.8 \mathrm{e0}$ & $<$ & $1.1 \mathrm{e} 1$ & $<$ & $1.1 \mathrm{e} 1$ & $\bar{k}$ & $2.2 \mathrm{e} 2$ & $<$ & $3.7 \mathrm{e}-6$ & $<$ & $2.9 e-5$ \\
\hline N-Nitroso-di-n-propylamine & $<$ & $2.5 \mathrm{e} 0$ & $<$ & $3.6 \mathrm{e} 0$ & $<$ & $3.6 \mathrm{e} 0$ & $<$ & $7.1 \mathrm{e} 1$ & $\mid<$ & 1. $2 \mathrm{e}-6$ & $<$ & $9.4 \theta-6$ \\
\hline N-Nitrosodimethylamine & $<$ & $2.5 \mathrm{e} 0$ & $<$ & $3.6 \mathrm{eo}$ & $<$ & $3.6 \mathrm{e} 0$ & $<$ & $7.1 \mathrm{e} 1$ & $\mid<$ & 1.2e-6 & $<$ & $9.4 e-6$ \\
\hline N-Nitrosodiphenylamine & $<$ & $3.5 \mathrm{eo}$ & $\leq$ & $4.9 e 0$ & $<$ & $4.9 \mathrm{e} 0$ & $<$ & $9.7 \mathrm{e} 1$ & $\mid<$ & $1.6 e-6$ & $<$ & $1.3 e-5$ \\
\hline Naphthalene & $<$ & $2.5 \mathrm{e}$ & $<$ & $3.6 \mathrm{eo}$ & $<$ & $3.6 \mathrm{e} 0$ & $<$ & $7.1 \mathrm{e} 1$ & $<$ & $1.2 \mathrm{e}-6$ & $<$ & $94 \mathrm{e}-6$ \\
\hline 2-Nitroaniline & $<$ & $2.5 \mathrm{e} 0$ & $<$ & $3.6 \mathrm{e} 0$ & $<$ & $3.6 \mathrm{e} 0$ & $<$ & $7.1 \mathrm{e} 1$ & $\mid<$ & $1.2 \mathrm{e}-6$ & $<$ & $9.4 e-6$ \\
\hline 3-Nitroaniline & $<<$ & 9.500 & $<$ & $1.3 e^{1}$ & $<$ & $1.3 \mathrm{e} 1$ & $<$ & $2.7 \mathrm{e} 2$ & $2<<$ & $4,4 e-6$ & $<$ & $3.5 e-5$ \\
\hline 4-Nitroaniline & $<$ & $8.3 \mathrm{e} 0$ & $<$ & $1.2 \mathrm{e} 1$ & $<$ & $1.2 \mathrm{e} 1$ & $<$ & $2.3 \mathrm{e} 2$ & $<$ & $3.9 \mathrm{e}-6$ & $<$ & $3.1 e-5$ \\
\hline Nitrobenzene & $<, j$ & $3.5 \mathrm{e} 0$ & $<\rfloor$, & $4.9 \mathrm{e} 0$ & $\langle, J$ & $4.9 \mathrm{e} 0$ & $<, J$ & $9.7 \mathrm{e} 1$ & $<, J$ & $1.6 e-6$ & $<, J$ & $1.3 e-5$ \\
\hline 2-Nitrophenol & |<.J & $9.5 e 0$ & $<, J$ & $13 \mathrm{e} 1$ & $<. J$ & $13 \mathrm{e} 1$ & $<. J$ & $2.7 \mathrm{e} 2$ & $2<. J$ & $4.4 e-6$ & $<, J$ & $3.5 e-5$ \\
\hline 4-Nitrophenol & k & $1.3 \mathrm{e} 1$ & $<$ & $1.8 \mathrm{e} 1$ & $<$ & $1.8 \mathrm{e} 1$ & k & $3.6 \mathrm{e} 2$ & $2<$ & $5.9 e-6$ & $<$ & $4.7 e-5$ \\
\hline 2.2'-Oxybis (1-chloropr & |< & $3.2 \mathrm{eO}$ & $<$ & $4.5 \mathrm{e} 0$ & is & $4.6 \mathrm{e} 0$ & i & $9.1 \mathrm{e} 1$ & | $<$ & $1.5 e-6$ & $<$ & $1.2 e-5$ \\
\hline
\end{tabular}


Table B-2. SVOC-END-1.

\begin{tabular}{|c|c|c|c|c|c|c|c|c|c|c|c|c|}
\hline \multirow[t]{3}{*}{$\begin{array}{r}\text { Project: } \\
\text { Run Date: } \\
\text { Run Identification: } \\
\text { Run Type: } \\
\text { Lab Report Date } \\
\text { Lab Report Status: (preliminary or } \\
\text { final) } \\
\end{array}$} & \multicolumn{2}{|c|}{$\begin{array}{c}01-1062-01- \\
0866 \\
6 / 18 / 2001 \\
0010-E N D-1 \\
\text { test } \\
8 / 28 / 2001 \\
\text { Final }\end{array}$} & \multicolumn{10}{|c|}{$\begin{array}{l}\text { RESULTS } \\
\text { - without blank corrections } \\
\text { - final presentation should be rounded to two significant digits }\end{array}$} \\
\hline & \multicolumn{6}{|c|}{ CONCENTRATIONS } & \multicolumn{6}{|c|}{ MASS FLOW RATES } \\
\hline & \multicolumn{2}{|c|}{$\begin{array}{c}\text { Actual } \\
\text { ( } \mu \mathrm{g} / \mathrm{acm})\end{array}$} & \multicolumn{2}{|c|}{$\begin{array}{l}\text { Standard } \\
(\mu \mathrm{g} / \mathrm{scm})\end{array}$} & \multicolumn{2}{|c|}{$\begin{array}{l}\text { Dry Standard } \\
(\mu \mathrm{g} / \mathrm{ds} \mathrm{cm})\end{array}$} & \multicolumn{2}{|c|}{$\mu g / \min$} & \multicolumn{2}{|c|}{ grams $/ \mathrm{sec}$} & \multicolumn{2}{|c|}{$\mathrm{lb} / \mathrm{h}$} \\
\hline Pentachlorobenzene & $<$ & $2.3 \mathrm{e} 0$ & $<$ & $3.2 \mathrm{e} 0$ & $<$ & $3.3 \mathrm{e} 0$ & $<$ & $6.5 \mathrm{e} 1$ & $<$ & $1.1 \mathrm{e}-6$ & $<$ & $8.6 \mathrm{e}-6$ \\
\hline Pentachloronitrobenzene & $<$ & $2.5 e 0$ & $<$ & $3.6 e 0$ & k & $3.6 \mathrm{e} 0$ & $<$ & $7.1 \mathrm{e} 1$ & $<$ & $1.2 \mathrm{e}-6$ & $<$ & $9.4 e-6$ \\
\hline Pentachiorophenol & $<$ & $6.9 e 1$ & $<$ & $9.7 \mathrm{e} 1$ & $<$ & $9.8 \mathrm{e} 1$ & $<$ & $1.9 \mathrm{e} 3$ & < & $3.2 e-5$ & $<$ & $2.6 \mathrm{e}-4$ \\
\hline Phenanthrene & $<$ & $2.3 e 0$ & $<$ & $3.2 \mathrm{e} 0$ & $<$ & $3.2 \mathrm{e} 0$ & $<$ & $6.4 \mathrm{e} 1$ & $<<$ & $1.1 e-6$ & $<$ & $8.4 \mathrm{e}-6$ \\
\hline Phenol & $\langle, J$ & $8.5 \mathrm{e}$ & $<, J$ & $1.2 \mathrm{e} 1$ & $<, J$ & $1.2 \mathrm{e} 1$ & $<, J$ & $2.4 \mathrm{e} 2$ & $<, j$ & $4.0 e-6$ & $<, J$ & $3.2 \mathrm{e}-5$ \\
\hline Pyrene & $<$ & $2.3 \mathrm{e} 0$ & $<$ & $3.2 \mathrm{e} 0$ & $<$ & $3.3 \mathrm{e} 0$ & $<$ & $6.5 \mathrm{e} 1$ & $<$ & $1.1 \mathrm{e}-6$ & $<$ & $8.6 e-6$ \\
\hline Pyridine & $<$ & $3.9 \mathrm{e} 0$ & $<$ & $5.5 \mathrm{e} 0$ & $<$ & $5.6 \mathrm{e} 0$ & $<$ & $1.1 \mathrm{e} 2$ & $<$ & $1.8 \mathrm{e}-6$ & $<$ & $1.5 e-5$ \\
\hline 1,2,4,5-Tetrachlorobenzene & $\leq$ & $2.5 \mathrm{e} 0$ & $<$ & $36 \mathrm{e} 0$ & $<$ & $3.6 \mathrm{e} 0$ & $<$ & $7.1 \mathrm{e} 1$ & $<$ & $1.2 \mathrm{e}-6$ & $\leq$ & $9.4 e-6$ \\
\hline $1,2,4-$ Trichlorobenzene & $<$ & $2.8 \mathrm{e} 0$ & $<$ & $3.9 \mathrm{e} 0$ & $<$ & $3.9 \mathrm{e} 0$ & $<$ & $7.8 \mathrm{e} 1$ & $<$ & $1.3 e-6$ & $<$ & $1.0 \mathrm{e}-5$ \\
\hline 2,4,5-Trichlorophenol & $<$ & $5.8 \mathrm{e} 0$ & $<$ & $8.1 \mathrm{e} 0$ & k & $8.2 \mathrm{e} 0$ & $<$ & $1.6 \mathrm{e} 2$ & $<$ & $2.7 e-6$ & $<$ & $2.1 e-5$ \\
\hline $\begin{array}{c}\text { 2,4,6-Trichlorophenol } \\
\text { TICs }\end{array}$ & $<$ & $3.7 \mathrm{e} 0$ & $<$ & $5.2 \mathrm{e} 0$ & $<$ & $5.2 \mathrm{e} 0$ & $<$ & $1.0 \mathrm{e} 2$ & $<$ & $1.7 \mathrm{e}-6$ & $<$ & $1.4 e-5$ \\
\hline Furan, 2,5-dimethyl- & $\bar{N}, J, \bar{M}$ & $1.8 \mathrm{e} 0$ & $\bar{N}, J, M$ & $2.5 \mathrm{e} 0$ & $N, J, M$ & $2.6 \mathrm{e} 0$ & $\mathrm{~N}, \mathrm{~J}, \mathrm{M}$ & $5.1 \mathrm{e} 1$ & $N, J, M$ & $8.4 e-7$ & $N, J, M$ & $6.7 \ddot{e}-\overline{6}$ \\
\hline 3-Hexanone & $N, J, M$ & $2.2 e 1$ & $\mathrm{~N}, \mathrm{~J}, \mathrm{M}$ & $3.1 \mathrm{e} 1$ & $N, J, M$ & $3.1 \mathrm{e}^{1}$ & $\mathrm{~N}, \mathrm{~J}, \mathrm{M}$ & $6.2 \mathrm{e} 2$ & N.J.M & $1.0 e-5$ & $\mathrm{~N}, J, \mathrm{M}$ & $8.2 e-5$ \\
\hline 2-Hexanone & $N, J, M$ & $2.8 \mathrm{e} 1$ & $\mathrm{~N}, J, M$ & $3.9 \mathrm{e} 1$ & $N, J, M$ & $3.9 \mathrm{e} 1$ & $\mathrm{~N}, \mathrm{~J}, \mathrm{M}$ & $7.8 \mathrm{e} 2$ & $\mathrm{~N}, \mathrm{~J}, \mathrm{M}$ & $1.3 e-5$ & $\mathrm{~N}, J, \mathrm{M}$ & $1,0 e-4$ \\
\hline Heptane, 2,3-dimethyl- & N.J,M & $2.3 \mathrm{e} 0$ & $\mathrm{~N}, J, M$ & $3.2 \mathrm{e0}$ & N.J.M & $3.2 e 0$ & N.J,M & $6.4 \mathrm{e} 1$ & N,J,M & $1.1 \mathrm{e}-6$ & N.J.M & $8.5 e-6$ \\
\hline Benzaldehyde & $N, J, M$ & $1.7 \mathrm{e} 2$ & $N, J, M$ & $2.4 \mathrm{e} 2$ & $N, J, M$ & $2.4 \mathrm{e} 2$ & $\mathrm{~N}, \mathrm{~J}, \mathrm{M}$ & $4.8 \mathrm{e} 3$ & $\mathrm{~N}, \mathrm{~J}, \mathrm{M}$ & $8.0 e-5$ & $N, J, M$ & $6.3 e-4$ \\
\hline Formic acid, phenylmethyl ester & $N, J, M$ & $1.7 \mathrm{e} 1$ & $N, J, M$ & $2.3 \mathrm{e} 1$ & $\mathrm{~N}, J, M$ & $2.4 \mathrm{e} 1$ & $\mathrm{~N}, \mathrm{~J}, \mathrm{M}$ & $4.7 \mathrm{e} 2$ & N,J,M & $7.8 e-6$ & N,J,M & $6.2 e-5$ \\
\hline Benzaldehyde, 4-ethyl- & $N, J, M$ & $1.3 \mathrm{e} 1$ & $N, J, M$ & $1.8 \mathrm{e} 1$ & $N, J, M$ & $1.9 \mathrm{e} 1$ & N,J,M & $3.7 \mathrm{e} 2$ & N,J.M & $6.2 e-6$ & $\mathrm{~N}, \mathrm{~J}, \mathrm{M}$ & $4.9 \mathrm{e}-5$ \\
\hline Dodecane & $N, J, M$ & $1.1 \mathrm{e} 1$ & $N, J, M$ & $16 \mathrm{e} 1$ & $N, J, M$ & $1.6 \mathrm{e} 1$ & $\mathrm{~N}, \mathrm{~J}, \mathrm{M}$ & 3.1e2 & $\mathrm{N}, \mathrm{J}, \mathrm{M}$ & $5.2 \mathrm{e}-6$ & $N, J, M$ & $4.1 e-5$ \\
\hline Tridecane & $\bar{N}, J, M$ & $4.8 \mathrm{e}$ & $\mathrm{N}, \mathrm{J}, \mathrm{M}$ & $6.8 \mathrm{e} 0$ & $N, J, M$ & $6.9 \mathrm{e} 0$ & $\mathrm{~N}, J, M$ & $1.4 \mathrm{e} 2$ & $\mathrm{~N}, \mathrm{~J}, \mathrm{M}$ & $2.3 e-6$ & $N, J, M$ & $1.8 \mathrm{e}-5$ \\
\hline 2,4-Hexadiene & $N, J, M$ & $3.7 e 1$ & $\mathrm{~N}, \mathrm{~J}, \mathrm{M}$ & $5.2 \mathrm{e} 1$ & $N, J, M$ & $5.2 \mathrm{e} 1$ & $\mathrm{~N}, \mathrm{~J}, \mathrm{M}$ & $1.0 \mathrm{e} 3$ & $\mathrm{~N}, J, M$ & $1.7 e-5$ & $\mathrm{~N}, \mathrm{~J}, \mathrm{M}$ & $1.4 \mathrm{e}-4$ \\
\hline Tetradecane & $\mathrm{N}, J, M$ & $2.2 \mathrm{e} 1$ & $\mathrm{~N}, \mathrm{~J}, \mathrm{M}$ & 3.1e1 & $\mathrm{N}, J, M$ & 3.1e1 & $\mathrm{N}, \mathrm{J}, \mathrm{M}$ & $6.2 \mathrm{e} 2$ & $\mathrm{~N}, \mathrm{~J}, \mathrm{M}$ & $1.0 \mathrm{e}-5$ & $\mathrm{~N}, \mathrm{~J}, \mathrm{M}$ & $8.2 e-5$ \\
\hline Phosphoric acid tributyl ester & $N, J, M$ & $3.7 \mathrm{e} 0$ & $N, J, M$ & $5.2 \mathrm{e} 0$ & $N, J, M$ & $5.2 \mathrm{e} 0$ & $\mathrm{~N}, \mathrm{~J}, \mathrm{M}$ & $1.0 \mathrm{e} 2$ & $\mathrm{~N}, \mathrm{~J}, \mathrm{M}$ & $1.7 \mathrm{e}-6$ & N,J,M & $1.4 \mathrm{e}-5$ \\
\hline Cyclododecane & $N, J, M$ & $6.9 \mathrm{e} 0$ & $\mathrm{~N}, \mathrm{~J}, \mathrm{M}$ & $9.7 \mathrm{e} 0$ & $N, J, M$ & $9.8 \mathrm{e} 0$ & $\mathrm{~N}, \mathrm{~J}, \mathrm{M}$ & $1.9 \mathrm{e} 2$ & N,J,M & $3.2 e-6$ & N.J,M & $2.6 \mathrm{e}-5$ \\
\hline Heptadecane & $N, J, M$ & $9.2 \mathrm{e}-1$ & $N, J, M$ & $1.3 \mathrm{e} 0$ & $N, J, M$ & $1.3 \mathrm{e} 0$ & $\mathrm{~N}, \mathrm{~J}, \mathrm{M}$ & $2.6 \mathrm{e} 1$ & N,J,M & $4.3 e-7$ & N.J,M & $3.4 e-6$ \\
\hline $\begin{array}{l}\text { Octadecanoic acid } \\
\text { Heplane, 2,5-dimethyl- }\end{array}$ & N,J,M & $46 \mathrm{e}-1$ & $\mathrm{~N}, \mathrm{~J}, \mathrm{M}$ & $6.5 e-1$ & $N, J, M$ & $6.5 \mathrm{e}-1$ & $\mathrm{~N}, J, \mathrm{M}$ & $1.3 \mathrm{e} 1$ & N,J,M & $2.2 e-7$ & N,J,M & $1.7 e-6$ \\
\hline $\begin{array}{l}\text { Benzoic acid, methyl ester } \\
\text { Benzaldehyde, ethyl- } \\
\text { Pentadecane } \\
\text { Eicosane }\end{array}$ & & & & & & & & & & & & \\
\hline $\begin{array}{l}\text { Heneicosane } \\
\text { Octodecane } \\
\text { Phosphine oxide, triphenyl- } \\
\text { Nonacosane } \\
\text { Hexatriacontane } \\
\text { Tetracosane } \\
\text { Tetratriacontane }\end{array}$ & & & & & & & & & & & & \\
\hline
\end{tabular}





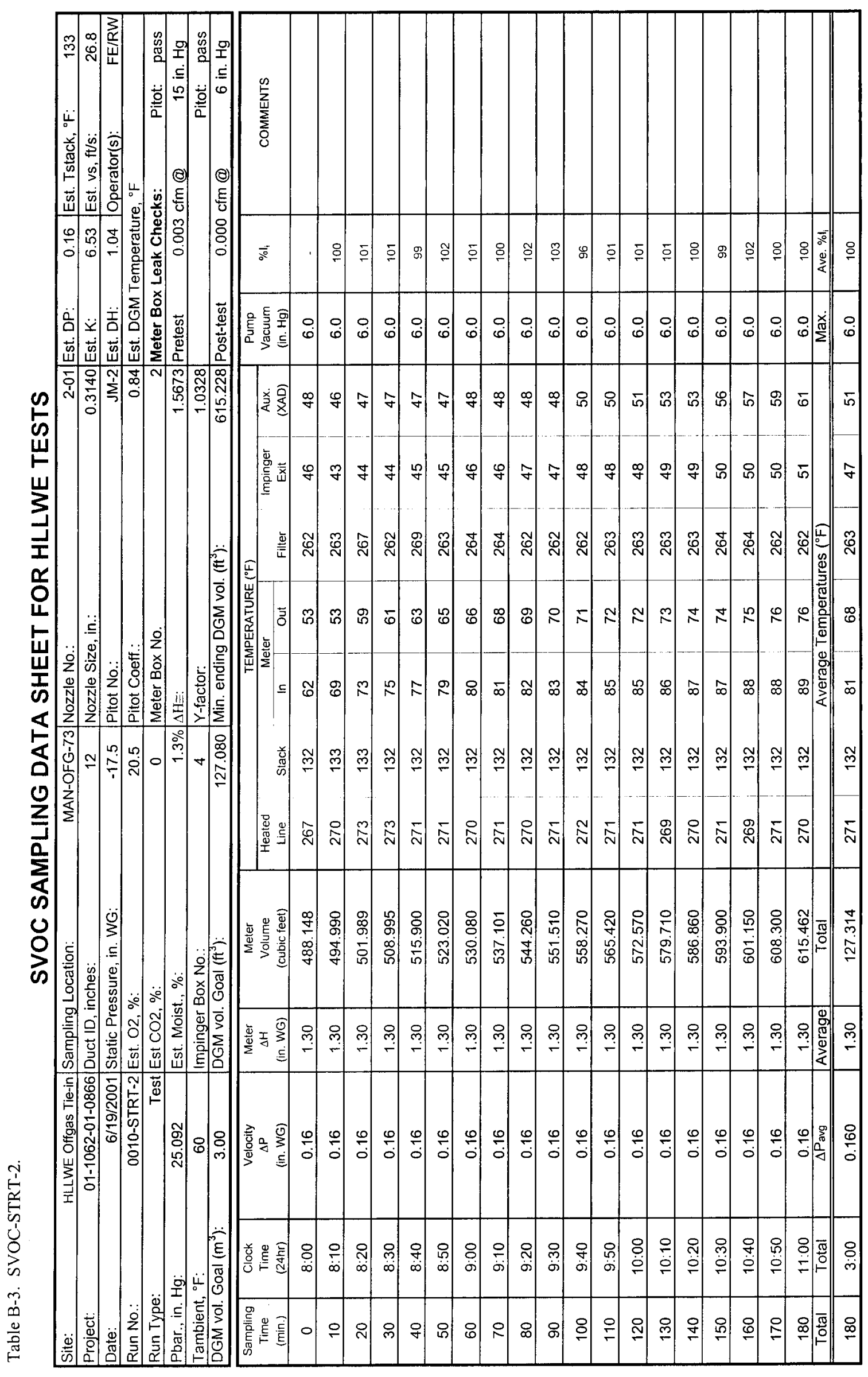




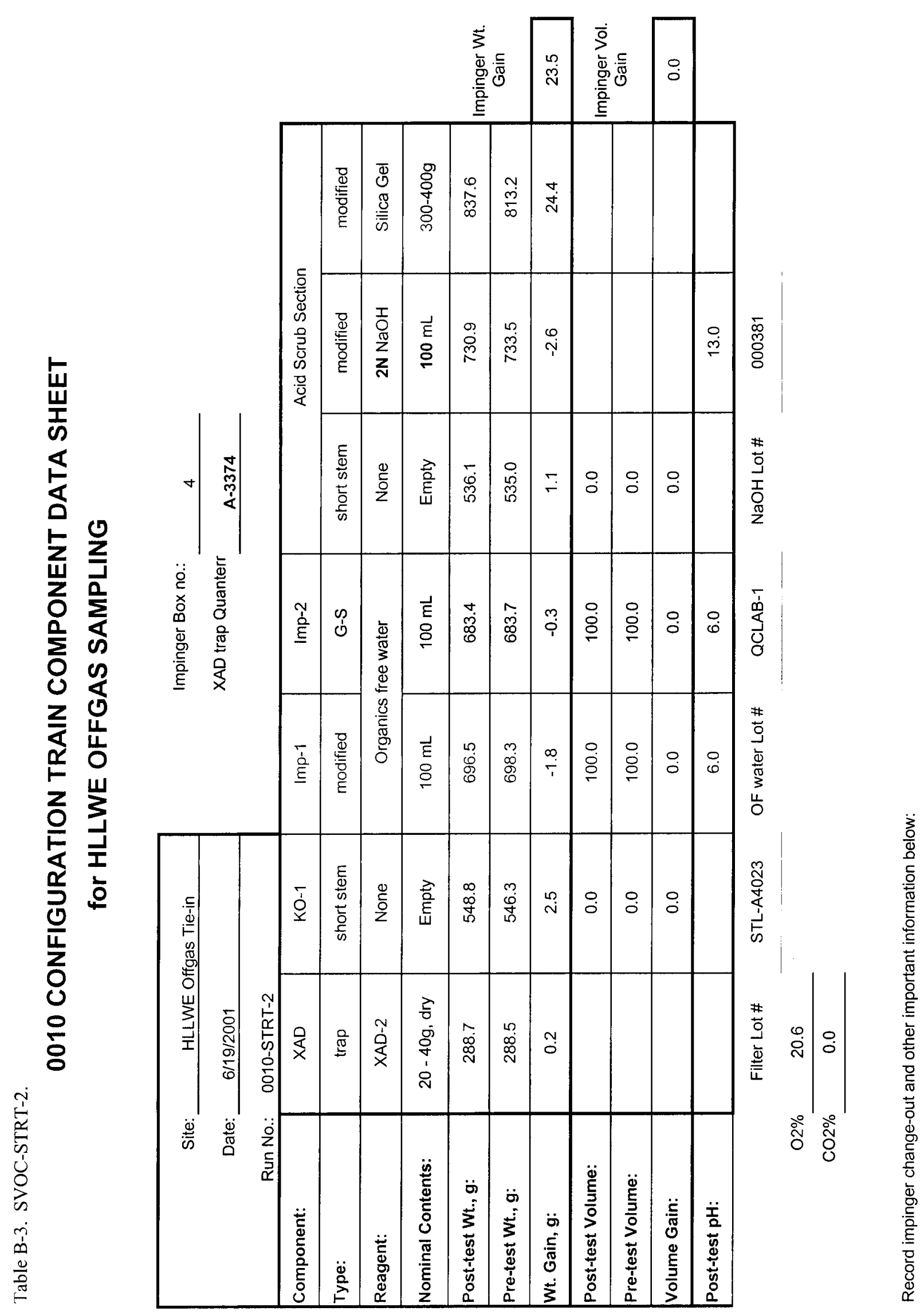


Table B-3. SVOC-STRT-2.

\section{FIELD DATA CALCULATIONS}

\begin{tabular}{|c|c|c|c|}
\hline $\begin{array}{r}\text { Project: } \\
\text { Run Date: } \\
\text { Run Identification: }\end{array}$ & $\begin{array}{r}01-1062 \\
6 / 19 / \\
0010-5\end{array}$ & $\begin{array}{l}01-0866 \\
001 \\
\text { TRT-2 }\end{array}$ & \\
\hline PARAMETER & SYMBOL & UNITS & \\
\hline Absolute Pressure in the Duct & Pabs & in. $\mathrm{Hg}$ & 23.805 \\
\hline Average Duct Gas Temperature & Ts & $\mathrm{R}$ & 592 \\
\hline Average Meter Temperature & $\mathrm{Tm}$ & $\mathrm{R}$ & 534 \\
\hline Average Gas Oxygen Content & $\mathrm{Co} 2, \mathrm{~m}$ & $\%$ & 20.6 \\
\hline Average Gas Carbon Dioxide Content & $\mathrm{Cco} 2, \mathrm{~m}$ & $\%$ & 0.0 \\
\hline Total Impinger Weight Gain (water) & Ww & grams & 23.5 \\
\hline Nozzle Area & $A n$ & $\mathrm{ft}^{2}$ & 0.000538 \\
\hline Duct Area & As & $\mathrm{ft}^{2}$ & 0.7854 \\
\hline Sample Volume & VmStd & dscf & 109.351 \\
\hline Sample Volume (SI) & VmStdm & dscm & 3.096 \\
\hline Average Sampling Rate & Qm & $\mathrm{dscf} / \mathrm{m}$ & 0.608 \\
\hline Volume of Water Vapor & VwStd & scf & 1.108 \\
\hline Volume of Water Vapor (SI) & VwStdm & $\mathrm{scm}$ & 0.0314 \\
\hline Moisture Fraction & Bws & - & 0.010 \\
\hline Dry Gas Molecular Weight & $\mathrm{Md}$ & $\mathrm{g} / \mathrm{g}-\mathrm{mol}$ & 28.82 \\
\hline Wet Gas Molecular Weight & Ms & $\mathrm{g} / \mathrm{g}-\mathrm{mol}$ & 28.72 \\
\hline Gas Velocity at Nozzle & $\mathrm{vn}$ & $\mathrm{ft} / \mathrm{s}$ & 26.7 \\
\hline Gas Velocity at Nozzle (SI) & $\mathrm{vnm}$ & $\mathrm{m} / \mathrm{s}$ & 8.15 \\
\hline Average Gas Velocity & vncor & $\mathrm{ft} / \mathrm{s}$ & 22.56 \\
\hline Dry Offgas Flow Rate & Qsd & $\mathrm{dscf} / \mathrm{h}$ & 44,791 \\
\hline Dry Offgas Flow Rate (SI) & Qsdm & $\mathrm{dscm} / \mathrm{h}$ & $1,268.3$ \\
\hline Actual Offgas Flow Rate & Q & acf $/ \mathrm{h}$ & 63,775 \\
\hline Intermediate Isokinetic Rate & li & $\%$ & 100.6 \\
\hline Final Isokinetic Rate & 1 & $\%$ & 100.3 \\
\hline
\end{tabular}

B3. 3 - 5 
Table B-3. SVOC-STRT-2.

\begin{tabular}{|c|c|c|c|c|c|c|c|c|c|c|c|c|}
\hline \multirow[t]{3}{*}{$\begin{array}{r}\text { Project: } \\
\text { Run Date: } \\
\text { Run Identification: } \\
\text { Run Type: } \\
\text { Lab Report Date: } \\
\text { Lab Report Status: (preliminary or } \\
\text { final) }\end{array}$} & \multicolumn{2}{|c|}{$\begin{array}{c}\text { 01-1062-01-0866 } \\
6 / 19 / 2001 \\
0010-S T R T-2 \\
\text { Test } \\
8 / 28 / 2001 \\
\text { Final }\end{array}$} & \multicolumn{10}{|c|}{$\begin{array}{l}\text { RESULTS } \\
\text { - without blank corrections } \\
\text { - final presentation should be rounded to two significant digits }\end{array}$} \\
\hline & \multicolumn{6}{|c|}{ CONCENTRATIONS } & \multicolumn{6}{|c|}{ MASS FLOW RATES } \\
\hline & \multicolumn{2}{|c|}{$\begin{array}{c}\text { Actual } \\
(\mu \mathrm{g} / \mathrm{acm})\end{array}$} & \multicolumn{2}{|c|}{$\begin{array}{l}\text { Standard } \\
(\mu \mathrm{g} / \mathrm{scm})\end{array}$} & \multicolumn{2}{|c|}{$\begin{array}{c}\text { Dry Standard } \\
(\mu \mathrm{g} / \mathrm{dscm})\end{array}$} & \multicolumn{2}{|c|}{$\mu \mathrm{g} / \mathrm{min}$} & \multicolumn{2}{|c|}{ grams/sec } & \multicolumn{2}{|c|}{$\mathrm{lb} / \mathrm{h}$} \\
\hline Acenaphthene & $<, J$ & $3.6 \mathrm{e} 0$ & $<, J$ & $5.1 \mathrm{e0}$ & $<, J$ & $5.2 \mathrm{e0}$ & $<, J$ & $1.1 \mathrm{e} 2$ & $<, J$ & $1.8 \mathrm{e}-6$ & $<, J$ & $1.4 e-5$ \\
\hline Acenaphthylene & $<, J$ & $3.6 \mathrm{e} 0$ & $<, J$ & $5.1 \mathrm{e} 0$ & $<, J$ & $5.2 \mathrm{e} 0$ & $<, J$ & $1.1 \mathrm{e} 2$ & $<, \sqrt{ }$ & $1.8 \mathrm{e}-6$ & $<, J$ & $1.4 \mathrm{e}-5$ \\
\hline Acetophenone & $<, J$ & $9.5 \mathrm{e} 0$ & $<, J$ & $1.3 e 1$ & $<, J$ & $1.4 \mathrm{e} 1$ & $<, \mathrm{J}$ & $2.9 \mathrm{e} 2$ & $<, J$ & $4.8 e-6$ & $<, J$ & $3.8 e-5$ \\
\hline Aniline & $<$ & $3.4 \mathrm{e} 1$ & $<$ & $4.8 e 1$ & $<$ & $4.8 \in 1$ & $<$ & $1.0 e 3$ & $<$ & $1.7 e-5$ & $<$ & $1.4 e-4$ \\
\hline Anthracene & $<, J$ & $3.6 \mathrm{e} 0$ & $<, J$ & $5.1 \mathrm{e} 0$ & $<, J$ & $5.2 \mathrm{e} 0$ & $<, J$ & $1.1 \mathrm{e} 2$ & $<, J$ & $1.8 e-6$ & $<, J$ & $1.4 \mathrm{e}-5$ \\
\hline Benzidine & $<$ & $1.8 \mathrm{e} 2$ & $<$ & $2.6 \mathrm{e} 2$ & $<$ & $2.6 \mathrm{e} 2$ & $<$ & $5.5 \mathrm{e} 3$ & $<$ & $9.1 e-5$ & $<$ & $7.2 e-4$ \\
\hline Benzoic acid & $E$ & $5.2 e 2$ & $E$ & $7.4 \mathrm{e} 2$ & $E$ & $7.4 \mathrm{e} 2$ & $E$ & $1.6 \mathrm{e} 4$ & E & $2.6 e-4$ & $E$ & $2.1 e-3$ \\
\hline Benzo(a)anthracene & $<, j$ & $4.3 \mathrm{e} 0$ & $<, J$ & $6.1 \mathrm{e} 0$ & $<J$ & $6.1 \mathrm{e} 0$ & $<, J$ & $1.3 \mathrm{e} 2$ & $<, J$ & $2.2 e-6$ & $<, J$ & $1.7 e-5$ \\
\hline Benzo(a)pyrene & $<, j$ & $3.2 \mathrm{e} 1$ & $<, J$ & $4.5 \mathrm{e} 1$ & $<, j$ & $4.5 \mathrm{e} 1$ & $<, j$ & $9.6 \mathrm{e} 2$ & $<, j$ & $1.6 \mathrm{e}-5$ & $<, J$ & $1.3 e-4$ \\
\hline Benzo(b)fluoranthene & $<, J$ & $6.8 \mathrm{e} 1$ & $<, J$ & $9.6 e 1$ & $<, J$ & $9.7 e 1$ & $<, J$ & $2.0 \mathrm{e} 3$ & $<, J$ & $3.4 e-5$ & $<, J$ & $2.7 e-4$ \\
\hline Benzo $(g, h, i)$ perylene & $<, J$ & $4.1 \mathrm{e} 1$ & $<, J$ & $5.8 \mathrm{e} 1$ & $<, d$ & $5.8 e 1$ & $<, \mathrm{J}$ & $1.2 \mathrm{e} 3$ & $<, J$ & $2.0 e-5$ & $<, J$ & $1.6 e-4$ \\
\hline Benzo(k)fluoranthene & $<, J$ & $9.8 \mathrm{e} 1$ & $<, J$ & $1.4 \mathrm{e} 2$ & $\leq J$ & $1.4 \mathrm{e} 2$ & $<, \mathrm{J}$ & $2.9 \mathrm{e} 3$ & $<, J$ & $4.9 \mathrm{e}-5$ & $<, J$ & $3.9 \mathrm{e}-4$ \\
\hline Benzyl alcohol & $<$ & $1.3 \mathrm{e} 2$ & $<$ & $1.8 \mathrm{e} 2$ & $<$ & $1.8 \mathrm{e} 2$ & $<$ & $3.9 \mathrm{e} 3$ & $<$ & $6.5 e-5$ & $<$ & $5.1 e-4$ \\
\hline bis(2-Chloroethoxy)methane & |< & $3.2 \mathrm{e} 0$ & $<$ & $4.5 \mathrm{e} 0$ & $<$ & $4.5 \mathrm{e} 0$ & $<$ & $9.6 \mathrm{e} 1$ & $<$ & $1.6 e-6$ & $<$ & $1.3 e-5$ \\
\hline bis(2-Chloroethyl)ether & $1<J$ & $3.6 \mathrm{eo}$ & $<, J$ & $5.1 \mathrm{e} 0$ & $<, \sqrt{ }$ & $5.2 \mathrm{e} 0$ & $<, \sqrt{ }$ & $1.1 \mathrm{e} 2$ & $<, J$ & $1.8 e-6$ & $<, J$ & $1.4 \mathrm{e}-5$ \\
\hline bis(2-Ethylhexyl)phthalate & $<, J$ & $2.9 e 1$ & $<, J$ & $4.2 \mathrm{e} 1$ & $<, J$ & $4.2 e 1$ & $<4$ & $8.9 \mathrm{e} 2$ & $<$ & $1.5 \mathrm{e}-5$ & $<, 3$ & $12 e-4$ \\
\hline 4-Bromophenyl-phenylether & $<, J$ & $4.1 \mathrm{e} 0$ & $<, J$ & $5.8 \mathrm{e} 0$ & $<, d$ & $5.8 \mathrm{e} 0$ & $<, \mathrm{J}$ & $1.2 \mathrm{e} 2$ & $<, J$ & $2.0 \mathrm{e}-\overline{6}$ & $<, J$ & $1.6 \mathrm{e}-5$ \\
\hline Butylbenzylphthalate & $<, J$ & $4.3 \mathrm{e} 0$ & $<, J$ & $6.1 \mathrm{e} 0$ & $<, J$ & $6.1 \mathrm{e} 0$ & $<, J$ & $1.3 \mathrm{e} 2$ & $<, J$ & $2.2 e-6$ & $<, J$ & $1.7 \mathrm{e}-5$ \\
\hline Carbazole & $<, J$ & $4.1 \mathrm{eo}$ & $<, J$ & $5.8 \mathrm{e} 0$ & $<, J$ & $5.8 \mathrm{e} 0$ & $<, J$ & $1.2 \mathrm{e} 2$ & $<, J$ & $2.0 e-6$ & $<, J$ & $1.6 \mathrm{e}-5$ \\
\hline 4-Chloro-3-methylphenol & $<, J$ & $7.0 \mathrm{e} 0$ & $<, J$ & $9.9 \mathrm{e} 0$ & $<, J$ & $1.0 \mathrm{e} 1$ & $<, J$ & $2.1 \mathrm{e} 2$ & $<, J$ & $3.5 e-6$ & $<, J$ & $2.8 e-5$ \\
\hline 4-Chloroaniline & $<$ & $2.5 \mathrm{e} 1$ & $<$ & $3.5 \mathrm{e} 1$ & $<$ & $3.6 \mathrm{e} 1$ & $<$ & $7.5 \mathrm{e} 2$ & $<$ & $1.3 e-5$ & $<$ & $9.9 e-5$ \\
\hline 2-Chloronaphthalene & $<, J$ & $3.4 \mathrm{eo}$ & $<, J$ & $4.8 \mathrm{e} 0$ & $<, J$ & $4.8 \mathrm{e} 0$ & $<, J$ & $1.0 \mathrm{e} 2$ & $<, J$ & $1.7 e-6$ & $<, J$ & $1.4 \mathrm{e}-5$ \\
\hline 2-Chlorophenol & $<, J$ & $3.4 \mathrm{e} 0$ & $<, J$ & $4.8 \mathrm{e} 0$ & $<, J$ & $4.8 \mathrm{e} 0$ & $<, J$ & $1.0 \mathrm{e} 2$ & $<, J$ & $1.7 e-6$ & $<, J$ & $1.4 \mathrm{e}-5$ \\
\hline 4-Chlorophenyl phenyl ether & $\leq \mathrm{J}$ & $3.9 \mathrm{e} 0$ & $s_{1} J$ & $5.4 \mathrm{e} 0$ & $<, \mathrm{J}$ & $5.5 \mathrm{e} 0$ & $<, J$ & $1.2 \mathrm{e} 2$ & $<, J$ & $1.9 e-6$ & $<, J$ & $1.5 \mathrm{e}-5$ \\
\hline Chrysene & $<, J$ & $4.8 \mathrm{e} 0$ & $<, J$ & $6.7 \mathrm{e} 0$ & $<, J$ & $6.8 \mathrm{e} 0$ & $<, J$ & $1.4 \mathrm{e} 2$ & $\overline{<, J}$ & $2.4 e-6$ & $<, J$ & $1.9 \mathrm{e}-5$ \\
\hline Di-n-buty|phthalate & $<, \mathrm{J}$ & $2.5 e 1$ & $<, J$ & $3.5 e 1$ & $<, \downarrow$ & $3.6 \mathrm{e} 1$ & $<, J$ & $7.5 \mathrm{e} 2$ & $<, J$ & $1.3 e-5$ & $<, J$ & $9.9 e-5$ \\
\hline Di-n-octylphthalate & $<, J$ & $3.6 \mathrm{e} 1$ & $<, J$ & $5.1 e 1$ & $<, J$ & $5.2 \mathrm{e} 1$ & $<, j$ & $1.1 \mathrm{e} 3$ & $<, J$ & $1.8 e-5$ & $<, J$ & $1.4 \mathrm{e}-4$ \\
\hline Dibenz $(a, h)$ anthracene & $<$ & $3.9 \mathrm{e} 1$ & $<, J$ & $5.4 e 1$ & $<, J$ & $5.5 \mathrm{e} 1$ & $<$. & $1.2 \mathrm{e} 3$ & $\leq-J$ & $1.9 e-5$ & $<, 3$ & $1.5 \mathrm{e}-4$ \\
\hline Dibenzofuran & $<, J$ & $3.9 \mathrm{e} 0$ & $\overline{<, J}$ & $5.4 € 0$ & $<, J$ & $5.5 \mathrm{e} 0$ & $<, j$ & $1.2 \mathrm{e} 2$ & $<, J$ & $1.9 e-6$ & $<, J$ & $1.5 \mathrm{e}-5$ \\
\hline 1,2-Dichlorobenzene & $<, J$ & $3.6 \mathrm{eo}$ & $<, J$ & $5.1 \mathrm{e} 0$ & $<, J$ & $5.2 \mathrm{e} 0$ & $<, J$ & $1.1 \mathrm{e} 2$ & $<, J$ & $1.8 e-6$ & $<, J$ & $1.4 \mathrm{e}-5$ \\
\hline 1,3-Dichlorobenzene & $<, J$ & $3.9 \mathrm{e} 0$ & $<, J$ & $5.4 \mathrm{e} 0$ & $<, \sqrt{ }$ & $5.5 \mathrm{e} 0$ & $<, J$ & $1.2 \mathrm{e} 2$ & $<, J$ & $1.9 e-6$ & $<, J$ & $1.5 e-5$ \\
\hline 1,4-Dichlorobenzene & $J$ & $4.5 \mathrm{e} 0$ & $J$ & $6.4 \mathrm{e} 0$ & $\mathrm{~J}$ & $6.5 \mathrm{e} 0$ & $\mathrm{~J}$ & $1.4 \mathrm{e} 2$ & $\mathrm{~J}$ & $2.3 e-6$ & J & $1.8 \mathrm{e}-5$ \\
\hline 3,3'-Dichlorobenzidine & $<$ & $2.9 \mathrm{e} 1$ & $<$ & $4.2 \mathrm{e} 1$ & $<$ & $4.2 \mathrm{e} 1$ & $<$ & $8.9 \mathrm{e} 2$ & $<$ & $1.5 \mathrm{e}-5$ & $<$ & $1.2 \mathrm{e}-4$ \\
\hline 2,4-Dichlorophenol & $<$ & $4.1 \mathrm{e} 0$ & $<$ & $5.8 e 0$ & $<$ & $5.8 \mathrm{e} 0$ & $<$ & $1.2 \mathrm{e} 2$ & $<$ & $2.0 e-6$ & $<$ & $1.6 \mathrm{e}-5$ \\
\hline Diethylphthalate & $<, J$ & $5.0 \mathrm{e} 0$ & $<, J$ & $7.0 \mathrm{e} 0$ & $<, J$ & $7.1 \mathrm{e} 0$ & $<, J$ & $1.5 \mathrm{e} 2$ & $<, J$ & $2.5 e-6$ & $<, J$ & $2.0 e-5$ \\
\hline Dimethyl phthalate & $\leq \leq J$ & $3.6 \mathrm{e} 0$ & $<, J$ & $5.1 \mathrm{e} 0$ & $<, \mathrm{J}$ & $5.2 \mathrm{e} 0$ & $\leq, J$ & $1.1 \mathrm{e} 2$ & $\leq, \mathrm{J}$ & $1.8 \mathrm{e}-6$ & $<, \mathrm{J}$ & $1.4 \mathrm{e}-5$ \\
\hline 2,4-Dimethyłphenol & $<$ & $1.4 \mathrm{e} 1$ & $<$ & $2.0 e 1$ & $<$ & $2.0 \mathrm{e} 1$ & $<$ & $4.2 \mathrm{e} 2$ & $<$ & $7.1 \mathrm{e}-6$ & $<$ & $5.6 e-5$ \\
\hline 4,6-Dinitro-2-methylphenol & $<$ & $3.2 \mathrm{e} 1$ & $<$ & $4.5 e 1$ & $<$ & $4.5 \mathrm{e} 1$ & $<$ & $9.6 e 2$ & $<$ & $1.6 e-5$ & $<$ & $1.3 e-4$ \\
\hline 2,4-Dinitrophenol & $<$ & $6.4 \mathrm{e} 1$ & $<$ & $9.0 \mathrm{e} 1$ & $<$ & $9.0 \mathrm{e} 1$ & $<$ & $1.9 \mathrm{e} 3$ & $<$ & $3.2 \mathrm{e}-5$ & $<$ & $2.5 e-4$ \\
\hline 2,4-Dinitrotoluene & $<, J$ & $4.3 \mathrm{e} 0$ & $<, J$ & $6.1 \mathrm{e} 0$ & $<, J$ & $6.1 \mathrm{eO}$ & $<, J$ & $1.3 e 2$ & $<, J$ & $2.2 \mathrm{e}-6$ & $<. J$ & $1.7 e-5$ \\
\hline 2,6-Dinitrotoluene & $<, J$ & $3.6 \mathrm{e} 0$ & $<, J$ & $5.1 \mathrm{e} 0$ & $<, J$ & 5.200 & $<, j$ & $1.1 \mathrm{e} 2$ & $<, j$ & $1.8 \mathrm{e}-6$ & $<, J$ & $1.4 \mathrm{e}-5$ \\
\hline 1,2-Diphenylhydrazine & $<, J$ & $3.6 \mathrm{e} 0$ & $<, J$ & $5.1 \mathrm{e} 0$ & $<, J$ & $5.2 \mathrm{eO}$ & $<, \sqrt{ }$ & $1.1 \mathrm{e} 2$ & $<, J$ & $1.8 \mathrm{e}-6$ & $<, J$ & $1.4 \mathrm{e}-5$ \\
\hline Fluoranthene & $<, J$ & $3.9 \mathrm{e} 0$ & $<, J$ & $5.4 \mathrm{e} 0$ & $<, J$ & $5.5 \mathrm{e} 0$ & $<, J$ & $1.2 \mathrm{e} 2$ & $<, J$ & $1.9 e-6$ & $<, J$ & $1.5 e-5$ \\
\hline Fluorene. & $<, J$ & $3.6 \mathrm{e} 0$ & $<, J$ & $5.1 \mathrm{e} 0$ & $<, J$ & $5.2 \mathrm{e} 0$ & $<, J$ & $1.1 e 2$ & $<, J$ & $1.8 \mathrm{e}-6$ & $<, \mathrm{J}$ & $1.4 e-5$ \\
\hline Hexachlorocyclopentadiene & $<$ & $4.1 e 1$ & $<$ & $5.8 \mathrm{e} 1$ & $<$ & $5.8 \mathrm{e} 1$ & $<$ & $1.2 \mathrm{e} 3$ & $<$ & $2.0 \mathrm{e}-5$ & $<$ & $1.6 e-4$ \\
\hline Hexachlorobenzene & $<, J$ & $3.6 \mathrm{e} 0$ & $<, J$ & $5.1 \mathrm{e} 0$ & $<, J$ & $5.2 e 0$ & $<, J$ & $1.1 \in 2$ & $<, J$ & $1.8 \mathrm{e}-6$ & $<, J$ & $1.4 e-5$ \\
\hline Hexachlorobutadiene & $<, J$ & $4.5 € 0$ & $<, J$ & $6.4 \mathrm{e} 0$ & $<, J$ & 6.500 & $<, J$ & $1.4 € 2$ & $<, j$ & $2.3 e-6$ & $<. J$ & $1.8 \mathrm{e}-5$ \\
\hline Hexachloroethane & $\leq, J$ & $4.5 \mathrm{e} 0$ & $<1$ & $6.4 \mathrm{e} 0$ & $<, 1$ & $6.5 \mathrm{e} 0$ & $<, J$ & $1.4 \mathrm{e} 2$ & $<J$ & $2.3 e-6$ & $<, J$ & $1.8 \mathrm{e}-5$ \\
\hline Indeno(1,2,3-cd)pyrene & $<, J$ & $3.4 \mathrm{e} 1$ & $<, j$ & $4.8 \mathrm{e} 1$ & $<, J$ & $4.8 \mathrm{e} 1$ & $<, J$ & $1.0 \mathrm{e} 3$ & $<, J$ & $1.7 e-5$ & $<, J$ & $1.4 \mathrm{e}-4$ \\
\hline Isophorone & $<, J$ & $3.6 \mathrm{e} 0$ & 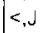 & $5.1 \mathrm{eo}$ & $<, J$ & $5.2 \mathrm{e} 0$ & $<, J$ & $1.1 \mathrm{e} 2$ & $<, J$ & $1.8 e-6$ & $<, J$ & $1.4 e-5$ \\
\hline 2-Methylnaphthalene & $<, J$ & $3.4 \mathrm{e} 0$ & $<, J$ & $4.8 \mathrm{e} 0$ & $<, J$ & $4.8 \mathrm{e} 0$ & $<, J$ & $1.0 \mathrm{e} 2$ & $<, J$ & $1.7 \mathrm{e}-6$ & $<, J$ & $1.4 e-5$ \\
\hline 2-Methylphenol & $<$ & $1.2 e 1$ & $<$ & $1.6 \mathrm{e} 1$ & $<$ & $1.6 \mathrm{e} 1$ & $<$ & $3.5 \mathrm{e} 2$ & $<$ & $5.8 \mathrm{e}-6$ & $<$ & $4.6 \mathrm{e}-5$ \\
\hline 3-Methylphenol \& 4-Methyiphenol & $<$ & $8.8 \mathrm{e} 0$ & $<$ & $1.2 \mathrm{e} 1$ & $<$ & $1.3 e 1$ & $<$ & $2.7 e 2$ & $<$ & $4.4 \mathrm{e}-6$ & $<$ & $3.5 e-5$ \\
\hline N-Nitroso-di-n-propylamine & $<$ & $3.4 \mathrm{e} 0$ & $<$ & $4.8 \mathrm{e} 0$ & $<$ & $4.8 \mathrm{e} 0$ & $<$ & $1.0 \mathrm{e} 2$ & $<$ & $1.7 \mathrm{e}-6$ & $<$ & $1.4 e-5$ \\
\hline $\mathrm{N}$-Nitrosodimethylamine & $<, J$ & $3.4 \mathrm{eo}$ & $<, J$ & $4.8 \mathrm{e} 0$ & $<, J$ & $4.8 \mathrm{eo}$ & $<, J$ & $1.0 \mathrm{e} 2$ & $<, J$ & $1.7 e-6$ & $<, J$ & $1.4 e-5$ \\
\hline N-Nitrosodiphenylamine. & $<, j$ & 4.800 & $\leq, j$ & $6.7 \mathrm{e} 0$ & $<, J$ & $6.8 e 0$ & $<, \mathrm{J}$ & $1.4 \mathrm{e}$ & $<, d$ & $2.4 e-6$ & $<, J$ & $1.9 e-5$ \\
\hline Naphthalene & $<, J$ & $3.9 \mathrm{e} 0$ & $<, J$ & $5.4 \mathrm{e} 0$ & $<, J$ & $5.5 \mathrm{e} 0$ & $<, J$ & $1.2 \mathrm{e} 2$ & $<, J$ & $1.9 e-6$ & $<, J$ & $1.5 \mathrm{e}-5$ \\
\hline 2-Nitroaniline & $<$ & $3.9 \mathrm{e0}$ & $<$ & $5.4 \mathrm{e} 0$ & $<$ & $5.5 e 0$ & $<$ & $1.2 \mathrm{e} 2$ & $<$ & $1.9 e-6$ & $<$ & $1.5 e-5$ \\
\hline 3-Nitroaniline & $<$ & $1.1 \mathrm{e} 1$ & $<$ & $1.6 \mathrm{e} 1$ & $<$ & $1.6 e 1$ & $<$ & $3.4 \mathrm{e} 2$ & $<$ & $5.7 e-6$ & $<$ & $4.5 e-5$ \\
\hline 4-Nitroaniline & $1<$ & $1.0 \mathrm{e} 1$ & $<$ & $1.4 \mathrm{e} 1$ & $<$ & $1.4 e 1$ & < & $3.0 \mathrm{e} 2$ & $<$ & $5.0 e-6$ & $1<$ & $4.0 e-5$ \\
\hline
\end{tabular}


Table B-3. SVOC-STRT-2.

\begin{tabular}{|c|c|c|c|c|c|c|c|c|c|c|c|c|}
\hline \multirow{3}{*}{$\begin{array}{r}\text { Project: } \\
\text { Run Date: } \\
\text { Run Identification: } \\
\text { Run Type: } \\
\text { Lab Report Date: } \\
\text { Lab Report Status: (preliminary or } \\
\text { final) } \\
\end{array}$} & \multicolumn{2}{|c|}{$\begin{array}{c}01-1062-01-0866 \\
6 / 19 / 2001 \\
0010-S T R T-2 \\
\text { Test } \\
8 / 28 / 2001 \\
\text { Final }\end{array}$} & & \multicolumn{9}{|c|}{$\begin{array}{l}\text { RESULTS } \\
\text { - without blank corrections } \\
\text { - final presentation should be rounded to two significant digits }\end{array}$} \\
\hline & \multicolumn{6}{|c|}{ CONCENTRATIONS } & \multicolumn{6}{|c|}{ MASS FLOW RATES } \\
\hline & \multicolumn{2}{|c|}{$\begin{array}{c}\text { Actual } \\
(\mu \mathrm{g} / \mathrm{acm})\end{array}$} & \multicolumn{2}{|c|}{$\begin{array}{l}\text { Standard } \\
(\mu \mathrm{g} / \mathrm{scm})\end{array}$} & \multicolumn{2}{|c|}{$\begin{array}{l}\text { Dry Standard } \\
(\mu \mathrm{g} / \mathrm{dscm})\end{array}$} & \multicolumn{2}{|c|}{$\mu \mathrm{g} / \mathrm{min}$} & \multicolumn{2}{|c|}{ grams/sec } & \multicolumn{2}{|c|}{$\mathrm{lb} / \mathrm{h}$} \\
\hline Nitrobenzene & $<, J$ & $4.5 \mathrm{e} 0$ & $<, J$ & $6.4 \mathrm{e} 0$ & $<, J$ & $6.5 \mathrm{e} 0$ & $<, J$ & $1.4 \mathrm{e} 2$ & $<, J$ & $2.3 e-6$ & $\overline{<, J}$ & $1.8 \mathrm{e}-5$ \\
\hline 2-Nitrophenol & $<, J$ & $8.4 \mathrm{e} 0$ & $<, J$ & $1.2 \mathrm{e} 1$ & $<, J$ & $1.2 \mathrm{e} 1$ & $<, J$ & $2.5 \mathrm{e} 2$ & $<, J$ & $4.2 e-6$ & $<, J$ & $3.3 e-5$ \\
\hline 4-Nitrophenol & $<$ & $1.4 \mathrm{e} 1$ & $<$ & $2.0 \mathrm{e} 1$ & $<$ & $2.0 \mathrm{e} 1$ & $<$ & $4.3 \mathrm{e} 2$ & $<$ & $7.2 e-6$ & $<$ & $5.7 e-5$ \\
\hline 2,2'-Oxybis (1-chloropropane) & $<, J$ & $5.0 \mathrm{e} 0$ & $<, J$ & $7.0 \mathrm{e} 0$ & $<, J$ & $7,1 \mathrm{eo}$ & $<, J$ & $1.5 \mathrm{e} 2$ & $\leq, J$ & $2.5 e-6$ & $\leq, J$ & $2.0 e-5$ \\
\hline Pentachiorobenzene & $<$ & $3.4 \mathrm{e} 0$ & $<$ & $4.8 \mathrm{e} 0$ & $<$ & $4.8 \mathrm{e} 0$ & $<$ & $1.0 \mathrm{e} 2$ & $<$ & $1.7 e-6$ & $<$ & $1.4 \mathrm{e}-5$ \\
\hline Pentachloronitrobenzene & $<$ & $3.6 \mathrm{e} 0$ & $<$ & $5.1 \mathrm{e} 0$ & $<$ & $5.2 \mathrm{e} 0$ & $<$ & $1.1 \mathrm{e} 2$ & $<$ & $1.8 e-6$ & $<$ & $1.4 e-5$ \\
\hline Pentachlorophenol & & $7.0 \mathrm{e} 1$ & $<$ & $9.9 \mathrm{e} 1$ & $<$ & $1.0 \mathrm{e} 2$ & $<$ & $2.1 \mathrm{e} 3$ & $<$ & $3.5 e-5$ & $<$ & $2.8 \mathrm{e}-4$ \\
\hline Phenanthrene & $<, J$ & $3.9 \mathrm{eo}$ & $<, J$ & $5.4 \mathrm{eo}$ & $<, J$ & $5.5 \mathrm{e} 0$ & $<, J$ & $1.2 \mathrm{e} 2$ & $<, J$ & $1.9 e-6$ & $<, J$ & $1.5 e-5$ \\
\hline Phenol & $<, J$ & $7.5 \mathrm{e} 0$ & $<, J$ & $1.1 \mathrm{e} 1$ & $<, J$ & $1.1 \mathrm{e} 1$ & $<, j$ & $2.3 \mathrm{e} 2$ & $<, J$ & $3.8 e-6$ & $<, J$ & $3.0 e-5$ \\
\hline Pyrene & $<, J$ & $4.1 \mathrm{e0}$ & $<, J$ & $5.8 \mathrm{eo}$ & $<, J$ & $5.8 \mathrm{e} 0$ & $<, J$ & $1.2 \mathrm{e} 2$ & $<, J$ & $2.0 e-6$ & $<, J$ & $1.6 e-5$ \\
\hline Pyridine & $<$ & $6.4 \mathrm{e} 0$ & $<$ & $9.0 \mathrm{e} 0$ & $<$ & $9.0 \mathrm{e} 0$ & $<$ & $1.9 \mathrm{e} 2$ & $<$ & $3.2 e-6$ & $<$ & $2.5 e-5$ \\
\hline 1,2,4,5-Tetrachlorobenzene & $<$ & $3.6 \mathrm{e} 0$ & $\leq$ & $5.1 \mathrm{e} 0$ & $<$ & $5.2 \mathrm{e} 0$ & $\leq$ & $1.1 \mathrm{e} 2$ & $\leq$ & $1.8 \mathrm{e}-6$ & $<$ & $1.4 e-5$ \\
\hline $1,2,4$-Trichlorobenzene & $<, J$ & $3.9 \mathrm{e} 0$ & $\overline{<, j}$ & $5.4 \mathrm{e} 0$ & $<, J$ & $5.5 \mathrm{e} 0$ & $<, J$ & $1.2 \mathrm{e} 2$ & $<, J$ & $1.9 \mathrm{e}-\overline{6}$ & $<, J$ & $1.5 e-5$ \\
\hline 2,4,5-Trichlorophenol & $<$ & $6.6 \mathrm{e} 0$ & $<$ & $9.3 \mathrm{eO}$ & $<$ & $9.4 \mathrm{e} 0$ & $<$ & $2.0 \mathrm{e} 2$ & $<$ & $3.3 e-6$ & $<$ & $2.6 e-5$ \\
\hline $\begin{array}{r}\text { 2,4,6-Trichlorophenol } \\
\text { TICs }\end{array}$ & $<$ & $4.8 \mathrm{eo}$ & $<$ & $6.7 \mathrm{eo}$ & $<$ & $6.8 \mathrm{e} 0$ & $<$ & $1.4 \mathrm{e} 2$ & $<$ & $2.4 e-6$ & $<$ & $1.9 e-5$ \\
\hline Furan, 2,5-dimethyl- & $\mathrm{N}, \mathrm{J}, \overline{\mathrm{M}}$ & $2.2 \mathrm{eo}$ & $N, J, M$ & $3.1 \mathrm{eo}$ & $\mathrm{N}, \mathrm{J}, \mathrm{M}$ & $3.2 \mathrm{e} 0$ & $\bar{N}, J, \bar{M}$ & $6.7 e 1$ & $\bar{N}, J, M$ & $1.1 e-6$ & N,J,M & $8.8 \mathrm{e}-6$ \\
\hline 3-Hexanone & $N, J, M$ & $2.2 \mathrm{e} 1$ & $N, J, M$ & $3.1 \mathrm{e} 1$ & $N, J, M$ & $3.1 \mathrm{e} 1$ & $N, J, M$ & $6.6 \mathrm{e} 2$ & $N, J, M$ & $1.1 e-5$ & $N, J, M$ & $8.7 e-5$ \\
\hline Heptane, 2,5-dimethyl- & N,J,M & $1.6 \mathrm{eo}$ & N,J,M & $2.3 \mathrm{eo}$ & $\mathrm{N}, \mathrm{J}, \mathrm{M}$ & $2.3 \mathrm{e} 0$ & $N, J, M$ & $4.8 \mathrm{e} 1$ & $\mathrm{~N}, \mathrm{~J}, \mathrm{M}$ & $8.1 \mathrm{e}-7$ & $\mathrm{~N}, \mathrm{~J}, \mathrm{M}$ & $6.4 \mathrm{e}-6$ \\
\hline Benzaldehyde & $\mathrm{N}, \mathrm{J}, \mathrm{M}$ & $1.5 \mathrm{e} 2$ & $N, J, M$ & 2.102 & $\mathrm{~N}, \mathrm{~J}, \mathrm{M}$ & $2.2 \mathrm{e} 2$ & $N, J, M$ & $4.6 \mathrm{e} 3$ & N,J,M & $7.6 e-5$ & $\mathrm{~N}, \mathrm{~J}, \mathrm{M}$ & $6.1 e-4$ \\
\hline Formic acid, phenylmethyl este & $N, J, M$ & $1.2 \mathrm{e} 1$ & $N, J, M$ & $1.7 \mathrm{e1}$ & $N, J, M$ & $1.7 \mathrm{e} 1$ & $N, J, M$ & $3.5 \mathrm{e} 2$ & $\mathrm{~N}, J, M$ & $5.9 e-6$ & $\mathrm{~N}, \mathrm{~J}, \mathrm{M}$ & $4.7 e-5$ \\
\hline Dodecane & $N, J, M$ & $1.2 \mathrm{e} 1$ & N,J,M & $1.8 \mathrm{e} 1$ & N,J,M & $1.8 \mathrm{e} 1$ & $N, J, M$ & $3.8 \mathrm{e} 2$ & $N, J, M$ & $6.3 e-6$ & $\mathrm{~N}, \mathrm{~J}, \mathrm{M}$ & $5.0 e-5$ \\
\hline Tridecane & $N, J, M$ & $4.1 \mathrm{eo}$ & $N, J, M$ & $5.8 \mathrm{eo}$ & $\mathrm{N}, \mathrm{J}, \mathrm{M}$ & $5.8 \mathrm{e} 0$ & $N, J, M$ & $1.2 \mathrm{e} 2$ & N,J,M & $2.0 e-6$ & $\mathrm{~N}, \mathrm{~J}, \mathrm{M}$ & $1.6 e-5$ \\
\hline Naphthalene, 1-methyl- & $N, J, Q$ & $1.8 \mathrm{eo}$ & $N, J, Q$ & $2.5 \mathrm{eo}$ & $N, J, Q$ & $2.5 \mathrm{e} 0$ & $N, J, Q$ & $5.3 e 1$ & $N, J, Q$ & $8.9 e-7$ & $N, J, Q$ & $7.0 \mathrm{e}-6$ \\
\hline Tetradecane & N,J,M & $1.3 e 1$ & $\mathrm{~N}, \mathrm{~J}, \mathrm{M}$ & $1.8 \mathrm{e} 1$ & $\mathrm{~N}, \mathrm{~J}, \mathrm{M}$ & $1.8 \mathrm{e} 1$ & $N, J, M$ & $3.8 \mathrm{e} 2$ & $N, J, M$ & $6.4 \mathrm{e}-6$ & $\mathrm{~N}, \mathrm{~J}, \mathrm{M}$ & $5.1 e-5$ \\
\hline Cyclododecane & $N, J, M$ & $1.3 \mathrm{e} 0$ & $N, J, M$ & $1.8 \mathrm{eo}$ & $\mathrm{N}, \mathrm{J}, \mathrm{M}$ & 1.800 & $\mathrm{~N}, \mathrm{~J}, \mathrm{M}$ & $3.8 \mathrm{e} 1$ & $\mathrm{~N}, J, M$ & $6.4 \mathrm{e}-7$ & $\mathrm{~N}, \mathrm{~J}, \mathrm{M}$ & $5.1 e-6$ \\
\hline Hexanedioic acid, bis(2-ethylh) & $N, J, Q$ & $2.3 \mathrm{e} 0$ & $N, J, Q$ & $3.2 \mathrm{eo}$ & $N, J, Q$ & $3.2 \mathrm{e} 0$ & $N, J, Q$ & $6.8 \mathrm{e} 1$ & $N, J, Q$ & $1.1 e-6$ & $N, J, Q$ & $9.0 e-6$ \\
\hline 1,2-Benzenedicarboxylic acid, & $N, J, Q$ & $1.9 \mathrm{eo}$ & $N, J, Q$ & $2.7 \mathrm{eo}$ & $N, J, Q$ & $2.7 \mathrm{e} 0$ & $N, J, Q$ & $5.7 \mathrm{e} 1$ & $N, J, Q$ & $9.6 \mathrm{e}-7$ & $\mathrm{~N}, \mathrm{~J}, \mathrm{Q}$ & $7.6 \mathrm{e}-6$ \\
\hline $\begin{array}{l}\text { Benzo(e)pyrene } \\
\text { Heptane, } 2,3 \text {-dimethyl- } \\
\text { Benzoic acid, methyl ester } \\
\text { Benzaldehyde, ethyl- }\end{array}$ & $N, J, Q$ & $3.2 \mathrm{eo}$ & $\mathrm{N}, \mathrm{J}, \mathrm{Q}$ & $4.5 \mathrm{eo}$ & $\bar{N}, J, Q$ & 4.500 & $\mathrm{~N}, \mathrm{~J}, \mathrm{Q}$ & $9.6 \mathrm{e} 1$ & $\mathrm{~N}, \mathrm{~J}, \mathrm{Q}$ & $1.6 e-6$ & $\mathrm{~N}, \mathrm{~J}, \mathrm{Q}$ & $1.3 e-5$ \\
\hline $\begin{array}{l}\text { Pentadecane } \\
\text { Heptadecane } \\
\text { Eicosane } \\
\text { Heneicosane }\end{array}$ & & & & & & & & & & & & \\
\hline $\begin{array}{l}\text { Octodecane } \\
\text { Phosphine oxide, triphenyl- } \\
\text { Nonacosane } \\
\text { Hexatriacontane }\end{array}$ & & & & & & & & & & & & \\
\hline $\begin{array}{l}\text { Tetracosane } \\
\text { Tetratriacontane }\end{array}$ & & & & & & & & & & & & \\
\hline
\end{tabular}





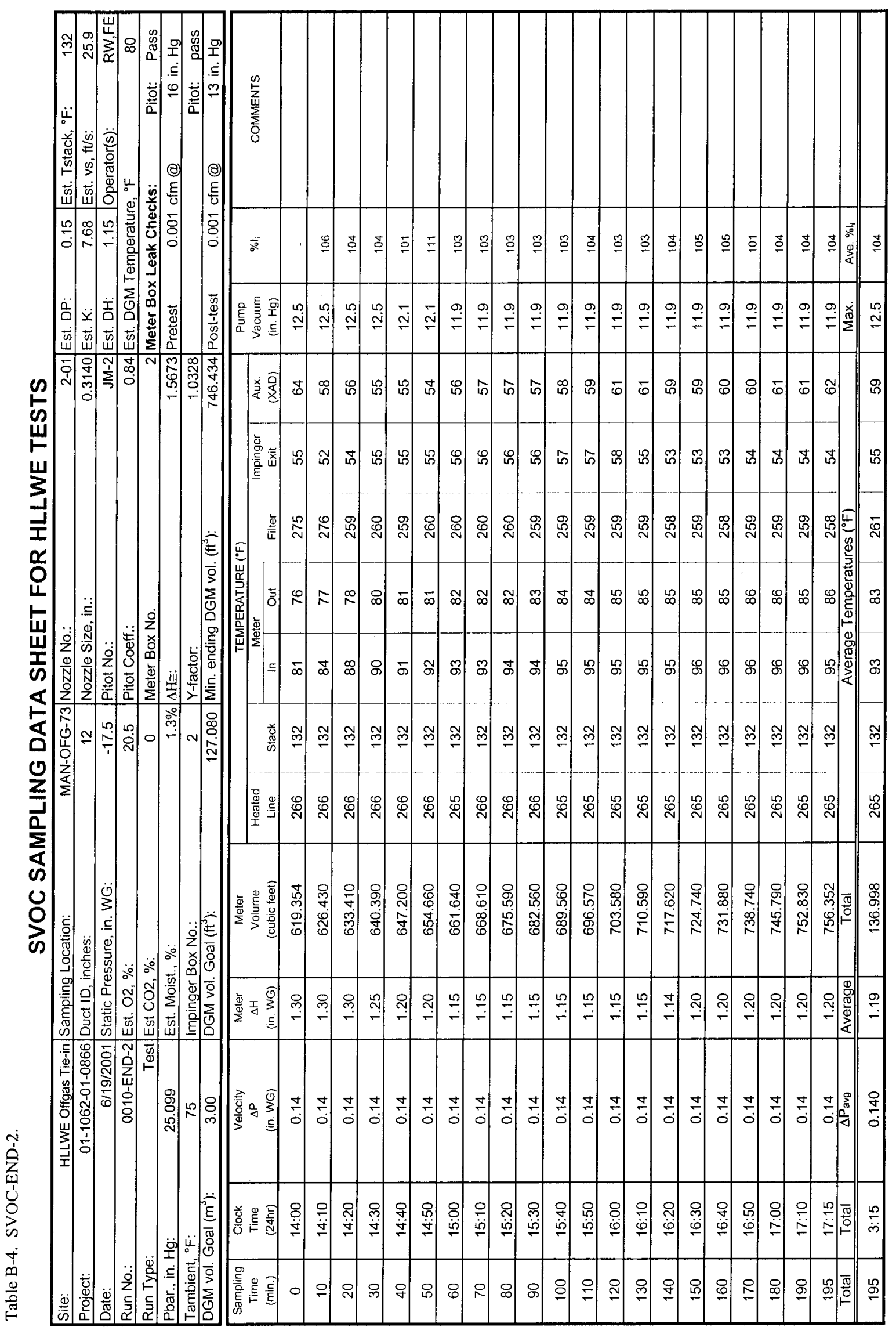




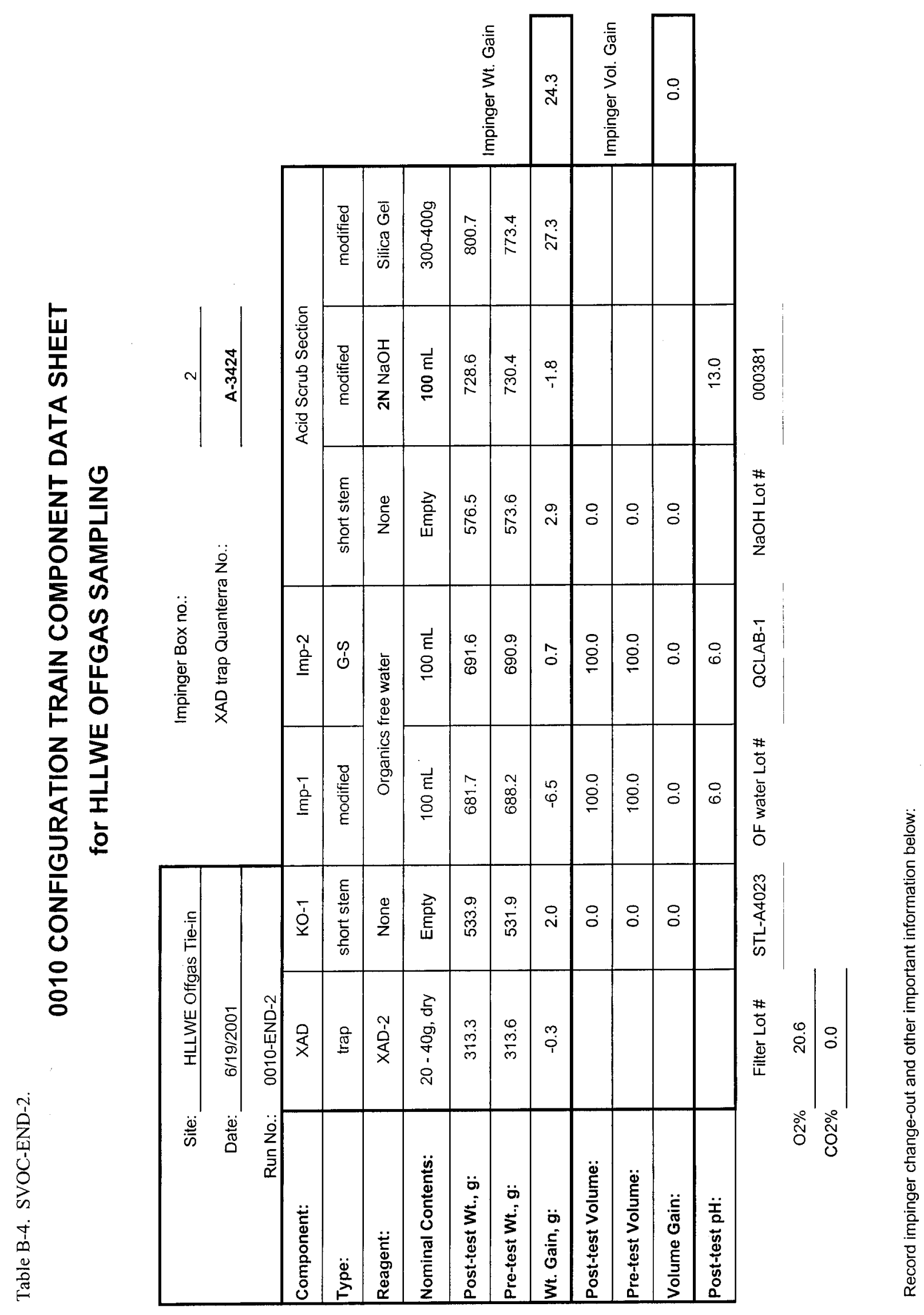


Table B-4. SVOC-END-2.

FIELD DATA CALCULATIONS

\begin{tabular}{|c|c|c|c|}
\hline $\begin{array}{r}\text { Project: } \\
\text { Run Date: } \\
\text { Run Identification: }\end{array}$ & $\begin{array}{r}01-1062-( \\
6 / 19 / 2 \\
\text { 0010-E } \\
\end{array}$ & $\begin{array}{l}01-0866 \\
001 \\
\text { ND-2 } \\
\end{array}$ & \\
\hline PARAMETER & SYMBOL & UNITS & \\
\hline Absolute Pressure in the Duct & Pabs & in. $\mathrm{Hg}$ & 23.812 \\
\hline Average Duct Gas Temperature & Ts & $\mathrm{R}$ & 592 \\
\hline Average Meter Temperature & $\mathrm{Tm}$ & $\mathrm{R}$ & 547 \\
\hline Average Gas Oxygen Content & $\mathrm{Co} 2, \mathrm{~m}$ & $\%$ & 20.6 \\
\hline Average Gas Carbon Dioxide Content & $\mathrm{Cco} 2, \mathrm{~m}$ & $\%$ & 0.0 \\
\hline Total Impinger Weight Gain (water) & Ww & grams & 24.3 \\
\hline Nozzle Area & An & $\mathrm{ft}^{2}$ & 0.000538 \\
\hline Duct Area & As & $\mathrm{ft}^{2}$ & 0.7854 \\
\hline Sample Volume & VmStd & dscf & 114.799 \\
\hline Sample Volume (SI) & VmStdm & $\mathrm{dscm}$ & 3.251 \\
\hline Average Sampling Rate & Qm & dscf/m & 0.589 \\
\hline Volume of Water Vapor & VwStd & scf & 1.146 \\
\hline Volume of Water Vapor (SI) & VwStdm & $\mathrm{scm}$ & 0.0324 \\
\hline Moisture Fraction & Bws & - & 0.010 \\
\hline Dry Gas Molecular Weight & Md & $\mathrm{g} / \mathrm{g}-\mathrm{mol}$ & 28.82 \\
\hline Wet Gas Molecular Weight & Ms & $\mathrm{g} / \mathrm{g}-\mathrm{mol}$ & 28.72 \\
\hline Gas Velocity at Nozzle & vn & $\mathrm{ft} / \mathrm{s}$ & 25.0 \\
\hline Gas Velocity at Nozzle (SI) & vnm & $\mathrm{m} / \mathrm{s}$ & 7.62 \\
\hline Average Gas Velocity & vncor & $\mathrm{ft} / \mathrm{s}$ & 21.09 \\
\hline Dry Offgas Flow Rate & Qsd & $\mathrm{dscf} / \mathrm{h}$ & 41,913 \\
\hline Dry Offgas Flow Rate (SI) & Qsdm & $\mathrm{dscm} / \mathrm{h}$ & $1,186.9$ \\
\hline Actual Offgas Flow Rate & Q & acf $/ \mathrm{h}$ & 59,641 \\
\hline Intermediate Isokinetic Rate & li & $\%$ & 104.2 \\
\hline Final Isokinetic Rate & 1 & $\%$ & 103.9 \\
\hline
\end{tabular}


Table B-4. SVOC-END-2

\begin{tabular}{|c|c|c|c|c|c|c|c|c|c|c|c|c|}
\hline \multirow[t]{3}{*}{$\begin{array}{r}\text { Project: } \\
\text { Run Date: } \\
\text { Run Identification: } \\
\text { Run Type: } \\
\text { Lab Report Date: } \\
\text { Lab Report Status: (preliminary or } \\
\text { final) } \\
\end{array}$} & \multicolumn{2}{|c|}{$\begin{array}{c}\text { 01-1062-01-0866 } \\
\text { 6/19/2001 } \\
\text { 0010-END-2 } \\
\text { Test } \\
\text { 8/28/2001 } \\
\text { Final }\end{array}$} & \multicolumn{10}{|c|}{$\begin{array}{l}\text { RESULTS } \\
\text { - without blank corrections } \\
\text { - final presentation should be rounded to two significant digits }\end{array}$} \\
\hline & \multicolumn{6}{|c|}{ CONCENTRATIONS } & \multicolumn{6}{|c|}{ MASS FLOW RATES } \\
\hline & \multicolumn{2}{|c|}{$\begin{array}{c}\text { Actual } \\
(\mu \mathrm{g} / \mathrm{acm})\end{array}$} & \multicolumn{2}{|c|}{$\begin{array}{l}\text { Standard } \\
(\mu \mathrm{g} / \mathrm{scm})\end{array}$} & \multicolumn{2}{|c|}{$\begin{array}{c}\text { Dry Standard } \\
(\mu \mathrm{g} / \mathrm{dscm})\end{array}$} & \multicolumn{2}{|c|}{$\mu \mathrm{g} / \mathrm{min}$} & \multicolumn{2}{|c|}{ grams/sec } & \multicolumn{2}{|c|}{$\mathrm{lb} / \mathrm{h}$} \\
\hline Acenaphthene & $<$ & $2.1 \mathrm{e0}$ & $<$ & $3.0 e 0$ & $<$ & $3.0 \mathrm{e} 0$ & $<$ & $6.0 \mathrm{e} 1$ & $<$ & $9.9 \mathrm{e}-7$ & $<$ & $7.9 e-6$ \\
\hline Acenaphthylene & $<$ & $2.1 \mathrm{e} 0$ & $<$ & $2.9 e 0$ & $<$ & $2.9 \mathrm{e} 0$ & $<$ & $5.8 \mathrm{e} 1$ & $<$ & $9.6 e-7$ & $<$ & $7.6 \mathrm{e}-6$ \\
\hline Acetophenone & $<, J$ & $8.0 \mathrm{e} 0$ & $<, J$ & $1.1 \mathrm{e} 1$ & $<, \mathrm{J}$ & $1.1 e^{1}$ & $<, J$ & $2.3 \mathrm{e} 2$ & $<, J$ & $3.8 \mathrm{e}-6$ & $<, J$ & $3.0 \mathrm{e}-5$ \\
\hline Aniline & $<$ & $2.4 \mathrm{e} 1$ & $<$ & $3.4 \mathrm{e} 1$ & $<$ & $3.4 \mathrm{e} 1$ & $<$ & $6.7 \mathrm{e} 2$ & $<$ & $1.1 \mathrm{e}-5$ & $<$ & $8.9 e-5$ \\
\hline Anthracene & $<$ & 2.100 & $<$ & $2.9 e 0$ & $<$ & $2.9 \mathrm{e} 0$ & $<$ & $5.8 \mathrm{e} 1$ & $<$ & $9.6 e-7$ & $<$ & $7.6 \mathrm{e}-6$ \\
\hline Benzidine & $<$ & $1.4 \mathrm{e} 2$ & $<$ & $2.0 e 2$ & $<$ & $2.1 \mathrm{e} 2$ & $<$ & $4.1 \mathrm{e} 3$ & $<$ & $6.8 e-5$ & $<$ & $5.4 e-4$ \\
\hline Benzoic acid & E & $5.8 \mathrm{e} 2$ & E & $8.2 \mathrm{e} 2$ & E & $8.3 \mathrm{e} 2$ & E & $1.6 \mathrm{e} 4$ & E & $2.7 e-4$ & $E$ & $2.2 e-3$ \\
\hline Benzo(a)anthracene & $<$ & $2.6 \mathrm{e} 0$ & $<$ & $3.7 \mathrm{eo}$ & $<$ & $3.7 \mathrm{e} 0$ & $<$ & $7.3 \mathrm{e} 1$ & $<$ & $1.2 e-6$ & $<$ & $9.7 \mathrm{e}-6$ \\
\hline Benzo(a)pyrene & $<$ & $2.8 \mathrm{e} 1$ & $<$ & $4.0 \mathrm{e} 1$ & $<$ & $4.0 \mathrm{e} 1$ & $<$ & $7.9 \mathrm{e} 2$ & $<$ & $1.3 e-5$ & $<$ & $1.0 \mathrm{e}-4$ \\
\hline Benzo(b)fluoranthene & $<$ & $6.5 \mathrm{e} 1$ & $<$ & $9.1 \mathrm{e} 1$ & $<$ & $9.2 e 1$ & $<$ & $1.8 \mathrm{e} 3$ & $<$ & $3.0 e-5$ & $<$ & $2.4 \mathrm{e}-4$ \\
\hline Benzo(g,h,i)perylene & $<$ & $3.7 \mathrm{e} 1$ & $<$ & $5.2 \mathrm{e} 1$ & $<$ & $5.2 \mathrm{e} 1$ & $<$ & $1.0 \mathrm{e} 3$ & $<$ & $1.7 \mathrm{e}-5$ & $<$ & $1.4 \mathrm{e}-4$ \\
\hline Benzo(k)fluoranthene & $<$ & $9.3 e 1$ & $<$ & $1.3 \mathrm{e} 2$ & $<$ & $1.3 \mathrm{e} 2$ & $<$ & $2.6 \mathrm{e} 3$ & $<$ & $4.4 e-5$ & $<$ & $3.5 \mathrm{e}-4$ \\
\hline Benzyl alcohol & $<$ & $1.2 \mathrm{e} 2$ & $<$ & $1.7 \mathrm{e} 2$ & $<$ & $1.8 \mathrm{e} 2$ & $<$ & $3.5 \mathrm{e} 3$ & $<$ & $5.8 \mathrm{e}-5$ & $<$ & $4.6 e-4$ \\
\hline bis(2-Chloroethoxy)methane & $<$ & $2.2 \mathrm{e} 0$ & $<$ & $3.0 \mathrm{e} 0$ & $<$ & $3.1 \mathrm{e} 0$ & $<$ & $6.1 \mathrm{e} 1$ & $<$ & $1.0 e-6$ & $<$ & $8.0 \mathrm{e}-6$ \\
\hline bis(2-Chloroethyl)ether & $<$ & $2.4 \mathrm{e} 0$ & $<$ & $3.4 \mathrm{e} 0$ & $<$ & $3.4 \mathrm{e} 0$ & $<$ & $6.7 \mathrm{e} 1$ & $<$ & $1.1 \mathrm{e}-6$ & $<$ & $8.9 e-6$ \\
\hline bis(2-Ethy|hexyl)phthalate & $<, J$ & $3.5 \mathrm{e} 1$ & $<, \mathrm{J}$ & $4.9 \mathrm{e} 1$ & $<, \mathrm{J}$ & $4.9 e 1$ & $<, \mathrm{J}$ & $9.7 e 2$ & $<, \mathrm{J}$ & $1.6 \mathrm{e}-5$ & $<, J$ & $1.3 e-4$ \\
\hline 4-Bromophenyl-phenylether & $<$ & $2.0 \mathrm{e} 0$ & $<$ & $2.9 \mathrm{e} 0$ & $<$ & $2.9 \mathrm{e} 0$ & $<$ & $5.7 \mathrm{e} 1$ & $<$ & $9.5 \mathrm{e}-7$ & $<$ & $7.6 e-6$ \\
\hline Butylbenzylphthalate & $<$ & $2.8 \mathrm{e} 0$ & $<$ & $4.0 \mathrm{e} 0$ & $<$ & $4.0 \mathrm{e} 0$ & $<$ & $7.9 \mathrm{e} 1$ & $<$ & $1.3 e-6$ & $<$ & $1.0 e-5$ \\
\hline Carbazole & $<$ & $2.8 \mathrm{e} 0$ & $<$ & $4.0 \mathrm{e} 0$ & $<$ & $4.0 \mathrm{e} 0$ & $<$ & $7.9 \mathrm{e} 1$ & $<$ & $1.3 e-6$ & $<$ & $1.0 e-5$ \\
\hline 4-Chloro-3-methylphenol & $<$ & $3.7 \mathrm{e} 0$ & $<$ & $5.2 \mathrm{eO}$ & $<$ & $5.2 \mathrm{e} 0$ & $<$ & $1.0 \mathrm{e} 2$ & $<$ & $1.7 e-6$ & $<$ & $1.4 e-5$ \\
\hline 4-Chloroaniline & $<$ & $1.9 \mathrm{e} 1$ & $<$ & $2.7 \mathrm{e} 1$ & $<$ & $2.8 \mathrm{e} 1$ & $<$ & $5.5 e 2$ & $<$ & $9.1 \mathrm{e}-6$ & $<$ & $7.2 e-5$ \\
\hline 2-Chloronaphthalene & $<$ & $2.0 \mathrm{e} 0$ & $<$ & $2.8 \mathrm{e} 0$ & $<$ & $2.9 e 0$ & $<$ & $5.7 \mathrm{e} 1$ & $<$ & $9.4 \mathrm{e}-7$ & $<$ & $7.5 e-6$ \\
\hline 2-Chlorophenol & $<$ & $2.4 \mathrm{e} 0$ & $<$ & $3.4 \mathrm{eO}$ & $<$ & $3.4 \mathrm{eo}$ & $<$ & $6.7 \mathrm{e} 1$ & $<$ & $1.1 \mathrm{e}-6$ & $<$ & $8.9 e-6$ \\
\hline 4-Chlorophenyl phenyl ether & $<$ & $2.4 \mathrm{e} 0$ & $<$ & $3.4 \mathrm{eO}$ & $<$ & $3.4 \mathrm{eO}$ & $<$ & $6.7 \mathrm{e} 1$ & $<$ & $1.1 \mathrm{e}-6$ & $<$ & $8.9 e-6$ \\
\hline Chrysene & $<$ & $2.6 \mathrm{e} 0$ & $<$ & $3.7 \mathrm{eo}$ & $<$ & $3.7 \mathrm{eo}$ & $<$ & $7.3 \mathrm{e} 1$ & $<$ & $1.2 \mathrm{e}-6$ & $<$ & $9.7 e-6$ \\
\hline Di-n-butylphthalate & $<, J$ & $2.2 \mathrm{e} 1$ & $<, J$ & $3.0 \mathrm{e} 1$ & $<, J$ & $3.1 \mathrm{e} 1$ & $<, J$ & $6.1 \mathrm{e} 2$ & $<, J$ & $1.0 \mathrm{e}-5$ & $<, J$ & $8.0 \mathrm{e}-5$ \\
\hline Di-n-octylphthalate & $<, J$ & $3.5 \mathrm{e} 1$ & $<, J$ & $4.9 \mathrm{e} 1$ & $<, J$ & $4.9 \mathrm{e} 1$ & $<, J$ & $9.7 e 2$ & $<, J$ & $1.6 \mathrm{e}-5$ & $<, J$ & $1.3 e-4$ \\
\hline Dibenz $(a, h)$ anthracene & $<$ & $3.7 \mathrm{e} 1$ & $<$ & $5.2 \mathrm{e} 1$ & $<$ & $5.2 \mathrm{e} 1$ & $<$ & $1.0 \mathrm{e} 3$ & $<$ & $1.7 e-5$ & $<$ & $1.4 \mathrm{e}-4$ \\
\hline Dibenzofuran & $<$ & $2.4 \mathrm{e} 0$ & $<$ & $3.4 \mathrm{e} 0$ & $<$ & $3.4 \mathrm{eO}$ & $<$ & $6.7 \mathrm{e} 1$ & $<$ & $1.1 \mathrm{e}-6$ & $<$ & $8.9 \mathrm{e}-6$ \\
\hline 1,2-Dichlorobenzene & $<$ & $2.4 \mathrm{e} 0$ & $\mid<$ & $3.4 \mathrm{e} 0$ & $<$ & $3.4 \mathrm{e} 0$ & $<$ & $6.7 \mathrm{e} 1$ & $<$ & $1.1 \mathrm{e}-6$ & $\mid<$ & $8.9 \mathrm{e}-6$ \\
\hline 1,3-Dichlorobenzene & $<$ & $2.6 \mathrm{e} 0$ & $<$ & $3.7 \mathrm{e} 0$ & $<$ & $3.7 \mathrm{e} 0$ & $<$ & $7.3 e 1$ & $<$ & $1.2 \mathrm{e}-6$ & $<$ & $9.7 e-6$ \\
\hline 1,4-Dichlorober & $<, \mathrm{J}$ & $4.5 \mathrm{e} 0$ & $<, J$ & $6.4 \mathrm{e} 0$ & $<, \mathrm{J}$ & $6.5 \mathrm{e} 0$ & $<, J$ & $1.3 e 2$ & $<, J$ & 2.1e-6 & $<, J$ & $1.7 \mathrm{e}-5$ \\
\hline 3,3'-Dichlorobenzidine & $<$ & $2.4 \mathrm{e} 1$ & $<$ & $3.4 \mathrm{e} 1$ & $<$ & $3.4 \mathrm{e} 1$ & $<$ & $6.7 \mathrm{e} 2$ & $<$ & $1.1 \mathrm{e}-5$ & $<$ & $8.9 e-5$ \\
\hline 2,4-Dichlorophenol & $<$ & $2.8 \mathrm{e} 0$ & $<$ & 4.000 & $<$ & $4.0 \mathrm{e} 0$ & $<$ & $7.9 \mathrm{e} 1$ & $<$ & $1.3 e-6$ & $<$ & $1.0 \mathrm{e}-5$ \\
\hline Diethylphthalate & $<$ & $3.2 \mathrm{e} 0$ & $<$ & $4.6 \mathrm{e} 0$ & $<$ & $4.6 \mathrm{e} 0$ & $<$ & $9.1 \mathrm{e} 1$ & $<$ & $1.5 e-6$ & i & $1.2 \mathrm{e}-5$ \\
\hline hyl phthalate & $<$ & $2.1 \mathrm{e} 0$ & $<$ & $2.9 \mathrm{e} 0$ & $<$ & $3.0 \mathrm{e} 0$ & $<$ & $5.8 \mathrm{e} 1$ & $<$ & $9.7 e-7$ & $<$ & $7.7 e-6$ \\
\hline 2,4-Dimethylphenol & $<$ & $1.3 e 1$ & $<$ & $1.8 \mathrm{e} 1$ & $<$ & $1.8 \mathrm{e} 1$ & $<$ & $3.6 \mathrm{e} 2$ & $<$ & $6.0 \mathrm{e}-6$ & $<$ & $4.7 e-5$ \\
\hline 4,6-Dinitro-2-methylphenol & $<$ & $2.8 e 1$ & $\mid<$ & $4.0 \mathrm{e} 1$ & $<$ & $4.0 \mathrm{e} 1$ & $<$ & $7.9 e 2$ & $<$ & $1.3 e-5$ & $<$ & $1.0 \mathrm{e}-4$ \\
\hline 2,4-Dinitrophenol & $<$ & $5.8 \mathrm{e} 1$ & $<$ & $8.2 \mathrm{e} 1$ & $<$ & $8.3 e 1$ & $<$ & $1.6 \mathrm{e} 3$ & $<$ & $2.7 e-5$ & $<$ & $2.2 \mathrm{e}-4$ \\
\hline initrotoluene & $<$ & $2.8 \mathrm{e} 0$ & $<<$ & 4.000 & $<$ & $4.0 \mathrm{e} 0$ & $<$ & $7.9 \mathrm{e} 1$ & $<$ & $1.3 \mathrm{e}-6$ & $<$ & $1.0 \mathrm{e}-5$ \\
\hline 2,6-Dinitrotoluene & $<$ & $2.6 \mathrm{e} 0$ & $\mid<$ & $3.7 \mathrm{e} 0$ & $<$ & $3.7 \mathrm{e} 0$ & $<$ & $7.3 \mathrm{e} 1$ & $<$ & $1.2 e-6$ & $<$ & $9.7 e-6$ \\
\hline 1,2-Diphenylhydrazine & $<$ & $2.1 \mathrm{e} 0$ & $<<$ & $3.0 \mathrm{e} 0$ & $<$ & $3.0 \mathrm{e} 0$ & $<$ & $6.0 \mathrm{e} 1$ & $<$ & $9.9 e-7$ & $<$ & $7.9 e-6$ \\
\hline Fluoranthene & $<$ & $2.2 \mathrm{e} 0$ & $<<$ & $3.0 \mathrm{e} 0$ & $<$ & $3.1 \mathrm{e} 0$ & $<$ & $6.1 \mathrm{e} 1$ & $<$ & $1.0 \mathrm{e}-6$ & $<$ & $8.0 \mathrm{e}-6$ \\
\hline Fluorene & $<$ & $2.2 \mathrm{e} 0$ & $<$ & $3.0 \mathrm{e} 0$ & $<$ & $3.1 \mathrm{e} 0$ & $<$ & $6.1 \mathrm{e} 1$ & $<$ & $1.0 e-6$ & $<$ & $8.0 \mathrm{e}-6$ \\
\hline Hexachlorocyclopentadiene & $<$ & $3.5 \mathrm{e} 1$ & $<$ & $4.9 \mathrm{e} 1$ & $<$ & $4.9 \mathrm{e} 1$ & $<$ & $9.7 \mathrm{e} 2$ & $<$ & $1.6 e-5$ & $<$ & $1.3 \mathrm{e}-4$ \\
\hline Hexachlorobenzene & $<$ & $2.4 \mathrm{e} 0$ & $<<$ & $3.4 \mathrm{e} 0$ & $<$ & $3.4 \mathrm{eO}$ & $<$ & $6.7 e 1$ & $<$ & $1.1 \mathrm{e}-6$ & $<$ & $8.9 e-6$ \\
\hline Hexachlorobutadiene & $<$ & $3.2 \mathrm{e} 0$ & $<$ & $4.6 \mathrm{e} 0$ & $<$ & $4.6 \mathrm{e} 0$ & $<$ & $9.1 \mathrm{e} 1$ & $<$ & $1.5 e-6$ & $<$ & $1.2 \mathrm{e}-5$ \\
\hline Hexachloroethane & $<$ & $3.5 \mathrm{e} 0$ & $<<$ & $4.9 \mathrm{e} 0$ & $<$ & $4.9 \mathrm{e} 0$ & $<$ & $9.7 \mathrm{e} 1$ & $<$ & $1.6 e-6$ & $<$ & $1.3 e-5$ \\
\hline Indeno(1,2,3-cd)pyrene & $<$ & $3.2 \mathrm{e} 1$ & $<<$ & $4.6 \mathrm{e} 1$ & $<$ & $4.6 \mathrm{e} 1$ & $<$ & $9.1 \mathrm{e} 2$ & $<$ & $1.5 e-5$ & $<$ & $1.2 e-4$ \\
\hline Isophorone & $<$ & $2.2 \mathrm{e} 0$ & $<$ & $3.0 \mathrm{e} 0$ & $<$ & $3.1 \mathrm{e} 0$ & $<$ & $6.1 \mathrm{e} 1$ & $\mid<$ & $1.0 e-6$ & $<$ & $8.0 \mathrm{e}-6$ \\
\hline 2-Methylnaphthalene & $<$ & $2.2 \mathrm{e} 0$ & $<$ & $3.0 e 0$ & $<$ & $3.1 \mathrm{e} 0$ & $<$ & $6.1 \mathrm{e} 1$ & $<$ & $1.0 \mathrm{e}-6$ & $<$ & $8.0 \mathrm{e}-6$ \\
\hline 2-Methylphenol & $<$ & $1.0 \mathrm{e} 1$ & $1<<$ & $1.4 \mathrm{e} 1$ & $\mid<$ & $1.4 \mathrm{e} 1$ & $k$ & $2.9 \mathrm{e} 2$ & $<$ & $4.8 e-6$ & $\leq$ & $3.8 \mathrm{e}-5$ \\
\hline
\end{tabular}


Table B-4. SVOC-END-2.

\begin{tabular}{|c|c|c|c|c|c|c|c|c|c|c|c|c|}
\hline \multirow[t]{3}{*}{$\begin{array}{r}\text { Project: } \\
\text { Run Date: } \\
\text { Run Identification: } \\
\text { Run Type: } \\
\text { Lab Report Date: } \\
\text { Lab Report Status: (preliminary or } \\
\text { final) } \\
\end{array}$} & \multicolumn{2}{|c|}{$\begin{array}{c}\text { 01-1062-01-0866 } \\
\text { 6/19/2001 } \\
\text { 0010-END-2 } \\
\text { Test } \\
\text { 8/28/2001 } \\
\text { Final }\end{array}$} & \multicolumn{10}{|c|}{$\begin{array}{l}\text { RESULTS } \\
\text { - without blank corrections } \\
\text { - final presentation should be rounded to two significant digits }\end{array}$} \\
\hline & \multicolumn{6}{|c|}{ CONCENTRATIONS } & \multicolumn{6}{|c|}{ MASS FLOW RATES } \\
\hline & \multicolumn{2}{|c|}{$\begin{array}{c}\text { Actual } \\
(\mu \mathrm{g} / \mathrm{acm})\end{array}$} & \multicolumn{2}{|c|}{$\begin{array}{l}\text { Standard } \\
(\mu \mathrm{g} / \mathrm{scm})\end{array}$} & \multicolumn{2}{|c|}{$\begin{array}{c}\text { Dry Standard } \\
(\mu \mathrm{g} / \mathrm{dscm})\end{array}$} & \multicolumn{2}{|c|}{$\mu \mathrm{g} / \mathrm{min}$} & \multicolumn{2}{|c|}{ grams/sec } & \multicolumn{2}{|c|}{$\mathrm{lb} / \mathrm{h}$} \\
\hline 3-Methylphenol \& 4-Methylphenol & $<$ & $7.4 \mathrm{e} 0$ & $<$ & $1.0 \mathrm{e} 1$ & $<$ & $1.0 \mathrm{e} 1$ & $<$ & $2.1 \mathrm{e2}$ & $<$ & $3.4 \mathrm{e}-6$ & $<$ & $2.7 e-5$ \\
\hline N-Nitroso-di-n-propylamine & $<$ & $2.4 \mathrm{e} 0$ & $<$ & $3.4 \mathrm{e} 0$ & $<$ & $3.4 \mathrm{eo}$ & $<$ & $6.7 \mathrm{e} 1$ & $<$ & $1.1 e-6$ & $<$ & $8.9 \mathrm{e}-6$ \\
\hline N-Nitrosodimethylamine & $<$ & $2.4 \mathrm{e} 0$ & $<$ & $3.4 \mathrm{eo}$ & $<$ & $3.4 \mathrm{e} 0$ & $<$ & $6.7 \mathrm{e} 1$ & $<$ & $1.1 e-6$ & $<$ & $8.9 e-6$ \\
\hline N-Nitrosodiphenylamine & $<$ & $3.2 \mathrm{e} 0$ & $<$ & $4.6 \mathrm{e} 0$ & $<$ & $4.6 \mathrm{e} 0$ & $<$ & $9.1 \mathrm{e} 1$ & $<$ & $1.5 e-6$ & $<$ & $1.2 e-5$ \\
\hline Naphthalene & $<$ & $2.4 \mathrm{e} 0$ & $<$ & $3.4 \mathrm{eo}$ & $<$ & $3.4 \mathrm{eO}$ & $<$ & $6.7 \mathrm{e} 1$ & $<$ & $1.1 \mathrm{e}-6$ & $<$ & $8.9 e-6$ \\
\hline 2-Nitroaniline & $<$ & $2.4 \mathrm{e} 0$ & $<$ & $3.4 \mathrm{eo}$ & $<$ & $3.4 \mathrm{e} 0$ & $<$ & $6.7 e 1$ & $<$ & $1,1 e-6$ & $<$ & $8.9 e-6$ \\
\hline 3-Nitroaniline & $<$ & $8.9 \mathrm{e} 0$ & $<$ & $1.2 \mathrm{e} 1$ & $<$ & $1.3 \mathrm{e} 1$ & $<$ & $2.5 \mathrm{e} 2$ & $<$ & $4.2 e-6$ & $<$ & $3.3 e-5$ \\
\hline 4-Nitroaniline & $<$ & $7.8 \mathrm{e} 0$ & $<$ & $1.1 \mathrm{e} 1$ & $<$ & $1.1 \mathrm{e} 1$ & $<$ & $2.2 \mathrm{e} 2$ & $<$ & $3.7 e-6$ & $<$ & $2.9 e-5$ \\
\hline Nitrobenzene & $<, J$ & $2.6 \mathrm{e} 0$ & $<, J$ & $3.7 e 0$ & $<, j$ & $3.7 \mathrm{e} 0$ & $<, \mathrm{J}$ & $7.3 \mathrm{e} 1$ & $<, J$ & $1.2 \mathrm{e}-6$ & $<, j$ & $9.7 e-6$ \\
\hline 2-Nitrophenol & $<, J$ & $1.1 \mathrm{e} 1$ & $<, J$ & $1.6 \mathrm{e} 1$ & $<, J$ & $1.6 \mathrm{e} 1$ & $<, \mathrm{J}$ & $3.1 \mathrm{e} 2$ & $<, J$ & $5.2 \mathrm{e}-6$ & $<, J$ & $4.1 e-5$ \\
\hline 4-Nitrophenol & $<, J$ & $1.2 \mathrm{e} 1$ & $<, J$ & $1.7 \mathrm{e} 1$ & $<, \mathrm{J}$ & $1.7 e 1$ & $<, J$ & $3.3 e 2$ & $<, J$ & $5.6 e-6$ & $<, J$ & $4.4 e-5$ \\
\hline 2,2'-Oxybis(1-chloropropane) & $<$ & $3.0 \mathrm{e} 0$ & $<$ & $4.3 \mathrm{eo}$ & $<$ & $4.3 \mathrm{e} 0$ & $<$ & $8.5 e 1$ & $\leq$ & $1.4 \mathrm{e}-6$ & $<$ & $1.1 e-5$ \\
\hline Pentachlorobenzene & $<$ & $\overline{2.2} \overline{\mathrm{e} 0}$ & $<$ & $3.0 \mathrm{e} 0$ & $<$ & $3.1 \mathrm{e} 0$ & $<$ & $6.1 \mathrm{e1}$ & $<$ & $1.0 \mathrm{e}-6$ & $<$ & $8.0 \mathrm{e}-6$ \\
\hline Pentachloronitrobenzene & $<$ & $2.4 \mathrm{e} 0$ & $<$ & $3.4 \mathrm{e} 0$ & $<$ & $3.4 \mathrm{e} 0$ & $<$ & $6.7 e 1$ & $<$ & $1.1 e-6$ & $<$ & $8.9 e-6$ \\
\hline Pentachlorophenol & $<$ & $8.0 \mathrm{e} 1$ & $<$ & $1.1 \mathrm{e} 2$ & $<$ & $1.1 \mathrm{e} 2$ & $<$ & $2.3 \mathrm{e} 3$ & $<$ & $3.8 e-5$ & $<$ & $3.0 \mathrm{e}-4$ \\
\hline Phenanthrene & $<$ & $2.1 \mathrm{e} 0$ & $<$ & $3.0 \mathrm{e} 0$ & $<$ & $3.0 \mathrm{e} 0$ & $<$ & $6.0 \mathrm{e} 1$ & $<$ & $9.9 \mathrm{e}-7$ & $<$ & $7.9 e-6$ \\
\hline Phenol & $<, J$ & $8.4 \mathrm{e} 0$ & $<, J$ & $1.2 \mathrm{e} 1$ & $<, J$ & $1.2 \mathrm{e} 1$ & $<, J$ & $2.4 \mathrm{e} 2$ & $<, \mathrm{J}$ & $4.0 \mathrm{e}-6$ & $<, J$ & $3.1 \mathrm{e}-5$ \\
\hline Pyrene & $<$ & $2.2 \mathrm{e} 0$ & $<$ & $3.0 \mathrm{e} 0$ & $<$ & $3.1 \mathrm{e} 0$ & $<$ & $6.1 \mathrm{e} 1$ & $<$ & $1.0 e-6$ & $<$ & $8.0 \mathrm{e}-6$ \\
\hline Pyridine & $<$ & $3.7 \mathrm{e} 0$ & $<$ & $5.2 \mathrm{e} 0$ & $<$ & $5.2 \mathrm{e} 0$ & $<$ & $1.0 \mathrm{e} 2$ & $<$ & $1.7 e-6$ & $<$ & $1.4 \mathrm{e}-5$ \\
\hline 1,2,4,5-Tetrachlorobenzene & $<$ & $2.4 \mathrm{e} 0$ & $<$ & $3.4 \mathrm{eo}$ & $<$ & $3.4 \mathrm{e} 0$ & $<$ & $6.7 \mathrm{e} 1$ & $<$ & $1.1 \mathrm{e}-6$ & $<$ & $8.9 \mathrm{e}-6$ \\
\hline 1,2,4-Trichlorobenzene & $<$ & $2.6 \mathrm{e} 0$ & $<$ & $3.7 \mathrm{eo}$ & $<$ & $3.7 \mathrm{e} 0$ & $<$ & $7.3 \mathrm{e} 1$ & $<$ & $1.2 \mathrm{e}-6$ & $<$ & $9.7 e-6$ \\
\hline 2,4,5-Trichlorophenol & $<$ & $5.4 \mathrm{e} 0$ & $<$ & $7.6 \mathrm{e} 0$ & $<$ & $7.7 \mathrm{e} 0$ & $<$ & $1.5 \mathrm{e} 2$ & $<$ & $2.5 e-6$ & $<$ & $2.0 \mathrm{e}-5$ \\
\hline $\begin{array}{r}2,4,6 \text {-Trichlorophenol } \\
\text { TICs }\end{array}$ & $<$ & $3.5 \mathrm{e} 0$ & $<$ & $4.9 \mathrm{e} 0$ & $<$ & $4.9 \mathrm{e} 0$ & $<$ & $9.7 \mathrm{e} 1$ & $<$ & $1.6 e-6$ & $<$ & $1.3 e-5$ \\
\hline 3-Hexanone & $\bar{N}, J_{1}$ & $1.5 \mathrm{e} 1$ & $\mathrm{~N}, \mathrm{~J}$ & $2.1 \mathrm{e} 1$ & $\bar{N}, \bar{J}$ & $2.2 \mathrm{e} 1$ & $\bar{N}, \mathrm{~J}$ & $4.3 \mathrm{e} 2$ & $\mathrm{~N}, \mathrm{~J}$ & $7.1 \mathrm{e}-6$ & N,J & $5.6 e-5$ \\
\hline Benzaldehyde & $\mathrm{N}, \mathrm{J}$ & $1.6 \mathrm{e} 2$ & $\mathrm{~N}, \mathrm{~J}$ & $2.2 e 2$ & $\mathrm{~N}, \mathrm{~J}$ & $2.2 \mathrm{e} 2$ & $\mathrm{~N}, \mathrm{~J}$ & $4.4 \mathrm{e} 3$ & $\mathrm{~N}, \mathrm{~J}$ & $7.4 \mathrm{e}-5$ & N.J & $5.9 e-4$ \\
\hline 2-Cyclohexene-1-one, 3-methyl- & $\mathrm{N}, \mathrm{J}$ & $6.9 e-1$ & $\mathrm{~N}, \mathrm{~J}$ & $9.7 e-1$ & $N, J$ & $9.8 \mathrm{e}-1$ & N,J & $1.9 \mathrm{e} 1$ & $\mathrm{~N}, \mathrm{~J}$ & $3.2 \mathrm{e}-7$ & $N, J$ & $2.6 e-6$ \\
\hline Formic acid, phenylmethyl ester & $\mathrm{N}, \mathrm{J}_{1}$ & $2.1 \mathrm{e} 1$ & $\mathrm{~N}_{1} \mathrm{~J}$ & $2.9 \mathrm{e} 1$ & $\mathrm{~N}, \mathrm{~J}$ & $2.9 \mathrm{e} 1$ & $\mathrm{~N}, \mathrm{~J}$ & $5.8 \mathrm{e} 2$ & $N, J$ & $9.6 \mathrm{e}-6$ & $N, J$ & $7.6 e-5$ \\
\hline Benzaldehyde, ethyl- & $\mathrm{N}, \mathrm{J}$ & $1.3 \mathrm{e} 1$ & $N_{1} J$ & $1.9 \mathrm{e} 1$ & $\mathrm{~N}, \mathrm{~J}$ & $1.9 \mathrm{e} 1$ & $\mathrm{~N}, \mathrm{~J}$ & $3.7 \mathrm{e} 2$ & $\mathrm{~N}, \mathrm{~J}$ & $6.2 \mathrm{e}-6$ & $\bar{N}, J$ & $4.9 e-5$ \\
\hline Dodecane & $\mathrm{N}, \mathrm{J}$ & $8.0 \mathrm{e} 0$ & $N_{1} J$ & $1.1 \mathrm{e} 1$ & $N, J$ & $1.1 \mathrm{e} 1$ & $\mathrm{~N}, \mathrm{~J}$ & $2.3 e 2$ & $N, J$ & $3.8 \mathrm{e}-6$ & $N, J$ & $3.0 \mathrm{e}-5$ \\
\hline Tridecane & $\mathrm{N}, \mathrm{J}$ & $4.5 \mathrm{e} 0$ & $\mathrm{~N}, \mathrm{~J}$ & $6.4 \mathrm{eo}$ & $\mathrm{N}, \mathrm{J}$ & $6.5 \mathrm{e} 0$ & $\mathrm{~N}, \mathrm{~J}$ & $1.3 \mathrm{e} 2$ & $\mathrm{~N}, \mathrm{~J}$ & $2.1 e-6$ & N.J & $1.7 e-5$ \\
\hline 2,4-Hexadiene & $N, J_{1}$ & $6.1 \mathrm{e} 0$ & $\mathrm{~N}, \mathrm{~J}$ & $8.5 \mathrm{eo}$ & $N, J$ & $8.6 \mathrm{e} 0$ & N,J & $1.7 \mathrm{e} 2$ & $\mathrm{~N}, \mathrm{~J}$ & $2.8 \mathrm{e}-6$ & $N, J$ & $2.3 e-5$ \\
\hline 2,5-Diethylphenol & $\bar{N}, \mathrm{~J}$, & $2.2 \mathrm{e} 1$ & $\mathbf{N}, \mathrm{J}$ & $3.0 \mathrm{e} 1$ & $\mathrm{~N}, \mathrm{~J}$ & $3.1 \mathrm{e} 1$ & $\bar{N}, \mathrm{~J}$ & $6.1 \mathrm{e} 2$ & $\mathrm{~N}, \mathrm{~J}$ & $1.0 \mathrm{e}-5$ & $N, J$ & $8.0 \mathrm{e}-5$ \\
\hline Tetradecane & $\mathrm{N}, \mathrm{J}$ & $2.1 \mathrm{e} 1$ & $\mathrm{~N}, \mathrm{~J}$ & $3.0 \mathrm{e} 1$ & $\mathrm{~N}, \mathrm{~J}$ & $3.0 \mathrm{e} 1$ & $\mathrm{~N}, \mathrm{~J}$ & $6.0 \mathrm{e} 2$ & $\mathrm{~N}, \mathrm{~J}$ & $1.0 \mathrm{e}-5$ & $N, J$ & $8.0 e-5$ \\
\hline Hexatriacontane & $N_{1} J$ & $1.2 \mathrm{e} 0$ & $\mathrm{~N}, \mathrm{~J}$ & $1.7 \mathrm{e} 0$ & $N, J$ & $1.8 \mathrm{e} 0$ & $\mathrm{~N}, \mathrm{~J}$ & $3.5 \mathrm{e} 1$ & $\mathrm{~N}, \mathrm{~J}$ & $5.8 \mathrm{e}-7$ & N,J & $4.6 e-6$ \\
\hline Phosphoric acid tributyl ester & $N, J$ & $5.2 \mathrm{e} 0$ & $\mathrm{~N}, \mathrm{~J}$ & $7.3 \mathrm{eo}$ & $N, j$ & $7.4 \mathrm{e} 0$ & $N_{1} J$ & $1.5 \mathrm{e} 2$ & N.J & $2.4 e-6$ & $N, J$ & $1.9 e-5$ \\
\hline Cyclododecane & $\mathrm{N}, \mathrm{J}$ & $2.8 \mathrm{e} 0$ & $\mathrm{~N}, \mathrm{~J}$ & $4.0 \mathrm{e} 0$ & $N, J$ & $4.0 \mathrm{e} 0$ & $\mathrm{~N}, \mathrm{~J}$ & $7.9 \mathrm{e} 1$ & $\mathrm{~N}, \mathrm{~J}$ & $1.3 \mathrm{e}-6$ & $\bar{N}, J$ & $1.0 \mathrm{e}-5$ \\
\hline Pentadecane & $N_{1} J$ & $9.7 e-1$ & N.J & $1.4 \mathrm{eo}$ & $N, J$ & $1.4 \mathrm{e} 0$ & $N, J$ & $2.7 \mathrm{e} 1$ & $N, J$ & $4.6 e-7$ & $N, J$ & $3.6 \mathrm{e}-6$ \\
\hline Heneicosane & $N, J$ & $2.0 \mathrm{e} 0$ & $\mathrm{~N}, \mathrm{~J}$ & $2.8 \mathrm{e} 0$ & $N, J$ & $2.8 \mathrm{e} 0$ & $N, J$ & $5.5 \mathrm{e} 1$ & $N, J$ & $9.2 \mathrm{e}-7$ & $N, J$ & $7.3 e-6$ \\
\hline Tetracosane & $\mathrm{N}, \mathrm{J}$ & $5.6 \mathrm{e} 0$ & $N, J$ & $7.9 \mathrm{eo}$ & $N, J$ & 8.000 & $N, J$ & $1.6 \mathrm{e} 2$ & $N, J$ & $2.6 \mathrm{e}-6$ & $N, \mathrm{~J}$ & $2.1 e-5$ \\
\hline Pentacosane & $\mathrm{N}, \mathrm{J}$ & $8.0 \mathrm{e} 0$ & $\mathrm{~N}, \mathrm{~J}$ & $1.1 \mathrm{e} 1$ & $N, J$ & $1.1 \mathrm{e} 1$ & $N, J$ & $2.3 \mathrm{e} 2$ & $N, j$ & $3.8 \mathrm{e}-6$ & $\bar{N}, \mathrm{~J}$ & $3.0 \mathrm{e}-5$ \\
\hline Hexacosane & $N, J$ & $1.4 \mathrm{e} 1$ & $\mathrm{~N}, \mathrm{~J}$ & $1.9 \mathrm{e} 1$ & $N, J$ & 2.001 & $N, J$ & $3.9 \mathrm{e} 2$ & $N, J$ & $6.5 e-6$ & $N, J$ & $5.2 \mathrm{e}-5$ \\
\hline Heptacosane & $\mathrm{N}, \mathrm{J}$ & $1.8 \mathrm{e} 1$ & $\mathrm{~N}, \mathrm{~J}$ & $2.5 \mathrm{e} 1$ & $N, J$ & $2.6 \mathrm{e} 1$ & N.J & $5.1 \mathrm{e} 2$ & $\mathrm{~N}, \mathrm{~J}$ & $8.4 \mathrm{e}-6$ & $\mathrm{~N}, \mathrm{~J}$ & $6.7 e-5$ \\
\hline Hexatriacontane & & $4.3 \mathrm{e} 1$ & $\mathrm{~N}, \mathrm{~J}$ & $6.1 \mathrm{e} 1$ & $N, J$ & $6.2 \mathrm{e} 1$ & N,J & $1.2 \mathrm{e} 3$ & $\mathrm{~N}, \mathrm{~J}$ & $2.0 \mathrm{e}-5$ & $\mathrm{~N}, \mathrm{~J}$ & $1.6 \mathrm{e}-4$ \\
\hline $\begin{array}{l}\text { Eicosane } \\
\text { Furan, 2,5-dimethyl- } \\
\text { Heptane, 2,5-dimethyl- } \\
\text { Heptane, 2,3-dimethyl- }\end{array}$ & $\bar{N}, \mathrm{~J}$ & $4.1 \mathrm{eo}$ & $\mathrm{N}, \mathrm{J}$ & $5.8 \mathrm{e} 0$ & $N, J$ & $5.8 \mathrm{e} 0$ & $\bar{N}, \mathrm{~J}$ & $1.2 \mathrm{e} 2$ & $N, J$ & $1.9 e-6$ & $\mathrm{~N}, \mathrm{~J}$ & $1.5 \mathrm{e}-5$ \\
\hline $\begin{array}{l}\text { Benzoic acid, methyl ester } \\
\text { Heptadecane } \\
\text { Octodecane } \\
\text { Phosphine oxide, triphenyl- }\end{array}$ & & & & & & & & & & & & \\
\hline $\begin{array}{l}\text { Nonacosane } \\
\text { Tetratriacontane }\end{array}$ & & & & & & & & & & & & \\
\hline
\end{tabular}





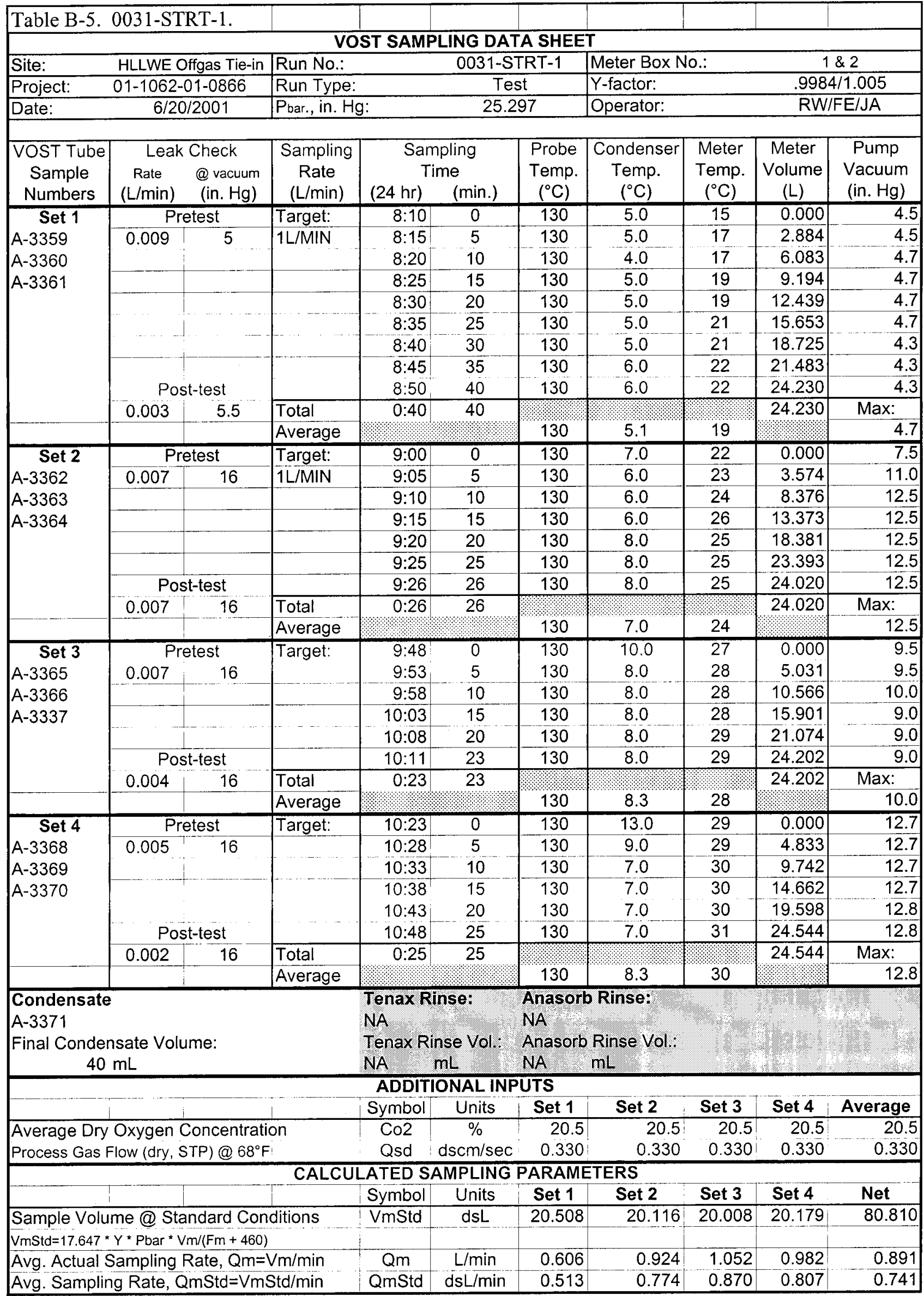

B5. 1 - 5 


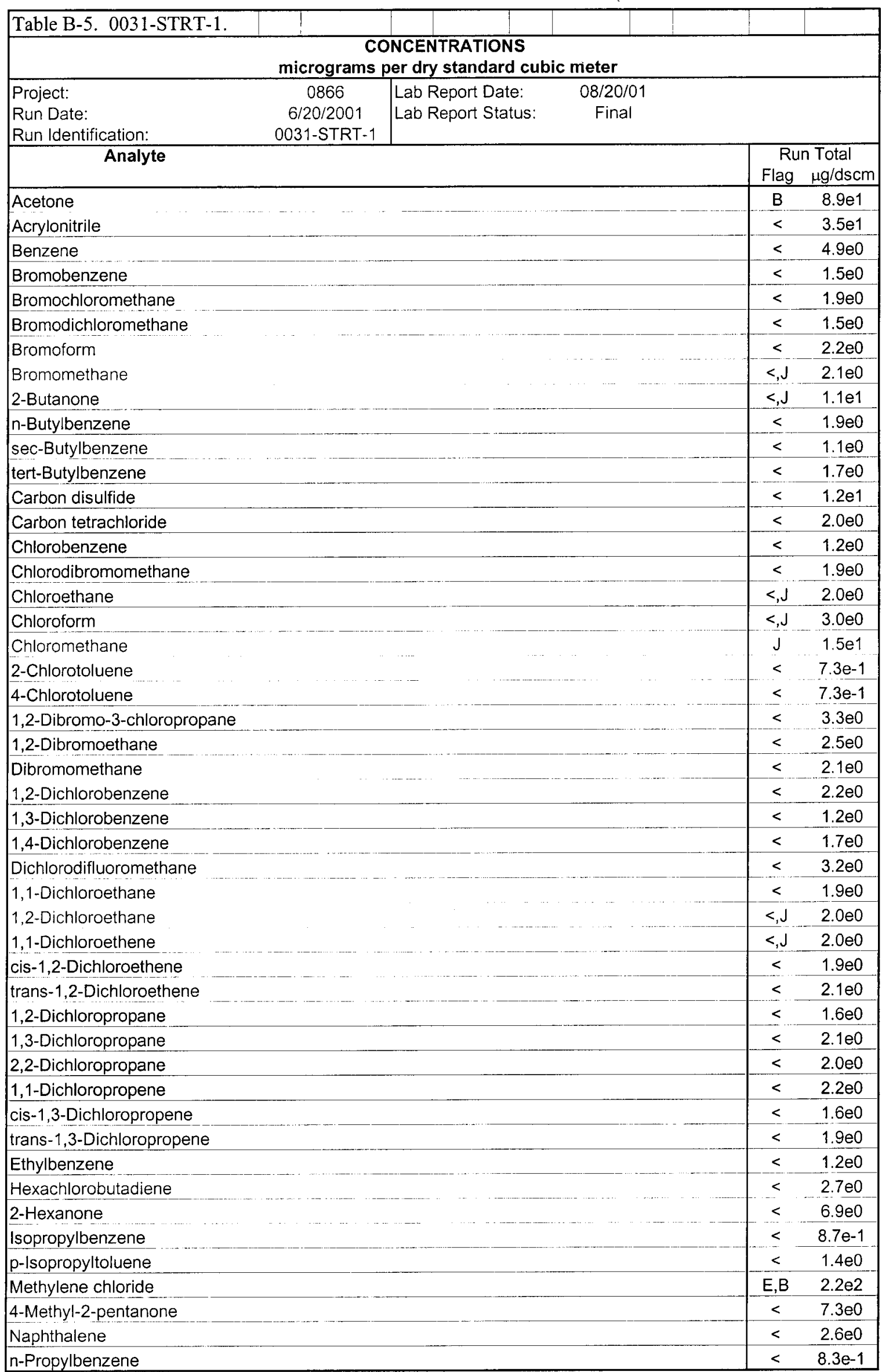

B5. 2 - 5 


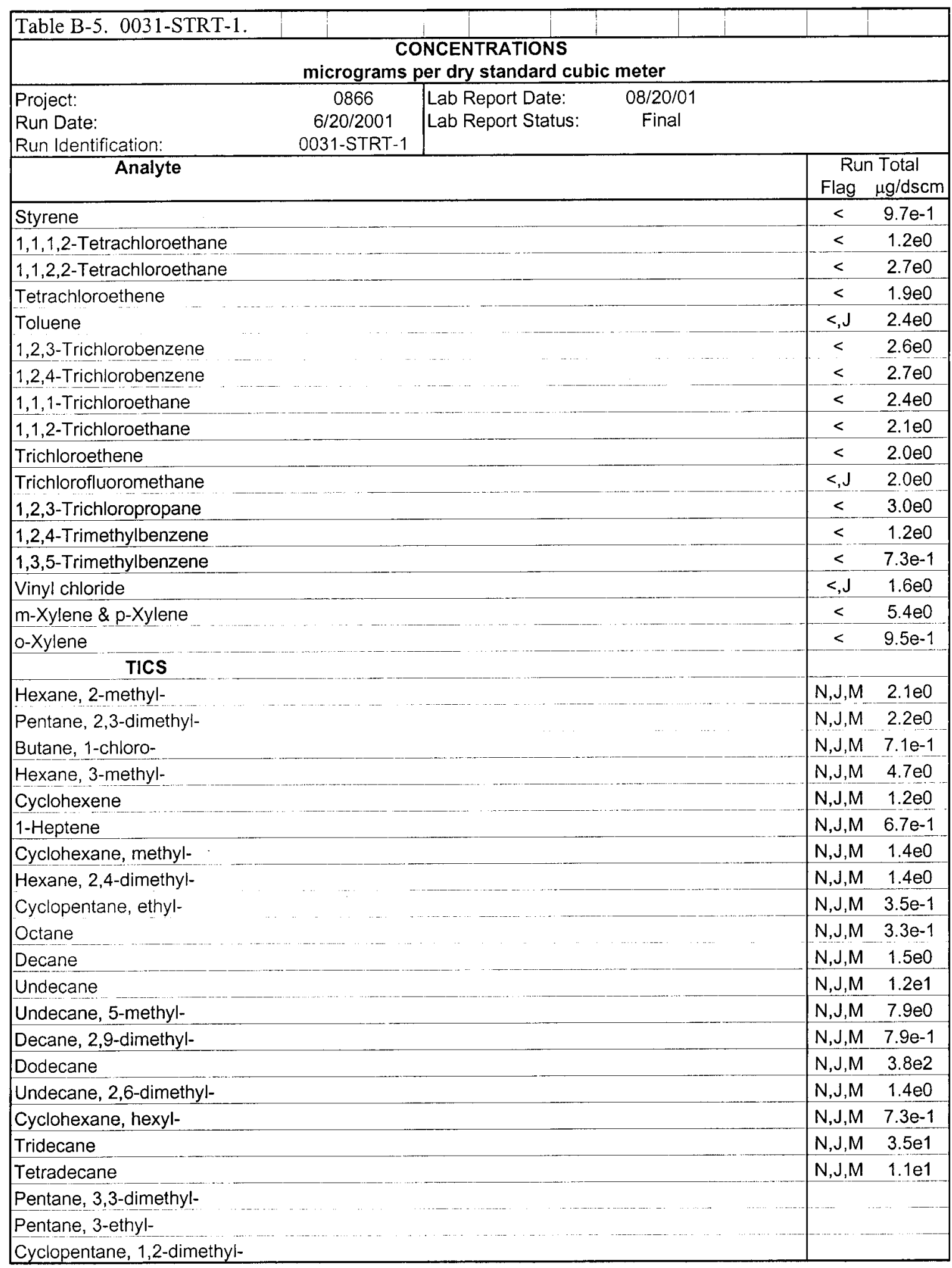




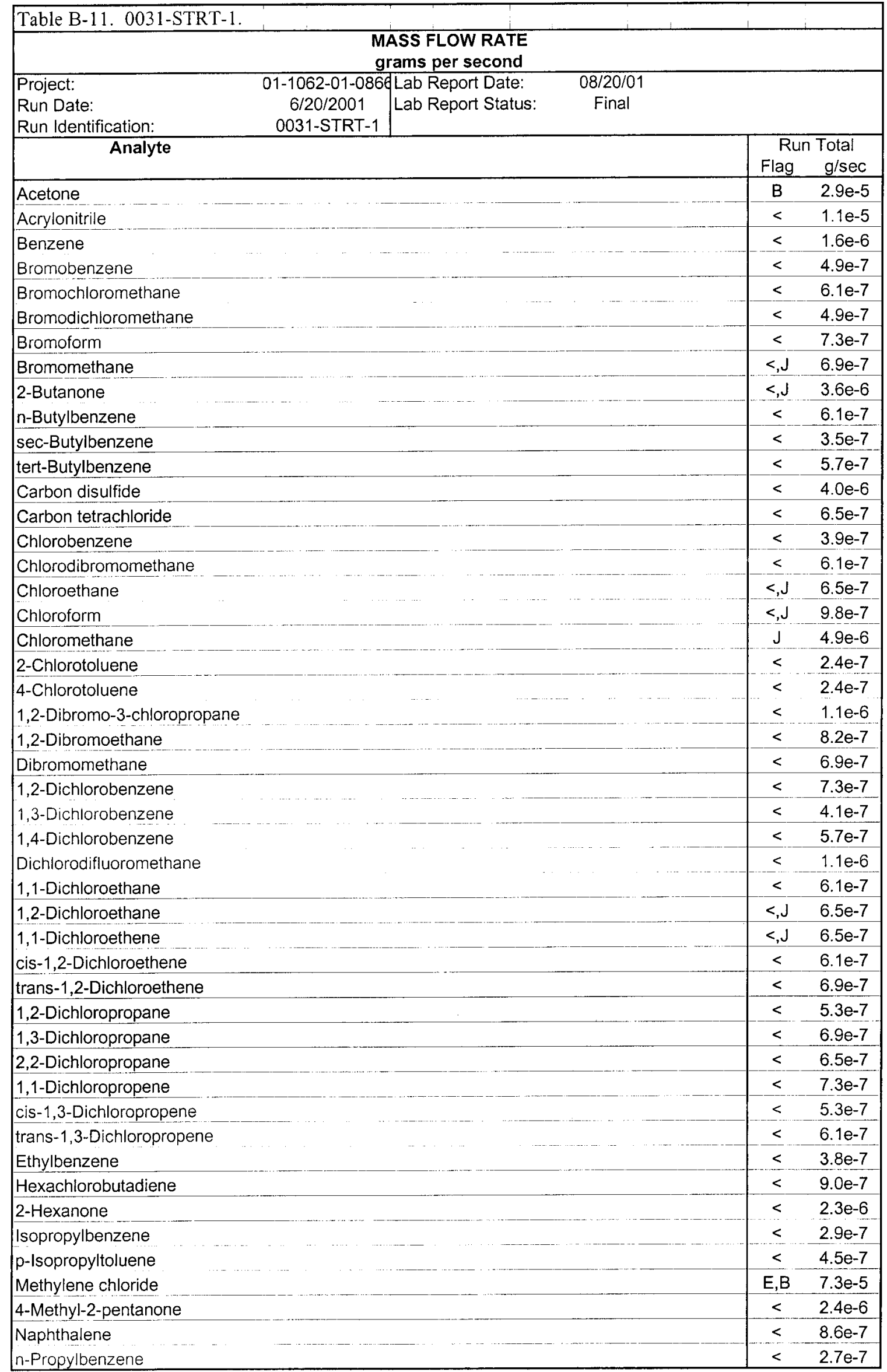




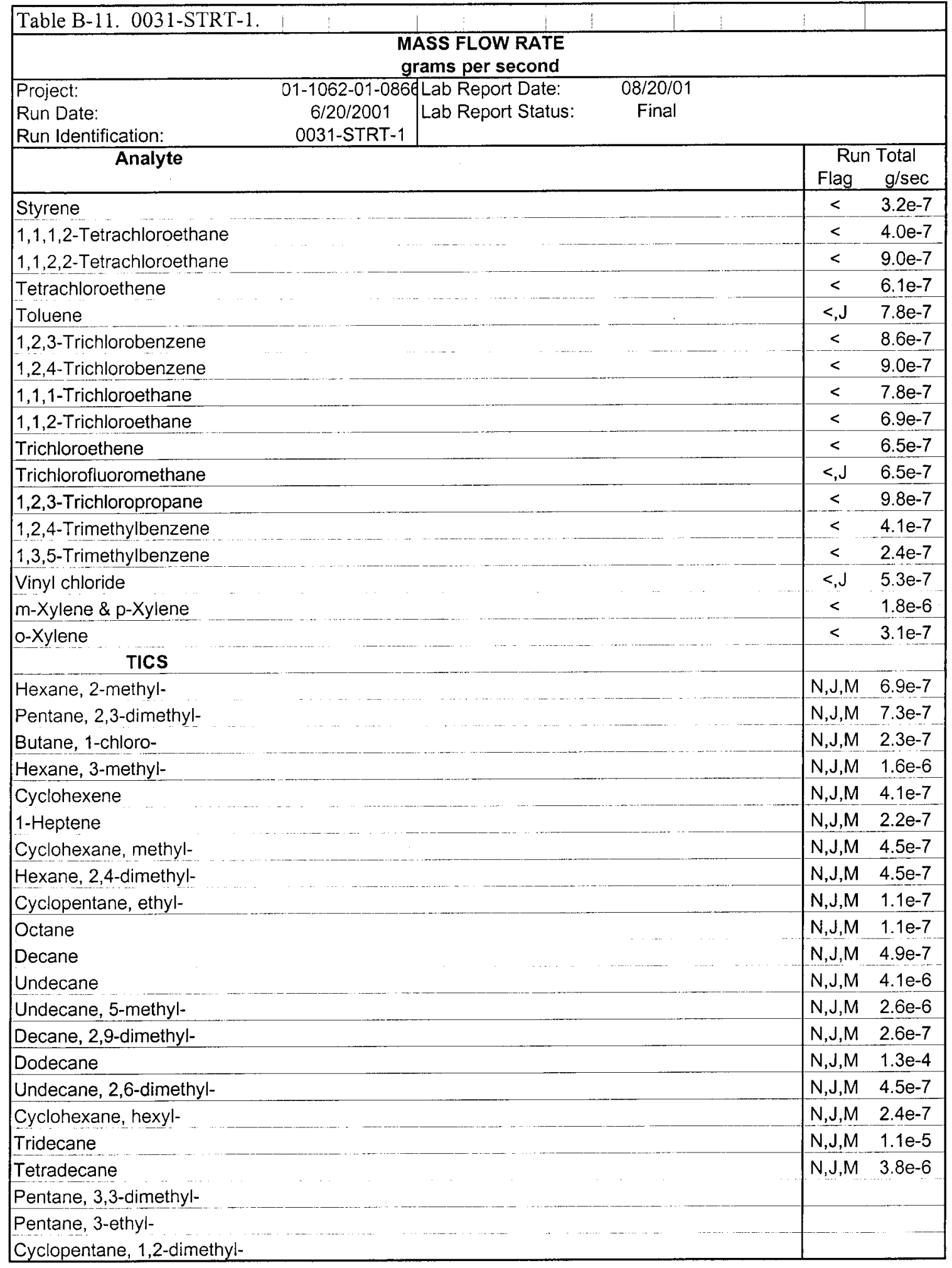





\begin{tabular}{|c|c|c|c|c|c|c|c|c|c|c|}
\hline \multicolumn{3}{|c|}{ Table B-6. 0031-END-1. } & & & \multicolumn{2}{|c|}{ i } & & & \multicolumn{2}{|r|}{ 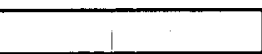 } \\
\hline \multicolumn{11}{|c|}{ VOST SAMPLING DATA SHEET } \\
\hline Site: & \multicolumn{2}{|c|}{ HLLWE Offgas Tie-in } & \multicolumn{2}{|l|}{ Run No.: } & \multicolumn{2}{|c|}{ 0031-END-1 } & \multicolumn{2}{|c|}{ Meter Box No.: } & \multicolumn{2}{|c|}{1} \\
\hline Project: & \multicolumn{2}{|c|}{$01-1062-01-0866$} & \multicolumn{2}{|l|}{ Run Type: } & \multicolumn{2}{|c|}{ Test } & Y-factor: & & & 398 \\
\hline Date: & & $20 / 01$ & Pbar., in. $\mathrm{Hg}$ & & 25.2 & & Operator: & & RW, & $E, J A$ \\
\hline VOST Tube & Leal & Check & Sampling & San & npling & Probe & Condenser & Meter & Meter & Pump \\
\hline $\begin{array}{l}\text { Sample } \\
\text { Numbers }\end{array}$ & $\begin{array}{c}\text { Rate } \\
(\mathrm{L} / \mathrm{min})\end{array}$ & $\begin{array}{l}\text { @ vacuum } \\
\text { (in. } \mathrm{Hg} \text { ) }\end{array}$ & $\begin{array}{l}\text { Rate } \\
\text { (L/min) }\end{array}$ & $(24 \mathrm{hr})^{\mathrm{Ti}}$ & ime & $\begin{array}{l}\text { Temp. } \\
\left({ }^{\circ} \mathrm{C}\right)\end{array}$ & $\begin{array}{c}\text { Temp. } \\
\left({ }^{\circ} \mathrm{C}\right)\end{array}$ & $\begin{array}{l}\text { Temp. } \\
\left({ }^{\circ} \mathrm{C}\right)\end{array}$ & $\begin{array}{c}\text { Volume } \\
\text { (L) }\end{array}$ & $\begin{array}{l}\text { Vacuum } \\
\text { (in. } \mathrm{Hg} \text { ) }\end{array}$ \\
\hline Set 1 & & etest & Target: & 14:00 & 0 & 130 & 11.0 & 31 & 0.000 & 13.0 \\
\hline A-3409 & 0.001 & 16 & 1 & $14: 05$ & 5 & 130 & 10.0 & 31 & 4.788 & 13.0 \\
\hline A-3410 & & & & $14: 10$ & 10 & 130 & 10.0 & 31 & 9.586 & 13.0 \\
\hline A-3411 & & & & $14: 15$ & 15 & 130 & 11.0 & 33 & 14.413 & 13.0 \\
\hline & & & & $14: 20$ & 20 & 130 & 11.0 & 33 & 19.260 & 13.0 \\
\hline & & t-test & & $14: 25$ & 25 & 130 & 11.0 & 34 & 24.164 & 13.0 \\
\hline .... & 0.003 & 16 & $\begin{array}{l}\text { Total } \\
\text { Average }\end{array}$ & $0: 25$ & 25 & 130 & 10.7 & 32 & 24.164 & $\begin{array}{r}\text { Max: } \\
13.0\end{array}$ \\
\hline Set 2 & & etest & Target: & $14: 36$ & 0 & 130 & 10.0 & 34 & 0.000 & 12.0 \\
\hline A-3412 & 0.000 & 16 & 1 & $14: 41$ & 5 & 130 & 10.0 & 35 & 4.994 & 12.0 \\
\hline A-3413 & & & & $14: 46$ & 10 & 130 & 10.0 & 35 & 10.001 & 12.0 \\
\hline A-3414 & & & & $14: 51$ & 15 & 130 & 10.0 & 35 & 15.128 & 12.0 \\
\hline & & & & $14: 56$ & 20 & 130 & 10.0 & 36 & 20.296 & 12.0 \\
\hline & & t-test & & $15: 00$ & 24 & 130 & 10.0 & 35 & 24.452 & 12.0 \\
\hline & 0.008 & 16 & Total & $0: 24$ & 24 & & & & 24.452 & Max: \\
\hline & & & Average & & & 130 & 10.0 & 35 & & 12.0 \\
\hline Set 3 & & etest & Target: & $15: 10$ & 0 & 130 & 12.0 & 35 & 0.000 & 5.0 \\
\hline A-3415 & 0.001 & 16 & & $15: 15$ & 5 & 130 & 11.0 & 35 & 1.909 & 5.0 \\
\hline A-3416 & & & & $15: 20$ & 10 & 130 & 11.0 & 35 & 3.721 & 5.0 \\
\hline A-3417 & & & & $15: 25$ & 15 & 130 & 11.0 & 35 & 5.650 & 5.0 \\
\hline & & & & $15: 30$ & $20^{-}$ & 130 & 11.0 & 35 & 7.605 & 5.0 \\
\hline & & & & $15: 35$ & 25 & 130 & 11.0 & 35 & 9.514 & 5.0 \\
\hline & & & & $15: 40$ & 30 & 130 & 11.0 & 35 & 11.410 & 5.0 \\
\hline & & & & $15: 45$ & 35 & 130 & 11.0 & 35 & 13.348 & 5.0 \\
\hline & & & & $15: 50$ & 40 & 130 & 11.0 & 35 & 15.321 & 5.0 \\
\hline & & & & $15: 55$ & 45 & 130 & 11.0 & 35 & 17.238 & 5.0 \\
\hline & & & & $16: 00$ & 50 & 130 & 11.0 & 35 & 19.154 & 5.0 \\
\hline & & & & $16: 05$ & 55 & 130 & 11.0 & 35 & 21.118 & 5.0 \\
\hline & & t-test & & $16: 10$ & 60 & 130 & 12.0 & 35 & 23.165 & 5.0 \\
\hline & 0.000 & 6 & & $16: 15$ & 65 & 130 & 12.0 & 35 & 24.206 & 5.0 \\
\hline & & & Total & $1: 05$ & 65 & & & & 24.206 & Max: \\
\hline & & & Average & & & 130 & 11.2 & 35 & & 5.0 \\
\hline Set 4 & & etest & Target: & $16: 29$ & 0 & 130 & 11.0 & 31 & 0.000 & 4.0 \\
\hline$A-3418$ & 0.000 & & 1 & $16: 34$ & 5 & 130 & 11.0 & 31 & 2.646 & 4.0 \\
\hline A-3419 & & & & $16: 39$ & 10 & 130 & 11.0 & 31 & 5.400 & 4.0 \\
\hline A-3420 & & & & $16: 44$ & 15 & 130 & 11.0 & 32 & 7.965 & 4.0 \\
\hline & & & & $16: 49$ & 20 & 130 & 11.0 & 33 & 10.607 & 4.0 \\
\hline & & & & $16: 54$ & 25 & 130 & 11.0 & 33 & 13.270 & 4.0 \\
\hline & & & & $16: 59$ & 30 & 130 & 11.0 & 34 & 15.873 & 4.0 \\
\hline & & & & $17: 04$ & 35 & 130 & 11.0 & 34 & 18.500 & 4.0 \\
\hline & & & & $17: 09$ & 40 & 130 & 12.0 & 34 & 21.135 & 4.0 \\
\hline & & t-test & & $17: 14$ & 45 & 130 & 11.0 & 35 & 23.825 & 4.0 \\
\hline & 0.000 & 5 & & $17: 15$ & 46 & 130 & 11.0 & 35 & 24.404 & 4.0 \\
\hline & & & Total & $0: 46$ & 46 & & & & 24.404 & Max: \\
\hline & & & Average & & & 130 & 11.1 & 33 & & 4.0 \\
\hline Condensate & & & & Tenax R & se: & Anasorb & b Rinse: & & & \\
\hline A-3421 & & & & & & & & & t & \\
\hline $\begin{array}{r}\text { Final Conder } \\
40\end{array}$ & $\begin{array}{l}\text { isate Vol } \\
\mathrm{mL}\end{array}$ & me: & & $\begin{array}{l}\text { Tenax R } \\
\text { NA }\end{array}$ & $\begin{array}{l}\text { nse Vol: } \\
\text { mL. }\end{array}$ & $\begin{array}{l}\text { Anasorb } \\
\text { NA }\end{array}$ & $\begin{array}{l}\text { Rinse Vol } \\
\mathrm{mL} \text {. }\end{array}$ & & & \\
\hline & & & & ADDITI & ONAL INP & UTS & & & & \\
\hline & & & & Symbol & Units & Set 1 & Set 2 & Set 3 & Set 4 & Average \\
\hline Average Dry & Oxygen & Joncentratio & & $\mathrm{Co} 2$ & $\%$ & 20.5 & 20.5 & 20.5 & 20.5 & 20.5 \\
\hline Process Gas $F$ & low (dry, & TTP)@68 $\mathrm{F}$ & & Qsd & $\mathrm{dscm} / \mathrm{sec}$ & 0.330 & 0.330 & 0.330 & 0.330 & 0.330 \\
\hline & & & CALCUL & ATED SA & AMPLING & PARAME & ETERS & & & \\
\hline & & & & Symbol & Units & Set 1 & Set 2 & Set 3 & Set 4 & Net \\
\hline Sample Volu & Ime@ @t & indard Cond & ditions & VmStd & dsL & 19.577 & 19.629 & 19.431 & 19.718 & 78.355 \\
\hline$V m S t d=17.647$ & $Y * \mathrm{Pbar}^{*} \mathrm{Y}$ & $\mathrm{m} / \mathrm{Fm}+460)$ & & & & & & & & \\
\hline Avg. Actual s & Sampling & Rate, $Q m=V$ & $/ \mathrm{m} / \mathrm{min}$ & $\mathrm{Qm}$ & $\mathrm{L} / \mathrm{min}$ & 0.967 & 1.019 & 0.372 & 0.531 & 0.722 \\
\hline Avg. Samplin & g Rate, & $\mathrm{mStd}=\mathrm{VmS}$ & $\mathrm{std} / \mathrm{min}$ & QmStd & $\mathrm{dsL} / \mathrm{min}$ & 0.783 & 0.818 & 0.299 & 0.429 & 0.582 \\
\hline
\end{tabular}




\begin{tabular}{|c|c|c|c|c|}
\hline Table B-6. 0031-END-1. & micrograms & $\begin{array}{l}\text { ONCENTRATIONS } \\
\text { per dry standard cubic meter }\end{array}$ & & \\
\hline $\begin{array}{l}\text { Project: } \\
\text { Run Date: } \\
\text { Run Identification: }\end{array}$ & $\begin{array}{c}01-1062-01- \\
6 / 20 / 2001 \\
0031-E N D-1\end{array}$ & $\begin{array}{lc}\text { Lab Report Date: } & 08 / 20 / 01 \\
\text { Lab Report Status: } & \text { Final }\end{array}$ & & \\
\hline Analyte & & & $\begin{array}{r}\text { Ru } \\
\text { Flag }\end{array}$ & $\begin{array}{l}\text { Total } \\
\mu \mathrm{g} / \mathrm{dscm}\end{array}$ \\
\hline Acetone & & & $\mathrm{B}$ & $9.2 \mathrm{e} 1$ \\
\hline Acrylonitrile & & & $<$ & $3.6 \mathrm{e} 1$ \\
\hline Benzene & & & $<$ & $3.1 \mathrm{e} 0$ \\
\hline Bromobenzene & & & $<$ & $1.5 \mathrm{e} 0$ \\
\hline Bromochloromethane & & & $<$ & $1.9 \mathrm{e} 0$ \\
\hline Bromodichloromethane & & & $<$ & $1.5 \mathrm{e} 0$ \\
\hline Bromoform & & & $<$ & $2.3 \mathrm{e} 0$ \\
\hline Bromomethane & & & $<, J$ & $2.9 e 0$ \\
\hline 2-Butanone & & & $<, \mathrm{J}$ & $1.1 \mathrm{e} 1$ \\
\hline n-Butylbenzene & & & $<$ & $1.9 \mathrm{e} 0$ \\
\hline sec-Butylbenzene & & & $<$ & $1.1 \mathrm{e} 0$ \\
\hline tert-Butylbenzene & & & $<$ & $1.8 \mathrm{e} 0$ \\
\hline Carbon disulfide & & & $<$ & $1.5 \mathrm{e} 1$ \\
\hline Carbon tetrachloride & & & $<$ & $2.0 \mathrm{e} 0$ \\
\hline Chlorobenzene & & & $<, \mathrm{J}$ & $1.2 \mathrm{e} 0$ \\
\hline Chlorodibromomethane & & & $<$ & $1.9 \mathrm{e} 0$ \\
\hline Chloroethane & & & $<, \mathrm{J}$ & $2.6 \mathrm{e} 0$ \\
\hline Chloroform & & & $<$ & $5.1 \mathrm{e} 0$ \\
\hline Chloromethane & & & $<$ & $3.7 \mathrm{e} 1$ \\
\hline 2-Chlorotoluene & & & $<$ & $7.5 e-1$ \\
\hline 4-Chlorotoluene & & & $<$ & $7.5 \mathrm{e}-1$ \\
\hline 1,2-Dibromo-3-chloropropane & & & $<$ & $3.6 \mathrm{e} 0$ \\
\hline 1,2-Dibromoethane & & & $<$ & $2.6 \mathrm{e} 0$ \\
\hline Dibromomethane & & & $<$ & $2.2 \mathrm{e} 0$ \\
\hline 1,2-Dichlorobenzene & & & $<$ & $2.3 \mathrm{e} 0$ \\
\hline 1,3-Dichlorobenzene & & & $<$ & $1.3 \mathrm{eo}$ \\
\hline 1,4-Dichlorobenzene & & & $<$ & $1.8 \mathrm{e} 0$ \\
\hline Dichlorodifluoromethane & & & $<, J$ & $2.0 \mathrm{e} 0$ \\
\hline 1,1-Dichloroethane & & & $<$ & $1.9 \mathrm{e} 0$ \\
\hline 1,2-Dichloroethane & & & $<, J$ & $2.0 \mathrm{e} 0$ \\
\hline 1,1-Dichloroethene & & & $<, J$ & $2.3 \mathrm{e} 0$ \\
\hline cis-1,2-Dichloroethene & & & $<$ & $1.9 \mathrm{e} 0$ \\
\hline trans-1,2-Dichloroethene & & & $<$ & $2.0 \mathrm{e} 0$ \\
\hline 1,2-Dichloropropane & & & $<, J$ & $1.7 \mathrm{e} 0$ \\
\hline 1,3-Dichloropropane & & & $<$ & $2.2 \mathrm{e} 0$ \\
\hline 2,2-Dichloropropane & & & $<$ & $2.0 \mathrm{e} 0$ \\
\hline 1,1-Dichloropropene & & & $<$ & $2.3 \mathrm{e} 0$ \\
\hline cis-1,3-Dichloropropene & & .. & $<$ & $1.7 \mathrm{e} 0$ \\
\hline trans-1,3-Dichloropropene & & & $<$ & $1.9 \mathrm{e} 0$ \\
\hline Ethylbenzene & & & $<$ & $1.2 \mathrm{e} 0$ \\
\hline Hexachlorobutadiene & & & $<$ & $2.8 \mathrm{e} 0$ \\
\hline 2-Hexanone & & & $<$ & $7.1 \mathrm{e} 0$ \\
\hline Isopropylbenzene & & & $<$ & $8.9 \mathrm{e}-1$ \\
\hline p-Isopropyltoluene & & & $<$ & $1.4 \mathrm{e} 0$ \\
\hline Methylene chloride & & & $\mathrm{B}$ & $2.2 \mathrm{e} 1$ \\
\hline 4-Methyl-2-pentanone & & & $<$ & $7.5 \mathrm{e} 0$ \\
\hline Naphthalene & & & $<$ & $2.7 \mathrm{e} 0$ \\
\hline
\end{tabular}

B6. 2 - 5 


\begin{tabular}{|c|c|c|c|c|}
\hline \multicolumn{5}{|c|}{$\begin{array}{l}\text { CONCENTRATIONS } \\
\text { micrograms per dry standard cubic meter }\end{array}$} \\
\hline $\begin{array}{l}\text { Project: } \\
\text { Run Date: } \\
\text { Run Identification: }\end{array}$ & $\begin{array}{c}01-1062-01- \\
6 / 20 / 2001 \\
0031-E N D-1\end{array}$ & $\begin{array}{lc}\text { Lab Report Date: } & 08 / 20 / 01 \\
\text { Lab Report Status: } & \text { Final }\end{array}$ & & \\
\hline \multicolumn{3}{|l|}{ Analyte } & \multicolumn{2}{|c|}{$\begin{array}{c}\text { Run Total } \\
\text { Flag } \mu \mathrm{g} / \mathrm{dscm} \\
\end{array}$} \\
\hline \multicolumn{3}{|l|}{ n-Propylbenzene } & $<$ & $8.4 \mathrm{e}-1$ \\
\hline \multicolumn{3}{|l|}{ Styrene } & $<$ & $1.0 \mathrm{e} 0$ \\
\hline \multicolumn{3}{|l|}{$1,1,1,2$-Tetrachloroethane } & $<$ & $1.3 \mathrm{e} 0$ \\
\hline \multicolumn{3}{|l|}{ 1,1,2,2-Tetrachloroethane } & $<$ & $2.8 \mathrm{e} 0$ \\
\hline \multicolumn{3}{|l|}{ Tetrachloroethene } & $<$ & $1.9 \mathrm{e} 0$ \\
\hline \multicolumn{3}{|l|}{ Toluene } & $<, J$ & $3.6 \mathrm{e} 0$ \\
\hline \multicolumn{3}{|l|}{ 1,2,3-Trichlorobenzene } & $<$ & $2.7 \mathrm{e} 0$ \\
\hline \multicolumn{3}{|l|}{ 1,2,4-Trichlorobenzene } & $<$ & $2.8 \mathrm{e} 0$ \\
\hline \multicolumn{3}{|l|}{ 1,1,1-Trichloroethane } & $<$ & $2.4 \mathrm{e} 0$ \\
\hline \multicolumn{3}{|l|}{ 1,1,2-Trichloroethane } & $<$ & $2.2 \mathrm{e} 0$ \\
\hline \multicolumn{3}{|l|}{ Trichloroethene } & $<$ & $2.0 \mathrm{e} 0$ \\
\hline \multicolumn{3}{|l|}{ Trichlorofluoromethane } & $<, \mathrm{J}$ & $2.0 \mathrm{e} 0$ \\
\hline \multicolumn{3}{|l|}{ 1,2,3-Trichloropropane } & $<$ & $3.1 \mathrm{e} 0$ \\
\hline \multicolumn{3}{|l|}{ 1,2,4-Trimethylbenzene } & $<$ & $1.3 \mathrm{e} 0$ \\
\hline \multicolumn{3}{|l|}{$1,3,5$-Trimethylbenzene } & $<$ & $7.5 \mathrm{e}-1$ \\
\hline \multicolumn{3}{|l|}{ Vinyl chloride } & $<, J$ & $2.3 \mathrm{e} 0$ \\
\hline \multicolumn{3}{|l|}{ m-Xylene \& $p$-Xylene } & $<$ & $5.6 \mathrm{e0}$ \\
\hline \multicolumn{3}{|l|}{ o-Xylene } & $<, J$ & $1.0 \mathrm{e} 0$ \\
\hline \multicolumn{5}{|l|}{ TICs } \\
\hline \multicolumn{3}{|l|}{ Pentane, 3,3-dimethyl- } & $N, J, M$ & $7.5 \mathrm{e}-1$ \\
\hline \multicolumn{3}{|l|}{ Hexane, 2-methyl- } & $N, J, M$ & $4.0 \mathrm{e} 0$ \\
\hline \multicolumn{3}{|l|}{ Pentane, 2,3-dimethyl- } & $N, J, M$ & $4.0 e-1$ \\
\hline \multicolumn{3}{|l|}{ Hexane, 3-methyl- } & $\mathrm{N}, \mathrm{J}, \mathrm{M}$ & $9.7 \mathrm{e} 0$ \\
\hline Cyclohexene & & & $\mathrm{N}, \mathrm{J}, \mathrm{M}$ & $4.6 \mathrm{e}-1$ \\
\hline Cyclobutane, ethenyl- & & & $\mathrm{N}, \mathrm{J}, \mathrm{M}$ & $1.5 \mathrm{e} 0$ \\
\hline Cyclopentane, 1,2-dimethyl-, t & & & $\mathrm{N}, \mathrm{J}, \mathrm{M}$ & $1.0 \mathrm{e} 0$ \\
\hline Cyclohexane, methyl- & & & $N, J, M$ & $2.6 \mathrm{e} 0$ \\
\hline Hexane, 2,4-dimethyl- & & & $\mathrm{N}, \mathrm{J}, \mathrm{M}$ & $2.3 \mathrm{e} 0$ \\
\hline Cyclopentane, ethyl- & & & $\mathrm{N}, J, M$ & $5.2 \mathrm{e}-1$ \\
\hline Methane, trichloronitro- & & & $\mathrm{N}, \mathrm{J}, \mathrm{M}$ & $4.6 \mathrm{e} 0$ \\
\hline Benzonitrile & & & $\mathrm{N}, \mathrm{J}, \mathrm{M}$ & $2.4 \mathrm{e} 0$ \\
\hline Undecane & & & $\mathrm{N}, \mathrm{J}, \mathrm{M}$ & $6.4 \mathrm{e} 0$ \\
\hline Undecane, 5-methyl- & & & $\mathrm{N}, \mathrm{J}, \mathrm{M}$ & $2.3 \mathrm{e} 0$ \\
\hline Dodecane & & & $\mathrm{N}, J, M$ & $3.3 \mathrm{e} 2$ \\
\hline Tridecane & & & $\mathrm{N}, \mathrm{J}, \mathrm{M}$ & $4.0 \mathrm{e} 1$ \\
\hline Tetradecane & & & $\mathrm{N}, \mathrm{J}, \mathrm{M}$ & $1.3 \mathrm{e} 1$ \\
\hline Hexadecane & & & $N, J, M$ & $2.7 \mathrm{e} 0$ \\
\hline Pentane, 3-ethyl- & & & & \\
\hline Cyclopentane, 1,2-dimethyl- & & & & \\
\hline
\end{tabular}




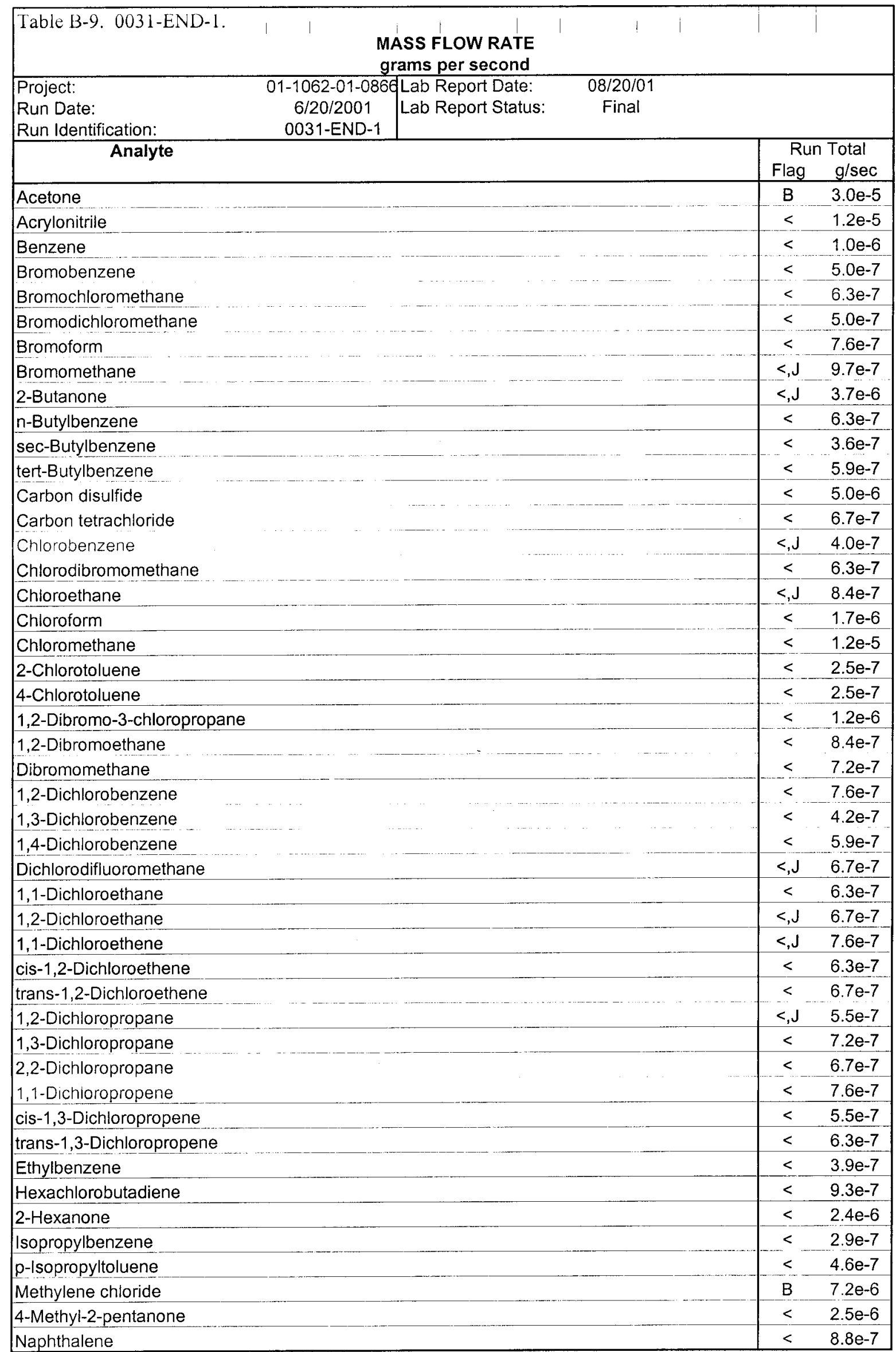




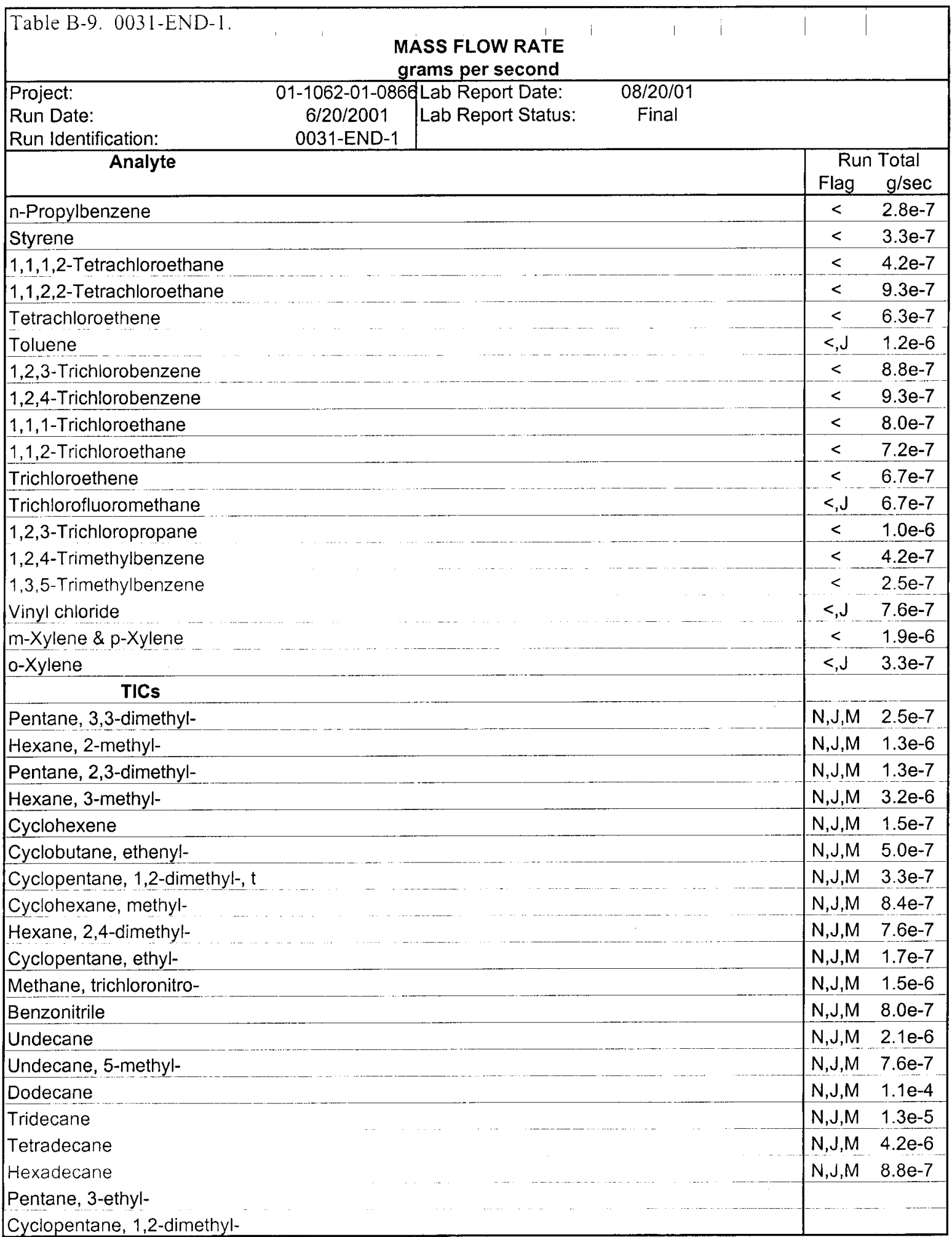





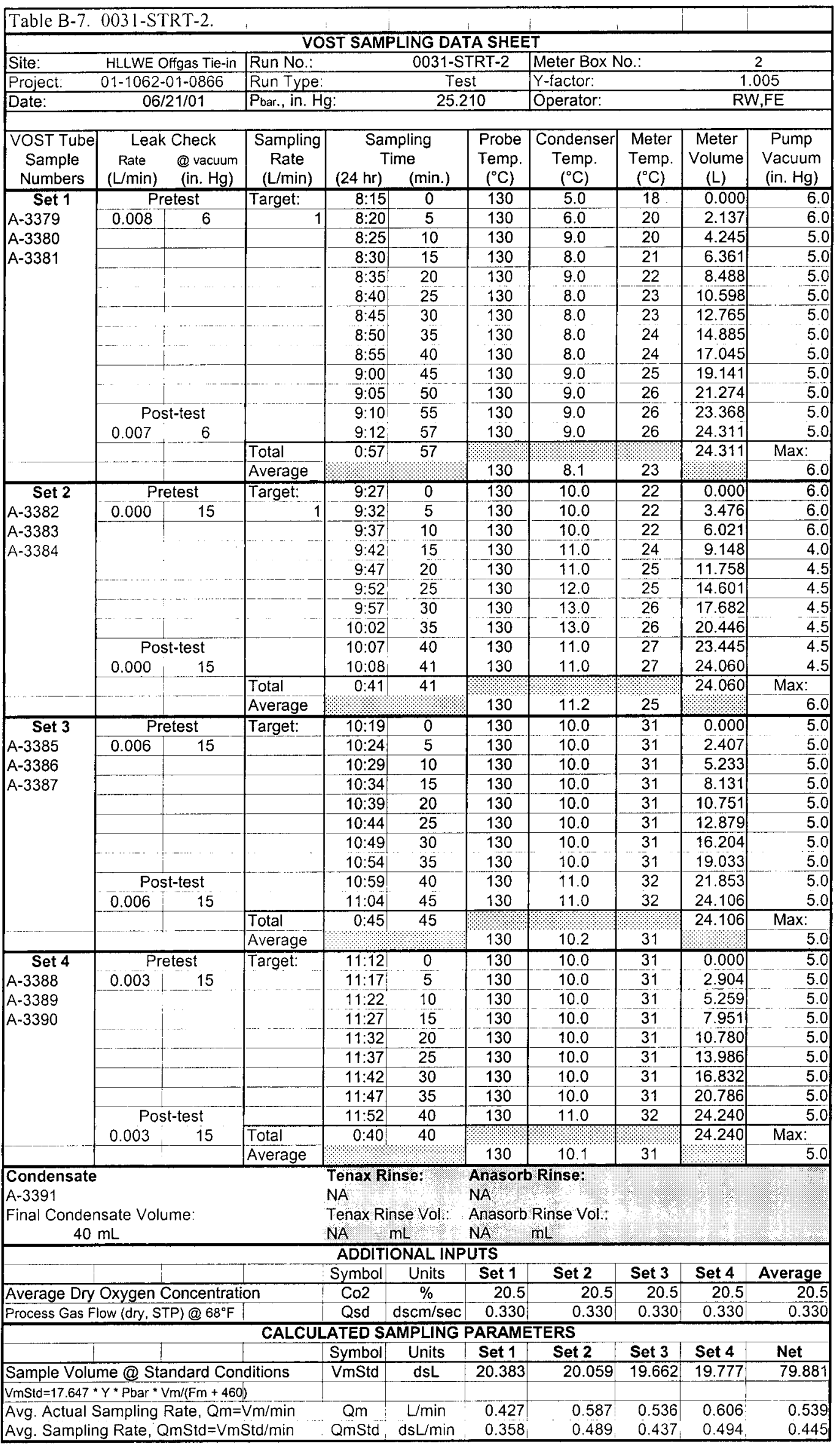




\begin{tabular}{|c|c|c|c|c|}
\hline Table B-7. 0031-STRT-2. & $\begin{array}{r}\mathrm{CC} \\
\text { micrograms }\end{array}$ & $\begin{array}{l}\text { ONCENTRATIONS } \\
\text { per dry standard cubic meter }\end{array}$ & & \\
\hline $\begin{array}{l}\text { Project: } \\
\text { Run Date: } \\
\text { Run Identification: }\end{array}$ & $\begin{array}{l}\text { 01-1062-01- } \\
\text { 6/21/2001 } \\
\text { 0031-STRT-2 }\end{array}$ & $\begin{array}{lc}\text { Lab Report Date: } & 08 / 20 / 01 \\
\text { Lab Report Status: } & \text { Final }\end{array}$ & & \\
\hline Analyte & & & $\begin{array}{r}\text { Ru } \\
\text { Flag } \\
\end{array}$ & $\begin{array}{l}\text { Total } \\
\mu \mathrm{g} / \mathrm{dscm}\end{array}$ \\
\hline Acetone & & & $\mathrm{J}, \mathrm{B}$ & $6.3 \mathrm{e} 1$ \\
\hline Acrylonitrile & & & $<$ & $3.5 \mathrm{e} 1$ \\
\hline Benzene & & & $<, J$ & $2.8 \mathrm{e} 0$ \\
\hline Bromobenzene & & & $<$ & $1.5 \mathrm{e} 0$ \\
\hline Bromochloromethane & & & $<$ & $1.9 \mathrm{e} 0$ \\
\hline Bromodichloromethane & & & $<$ & $1.5 \mathrm{e} 0$ \\
\hline Bromoform & & & $<$ & $2.3 \mathrm{e} 0$ \\
\hline Bromomethane & & $\cdots$ & $<, J$ & $1.9 \mathrm{e} 0$ \\
\hline 2-Butanone & & & $<, J$ & $1.1 \mathrm{e} 1$ \\
\hline n-Butylbenzene & & $\ldots$ & $<$ & $1.9 \mathrm{e} 0$ \\
\hline sec-Butylbenzene & & & $<$ & $1.1 \mathrm{e} 0$ \\
\hline tert-Butylbenzene & & & $<$ & $1.8 \mathrm{e} 0$ \\
\hline Carbon disulfide & & & $<$ & $1.6 \mathrm{e} 1$ \\
\hline Carbon tetrachloride & & & $<$ & $2.0 \mathrm{e} 0$ \\
\hline Chlorobenzene & & & $<$ & $1.2 \mathrm{e} 0$ \\
\hline Chlorodibromomethane & & & $<$ & $1.9 \mathrm{e} 0$ \\
\hline Chloroethane & & & $<, J$ & $2.1 \mathrm{e} 0$ \\
\hline Chloroform & & & $<$ & $4.1 \mathrm{e} 0$ \\
\hline Chloromethane & & & $<, J$ & $1.5 \mathrm{e} 1$ \\
\hline 2-Chlorotoluene & & & $<$ & $7.4 \mathrm{e}-1$ \\
\hline 4-Chlorotoluene & & & $<$ & $7.4 \mathrm{e}-1$ \\
\hline 1,2-Dibromo-3-chloropropane & & & $<$ & $3.5 \mathrm{e} 0$ \\
\hline 1,2-Dibromoethane & & & $<$ & $2.5 \mathrm{e} 0$ \\
\hline Dibromomethane & & & $<$ & $2.1 \mathrm{e} 0$ \\
\hline 1,2-Dichlorobenzene & & & $<$ & $2.3 \mathrm{e} 0$ \\
\hline 1,3-Dichlorobenzene & & & $<$ & $1.3 \mathrm{e} 0$ \\
\hline 1,4-Dichlorobenzene & & 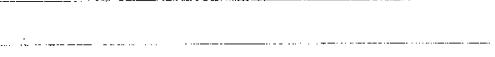 & $<$ & $1.8 \mathrm{e} 0$ \\
\hline Dichlorodifluoromethane & & $\ldots$ & $<$ & $2.5 \mathrm{e} 0$ \\
\hline 1,1-Dichloroethane & & & $<$ & $1.9 \mathrm{e} 0$ \\
\hline 1,2-Dichloroethane & & & $<$ & $2.0 \mathrm{e} 0$ \\
\hline 1,1-Dichloroethene & & & $<, J$ & $2.0 \mathrm{e} 0$ \\
\hline cis-1,2-Dichloroethene & & & $<$ & $1.9 \mathrm{e} 0$ \\
\hline trans-1,2-Dichloroethene & & $-\ldots$ & $<$ & $2.0 \mathrm{e} 0$ \\
\hline 1,2-Dichloropropane & & .. & $<$ & $1.6 \mathrm{e} 0$ \\
\hline 1,3-Dichloropropane & & & $<$ & $2.1 \mathrm{e} 0$ \\
\hline 2,2-Dichloropropane & & & $<$ & $2.0 \mathrm{e} 0$ \\
\hline 1,1-Dichloropropene & & _. & $<$ & $2.3 \mathrm{e} 0$ \\
\hline cis-1,3-Dichloropropene & & & $<$ & $1.6 \mathrm{e} 0$ \\
\hline trans-1,3-Dichloropropene & & & $<$ & $1.9 \mathrm{e} 0$ \\
\hline Ethylbenzene & & 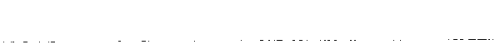 & $<$ & $1.2 \mathrm{e} 0$ \\
\hline Hexachlorobutadiene & & & $<$ & $2.8 \mathrm{e} 0$ \\
\hline 2-Hexanone & & & $<$ & $7.0 \mathrm{e} 0$ \\
\hline Isopropylbenzene & & & $<$ & $8.8 \mathrm{e}-1$ \\
\hline p-Isopropyltoluene & & & $<$ & $1.4 \mathrm{e} 0$ \\
\hline Methylene chloride & & & $<, \mathrm{B}$ & $1.4 \mathrm{e} 1$ \\
\hline 4-Methyl-2-pentanone & & & $<$ & $7.4 \mathrm{e} 0$ \\
\hline Naphthalene & & & $<$ & $2.6 \mathrm{e} 0$ \\
\hline
\end{tabular}




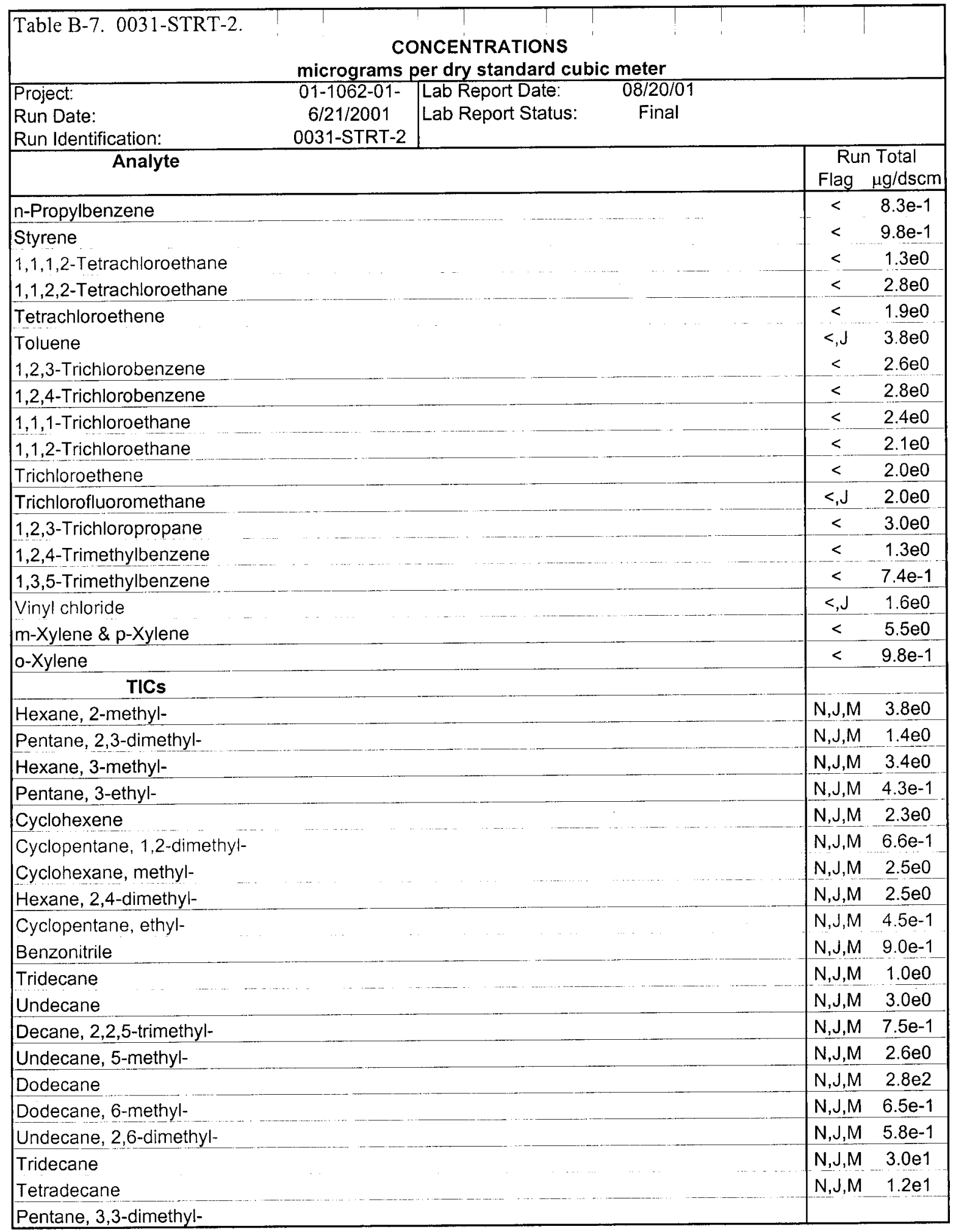




\begin{tabular}{|c|c|c|c|c|}
\hline Table B-7. 0031-STRT-2. & \multicolumn{2}{|l|}{$\begin{array}{l}\text { MASS FLOW RATE } \\
\text { grams per second }\end{array}$} & & \\
\hline $\begin{array}{l}\text { Project: } \\
\text { Run Date: } \\
\text { Run Identification: } \\
\end{array}$ & \begin{tabular}{c|c} 
01-1062-01-0866 Lab Report Date: \\
6/21/2001 \\
0031-STRT-2
\end{tabular} & $\begin{array}{l}08 / 20 / 01 \\
\text { Final }\end{array}$ & & \\
\hline $\begin{array}{r}\text { Analyte } \\
\end{array}$ & & & $\begin{array}{r}\text { Ru } \\
\text { Flag } \\
\end{array}$ & $\begin{array}{l}\text { Total } \\
\mathrm{g} / \mathrm{sec} \\
\end{array}$ \\
\hline Acetone & & & $J, B$ & $2.1 \mathrm{e}-5$ \\
\hline Acrylonitrile & & & $<$ & $1.2 \mathrm{e}-5$ \\
\hline Benzene & & $\ldots$ & $<, \mathrm{J}$ & $9.1 \mathrm{e}-7$ \\
\hline Bromobenzene & & & $<$ & $5.0 \mathrm{e}-7$ \\
\hline Bromochloromethane & & & $<$ & $6.2 \mathrm{e}-7$ \\
\hline Bromodichloromethane & & & $<$ & $5.0 \mathrm{e}-7$ \\
\hline Bromoform & & & $<$ & $7.4 e-7$ \\
\hline Bromomethane & & & $<, \mathrm{J}$ & $6.2 \mathrm{e}-7$ \\
\hline 2-Butanone & & & $<, \mathrm{J}$ & $3.7 e-6$ \\
\hline n-Butylbenzene & & & $<$ & $6.2 e-7$ \\
\hline sec-Butylbenzene & $\cdot$ & & $<$ & $3.5 e-7$ \\
\hline tert-Butylbenzene & & $\ldots$ & $<$ & $5.8 \mathrm{e}-7$ \\
\hline Carbon disulfide & & ..... & $<$ & $5.4 e-6$ \\
\hline Carbon tetrachloride & & - & $<$ & $6.6 e-7$ \\
\hline Chlorobenzene & & & $<$ & $3.9 \mathrm{e}-7$ \\
\hline Chlorodibromomethane & & & $<$ & $6.2 e-7$ \\
\hline Chloroethane & & & $<, \mathrm{J}$ & $7.0 \mathrm{e}-7$ \\
\hline Chloroform & & & $<$ & $1.4 \mathrm{e}-6$ \\
\hline Chloromethane & & & $<, \mathrm{J}$ & $5.0 \mathrm{e}-6$ \\
\hline 2-Chlorotoluene & & & $<$ & $2.4 \mathrm{e}-7$ \\
\hline 4-Chlorotoluene & & & $<$ & $2.4 \mathrm{e}-7$ \\
\hline 1,2-Dibromo-3-chloropropane & & & $<$ & $1.2 e-6$ \\
\hline 1,2-Dibromoethane & & & $<$ & $8.3 e-7$ \\
\hline Dibromomethane & & & $<$ & $7.0 \mathrm{e}-7$ \\
\hline 1,2-Dichlorobenzene & & & $<$ & $7.4 \mathrm{e}-7$ \\
\hline 1,3-Dichlorobenzene & & & $<$ & $4.1 \mathrm{e}-7$ \\
\hline 1,4-Dichlorobenzene & & & $<$ & $5.8 \mathrm{e}-7$ \\
\hline Dichlorodifluoromethane & & & $<$ & $8.3 e-7$ \\
\hline 1,1-Dichloroethane & & & $<$ & $6.2 \mathrm{e}-7$ \\
\hline 1,2-Dichloroethane & & & $<$ & $6.6 \mathrm{e}-7$ \\
\hline 1,1-Dichloroethene & & & $<, J$ & $6.6 \mathrm{e}-7$ \\
\hline cis-1,2-Dichloroethene & & & $<$ & $6.2 \mathrm{e}-7$ \\
\hline trans-1,2-Dichloroethene & & & $<$ & $6.6 \mathrm{e}-7$ \\
\hline 1,2-Dichloropropane & & & $<$ & $5.4 e-7$ \\
\hline 1,3-Dichloropropane & & & $<$ & $7.0 \mathrm{e}-7$ \\
\hline 2,2-Dichloropropane & & $\ldots$ & $<$ & $6.6 \mathrm{e}-7$ \\
\hline 1,1-Dichloropropene & & & $<$ & $7.4 \mathrm{e}-7$ \\
\hline cis-1,3-Dichloropropene & & & $<$ & $5.4 e-7$ \\
\hline trans-1,3-Dichloropropene & & & $<$ & $6.2 e-7$ \\
\hline Ethylbenzene & & & $<$ & $3.8 \mathrm{e}-7$ \\
\hline Hexachlorobutadiene & & & $<$ & $9.1 \mathrm{e}-7$ \\
\hline 2-Hexanone & & & $<$ & $2.3 e-6$ \\
\hline Isopropylbenzene & & & $<$ & $2.9 \mathrm{e}-7$ \\
\hline p-Isopropyltoluene & & & $<$ & $4.5 e-7$ \\
\hline Methylene chloride & & & $<, B$ & $4.5 e-6$ \\
\hline 4-Methyl-2-pentanone & & & $<$ & $2.4 e-6$ \\
\hline Naphthalene & & & $<$ & $8.7 e-7$ \\
\hline
\end{tabular}

B7. 4 - 5 


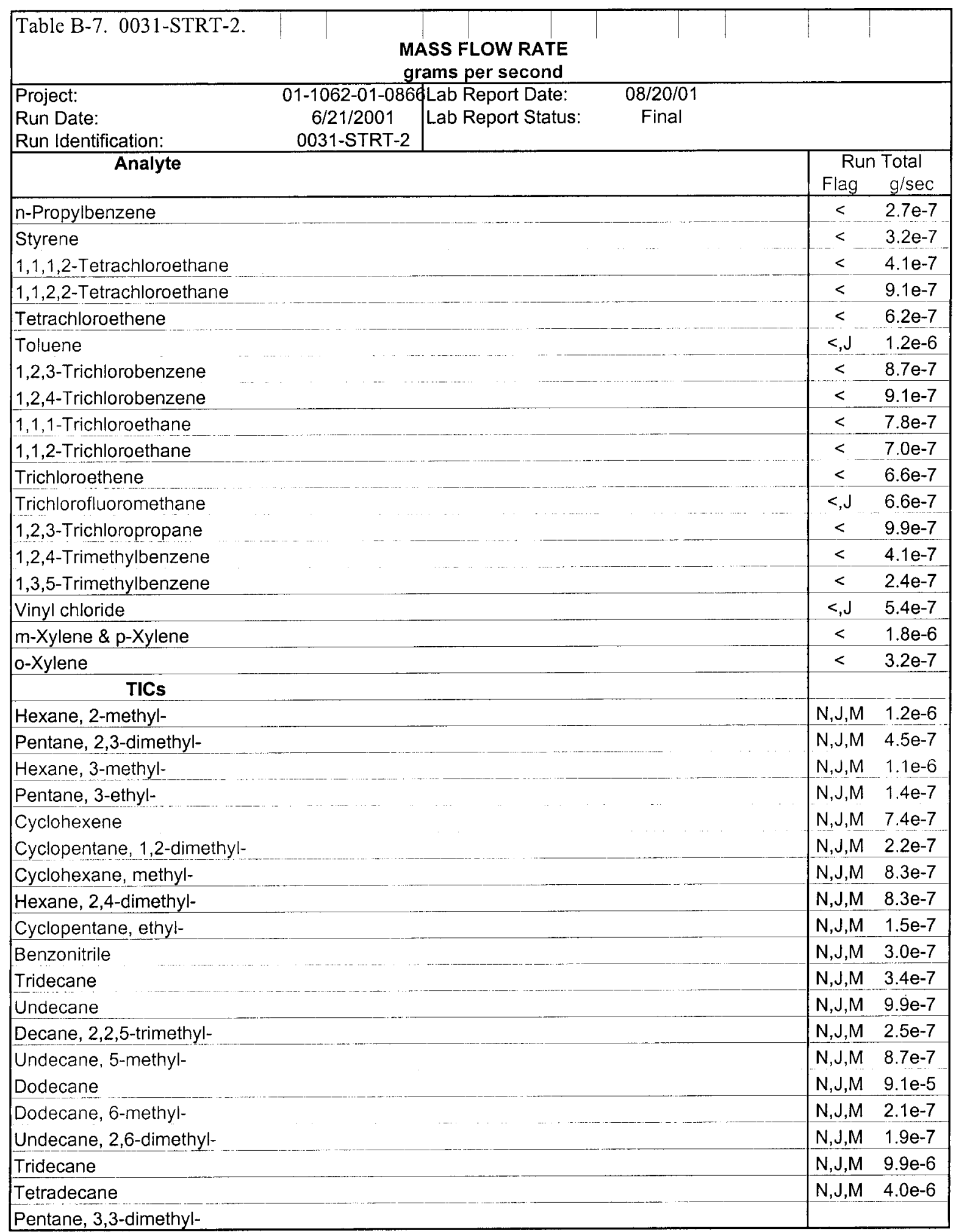





\begin{tabular}{|c|c|c|c|c|c|c|c|c|c|c|}
\hline \multicolumn{3}{|c|}{ Table B-8. 0031-END-2. } & & & & & & & & \\
\hline \multicolumn{11}{|c|}{ VOST SAMPLING DATA SHEET } \\
\hline Site: & \multicolumn{2}{|c|}{ HLLWE Offgas Tie-in } & \multicolumn{2}{|l|}{ Run No.: } & \multicolumn{2}{|c|}{ 0031-END-2 } & \multicolumn{2}{|c|}{ Meter Box No.: } & \multicolumn{2}{|r|}{2} \\
\hline Project: & \multicolumn{2}{|c|}{$01-1062-01-0866$} & \multicolumn{2}{|l|}{ Run Type: } & \multicolumn{2}{|c|}{ Test } & Y-factor: & & & .005 \\
\hline Date: & & un-01 & Pbar., in. $\mathrm{Hg}$ & & 25.2 & & Operator: & & & $\mathrm{e} / \mathrm{rw}$ \\
\hline VOST Tube & Lea & Check & Sampling & San & ppling & Probe & Condenser & Meter & Meter & Pump \\
\hline $\begin{array}{c}\text { Sample } \\
\text { Numbers }\end{array}$ & $\begin{array}{l}\text { Rate } \\
\text { (L/min) }\end{array}$ & $\begin{array}{l}\text { @ vacuum } \\
\text { (in. } \mathrm{Hg} \text { ) }\end{array}$ & $\begin{array}{l}\text { Rate } \\
(\mathrm{L} / \mathrm{min})\end{array}$ & $(24 \mathrm{hr})^{\mathrm{T}}$ & $\begin{array}{l}\text { me } \\
\text { (min.) }\end{array}$ & $\begin{array}{l}\text { Temp. } \\
\left({ }^{\circ} \mathrm{C}\right)\end{array}$ & $\begin{array}{c}\text { Temp. } \\
\left({ }^{\circ} \mathrm{C}\right)\end{array}$ & $\begin{array}{c}\text { Temp. } \\
\left({ }^{\circ} \mathrm{C}\right)\end{array}$ & $\begin{array}{c}\text { Volume } \\
(L)\end{array}$ & $\begin{array}{l}\text { Vacuum } \\
\text { (in. } \mathrm{Hg} \text { ) }\end{array}$ \\
\hline Set 1 & & test & Target: & $13: 50$ & 0 & 130 & 14.0 & 36 & 0.000 & 5.0 \\
\hline A-3428 & 0.003 & 15 & 1 & $13: 55$ & 5 & 130 & 15.0 & 36 & 2.913 & 5.0 \\
\hline A-3429 & & & & $14: 00$ & 10 & 130 & 15.0 & 36 & 5.730 & $4 . \overline{5}$ \\
\hline A-3430 & & & & $14: 05$ & 15 & 130 & 16.0 & 36 & 8.442 & $4 . \overline{5}$ \\
\hline & & & & $14: 10$ & 20 & 130 & 17.0 & 36 & 11.143 & 4.5 \\
\hline & & & & $14: 15$ & 25 & 130 & 14.0 & 36 & 13.890 & 4.5 \\
\hline & & & & $14: 20$ & 30 & 130 & 13.0 & 37 & 16.475 & 4.5 \\
\hline & & & & $14: 25$ & 35 & 130 & 13.0 & 37 & 19.112 & 4.5 \\
\hline & & & & $14: 30$ & 40 & 130 & 12.0 & 37 & 21.676 & 4.5 \\
\hline & & t-test & & $14: 35$ & 45 & 130 & 12.0 & 37 & 24.121 & 4.5 \\
\hline & 0.000 & 15 & Total & $0: 45$ & 45 & & & & 24.121 & Max: \\
\hline & & & Average & & & 130 & 14.1 & 36 & & 5.0 \\
\hline Set 2 & & test & Target: & $14: 45$ & 0 & 130 & 14.0 & 37 & 0 & 4.7 \\
\hline A-3431 & 0.003 & 15 & 1 & $14: 50$ & 5 & 130 & 13.0 & 37 & 2.884 & 4.8 \\
\hline A-3432 & & & & $14: 55$ & 10 & 130 & 12.0 & 37 & 5.986 & 4.8 \\
\hline A-3433 & & & & $15: 00$ & 15 & 130 & 13.0 & 37 & 8.994 & 4.8 \\
\hline & & & & $15: 05$ & 20 & 130 & 14.0 & 37 & 12.108 & 4.8 \\
\hline & & & & $15: 10$ & 25 & 130 & 13.0 & 38 & 16.982 & 4.8 \\
\hline & & & & $15: 15$ & $\overline{30}$ & 130 & 14.0 & 37 & 18.303 & 4.8 \\
\hline & & & & $15: 20$ & 35 & 130 & 13.0 & 37 & 21.394 & 4.8 \\
\hline & & t-test & & $15: 25$ & 40 & 130 & 13.0 & 37 & 25.561 & 4.8 \\
\hline & 0.000 & 15 & Total & $0: 40$ & 40 & & & & 25.561 & Max: \\
\hline & & & Average & & & 130 & 13.2 & 37 & & $4 . \overline{8}$ \\
\hline Set 3 & & test & Target: & $15: 34$ & 0 & 130 & 15.0 & 37 & 0.000 & 4.8 \\
\hline A-3434 & 0.000 & 15 & & $15: 39$ & 5 & 130 & 14.0 & 37 & $3 . \overline{251}$ & 4.8 \\
\hline A-3435 & & & & $15: 44$ & 10 & 130 & 13.0 & 38 & 6.003 & 4.8 \\
\hline A-3436 & & & & $15: 49$ & 15 & 130 & 13.0 & 38 & 9.136 & $4 . \overline{8}$ \\
\hline & & & & $15: 54$ & 20 & 130 & 14.0 & 38 & 11.487 & 4.8 \\
\hline & & & & $15: 59$ & 25 & 130 & 14.0 & 38 & 13.953 & 4.8 \\
\hline & & & & $16: 04$ & 30 & 130 & 14.0 & 38 & 16.616 & $4 . \overline{8}$ \\
\hline & & & & $16: 09$ & 35 & 130 & 14.0 & 38 & 19.331 & 4.8 \\
\hline & & & & $16: 14$ & 40 & 130 & 14.0 & 38 & 22.039 & 4.8 \\
\hline & & t-test & & $16: 18$ & 44 & 130 & 14.0 & 38 & 24.214 & 4.8 \\
\hline & 0.006 & 15 & Total & $0: 44$ & 44 & & & & 24.214 & Max: \\
\hline & & & Average & & & 130 & 13.9 & 38 & & 4.8 \\
\hline Set 4 & & test & Target: & $16: 26$ & 0 & 130 & 15.0 & 38 & 0.000 & 4.0 \\
\hline$A-3437$ & 0.006 & 15 & & $16: 31$ & 5 & 130 & 15.0 & 38 & 2.624 & 4.0 \\
\hline A-3438 & & & & $16: 36$ & 10 & 130 & 15.0 & 38 & 5.801 & 4.0 \\
\hline A-3439 & & & & $16: 41$ & 15 & 130 & 15.0 & 38 & 8.223 & 4.0 \\
\hline & & & & $16: 46$ & 20 & 130 & 15.0 & 39 & 10.998 & 4.0 \\
\hline & & & & $16: 51$ & 25 & 130 & 15.0 & 39 & 13.737 & 4.0 \\
\hline & & & & $16: 56$ & 30 & 130 & 15.0 & 39 & 16.501 & 4.0 \\
\hline & & & & $17: 01$ & 35 & 130 & 15.0 & 39 & 19.272 & 4.0 \\
\hline & & & & $17: 06$ & 40 & 130 & 15.0 & 40 & 22.038 & 4.0 \\
\hline & & t-test & & $17: 10$ & 44 & 130 & 15.0 & 40 & 24.227 & 4.0 \\
\hline & 0.006 & 15 & Total & $0: 44$ & 44 & & & & 24.227 & Max: \\
\hline & & & Average & & & 130 & 15.0 & 39 & & 4.0 \\
\hline Condensate & & & & Tonax R & nse: & Anasort & Rinse: & & & \\
\hline$A-3440$ & & & & & & NA & & & & \\
\hline $\begin{array}{r}\text { Final Conder } \\
40\end{array}$ & $\begin{array}{l}\text { isate Volt } \\
\mathrm{mL}\end{array}$ & & & $\begin{array}{l}\text { Tenax R } \\
\text { NA. }\end{array}$ & ase Vol.: & $\begin{array}{l}\text { Anasorb } \\
\text { NA }\end{array}$ & $\begin{array}{l}\text { Rinse Vol } \\
\text { mL }\end{array}$ & & & \\
\hline & & & & ADDITI & NAL INF & UTS & & & & \\
\hline & & & & Symbol & Units & Set 1 & Set 2 & Set 3 & Set 4 & Average \\
\hline Average Dr & Oxygen & oncentr & & Co2 & $\%$ & 20.5 & 20.5 & 20.5 & 20.5 & 20.5 \\
\hline Process Gas $F$ & flow (dry, & P) @ $68^{\circ} \mathrm{F}$ & & Qsd & $\mathrm{dscm} / \mathrm{sec}$ & 0.330 & 0.330 & 0.330 & 0.330 & 0.330 \\
\hline & & & $\overline{\mathbf{C}}$ & $\overline{A T E D ~ S A}$ & MPLING & $\overline{\text { ARAME }}$ & TERS & & & \\
\hline & & & & Symbol & Units & Set 1 & Set 2 & Set 3 & Set 4 & Net \\
\hline Sample Volu & me@ @t & dard Condi & tions & VmStd & dsL & 19.344 & 20.452 & 19.331 & 19.280 & 78.406 \\
\hline$V m S t d=17.647$ & ${ }^{*}{ }^{*} \mathrm{Pbar}{ }^{*}$ & $\mathrm{n} /(\mathrm{Fm}+460)$ & & & & & & & & \\
\hline Avg. Actuals & Sampling & ate, $Q m=V r$ & /min & $Q m$ & $\mathrm{~L} / \mathrm{min}$ & 0.536 & 0.639 & 0.550 & 0.551 & 0.569 \\
\hline Avg. Samplin & g Rate, & $\mathrm{nStd}=\mathrm{VmSt}$ & $\mathrm{d} / \mathrm{min}$ & QmStd & $\mathrm{dsL} / \mathrm{min}$ & 0.430 & 0.511 & 0.439 & 0.438 & 0.455 \\
\hline
\end{tabular}




\begin{tabular}{|c|c|c|c|c|c|}
\hline Table B-8. 0031-END-2. & $\begin{array}{r}\mathrm{C} \\
\text { micrograms }\end{array}$ & $\begin{array}{l}\text { DNCENTRATIONS } \\
\text { per dry standard cubi }\end{array}$ & meter & & \\
\hline $\begin{array}{l}\text { Project: } \\
\text { Run Date: } \\
\text { Run Identification: }\end{array}$ & $\begin{array}{c}01-1062-01- \\
6 / 21 / 2001 \\
0031-E N D-2\end{array}$ & $\begin{array}{l}\text { Lab Report Date: } \\
\text { Lab Report Status: }\end{array}$ & $\begin{array}{c}08 / 20 / 01 \\
\text { Final }\end{array}$ & & \\
\hline Analyte & & & & $\begin{array}{l}\text { Rur } \\
\text { Flag } \\
\end{array}$ & $\begin{array}{l}\text { Total } \\
\mu \mathrm{g} / \mathrm{dscm}\end{array}$ \\
\hline Acetone & & & & $<, J, B$ & $4.8 \mathrm{e} 1$ \\
\hline Acrylonitrile & & & & $<$ & $3.6 \mathrm{e} 1$ \\
\hline Benzene & & & & $<$ & $2.6 \mathrm{e} 0$ \\
\hline Bromobenzene & & & & $<$ & $1.5 \mathrm{e} 0$ \\
\hline Bromochloromethane & & & & $<$ & $1.9 \mathrm{e} 0$ \\
\hline Bromodichloromethane & & & & $<$ & $1.5 \mathrm{e} 0$ \\
\hline Bromoform & & & & $<$ & $2.3 \mathrm{e} 0$ \\
\hline Bromomethane & & & & $\mathrm{J}$ & $2.6 \mathrm{e} 0$ \\
\hline 2-Butanone & & & & $<$ & $1.1 \mathrm{e} 1$ \\
\hline n-Butylbenzene & & & & $<$ & $1.9 \mathrm{e} 0$ \\
\hline sec-Butylbenzene & & & & $<$ & $1.1 \mathrm{e} 0$ \\
\hline tert-Butylbenzene & & & & $<$ & $1.8 \mathrm{e} 0$ \\
\hline Carbon disulfide & & & & $<$ & $8.2 \mathrm{e} 0$ \\
\hline Carbon tetrachloride & & & & $<, \mathrm{J}$ & $2.0 \mathrm{e} 0$ \\
\hline Chlorobenzene & & & & $<, \mathrm{J}$ & $1.2 \mathrm{e} 0$ \\
\hline Chlorodibromomethane & & & & $<$ & $1.9 \mathrm{e} 0$ \\
\hline Chloroethane & & & & $<, \mathrm{J}$ & $2.0 \mathrm{e} 0$ \\
\hline Chloroform & & & & $<$ & $5.0 \mathrm{e} 0$ \\
\hline Chloromethane & & & $\cdots$ & $<, \mathrm{J}$ & $3.2 \mathrm{e} 1$ \\
\hline 2-Chlorotoluene & & & & $<$ & $7.5 \mathrm{e}-1$ \\
\hline 4-Chlorotoluene & & & & $\leq$ & $7.5 \mathrm{e}-1$ \\
\hline 1,2-Dibromo-3-chloropropane & & & 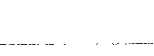 & $<$ & $3.4 \mathrm{e} 0$ \\
\hline 1,2-Dibromoethane & & & & $<$ & $2.6 \mathrm{e} 0$ \\
\hline Dibromomethane & & & & $<$ & $2.2 \mathrm{e} 0$ \\
\hline 1,2-Dichlorobenzene & & & - & $<$ & $2.3 \mathrm{e} 0$ \\
\hline 1,3-Dichlorobenzene & & & & $<$ & $1.3 \mathrm{e} 0$ \\
\hline 1,4-Dichlorobenzene & & & $\ldots$ & $<$ & $1.8 \mathrm{e} 0$ \\
\hline $\begin{array}{l}\text { Dichlorodifluoromethane } \\
\text { 1,1-Dichloroethane }\end{array}$ & & & & $\begin{array}{l}<, J \\
< \\
<\end{array}$ & $\begin{array}{l}2.0 \mathrm{e} 0 \\
1.9 \mathrm{e} 0\end{array}$ \\
\hline 1,2-Dichloroethane & & & & $<$ & $2.0 \mathrm{e} 0$ \\
\hline 1,1-Dichloroethene & & & & $<, J$ & $2.0 \mathrm{e} 0$ \\
\hline cis-1,2-Dichloroethene & & & & $<$ & $1.9 \mathrm{e} 0$ \\
\hline trans-1,2-Dichloroethene & & & & $<$ & $2.2 \mathrm{e} 0$ \\
\hline 1,2-Dichloropropane & & & & $<$ & $1.7 \mathrm{e} 0$ \\
\hline 1,3-Dichloropropane & & & & $<$ & $2.2 \mathrm{e} 0$ \\
\hline 2,2-Dichloropropane & & & & $<$ & $2.0 \mathrm{e} 0$ \\
\hline 1,1-Dichloropropene & & & & $<$ & $2.3 \mathrm{e} 0$ \\
\hline cis-1,3-Dichloropropene & & & & $<$ & $1.7 \mathrm{e} 0$ \\
\hline trans-1,3-Dichloropropene & & & & $<$ & $1.9 \mathrm{e} 0$ \\
\hline Ethylbenzene & & & & $<$ & $1.2 \mathrm{e} 0$ \\
\hline Hexachlorobutadiene & & & & $<$ & $2.9 \mathrm{e} 0$ \\
\hline 2-Hexanone & & & & $<$ & $7.1 \mathrm{e} 0$ \\
\hline Isopropylbenzene & & & & $<$ & $8.9 e-1$ \\
\hline p-Isopropyltoluene & & & & $<$ & $1.4 \mathrm{e} 0$ \\
\hline Methylene chloride & & & & $<, \mathrm{J}, \mathrm{B}$ & $5.5 \mathrm{e} 0$ \\
\hline
\end{tabular}

B8. 2 - 5 


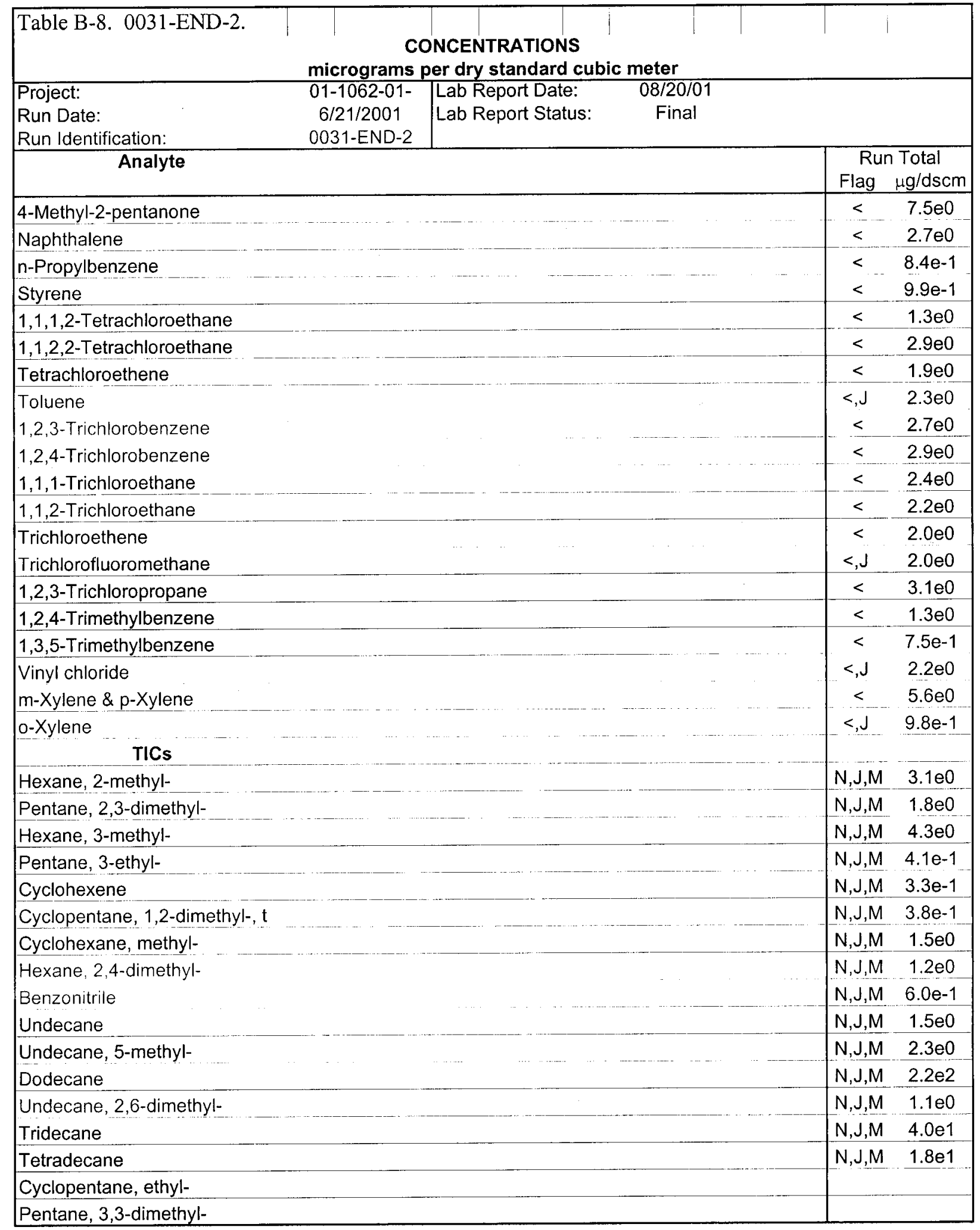

B8. 3 - 5 


\begin{tabular}{|c|c|c|c|c|}
\hline Table B-8. 0031-END-2. & $\begin{array}{l}\text { MASS FLOW RATE } \\
\text { grams per second }\end{array}$ & & & \\
\hline $\begin{array}{l}\text { Project: } \\
\text { Run Date: } \\
\text { Run Identification: } \\
\end{array}$ & 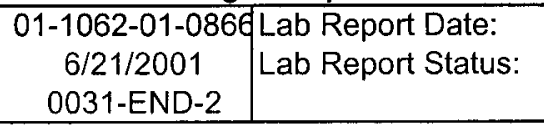 & $\begin{array}{c}08 / 20 / 01 \\
\text { Final }\end{array}$ & & \\
\hline Analyte & & & \begin{tabular}{|l} 
Run \\
Flag \\
\end{tabular} & $\begin{array}{l}\text { Total } \\
\text { g/sec }\end{array}$ \\
\hline Acetone & & & $<, \mathrm{J}, \mathrm{B}$ & $1.6 e-5$ \\
\hline Acrylonitrile & & & $<$ & $1.2 e-5$ \\
\hline Benzene & & & $<$ & $8.4 e-7$ \\
\hline Bromobenzene & & & $<$ & $5.0 \mathrm{e}-7$ \\
\hline Bromochloromethane & & & $<$ & $6.3 e-7$ \\
\hline Bromodichloromethane & & & $<$ & $5.0 \mathrm{e}-7$ \\
\hline Bromoform & & & $<$ & $7.6 \mathrm{e}-7$ \\
\hline Bromomethane & & & J & $8.4 \mathrm{e}-7$ \\
\hline 2-Butanone & & & $<$ & $3.7 \mathrm{e}-6$ \\
\hline n-Butylbenzene & & & $<$ & $6.3 e-7$ \\
\hline sec-Butylbenzene & & & $<$ & $3.6 \mathrm{e}-7$ \\
\hline tert-Butylbenzene & & & $<$ & $5.9 e-7$ \\
\hline Carbon disulfide & & & $<$ & $2.7 e-6$ \\
\hline Carbon tetrachloride & & & $<, \mathrm{J}$ & $6.7 e-7$ \\
\hline Chlorobenzene & & & $<, \mathrm{J}$ & $4.0 \mathrm{e}-7$ \\
\hline Chlorodibromomethane & & & $<$ & $6.3 e-7$ \\
\hline Chloroethane & & & $<, J$ & $6.7 e-7$ \\
\hline Chloroform & & & $<$ & $1.6 \mathrm{e}-6$ \\
\hline Chloromethane & & & $<, \mathrm{J}$ & $1.1 e-5$ \\
\hline 2-Chlorotoluene & & & $<$ & $2.5 \mathrm{e}-7$ \\
\hline 4-Chlorotoluene & & & $<$ & $2.5 \mathrm{e}-7$ \\
\hline 1,2-Dibromo-3-chloropropane & & & $<$ & $1.1 \mathrm{e}-6$ \\
\hline 1,2-Dibromoethane & & & $<$ & $8.4 \mathrm{e}-7$ \\
\hline Dibromomethane & & & $<$ & $7.1 \mathrm{e}-7$ \\
\hline 1,2-Dichlorobenzene & & & $<$ & $7.6 \mathrm{e}-7$ \\
\hline 1,3-Dichlorobenzene & & & $<$ & $4.2 e-7$ \\
\hline 1,4-Dichlorobenzene & & & $<$ & $5.9 \mathrm{e}-7$ \\
\hline Dichlorodifluoromethane & & & $<, J$ & $6.7 \mathrm{e}-7$ \\
\hline 1,1-Dichloroethane & & & $<$ & $6.3 \mathrm{e}-7$ \\
\hline 1,2-Dichloroethane & & & $<$ & $6.7 e-7$ \\
\hline 1,1-Dichloroethene & & & $<, \mathrm{J}$ & $6.7 \mathrm{e}-7$ \\
\hline cis-1,2-Dichloroethene & & & $<$ & $6.3 e-7$ \\
\hline trans-1,2-Dichloroethene & & & $<$ & $7.1 e-7$ \\
\hline 1,2-Dichloropropane & & & $<$ & $5.5 e-7$ \\
\hline 1,3-Dichloropropane & & & $<$ & $7.1 \mathrm{e}-7$ \\
\hline 2,2-Dichloropropane & & & $<$ & $6.7 \mathrm{e}-7$ \\
\hline 1,1-Dichloropropene & & & $<$ & $7.6 e-7$ \\
\hline cis-1,3-Dichloropropene & & & $<$ & $5.5 e-7$ \\
\hline trans-1,3-Dichloropropene & & & $<$ & $6.3 e-7$ \\
\hline Ethylbenzene & & & $<$ & $3.9 e-7$ \\
\hline Hexachlorobutadiene & & & $<$ & $9.7 e-7$ \\
\hline 2-Hexanone & & & $<$ & $2.4 \mathrm{e}-6$ \\
\hline Isopropylbenzene & & & $<$ & $2.9 \mathrm{e}-7$ \\
\hline p-Isopropyltoluene & & & $<$ & $4.6 \mathrm{e}-7$ \\
\hline Methylene chloride & & & $<, \mathrm{J}, \mathrm{B}$ & $1.8 \mathrm{e}-6$ \\
\hline
\end{tabular}

B8. 4 - 5 


\begin{tabular}{|c|c|c|c|c|}
\hline Table B-8. 0031-END-2. & $\begin{array}{l}\text { MASS FLOW RATE } \\
\text { grams per second }\end{array}$ & & & \\
\hline $\begin{array}{l}\text { Project: } \\
\text { Run Date: } \\
\text { Run Identification: } \\
\end{array}$ & \begin{tabular}{c|c}
$01-1062-01-0866$ & Lab Report Date: \\
$6 / 21 / 2001$ & Lab Report Status: \\
$0031-E N D-2$ & \\
\end{tabular} & $\begin{array}{c}08 / 20 / 01 \\
\text { Final }\end{array}$ & & \\
\hline Analyte & & & $\begin{array}{l}\text { Rur } \\
\text { Flag }\end{array}$ & $\begin{array}{l}\text { Total } \\
\text { g/sec }\end{array}$ \\
\hline $\begin{array}{l}\text { 4-Methyl-2-pentanone } \\
\text { Naphthalene }\end{array}$ & & & $\begin{array}{l}< \\
<\end{array}$ & $\begin{array}{l}2.5 e-6 \\
8.8 e-7\end{array}$ \\
\hline n-Propylbenzene & & & $<$ & $2.8 \mathrm{e}-7$ \\
\hline Styrene & & & $<$ & $3.3 e-7$ \\
\hline 1,1,1,2-Tetrachloroethane & & & $<$ & $4.2 \mathrm{e}-7$ \\
\hline 1,1,2,2-Tetrachloroethane & & & $<$ & $9.7 e-7$ \\
\hline Tetrachloroethene & & & $<$ & $6.3 e-7$ \\
\hline Toluene & & & $<, J$ & $7.6 e-7$ \\
\hline 1,2,3-Trichlorobenzene & & & $<$ & $8.8 \mathrm{e}-7$ \\
\hline 1,2,4-Trichlorobenzene & & & $<$ & $9.7 e-7$ \\
\hline 1,1,1-Trichloroethane & & & $<$ & $8.0 \mathrm{e}-7$ \\
\hline 1,1,2-Trichloroethane & & & $<$ & $7.1 \mathrm{e}-7$ \\
\hline Trichloroethene & & & $<$ & $6.7 e-7$ \\
\hline Trichlorofluoromethane & & & $<, J$ & $6.7 \mathrm{e}-7$ \\
\hline 1,2,3-Trichloropropane & & & $<$ & $1.0 \mathrm{e}-6$ \\
\hline 1,2,4-Trimethylbenzene & & & $<$ & $4.2 \mathrm{e}-7$ \\
\hline 1,3,5-Trimethylbenzene & & & $<$ & $2.5 \mathrm{e}-7$ \\
\hline $\begin{array}{l}\text { Vinyl chloride } \\
\text { m-Xylene \& p-Xylene }\end{array}$ & & 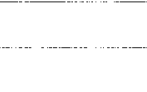 & {$\left[\begin{array}{c}<, J \\
<\end{array}\right.$} & $\begin{array}{l}7.1 e-7 \\
1.9 e-6\end{array}$ \\
\hline o-Xylene & & & $<, \mathrm{J}$ & $3.2 \mathrm{e}-7$ \\
\hline $\begin{array}{r}\text { TICs } \\
\text { Hexane, 2-methyl- }\end{array}$ & & & $\mathrm{N}, \mathrm{J}, \mathrm{M}$ & $1.0 e-6$ \\
\hline $\begin{array}{l}\text { Pentane, 2,3-dimethyl- } \\
\text { Hexane, 3-methyl- }\end{array}$ & & & $\begin{array}{l}N, J, M \\
N, J, M\end{array}$ & $\begin{array}{l}5.9 \mathrm{e}-7 \\
1.4 \mathrm{e}-6\end{array}$ \\
\hline Pentane, 3-ethyl- & & & $N, J, M$ & $1.3 e-7$ \\
\hline Cyclohexene & & & $\mathrm{N}, \mathrm{J}, \mathrm{M}$ & $1.1 \mathrm{e}-7$ \\
\hline Cyclopentane, 1,2-dimethyl-, $t$ & & & $\mathrm{~N}, \mathrm{~J}, \mathrm{M}$ & $1.3 \mathrm{e}-7$ \\
\hline Cyclohexane, methyl- & & & $\mathrm{N}, \mathrm{J}, \mathrm{M}$ & $5.0 \mathrm{e}-7$ \\
\hline Hexane, 2,4-dimethyl- & & & $N, J, M$ & $4.0 \mathrm{e}-7$ \\
\hline Benzonitrile & & & $\mathrm{N}, \mathrm{J}, \mathrm{M}$ & $2.0 \mathrm{e}-7$ \\
\hline Undecane & & & $N, J, M$ & $5.0 \mathrm{e}-7$ \\
\hline Undecane, 5-methyl- & & & $\mathrm{N}, \mathrm{J}, \mathrm{M}$ & $7.6 e-7$ \\
\hline Dodecane & & & $\mathrm{N}, \mathrm{J}, \mathrm{M}$ & $7.1 \mathrm{e}-5$ \\
\hline Undecane, 2,6-dimethyl- & & & $\mathrm{N}, \mathrm{J}, \mathrm{M}$ & $3.5 \mathrm{e}-7$ \\
\hline Tridecane & & & $\mathrm{N}, \mathrm{J}, \mathrm{M}$ & $1.3 e-5$ \\
\hline Tetradecane & & & $\mathrm{N}, \mathrm{J}, \mathrm{M}$ & $5.9 e-6$ \\
\hline Cyclopentane, ethyl- & & & & \\
\hline Pentane, 3,3-dimethyl- & & & & \\
\hline
\end{tabular}

B8. 5 - 5 



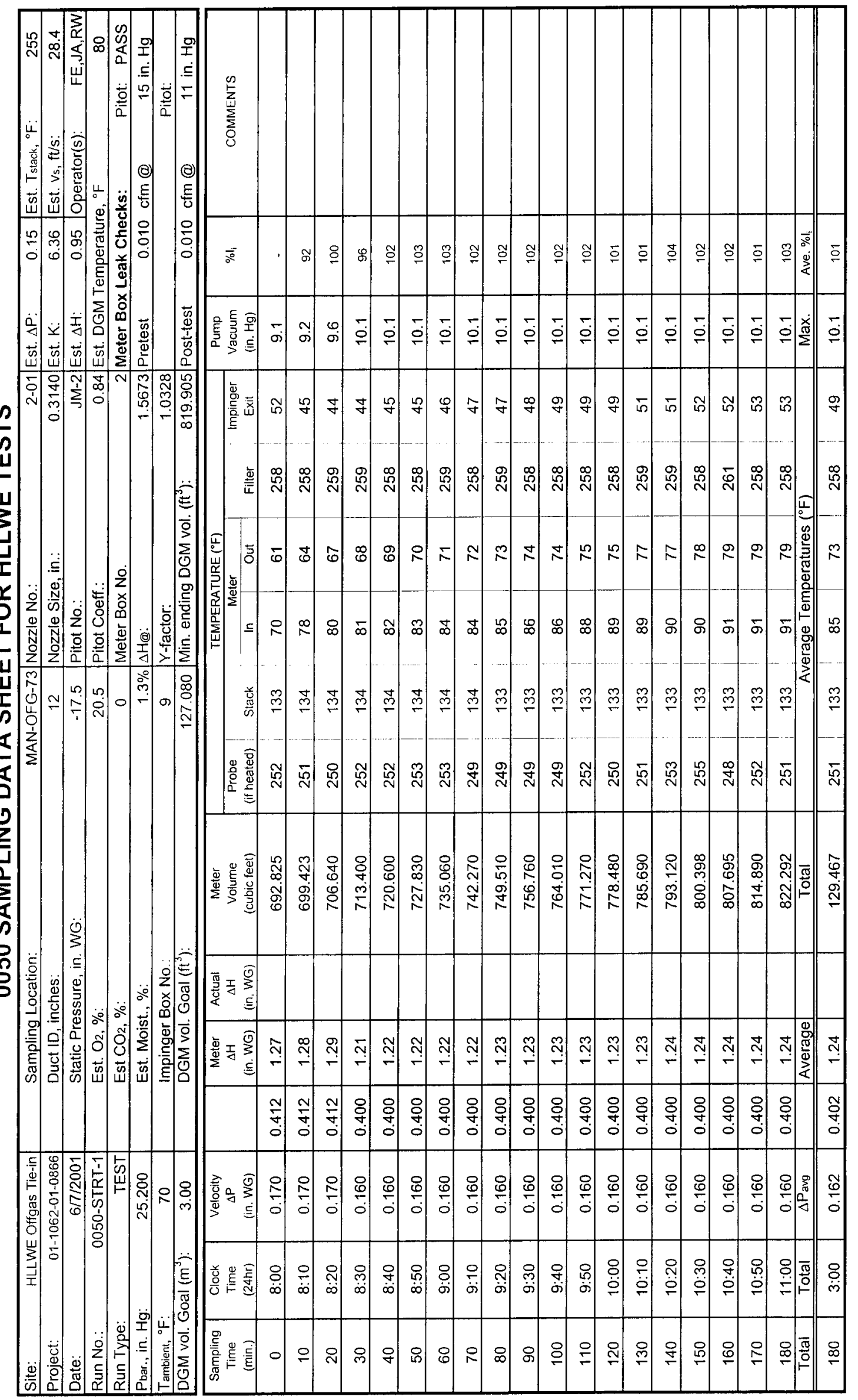




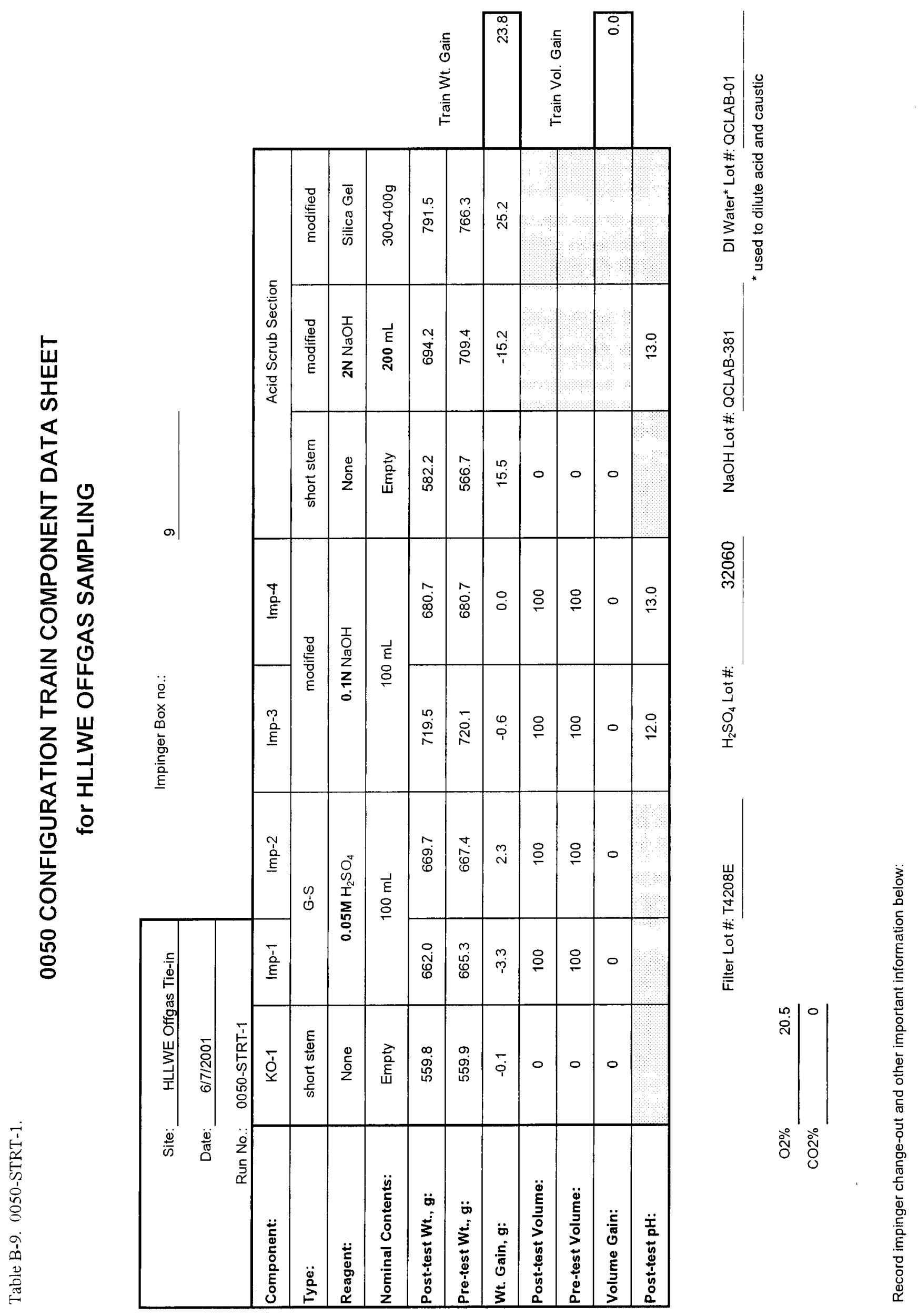


Table B-9. 0050-STRT-1.

0050 FIELD DATA CALCULATIONS

\begin{tabular}{|c|c|c|c|}
\hline $\begin{array}{r}\text { Project: } \\
\text { Run Date: } \\
\text { Run Identification: }\end{array}$ & $\begin{array}{r}01-106 \\
6 / 7 \\
0050-\end{array}$ & $\begin{array}{l}\text { D1-0866 } \\
001 \\
\text { TRT-1 }\end{array}$ & \\
\hline PARAMETER & SYMBOL & UNITS & \\
\hline Absolute Pressure in the Duct & Pabs & in. $\mathrm{Hg}$ & 23.913 \\
\hline Average Duct Gas Temperature & Ts & $\mathrm{R}$ & 593 \\
\hline Average Meter Temperature & $\mathrm{Tm}$ & $\mathrm{R}$ & 539 \\
\hline Average Gas Oxygen Content & $\mathrm{Co} 2, \mathrm{~m}$ & $\%$ & 20.5 \\
\hline Average Gas Carbon Dioxide Content & $\mathrm{Cco} 2, \mathrm{~m}$ & $\%$ & 0.0 \\
\hline Total Impinger Weight Gain (water) & WW & grams & 23.8 \\
\hline Nozzle Area & An & $\mathrm{ft}^{2}$ & 0.000538 \\
\hline Duct Area & As & $\mathrm{ft}^{2}$ & 0.7854 \\
\hline Sample Volume & VmStd & dscf & 110.729 \\
\hline Sample Volume (SI) & VmStdm & dscm & 3.136 \\
\hline Average Sampling Rate & Qm & $\mathrm{dscf} / \mathrm{m}$ & 0.615 \\
\hline Volume of Water Vapor & VwStd & scf & 1.122 \\
\hline Volume of Water Vapor (SI) & VwStdm & $\mathrm{scm}$ & 0.0318 \\
\hline Moisture Fraction & Bws & - & 0.010 \\
\hline Dry Gas Molecular Weight & Md & $\mathrm{g} / \mathrm{g}-\mathrm{mol}$ & 28.82 \\
\hline Wet Gas Molecular Weight & Ms & $\mathrm{g} / \mathrm{g}-\mathrm{mol}$ & 28.71 \\
\hline Gas Velocity at Nozzle & vn & $\mathrm{ft} / \mathrm{s}$ & 26.8 \\
\hline Gas Velocity at Nozzle (SI) & vnm & $\mathrm{m} / \mathrm{s}$ & 8.18 \\
\hline Average Gas Velocity & vncor & $\mathrm{ft} / \mathrm{s}$ & 22.64 \\
\hline Dry Offgas Flow Rate & Qsd & $\mathrm{dscf} / \mathrm{h}$ & 45,066 \\
\hline Dry Offgas Flow Rate (SI) & Qsdm & $\mathrm{dscm} / \mathrm{h}$ & 1,276 \\
\hline Actual Offgas Flow Rate & $Q$ & acf/h & 64,013 \\
\hline Intermediate Isokinetic Rate & li & $\%$ & 101.3 \\
\hline Final Isokinetic Rate & I & $\%$ & 101.0 \\
\hline
\end{tabular}


Table B-9. 0050-STRT-1.

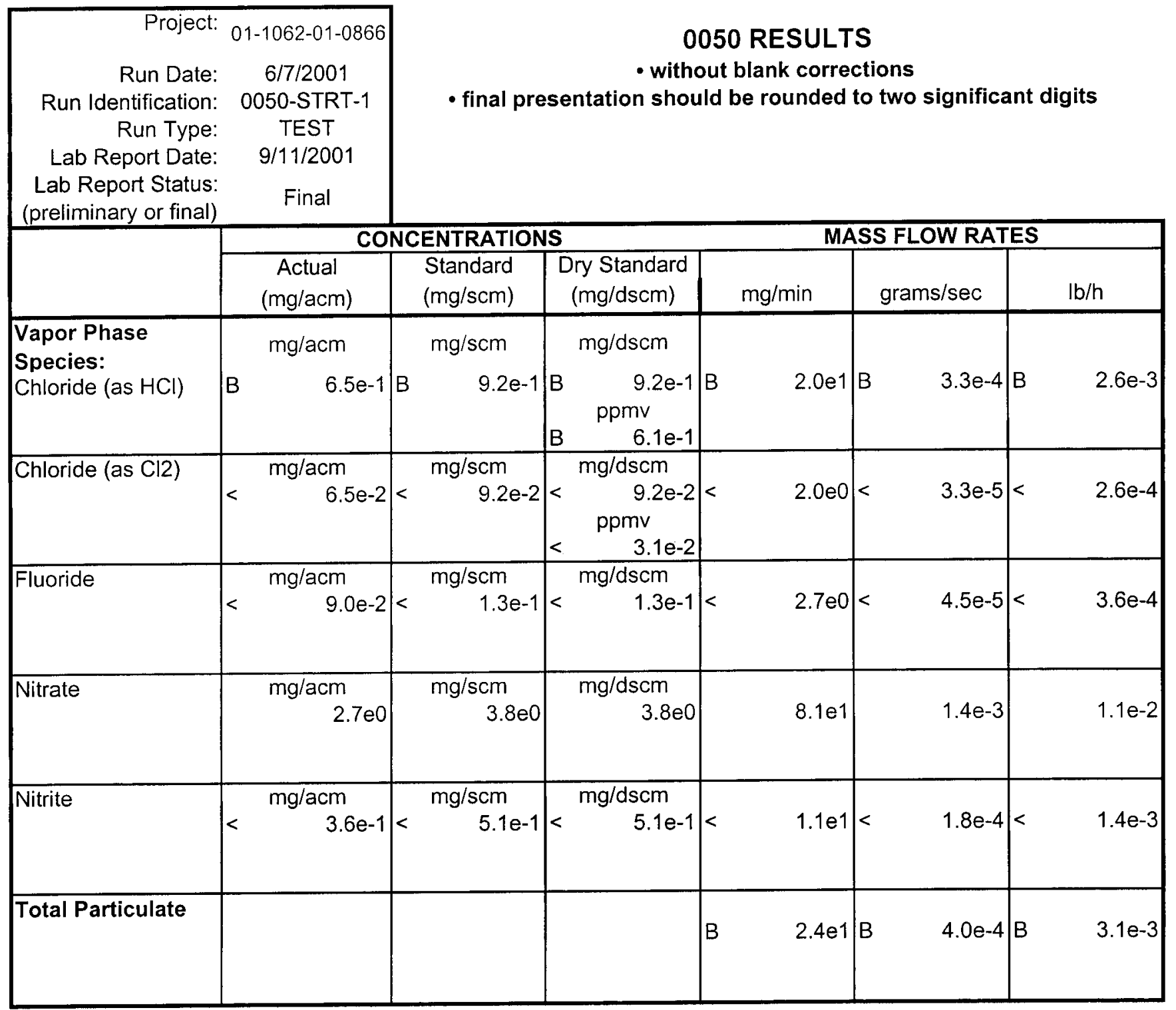




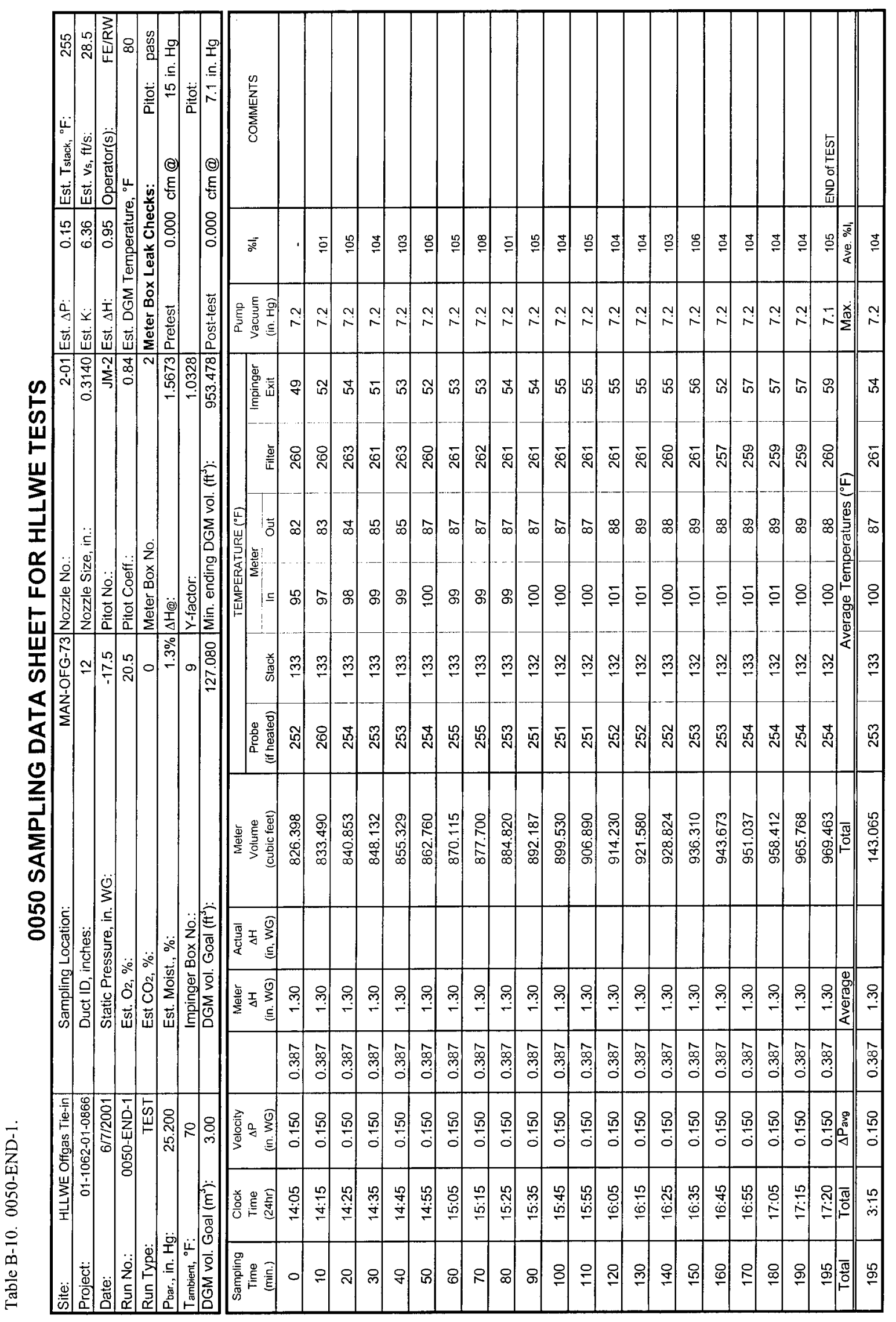




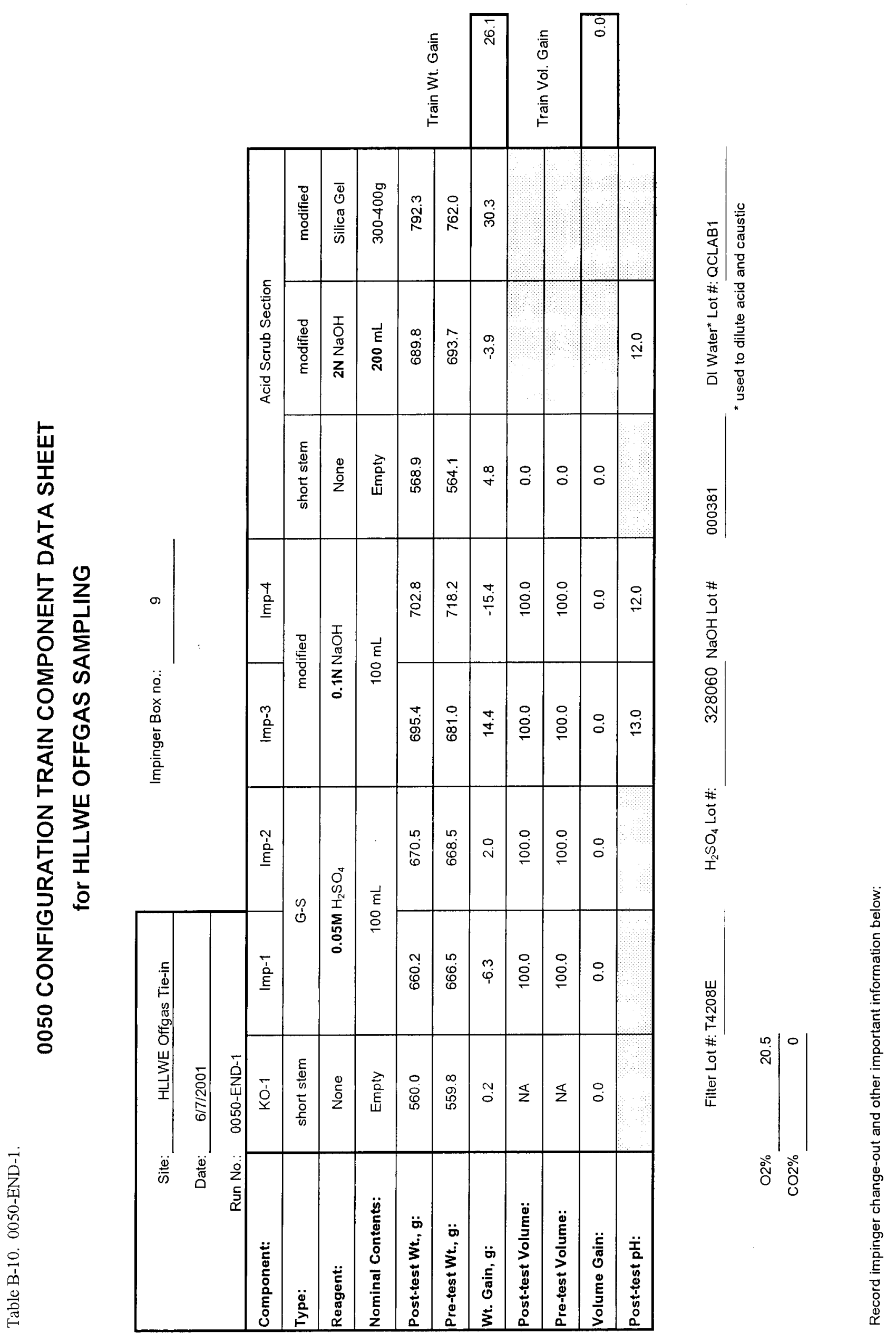


Table B-10. 0050-END-1.

\section{FIELD DATA CALCULATIONS}

\begin{tabular}{|c|c|c|c|}
\hline $\begin{array}{r}\text { Project: } \\
\text { Run Date: } \\
\text { Run Identification: }\end{array}$ & $\begin{array}{r}01-1062 \\
6 / 7 / \\
0050-\end{array}$ & $\begin{array}{l}01-0866 \\
001 \\
\text { ND-1 }\end{array}$ & \\
\hline PARAMETER & SYMBOL & UNITS & \\
\hline Absolute Pressure in the Duct & Pabs & in. $\mathrm{Hg}$ & 23.913 \\
\hline Average Duct Gas Temperature & Ts & $\mathrm{R}$ & 592 \\
\hline Average Meter Temperature & $\mathrm{Tm}$ & $\mathrm{R}$ & 553 \\
\hline Average Gas Oxygen Content & $\mathrm{Co} 2, \mathrm{~m}$ & $\%$ & 20.5 \\
\hline Average Gas Carbon Dioxide Content & $\mathrm{Cco} 2, \mathrm{~m}$ & $\%$ & 0.0 \\
\hline Total Impinger Weight Gain (water) & Ww & grams & 26.1 \\
\hline Nozzle Area & An & $\mathrm{ft}^{2}$ & 0.000538 \\
\hline Duct Area & As & $\mathrm{ft}^{2}$ & 0.7854 \\
\hline Sample Volume & VmStd & dscf & 119.218 \\
\hline Sample Volume (SI) & VmStdm & $\mathrm{dscm}$ & 3.376 \\
\hline Average Sampling Rate & Qm & $\mathrm{dscf} / \mathrm{m}$ & 0.611 \\
\hline Volume of Water Vapor & VwStd & scf & 1.231 \\
\hline Volume of Water Vapor (SI) & VwStdm & $\mathrm{scm}$ & 0.0348 \\
\hline Moisture Fraction & Bws & - & 0.010 \\
\hline Dry Gas Molecular Weight & Md & $\mathrm{g} / \mathrm{g}-\mathrm{mol}$ & 28.82 \\
\hline Wet Gas Molecular Weight & Ms & $\mathrm{g} / \mathrm{g}-\mathrm{mol}$ & 28.71 \\
\hline Gas Velocity at Nozzle & $\mathrm{vn}$ & $\mathrm{ft} / \mathrm{s}$ & 25.8 \\
\hline Gas Velocity at Nozzle (SI) & $\mathrm{vnm}$ & $\mathrm{m} / \mathrm{s}$ & 7.87 \\
\hline Average Gas Velocity & vncor & $\mathrm{ft} / \mathrm{s}$ & 21.80 \\
\hline Dry Offgas Flow Rate & Qsd & $\mathrm{dscf} / \mathrm{h}$ & 43,448 \\
\hline Dry Offgas Flow Rate (SI) & Qsdm & $\mathrm{dscm} / \mathrm{h}$ & 1,230 \\
\hline Actual Offgas Flow Rate & $\mathrm{Q}$ & acf/h & 61,639 \\
\hline Intermediate Isokinetic Rate & li & $\%$ & 104.4 \\
\hline Final Isokinetic Rate & I & $\%$ & 104.1 \\
\hline
\end{tabular}


Table B-10. 0050-END-1.

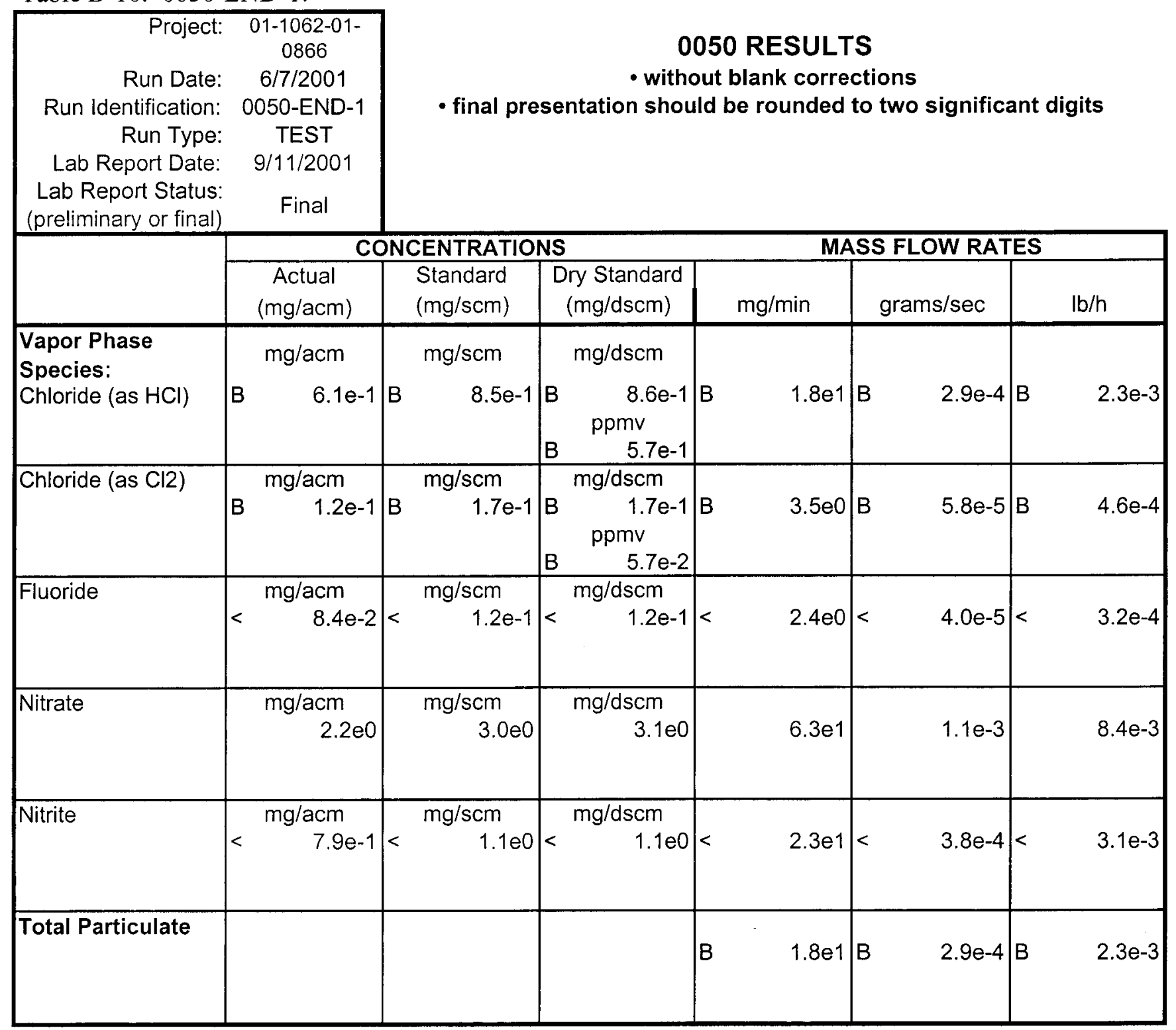




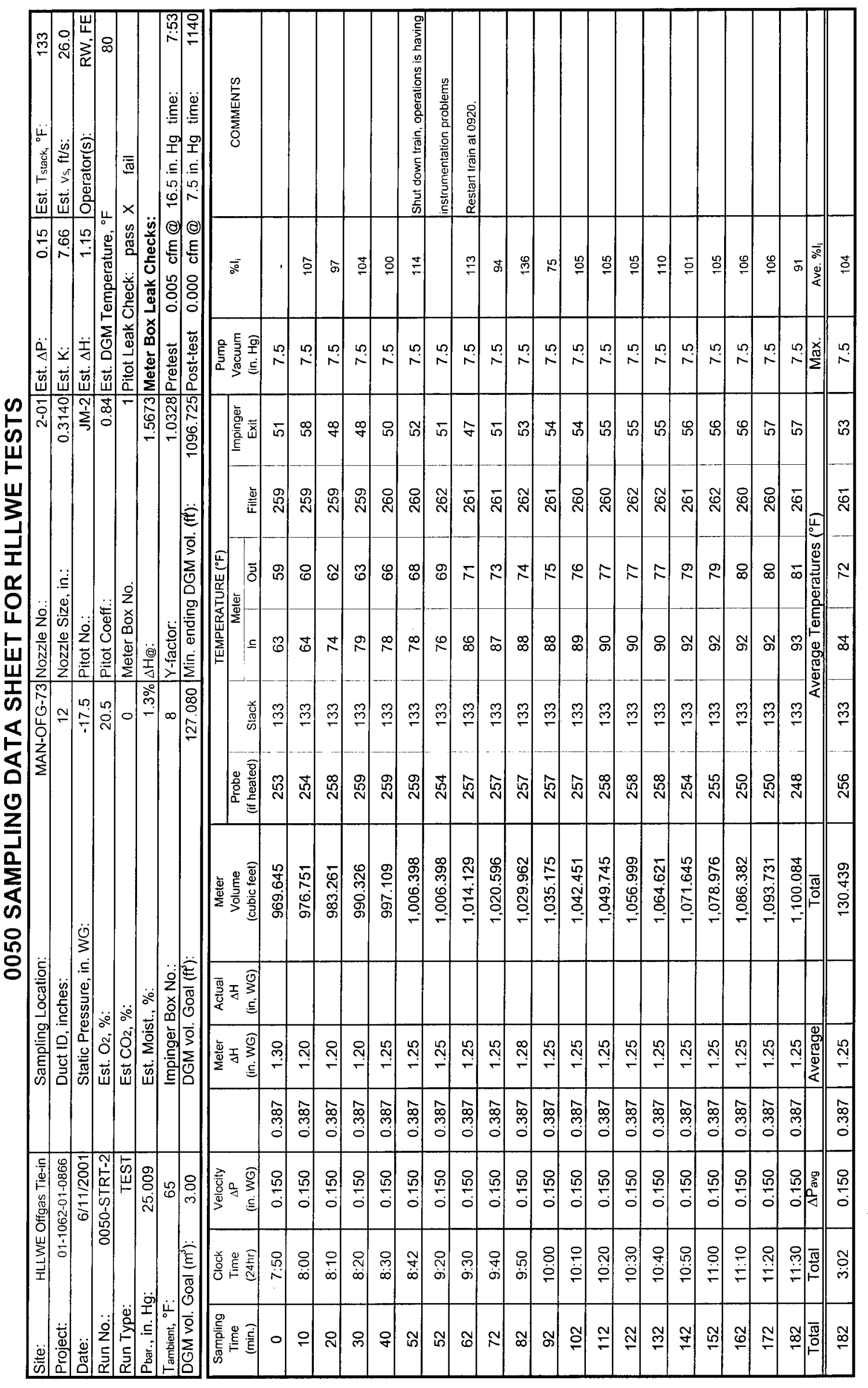




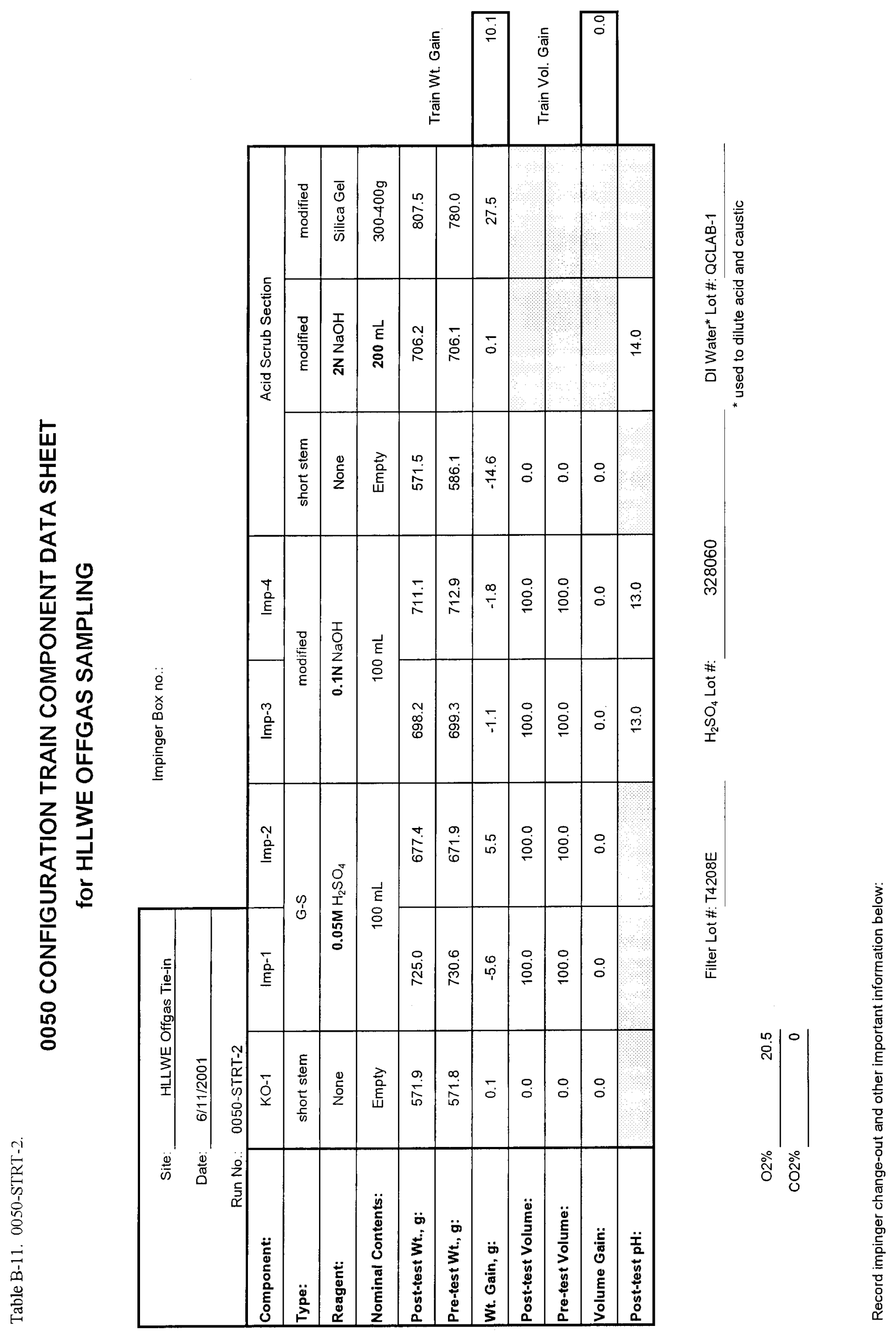


Table B-11. 0050-STRT-2.

\section{FIELD DATA CALCULATIONS}

\begin{tabular}{|c|c|c|c|}
\hline $\begin{array}{r}\text { Project: } \\
\text { Run Date: } \\
\text { Run Identification: }\end{array}$ & $\begin{array}{r}01-1062 \\
6 / 11 \\
0050-5\end{array}$ & $\begin{array}{l}01-0866 \\
001 \\
\text { TRT-2 }\end{array}$ & \\
\hline PARAMETER & SYMBOL & UNITS & \\
\hline Absolute Pressure in the Duct & Pabs & in. $\mathrm{Hg}$ & 23.722 \\
\hline Average Duct Gas Temperature & Ts & $\mathrm{R}$ & 593 \\
\hline Average Meter Temperature & $\mathrm{Tm}$ & $\mathrm{R}$ & 538 \\
\hline Average Gas Oxygen Content & $\mathrm{Co} 2, \mathrm{~m}$ & $\%$ & 20.5 \\
\hline Average Gas Carbon Dioxide Content & $\mathrm{Cco} 2, \mathrm{~m}$ & $\%$ & 0.0 \\
\hline Total Impinger Weight Gain (water) & Ww & grams & 10.1 \\
\hline Nozzle Area & An & $\mathrm{ft}^{2}$ & 0.000538 \\
\hline Duct Area & As & $\mathrm{ft}^{2}$ & 0.7854 \\
\hline Sample Volume & VmStd & dscf & 110.880 \\
\hline Sample Volume (SI) & VmStdm & $\mathrm{dscm}$ & 3.140 \\
\hline Average Sampling Rate & Qm & $\mathrm{dscf} / \mathrm{m}$ & 0.609 \\
\hline Volume of Water Vapor & VwStd & scf & 0.476 \\
\hline Volume of Water Vapor (SI) & VwStdm & $\mathrm{scm}$ & 0.0135 \\
\hline Moisture Fraction & Bws & - & 0.004 \\
\hline Dry Gas Molecular Weight & Md & $\mathrm{g} / \mathrm{g}-\mathrm{mol}$ & 28.82 \\
\hline Wet Gas Molecular Weight & Ms & $\mathrm{g} / \mathrm{g}-\mathrm{mol}$ & 28.77 \\
\hline Gas Velocity at Nozzle & $\mathrm{vn}$ & $\mathrm{ft} / \mathrm{s}$ & 25.9 \\
\hline Gas Velocity at Nozzle (SI) & vnm & $\mathrm{m} / \mathrm{s}$ & 7.90 \\
\hline Average Gas Velocity & vncor & $\mathrm{ft} / \mathrm{s}$ & 21.87 \\
\hline Dry Offgas Flow Rate & Qsd & $\mathrm{dscf} / \mathrm{h}$ & 43,468 \\
\hline Dry Offgas Flow Rate (SI) & Qsdm & $\mathrm{dscm} / \mathrm{h}$ & 1,231 \\
\hline Actual Offgas Flow Rate & $Q$ & acf $/ \mathrm{h}$ & 61,842 \\
\hline Intermediate Isokinetic Rate & li & $\%$ & 104.6 \\
\hline Final Isokinetic Rate & I & $\%$ & 103.7 \\
\hline
\end{tabular}


Table B-11. 0050-STRT-2.

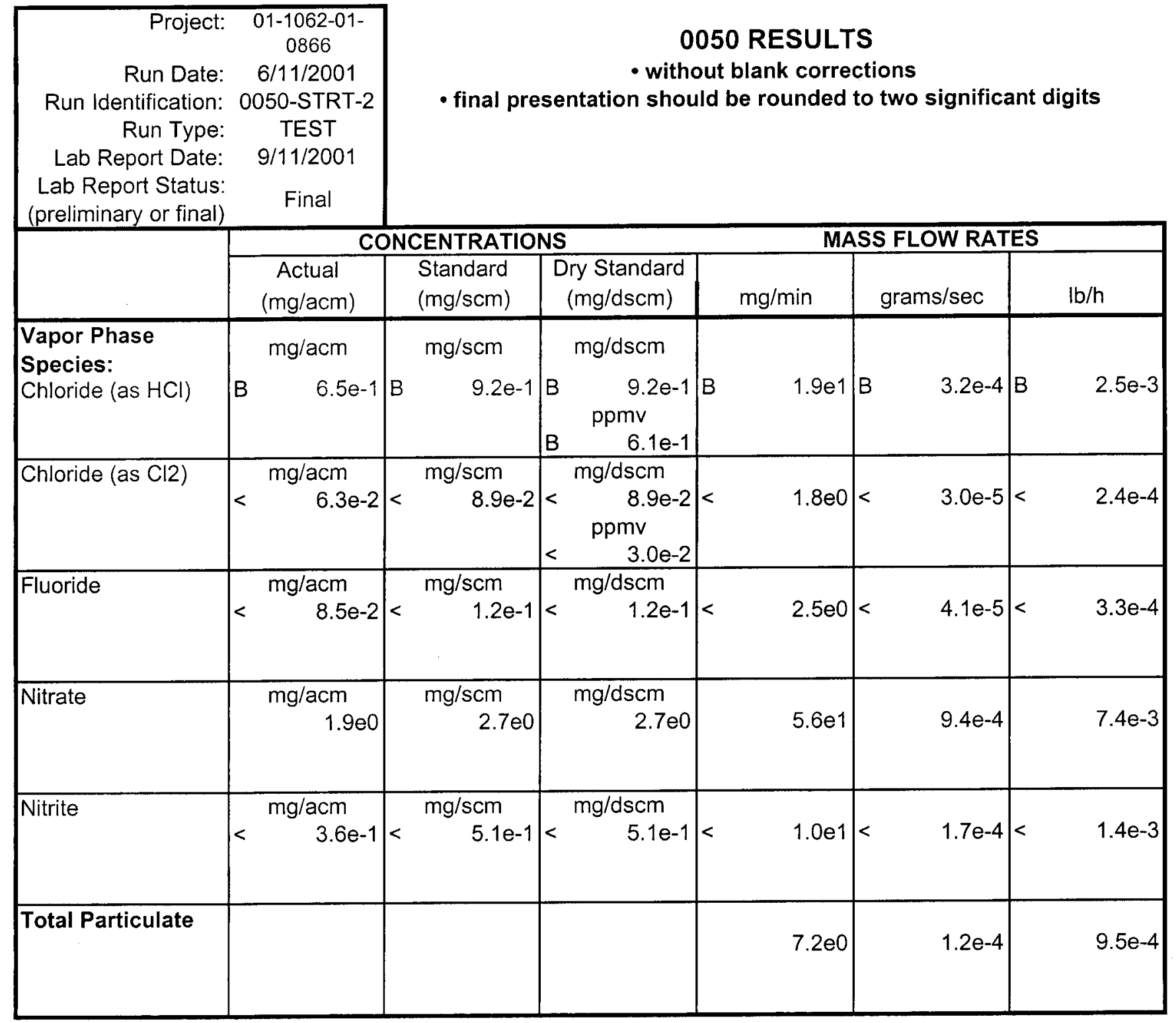




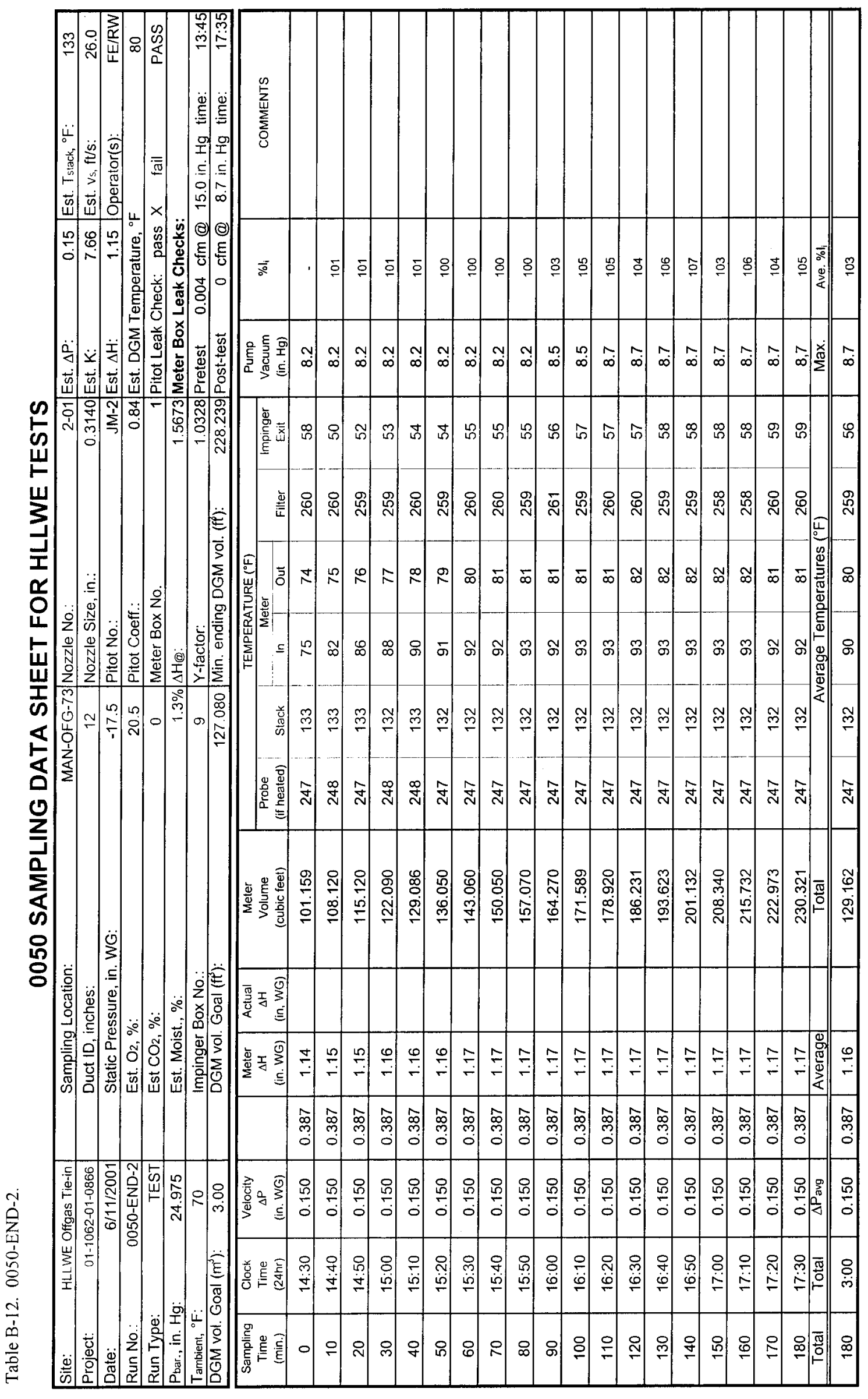




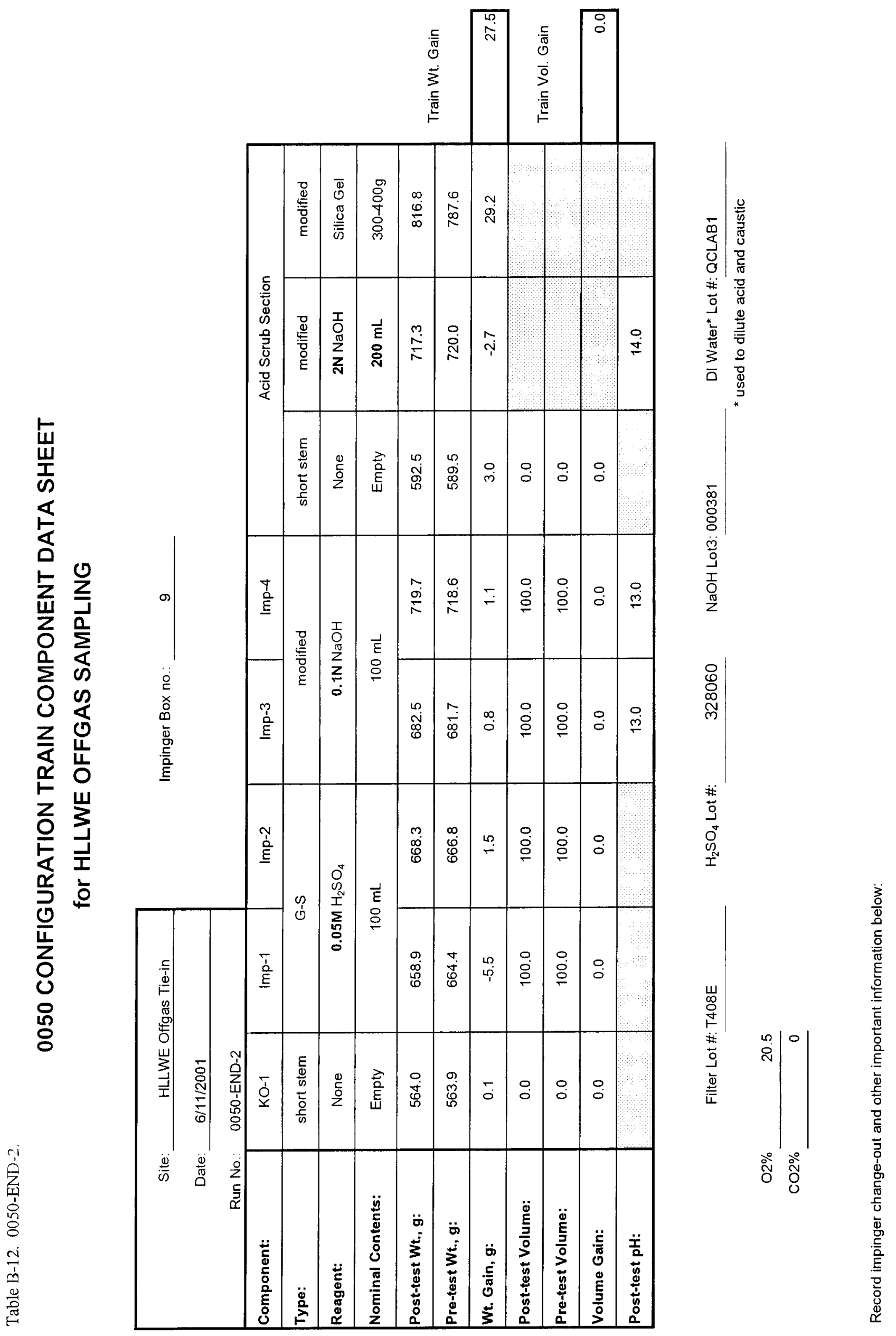


Table B-12. 0050-END-2.

\section{FIELD DATA CALCULATIONS}

\begin{tabular}{|c|c|c|c|}
\hline $\begin{array}{r}\text { Project: } \\
\text { Run Date: } \\
\text { Run Identification: }\end{array}$ & $\begin{array}{r}01-1062 \\
6 / 11 \\
0050\end{array}$ & $\begin{array}{l}11-0866 \\
001 \\
\text { ND-2 }\end{array}$ & \\
\hline PARAMETER & SYMBOL & UNITS & \\
\hline Absolute Pressure in the Duct & Pabs & in. $\mathrm{Hg}$ & 23.688 \\
\hline Average Duct Gas Temperature & Ts & $\mathrm{R}$ & 592 \\
\hline Average Meter Temperature & $\mathrm{Tm}$ & $\mathrm{R}$ & 545 \\
\hline Average Gas Oxygen Content & $\mathrm{Co} 2, \mathrm{~m}$ & $\%$ & 20.5 \\
\hline Average Gas Carbon Dioxide Content & $\mathrm{Cco} 2, \mathrm{~m}$ & $\%$ & 0.0 \\
\hline Total Impinger Weight Gain (water) & Ww & grams & 27.5 \\
\hline Nozzle Area & An & $\mathrm{ft}^{2}$ & 0.000538 \\
\hline Duct Area & As & $\mathrm{ft}^{2}$ & 0.7854 \\
\hline Sample Volume & VmStd & dscf & 108.235 \\
\hline Sample Volume (SI) & VmStdm & dscm & 3.065 \\
\hline Average Sampling Rate & Qm & $\mathrm{dscf} / \mathrm{m}$ & 0.601 \\
\hline Volume of Water Vapor & VwStd & scf & 1.297 \\
\hline Volume of Water Vapor (SI) & VwStdm & $\mathrm{scm}$ & 0.0367 \\
\hline Moisture Fraction & Bws & - & 0.012 \\
\hline Dry Gas Molecular Weight & Md & g/g-mol & 28.82 \\
\hline Wet Gas Molecular Weight & Ms & g/g-mol & 28.69 \\
\hline Gas Velocity at Nozzle & $\mathrm{vn}$ & $\mathrm{ft} / \mathrm{s}$ & 26.0 \\
\hline Gas Velocity at Nozzle (SI) & $\mathrm{vnm}$ & $\mathrm{m} / \mathrm{s}$ & 7.91 \\
\hline Average Gas Velocity & vncor & $\mathrm{ft} / \mathrm{s}$ & 21.90 \\
\hline Dry Offgas Flow Rate & Qsd & dscf/h & 43,197 \\
\hline Dry Offgas Flow Rate (SI) & Qsdm & $\mathrm{dscm} / \mathrm{h}$ & 1,223 \\
\hline Actual Offgas Flow Rate & $Q$ & acf/h & 61,934 \\
\hline Intermediate Isokinetic Rate & li & $\%$ & 103.1 \\
\hline Final Isokinetic Rate & 1 & $\%$ & 103.0 \\
\hline
\end{tabular}


Table B-12. 0050-END-2.

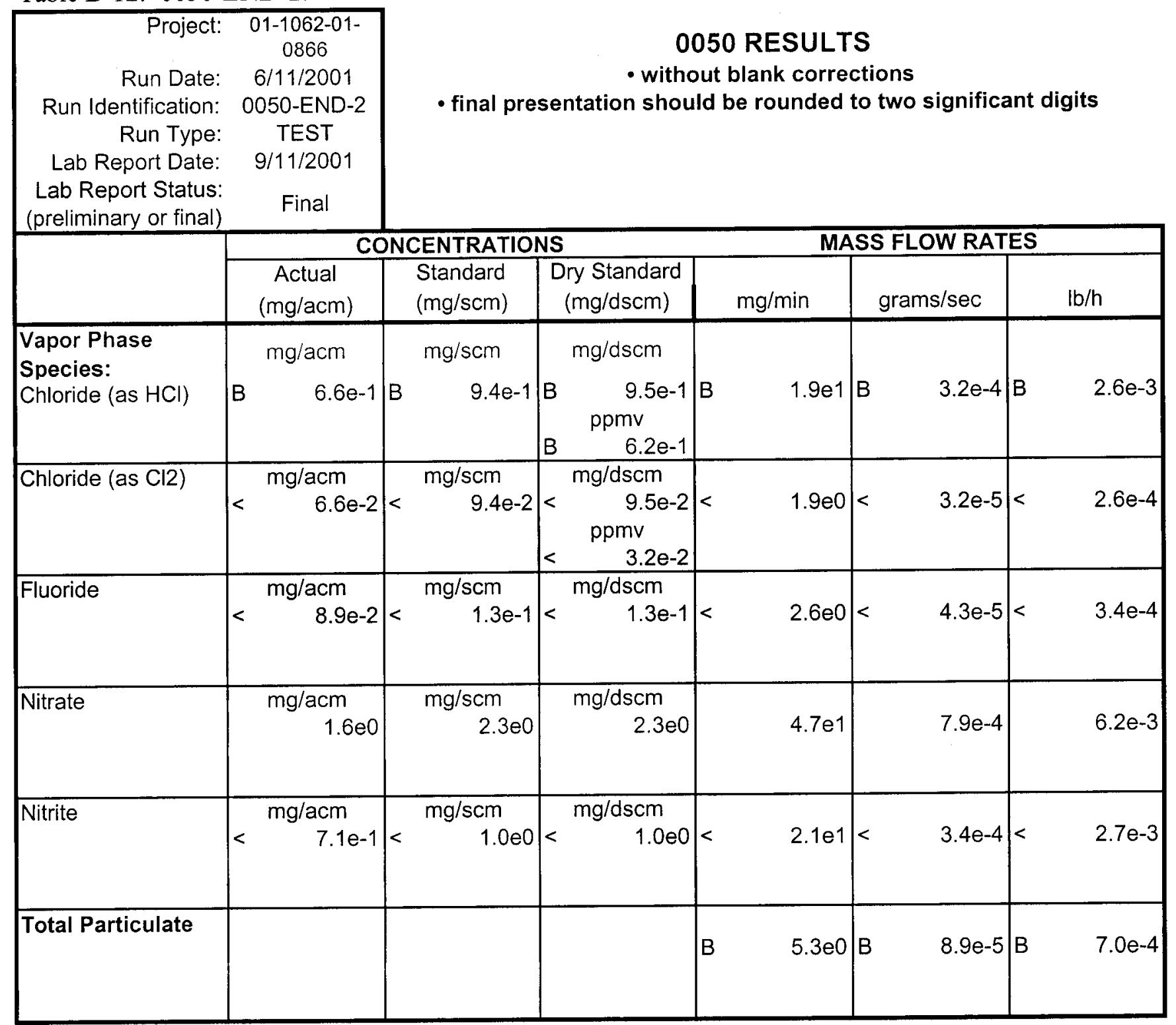




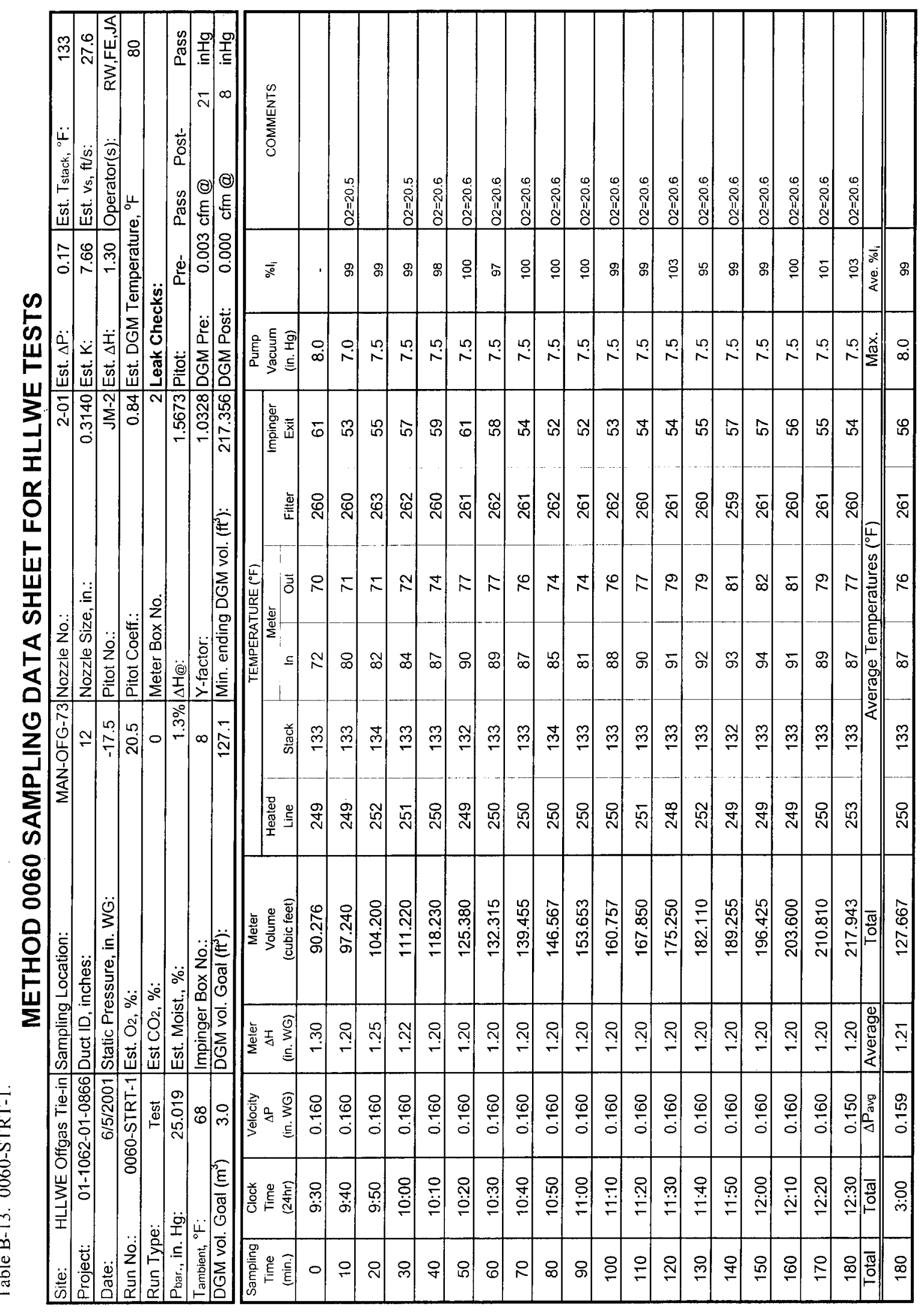

5
1
$\dot{m}$
$\dot{m}$ 


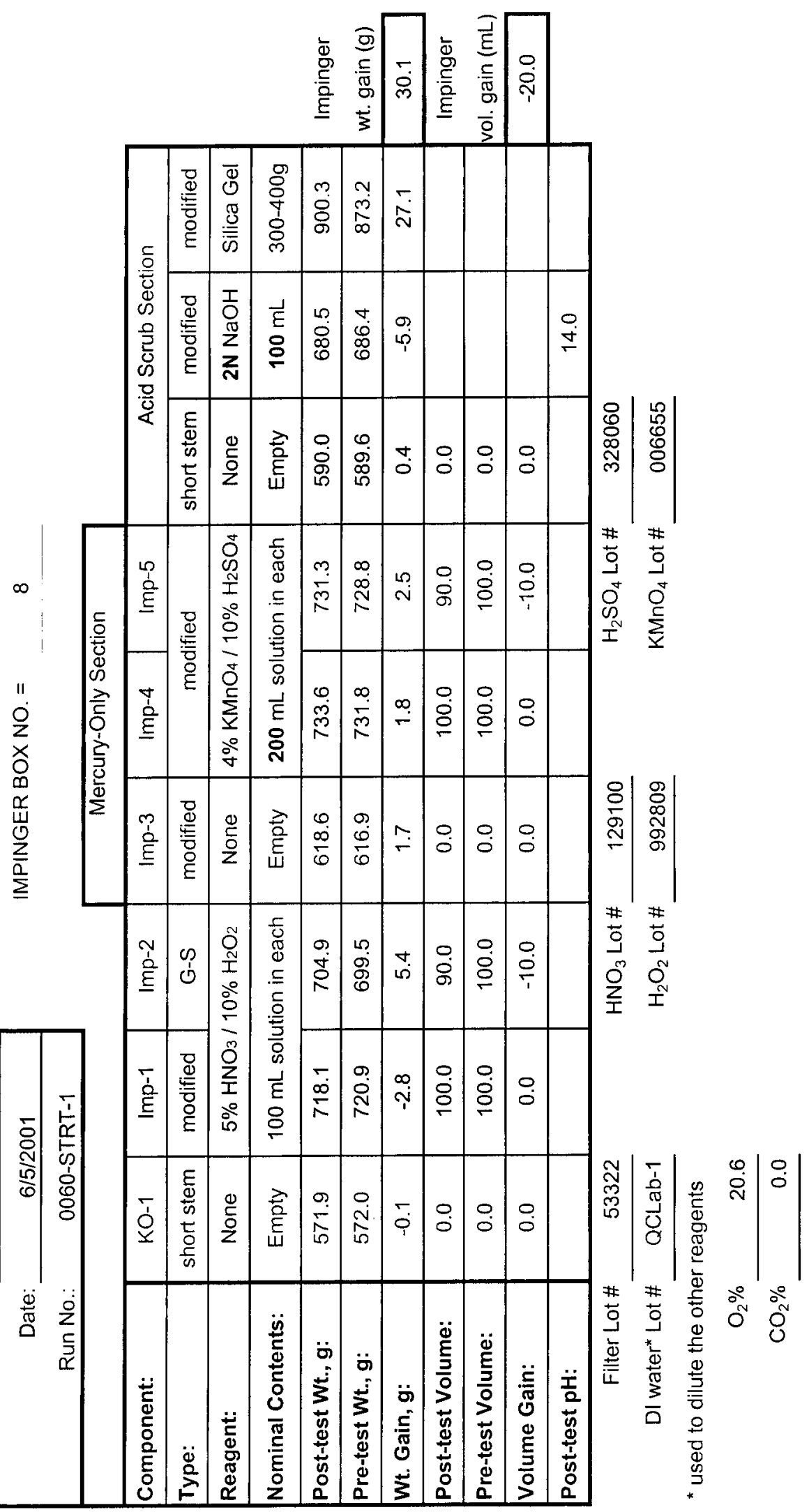

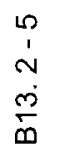


Table B-13. 0060-STRT-1.

\section{FIELD DATA CALCULATIONS}

\begin{tabular}{|c|c|c|c|}
\hline $\begin{array}{r}\text { Project: } \\
\text { Run Date } \\
\text { Run Identification: }\end{array}$ & $\begin{array}{r}01-1062 \\
6 / 5 \\
0060-\end{array}$ & $\begin{array}{l}\text { D1-0866 } \\
001 \\
\text { TRT-1 }\end{array}$ & \\
\hline PARAMETER & SYMBOL & UNITS & \\
\hline Absolute Pressure in the Duct & Pabs & in. $\mathrm{Hg}$ & 23.732 \\
\hline Average Duct Gas Temperature & Ts & $\mathrm{R}$ & 593 \\
\hline Average Meter Temperature & $\mathrm{Tm}$ & $\mathrm{R}$ & 541 \\
\hline Average Gas Oxygen Content & $\mathrm{Co} 2, \mathrm{~m}$ & $\%$ & 20.6 \\
\hline Average Gas Carbon Dioxide Content & $\mathrm{Cco} 2, \mathrm{~m}$ & $\%$ & 0.0 \\
\hline Total Impinger Weight Gain (water) & WW & grams & 30.1 \\
\hline Nozzle Area & An & $\mathrm{ft}^{2}$ & 0.000538 \\
\hline Duct Area & As & $\mathrm{ft}^{2}$ & 0.785 \\
\hline Sample Volume & VmStd & dscf & 107.88 \\
\hline Sample Volume (SI) & VmStdm & dscm & 3.055 \\
\hline Average Sampling Rate & Qm & $\mathrm{dscf} / \mathrm{m}$ & 0.599 \\
\hline Volume of Water Vapor & VwStd & scf & 1.419 \\
\hline Volume of Water Vapor (SI) & VwStdm & $\mathrm{scm}$ & 0.0402 \\
\hline Moisture Fraction & Bws & - & 0.013 \\
\hline Dry Gas Molecular Weight & Md & $\mathrm{g} / \mathrm{g}-\mathrm{mol}$ & 28.82 \\
\hline Wet Gas Molecular Weight & Ms & $\mathrm{g} / \mathrm{g}-\mathrm{mol}$ & 28.68 \\
\hline Gas Velocity at Nozzle & vn & $\mathrm{ft} / \mathrm{s}$ & 26.8 \\
\hline Gas Velocity at Nozzle (SI) & $\mathrm{vnm}$ & $\mathrm{m} / \mathrm{s}$ & 8.16 \\
\hline Average Gas Velocity & vncor & $\mathrm{ft} / \mathrm{s}$ & 22.58 \\
\hline Dry Offgas Flow Rate & Qsd & dscf/h & 44,506 \\
\hline Dry Offgas Flow Rate (SI) & Qsdm & $\mathrm{dscm} / \mathrm{h}$ & 1,260 \\
\hline Actual Offgas Flow Rate & $Q$ & $\mathrm{acf} / \mathrm{h}$ & 63,850 \\
\hline Intermediate Isokinetic Rate & li & $\%$ & 99.6 \\
\hline Final Isokinetic Rate & I & $\%$ & 99.6 \\
\hline
\end{tabular}


Table B-13. 0060-STRT-1.

\begin{tabular}{|c|c|c|c|c|c|c|c|c|c|c|c|c|}
\hline \multirow[t]{3}{*}{$\begin{array}{r}\text { Project: } \\
\text { Run Date: } \\
\text { Run Identification: } \\
\text { Run Type: } \\
\text { Lab Report Date: } \\
\text { Lab Report Status: } \\
\text { (preliminary or final) }\end{array}$} & \multicolumn{2}{|c|}{$\begin{array}{c}\text { 01-1062-01-0866 } \\
\text { 6/5/2001 } \\
\text { 0060-STRT-1 } \\
\text { Test } \\
\text { 8/28/2001 } \\
\text { Final }\end{array}$} & \multicolumn{10}{|c|}{$\begin{array}{l}\text { - without blank corrections } \\
\text { - final presentation should be rounded to two significant digits }\end{array}$} \\
\hline & \multicolumn{6}{|c|}{ CONCENTRATIONS } & \multicolumn{6}{|c|}{ MASS FLOW RATES } \\
\hline & \multicolumn{2}{|c|}{$\begin{array}{c}\text { Actual } \\
(\mu \mathrm{g} / \mathrm{acm})\end{array}$} & \multicolumn{2}{|c|}{$\begin{array}{l}\text { Standard } \\
(\mu \mathrm{g} / \mathrm{scm})\end{array}$} & \multicolumn{2}{|c|}{$\begin{array}{l}\text { Dry Standard } \\
(\mu \mathrm{g} / \mathrm{dscm})\end{array}$} & \multicolumn{2}{|c|}{$\mu \mathrm{g} / \mathrm{min}$} & \multicolumn{2}{|c|}{ grams/sec } & \multicolumn{2}{|c|}{$\mathrm{lb} / \mathrm{h}$} \\
\hline Aluminum (Al) & & $3.2 \mathrm{e} 1$ & & $4.5 e 1$ & & $4.6 e 1$ & & $9.6 \mathrm{e} 2$ & & $1.6 e-5$ & & $1.3 e-4$ \\
\hline Antimony (Sb) & $B$ & $1.0 \mathrm{e} 0$ & $B$ & $1.5 \mathrm{e} 0$ & $B$ & $1.5 \mathrm{e} 0$ & $B$ & $3.2 \mathrm{e} 1$ & $B$ & $5.3 e-7$ & B & $4.2 e-6$ \\
\hline Arsenic (As) & $<, B$ & $3.7 \mathrm{e}-1$ & $<, \mathrm{B}$ & $5.2 e-1$ & $<, B$ & $5.2 \mathrm{e}-1$ & $<, B$ & $1.1 \mathrm{e} 1$ & $<, B$ & $1.8 \mathrm{e}-7$ & $<, B$ & $1.5 e-6$ \\
\hline Barium (Ba) & $B$ & $1.5 \mathrm{e} 0$ & $B$ & $2.2 \mathrm{e} 0$ & $B$ & $2.2 \mathrm{e} 0$ & B & $4.6 \mathrm{e} 1$ & $B$ & $7.7 e-7$ & B & $6.1 e-6$ \\
\hline Beryllium (Be) & $<, B$ & $1.5 e-1$ & $<, \mathrm{B}$ & $2.1 \mathrm{e}-1$ & $<, B$ & $2.1 \mathrm{e}-1$ & $<, B$ & $4.5 \mathrm{e} 0$ & $<, B$ & $7.4 e-8$ & $<, B$ & $5.9 e-7$ \\
\hline Cadmium (Cd) & B & $1.0 \mathrm{e}-1$ & $B$ & $1.5 e-1$ & $B$ & $1.5 \mathrm{e}-1$ & $B$ & $3.2 \mathrm{e} 0$ & B & $5.3 e-8$ & B & $4.2 \mathrm{e}-7$ \\
\hline Chromium (Cr) & & $6.8 \mathrm{e}-1$ & & $9.7 e-1$ & & $9.8 \mathrm{e}-1$ & & $2.1 \mathrm{e} 1$ & & $3.4 e-7$ & & $2.7 e-6$ \\
\hline Cobalt (Co) & $B$ & $6.2 \mathrm{e}-1$ & $B$ & $8.7 e-1$ & B & $8.8 \mathrm{e}-1$ & $B$ & $1.9 e 1$ & $B$ & $3.1 e-7$ & $B$ & $2.5 e-6$ \\
\hline Copper (Cu) & B & $9.8 e-1$ & $B$ & $1.4 \mathrm{e} 0$ & $B$ & $1.4 \mathrm{e} 0$ & $B$ & $3.0 \mathrm{e} 1$ & $B$ & $4.9 e-7$ & B & $3.9 e-6$ \\
\hline Lead (Pb) & $<, B$ & $3.0 e-1$ & $<, \mathrm{B}$ & $4.2 \mathrm{e}-1$ & $<, B$ & $4.3 e-1$ & $<, B$ & $8.9 \mathrm{e} 0$ & $<, B$ & $1.5 e-7$ & $<, B$ & $1.2 e-6$ \\
\hline Manganese (Mn) & & $4.3 e 0$ & & $6.1 \mathrm{e} 0$ & & $6.2 e 0$ & & $1.3 e 2$ & & $2.2 e-6$ & & $1.7 e-5$ \\
\hline Mercury $(\mathrm{Hg})$ & & $2.3 e 1$ & & $3.2 \mathrm{e} 1$ & & $3.3 e 1$ & & $6.9 \mathrm{e} 2$ & & $1.1 e-5$ & & $9.1 e-5$ \\
\hline Nickel (Ni) & $B$ & $1.1 \mathrm{eo}$ & $B$ & $1.6 e 0$ & $B$ & $1.6 \mathrm{e} 0$ & $B$ & $3.4 \mathrm{e} 1$ & B & $5.7 e-7$ & B & $4.5 e-6$ \\
\hline Selenium (Se) & $B$ & $7.3 e-1$ & $B$ & $1.0 \mathrm{e} 0$ & $B$ & $1.0 e 0$ & $B$ & $2.2 \mathrm{e} 1$ & $B$ & $3.7 e-7$ & $B$ & $2.9 e-6$ \\
\hline Silver $(\mathrm{Ag})$ & $<$ & $5.9 e-1$ & $<$ & $8.4 e-1$ & $<$ & $8.5 e-1$ & $<$ & $1.8 \mathrm{e} 1$ & $<$ & $3.0 e-7$ & $<$ & $2.4 \mathrm{e}-6$ \\
\hline Thallium (TI) & $<$ & $5.7 e-1$ & $<$ & $8.1 e-1$ & $<$ & $8.2 e-1$ & $<$ & $1.7 \mathrm{e} 1$ & $<$ & $2.9 e-7$ & $<$ & $2.3 e-6$ \\
\hline Vanadium (V) & $<$ & $6.2 e-1$ & $<$ & $8.7 e-1$ & $<$ & $8.8 e-1$ & $<$ & $1.9 e 1$ & $<$ & $3.1 e-7$ & $<$ & $2.5 e-6$ \\
\hline $\operatorname{Zinc}(\mathrm{Zn})$ & & $1.4 \mathrm{e} 1$ & & $2.0 \mathrm{e} 1$ & & $2.0 \mathrm{e} 1$ & & $4.2 \mathrm{e} 2$ & & $7.0 \mathrm{e}-6$ & & $5.5 e-5$ \\
\hline
\end{tabular}


Table B-13. 0060-STRT-1.

\begin{tabular}{|c|c|c|c|c|c|c|c|c|c|c|c|c|}
\hline \multirow[t]{3}{*}{$\begin{array}{r}\text { Project: } \\
\text { Run Date: } \\
\text { Run Identification: } \\
\text { Run Type: } \\
\text { Lab Report Date: } \\
\text { Lab Report Status: } \\
\text { (preliminary or final) }\end{array}$} & \multicolumn{2}{|c|}{$\begin{array}{c}\text { 6/5/2001 } \\
\text { 0060-STRT-1 } \\
\text { Test } \\
\text { 8/28/2001 } \\
\text { Final }\end{array}$} & \multicolumn{10}{|c|}{$\begin{array}{c}\text { RESULTS } \\
\text { CORRECTED FOR CONTRIBUTION FROM } \\
\text { Corrected for Reagent Blank per EPA SW-846 Test Method } 0060 \\
\text { - final presentation should be rounded to two significant digits }\end{array}$} \\
\hline & \multicolumn{6}{|c|}{ CONCENTRATIONS } & \multicolumn{6}{|c|}{ MASS FLOW RATES } \\
\hline & \multicolumn{2}{|c|}{$\begin{array}{c}\text { Actual } \\
(\mu \mathrm{g} / \mathrm{acm})\end{array}$} & \multicolumn{2}{|c|}{$\begin{array}{l}\text { Standard } \\
(\mu \mathrm{g} / \mathrm{scm})\end{array}$} & \multicolumn{2}{|c|}{$\begin{array}{c}\text { Dry Standard } \\
(\mu \mathrm{g} / \mathrm{dscm})\end{array}$} & \multicolumn{2}{|c|}{$\mu \mathrm{g} / \mathrm{min}$} & \multicolumn{2}{|c|}{ grams/sec } & \multicolumn{2}{|r|}{$\mathrm{Ib} / \mathrm{h}$} \\
\hline$\overline{\text { Jluminum (Al) }}$ & & $3.0 \mathrm{e} 1$ & & $4.2 \mathrm{e} 1$ & & $4.2 \mathrm{e} 1$ & & $8.9 \mathrm{e} 2$ & & $1.5 e-5$ & & $1.2 \mathrm{e}-4$ \\
\hline$y(S b)$ & B & $2.4 e-1$ & B & $3.4 e-1$ & $B$ & $3.4 \mathrm{e}-1$ & $B$ & $7.2 \mathrm{e} 0$ & B & $e-7$ & B & \\
\hline (As) & $<, B$ & $2.0 e-1$ & $<, B$ & $2.8 e-1$ & $<, B$ & $2.8 e-1$ & $<, B$ & $5.9 \mathrm{e} 0$ & $<, B$ & $9.9 e-8$ & $<, B$ & -7 \\
\hline & $B$ & $4.9 e-1$ & $B$ & $6.9 e-1$ & $B$ & $7.0 e-1$ & $B$ & $1.5 \mathrm{e} 1$ & B & -7 & B & -6 \\
\hline Ilium (Be) & $<, B$ & $1.0 \mathrm{e}-1$ & $<, B$ & $1.5 e-1$ & $<, B$ & $1.5 e-1$ & $<, B$ & $3.1 \mathrm{e} 0$ & $<, B$ & -8 & $<, B$ & e-7 \\
\hline dmium (Cd) & $B$ & $9.1 e-2$ & $B$ & $1.3 e-1$ & $B$ & $1.3 e-1$ & B & $2.8 \mathrm{e} 0$ & $B$ & $4.6 e-8$ & B & $3.6 e-7$ \\
\hline$n(\mathrm{Cr})$ & & $2.7 e-1$ & & $3.8 e-1$ & & $3.8 e-1$ & & $8.0 \mathrm{e} 0$ & & & & $e-6$ \\
\hline & $B$ & $6.2 e-1$ & $B$ & $8.7 e-1$ & $B$ & $8.8 e-1$ & B & $1.9 e 1$ & $B$ & & $B$ & $2.5 e-6$ \\
\hline (Cu) & $B$ & $9.9 e-1$ & $B$ & $1.4 \mathrm{e} 0$ & $B$ & $1.4 \mathrm{eO}$ & $B$ & $3.0 \mathrm{e} 1$ & $B$ & $5.0 e-7$ & B & $3.9 e-6$ \\
\hline & $<, B$ & $2.0 \mathrm{e}-1$ & $<, B$ & $2.8 e-1$ & $<, B$ & $2.8 e-1$ & $<, B$ & $6.0 e 0$ & $<, B$ & $1.0 e-7$ & $<, B$ & $7.9 e-7$ \\
\hline lese $(\mathrm{Mn})$ & & $3.9 \mathrm{e} 0$ & & $5.6 \mathrm{e} 0$ & & $5.7 \mathrm{e} 0$ & & $1.2 \mathrm{e} 2$ & & $2.0 e-6$ & & $1.6 e-5$ \\
\hline $\mathrm{Hg})$ & & $2.3 \mathrm{e} 1$ & & $3.2 \mathrm{e} 1$ & & $3.3 \mathrm{e} 1$ & & $6.9 \mathrm{e} 2$ & & $1.1 e-5$ & & $9.1 e-5$ \\
\hline & $B$ & $3.5 \mathrm{e}-1$ & $B$ & $5.0 e-1$ & $B$ & $5.0 \mathrm{e}-1$ & B & $1.1 \mathrm{e} 1$ & B & $1.8 e-7$ & B & $1.4 e-6$ \\
\hline (Se) & $B$ & $2.3 e-11$ & $B$ & $3.2 e-11$ & $B$ & $3.3 e-11$ & $B$ & $6.9 \mathrm{e}-10$ & B & $1.1 \mathrm{e}-17$ & B & $9.1 e-17$ \\
\hline & $<$ & $6.0 e-1$ & $<$ & & $<$ & $8.5 e-1$ & $<$ & $1.8 e 1$ & $<$ & $3.0 \mathrm{e}-7$ & $<$ & $2.4 \mathrm{e}-6$ \\
\hline & $<$ & $5.7 e-1$ & $<$ & $8.1 \mathrm{e}-1$ & $<$ & $8.2 e-1$ & $<$ & $1.7 e 1$ & $<$ & $2.9 e-7$ & $<$ & $2.3 e-6$ \\
\hline (V) & $<$ & $6.2 e-1$ & $<$ & $8.7 e-1$ & $<$ & $8.8 e-1$ & $<$ & $1.9 \mathrm{e} 1$ & $<$ & $3.1 e-7$ & $<$ & $2.5 e-6$ \\
\hline & & $1.3 \mathrm{e} 1$ & & & & $1.8 \mathrm{e} 1$ & & $3.8 \mathrm{e} 2$ & & $6.4 \mathrm{e}-6$ & & $5.1 e-5$ \\
\hline
\end{tabular}





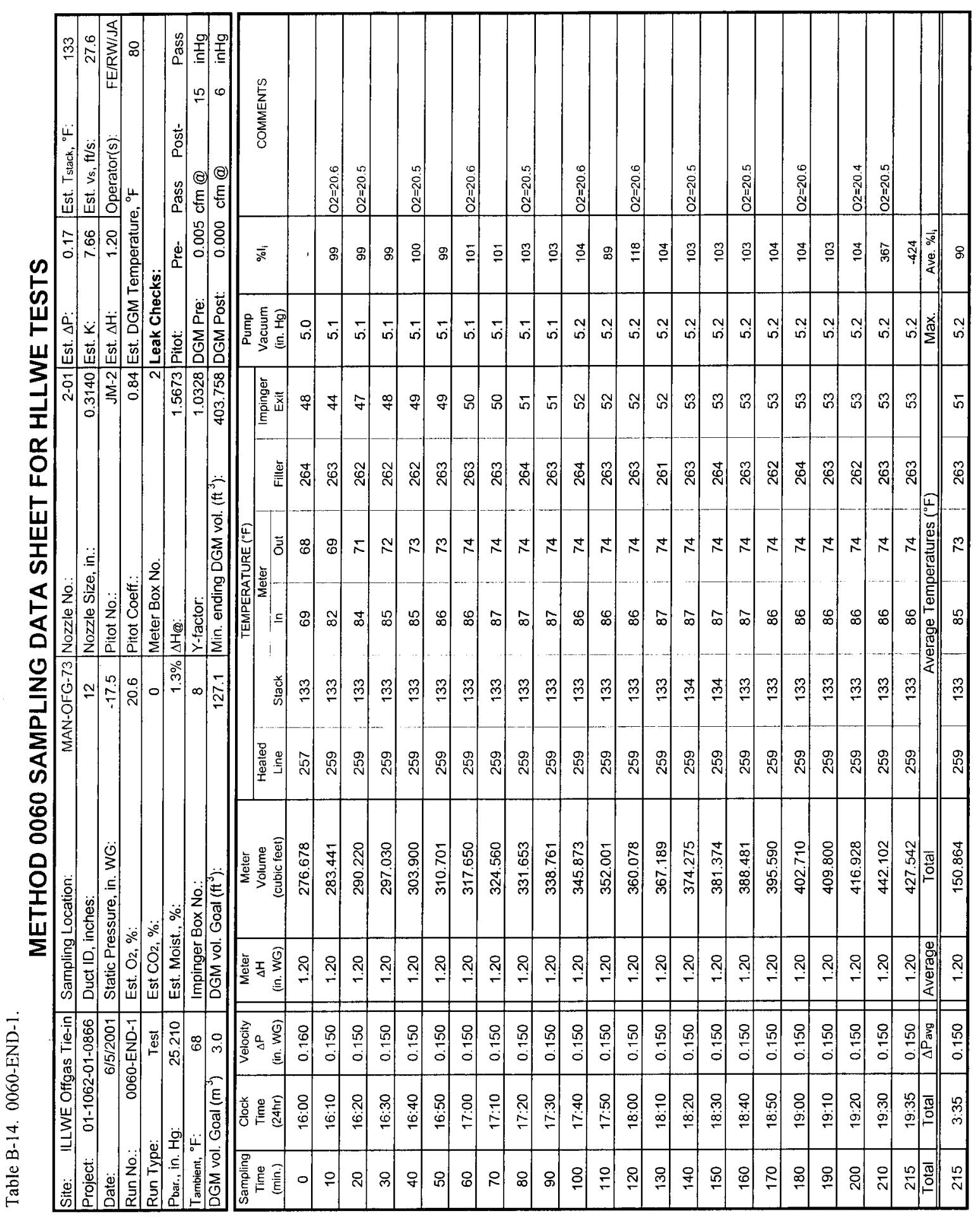



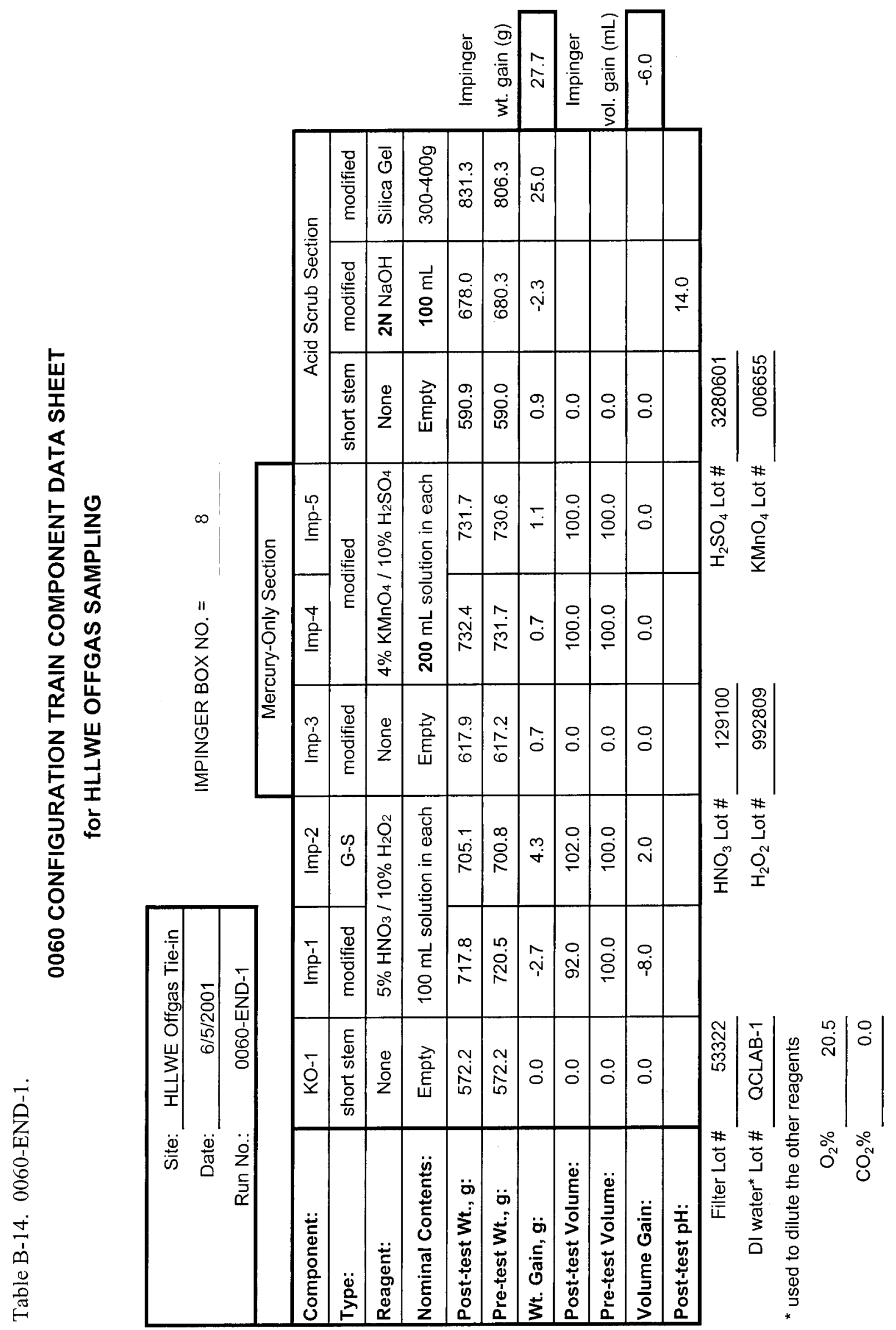

$n$
$\dot{v}$
$\frac{1}{m}$ 
Table B-14. 0060-END-1.

FIELD DATA CALCULATIONS

\begin{tabular}{|c|c|c|c|}
\hline $\begin{array}{r}\text { Project: } \\
\text { Run Date: } \\
\text { Run Identification: }\end{array}$ & $\begin{array}{r}01-1062 \\
6 / 5 / 2 \\
0060- \\
\end{array}$ & $\begin{array}{l}11-0866 \\
01 \\
\text { ND-1 }\end{array}$ & \\
\hline PARAMETER & SYMBOL & UNITS & \\
\hline Absolute Pressure in the Duct & Pabs & in. $\mathrm{Hg}$ & 23.923 \\
\hline Average Duct Gas Temperature & Ts & $\mathrm{R}$ & 593 \\
\hline Average Meter Temperature & $\mathrm{Tm}$ & $\mathrm{R}$ & 539 \\
\hline Average Gas Oxygen Content & $\mathrm{Co} 2, \mathrm{~m}$ & $\%$ & 20.5 \\
\hline Average Gas Carbon Dioxide Content & $\mathrm{Cco} 2, \mathrm{~m}$ & $\%$ & 0.0 \\
\hline Total Impinger Weight Gain (water) & Ww & grams & 27.7 \\
\hline Nozzle Area & An & $\mathrm{ft}^{2}$ & 0.000538 \\
\hline Duct Area & As & $\mathrm{ft}^{2}$ & 0.785 \\
\hline Sample Volume & VmStd & dscf & 129.00 \\
\hline Sample Volume (SI) & VmStdm & dscm & 3.653 \\
\hline Average Sampling Rate & Qm & $\mathrm{dscf} / \mathrm{m}$ & 0.600 \\
\hline Volume of Water Vapor & VwStd & scf & 1.306 \\
\hline Volume of Water Vapor (SI) & VwStdm & $\mathrm{scm}$ & 0.0370 \\
\hline Moisture Fraction & Bws & - & 0.010 \\
\hline Dry Gas Molecular Weight & Md & g/g-mol & 28.82 \\
\hline Wet Gas Molecular Weight & Ms & $\mathrm{g} / \mathrm{g}-\mathrm{mol}$ & 28.71 \\
\hline Gas Velocity at Nozzle & vn & $\mathrm{ft} / \mathrm{s}$ & 25.9 \\
\hline Gas Velocity at Nozzle (SI) & vnm & $\mathrm{m} / \mathrm{s}$ & 7.89 \\
\hline Average Gas Velocity & vncor & $\mathrm{ft} / \mathrm{s}$ & 21.84 \\
\hline Dry Offgas Flow Rate & Qsd & dscf/h & 43,505 \\
\hline Dry Offgas Flow Rate (SI) & Qsdm & $\mathrm{dscm} / \mathrm{h}$ & 1,232 \\
\hline Actual Offgas Flow Rate & Q & acf/h & 61,741 \\
\hline Intermediate Isokinetic Rate & li & $\%$ & 102.3 \\
\hline Final Isokinetic Rate & I & $\%$ & 102.0 \\
\hline
\end{tabular}


Table B-14. 0060-END-1.

\begin{tabular}{|c|c|c|c|c|c|c|c|c|c|c|c|c|}
\hline \multirow[t]{3}{*}{\begin{tabular}{|r} 
Project: \\
Run Date: \\
Run Identification: \\
Run Type: \\
Lab Report Date: \\
Lab Report Status: \\
(preliminary or final) \\
\end{tabular}} & \multicolumn{2}{|c|}{$\begin{array}{c}\text { 01-1062-01-0866 } \\
\text { 6/5/2001 } \\
\text { 0060-END-1 } \\
\text { Test } \\
\text { 8/28/2001 } \\
\text { Final }\end{array}$} & \multicolumn{10}{|c|}{$\begin{array}{c}\text { RESULTS } \\
\text { • without blank corrections } \\
\text { - final presentation should be rounded to two significant digits }\end{array}$} \\
\hline & \multicolumn{6}{|c|}{ CONCENTRATIONS } & \multicolumn{6}{|c|}{ MASS FLOW RATES } \\
\hline & & $\begin{array}{l}\text { ual } \\
\text { cm) }\end{array}$ & & $\begin{array}{l}\text { tandard } \\
\mathrm{g} / \mathrm{scm})\end{array}$ & & $\begin{array}{l}\text { ndard } \\
\mathrm{cm})\end{array}$ & & $\min$ & & $\mathrm{ns} / \mathrm{sec}$ & & $\mathrm{lb} / \mathrm{h}$ \\
\hline Aluminum (Al) & & $5.2 \mathrm{e} 1$ & & $7.3 \mathrm{e} 1$ & & $7.4 \mathrm{e} 1$ & & $1.5 \mathrm{e} 3$ & & $2.5 e-5$ & & $2.0 e-4$ \\
\hline Antimony (Sb) & $B$ & $9.8 e-1$ & $B$ & $1.4 \mathrm{e} 0$ & $B$ & $1.4 \mathrm{e} 0$ & $B$ & $2.9 \mathrm{e} 1$ & $B$ & $4.8 e-7$ & $B$ & $3.8 e-6$ \\
\hline Arsenic (As) & $<$ & $3.1 \mathrm{e}-1$ & $<$ & $4.3 e-1$ & $<$ & $4.4 \mathrm{e}-1$ & $<$ & $9.0 \mathrm{e} 0$ & $<$ & $1.5 e-7$ & $<$ & $1.2 \mathrm{e}-6$ \\
\hline Barium (Ba) & B & $1.9 \mathrm{e} 0$ & $B$ & $2.7 \mathrm{e} 0$ & B & $2.7 e 0$ & $B$ & $5.6 e 1$ & $B$ & $9.4 e-7$ & B & $7.4 e-6$ \\
\hline Beryllium (Be) & $<, \mathrm{B}$ & $1.3 e-1$ & $<, B$ & $1.8 \mathrm{e}-1$ & $<, B$ & $1.8 e-1$ & $<, B$ & $3.7 e 0$ & $<, B$ & $6.1 e-8$ & $<, B$ & $4.8 e-7$ \\
\hline Cadmium (Cd) & B & $1.6 e-1$ & B & $2.2 e-1$ & $B$ & $2.2 e-1$ & $B$ & $4.6 \mathrm{e} 0$ & $B$ & $7.7 e-8$ & $B$ & $6.1 e-7$ \\
\hline Chromium $(\mathrm{Cr})$ & & $9.1 e-1$ & & $1.3 \mathrm{e} 0$ & & $1.3 \mathrm{eo}$ & & $2.6 \mathrm{e} 1$ & & $4.4 e-7$ & & $3.5 e-6$ \\
\hline Cobalt (Co) & k & $5.2 e-1$ & $<$ & $7.3 e-1$ & $<$ & $7.4 e-1$ & $<$ & $1.5 \mathrm{e} 1$ & $<$ & $2.5 e-7$ & $<$ & $2.0 e-6$ \\
\hline Copp & $B$ & $6.9 e-1$ & $B$ & $9.8 e-1$ & $B$ & $9.9 e-1$ & $B$ & $2.0 \mathrm{e} 1$ & $B$ & $3.4 e-7$ & $B$ & $2.7 e-6$ \\
\hline Lead $(\mathrm{Pb})$ & B & $2.5 e-1$ & $B$ & $3.5 e-1$ & B & $3.6 e-1$ & $B$ & $7.3 \mathrm{e} 0$ & $B$ & $1.2 e-7$ & $B$ & $9.7 e-7$ \\
\hline Manganese (Mn) & & $4.4 \mathrm{eO}$ & & $6.2 \mathrm{e} 0$ & & $6.3 \mathrm{e} 0$ & & $1.3 \mathrm{e} 2$ & & $2.2 e-6$ & & $1.7 e-5$ \\
\hline Mercury $(\mathrm{Hg})$ & & $2.9 \mathrm{e} 1$ & & $4.1 \mathrm{e} 1$ & & $4.1 \mathrm{e} 1$ & & $8.4 \mathrm{e} 2$ & & $1.4 e-5$ & & $1.1 e-4$ \\
\hline Nick & $B$ & $1.2 \mathrm{eO}$ & $B$ & $1.6 \mathrm{e} 0$ & $B$ & $1.6 \mathrm{e} 0$ & B & $3.4 \mathrm{e} 1$ & $B$ & $5.6 e-7$ & $B$ & $4.5 e-6$ \\
\hline $\mathrm{um}(\mathrm{Se})$ & |< & $4.1 e-1$ & k & $5.7 e-1$ & < $<$ & $5.7 e-1$ & < & $1.2 \mathrm{e} 1$ & |< & $2.0 e-7$ & |< & $1.6 \mathrm{e}-6$ \\
\hline Silve & $<$ & $5.0 \mathrm{e}-1$ & $<$ & $7.0 e-1$ & < & $7.1 e-1$ & $<$ & $1.5 \mathrm{e} 1$ & $<$ & $2.4 e-7$ & $<$ & $1.9 e-6$ \\
\hline Thallium (TI) & $<$ & $4.6 e-1$ & $<$ & $6.5 e-1$ & < & $6.6 e-1$ & $<$ & $1.3 e 1$ & $<$ & $2.2 e-7$ & $<$ & $1.8 e-6$ \\
\hline Vanadium (V) & $<$ & $5.2 e-1$ & $<$ & $7.3 e-1$ & $<$ & $7.4 e-1$ & $<$ & $1.5 \mathrm{e} 1$ & $<$ & $2.5 e-7$ & $<$ & $2.0 e-6$ \\
\hline Zinc $(\mathrm{Zn})$ & & $2.3 e 1$ & & $3.3 \mathrm{e} 1$ & & $3.3 \mathrm{e} 1$ & & $6.7 \mathrm{e} 2$ & & $1.1 \mathrm{e}-5$ & & $8.9 e-5$ \\
\hline
\end{tabular}


Table B-14. 0060-END-1.

\begin{tabular}{|c|c|c|c|c|c|c|c|c|c|c|c|c|}
\hline \multirow[t]{3}{*}{$\begin{array}{r}\text { Project: } \\
\text { Run Date: } \\
\text { Run Identification: } \\
\text { Run Type: } \\
\text { Lab Report Date: } \\
\text { Lab Report Status: } \\
\text { (preliminary or final) } \\
\end{array}$} & \multicolumn{2}{|c|}{$\begin{array}{c}\text { 01-1062-01-0866 } \\
\text { 6/5/2001 } \\
\text { 0060-END-1 } \\
\text { Test } \\
\text { 8/28/2001 } \\
\text { Final } \\
\end{array}$} & \multicolumn{10}{|c|}{$\begin{array}{l}\text { CORRECTED FOR CONTRIBUTION FROM } \\
\text { Corrected for Reagent Blank per EPA SW-846 Test Method } 0060 \\
\text { - final presentation should be rounded to two significant digits }\end{array}$} \\
\hline & \multicolumn{6}{|c|}{ CONCENTRATIONS } & \multicolumn{6}{|c|}{ MASS FLOW RATES } \\
\hline & & $\begin{array}{l}\text { tual } \\
\text { acm) }\end{array}$ & & $\begin{array}{l}\text { landard } \\
\mathrm{g} / \mathrm{scm})\end{array}$ & & $\begin{array}{l}\text { ndard } \\
\mathrm{cm} \text { ) }\end{array}$ & & $\min$ & & /sec & & $\mathrm{lb} / \mathrm{h}$ \\
\hline$\overline{\text { Juminum (AI) }}$ & & $4.9 \mathrm{e} 1$ & & $6.9 \mathrm{e} 1$ & & $7.0 \mathrm{e} 1$ & & $1.4 \mathrm{e} 3$ & & $2.4 e-5$ & & $1.9 e-4$ \\
\hline ntimony (Sb) & B & $3.0 e-1$ & B & $4.2 \mathrm{e}-1$ & $B$ & $4.2 \mathrm{e}-1$ & $B$ & $8.7 \mathrm{eo}$ & $B$ & $1.4 e-7$ & $B$ & 1. \\
\hline senic (As) & $<$ & $1.7 e-1$ & $<$ & $2.3 e-1$ & $<$ & $2.4 e-1$ & $<$ & $4.8 \mathrm{eO}$ & $<$ & $8.1 e-8$ & $<$ & $6.4 e-7$ \\
\hline Barium $(\mathrm{Ba})$ & B & $1.1 \mathrm{e} 0$ & $B$ & $1.5 \mathrm{e} 0$ & B & $1.5 \mathrm{e} 0$ & $B$ & $3.1 \mathrm{e} 1$ & $B$ & $5.2 e-7$ & $B$ & $4.1 e-6$ \\
\hline Beryllium (Be) & $<, B$ & $8.7 e-2$ & $<, B$ & $1.2 e-1$ & $<, \mathrm{B}$ & $1.2 \mathrm{e}-1$ & $<, \mathrm{B}$ & $2.5 \mathrm{e} 0$ & $<, B$ & $4.2 e-8$ & $<, B$ & $3.3 e-7$ \\
\hline Cadmium (Cd) & $B$ & $1.5 e-1$ & B & $2.1 e-1$ & $B$ & $2.1 e-1$ & $B$ & $4.3 \mathrm{eO}$ & $B$ & $7.1 e-8$ & $B$ & $5.7 e-7$ \\
\hline Chromium (Cr) & & $5.5 \mathrm{e}-1$ & & $7.8 e-1$ & & $7.9 e-1$ & & $1.6 e 1$ & & e-7 & & $2.1 e-6$ \\
\hline & $<$ & $5.2 \mathrm{e}-1$ & 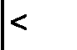 & $7.3 e-1$ & $<$ & $7.4 e-1$ & $<$ & $1.5 \mathrm{e} 1$ & $<$ & $e-7$ & $<$ & $2.0 e-6$ \\
\hline $\mathrm{Cu})$ & $B$ & $6.9 \mathrm{e}-1$ & B & $9.8 e-1$ & $B$ & $9.9 e-1$ & $B$ & $2.0 \mathrm{e} 1$ & $B$ & e-7 & B & $2.7 e-6$ \\
\hline Lea & B & $1.7 e-1$ & $B$ & $2.4 e-1$ & $B$ & $2.4 e-1$ & $B$ & $4.9 \mathrm{e} 0$ & $B$ & $8.2 e-8$ & B & $6.5 e-7$ \\
\hline nese $(M n)$ & & $4.2 e 0$ & & $5.9 e 0$ & & $6.0 \mathrm{e} 0$ & & $1.2 \mathrm{e} 2$ & & $2.0 e-6$ & & $1.6 e-5$ \\
\hline$(\mathrm{Hg})$ & & $2.9 e 1$ & & $4.1 \mathrm{e} 1$ & & $4.1 \mathrm{e} 1$ & & $8.4 \mathrm{e} 2$ & & $1.4 e-5$ & & $1.1 e-4$ \\
\hline & $B$ & $4.9 e-1$ & B & $6.9 \mathrm{e}-1$ & B & $7.0 \mathrm{e}-1$ & $B$ & $1.4 \mathrm{e} 1$ & $B$ & $2.4 e-7$ & B & $1.9 e-6$ \\
\hline (Se) & $<$ & $1.9 e-11$ & $<$ & $2.7 e-11$ & $<$ & $2.7 \mathrm{e}-11$ & $<$ & $5.6 e-10$ & $<$ & $9.4 e-18$ & $<$ & $7.4 e-17$ \\
\hline & $<$ & $5.0 e-1$ & $<$ & $7.1 e-1$ & $<$ & $7.1 \mathrm{e}-1$ & k & $1.5 \mathrm{e} 1$ & $<$ & $2.4 \mathrm{e}-7$ & $<$ & $1.9 e-6$ \\
\hline $\mathrm{Tr}$ & $<$ & $4.6 e-1$ & $<$ & $6.5 e-1$ & $<$ & $6.6 e-1$ & $<$ & $1.3 e 1$ & $<$ & $2.2 \mathrm{e}-7$ & $<$ & $1.8 e-6$ \\
\hline$m(V)$ & & $5.2 e-1$ & $<$ & $7.3 e-1$ & $<$ & $7.4 \mathrm{e}-1$ & $<$ & $1.5 \mathrm{e} 1$ & $<$ & $2.5 \mathrm{e}-7$ & $<$ & $2.0 e-6$ \\
\hline Zinc $(Z n)$ & & 2.2 & & & & $3.1 \mathrm{e} 1$ & & $6.4 \mathrm{e} 2$ & & -5 & & $0.0 \mathrm{e}=$ \\
\hline
\end{tabular}





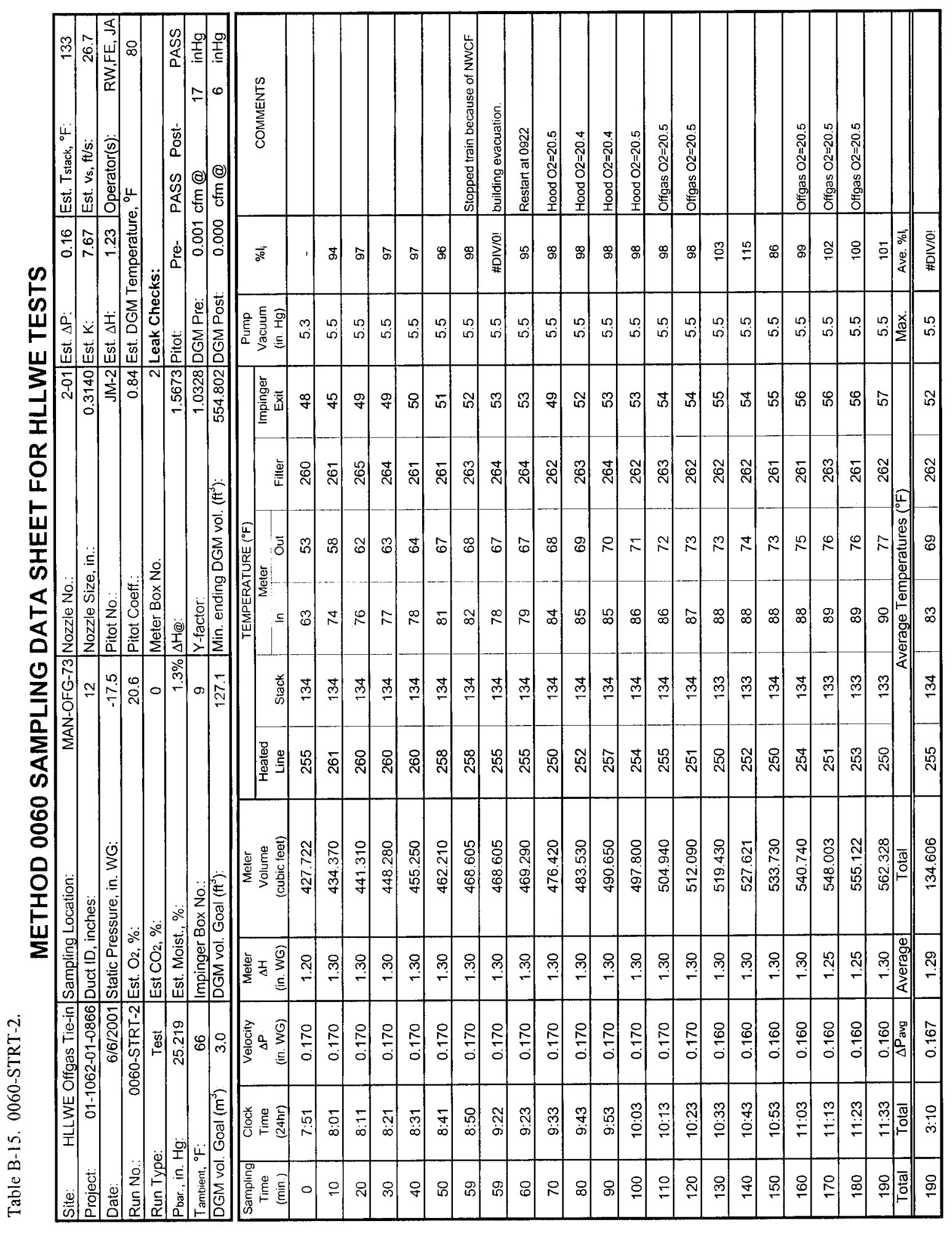




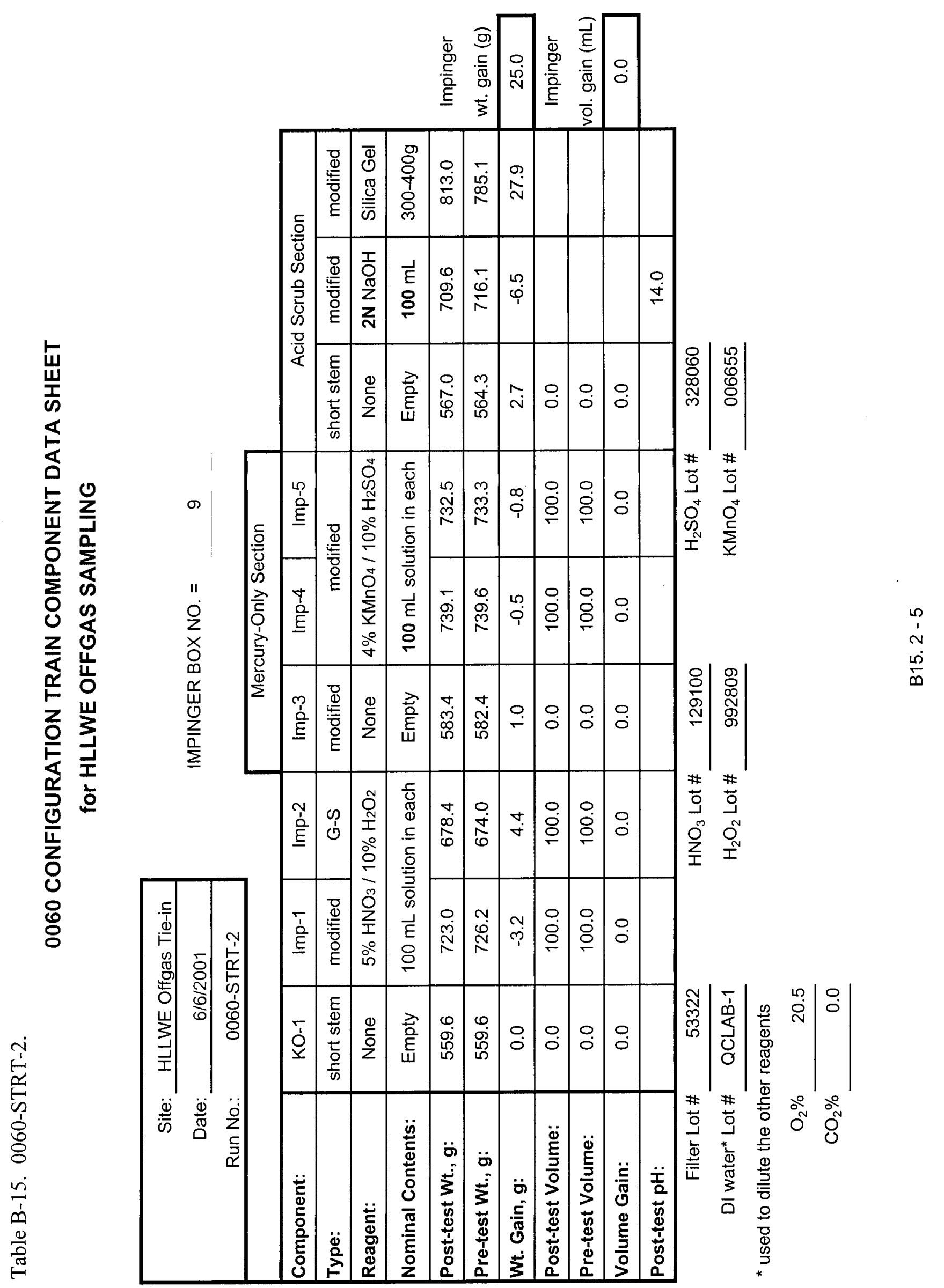


Table B-15. 0060-STRT-2.

\section{FIELD DATA CALCULATIONS}

\begin{tabular}{|c|c|c|c|}
\hline $\begin{array}{r}\text { Project: } \\
\text { Run Date: } \\
\text { Run Identification: }\end{array}$ & $\begin{array}{r}01-1062 \\
6 / 6 / \\
0060-\varsigma \\
\end{array}$ & $\begin{array}{l}01-0866 \\
001 \\
\text { TRT-2 }\end{array}$ & \\
\hline PARAMETER & SYMBOL & UNITS & \\
\hline Absolute Pressure in the Duct & Pabs & in. $\mathrm{Hg}$ & 23.932 \\
\hline Average Duct Gas Temperature & Ts & $\mathrm{R}$ & 593 \\
\hline Average Meter Temperature & $\mathrm{Tm}$ & $\mathrm{R}$ & 536 \\
\hline Average Gas Oxygen Content & $\mathrm{Co} 2, \mathrm{~m}$ & $\%$ & 20.5 \\
\hline Average Gas Carbon Dioxide Content & $\mathrm{Cco} 2, \mathrm{~m}$ & $\%$ & 0.0 \\
\hline Total Impinger Weight Gain (water) & Ww & grams & 25.0 \\
\hline Nozzle Area & An & $\mathrm{ft}^{2}$ & 0.000538 \\
\hline Duct Area & As & $\mathrm{ft}^{2}$ & 0.785 \\
\hline Sample Volume & VmStd & dscf & 115.90 \\
\hline Sample Volume (SI) & VmStdm & dscm & 3.282 \\
\hline Average Sampling Rate & Qm & $\mathrm{dscf} / \mathrm{m}$ & 0.610 \\
\hline Volume of Water Vapor & VwStd & scf & 1.179 \\
\hline Volume of Water Vapor (SI) & VwStdm & $\mathrm{scm}$ & 0.0334 \\
\hline Moisture Fraction & Bws & - & 0.010 \\
\hline Dry Gas Molecular Weight & Md & $\mathrm{g} / \mathrm{g}-\mathrm{mol}$ & 28.82 \\
\hline Wet Gas Molecular Weight & Ms & $\mathrm{g} / \mathrm{g}-\mathrm{mol}$ & 28.71 \\
\hline Gas Velocity at Nozzle & vn & $\mathrm{ft} / \mathrm{s}$ & 27.3 \\
\hline Gas Velocity at Nozzle (SI) & vnm & $\mathrm{m} / \mathrm{s}$ & 8.31 \\
\hline Average Gas Velocity & vncor & $\mathrm{ft} / \mathrm{s}$ & 23.00 \\
\hline Dry Offgas Flow Rate & Qsd & $\mathrm{dscf} / \mathrm{h}$ & 45,790 \\
\hline Dry Offgas Flow Rate (SI) & Qsdm & $\mathrm{dscm} / \mathrm{h}$ & 1,297 \\
\hline Actual Offgas Flow Rate & Q & acf/h & 65,037 \\
\hline Intermediate Isokinetic Rate & li & $\%$ & 98.8 \\
\hline Final Isokinetic Rate & $\mathbf{I}$ & $\%$ & 98.5 \\
\hline
\end{tabular}


Table B-15. 0060-STRT-2.

\begin{tabular}{|c|c|c|c|c|c|c|c|c|c|c|c|c|}
\hline \multirow[t]{3}{*}{$\begin{array}{r}\text { Project: } \\
\text { Run Date: } \\
\text { Run Identification: } \\
\text { Run Type: } \\
\text { Lab Report Date: } \\
\text { Lab Report Status: } \\
\text { (preliminary or final) } \\
\end{array}$} & \multicolumn{2}{|c|}{$\begin{array}{c}\text { 01-1062-01-0866 } \\
\text { 6/6/2001 } \\
\text { 0060-STRT-2 } \\
\text { Test } \\
8 / 28 / 2001 \\
\text { Final }\end{array}$} & \multicolumn{10}{|c|}{$\begin{array}{c}\text { RESULTS } \\
\text { - without blank corrections } \\
\text { - final presentation should be rounded to two significant digits }\end{array}$} \\
\hline & \multicolumn{6}{|c|}{ CONCENTRATIONS } & \multicolumn{6}{|c|}{ MASS FLOW RATES } \\
\hline & \multicolumn{2}{|c|}{$\begin{array}{l}\text { Actual } \\
(\mu \mathrm{g} / \mathrm{acm})\end{array}$} & \multicolumn{2}{|c|}{$\begin{array}{l}\text { Standard } \\
(\mu \mathrm{g} / \mathrm{scm})\end{array}$} & \multicolumn{2}{|c|}{$\begin{array}{c}\text { Dry Standard } \\
(\mu \mathrm{g} / \mathrm{dscm})\end{array}$} & \multicolumn{2}{|c|}{$\mu \mathrm{g} / \mathrm{min}$} & \multicolumn{2}{|c|}{ grams/sec } & \multicolumn{2}{|r|}{$\mathrm{lb} / \mathrm{h}$} \\
\hline Aluminum (Al) & & $1.6 \mathrm{e} 1$ & & $2.3 e 1$ & & $2.3 \mathrm{e} 1$ & & $5.0 \mathrm{e} 2$ & & $8.3 e-6$ & & $6.6 e-5$ \\
\hline Antimony (Sb) & $B$ & $9.7 e-1$ & $B$ & $1.4 \mathrm{e} 0$ & $B$ & $1.4 \mathrm{e} 0$ & B & $3.0 \mathrm{e} 1$ & B & $4.9 e-7$ & $B$ & $3.9 e-6$ \\
\hline Arsenic (As) & $<$ & $3.4 e-1$ & $<$ & $4.8 e-1$ & $<$ & $4.9 e-1$ & $<$ & $1.1 \mathrm{e} 1$ & $<$ & $1.8 \mathrm{e}-7$ & $<$ & $1.4 e-6$ \\
\hline Barium (Ba) & B & $1.1 \mathrm{e} 0$ & B & $1.5 \mathrm{e} 0$ & B & $1.6 \mathrm{eo}$ & B & $3.4 \mathrm{e} 1$ & B & $5.6 e-7$ & B & $4.4 e-6$ \\
\hline Beryllium (Be) & $<, \mathrm{B}$ & $1.4 \mathrm{e}-1$ & $<, B$ & $2.0 e-1$ & $<, \mathrm{B}$ & $2.0 e-1$ & $<, B$ & $4.3 \mathrm{eO}$ & $<, B$ & $7.1 e-8$ & $<, B$ & $5.7 e-7$ \\
\hline Cadmium (Cd) & $<, B$ & $5.8 e-2$ & $<, B$ & $8.1 \mathrm{e}-2$ & $<, \mathrm{B}$ & $8.2 e-2$ & $<, \mathrm{B}$ & $1.8 \mathrm{eO}$ & $<, B$ & $3.0 e-8$ & $<, B$ & $2.4 e-7$ \\
\hline Chromium (Cr) & & $5.4 e-1$ & & $7.5 e-1$ & & $7.6 e-1$ & & $1.6 \mathrm{e} 1$ & & $2.7 e-7$ & & $2.2 \mathrm{e}-6$ \\
\hline Cobalt (Co) & $<$ & $5.8 e-1$ & $<$ & $8.1 e-1$ & $<$ & $8.2 e-1$ & $<$ & $1.8 \mathrm{e} 1$ & $<$ & $3.0 e-7$ & $<$ & $2.4 e-6$ \\
\hline Copper (Cu) & B & $4.9 e-1$ & B & $6.9 e-1$ & $B$ & $7.0 e-1$ & B & $1.5 \mathrm{e} 1$ & B & $2.5 e-7$ & B & $2.0 e-6$ \\
\hline Lead $(\mathrm{Pb})$ & $<, \mathrm{B}$ & $3.0 e-1$ & $<, B$ & $4.2 e-1$ & $<, \mathrm{B}$ & $4.3 e-1$ & $<, B$ & $9.2 \mathrm{eo}$ & $<, B$ & $1.5 e-7$ & $<, B$ & $1.2 e-6$ \\
\hline Manganese (Mn) & & $8.2 \mathrm{e} 0$ & & $1.1 \mathrm{e} 1$ & & $1.2 \mathrm{e} 1$ & & $2.5 \mathrm{e} 2$ & & $4.2 e-6$ & & $3.3 e-5$ \\
\hline$(\mathrm{Hg})$ & & $2.4 \mathrm{e} 1$ & & $3.3 e 1$ & & $3.4 \mathrm{e} 1$ & & $7.2 \mathrm{e} 2$ & & $1.2 e-5$ & & $9.6 e-5$ \\
\hline & $B$ & $9.7 e-1$ & B & $1.4 \mathrm{e} 0$ & $B$ & $1.4 \mathrm{eO}$ & $B$ & $3.0 \mathrm{e} 1$ & $B$ & $4.9 e-7$ & $B$ & $3.9 e-6$ \\
\hline nium (Se) & B & $5.6 e-1$ & $B$ & $7.8 e-1$ & $B$ & $7.9 e-1$ & $B$ & $1.7 \mathrm{e} 1$ & $B$ & $2.9 e-7$ & B & $2.3 e-6$ \\
\hline & $<$ & $5.6 e-1$ & $<$ & $7.8 e-1$ & $<$ & $7.9 e-1$ & $<$ & $1.7 \mathrm{e} 1$ & $<$ & $2.9 e-7$ & < & $2.3 e-6$ \\
\hline ium (TI) & $<$ & $5.4 e-1$ & $<$ & $7.5 e-1$ & $<$ & $7.6 e-1$ & $<$ & $1.6 \mathrm{e} 1$ & $<$ & $2.7 e-7$ & $<$ & $2.2 e-6$ \\
\hline Vanadium (V) & $<$ & $5.8 e-1$ & $<$ & $8.1 \mathrm{e}-1$ & $<$ & $8.2 e-1$ & $<$ & $1.8 \mathrm{e} 1$ & $<$ & $3.0 e-7$ & $<$ & $2.4 \mathrm{e}-6$ \\
\hline Zinc $(Z n)$ & & $6.4 \mathrm{e} 0$ & & $9.0 e 0$ & & $9.1 \mathrm{e} 0$ & & $2.0 \mathrm{e} 2$ & & $3.3 e-6$ & & $2.6 \mathrm{e}-$ \\
\hline
\end{tabular}


Table B-15. 0060-STRT-2.

\begin{tabular}{|c|c|c|c|c|c|c|c|c|c|c|c|c|}
\hline \multirow[t]{3}{*}{$\begin{array}{r}\text { Project: } \\
\text { Run Date: } \\
\text { Run Identification: } \\
\text { Run Type: } \\
\text { Lab Report Date: } \\
\text { Lab Report Status: } \\
\text { (preliminary or final) } \\
\end{array}$} & \multicolumn{2}{|c|}{$\begin{array}{c}\text { 01-1062-01-0866 } \\
\text { 6/6/2001 } \\
\text { 0060-STRT-2 } \\
\text { Test } \\
8 / 28 / 2001 \\
\text { Final }\end{array}$} & \multicolumn{10}{|c|}{$\begin{array}{l}\text { CORRECTED FOR CONTRIBUTION FROM } \\
\text { Corrected for Reagent Blank per EPA SW-846 Test Method } 0060 \\
\text { - final presentation should be rounded to two significant digits }\end{array}$} \\
\hline & \multicolumn{6}{|c|}{ CONCENTRATIONS } & \multicolumn{6}{|c|}{ MASS FLOW RATES } \\
\hline & & $\begin{array}{l}\mathrm{ual} \\
\mathrm{cm})\end{array}$ & & $\begin{array}{l}\text { andard } \\
\mathrm{g} / \mathrm{scm})\end{array}$ & & $\begin{array}{l}\text { ndard } \\
\mathrm{cm})\end{array}$ & & $\min$ & & ec & & $\mathrm{lb} / \mathrm{h}$ \\
\hline Iuminum $(\mathrm{Al})$ & & $1.5 \mathrm{e} 1$ & & $2.1 \mathrm{e} 1$ & & $2.1 \mathrm{e1}$ & & $4.5 \mathrm{e} 2$ & & $7.5 e-6$ & & $6.0 \mathrm{e}-5$ \\
\hline & $B$ & $2.0 e-1$ & B & $2.8 e-1$ & $B$ & $2.9 e-1$ & B & $6.2 \mathrm{e} 0$ & $B$ & $1.0 \mathrm{e}-7$ & B & e-7 \\
\hline & $<$ & $1.8 e-1$ & $<$ & $2.6 e-1$ & $<$ & $2.6 e-1$ & $<$ & $5.7 \mathrm{eo}$ & $<$ & $9.4 e-8$ & $<$ & $7.5 e-7$ \\
\hline & B & $1.2 \mathrm{e}-1$ & B & $1.6 e-1$ & $B$ & $1.6 e-1$ & $B$ & $3.6 \mathrm{e} 0$ & $B$ & $5.9 e-8$ & $B$ & $4.7 e-7$ \\
\hline$n(B e)$ & $<, B$ & $9.7 e-2$ & $<, B$ & $1.4 \mathrm{e}-1$ & $<, \mathrm{B}$ & $1.4 \mathrm{e}-1$ & $<, B$ & $3.0 \mathrm{e} 0$ & $<, B$ & $4.9 e-8$ & $<, B$ & $3.9 e-7$ \\
\hline$(C d)$ & $<, B$ & $4.5 e-2$ & $<, B$ & $6.4 \mathrm{e}-2$ & $<, B$ & $6.4 \mathrm{e}-2$ & $<, B$ & $1.4 \mathrm{e} 0$ & $<, \mathrm{B}$ & $2.3 e-8$ & $<, B$ & $1.8 \mathrm{e}-7$ \\
\hline$n(C r)$ & & $1.4 \mathrm{e}-1$ & & $2.0 \mathrm{e}-1$ & & $2.0 \mathrm{e}-1$ & & $4.4 \mathrm{e} 0$ & & $7.4 e-8$ & & $5.8 e-7$ \\
\hline & $<$ & $5.8 e-1$ & $<$ & $8.1 e-1$ & $<$ & $8.2 e-1$ & $<$ & $1.8 \mathrm{e} 1$ & $<$ & $3.0 e-7$ & $<$ & $2.4 \mathrm{e}-6$ \\
\hline Cu) & B & $4.8 \mathrm{e}-1$ & B & $6.8 e-1$ & $B$ & $6.9 e-1$ & $B$ & $1.5 \mathrm{e} 1$ & $B$ & $2.5 e-7$ & $B$ & $2.0 e-6$ \\
\hline Lead & $<, B$ & $2.1 e-1$ & $<, B$ & $3.0 e-1$ & $<, B$ & $3.0 e-1$ & $<, B$ & $6.5 \mathrm{e} 0$ & $<, B$ & $1.1 \mathrm{e}-7$ & $<, B$ & $8.5 e-7$ \\
\hline ese $(M n)$ & & $7.8 \mathrm{e} 0$ & & $1.1 \mathrm{e} 1$ & & $1.1 \mathrm{e} 1$ & & $2.4 \mathrm{e} 2$ & & $4.0 e-6$ & & $3.2 e-5$ \\
\hline $\mathrm{Hg})$ & & $2.4 \mathrm{e} 1$ & & $3.3 e 1$ & & $3.4 \mathrm{e} 1$ & & $7.2 \mathrm{e} 2$ & & $1.2 e-5$ & & $9.6 e-5$ \\
\hline & $B$ & $2.2 e-1$ & B & $3.1 e-1$ & $B$ & $3.2 e-1$ & B & $6.8 \mathrm{e} 0$ & $B$ & $1.1 e-7$ & B & $9.1 e-7$ \\
\hline $\mathrm{Se})$ & $B$ & $2.1 e-11$ & B & $3.0 e-11$ & $B$ & -11 & $B$ & $6.6 e-10$ & B & $1.1 e-17$ & B & $8.7 e-17$ \\
\hline & $<$ & $5.6 e-1$ & $<$ & $7.9 e-1$ & $<$ & $8.0 e-1$ & $<$ & $1.7 \mathrm{e} 1$ & $<$ & $2.9 e-7$ & $<$ & $2.3 e-6$ \\
\hline & $<$ & $5.4 e-1$ & $<$ & $7.5 e-1$ & $<$ & $7.6 e-1$ & $<$ & $1.6 \mathrm{e} 1$ & $<$ & $2.7 e-7$ & $<$ & $2.2 e-6$ \\
\hline (V) & & $5.8 e-1$ & $<$ & $8.1 e-1$ & $<$ & $8.2 e-1$ & $<$ & $1.8 \mathrm{e} 1$ & $<$ & $3.0 e-7$ & $<$ & $2.4 e-6$ \\
\hline & & $5.6 \mathrm{eo}$ & & $7.8 \mathrm{e} 0$ & & $7.9 \mathrm{eo}$ & & $1.7 \mathrm{e} 2$ & & $2.8 e-6$ & & $2.3 e-5$ \\
\hline
\end{tabular}





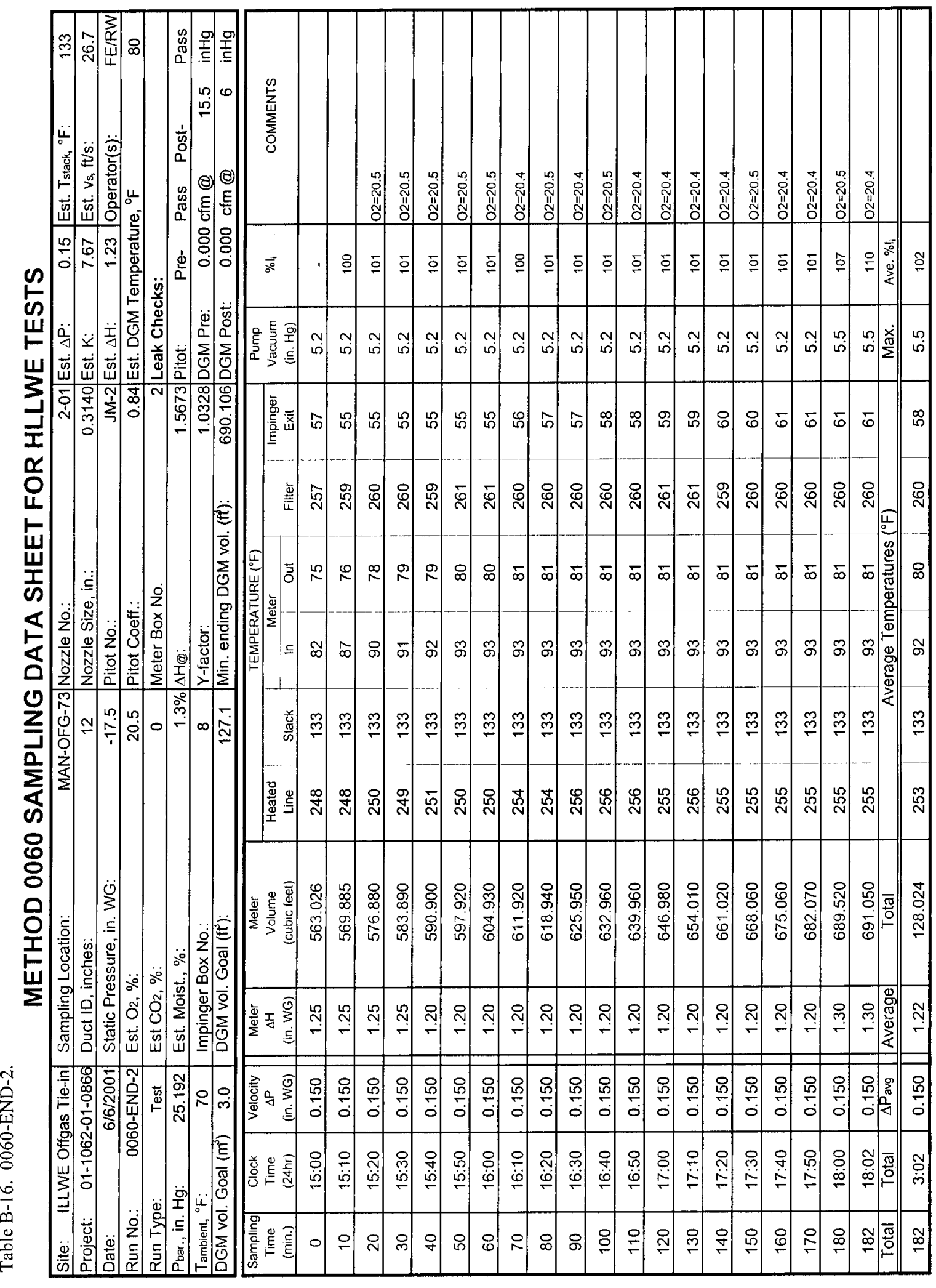




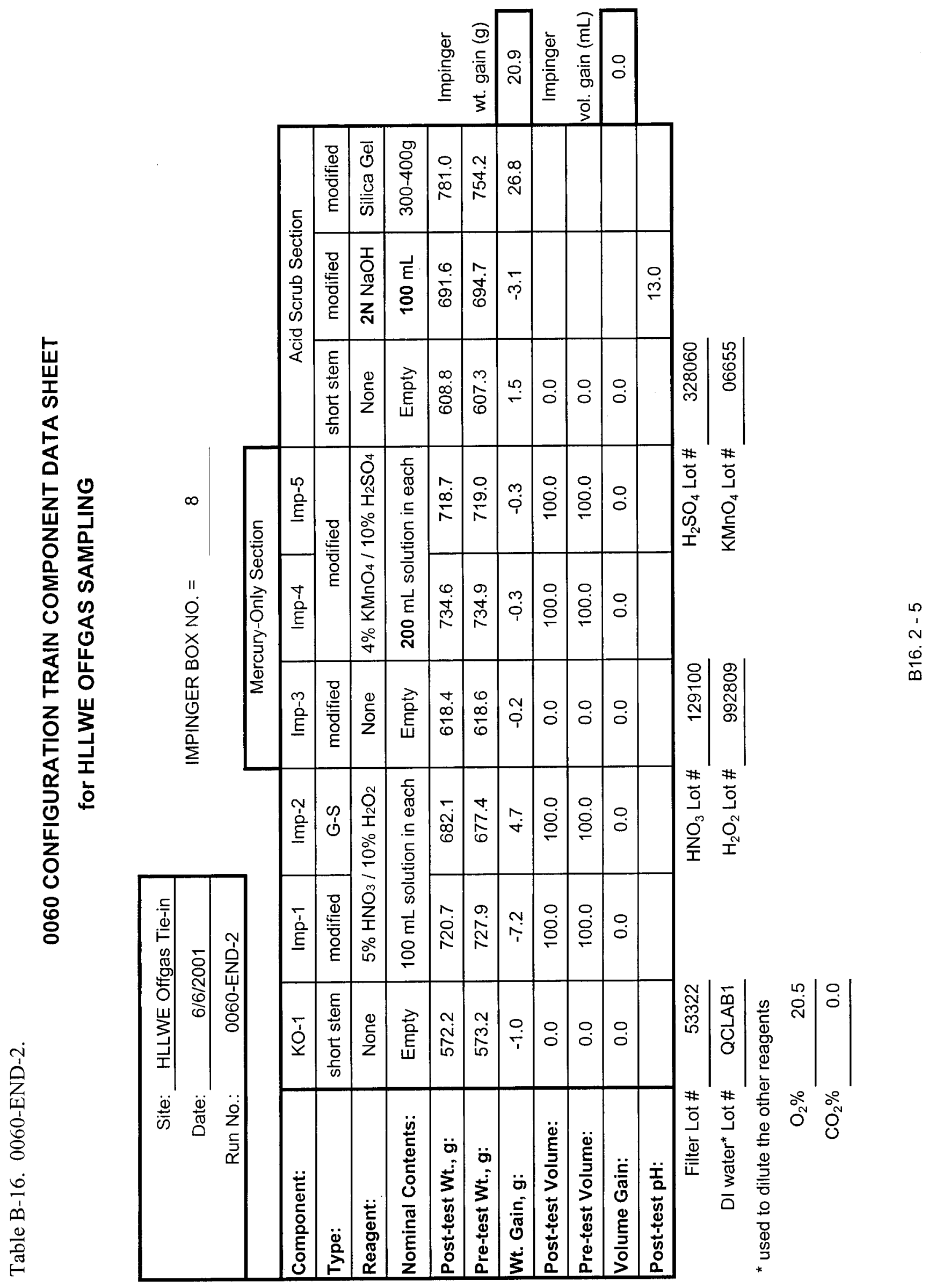


Table B-16. 0060-END-2.

\section{FIELD DATA CALCULATIONS}

\begin{tabular}{|c|c|c|c|}
\hline $\begin{array}{r}\text { Project: } \\
\text { Run Date: } \\
\text { Run Identification: }\end{array}$ & $\begin{array}{r}01-1062 \\
6 / 6 / \\
0060-\end{array}$ & $\begin{array}{l}1-0866 \\
01 \\
\text { ND-2 }\end{array}$ & \\
\hline PARAMETER & SYMBOL & UNITS & \\
\hline Absolute Pressure in the Duct & Pabs & in. $\mathrm{Hg}$ & 23.905 \\
\hline Average Duct Gas Temperature & Ts & $\mathrm{R}$ & 593 \\
\hline Average Meter Temperature & $\operatorname{Tm}$ & $\mathrm{R}$ & 546 \\
\hline Average Gas Oxygen Content & $\mathrm{Co} 2, \mathrm{~m}$ & $\%$ & 20.5 \\
\hline Average Gas Carbon Dioxide Content & $\mathrm{Cco} 2, \mathrm{~m}$ & $\%$ & 0.0 \\
\hline Total Impinger Weight Gain (water) & Ww & grams & 20.9 \\
\hline Nozzle Area & An & $\mathrm{ft}^{2}$ & 0.000538 \\
\hline Duct Area & As & $\mathrm{ft}^{2}$ & 0.785 \\
\hline Sample Volume & VmStd & dscf & 108.05 \\
\hline Sample Volume (SI) & VmStdm & dscm & 3.060 \\
\hline Average Sampling Rate & Qm & dscf/m & 0.594 \\
\hline Volume of Water Vapor & VwStd & scf & 0.985 \\
\hline Volume of Water Vapor (SI) & VwStdm & $\mathrm{scm}$ & 0.0279 \\
\hline Moisture Fraction & Bws & - & 0.009 \\
\hline Dry Gas Molecular Weight & Md & g/g-mol & 28.82 \\
\hline Wet Gas Molecular Weight & Ms & $\mathrm{g} / \mathrm{g}-\mathrm{mol}$ & 28.72 \\
\hline Gas Velocity at Nozzle & vn & $\mathrm{ft} / \mathrm{s}$ & 25.8 \\
\hline Gas Velocity at Nozzle (SI) & vnm & $\mathrm{m} / \mathrm{s}$ & 7.88 \\
\hline Average Gas Velocity & vncor & $\mathrm{ft} / \mathrm{s}$ & 21.81 \\
\hline Dry Offgas Flow Rate & Qsd & $\mathrm{dscf} / \mathrm{h}$ & 43,465 \\
\hline Dry Offgas Flow Rate (SI) & Qsdm & $\mathrm{dscm} / \mathrm{h}$ & 1,231 \\
\hline Actual Offgas Flow Rate & Q & acf/h & 61,660 \\
\hline Intermediate Isokinetic Rate & li & $\%$ & 101.4 \\
\hline Final Isokinetic Rate & I & $\%$ & 101.0 \\
\hline
\end{tabular}


Table B-16. 0060-END-2.

\begin{tabular}{|c|c|c|c|c|c|c|c|c|c|c|c|c|}
\hline \multirow[t]{3}{*}{$\begin{array}{r}\text { Project: } \\
\text { Run Date: } \\
\text { Run Identification: } \\
\text { Run Type: } \\
\text { Lab Report Date: } \\
\text { Lab Report Status: } \\
\text { (preliminary or final) } \\
\end{array}$} & \multicolumn{2}{|c|}{$\begin{array}{c}\text { 01-1062-01-0866 } \\
\text { 6/6/2001 } \\
\text { 0060-END-2 } \\
\text { Test } \\
\text { 8/28/2001 } \\
\text { Final }\end{array}$} & \multicolumn{10}{|c|}{$\begin{array}{c}\text { RESULTS } \\
\text { - without blank corrections } \\
\text { - final presentation should be rounded to two significant digits }\end{array}$} \\
\hline & \multicolumn{6}{|c|}{ CONCENTRATIONS } & \multicolumn{6}{|c|}{ MASS FLOW RATES } \\
\hline & \multicolumn{2}{|c|}{$\begin{array}{c}\text { Actual } \\
(\mu \mathrm{g} / \mathrm{acm})\end{array}$} & \multicolumn{2}{|c|}{$\begin{array}{l}\text { Standard } \\
(\mu \mathrm{g} / \mathrm{scm})\end{array}$} & \multicolumn{2}{|c|}{$\begin{array}{c}\text { Dry Standard } \\
(\mu \mathrm{g} / \mathrm{dscm})\end{array}$} & \multicolumn{2}{|c|}{$\mu \mathrm{g} / \mathrm{min}$} & \multicolumn{2}{|c|}{ grams/sec } & \multicolumn{2}{|c|}{$\mathrm{lb} / \mathrm{h}$} \\
\hline Aluminum (Al) & & $1.7 e 1$ & & $2.4 \mathrm{e} 1$ & & $2.4 \mathrm{e} 1$ & & $4.9 \mathrm{e} 2$ & & $8.2 e-6$ & & $6.5 e-5$ \\
\hline Antimony (Sb) & $B$ & $9.4 \mathrm{e}-1$ & $B$ & $1.3 \mathrm{e} 0$ & B & $1.3 \mathrm{e} 0$ & B & $2.7 e 1$ & B & $4.6 e-7$ & $B$ & $3.6 e-6$ \\
\hline Arsenic (As) & $<$ & $3.7 e-1$ & $<$ & $5.2 e-1$ & $<$ & $5.2 e-1$ & $<$ & $1.1 \mathrm{e} 1$ & $<$ & $1.8 e-7$ & $<$ & $1.4 e-6$ \\
\hline Barium (Ba) & B & $1.1 \mathrm{e} 0$ & $B$ & $1.5 \mathrm{eo}$ & $B$ & $1.5 \mathrm{e} 0$ & B & $3.2 e 1$ & $B$ & $5.3 e-7$ & $B$ & $4.2 e-6$ \\
\hline Beryllium (Be) & $<, B$ & $1.5 e-1$ & $<, B$ & $2.1 \mathrm{e}-1$ & $<, B$ & $2.1 e-1$ & $<, \mathrm{B}$ & $4.4 \mathrm{e} 0$ & $<, B$ & $7.3 e-8$ & $<, B$ & $5.8 e-7$ \\
\hline Cadmium $(\mathrm{Cd})$ & $<$ & $6.2 e-2$ & $<$ & $8.7 e-2$ & $<$ & $8.8 e-2$ & $<$ & $1.8 \mathrm{e} 0$ & $<$ & $3.0 \mathrm{e}-8$ & $<$ & $2.4 e-7$ \\
\hline Chromium ( $\mathrm{Cr}$ ) & & $1.2 \mathrm{e} 0$ & & $1.7 \mathrm{e} 0$ & & $1.7 \mathrm{eo}$ & & $3.4 \mathrm{e} 1$ & & $5.7 e-7$ & & $4.5 e-6$ \\
\hline Cobalt (Co) & $<$ & $6.2 e-1$ & $<$ & $8.7 e-1$ & $<$ & $8.8 \mathrm{e}-1$ & $<$ & $1.8 \mathrm{e} 1$ & $<$ & $3.0 \mathrm{e}-7$ & $<$ & $2.4 \mathrm{e}-6$ \\
\hline Copper (Cu) & $<, B$ & $3.2 e-1$ & $<, B$ & $4.5 e-1$ & $<, B$ & $4.6 e-1$ & $<, \mathrm{B}$ & $9.4 \mathrm{e} 0$ & $<, B$ & $1.6 e-7$ & $<, B$ & $1.2 \mathrm{e}-6$ \\
\hline Lead $(\mathrm{Pb})$ & $<, B$ & $2.8 e-1$ & $<, \mathrm{B}$ & $3.9 e-1$ & $<, B$ & $3.9 e-1$ & $<, \mathrm{B}$ & $8.0 \mathrm{e} 0$ & $<, B$ & $1.3 e-7$ & $<, B$ & $1.1 \mathrm{e}-6$ \\
\hline Manganese (Mn) & & $1.7 e 1$ & & $2.3 \mathrm{e} 1$ & & $2.4 \mathrm{e} 1$ & & $4.8 \mathrm{e} 2$ & & $8.0 e-6$ & & $6.4 e-5$ \\
\hline Mercury $(\mathrm{Hg})$ & & $2.5 \mathrm{e} 1$ & & $3.6 \mathrm{e} 1$ & & $3.6 \mathrm{e} 1$ & & $7.4 \mathrm{e} 2$ & & $1.2 e-5$ & & $9.8 e-5$ \\
\hline Nickel (Ni) & $B$ & $9.4 e-1$ & B & $1.3 \mathrm{e} 0$ & B & $1.3 e 0$ & B & $2.7 e 1$ & B & $4.6 e-7$ & B & $3.6 e-6$ \\
\hline Selenium (Se) & $B$ & $5.3 e-1$ & B & $7.4 e-1$ & B & $7.5 e-1$ & B & $1.5 \mathrm{e} 1$ & B & $2.6 e-7$ & B & $2.0 e-6$ \\
\hline Silver $(\mathrm{Ag})$ & k & $6.0 e-1$ & $<$ & $8.4 e-1$ & $<$ & $8.5 e-1$ & $<$ & $1.7 \mathrm{e} 1$ & $<$ & $2.9 e-7$ & $<$ & $2.3 e-6$ \\
\hline Thallium (TI) & $<$ & $5.5 e-1$ & $<$ & $7.8 e-1$ & $<$ & $7.8 e-1$ & $<$ & $1.6 \mathrm{e} 1$ & < & $2.7 e-7$ & |< & $2.1 e-6$ \\
\hline Vanadium $(\mathrm{V})$ & $<$ & $6.2 e-1$ & $<$ & $8.7 e-1$ & $<$ & $8.8 e-1$ & $<$ & $1.8 \mathrm{e} 1$ & $<$ & $3.0 e-7$ & $<$ & $2.4 \mathrm{e}-6$ \\
\hline Zinc $(Z n)$ & & $3.9 \mathrm{e} 0$ & & $5.5 \mathrm{e} 0$ & & $5.6 \mathrm{eO}$ & & $1.1 \mathrm{e} 2$ & & $1.9 e-6$ & & $1.5 e-5$ \\
\hline
\end{tabular}


Table B-16. 0060-END-2.

\begin{tabular}{|c|c|c|c|c|c|c|c|c|c|c|c|c|}
\hline \multirow[t]{3}{*}{$\begin{array}{r}\text { Project: } \\
\text { Run Date: } \\
\text { Run Identification: } \\
\text { Run Type: } \\
\text { Lab Report Date: } \\
\text { Lab Report Status: } \\
\text { (preliminary or final) } \\
\end{array}$} & \multicolumn{2}{|c|}{$\begin{array}{c}\text { 01-1062-01-0866 } \\
\text { 6/6/2001 } \\
\text { 0060-END-2 } \\
\text { Test } \\
\text { 8/28/2001 } \\
\text { Final }\end{array}$} & \multicolumn{10}{|c|}{$\begin{array}{l}\text { CORRECTED FOR CONTRIBUTION FROM } \\
\text { Corrected for Reagent Blank per EPA SW-846 Test Method } 0060 \\
\text { - final presentation should be rounded to two significant digits }\end{array}$} \\
\hline & \multicolumn{6}{|c|}{ CONCENTRATIONS } & \multicolumn{6}{|c|}{ MASS FLOW RATES } \\
\hline & & $\begin{array}{l}\text { ual } \\
\mathrm{cm} \text { ) }\end{array}$ & & $\begin{array}{l}\text { dard } \\
\text { scm) }\end{array}$ & & $\begin{array}{l}\text { andard } \\
\mathrm{scm} \text { ) }\end{array}$ & & $\min$ & & $/ \mathrm{sec}$ & & $\mathrm{Ib} / \mathrm{h}$ \\
\hline Alumin & & $1.5 \mathrm{e} 1$ & & $2.1 \mathrm{e} 1$ & & $2.2 \mathrm{e} 1$ & & $4.4 \mathrm{e} 2$ & & $7.4 e-6$ & & $5.9 e-5$ \\
\hline (Sb) & $B$ & $1.2 e-1$ & $B$ & $1.7 e-1$ & B & $1.8 \mathrm{e}-1$ & $B$ & $3.6 \mathrm{e} 0$ & $B$ & $6.0 e-8$ & $B$ & e-7 \\
\hline & $<$ & $2.0 \mathrm{e}-1$ & $<$ & $2.8 e-1$ & $<$ & $2.8 \mathrm{e}-1$ & $<$ & $5.8 \mathrm{e} 0$ & $<$ & $9.6 e-8$ & $<$ & $7.6 e-7$ \\
\hline & $B$ & $3.2 e-2$ & $B$ & $4.5 e-2$ & B & $4.6 e-2$ & $B$ & $9.4 e-1$ & $B$ & $1.6 e-8$ & $B$ & $1.2 e-7$ \\
\hline$(\mathrm{Be})$ & $<, B$ & $1.0 \mathrm{e}-1$ & $<, \mathrm{B}$ & $1.5 e-1$ & $<, B$ & $1.5 \mathrm{e}-1$ & $<, B$ & $3.0 \mathrm{e} 0$ & $<, B$ & $5.0 e-8$ & $<, B$ & $4.0 \mathrm{e}-7$ \\
\hline Cadmium (Cd) & $<$ & $4.9 e-2$ & $<$ & $6.8 e-2$ & $<$ & $6.9 e-2$ & $<$ & $1.4 \mathrm{e} 0$ & $<$ & $2.4 e-8$ & $<$ & $1.9 e-7$ \\
\hline Chromium (Cr) & & $7.5 e-1$ & & $1.1 \mathrm{e} 0$ & & $1.1 \mathrm{e} 0$ & & $2.2 \mathrm{e} 1$ & & 3. & & $2.9 e-6$ \\
\hline Cobalt (Co) & $<$ & $6.2 e-1$ & $<$ & $8.7 e-1$ & $<$ & $8.8 e-1$ & $<$ & $1.8 \mathrm{e} 1$ & $<$ & $3.0 e-7$ & $<$ & $2.4 \mathrm{e}-6$ \\
\hline Copper (Cu) & $<, B$ & $3.2 e-1$ & $<, B$ & $4.4 e-1$ & $<, B$ & $4.5 e-1$ & $<, B$ & $9.2 \mathrm{e} 0$ & $<, B$ & $1.5 e-7$ & $<, B$ & $1.2 e-6$ \\
\hline Lead $(\mathrm{Pb})$ & $<, B$ & $1.8 e-1$ & $<, B$ & $2.5 e-1$ & $<, B$ & $2.5 e-1$ & $<, B$ & $5.2 \mathrm{e} 0$ & $<, B$ & $8.6 e-8$ & $<, B$ & $6.8 e-7$ \\
\hline Manganese (Mn) & & $1.6 \mathrm{e} 1$ & & $2.2 \mathrm{e} 1$ & & $2.3 \mathrm{e} 1$ & & $4.6 \mathrm{e} 2$ & & $7.7 e-6$ & & $6.1 e-5$ \\
\hline Mercury $(\mathrm{Hg})$ & & $2.5 \mathrm{e} 1$ & & $3.6 \mathrm{e} 1$ & & $3.6 \mathrm{e} 1$ & & $7.4 \mathrm{e} 2$ & & $1.2 e-5$ & & $9.8 e-5$ \\
\hline Nickel (Ni) & $B$ & $1.5 e-1$ & $B$ & $2.1 \mathrm{e}-1$ & $B$ & $2.1 e-1$ & $B$ & $4.3 \mathrm{e} 0$ & $B$ & $7.2 e-8$ & $B$ & $5.7 e-7$ \\
\hline$n(\mathrm{Se})$ & $B$ & $2.3 e-11$ & B & $3.2 e-11$ & $B$ & $3.3 e-11$ & $B$ & $6.7 e-10$ & $B$ & $1.1 e-17$ & $B$ & $8.9 e-17$ \\
\hline Silver & $<$ & $6.0 e-1$ & $<$ & $8.5 e-1$ & $<$ & $8.5 e-1$ & $<$ & $1.7 \mathrm{e} 1$ & $<$ & $2.9 e-7$ & $<$ & $2.3 e-6$ \\
\hline Thalliu & $<$ & $5.5 e-1$ & $<$ & $7.8 e-1$ & $<$ & $7.8 e-1$ & k & $1.6 \mathrm{e} 1$ & $<$ & $2.7 e-7$ & $<$ & $2.1 e-6$ \\
\hline Vanadium & $<$ & $6.2 e-1$ & $<$ & $8.7 e-1$ & $<$ & $8.8 e-1$ & $<$ & $1.8 \mathrm{e} 1$ & $<$ & $3.0 e-7$ & $<$ & $2.4 e-6$ \\
\hline Zinc $(\mathrm{Zn})$ & & $2.9 \mathrm{e} 0$ & & $4.0 \mathrm{e} 0$ & & $4.1 \mathrm{e} 0$ & & $8.4 \mathrm{e} 1$ & & $1.4 \mathrm{e}-6$ & & $1.1 e-5$ \\
\hline
\end{tabular}




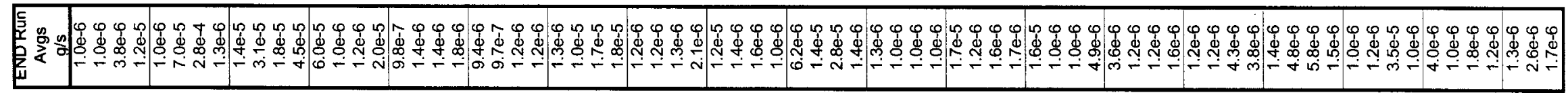

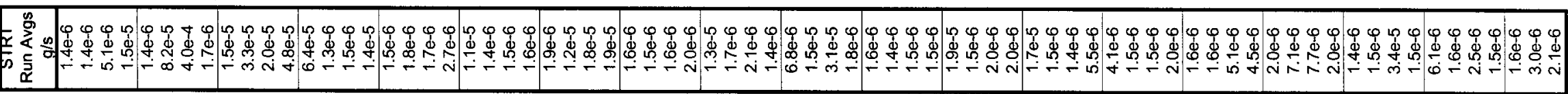

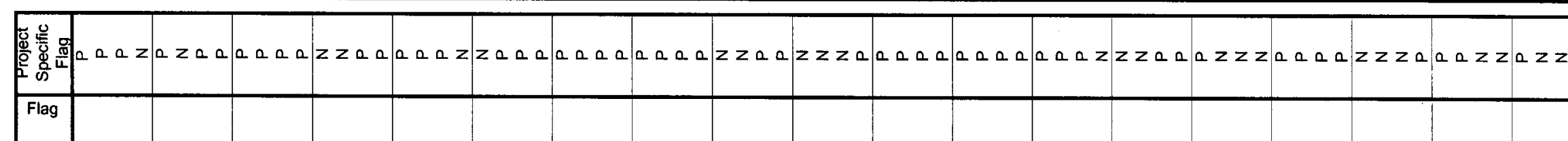

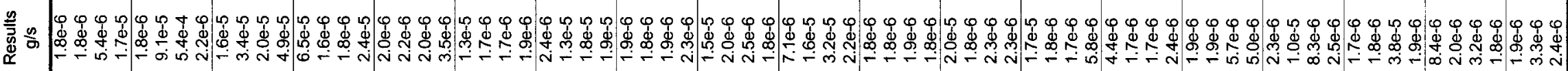

Flag

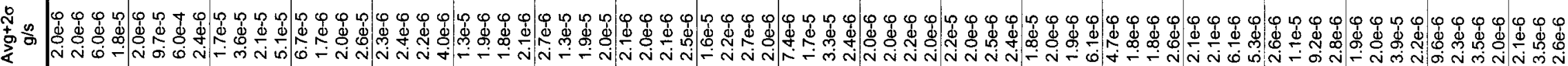

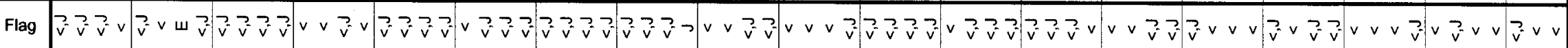

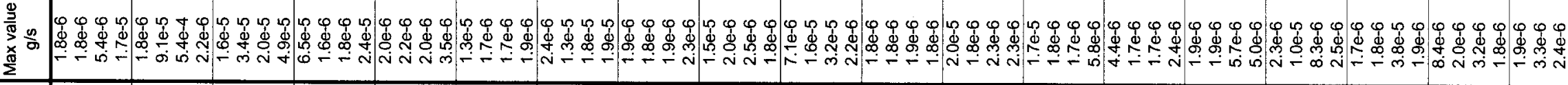

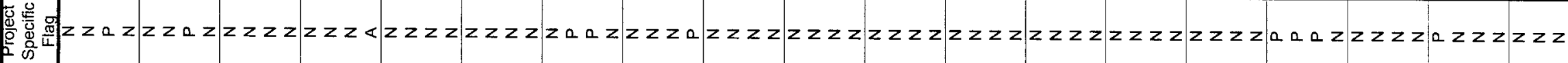

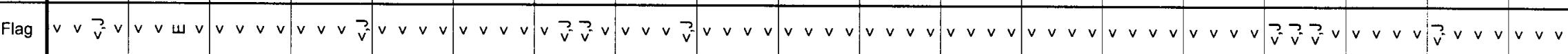

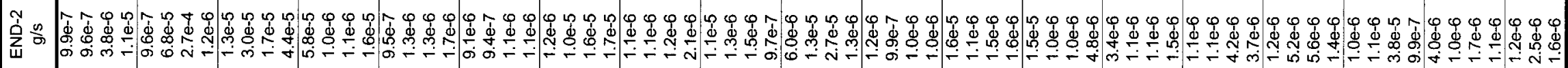

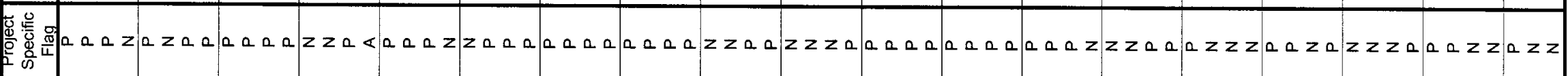

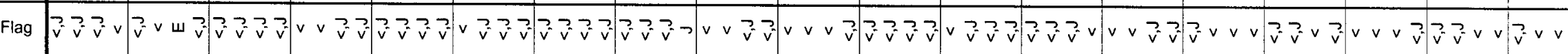

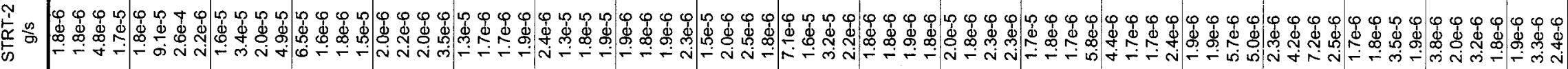

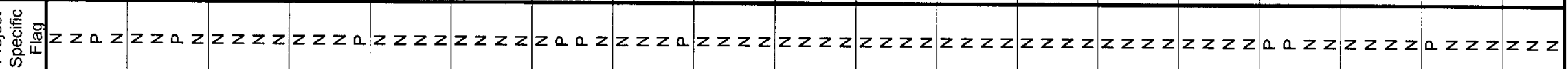

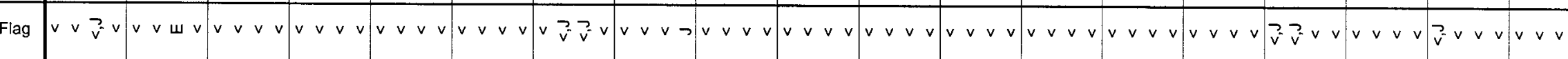

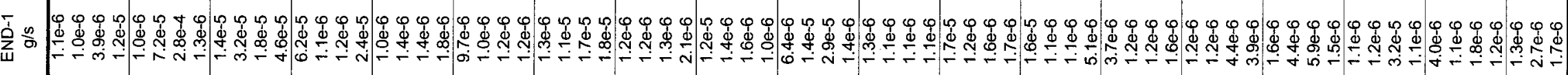

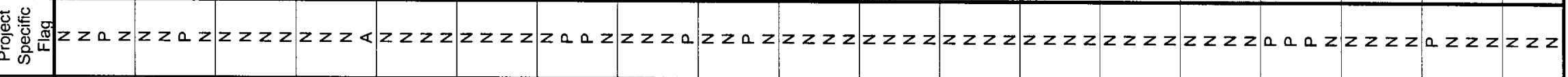

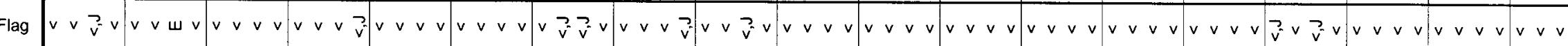

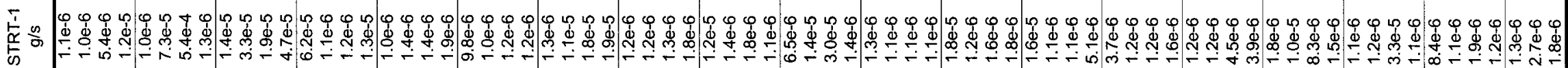
不 匹

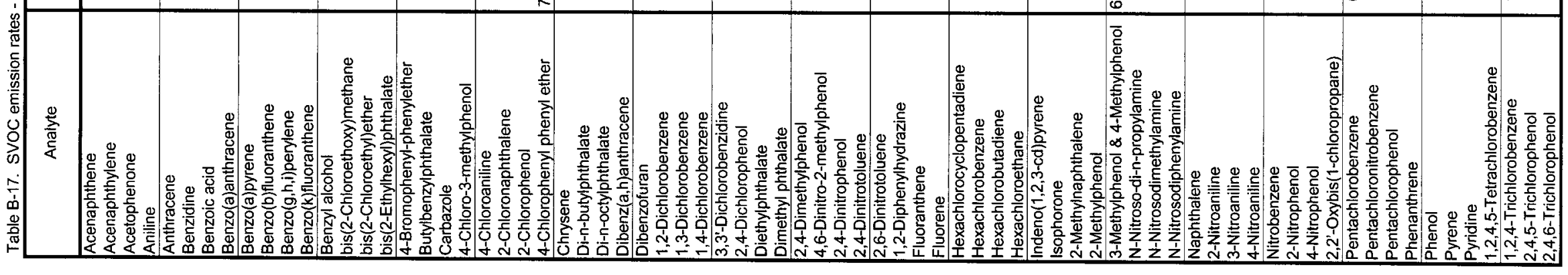




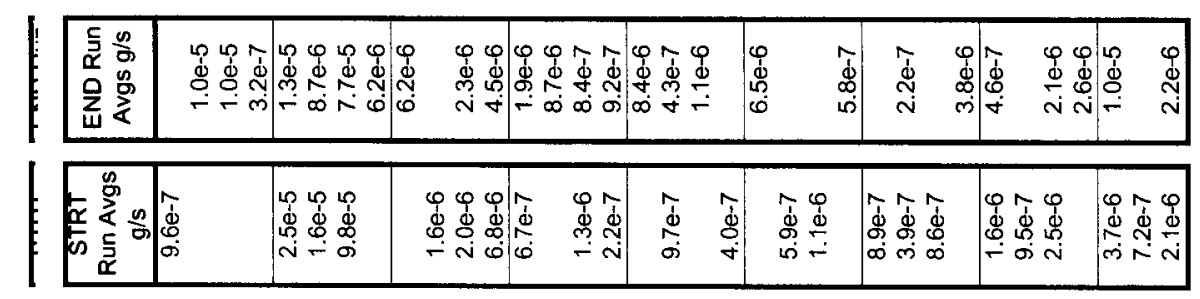

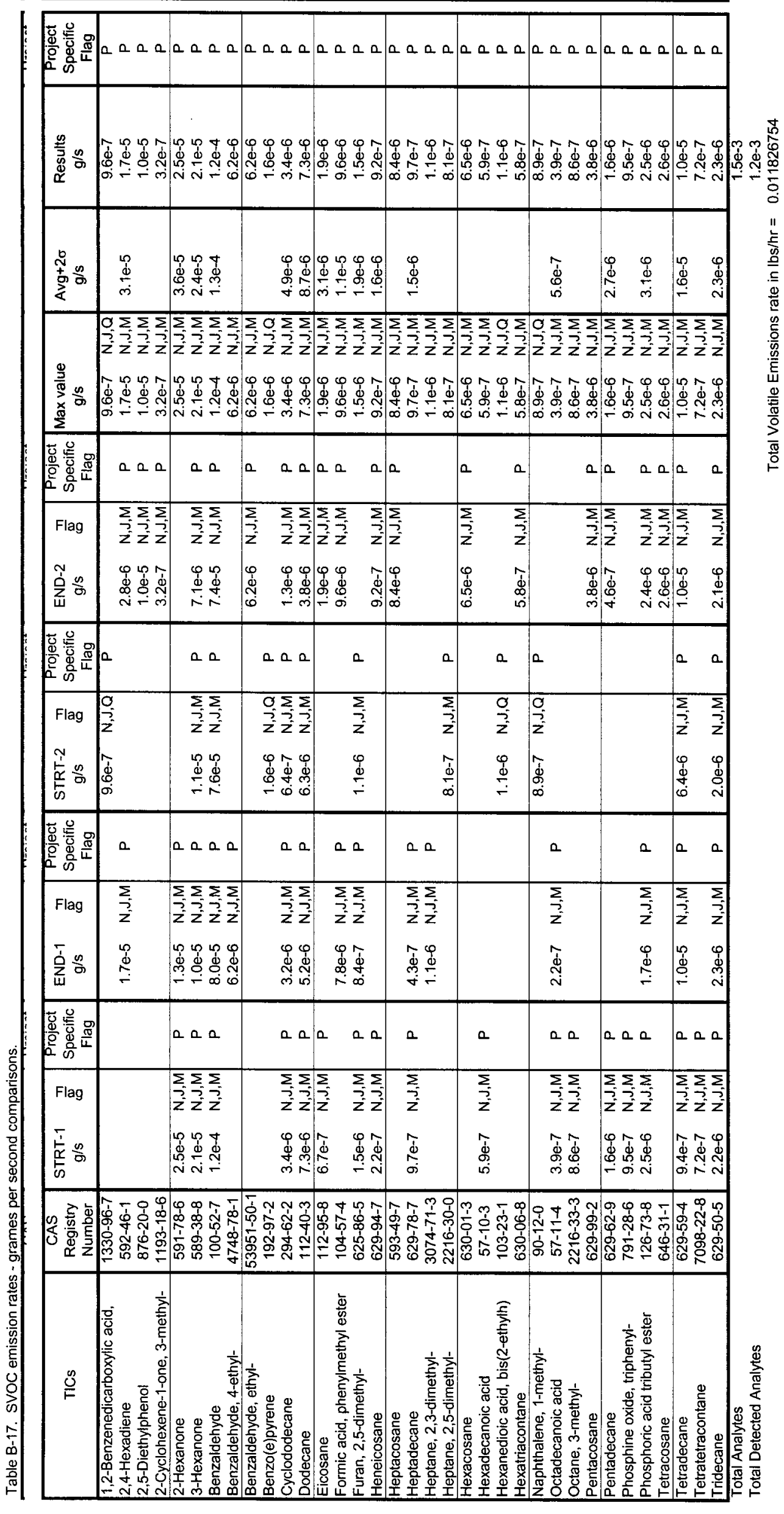




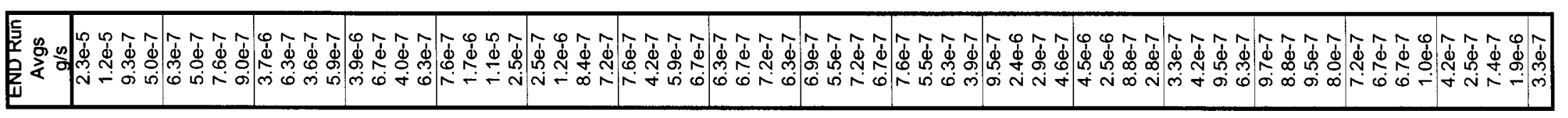

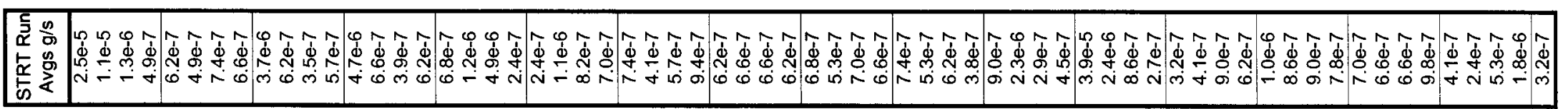

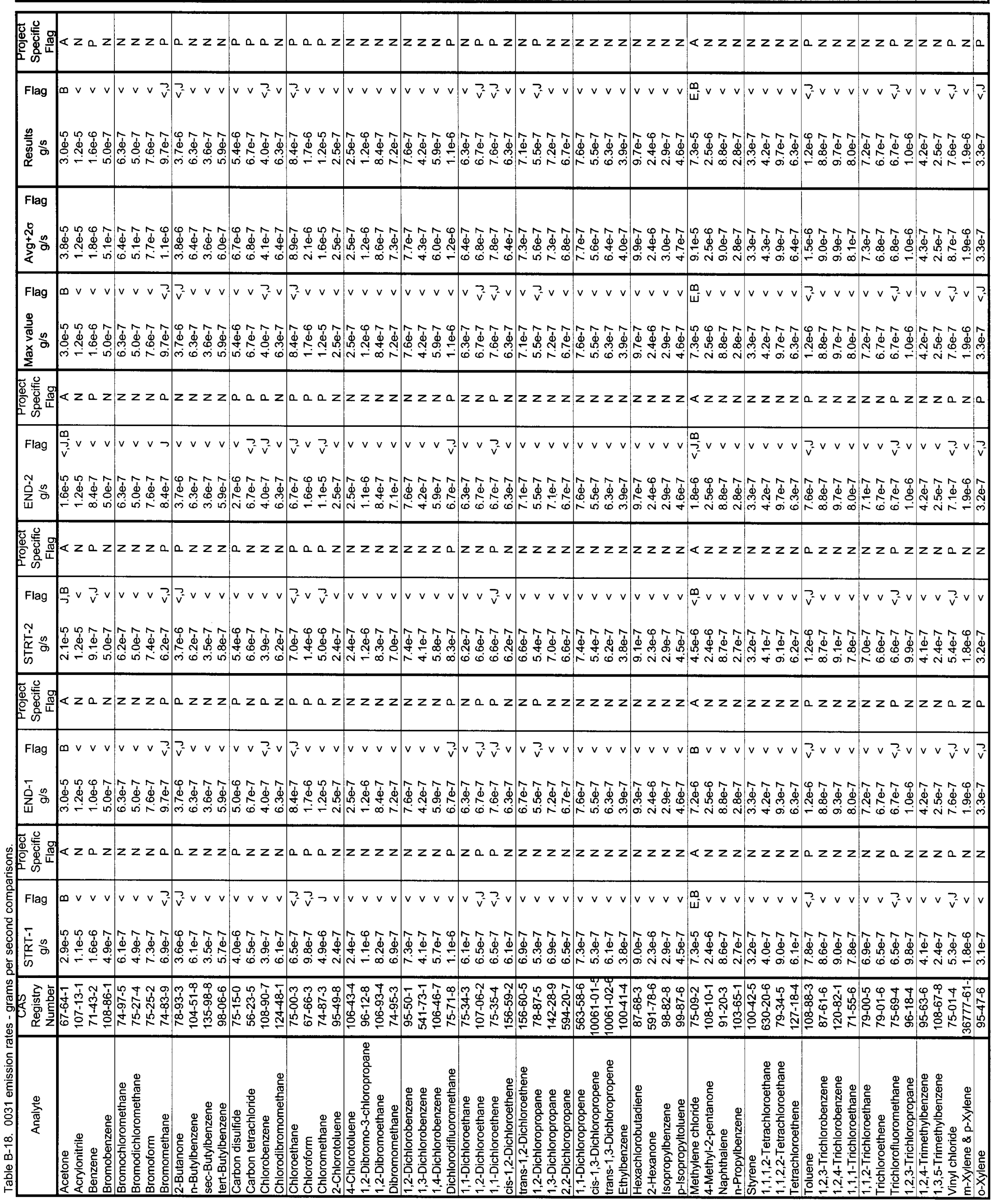




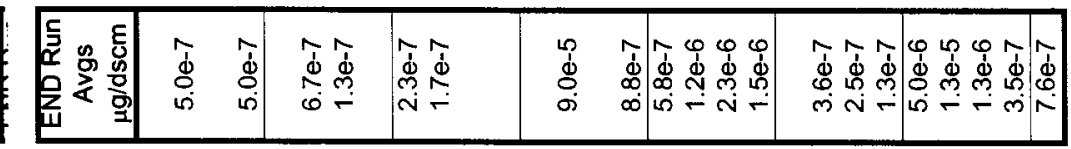

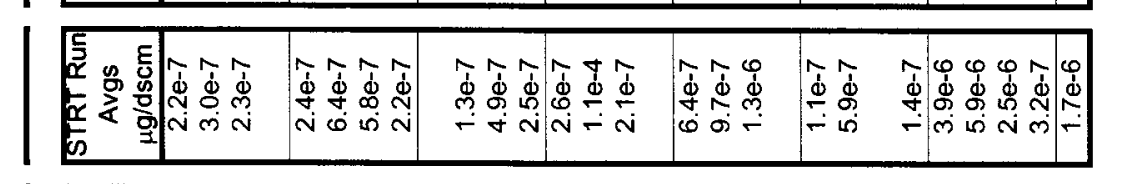

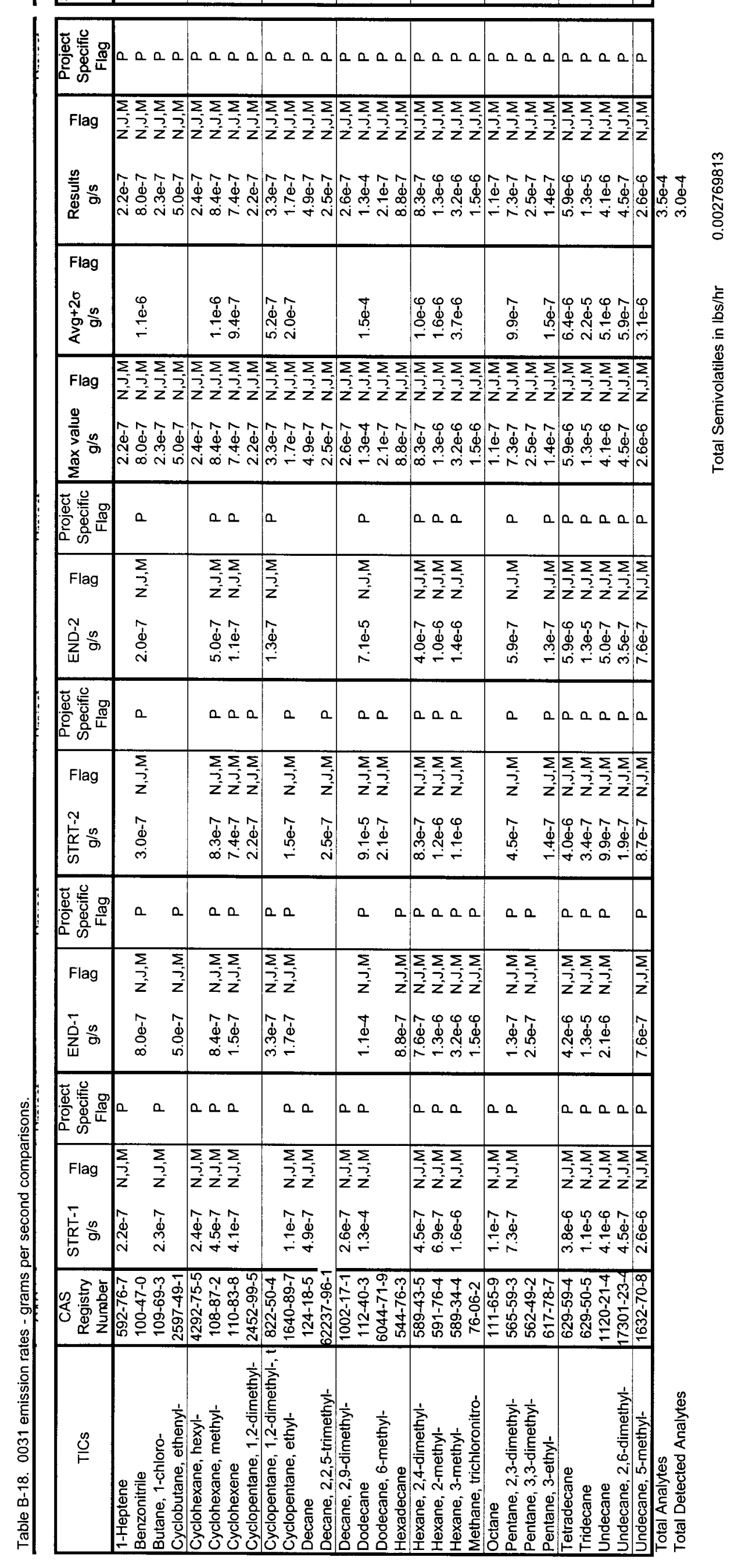



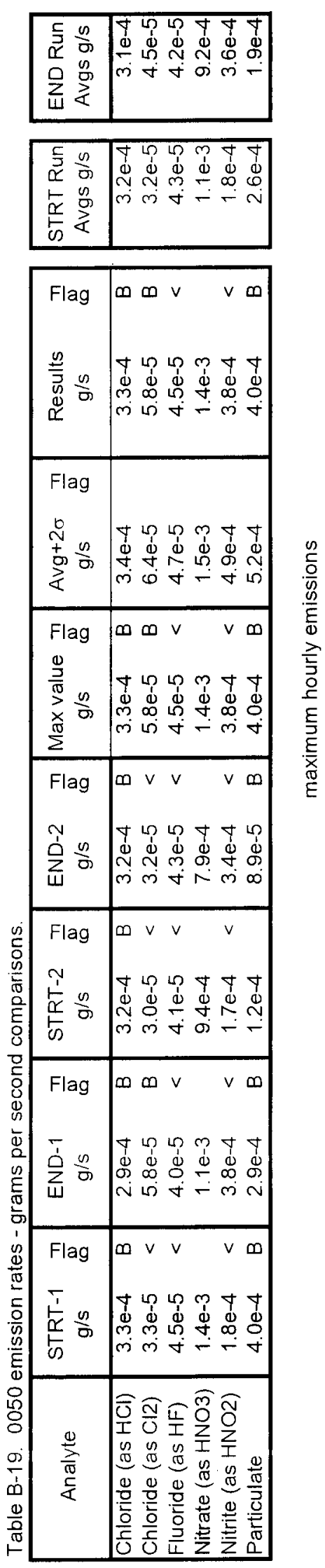

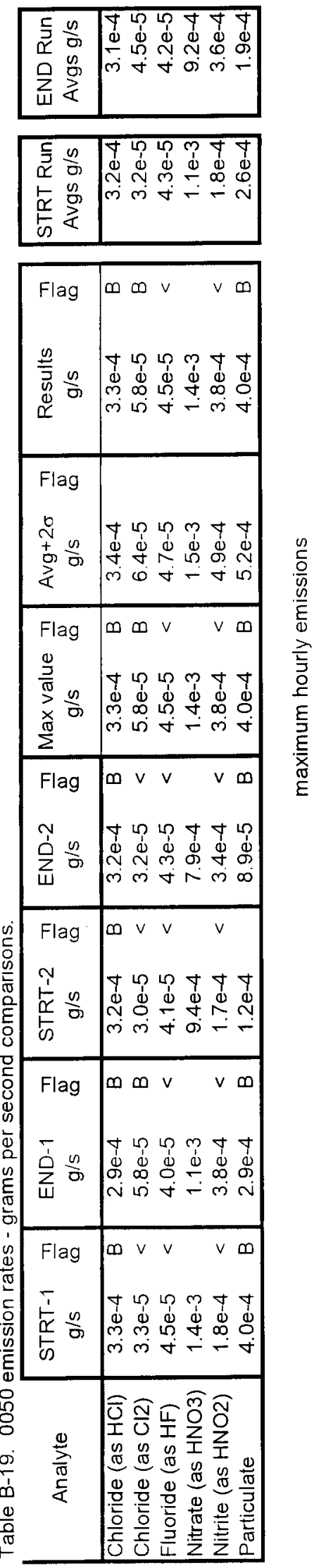


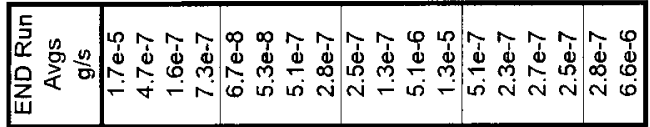

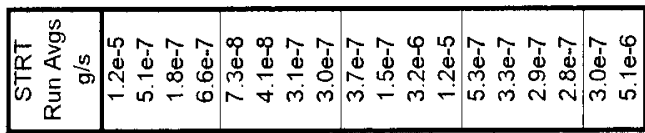

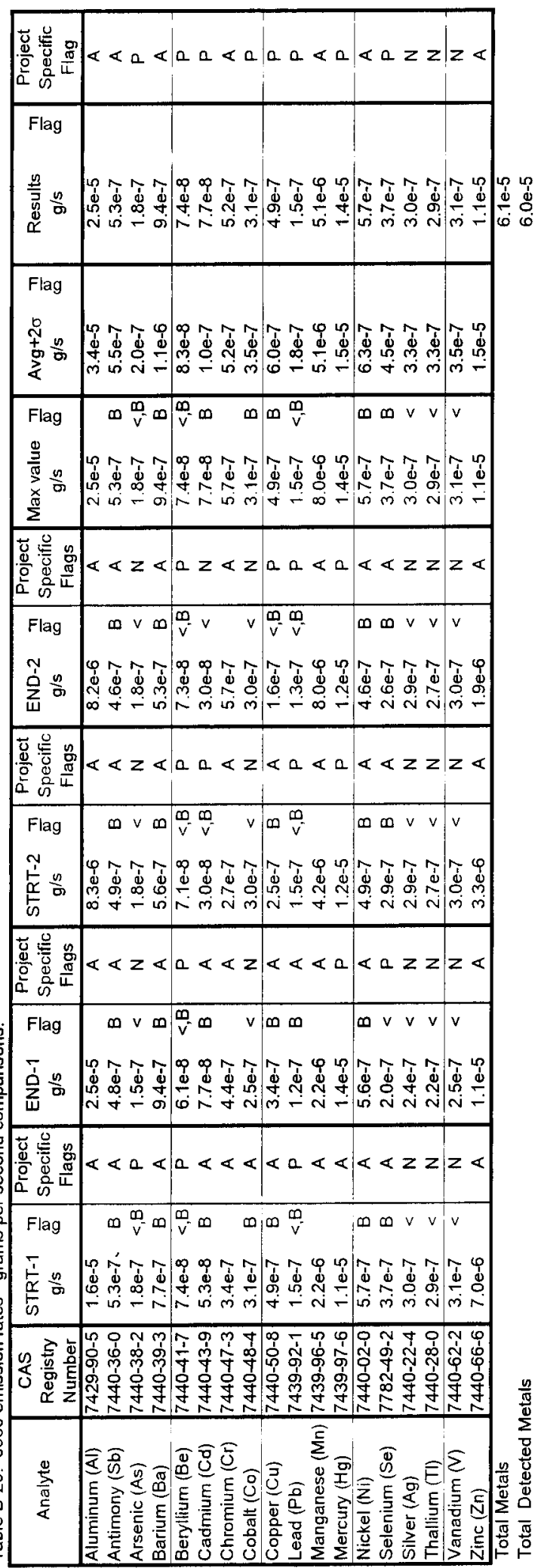




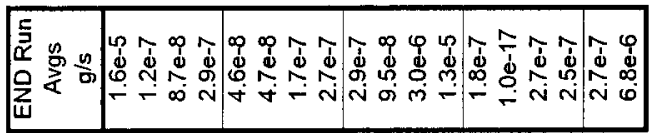

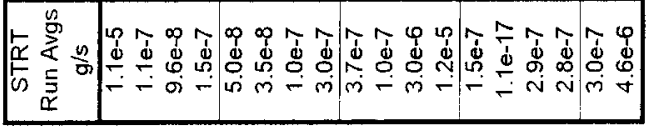
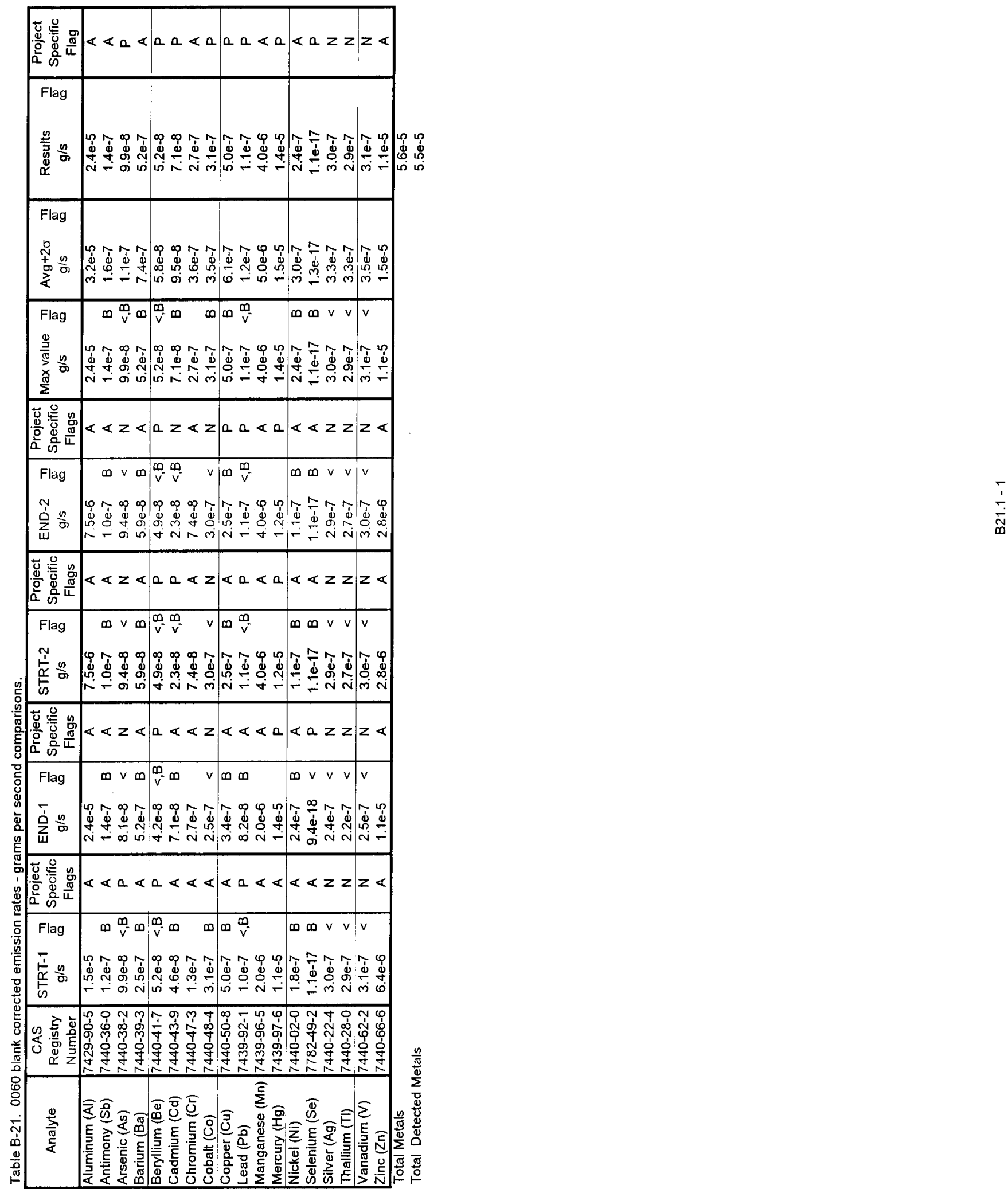


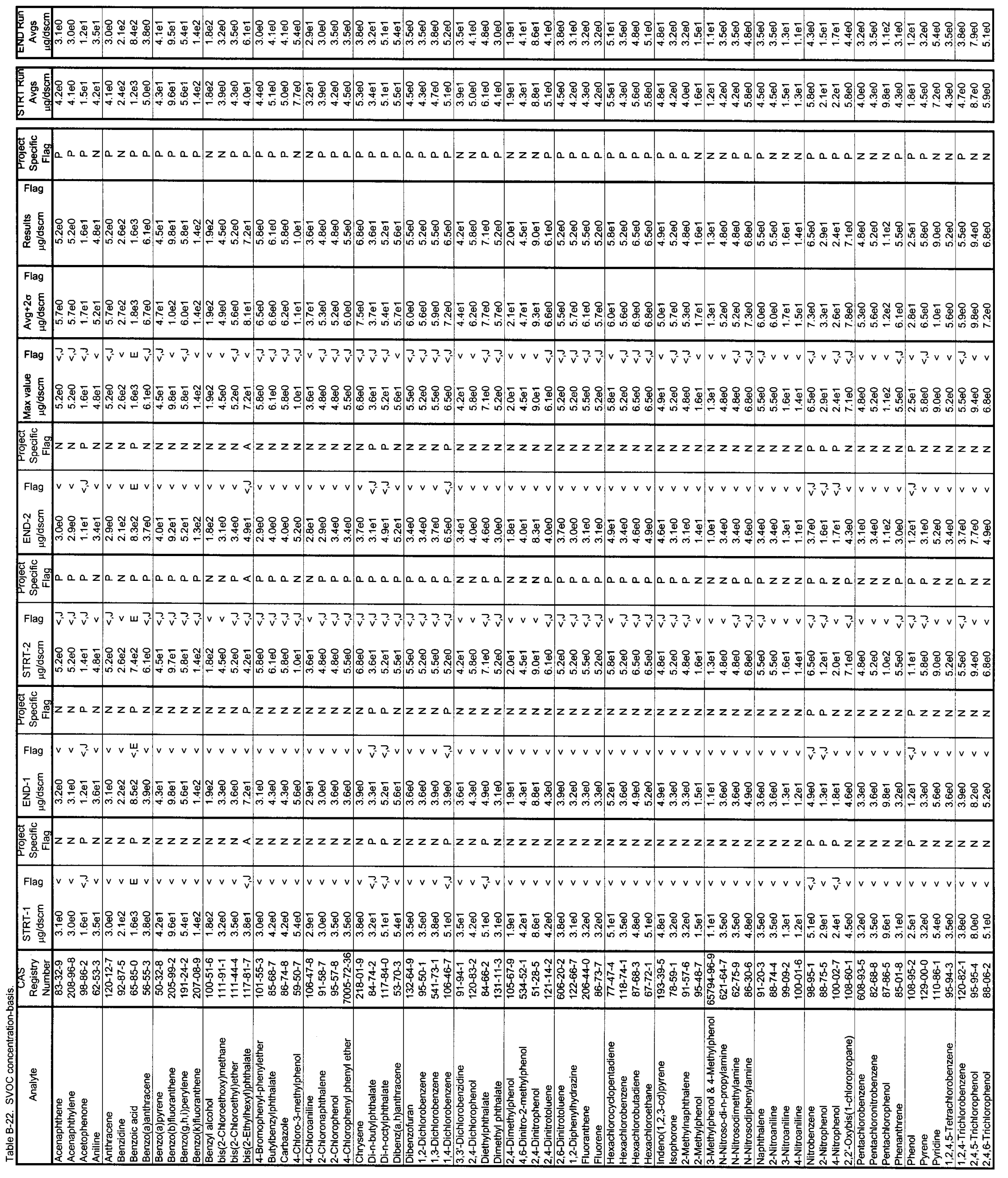


Table C-1. VOC target analyte list reported by the INTEC ALD.

\begin{tabular}{|c|c|c|c|}
\hline CAS \# & Compound & CAS \# & Compound \\
\hline $74-87-3$ & Chloromethane & $79-01-6$ & Trichloroethene \\
\hline $75-01-04$ & Vinyl Chloride & $78-87-5$ & 1,2-Dichloropropane \\
\hline $74-83-9$ & Bromomethane & $75-27-4$ & Bromodichloromethane \\
\hline $75-00-3$ & Chloroethane & $10061-01-5$ & Cis-1,3-dichloropropene \\
\hline $75-69-4$ & Trichlorofluoromethane & $108-10-1$ & 4-Methyl-2-pentanone \\
\hline $75-35-4$ & 1,1-Dichloroethene & $108-88-3$ & Toluene \\
\hline $76-13-1$ & $\begin{array}{l}1,1,2 \text {-Trichloro-1,2,2- } \\
\text { trifluoroethane }\end{array}$ & $10061-02-6$ & Trans-1,3-dichloropropene \\
\hline $75-15-0$ & Carbon disulfide & $79-00-5$ & 1,1,2-Trichloroethane \\
\hline $67-64-1$ & Acetone & $127-18-4$ & Tetrachloroethene \\
\hline $75-09-2$ & $\begin{array}{l}\text { Methylene chloride } \\
\text { (Dichloromethane) }\end{array}$ & $591-78-6$ & 2-Hexanone \\
\hline $156-60-5$ & Trans-1,2-dichloroethene & $124-48-1$ & Dibromochloromethane \\
\hline $75-34-3$ & 1,1-Dichloroethane & $108-90-7$ & Chlorobenzene \\
\hline $156-59-2$ & Cis-1,2-dichloroethene & $100-41-4$ & Ethylbenzene \\
\hline $78-93-3$ & 2-Butanone & $108-38-3$ & M-xylene and 106-42-3 p-xylene \\
\hline $67-66-3$ & Chloroform & $95-47-6$ & O-xylene \\
\hline $71-55-6$ & 1,1,1-Trichloroethane & $100-42-5$ & Styrene \\
\hline $56-23-5$ & Carbon tetrachloride & $75-25-2$ & Bromoform \\
\hline $71-43-2$ & Benzene & $79-34-5$ & 1,1,2,2-Tetrachloroethane \\
\hline $107-06-2$ & 1,2-Dichloroethane & & \\
\hline
\end{tabular}




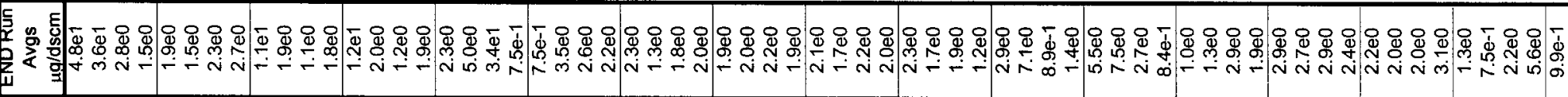

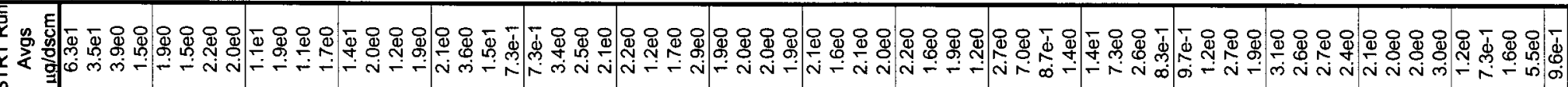

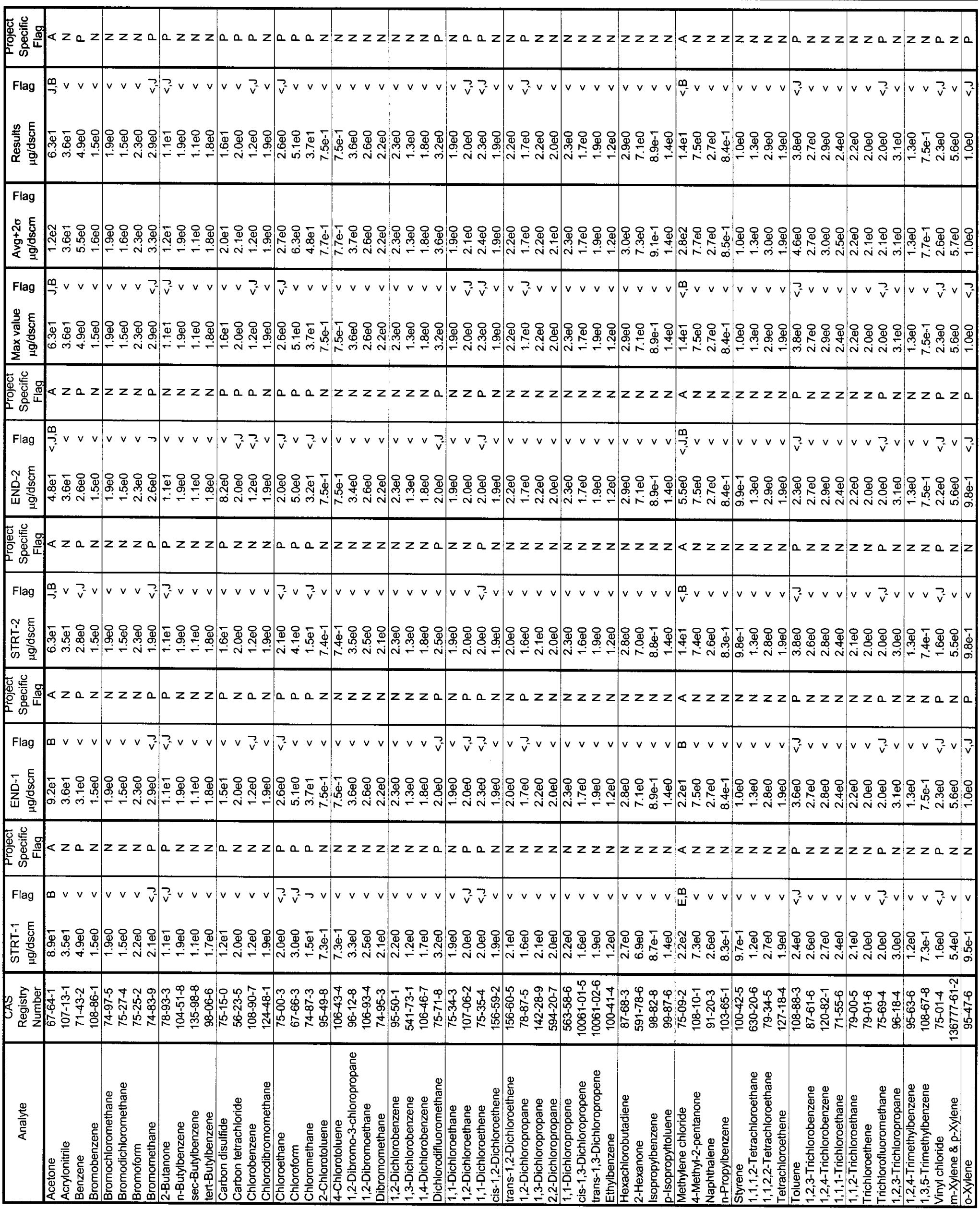




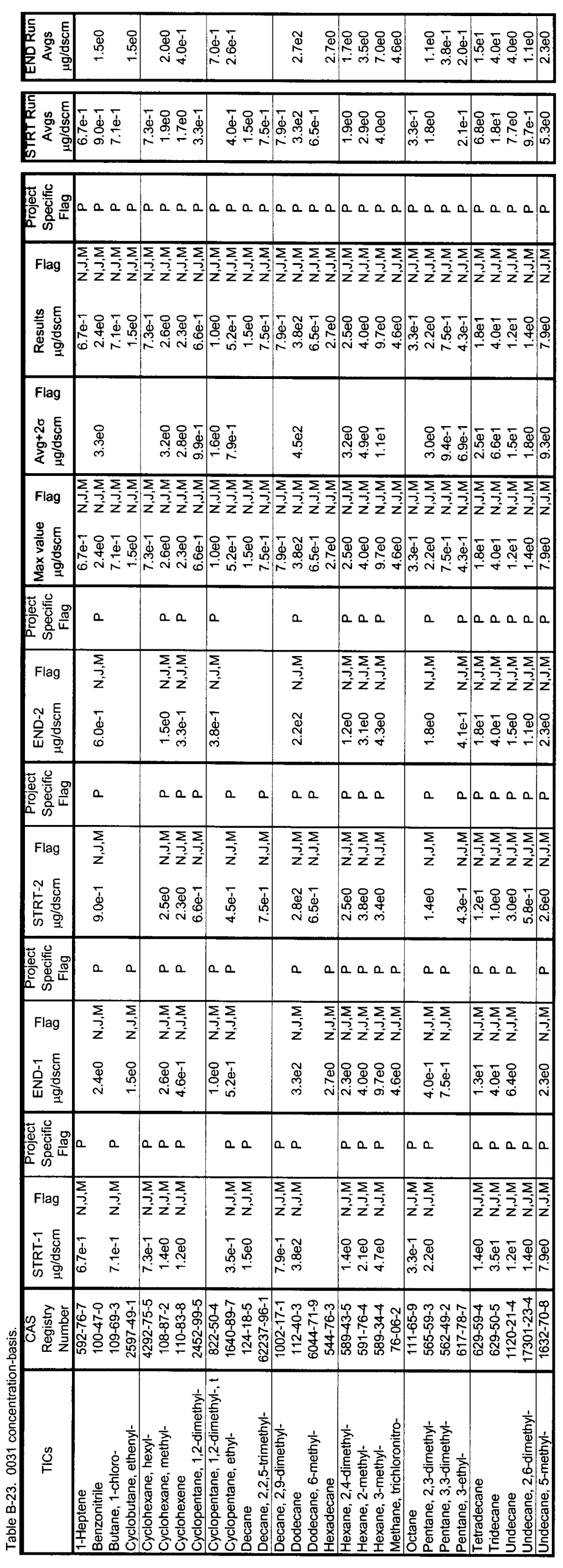



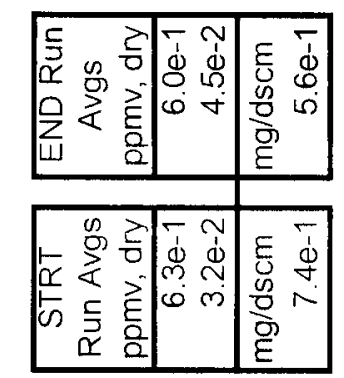

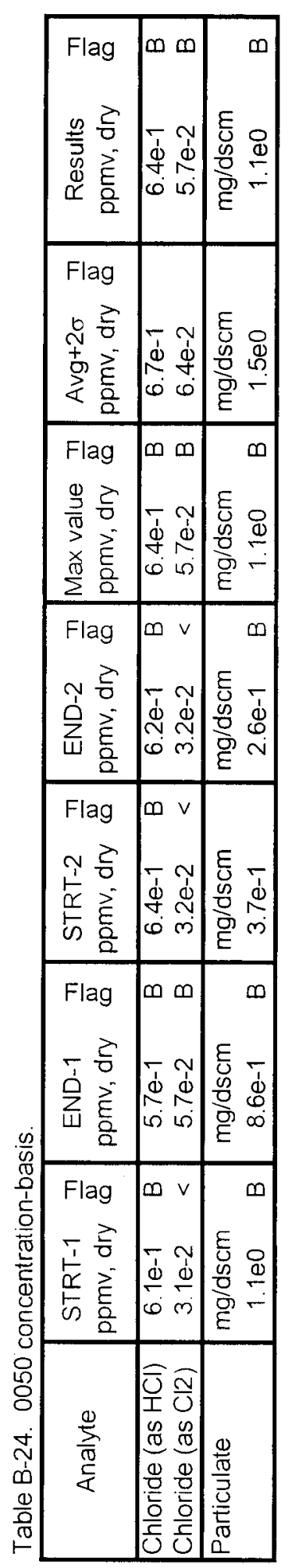

$\frac{i}{\stackrel{\Xi}{\Xi}}$ 


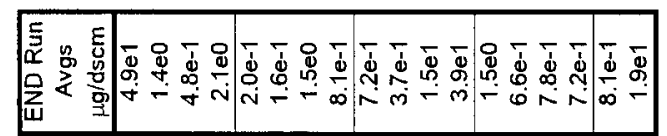

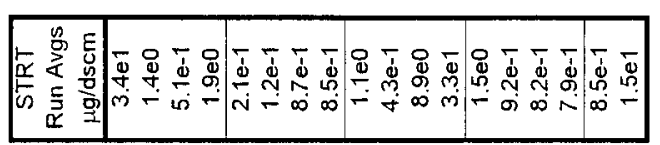

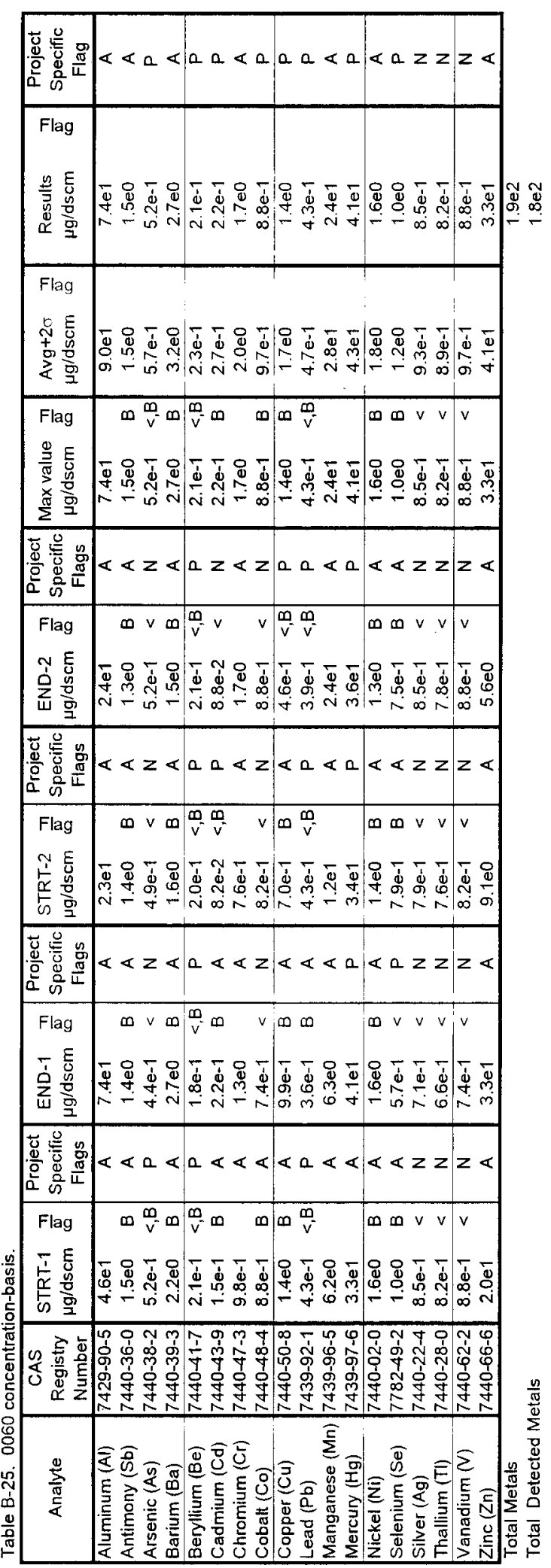




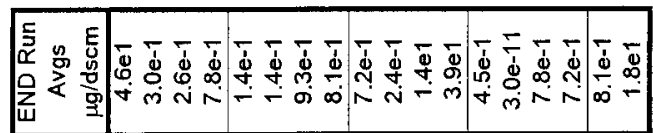

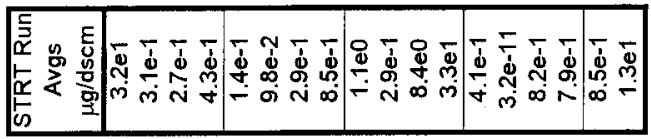

\begin{tabular}{|c|c|c|c|c|c|}
\hline 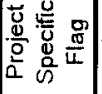 & $<\ll 0<$ & $a a<a$ & $a 0<a$ & $<a z z$ & $\mid z<$ \\
\hline 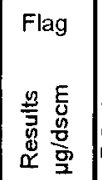 & 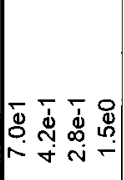 & & 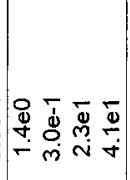 & 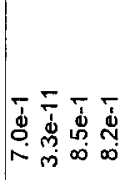 & 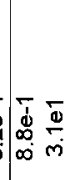 \\
\hline 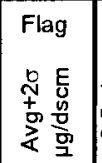 & 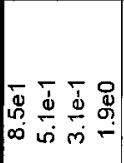 & 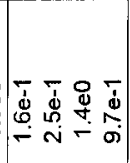 & 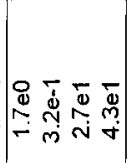 & 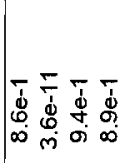 & 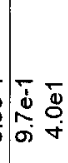 \\
\hline 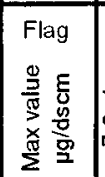 & 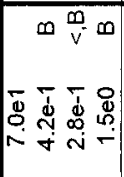 & 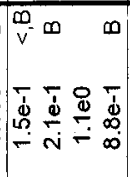 & 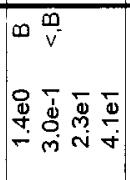 & 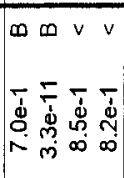 & 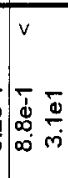 \\
\hline 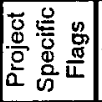 & $<<z<$ & $a z<z$ & $a \quad a<a$ & $<\ll z z$ & $z<$ \\
\hline 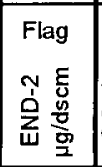 & 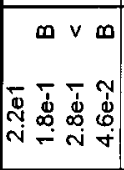 & 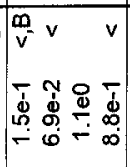 & 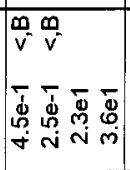 & 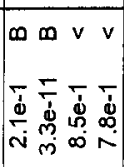 & $\mid \begin{array}{ll}v \\
-\bar{d} & 0 \\
\infty & \frac{8}{\sigma} \\
\infty & \dot{f}\end{array}$ \\
\hline 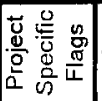 & $<<z<1$ & $\ln a<z$ & $|<a<a|$ & $\mid \varangle \varangle z z$ & $z<$ \\
\hline 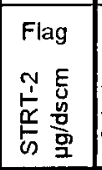 & 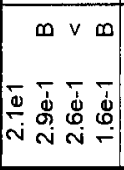 & 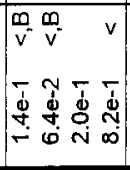 & 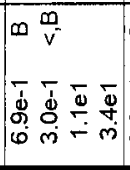 & 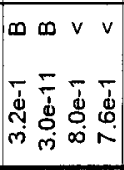 & 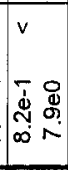 \\
\hline 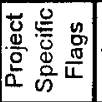 & $<<z<$ & $0<<z$ & $<<<0$ & $<a z z$ & $z<$ \\
\hline 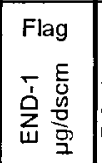 & 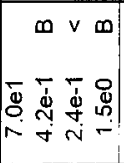 & 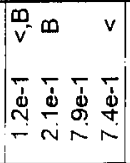 & 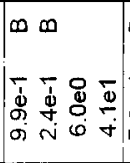 & 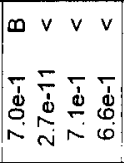 & $\frac{v}{d \dot{d}} \frac{\bar{\theta}}{m}$ \\
\hline 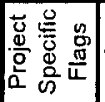 & $<<a<$ & $a<$ & $<a<<$ & $<<z z$ & $z<$ \\
\hline 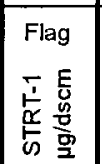 & 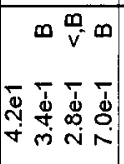 & 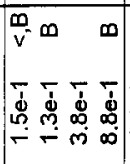 & 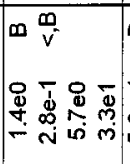 & 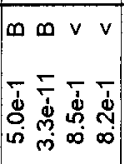 & $\begin{array}{ll}v \\
\bar{d} & \bar{d} \\
\infty & \infty \\
\infty & \stackrel{\infty}{-} \\
\infty & \end{array}$ \\
\hline 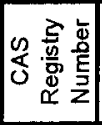 & 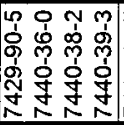 & 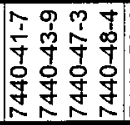 & 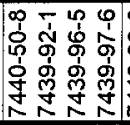 & 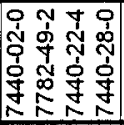 & 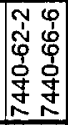 \\
\hline$\frac{\frac{9}{5}}{\frac{5}{\pi 0}}$ & 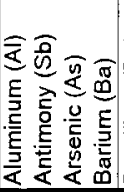 & 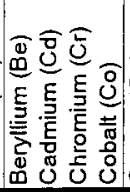 & 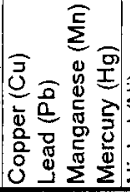 & 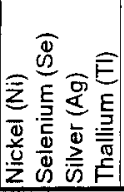 & 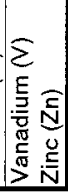 \\
\hline
\end{tabular}


APPENDIX C

\section{PROCESS STREAM SAMPLING DATA}



Table C-1. VOC target analyte list reported by the INTEC ALD.

\begin{tabular}{|c|c|c|c|}
\hline CAS \# & Compound & CAS \# & Compound \\
\hline $74-87-3$ & Chloromethane & $79-01-6$ & Trichloroethene \\
\hline $75-01-04$ & Vinyl Chloride & $78-87-5$ & 1,2-Dichloropropane \\
\hline $74-83-9$ & Bromomethane & $75-27-4$ & Bromodichloromethane \\
\hline $75-00-3$ & Chloroethane & $10061-01-5$ & Cis-1,3-dichloropropene \\
\hline $75-69-4$ & Trichlorofluoromethane & $108-10-1$ & 4-Methyl-2-pentanone \\
\hline $75-35-4$ & 1,1-Dichloroethene & $108-88-3$ & Toluene \\
\hline $76-13-1$ & $\begin{array}{l}1,1,2 \text {-Trichloro-1,2,2- } \\
\text { trifluoroethane }\end{array}$ & $10061-02-6$ & Trans-1,3-dichloropropene \\
\hline $75-15-0$ & Carbon disulfide & $79-00-5$ & 1,1,2-Trichloroethane \\
\hline $67-64-1$ & Acetone & $127-18-4$ & Tetrachloroethene \\
\hline $75-09-2$ & $\begin{array}{l}\text { Methylene chloride } \\
\text { (Dichloromethane) }\end{array}$ & $591-78-6$ & 2-Hexanone \\
\hline $156-60-5$ & Trans-1,2-dichloroethene & $124-48-1$ & Dibromochloromethane \\
\hline $75-34-3$ & 1,1-Dichloroethane & $108-90-7$ & Chlorobenzene \\
\hline $156-59-2$ & Cis-1,2-dichloroethene & $100-41-4$ & Ethylbenzene \\
\hline $78-93-3$ & 2-Butanone & $108-38-3$ & M-xylene and 106-42-3 p-xylene \\
\hline $67-66-3$ & Chloroform & $95-47-6$ & O-xylene \\
\hline $71-55-6$ & 1,1,1-Trichloroethane & $100-42-5$ & Styrene \\
\hline $56-23-5$ & Carbon tetrachloride & $75-25-2$ & Bromoform \\
\hline $71-43-2$ & Benzene & $79-34-5$ & 1,1,2,2-Tetrachloroethane \\
\hline $107-06-2$ & 1,2-Dichloroethane & & \\
\hline
\end{tabular}


Table C-2. SVOC target analyte list reported by the INTEC ALD.

\begin{tabular}{|c|c|c|c|}
\hline CAS \# & Compound & CAS \# & Compound \\
\hline $62-75-9$ & N-Nitrosodimethylamine & $83-32-9$ & Acenaphthene \\
\hline $110-86-1$ & Pyridine & $51-28-5$ & 2,4-Dinitrophenol \\
\hline $108-95-2$ & Phenol & $100-02-7$ & 4-Nitrophenol \\
\hline $111-44-4$ & bis(2-Chloroethyl)ether & $132-64-9$ & Dibenzofuran \\
\hline $95-57-8$ & 2-Chlorophenol & $121-14-2$ & 2,4-Dinitrotoluene \\
\hline $541-73-1$ & 1,3-Dichlorobenzene & $84-66-2$ & Diethylphthalate \\
\hline $106-46-7$ & 1,4-Dichlorobenzene & $7005-72-3$ & 4-Chlorophenyl-phenylether \\
\hline $95-50-1$ & 1,2-Dichlorobenzene & $86-73-7$ & Fluorene \\
\hline $95-48-7$ & 2-Methylphenol & $100-01-6$ & 4-Nitroaniline \\
\hline $108-60-1$ & bis(2-Chloroisopropyl)ether & $534-52-1$ & 4,6-Dinitro-2-methylphenol \\
\hline $106-44-5$ & $3 \& 4$-Methylphenol & $86-30-6$ & N-Nitrosodiphenylamine \\
\hline $621-64-7$ & N-Nitroso-di-n-propylamine & $126-73-8$ & Tri-n-butyl phosphate \\
\hline $67-72-1$ & Hexachloroethane & $103-33-3$ & Azobenzene \\
\hline $98-95-3$ & Nitrobenzene & $101-55-3$ & 4-Bromophenyl-phenylether \\
\hline $78-59-1$ & Isophorone & $118-74-1$ & Hexachlorobenzene \\
\hline $88-75-5$ & 2-Nitrophenol & $87-86-5$ & Pentachlorophenol \\
\hline $105-67-9$ & 2,4-Dimethylphenol & $85-01-8$ & Phenanthrene \\
\hline $111-91-1$ & bis(2-Chloroethoxy)methane) & $120-12-7$ & Anthracene \\
\hline $120-83-2$ & 2,4-Dichlorophenol & $86-74-8$ & Carbazole \\
\hline $120-82-1$ & 1,2,4-Trichlorobenzene & $84-74-2$ & Di-n-butylphthalate \\
\hline $91-20-3$ & Naphthalene & $206-44-0$ & Fluoranthene \\
\hline $106-47-8$ & 4-Chloroaniline & $129-00-0$ & Pyrene \\
\hline $87-68-3$ & Hexachlorobutadiene & $85-68-7$ & Butylbenzylphthalate \\
\hline $59-50-7$ & 4-Chloro-3-methylphenol & $91-94-1$ & 3,3'-Dichlorobenzidine \\
\hline $91-57-6$ & 2-Methylnaphthalene & $218-01-9$ & Chrysene \\
\hline $77-47-4$ & Hexachlorocyclopentadiene & $56-55-3$ & Benzo(a)anthracene \\
\hline $88-06-2$ & 2,4,6-Trichlorophenol & $117-81-7$ & bis(2-Ethylhexyl)phthalate \\
\hline $95-95-4$ & 2,4,5-Trichlorophenol & $117-84-0$ & Di-n-octylphthalate \\
\hline $91-58-7$ & 2-Chloronaphthalene & $205-99-2$ & Benzo(b)fluoranthene \\
\hline $88-74-4$ & 2-Nitroaniline & $207-08-9$ & Benzo(k)fluoranthene \\
\hline $131-11-3$ & Dimethylphthalate & $50-32-8$ & Benzo(a)pyrene \\
\hline $606-20-2$ & 2,6-Dinitrotoluene & $193-39-5$ & Indeno(1,2,3-cd)pyrene \\
\hline $208-96-8$ & Acenaphthylene & $53-70-3$ & Dibenzo(a,h)anthracene \\
\hline $99-09-2$ & 3-Nitroaniline & $191-24-2$ & Benzo(g,h,I)perylene \\
\hline
\end{tabular}




\section{FEED SAMPLES ANALYSIS REPORTS}


F I N A L R E P O R T for 150WM:181 198

Log Type: ** RCRA - PLANT * *

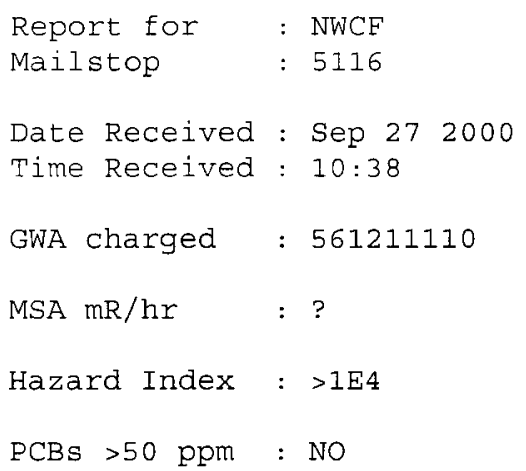

$\begin{array}{lcc} & \text { Lab } & \text { Field } \\ \text { Analysis } & \text { Spl ID Spl ID } \\ \ldots & -\ldots \text { OCD15 } & \text { 150WM/NEOPRENE } \\ \text { Total Sr } & \text { OCDI5 } & \text { 150wM/NEOPRENE } \\ \text { Tritium } & 2 \text { results. }\end{array}$

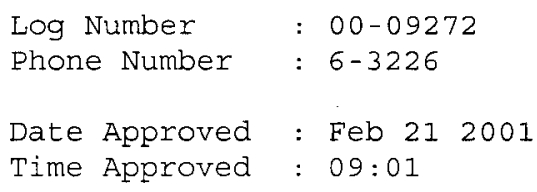


F I N A L R E P O R T for 150FEED:103 198/181

Log Type: * * PLANT * *

$\begin{array}{ll}\text { Report for } & : \text { NWCF } \\ \text { Mailstop } & : 5116 \\ \text { Date Received } & : \text { Sep } 272000 \\ \text { Time Received } & : 11: 11 \\ \text { GWA charged } & : 561211110 \\ \text { MSA mR/hr } & : \text { COLD } \\ \text { Hazard Index } & :<1 E 4 \\ \text { PCBs }>50 \text { ppm } & : \text { NO }\end{array}$

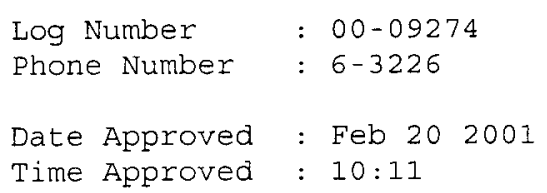

Reviewed by TIFFANY PARK

Signature

Lab QC/QA reviewed by

Signature

COMMENTS :

\begin{tabular}{|c|c|c|}
\hline & $\mathrm{Lab}$ & Field \\
\hline Analysis & Spl ID & Spl ID \\
\hline 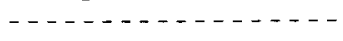 & ---- & $-\ldots-\ldots,-\ldots$ \\
\hline Acid & $0 \mathrm{CD} 21$ & $150 \mathrm{WM} / \mathrm{NEOPRENE}$ \\
\hline Aluminum & $0 \mathrm{CD} 25$ & FEED150/PLASTIC \\
\hline Boron & $0 \mathrm{CD} 25$ & FEED150/PLASTIC \\
\hline Cadmium & $0 \mathrm{CD} 25$ & FEED150/PLASTIC \\
\hline Calcium & OCD25 & FEED150/PLASTIC \\
\hline Chloride & $0 \mathrm{CD} 21$ & 150WM/NEOPRENE \\
\hline Chromium & $0 \mathrm{CD} 25$ & FEED150/PLASTIC \\
\hline $\operatorname{Co6} 0$ & OCD2 1 & $150 \mathrm{WM} / \mathrm{NEOPRENE}$ \\
\hline $\operatorname{Cs} 134$ & $\mathrm{OCD} 21$ & 150WM/NEOPRENE \\
\hline $\operatorname{CsI} 37$ & $0 \mathrm{CD} 21$ & 150WM/NEOPRENE \\
\hline Eu154 & $0 \mathrm{CD} 21$ & 150WM/NEOPRENE \\
\hline Eu155 & $0 \mathrm{CD} 21$ & 150WM/NEOPRENE \\
\hline Fluoride & $0 \mathrm{CD} 21$ & $150 \mathrm{WM} / \mathrm{NEOPRENE}$ \\
\hline Iron & OCD25 & FEED150/PLASTIC \\
\hline Mercury & $0 \mathrm{CD} 25$ & FEED150/PLASTIC \\
\hline NB9 4 & $0 \mathrm{CD} 21$ & $150 \mathrm{WM} / \mathrm{NEOPRENE}$ \\
\hline Nitrate & $0 C D 21$ & 150WM/NEOPRENE \\
\hline Potassium & $0 \operatorname{CD} 25$ & FEED150/PLASTIC \\
\hline Sodium & $0 C D 25$ & FEED150/PLASTIC \\
\hline SpGr & $0 C D 21$ & $150 \mathrm{WM} / \mathrm{NEOPRENE}$ \\
\hline Sulfate & $0 \mathrm{CD} 21$ & 150WM/NEOPRENE \\
\hline Uranium PreP & $0 C D 21$ & 150WM/NEOPRENE \\
\hline Pirconium & $0 \mathrm{CD} 25$ & FEEDI50/PLASTIC \\
\hline $\operatorname{Zrg5}$ & $0 \mathrm{CD} 21$ & $150 \mathrm{WM} / \mathrm{NEOPRENE}$ \\
\hline End of Report - - & $24 r$ & esults. \\
\hline
\end{tabular}

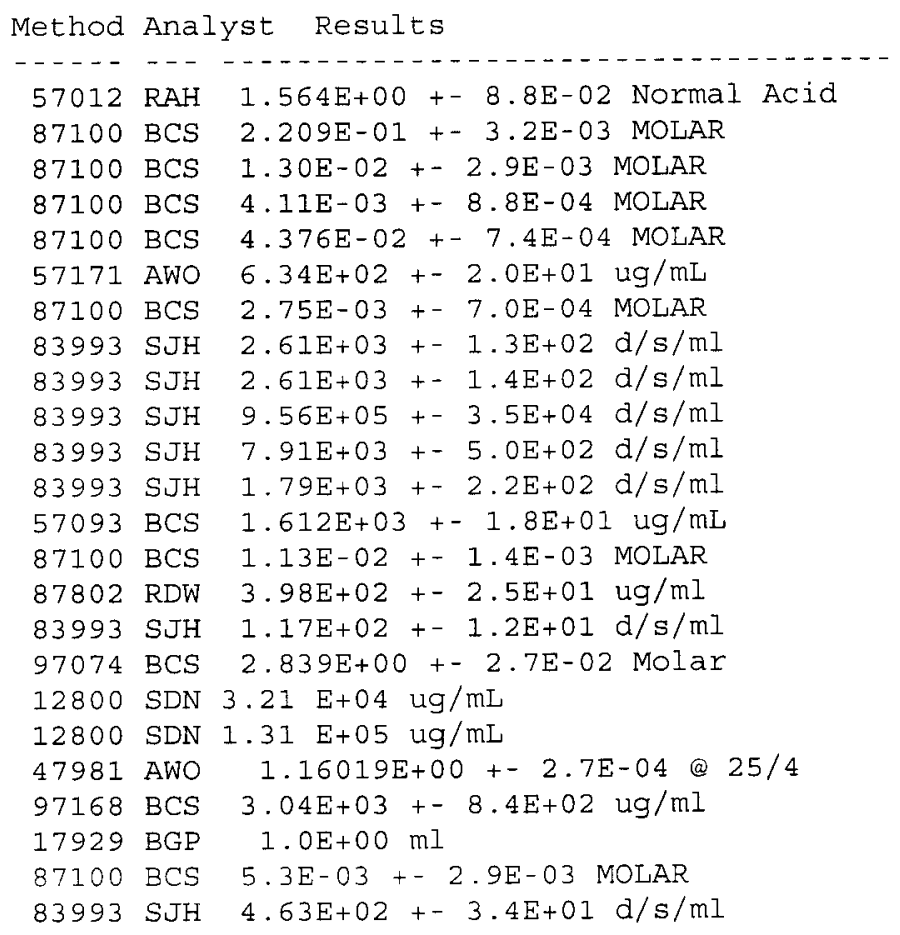


F I N A L R E P O R T for 150FEED:103 199

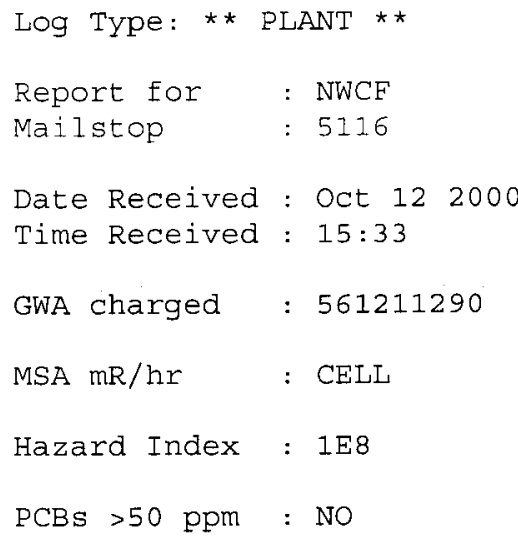

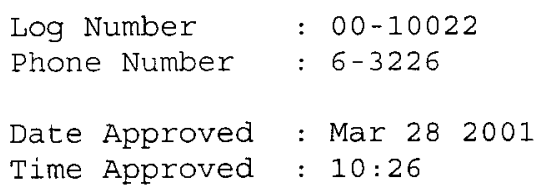

Signature

COMMENTS :

\begin{tabular}{|c|c|c|}
\hline & Lab & Field \\
\hline Analysis & Spl ID & Spl ID \\
\hline------------- & $--\cdot-$ & $-\ldots-\ldots,-\ldots$ \\
\hline Acid & OCD94 & FEED150/NEOPRENE \\
\hline Aluminum & 0CD95 & FEED150/PLASTIC \\
\hline Boron & OCD95 & FEED150/PLASTIC \\
\hline Cadmium & OCD95 & FEED150/PLASTIC \\
\hline Calcium & OCD95 & FEED150/PLASTIC \\
\hline Chloride & OCD9 4 & FEED150/NEOPRENE \\
\hline Chromium & $0 \mathrm{CD} 95$ & FEED150/PLASTIC \\
\hline Co60 & OCD9 4 & FEED150/NEOPRENE \\
\hline $\operatorname{Cs} 134$ & $0 \operatorname{CD} 94$ & FEED150/NEOPRENE \\
\hline $\operatorname{Cs} 137$ & OCD 94 & FEED150/NEOPRENE \\
\hline Eul 54 & $0 \mathrm{CD} 94$ & FEED150/NEOPRENE \\
\hline Eu155 & $0 \mathrm{CD} 94$ & FEED150/NEOPRENE \\
\hline Fluoride & $0 \mathrm{CD} 94$ & FEED150/NEOPRENE \\
\hline Iron & OCD95 & FEED150/PLASTIC \\
\hline Mercury & $0 C D 95$ & FEED150/PLASTIC \\
\hline NB94 & $0 \operatorname{CD} 94$ & FEED150/NEOPRENE \\
\hline Nitrate & $0 \mathrm{CD} 94$ & FEED150/NEOPRENE \\
\hline Potassium & $0 \mathrm{CD} 95$ & FEED150/PLASTIC \\
\hline $\mathrm{Sb} 125$ & $0 \mathrm{CD} 94$ & FEED150/NEOPRENE \\
\hline Sodium & OCD95 & FEED150/PLASTIC \\
\hline SpGr & OCD94 & FEED150/NEOPRENE \\
\hline Sulfate & OCD94 & FEED150/NEOPRENE \\
\hline Uranium & OCD 94 & FEED150/NEOPRENE \\
\hline zirconium & OCD95 & FEED150/PLASTIC \\
\hline $\operatorname{Zrg5}$ & OCD94 & FEED150/NEOPRENE \\
\hline
\end{tabular}

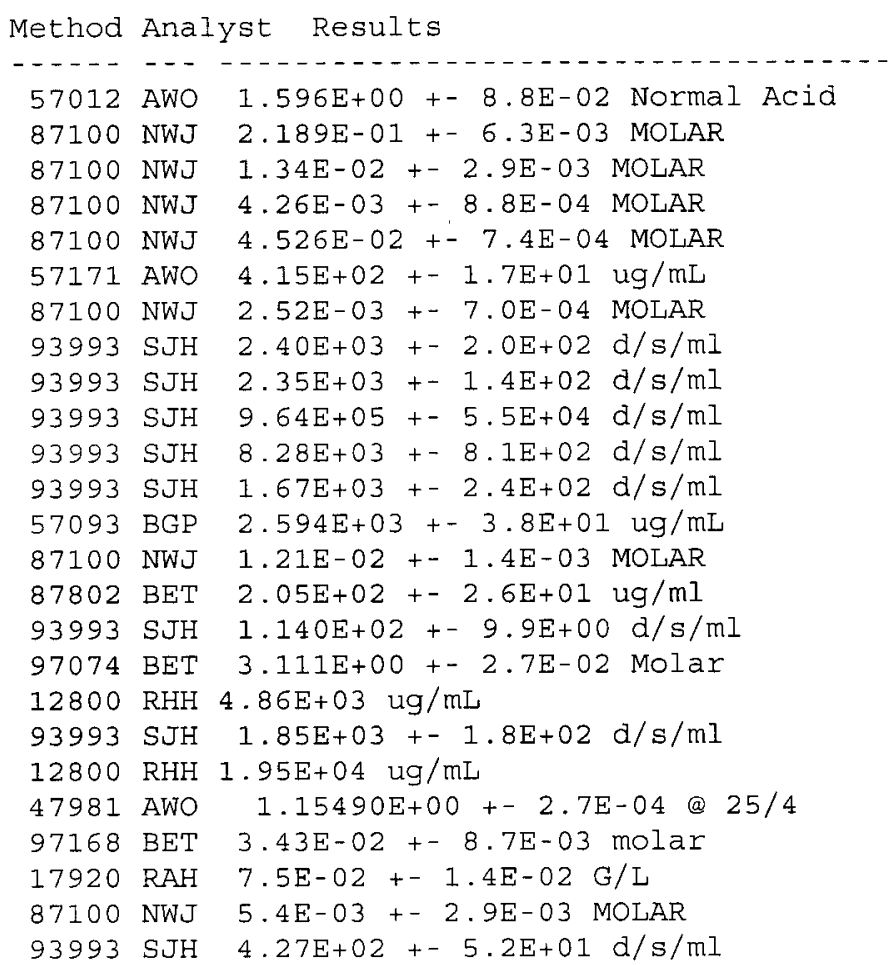


F I N A L R E P O R T for 150WM:181 199

Log Type: ** RCRA - PLANT * *

\begin{tabular}{|c|c|c|}
\hline Report for & : NWCF & $: 00-100415$ \\
\hline Mailstop & : 5116 & Phone Number \\
\hline Date Received & : Oct 052000 & Date Approved : Feb 212001 \\
\hline Time Received & $: 11: 35$ & Time Approved : 09:00 \\
\hline GWA charged & : 591211290 & Reviewed by BRIAN STORMS \\
\hline $\mathrm{MSA} \mathrm{mR} / \mathrm{hr}$ & : HOT & Signature \\
\hline Hazard Index & $:>1 \mathrm{E} 4$ & Lab QC/QA reviewed by \\
\hline PCBs $>50 \mathrm{ppm}$ & : NO & Signature \\
\hline
\end{tabular}

COMMENTS :

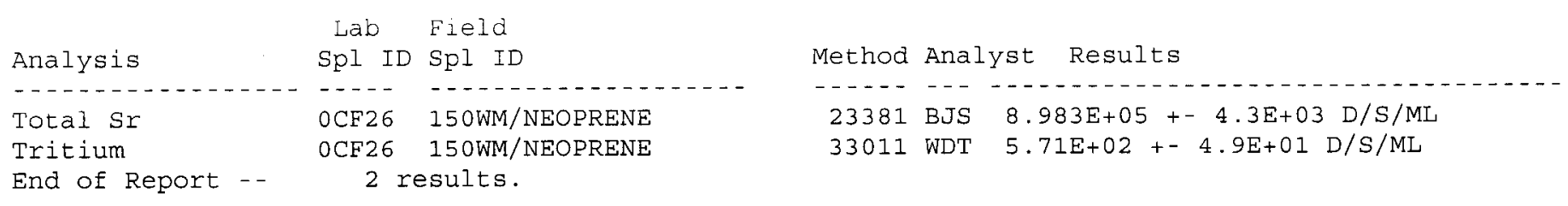


F I N A L R E P O R T for 150WM:181 200

Log Type: ** RCRA - PLANT * *

$\begin{array}{llll}\text { Report for } & : \text { NWCF } & \text { Log Number } & : 00-10164 \\ \text { Mailstop } & : 5116 & \text { Phone Number } & : 6-3226 \\ & & & \\ \text { Date Received : Oct } 172000 & \text { Date Approved } & : \text { Feb } 212001 \\ \text { Time Received : } 17: 04 & \text { Time Approved : } 08: 59\end{array}$

Iime Received : 17:04

Time Approved : 08:59

GWA charged : 561211290

Reviewed by BRIAN STORMS

MSA mR/hr : CELL

Signature

Hazard Index : 1E8

Lab QC/QA reviewed by

PCBs $>50 \mathrm{ppm}:$ NO

Signature

COMMENTS :

\begin{tabular}{|c|c|c|}
\hline & $\mathrm{Lab}$ & Field \\
\hline Analysis & Spl ID & Spl ID \\
\hline-------------- & ----- & 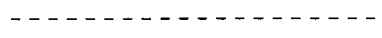 \\
\hline Total Sr & 0CG62 2 & WM1 81 / NEOPREN \\
\hline Tritium & 0CG62 & WM181/NEOPREN \\
\hline
\end{tabular}

Method Analyst Results
23381 BJS $7.656 \mathrm{E}+05+4.3 \mathrm{E}+03 \mathrm{D} / \mathrm{S} / \mathrm{ML}$
33011 WDT $4.66 \mathrm{E}+02+-4.0 \mathrm{E}+01 \mathrm{D} / \mathrm{S} / \mathrm{ML}$


F I N A L R E P O R T for 150FEED:103 200

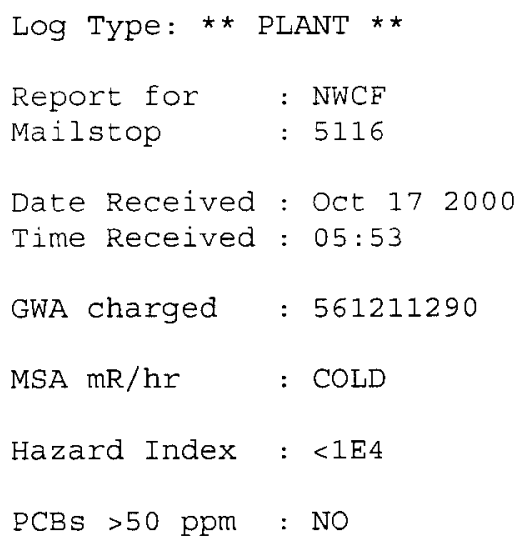

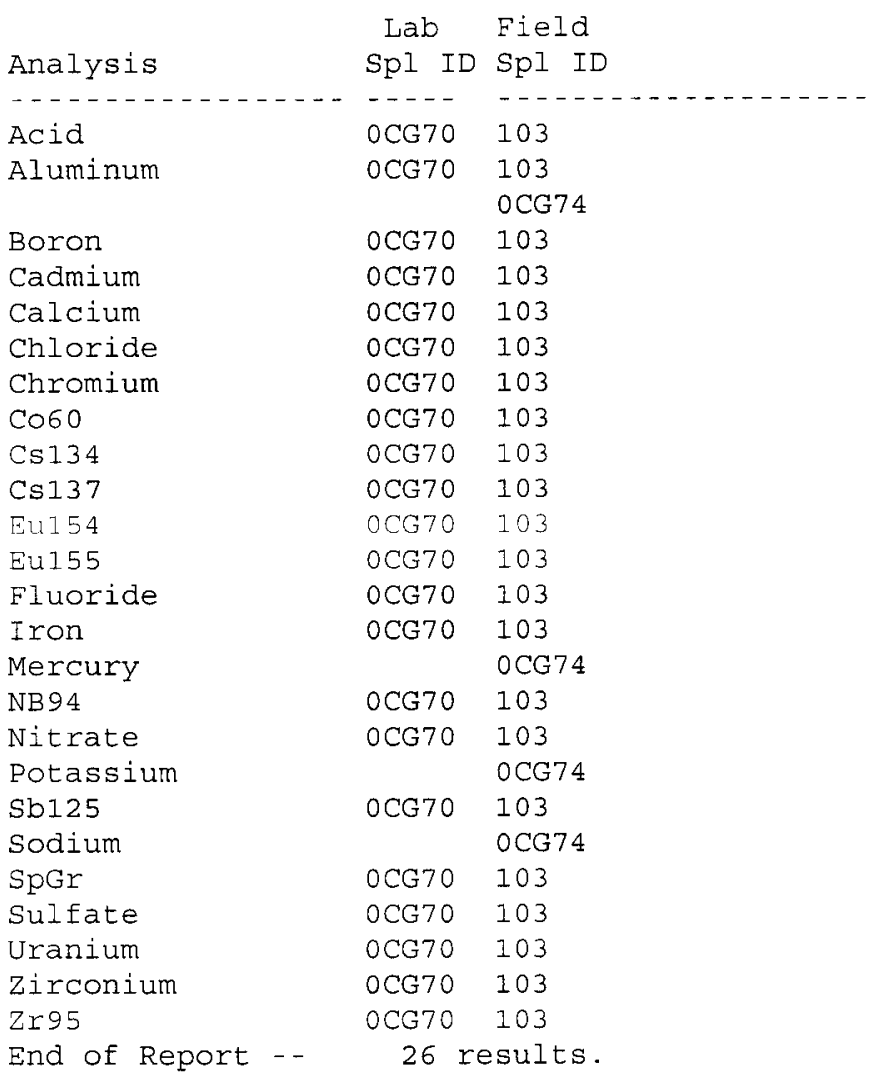

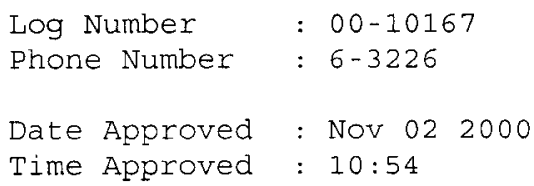

Signature

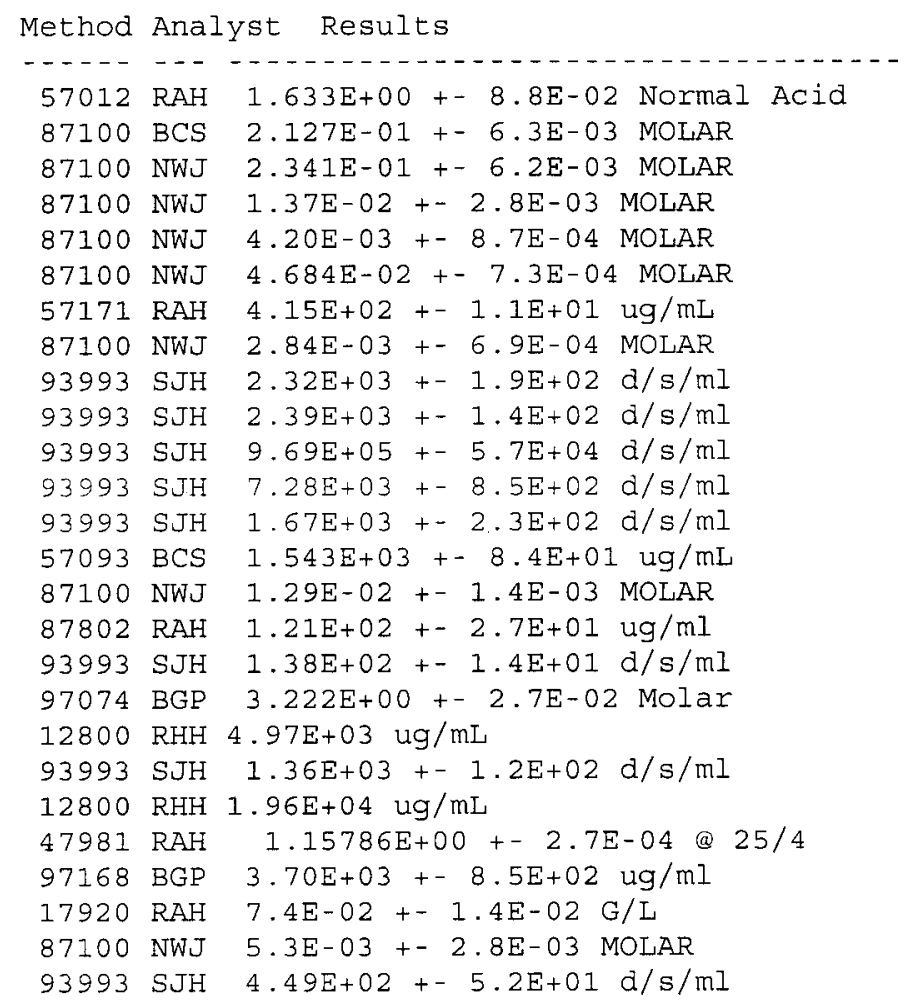


Log Type: ** PLANT **

$\begin{array}{ll}\text { Report for } & \text { NWCF } \\ \text { Mailstop } & : 5116 \\ \text { Date Received } & : \text { Apr } 102001 \\ \text { Time Received } & : 13: 42 \\ \text { GWA charged } & : 561211290 \\ \text { MSA mR/hr } & : \text { HOT } \\ \text { Hazard Index } & :<1 E 4 \\ \text { PCBs >50 ppm : NO }\end{array}$

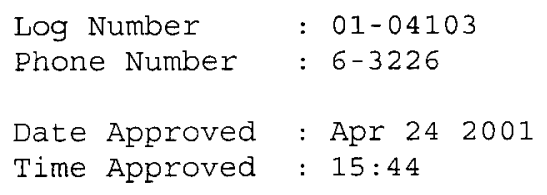

COMMENTS :

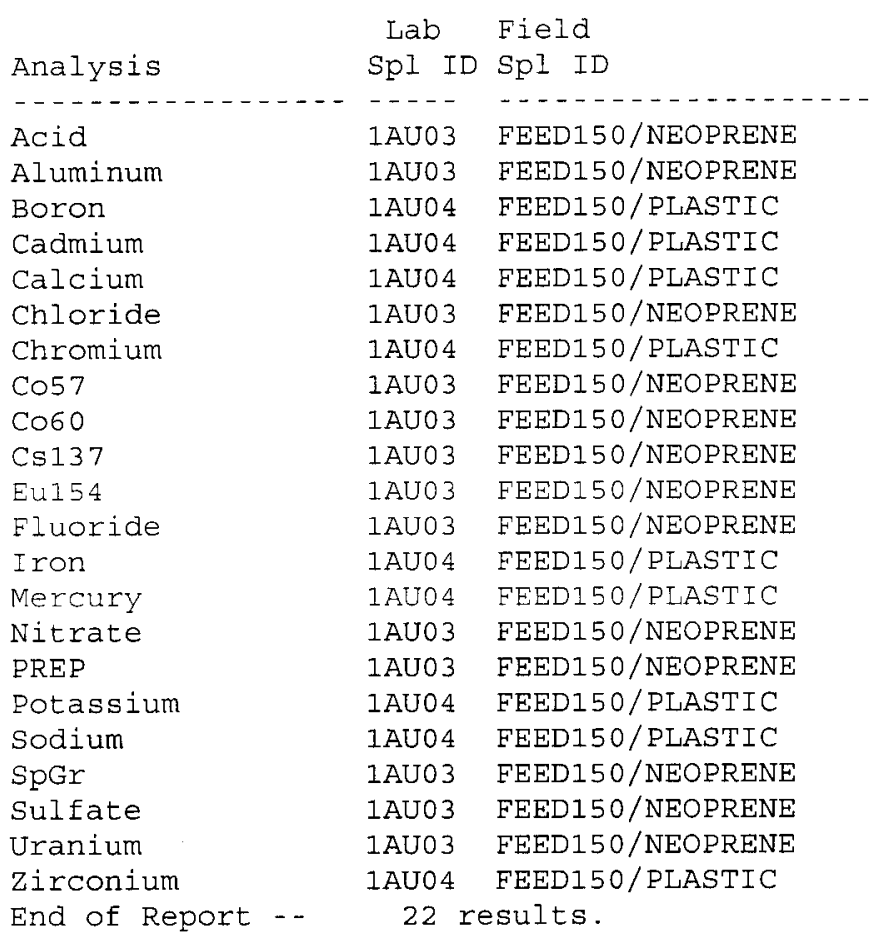


F I N A L R E P O R T for 150FEED:184 281

Log Type: * * PLANT * *

$\begin{array}{ll}\text { Report for } & : \text { NWCF } \\ \text { Mailstop } & : 5116 \\ \text { Date Received } & \text { : Apr } 132001 \\ \text { Time Received } & : 00: 15 \\ \text { GWA charged } & : 561211290 \\ \text { MSA mR/hr } & \text { COLD } \\ \text { Hazard Index } & :<1 E 4 \\ \text { PCBs }>50 \mathrm{ppm} & : \text { NO }\end{array}$

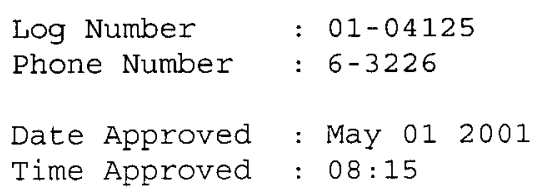

COMMENTS :

\begin{tabular}{|c|c|c|}
\hline & Lab & Field \\
\hline Analysis & Spl ID & Spl ID \\
\hline$---\cdots--$ & ---- & -------------- \\
\hline Acid & 1AU8 8 & FEED150/NEOPRENE \\
\hline \multirow[t]{2}{*}{ Aluminum } & 1AU89 & FEED150/PLASTIC \\
\hline & 1 AU89 & FEED150/PLASTIC \\
\hline Boron & 1 AU89 & FEED150/PLASTIC \\
\hline Cadmium & IAU89 & FEED150/PLASTIC \\
\hline Calcium & 1 AU89 & FEED150/PLASTIC \\
\hline Chloride & 1AU88 & FEED150/NEOPRENE \\
\hline Chromium & 1 AU89 & FEED150/PLASTIC \\
\hline Co6o & $1 \mathrm{AU} 88$ & FEED150/NEOPRENE \\
\hline $\operatorname{Cs} 134$ & 1AU88 & FEED150/NEOPRENE \\
\hline $\operatorname{Cs} 137$ & 1AU8 8 & FEED150/NEOPRENE \\
\hline Eul54 & IAU8 8 & FEED150/NEOPRENE \\
\hline Eluoride & 1AU88 & FEED150/NEOPRENE \\
\hline Iron & 1 AU89 & FEED150/PLASTIC \\
\hline Mercury & 1 AU89 & FEED150/PLASTIC \\
\hline Nitrate & 1 AU8 8 & FEED150/NEOPRENE \\
\hline Potassium & IAU89 & FEED150/PLASTIC \\
\hline Sodium & 1AU89 & FEED150/PLASTIC \\
\hline SpGr & IAU8 8 & FEED150/NEOPRENE \\
\hline Sulfate & 1 AU88 & FEED150/NEOPRENE \\
\hline Uranium & 1 AU88 & FEED150/NEOPRENE \\
\hline Zirconium & 1AU89 & FEED150/PLASTIC \\
\hline End of Rep & 22 & sults. \\
\hline
\end{tabular}

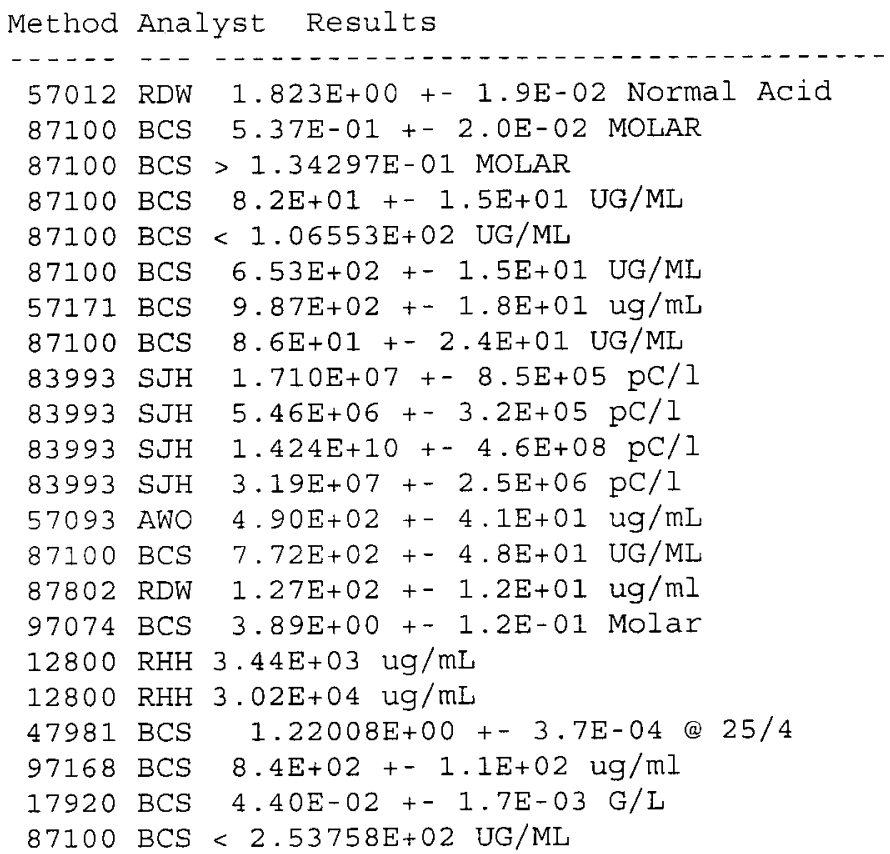


Log TYpe: ** PLANT **

\begin{tabular}{|c|c|c|}
\hline Report for & : NWCF & Log Number \\
\hline Mailstop & : 5116 & Phone Number \\
\hline Date Received & : Apr 142001 & Date Approved : May 012001 \\
\hline Time Received & $: 12: 25$ & Time Approved : $08: 16$ \\
\hline GWA charged & : 561211290 & Reviewed by TIFFANY PARK \\
\hline $\mathrm{MSA} \mathrm{mR} / \mathrm{hr}$ & : CELL & Signature \\
\hline Hazard Index & : $1 \mathrm{E7}$ & Lab QC/QA reviewed by \\
\hline PCBs $>50 \mathrm{ppm}$ & : NO & Signature \\
\hline
\end{tabular}

\section{COMMENTS :}

\begin{tabular}{|c|c|c|}
\hline & Lab & Field \\
\hline Analysis & Spl ID & Spl ID \\
\hline$-\ldots--$. & --- & $-\ldots-\ldots-\ldots$ \\
\hline Acid & IAU93 & FEED150/NEOPRENE \\
\hline Aluminum & 1 AU94 & FEED150/PLASTIC \\
\hline $\operatorname{Am} 241$ & 1 AU9 3 & FEED150/NEOPRENE \\
\hline Boron & 1 AU93 & FEED150/NEOPRENE \\
\hline Cadmium & $1 \mathrm{AU} 93$ & FEED150/NEOPRENE \\
\hline Calcium & IAU93 & FEED150/NEOPRENE \\
\hline Chloride & $1 \mathrm{AU} 93$ & FEED150/NEOPRENE \\
\hline Chromium & 1AU93 & FEED150/NEOPRENE \\
\hline Co60 & IAU93 & FEED150/NEOPRENE \\
\hline $\operatorname{Cs} 134$ & IAU93 & FEED150/NEOPRENE \\
\hline $\operatorname{Cs} 137$ & 1AU93 & FEED150/NEOPRENE \\
\hline Eu154 & 1 AU93 & FEED150/NEOPRENE \\
\hline Fluoride & $1 \mathrm{AU} 93$ & FEED150/NEOPRENE \\
\hline Iron & 1 AU93 & FEED150/NEOPRENE \\
\hline Mercury & 1 AU9 4 & FEED150/PLASTIC \\
\hline Nitrate & 1 AU9 3 & FEED150/NEOPRENE \\
\hline Potassium & 1AU94 & FEED150/PLASTIC \\
\hline Sodium & 1AU94 & FEED150/PLASTIC \\
\hline SpGr & 1AU93 & FEED150/NEOPRENE \\
\hline Sulfate & 1AU93 & FEED150/NEOPRENE \\
\hline Uranium & 1AU93 & FEED150/NEOPRENE \\
\hline Zirconium & 1AU93 & FEED150/NEOPRENE \\
\hline End of Report -- & $22 r$ & sults. \\
\hline
\end{tabular}

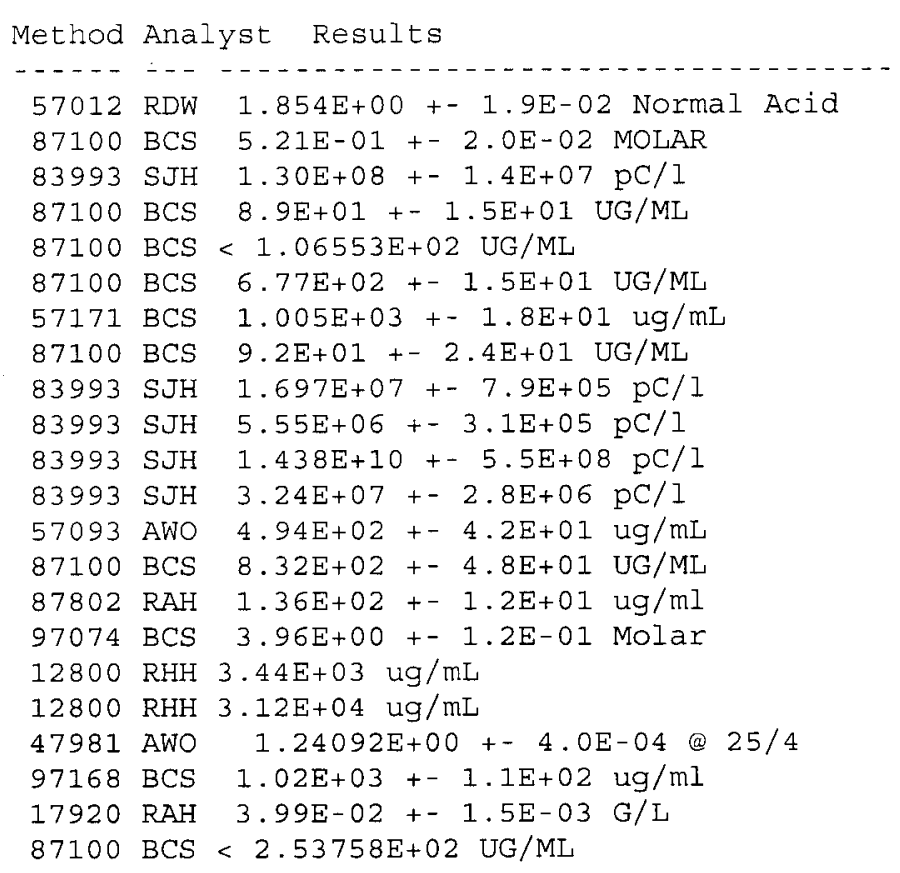


F I N A I R E P O R T for 150FEED:101 294

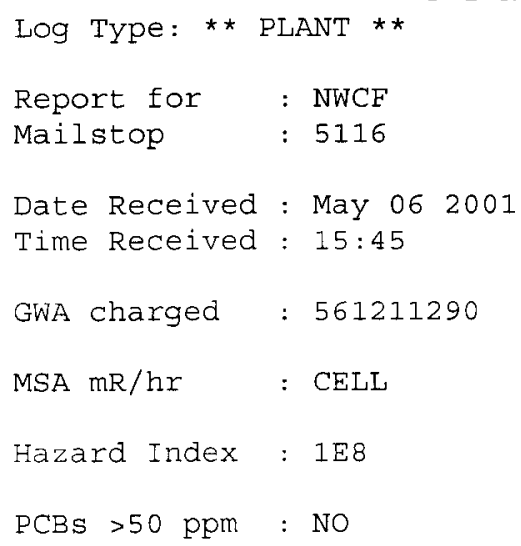

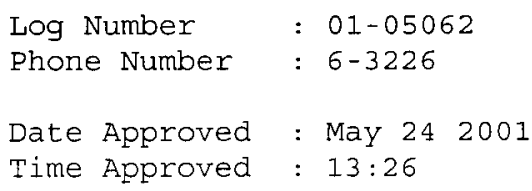

Signature
1BBO6 FEED150/NEOPRENE

1BB07 FEED150/PLASTIC

1BB07 FEED150/PLASTIC

1BB07 FEED150/PLASTIC

1BB07 FEED150/PLASTIC

1BB06 FEED150/NEOPRENE

1BB07 FEED150/PLASTIC

1BB06 FEED150/NEOPRENE

$1 B B 06$ FEED150/NEOPRENE

1BB06 FEED150/NEOPRENE

1BB07 FEED150/PLASTIC

1BB0 7 FEED150/PLASTIC

1BB06 FEED150/NEOPRENE

$1 B B 06$ FEED150/NEOPRENE

1BB06 FEED150/NEOPRENE

1BB07 FEED150/PLASTIC

1BB07 FEED150/PLASTIC

1BB06 FEED150/NEOPRENE

1BB06 FEED150/NEOPRENE

IBB06 FEED150/NEOPRENE

1BB07 FEED150/PLASTIC

1BB06 FEED150/NEOPRENE

22 results.

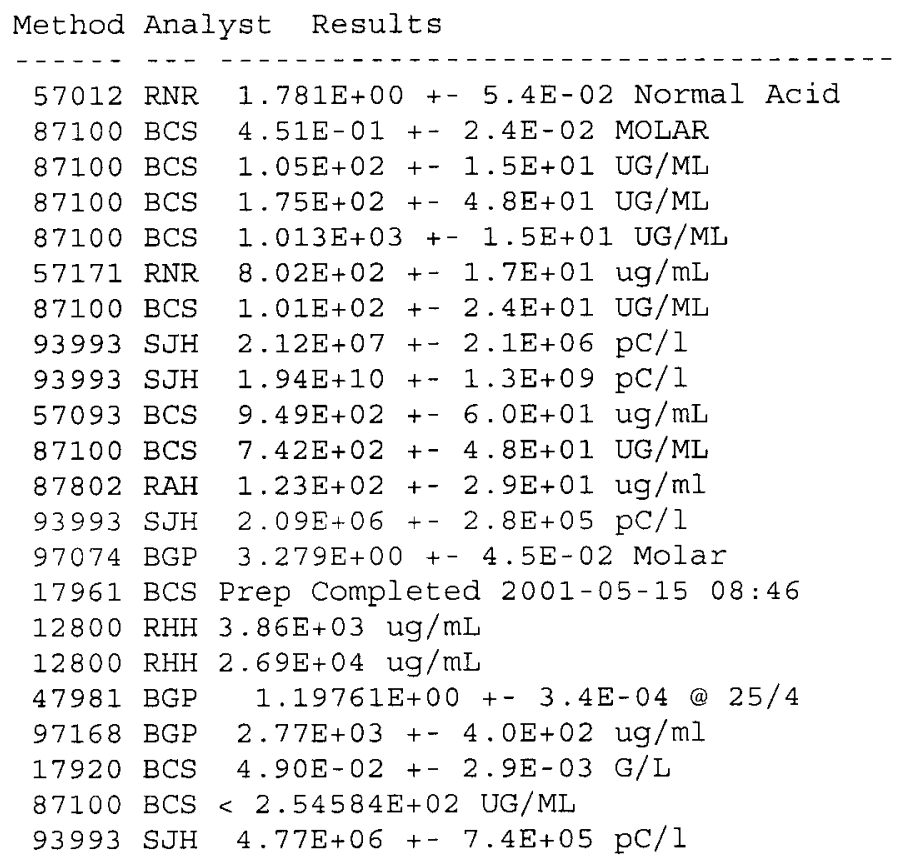


F I N A L R E P O R T for 150WM:101 294

Log Type: * RCRA - PLANT * *

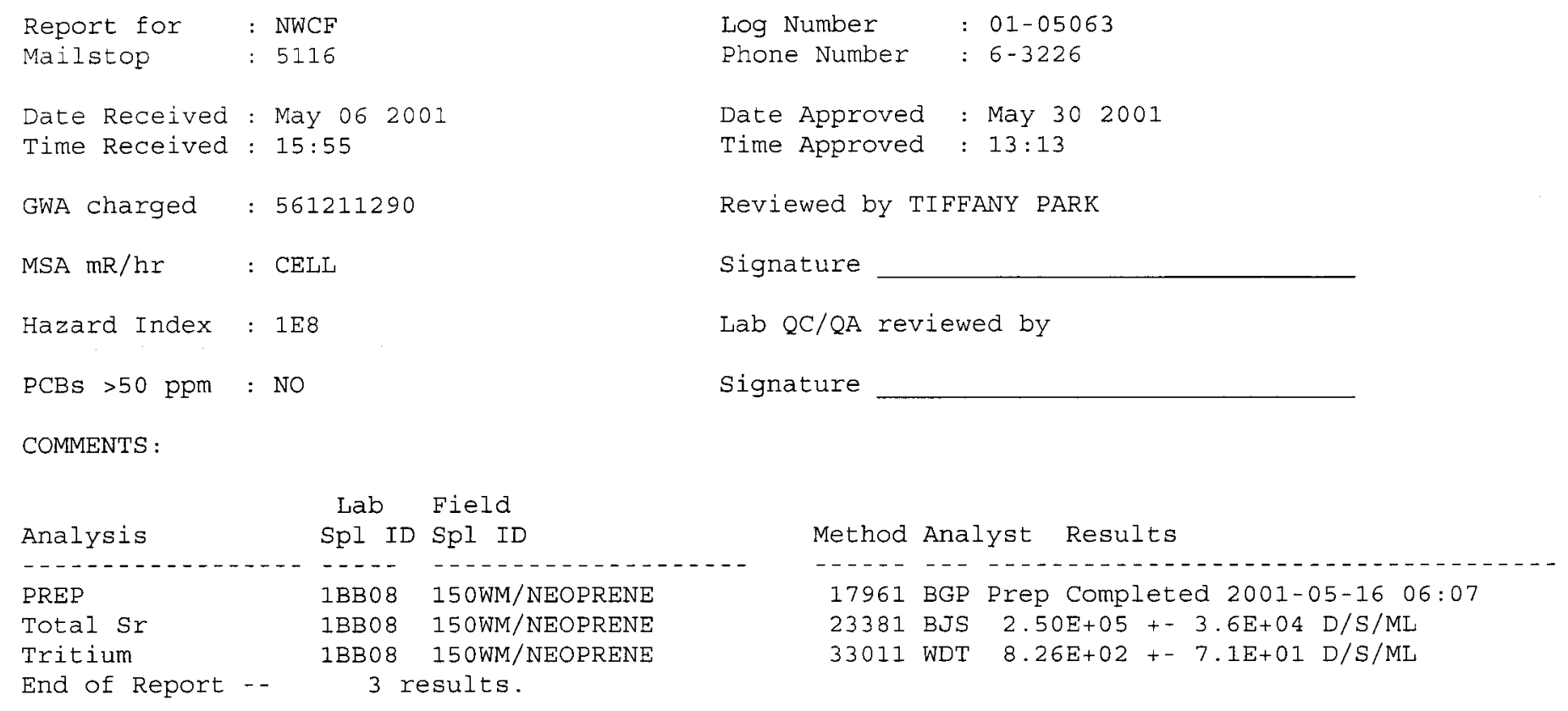


F I N A L R E P O R T for 150WM:101 298

Log Type: * * RCRA - PLANT * *

$\begin{array}{llll}\begin{array}{l}\text { Report for } \\ \text { Mailstop }\end{array} & : 5116 & \begin{array}{l}\text { Log Number } \\ \text { Phone Number }\end{array} & : 61-05112 \\ \text { Date Received }: \text { May } 112001 & \text { Date Approved }: \text { May } 302001 \\ \text { Time Received }: 09: 43 & \begin{array}{l}\text { Time Approved }: 13: 13 \\ \text { GWA charged }: 561211290\end{array} & \text { Reviewed by TIFFANY PARK } \\ \text { MSA mR/hr }: ? & \text { Signature } \\ \text { Hazard Index : }>1 E 4 & \text { Lab QC/QA reviewed by } \\ \text { PCBs }>50 \mathrm{ppm}: \text { NO } & \text { Signature }\end{array}$

COMMENTS :

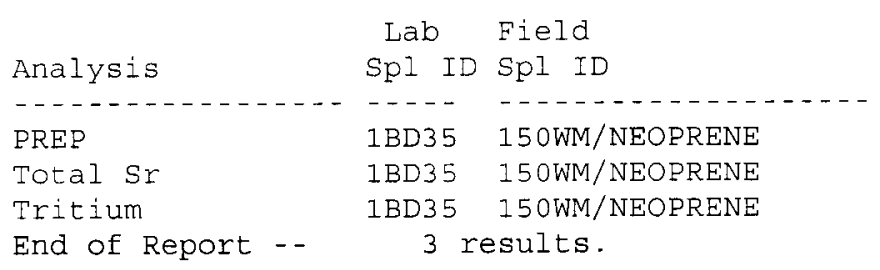

Method Analyst Results
17961 BCS Prep Completed $2001-05-1508: 48$
23381 BJS $6.19 E+05+-6.5 \mathrm{E}+04 \mathrm{D} / \mathrm{S} / \mathrm{ML}$
33011 WDT $8.11 \mathrm{E}+02+-6.9 \mathrm{E}+01 \mathrm{D} / \mathrm{S} / \mathrm{ML}$


Log Type: $\star \star$ RCRA $\star \star$

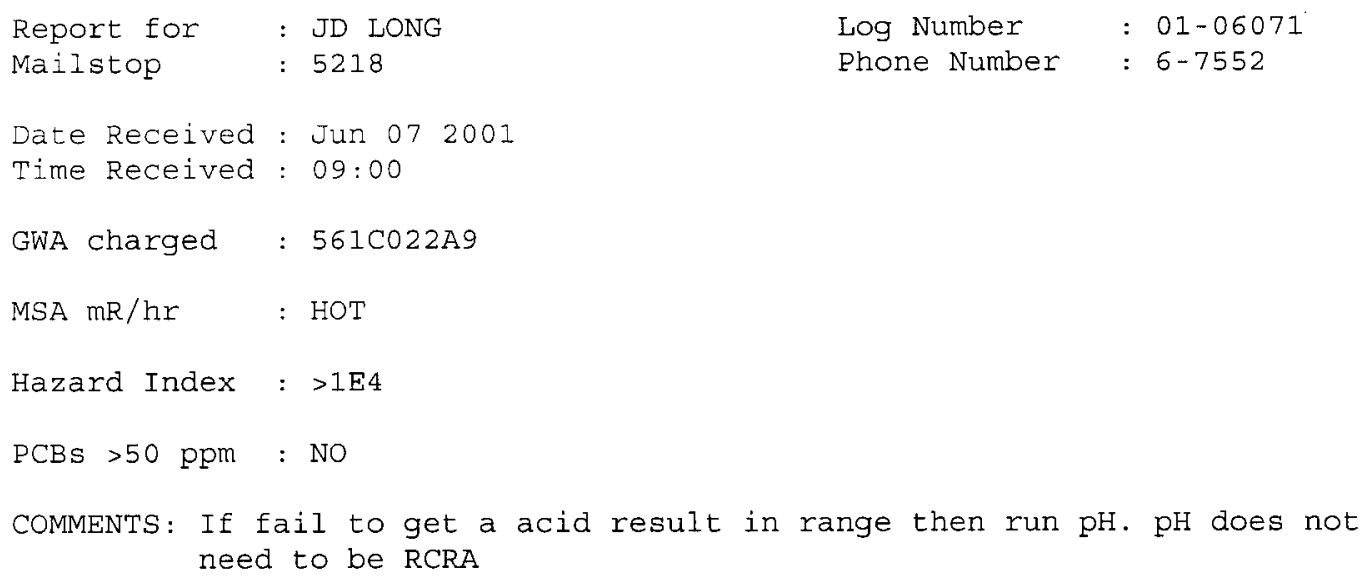

\begin{tabular}{|c|c|c|}
\hline & Lab & Field \\
\hline Analysis & Spl ID & Spl ID \\
\hline$-\ldots+-\ldots-\ldots-\ldots$ & ---- & $----\ldots \ldots---\ldots$ \\
\hline Acid & 1BI 26 & BP10120101 \\
\hline Aluminum & $1 B I 26$ & $\mathrm{BP} 10120101$ \\
\hline Antimony & $1 B I 26$ & BP10120101 \\
\hline Arsenic & $1 B I 26$ & BP10120101 \\
\hline Barium & 1BI 26 & $\mathrm{BP} 10120101$ \\
\hline Beryllium & $1 B I 26$ & $\mathrm{BP} 10120101$ \\
\hline Cadmium & $1 B I 26$ & BP10120101 \\
\hline Chromium & $1 B I 26$ & BP10120101 \\
\hline Cobalt & $1 B I 26$ & $\mathrm{BP} 10120101$ \\
\hline Copper & $1 B I 26$ & BP10120101 \\
\hline Fluoride & $1 \mathrm{BI} 26$ & BP10120101 \\
\hline Lead & $1 \mathrm{BI} 26$ & $\mathrm{BP} 10120101$ \\
\hline Manganese & $1 \mathrm{BI} 26$ & $\mathrm{BP} 10120101$ \\
\hline Mercury & $1 B I 26$ & BP10120101 \\
\hline Nickel & $1 B I 26$ & BP10120101 \\
\hline SVOA (TOTAL) & $1 B I 26$ & $\mathrm{BP} 10120101$ \\
\hline Selenium & $1 \mathrm{BI} 26$ & BP10120101 \\
\hline Silver & $1 B I 26$ & $\mathrm{BP} 10120101$ \\
\hline TIC & $1 B I 26$ & BP10120101 \\
\hline TOC & $1 B I 26$ & BP10120101 \\
\hline Thallium & $1 B I 26$ & $\mathrm{BP} 10120101$ \\
\hline UDS & $1 B I 26$ & BP10120101 \\
\hline Uranium & $1 B I 26$ & BP10120101 \\
\hline \multirow[t]{2}{*}{ VOA (TOTAL) } & $1 B I 26$ & BP10120101 \\
\hline & $1 B I 27$ & $\mathrm{BP} 10120201$ \\
\hline Vanadium & $1 B I 26$ & $\mathrm{BP} 10120101$ \\
\hline Zinc & $1 B I 26$ & BP10120101 \\
\hline
\end{tabular}

Method Analyst Results

\begin{tabular}{|c|c|c|}
\hline 57012 & RNR & $1.711 \mathrm{E}+00+-5.4 \mathrm{E}-02$ Normal \\
\hline 42900 & LAM & $1.14534 \mathrm{E}+07 \mathrm{ug} / \mathrm{L}$ \\
\hline 42900 & LAM & Not Detected: IDL $=235 \mathrm{ug} / \mathrm{L}$ \\
\hline 42900 & LAM & $3.35 \mathrm{E}+02 \mathrm{ug} / \mathrm{L}$ \\
\hline 42900 & LAM & $3.985 \mathrm{E}+03 \mathrm{ug} / \mathrm{L}$ \\
\hline 42900 & LAM & $9.5 \mathrm{E}+01 \mathrm{ug} / \mathrm{L}$ \\
\hline 42900 & LAM & $1.7555 \mathrm{E}+05 \mathrm{ug} / \mathrm{L}$ \\
\hline 42900 & LAM & $1.02 \mathrm{E}+05 \mathrm{ug} / \mathrm{L}$ \\
\hline 42900 & LAM & $1.67 \mathrm{E}+03 \mathrm{ug} / \mathrm{L}$ \\
\hline 42900 & LAM & $2.697 \mathrm{E}+04 \mathrm{ug} / \mathrm{L}$ \\
\hline 57093 & AWO & $8.26654 \mathrm{E}+02 \mathrm{ug} / \mathrm{mL}$ \\
\hline 42900 & LAM & $1.1125 \mathrm{E}+05 \mathrm{ug} / \mathrm{L}$ \\
\hline 42900 & LAM & $4.30765 E+05 \mathrm{ug} / \mathrm{L}$ \\
\hline 12800 & SDN & $1.49 \mathrm{E}+05 \mathrm{ug} / \mathrm{L}$ \\
\hline $\begin{array}{r}42900 \\
9270\end{array}$ & LAM & $6.64 \mathrm{E}+04 \mathrm{ug} / \mathrm{L}$ \\
\hline 42900 & LAM & Not Detected: IDL $=240 \mathrm{ug} / \mathrm{L}$ \\
\hline 42900 & LAM & 1.2E+02 ug/L \\
\hline 18060 & $\mathrm{RDW}$ & $\mathrm{MDL}=119.004 \mathrm{ug} / \mathrm{ml}$ \\
\hline 18060 & $\mathrm{RDW}$ & $6.08462 \mathrm{E}+02 \mathrm{ug} / \mathrm{ml}$ \\
\hline 42900 & LAM & Not Detected: $I D L=200 \mathrm{ug} / \mathrm{L}$ \\
\hline 17972 & BCS & $0.619 \mathrm{~g}(\mathrm{UDS}) / \mathrm{L}$ \\
\hline 17920 & $\mathrm{BCS}$ & $4.98 \mathrm{E}-02+-2.9 \mathrm{E}-03 \mathrm{G} / \mathrm{L}$ \\
\hline 9260 & & \\
\hline 9260 & & \\
\hline 42900 & LAM & $6.1 E+02 \mathrm{ug} / \mathrm{L}$ \\
\hline 42900 & LAM & $4.12784 E+04 \mathrm{ug} / \mathrm{L}$ \\
\hline
\end{tabular}


CONCENTRATED BOTTOMS SAMPLES ANALYSIS REPORTS 
F I N A L R E P O R T for 150BOT119 296/297

\author{
Log Type: ** PLANT **
}

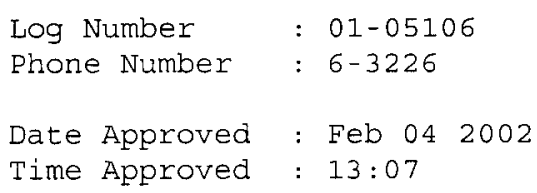

Signature

COMMENTS :

\begin{tabular}{|c|c|c|}
\hline & Lab & Field \\
\hline Analysis & Spl ID & Spl ID \\
\hline 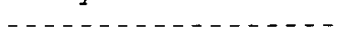 & $--\ldots$ & ----- \\
\hline Acid & $1 B D 27$ & 150BOT119PLAS \\
\hline Aluminum & $1 \mathrm{BD} 27$ & 150BOT119PLAS \\
\hline Chloride & $1 \mathrm{BD} 27$ & 150BOT119PLAS \\
\hline \multirow[t]{2}{*}{ Co60 } & $1 \mathrm{BD} 28$ & $150 \mathrm{BOT} 119$ \\
\hline & IBE80 & 150BOT119 DUP \\
\hline \multirow[t]{2}{*}{$\operatorname{Cs} 134$} & $1 \mathrm{BD} 28$ & $150 \mathrm{BOT} 119$ \\
\hline & $1 \mathrm{BE} 80$ & $150 B O T 119$ DUP \\
\hline \multirow[t]{2}{*}{$\operatorname{Cs} 137$} & $1 \mathrm{BD} 28$ & 150ВОТ119 \\
\hline & $1 B E 80$ & 150BOT119 DUP \\
\hline \multirow[t]{2}{*}{ Eu154 } & $1 \mathrm{BD} 28$ & 150 ВОT119 \\
\hline & $1 \mathrm{BE} 80$ & 150BOT119 DUP \\
\hline Fluoride & $1 \mathrm{BD} 27$ & 150BOT119PLAS \\
\hline Nitrate & $1 \mathrm{BD} 27$ & 150BOT119PLAS \\
\hline \multirow[t]{2}{*}{ PREP } & $1 \mathrm{BD} 27$ & 150BOT119PLAS \\
\hline & $1 \mathrm{BD} 28$ & $150 \mathrm{BOT} 119$ \\
\hline Phosphorous & $1 \mathrm{BD} 27$ & 150BOT119PLAS \\
\hline Potassium & $1 \mathrm{BD} 28$ & $150 \mathrm{BOT} 119$ \\
\hline Sodium & $1 \mathrm{BD} 28$ & $150 \mathrm{BOT} 119$ \\
\hline SpGr & $1 \mathrm{BD} 27$ & 150BOT119PLAS \\
\hline Tritium & $1 \mathrm{BD} 27$ & 150BOT119PLAS \\
\hline UDS & $1 \mathrm{BD} 27$ & 150BOT119PLAS \\
\hline Uranium & $1 \mathrm{BD} 28$ & 150 BОT119 \\
\hline \multirow{2}{*}{$\operatorname{Zr} 95$} & $1 \mathrm{BD} 28$ & 150ВОТ119 \\
\hline & $1 \mathrm{BE} 80$ & $150 B O T 119$ DUP \\
\hline
\end{tabular}

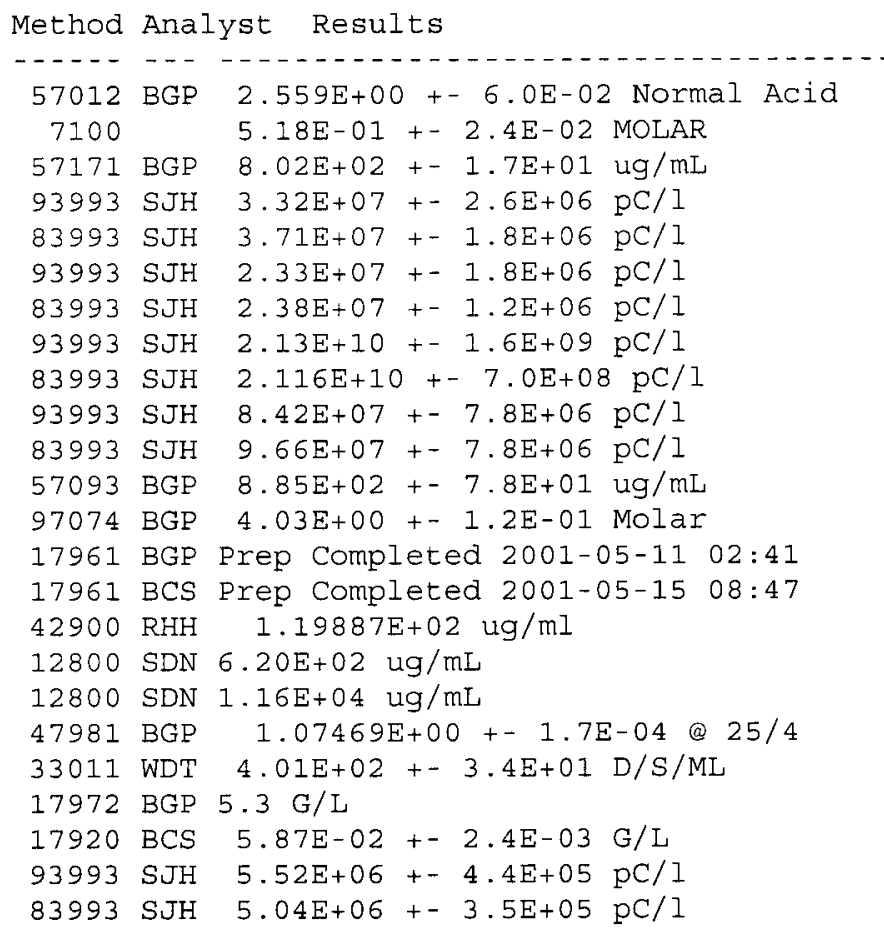


I N T E R I M R E P O R T for 150BOT119 324

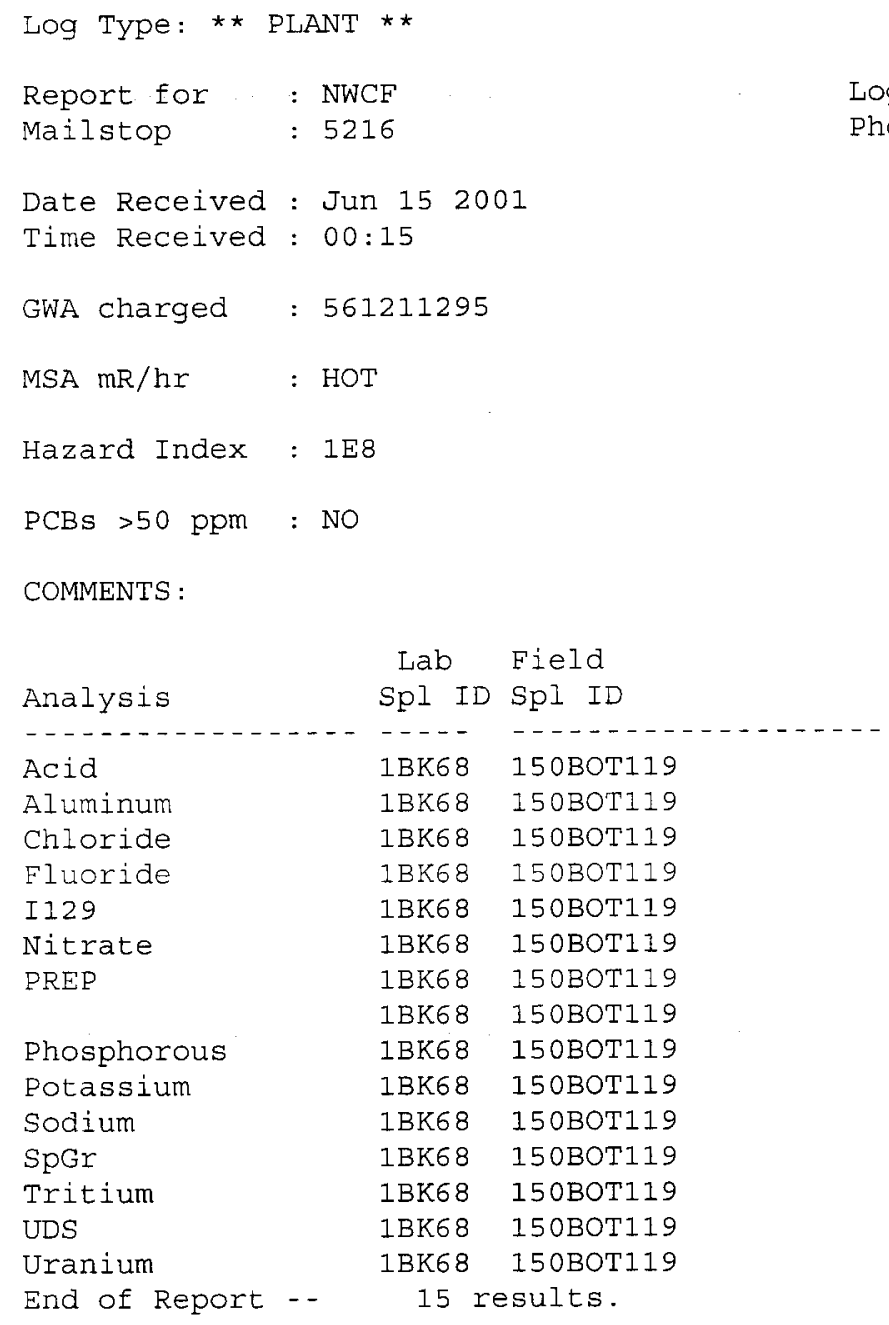

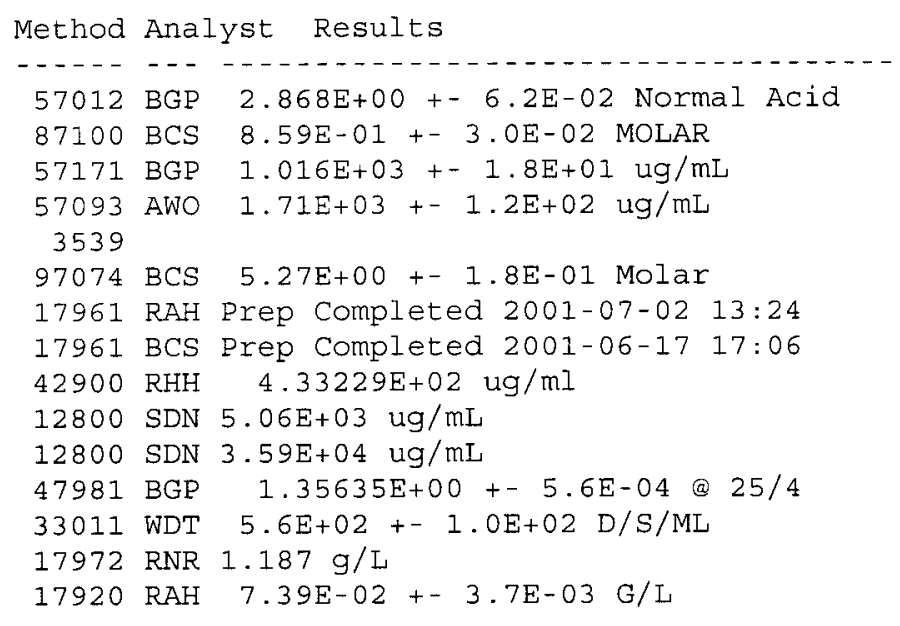




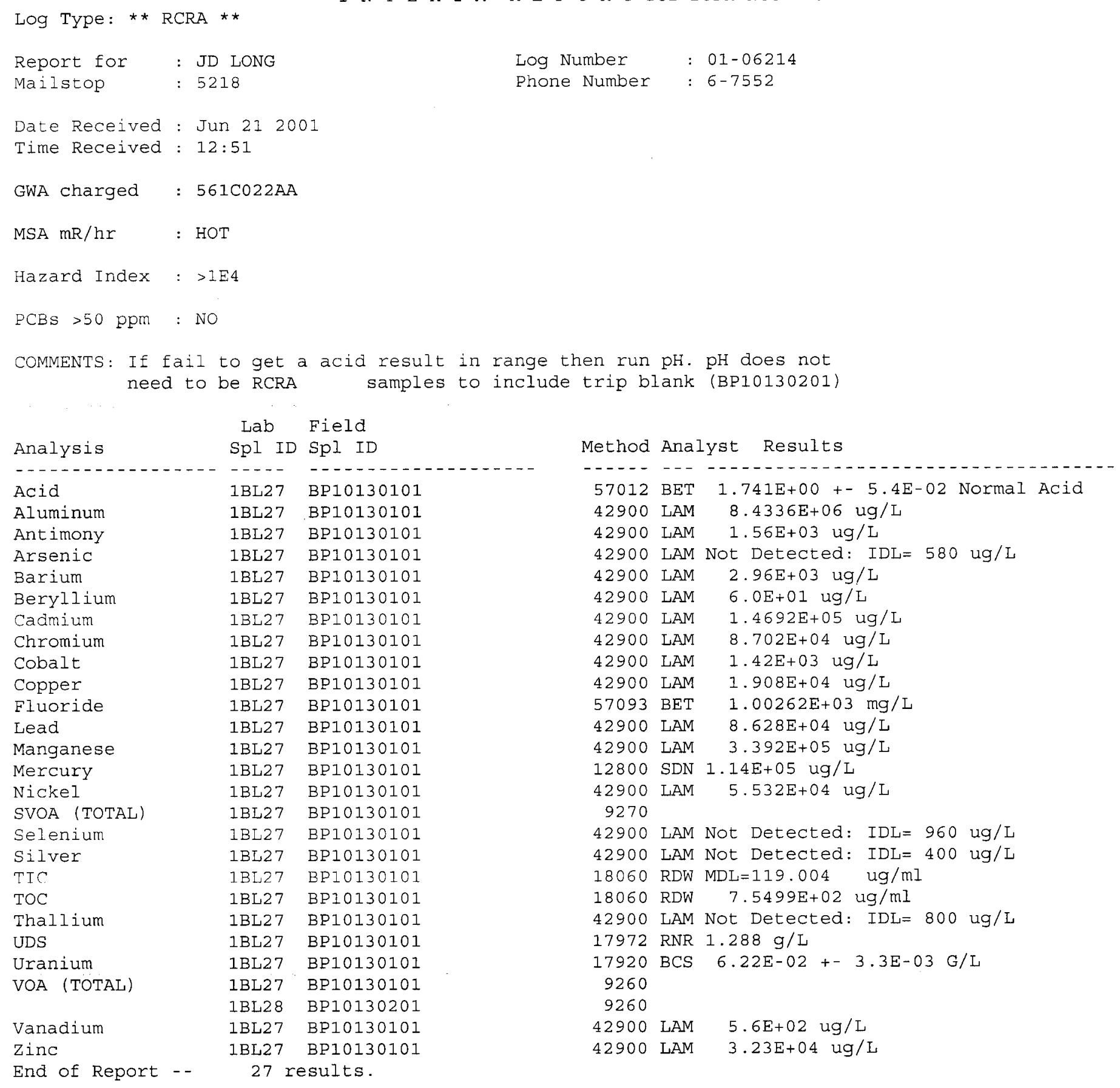


I $N$ T E R I M R E P O R T for 150BOT119 $333 / 334$

Log Type: * * PLANT * *

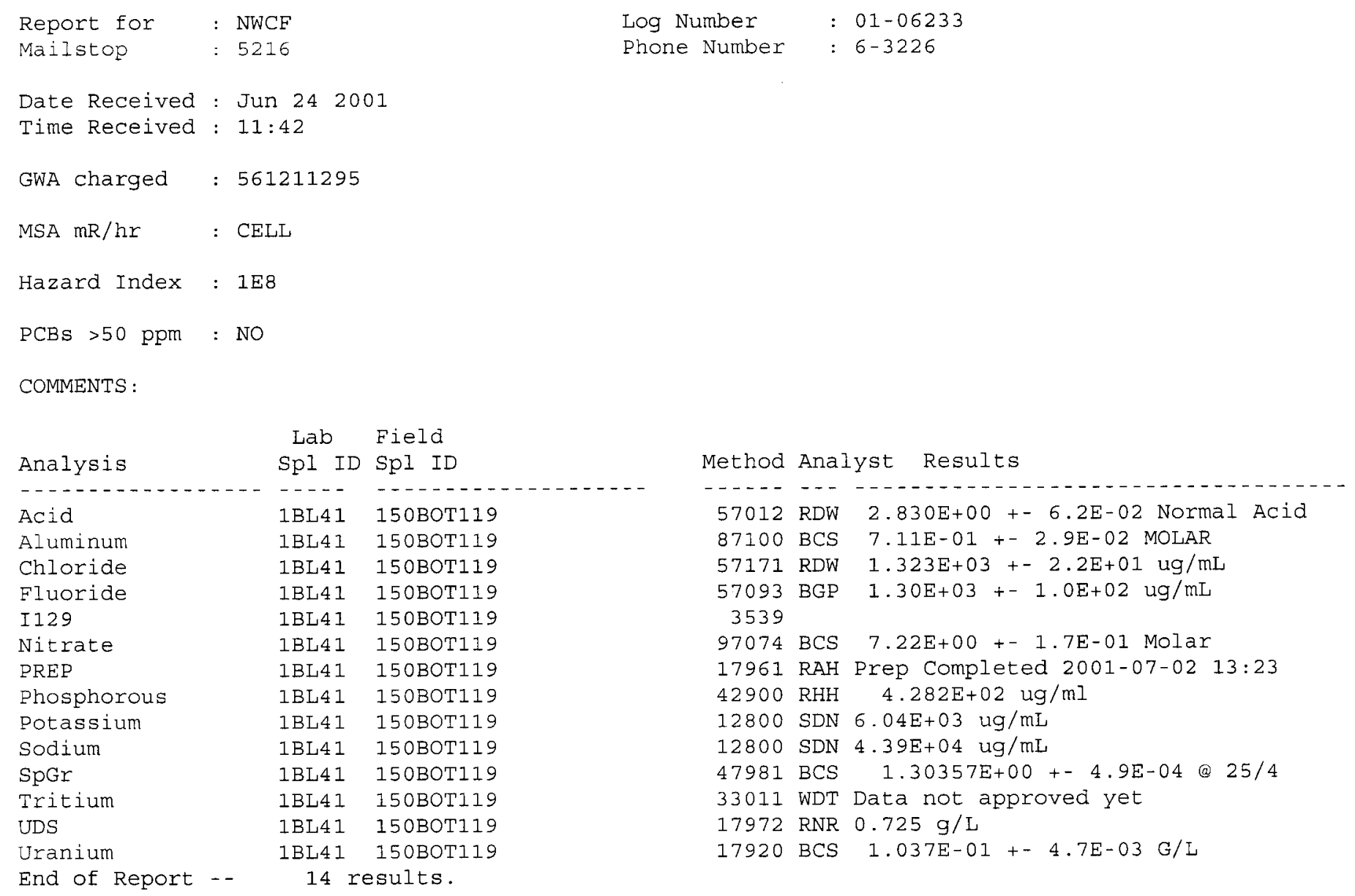


CONDENSED OVERHEADS SAMPLES ANALYSIS REPORTS 
Log Type: * * PLANT **

\begin{tabular}{|c|c|c|}
\hline Report for & : NWCF & Log Number \\
\hline Mailstop & : 5116 & Phone Number \\
\hline Date Received & : May 062001 & Date Approved \\
\hline Time Received & : $12: 17$ & Time Approved : 17:51 \\
\hline GWA charged & : 561211296 & Reviewed by W. (BILL) STRONG \\
\hline $\mathrm{MSA} \mathrm{mR} / \mathrm{hr}$ & : $\mathrm{HOT}$ & Signature \\
\hline Hazard Index & : $1 \mathrm{E5}$ & Lab QC/QA reviewed by \\
\hline PCBs $>50$ ppm & : NO & Signature \\
\hline
\end{tabular}

COMMENTS :

\begin{tabular}{|c|c|c|}
\hline & Lab & Field \\
\hline Analysis & Spl ID & Spl ID \\
\hline$-\cdots-\cdots-\cdots$ & ---- & ---- \\
\hline $\mathrm{AL} / \mathrm{F}$ RATIO & $1 \mathrm{BBO} 4$ & 150 COND122/NEOPRENE \\
\hline Acid & $1 \mathrm{BBO} 4$ & $150 \mathrm{COND} 122 / \mathrm{NEOPRENE}$ \\
\hline Aluminum & $1 \mathrm{BB} 05$ & $150 \mathrm{CONDI} 22$ / PLASTIC \\
\hline Chloride & $1 \mathrm{BBO} 4$ & $150 \mathrm{COND} 122 / \mathrm{NEOPRENE}$ \\
\hline Flash Point & $1 \mathrm{BB} 04$ & 150COND122/NEOPRENE \\
\hline Fluoride & $1 \mathrm{BBO} 4$ & $150 \mathrm{COND} 122 / \mathrm{NEOPRENE}$ \\
\hline GROSS BETA & $1 \mathrm{BBO} 4$ & 150COND122/NEOPRENE \\
\hline Mercury & $1 B B 05$ & 150COND122/PLASTIC \\
\hline Nitrate & 1BB0 4 & 150COND122/NEOPRENE \\
\hline SpGr & $1 \mathrm{BB} 04$ & 150 COND122/NEOPRENE \\
\hline Sulfate & 1BB0 4 & 150COND122/NEOPRENE \\
\hline TOC & 1BB04 & $150 C O N D 122 /$ NEOPRENE \\
\hline UDS & $1 \mathrm{BBO} 4$ & $150 C O N D 122 / N E O P R E N E$ \\
\hline Uranium & $1 B B 04$ & 150COND122/NEOPRENE \\
\hline & $1 \mathrm{BBO} 4$ & 150 COND122/NEOPRENE \\
\hline
\end{tabular}

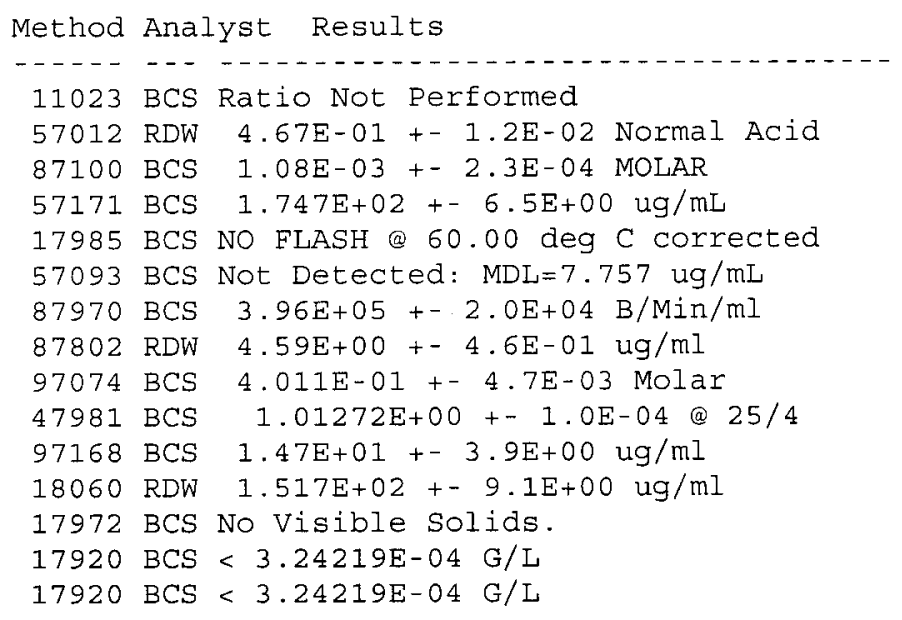


F I N A L R E P O R T for 150COND122 294

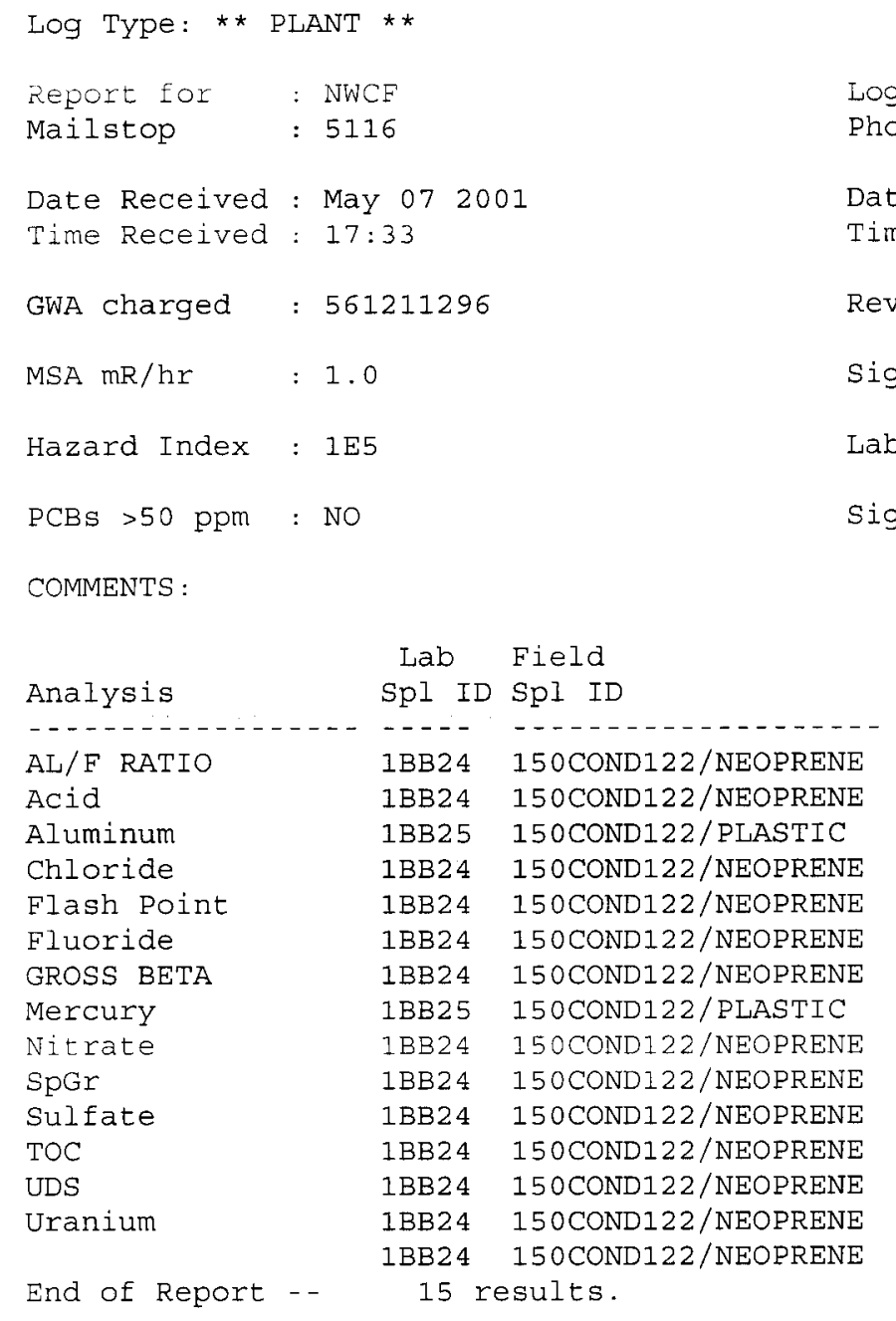

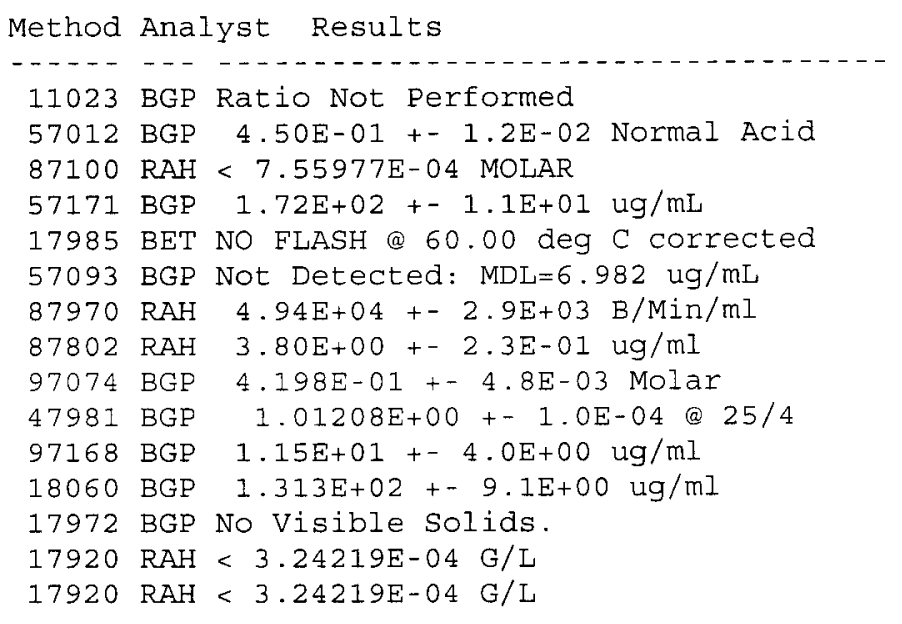


F I N A L R E P O R T for 150COND122 295

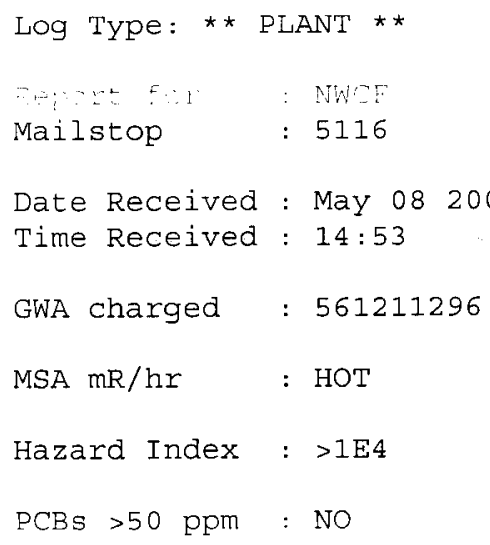

COMMENTS :

\begin{tabular}{|c|c|c|}
\hline & Lab & Field \\
\hline Analysis & Spl ID & Spl ID \\
\hline--------------- & $-\cdots-$ & -------- \\
\hline AL/F RATIO & $1 B B 54$ & 150COND122/NEOPRENE \\
\hline Acid & $1 B B 54$ & $150 C O N D 122 /$ NEOPRENE \\
\hline Aluminum & $1 B B 55$ & 150 COND $122 /$ PLASTIC \\
\hline Chloride & $1 \mathrm{BB} 54$ & 150COND122/NEOPRENE \\
\hline Flash Point & 1BB5 4 & 150 COND122/NEOPRENE \\
\hline Fluoride & $1 \mathrm{BB} 54$ & 150COND122/NEOPRENE \\
\hline SROSS BETA & $1 \mathrm{BB} 54$ & 150 COND $122 /$ NEOPRENE \\
\hline Mercury & $1 \mathrm{BB} 55$ & 150 COND122/PLASTIC \\
\hline Nitrate & $1 B B 54$ & $150 \mathrm{COND} 122 / \mathrm{NEOPRENE}$ \\
\hline SpGr & $1 B B 54$ & 150COND122/NEOPRENE \\
\hline Sulfate & 1BB54 & 150COND122/NEOPRENE \\
\hline TOC & $1 B B 54$ & 150 COND122/NEOPRENE \\
\hline UDS & $1 B B 54$ & 150COND122/NEOPRENE \\
\hline ranium & $1 B B 54$ & 150 COND122/NEOPRENE \\
\hline & $1 B B 54$ & 150COND122/NEOPRENE \\
\hline
\end{tabular}

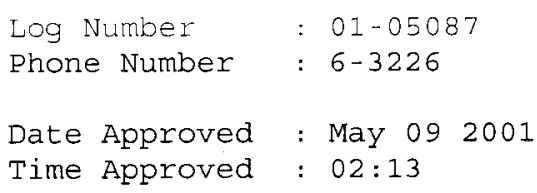

Signature

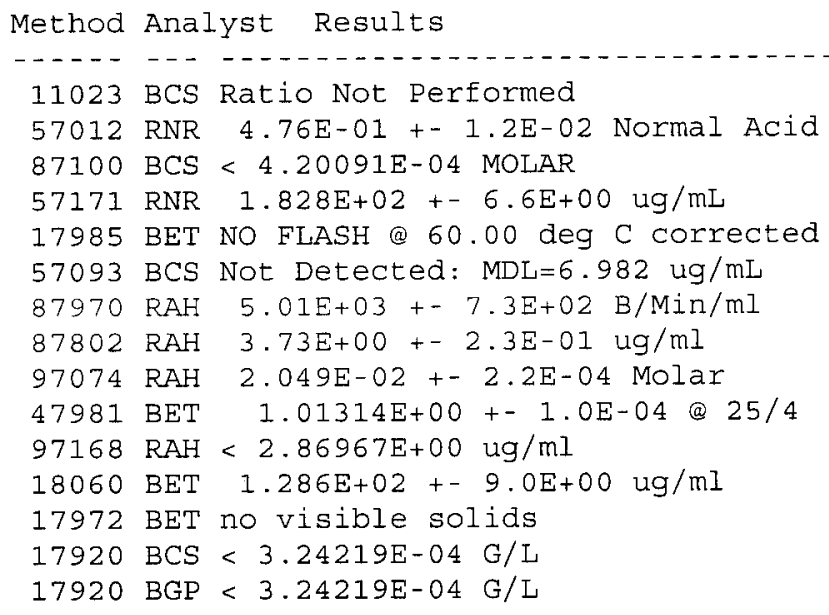




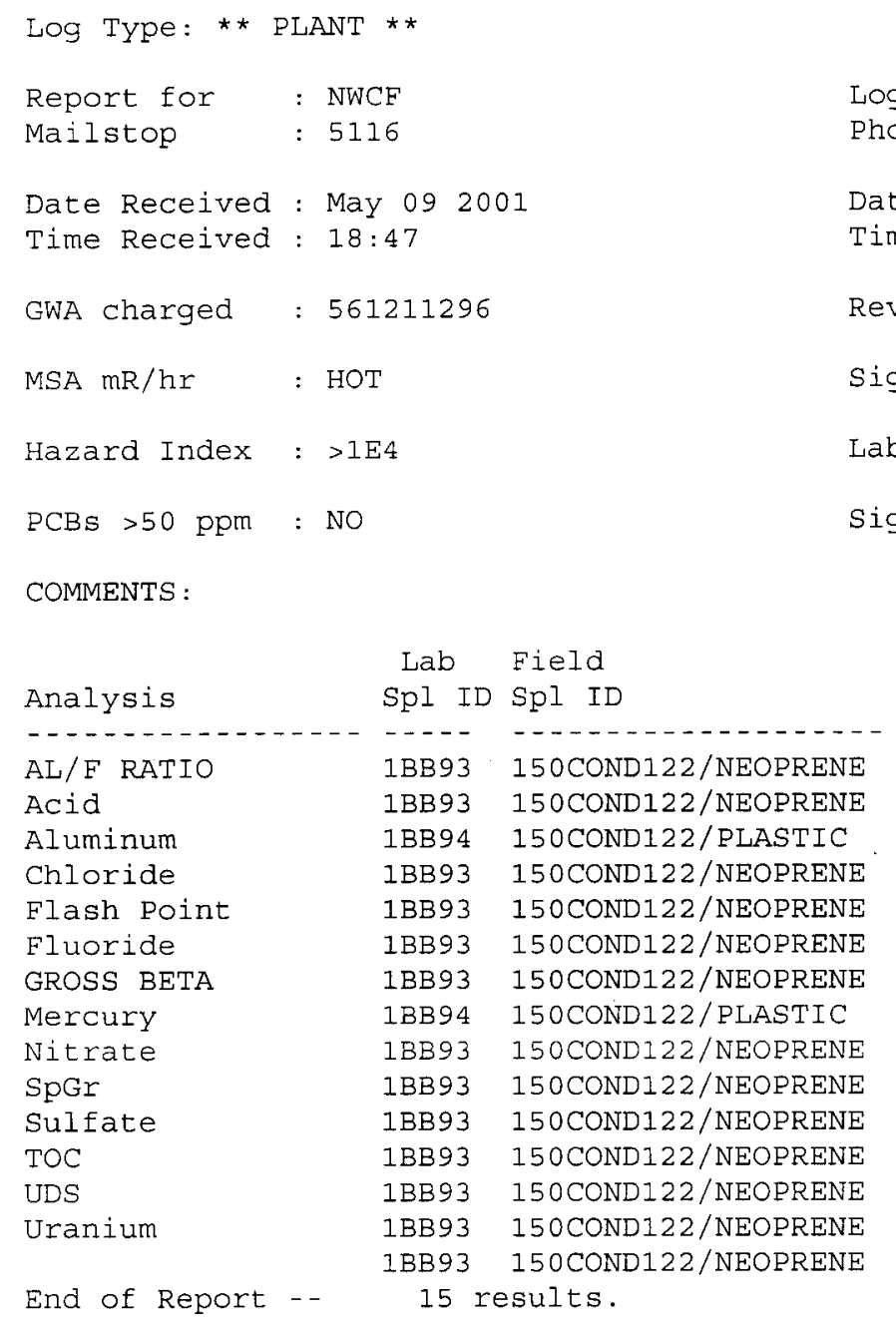

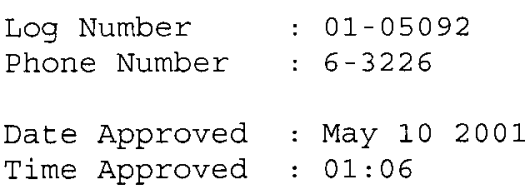

ignature

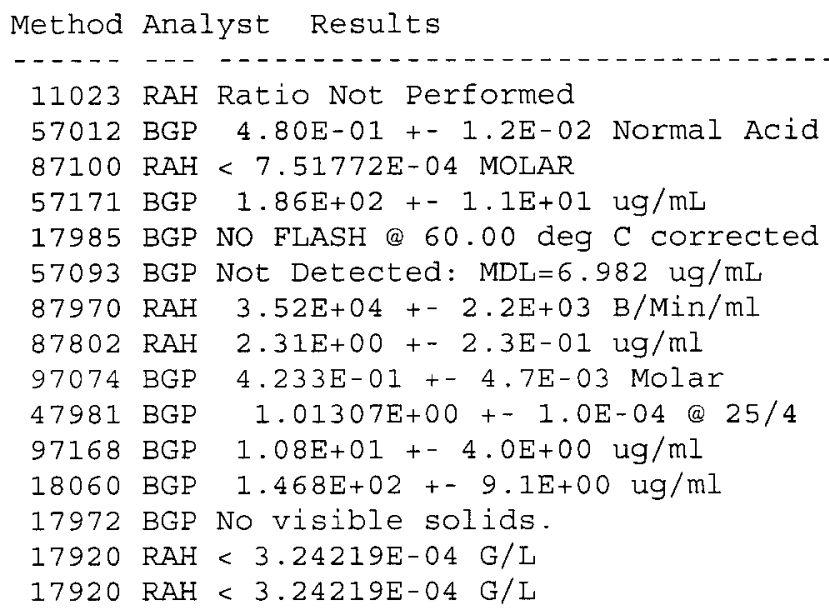


F I N A L R E P O R T for 150COND122 324

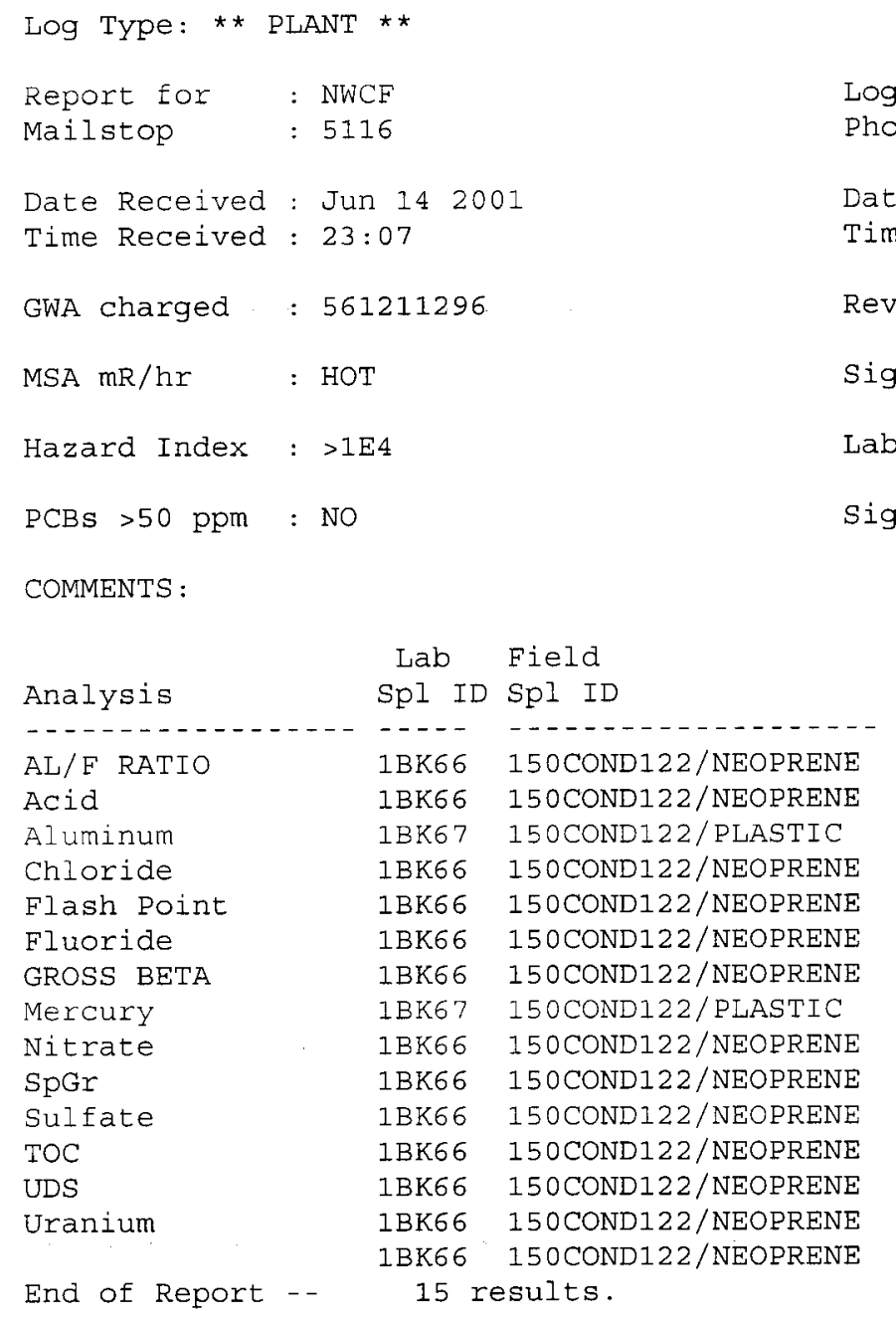

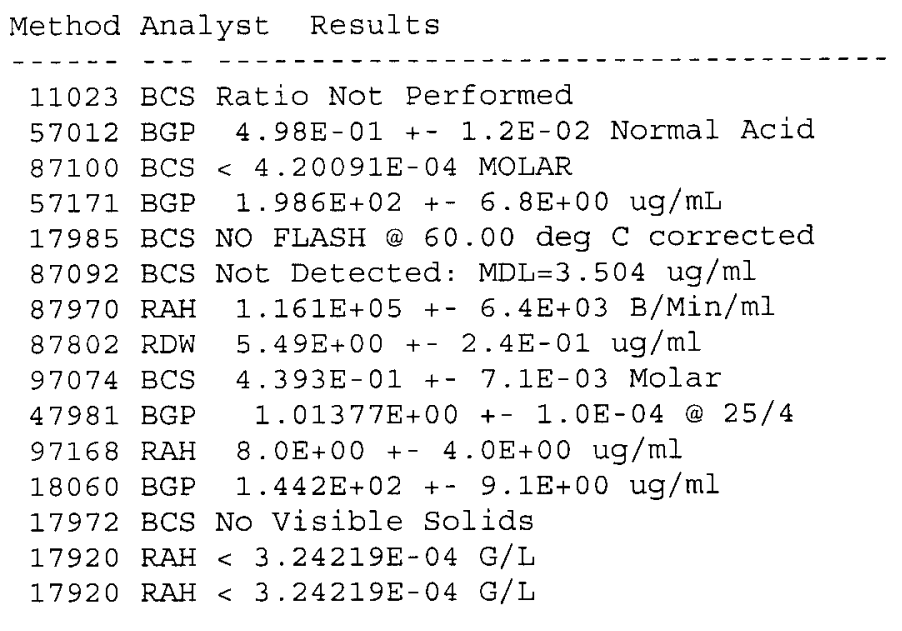


Log Type: * * RCRA * *

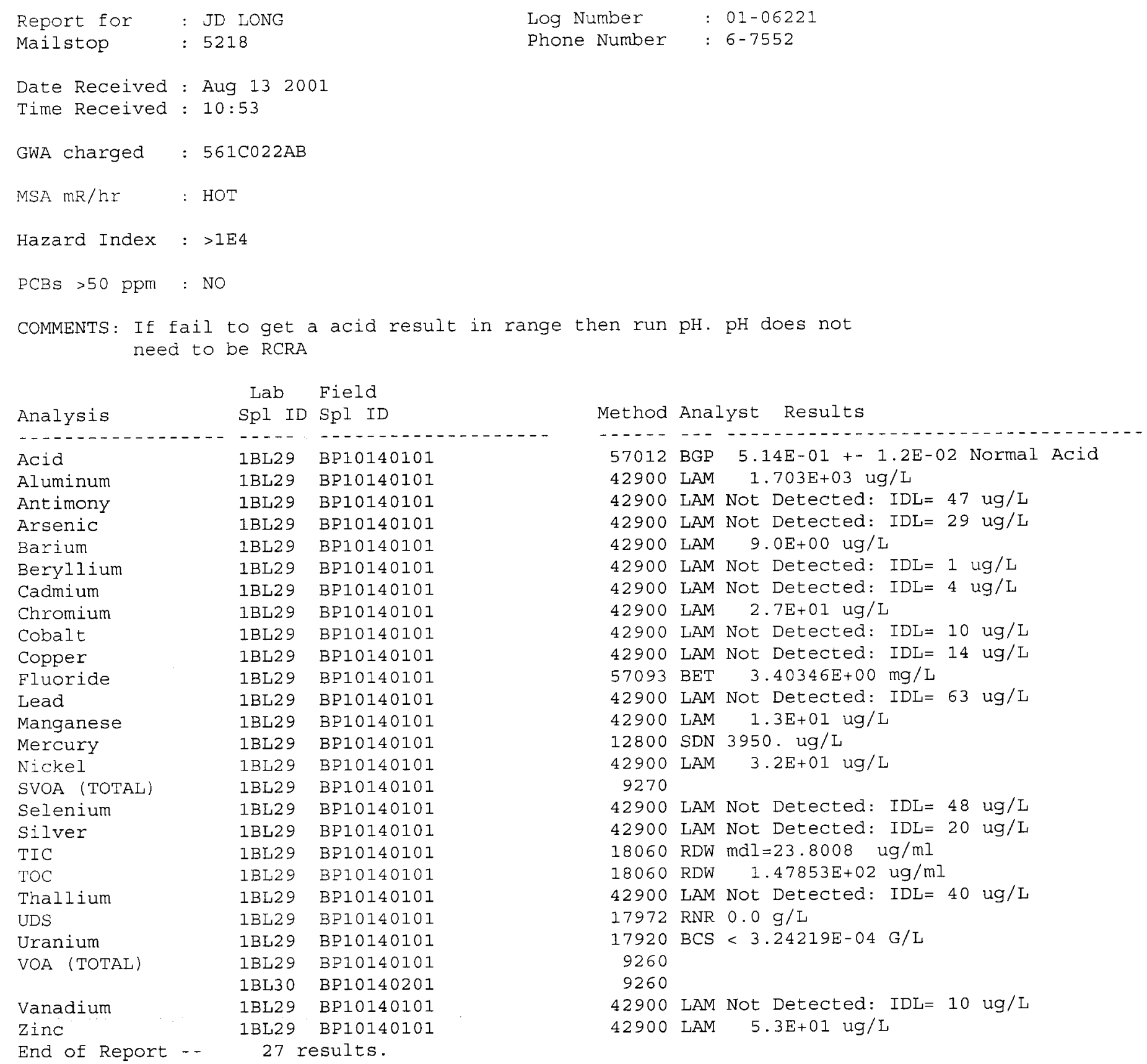


F I N A I R E P O R T for 150COND122 335

Log Type: * * PLANT * *

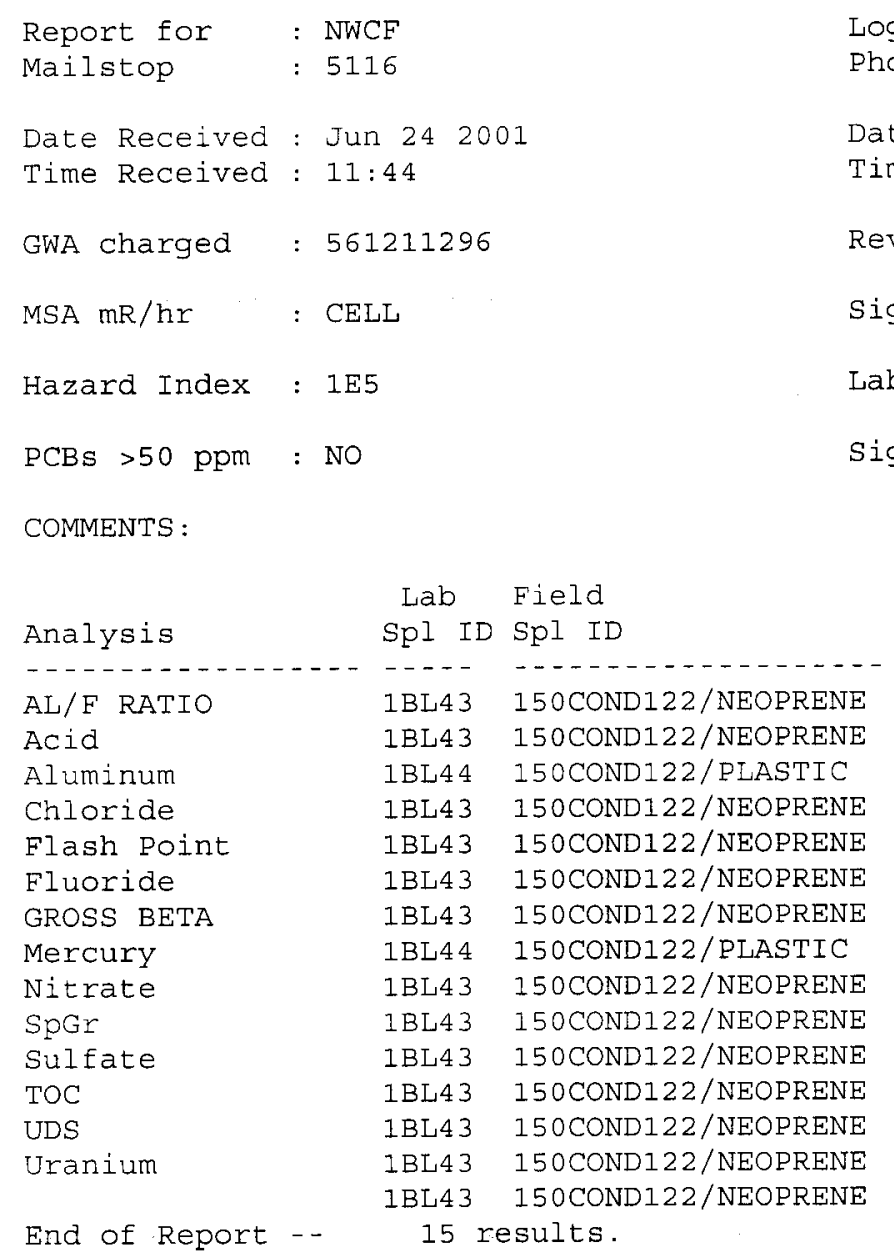

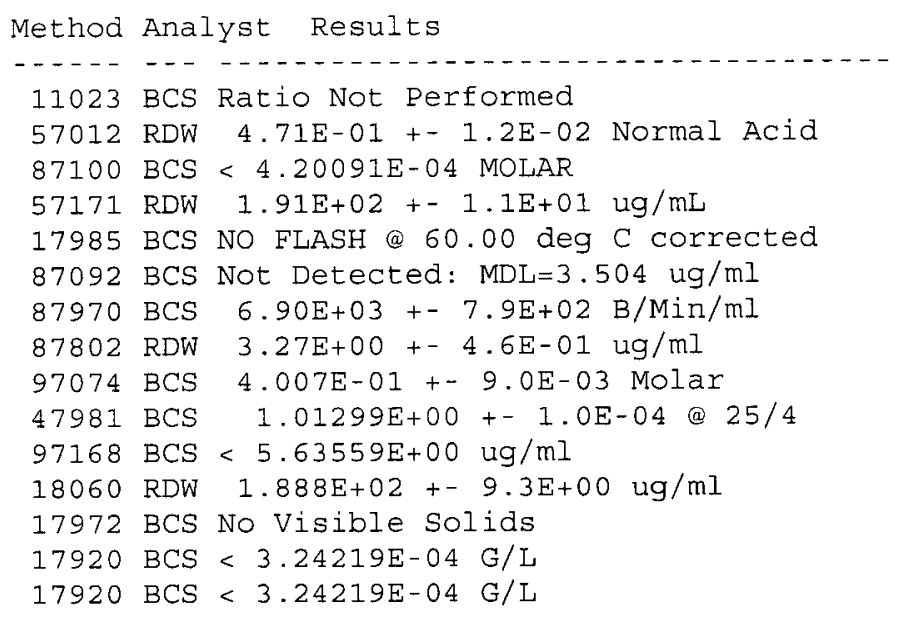




\section{LAB QUALIFIER LIST}

For volatile and semi-volatile organic analytical results, the INEEL qualifiers to be used are as follows:

$\mathrm{U}$ Indicates the compound was analyzed for but not detected. The sample quantification limit, or method detection limit (MDL) for EPA Method 524.2 (see Section C, Part I), must be corrected for dilution. For example, $10 \mathrm{U}$ for phenol in water if the sample final volume is the protocol-specified final volume and the method quantification limit for phenol is $10 \mu \mathrm{g} / \mathrm{L}$. If a 1 to 10 dilution of extract is necessary, the reported limit is $100 \mathrm{U}$.

If an analyte is detected at a concentration that is less than the MDL, it shall be reported at the estimated quantification limit (EQL) (concentration of the low standard in the initial calibration) and a "U" flag shall be used on the Form I.

J Indicates an estimated value. This flag is used under the following circumstances: 1) either when estimating a concentration for tentatively identified compounds where a $1: 1$ response is assumed, 2) when the mass spectral data indicate the presence of a compound that meets the identification criteria but the result is less than the sample estimated quantification limit but greater than the method detection limit (MDL), and 3) when the retention time data indicates the presence of a compound that meets the pesticide/polychlorinated bisphenyls (PCBs) identification criteria and the result is less that the EQL but greater than zero. For example, if the sample quantification limit is $10 \mu \mathrm{g} / \mathrm{L}$, but a concentration of $3 \mu \mathrm{g} / \mathrm{L}$ is calculated, report the result as $3 \mathrm{~J}$. The sample estimated quantification limit must be adjusted for the $\mathrm{U}$ flag, so that if a sample with $24 \%$ moisture and a 1 to 10 dilution factor has a calculated concentration of $300 \mu \mathrm{g} / \mathrm{kg}$ and a sample quantification limit of $4300 \mu \mathrm{g} / \mathrm{kg}$, report the concentration as $300 \mathrm{~J}$ on Form I. Note: The "J" code is not used and the compound is not reported as being identified for pesticide/PCBs results less than the ContractRequired Quantification Limit (CRQL), if the technical judgement of the pesticide residue analysis expert determines that the peaks used for compound identification resulted from instrument noise or other interferences (column bleed, solvent contamination, etc).

$\mathrm{N}$ Indicates presumptive evidence of a compound. This flag is only used for tentatively identified compounds, where the identification is based on a mass spectral library search. The $\mathrm{N}$ flag is applicable only to tentatively identified compound results. For generic characterization of a tentatively identified compound, such as chlorinated hydrocarbon, the $\mathrm{N}$ code is not used.

B This flag is used when the analyte is found in the associated blank as well as in the sample. It indicates possible/probable blank contamination and warns the data user to take appropriate action. This flag shall be used for a TIC as well as for a positively identified target compound. This flag shall not be used for flagging results on Forms I for the method blank analyses.

E This flag identifies compounds whose concentrations exceed the calibration range of the gas chromatography (GC) or gas chromatography/mass spectrometry (GC/MS) instrument for that specific analysis. If one or more compounds has a response representing a concentration greater than the highest concentration used in the initial calibration of the instrument, the sample or extract shall be diluted and re-analyzed. All such compounds with a response greater than the highest concentration used in the initial calibration shall have the 
concentration flagged with an "E" on the Form I for the original analysis. If the dilution of the extract causes any compounds identified in the first analysis to be below the calibration range in the second analysis, then the results of both analyses shall be reported on separate Forms I. The Form I for the diluted sample shall have the "DL" suffix appended to the sample number. NOTE: For total xylenes, where three isomers are quantified as two peaks when using capillary column chromatography, the calibration range of each peak should be considered separately, e.g., a diluted analysis is not required for total xylenes unless the concentration of either peak separately exceeds $200 \mu \mathrm{g} / \mathrm{L}$.

D This flag identifies all compounds identified in an analysis at a secondary dilution factor. If a sample or extract is re-analyzed at a higher dilution factor, as in the "E" flag above, the "DL" suffix is appended to the sample number on the Form I for the diluted sample, and all concentration and quantification limit values reported on that Form I are flagged with the "D" flag.

A This flag indicates that a TIC is a suspected aldol-condensation product.

$\mathrm{S}$ This optional flag indicates that the compound is a matrix spike and thus, the concentration is not reported on Form I.

$\mathrm{P}$ This flag is used for a pesticide/PCBs target analyte when there is greater than $25 \%$ difference for detected concentrations between the two GC Columns (See Form X). The lower of the two values is reported on the Form I and flagged with a "P".

M This flag indicates that the analyte was quantified using a calibration curve constructed using a first or higher order regression tit as specified in and allowed by SW-846 methods 8260A (paragraph 7.3.6.1) and 8270B (paragraph 7.3.5.1).

H The extraction holding time was exceeded.

$\mathrm{X}, \mathrm{Y}$, or Z Other specific flags may be required to properly define the results. If used, they must be fully described and such description attached to the Sample Data Summary Package and the Sample Delivery Group (SDG) Narrative. For the data contained in this report, "Y" indicates that the data is to be used for qualitative purposes only and " $Z$ " indicates that the initial calibration contains one less point than required by the method.

The combination of flags "BU" or "UB" is expressly prohibited. Blank contaminants are flagged "B" only when they are also detected in the sample. 
For the analyses, other than volatile and semi-volatile organic compounds the lab qualifiers are as follows:

$\mathrm{U}$ Used if the reported value is less than the instrument's detection limit (IDL).

B Used if the reported value is less than ten times the IDL, but is greater than or equal to the IDL.

$\mathrm{N}$ Used when spike recovery of either the matrix spike or matrix spike duplicate is not within the limits of 80 $120 \%$.

E Used when the serial dilution or analytical spike is not within the SOW-156 limits. 
APPENDIX D

\section{DCS DATA}




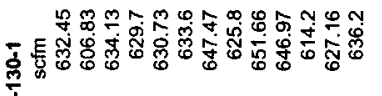

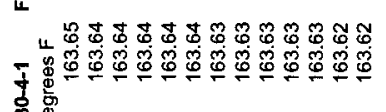

I

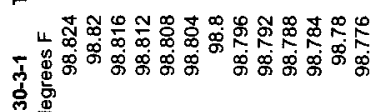

i

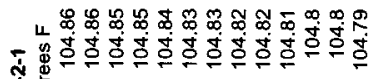

惫

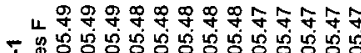

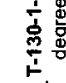

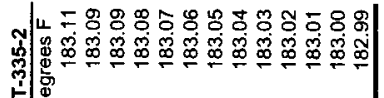

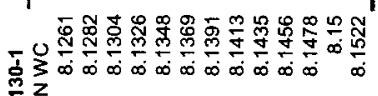

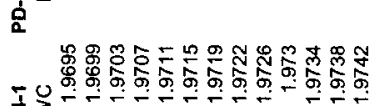

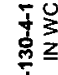

急总

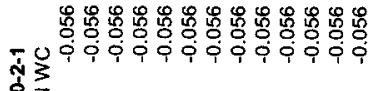

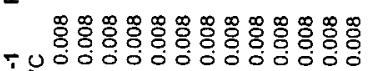

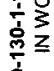

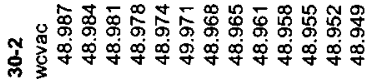

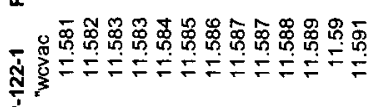

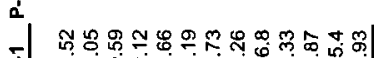

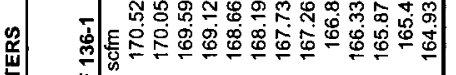

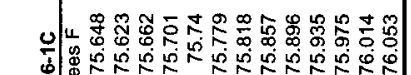
.

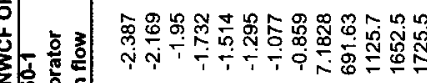
这

然 告

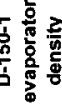

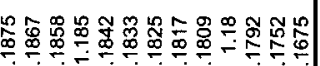
ชั

:

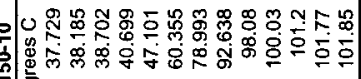

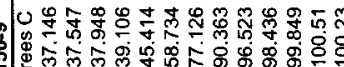

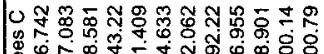

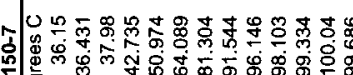

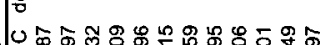

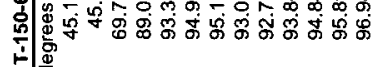

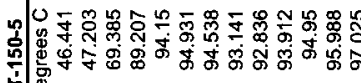

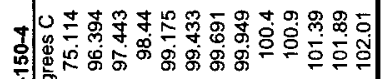

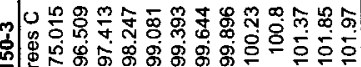

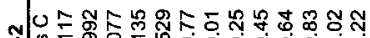

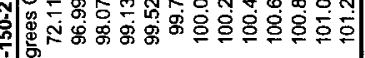

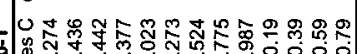

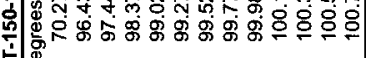

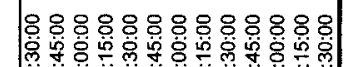
연

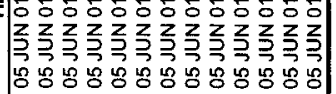

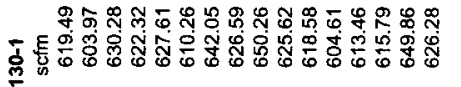

4

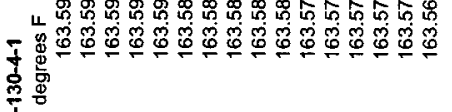

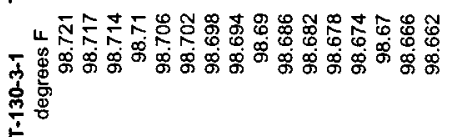

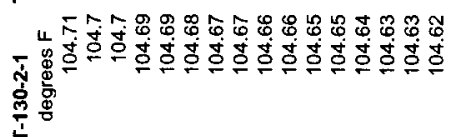

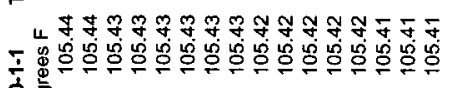
家悹

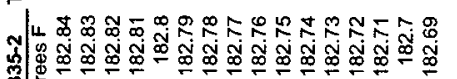
न

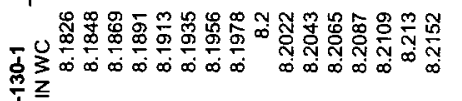

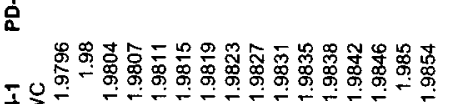
字

牙管

宫

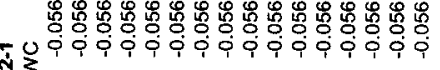
空交

家

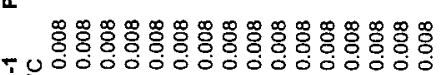

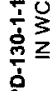

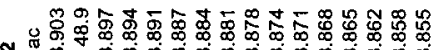

는

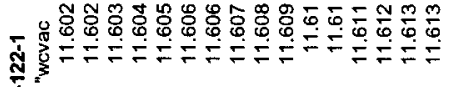

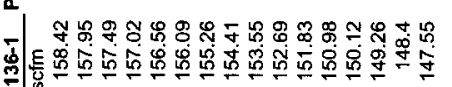

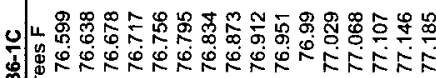

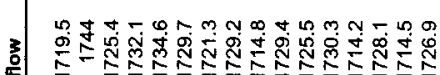

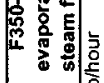

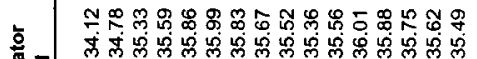

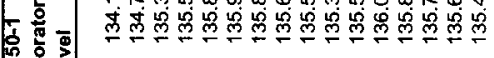

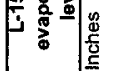

童 客商

ธิ

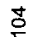

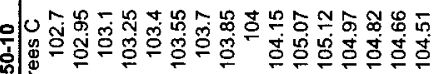

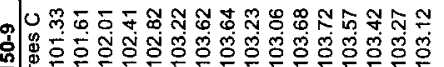
1\%

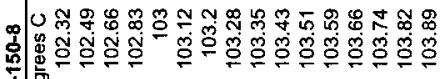

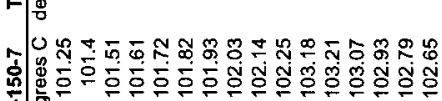

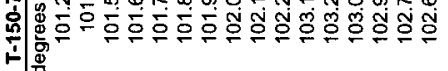

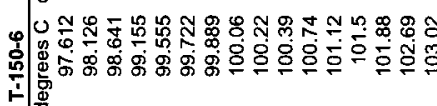

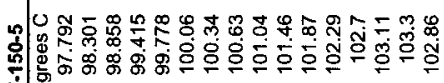

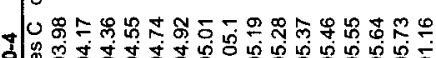
minnm

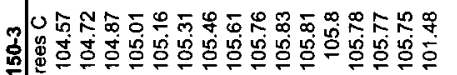
0

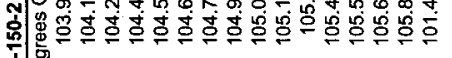

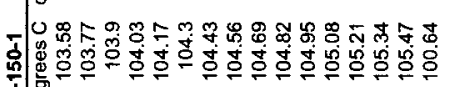

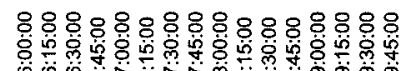

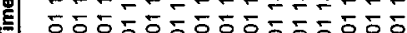

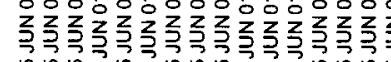

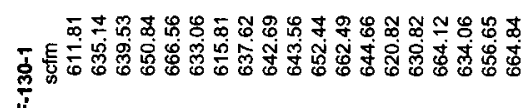

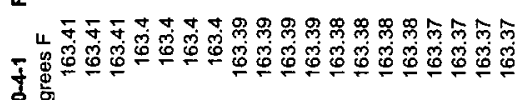
要

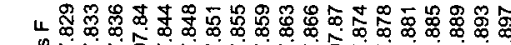

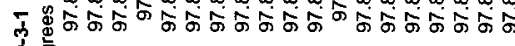
空

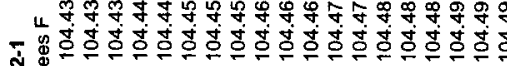
空密

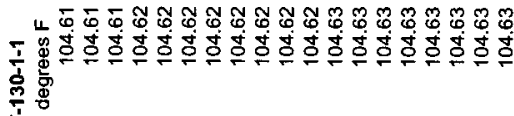

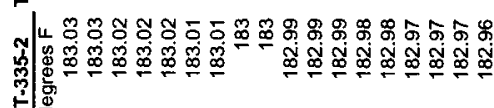

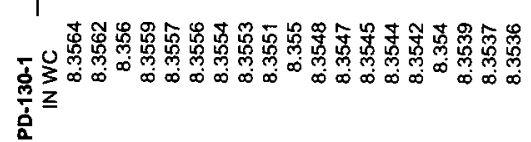

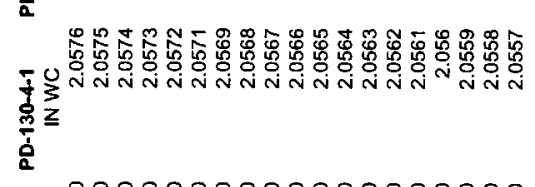

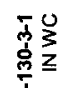

官 蔩 产

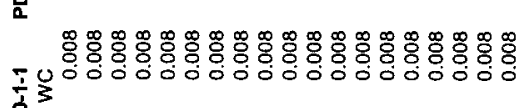
家

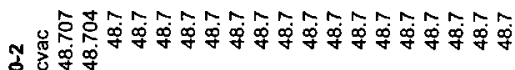

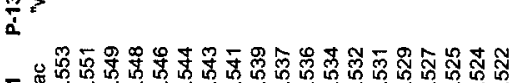

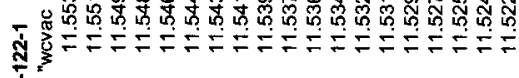

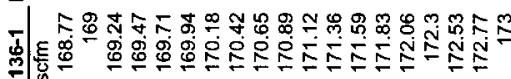

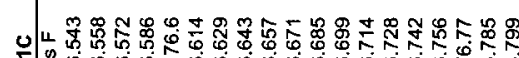

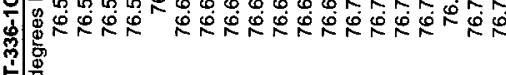

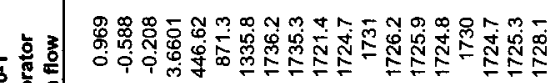

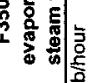

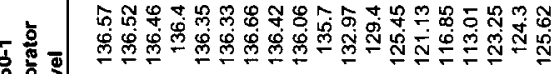

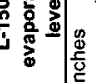

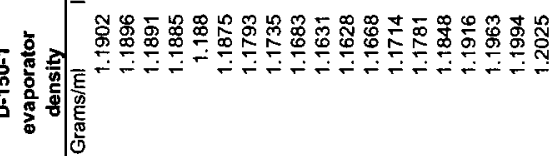

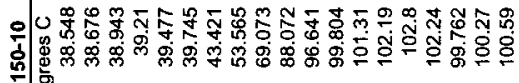

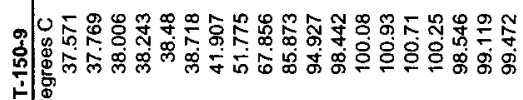

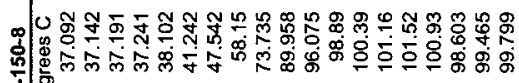
:

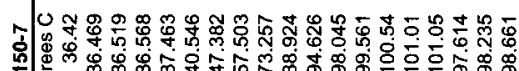

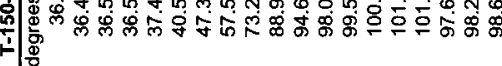

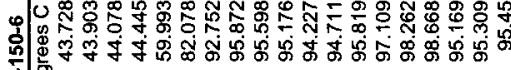

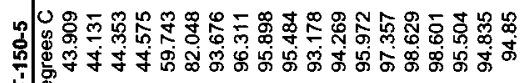

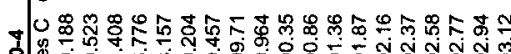
뉜.

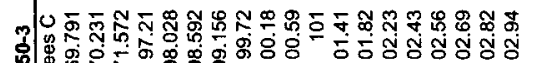

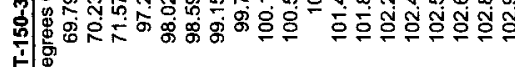
ํํำ

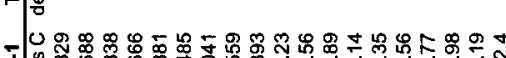

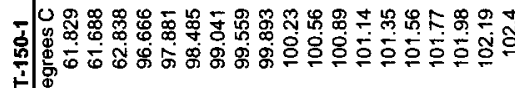

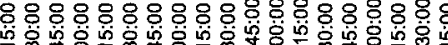

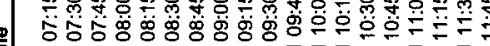

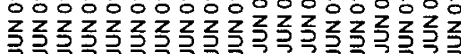
年

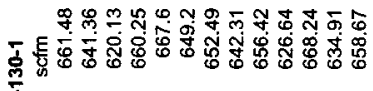

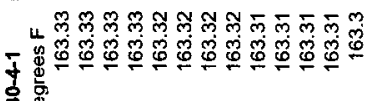

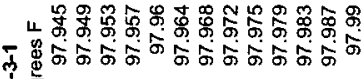

旁要

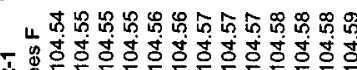
客要

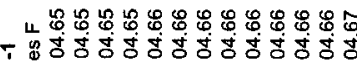

总

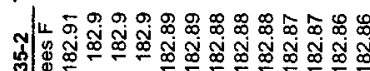
to

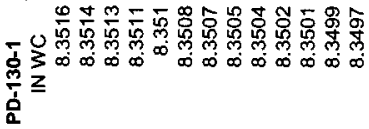

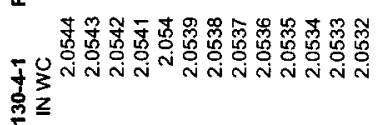
每

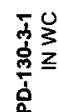

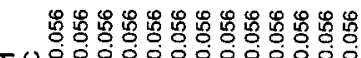
锌

à

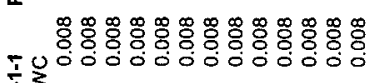

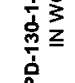

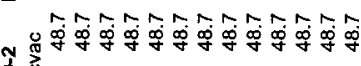

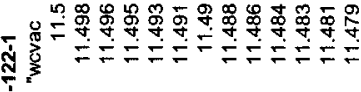

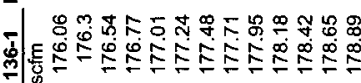
in

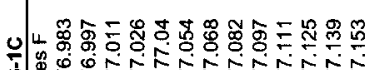

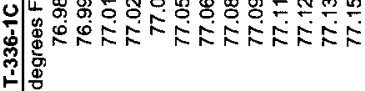

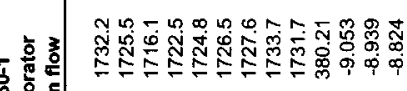

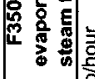

- ํㅡㄹ. (3)

毫

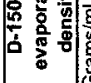

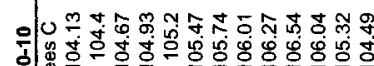

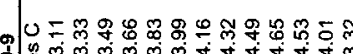

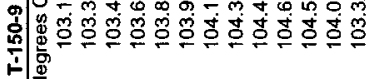

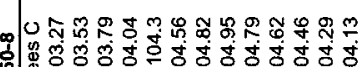
要

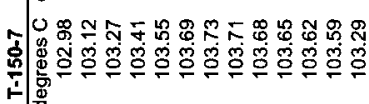

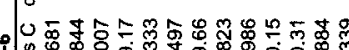
勻

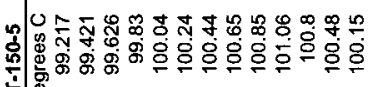

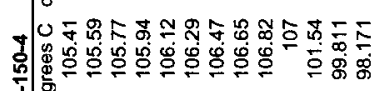
(2)

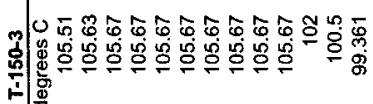

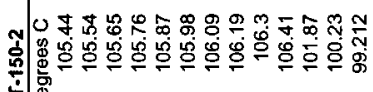

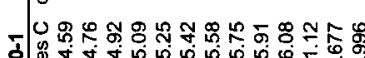

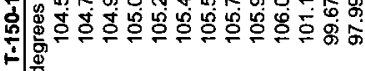

응응융유.

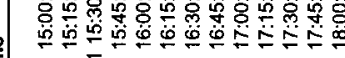

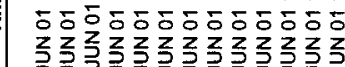

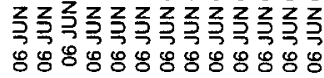




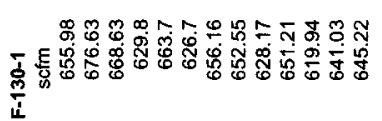

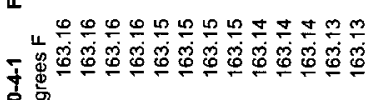
要

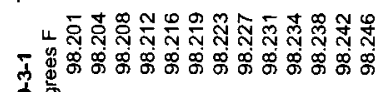
兽要

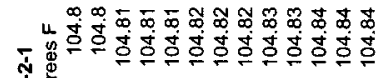
空要

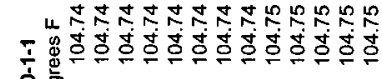
客要

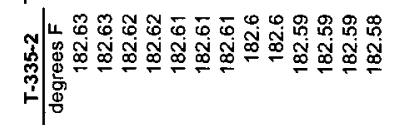

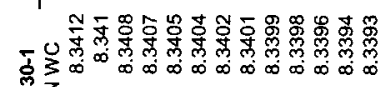
定

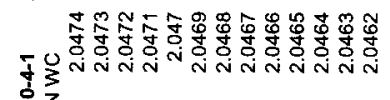
势 容总

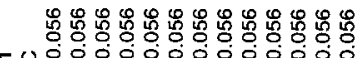

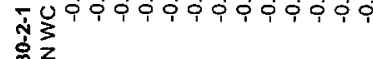
虽

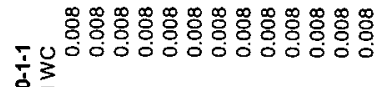

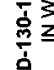

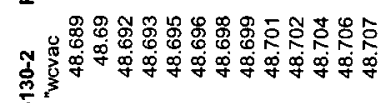

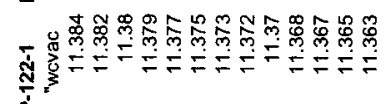

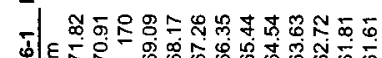

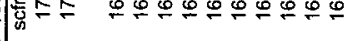

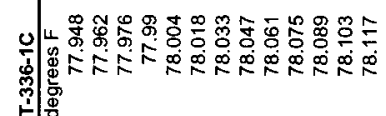

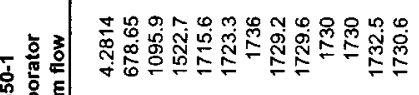

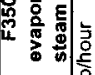

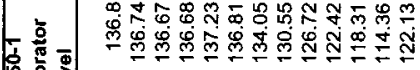

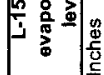

等

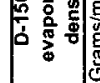

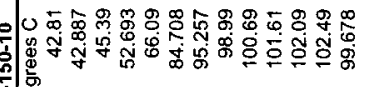

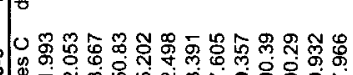

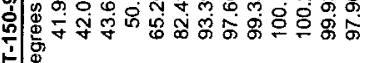

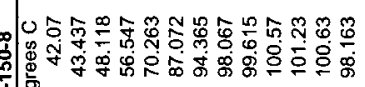

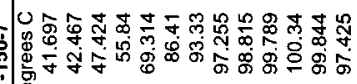

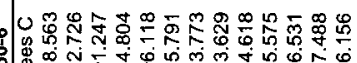

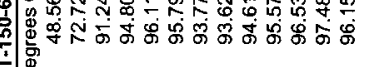

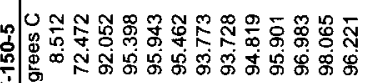

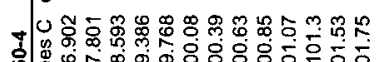

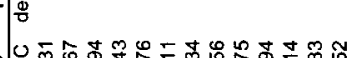

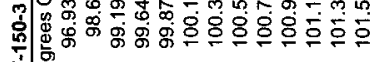

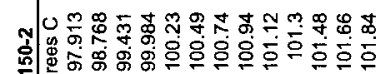

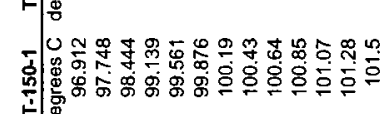
응응응응ㅇㅇㅇㅇㅇㅇㅇㅇㅇㅇㅇㅇ -

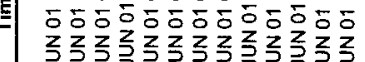

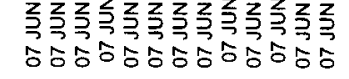

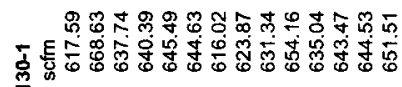
¿ 드요

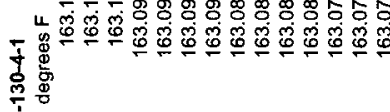

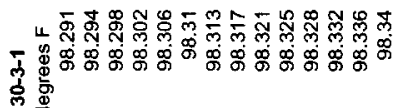

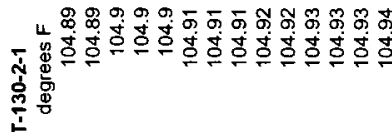

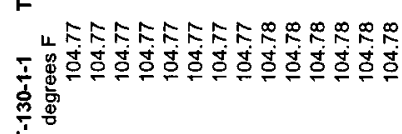

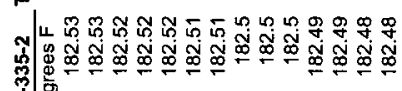

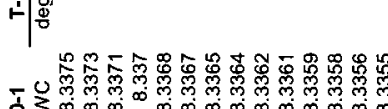

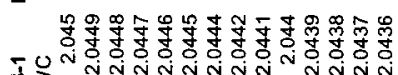

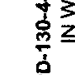
官 客离

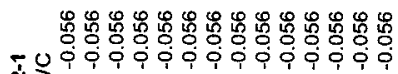

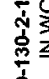
㝘竞

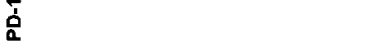

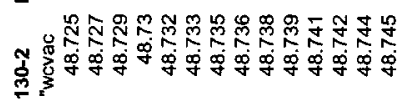

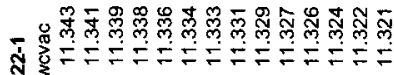

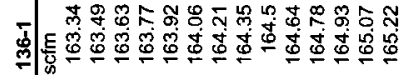

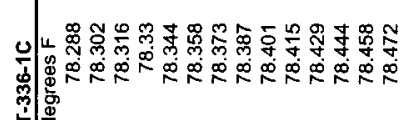

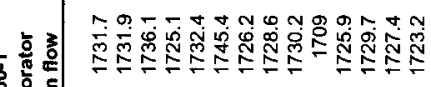
势

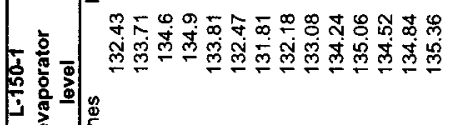

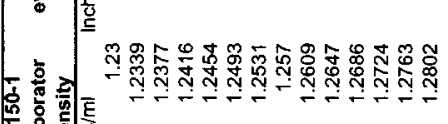

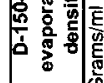

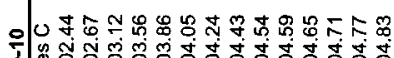

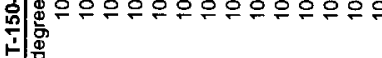

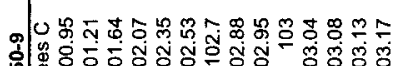

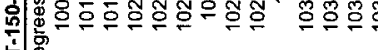

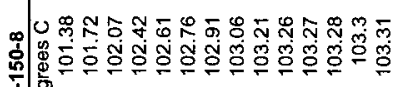
$r:$

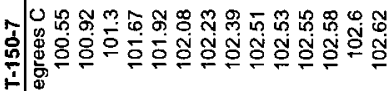

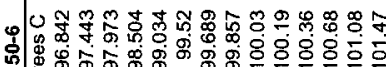
象害

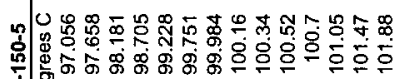

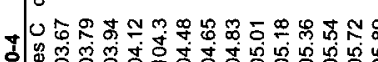

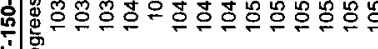

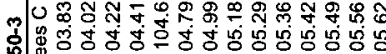
i

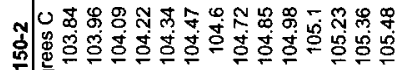
On

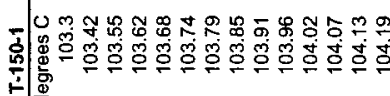

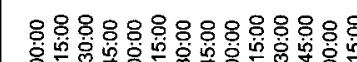

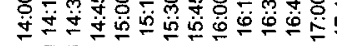
5050505050505

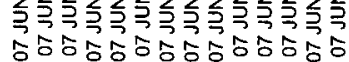

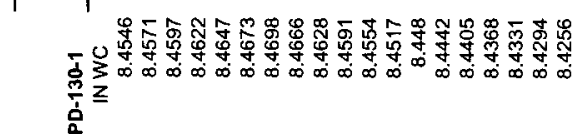

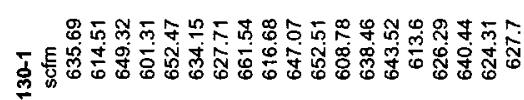

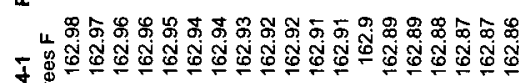
尊

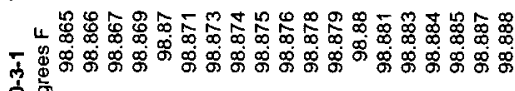
要

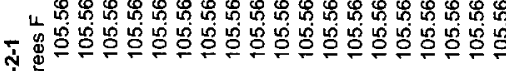

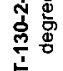

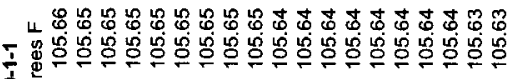
产

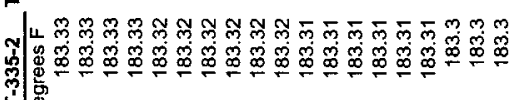

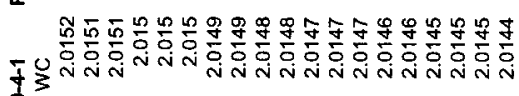
管 aे

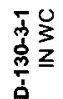

ì

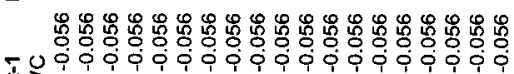

就

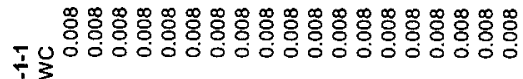
竞

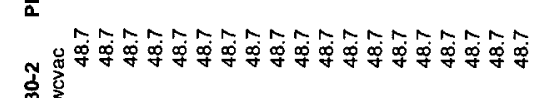

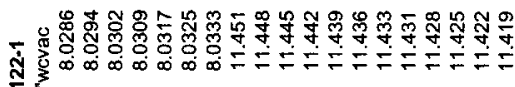

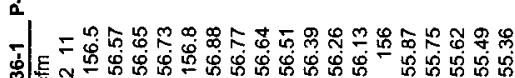

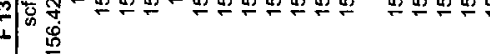

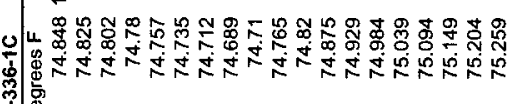

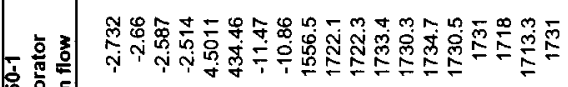

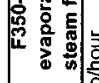

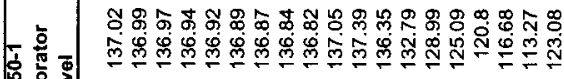

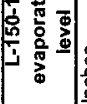

等

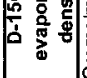

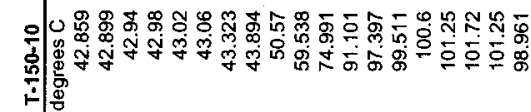

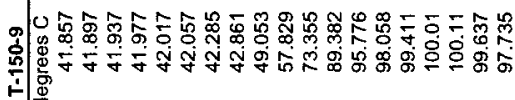

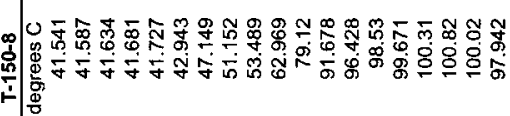

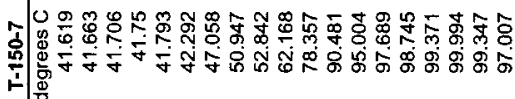

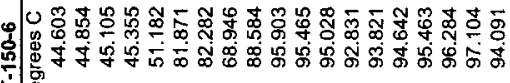

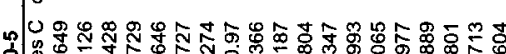

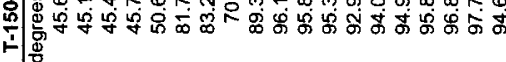

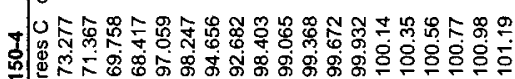

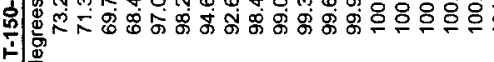

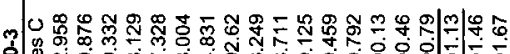

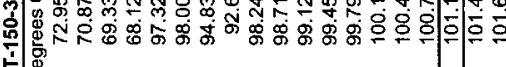

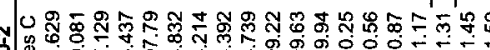

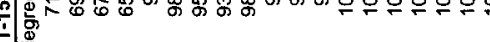

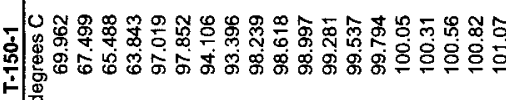

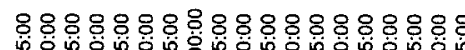

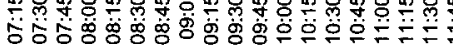

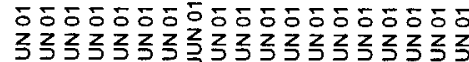

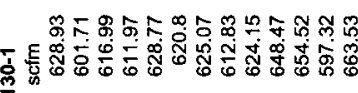

H

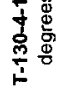

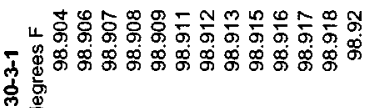
更

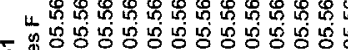
空

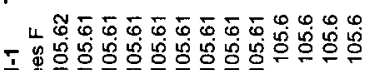

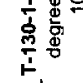

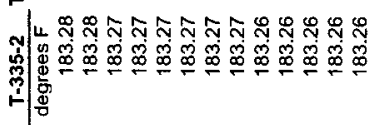

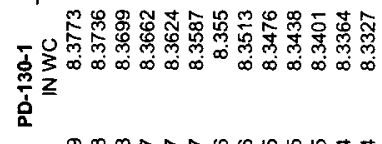

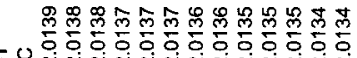

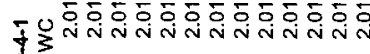
富

輬总

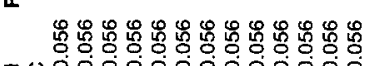

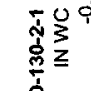

产

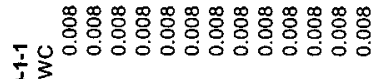
高富

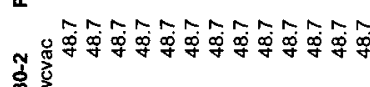
m.

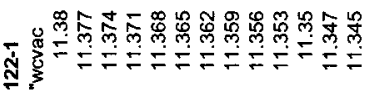

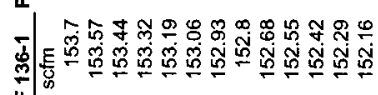

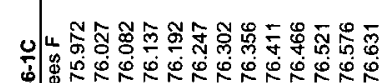
解

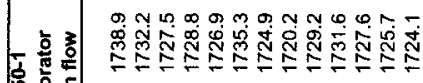

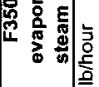

产

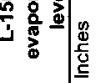

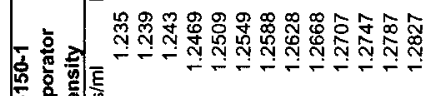

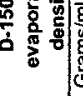

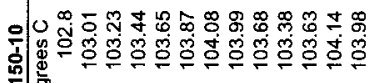
可高

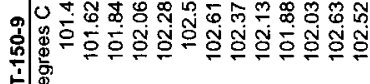

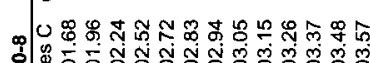

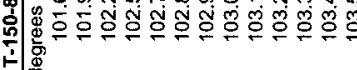

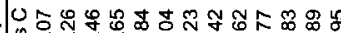

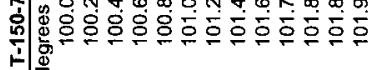

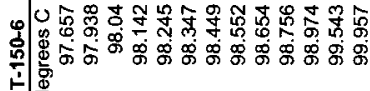

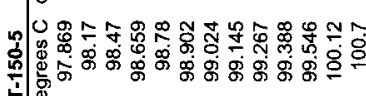

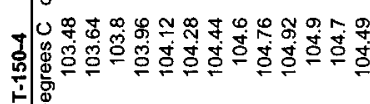
ت.

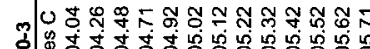

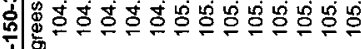

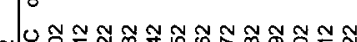

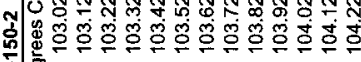

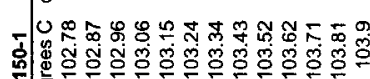

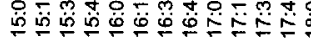

50.0.

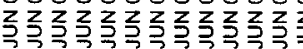

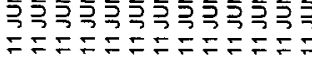




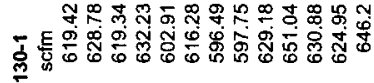

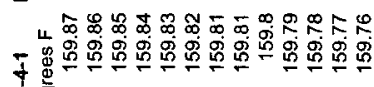

举密

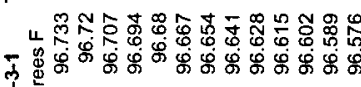

它

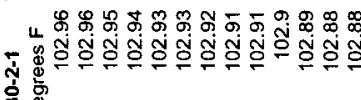

क्ष

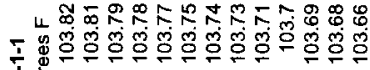

일

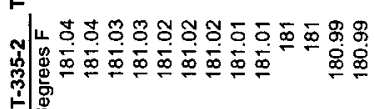

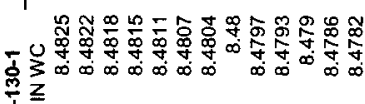

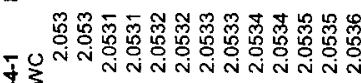

总竞

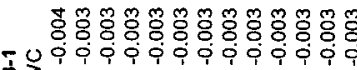

容艺

高

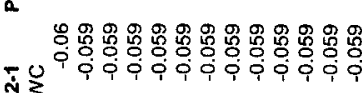
is

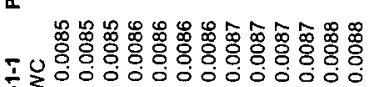
迹

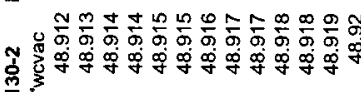

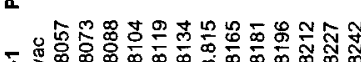

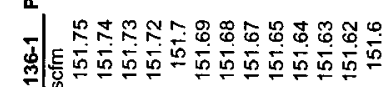

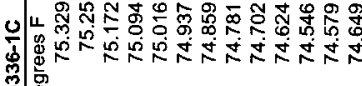

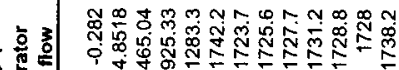

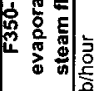

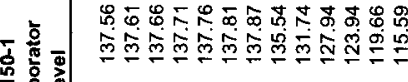

1.

촐

:

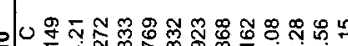

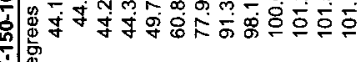

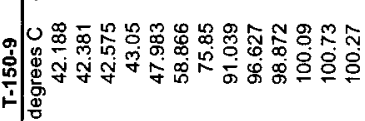

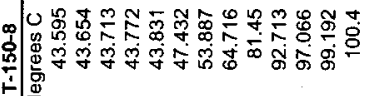

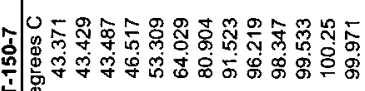

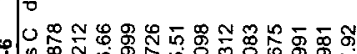

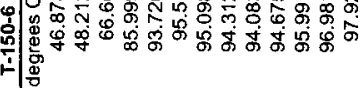

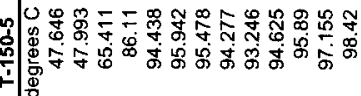

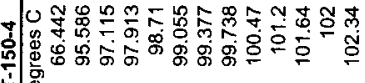

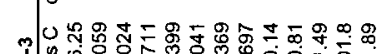

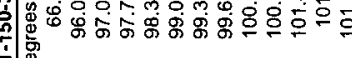
i

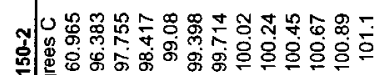
4

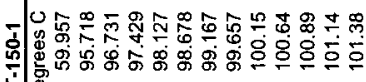

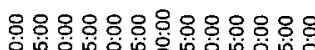

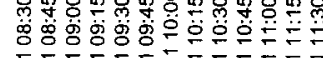

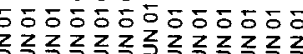

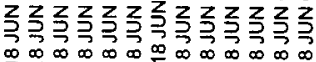

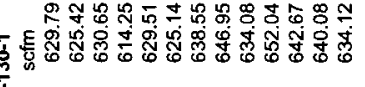

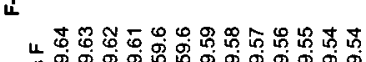

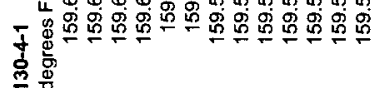

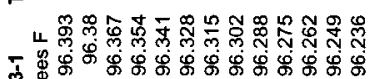
富要

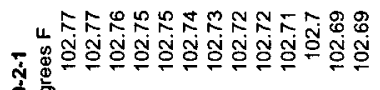
今े

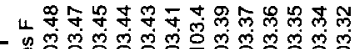
客密

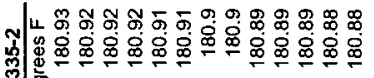

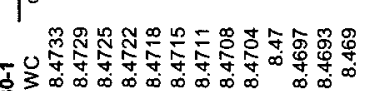

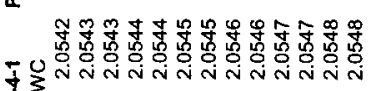
总

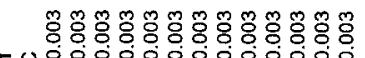
证窟

à

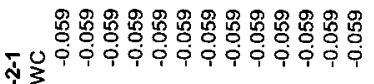
官

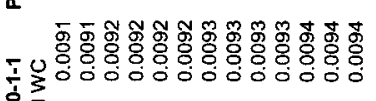
突

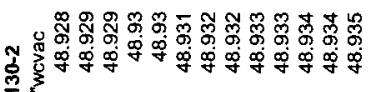

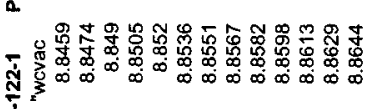

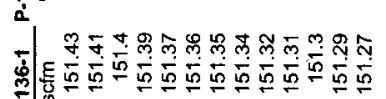

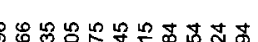

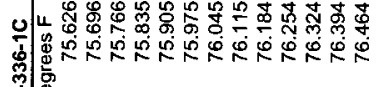

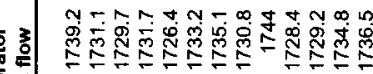

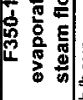
旅

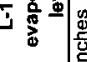

施

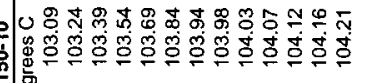

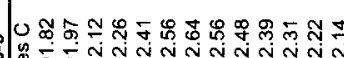

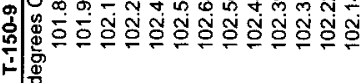

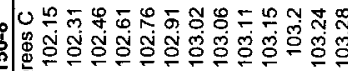
空 ํㅐㅁำ

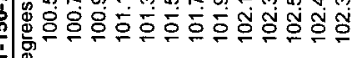

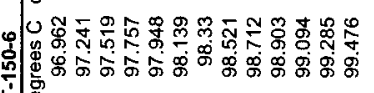

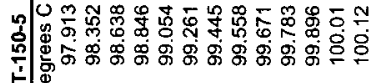

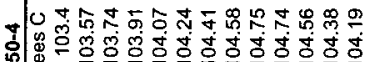
नो

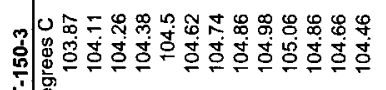

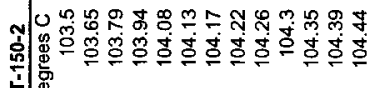
i)

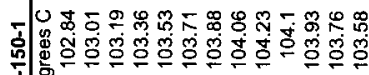

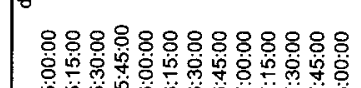

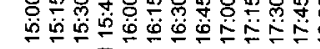

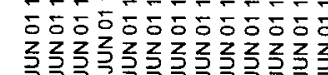

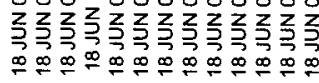

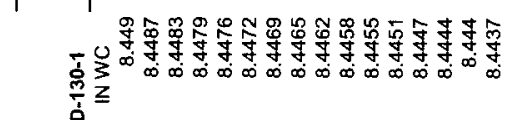

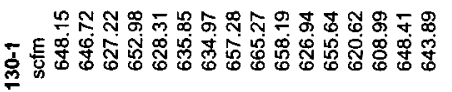

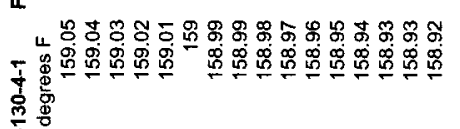

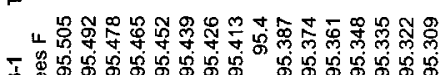

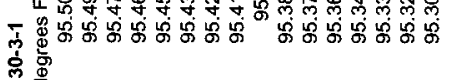

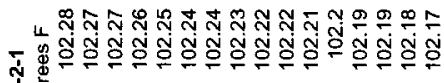
突密

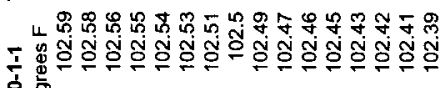

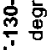

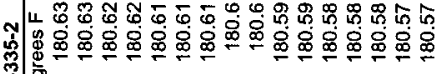
2 望

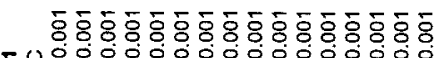

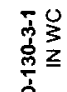

高

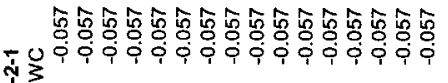
官

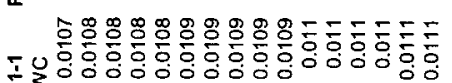
容

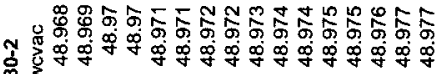

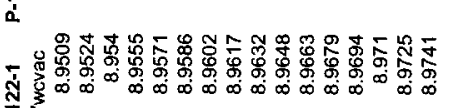

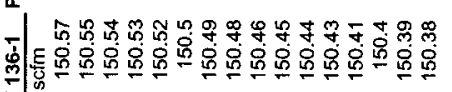

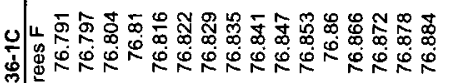

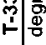

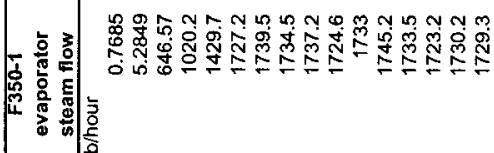

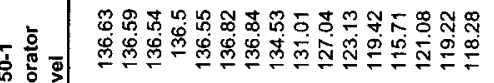

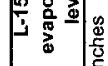

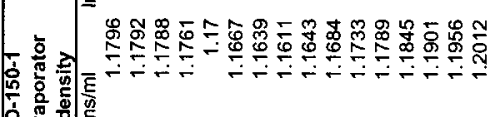
势

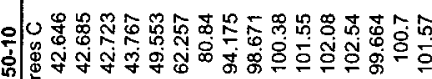

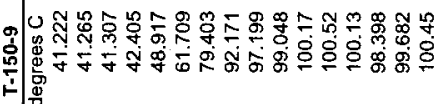

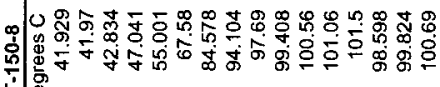

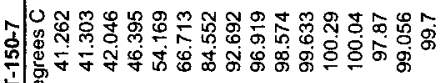

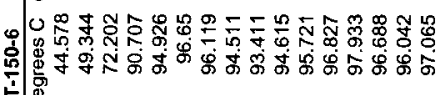

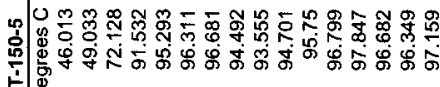

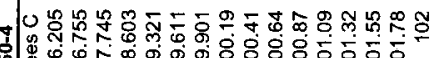

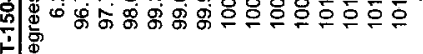

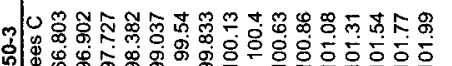

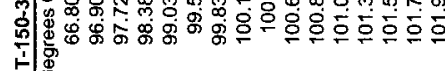

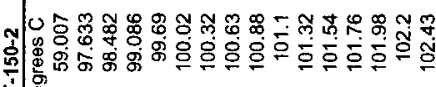

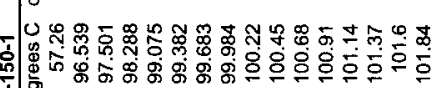
䉼

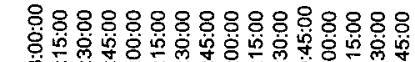

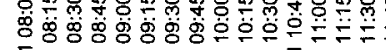

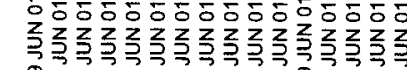

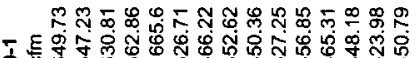

ป

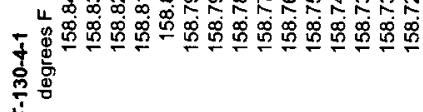

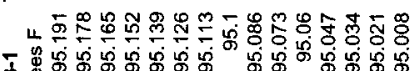
总

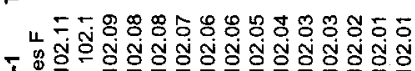
势

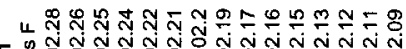

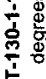

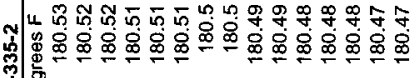

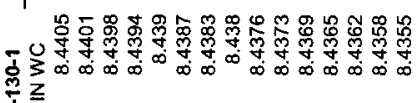
主

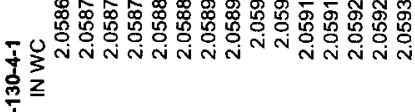

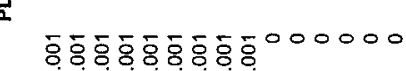

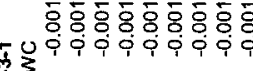

要

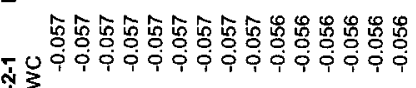
峞

à

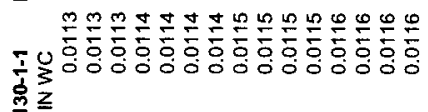
㝘

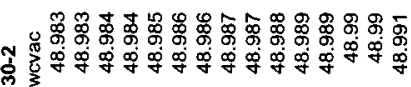

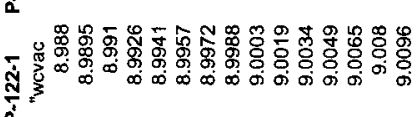

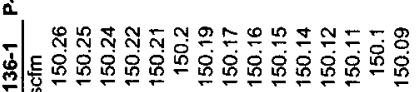

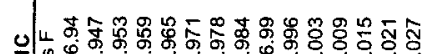
解

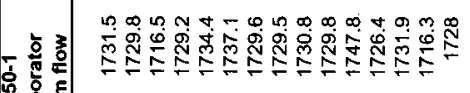

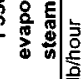

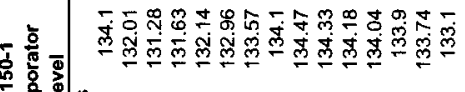

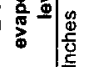

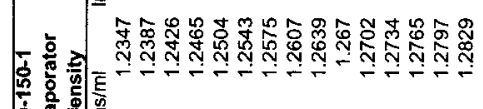

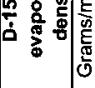

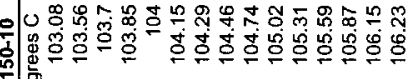

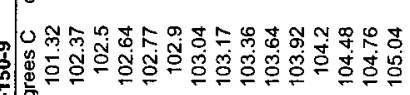

-

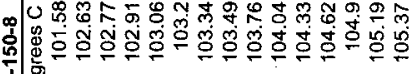

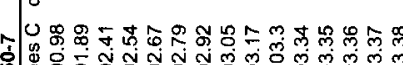

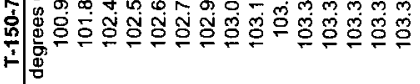

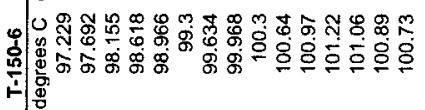

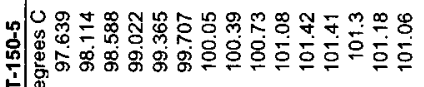

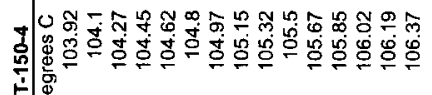

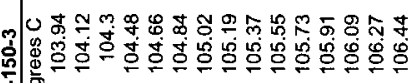
क्ष

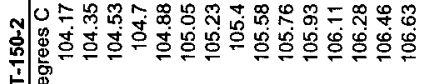

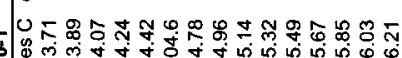

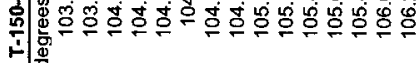

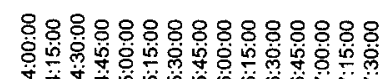
5

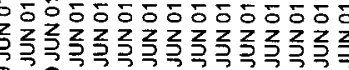

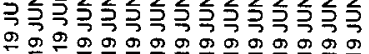




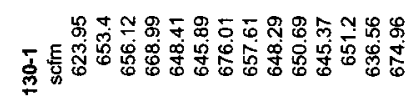

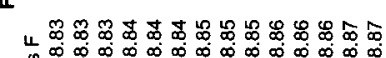

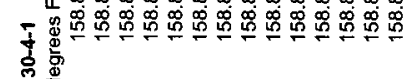

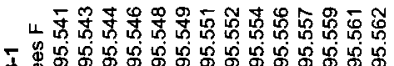
峞

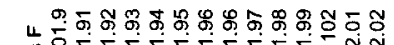

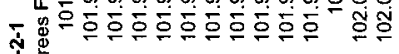
官

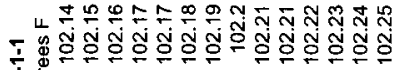
兽兽

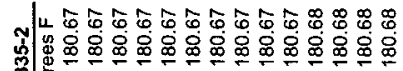

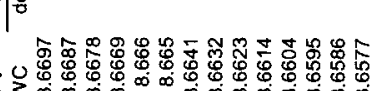
家

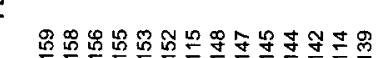

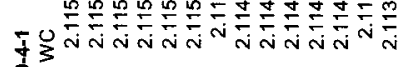

嚆总

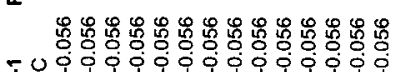

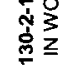
à

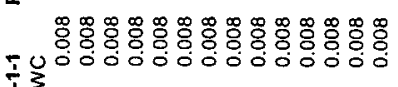

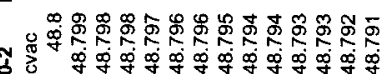

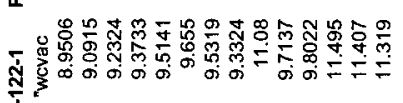
곤

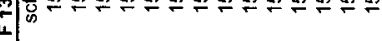

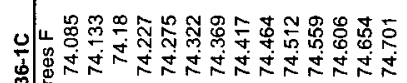

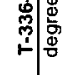

-

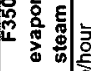

ํㅗㄹ है

等

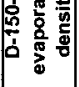

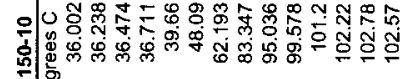

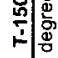

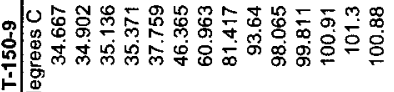

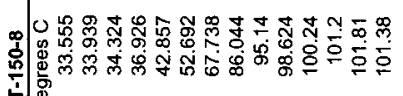

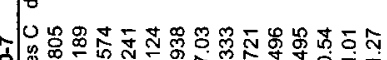

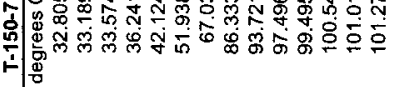

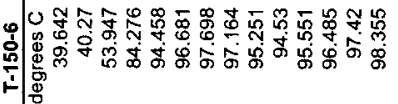

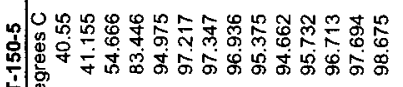

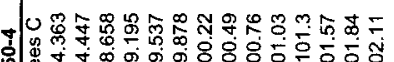
要

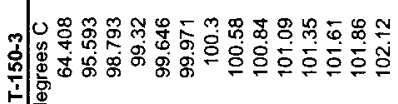

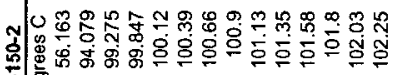
(3)

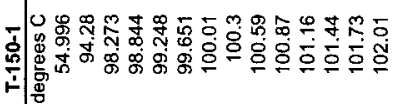

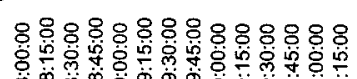
영

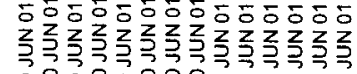

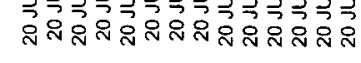

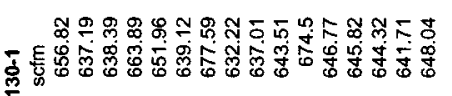

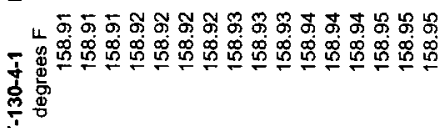

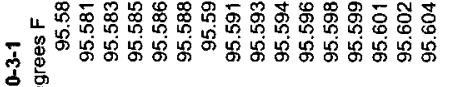

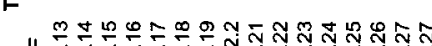

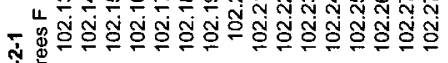
要

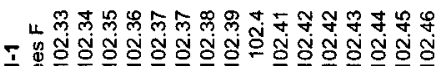
商整

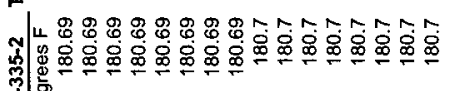
行

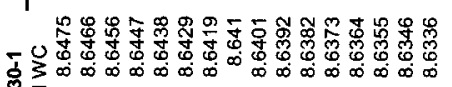

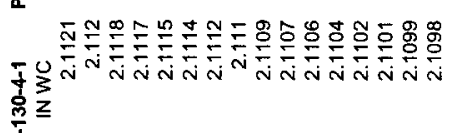

à่

要弯

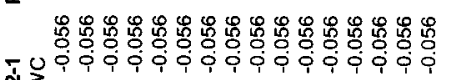
究芦

高

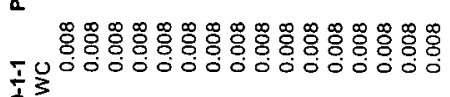
它荌

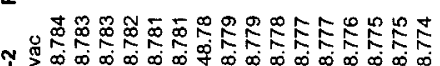
$\frac{m_{i}^{2}}{2}$

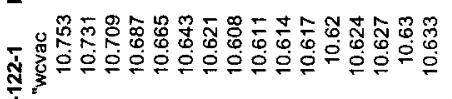

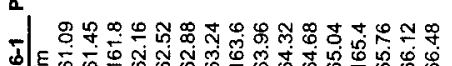

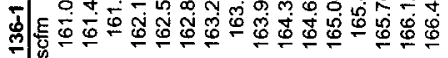

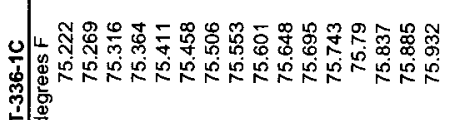

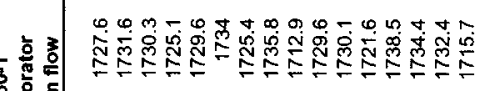
變衰言

等 竞旁

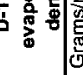

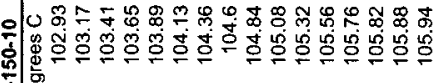

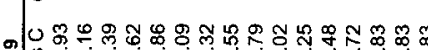

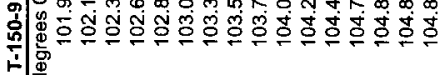

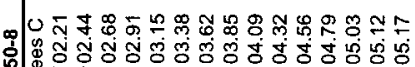
납

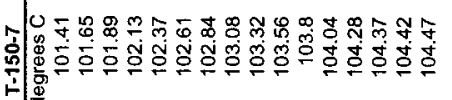

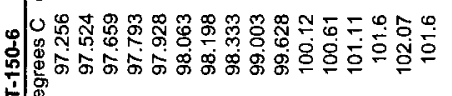

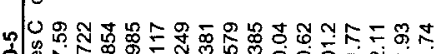

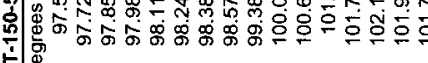

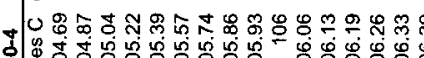

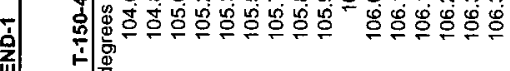

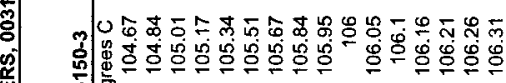
The

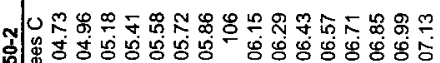
家

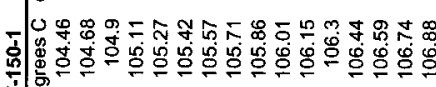

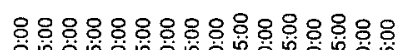

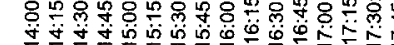

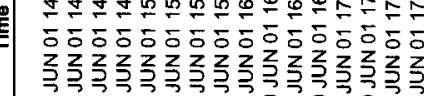

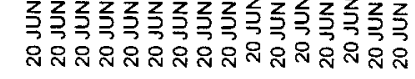

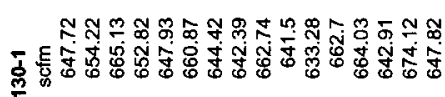

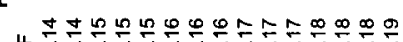

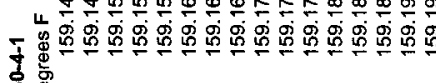
要

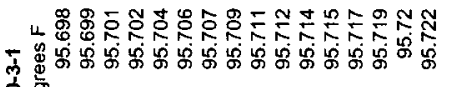
客

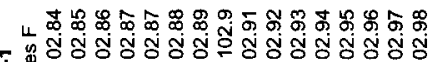

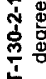

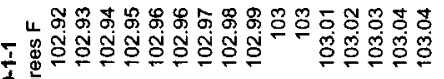
容

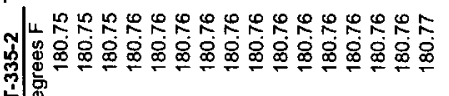

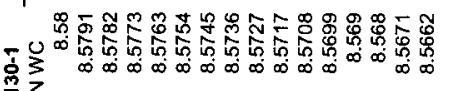
官

\begin{tabular}{rl}
0 & 0 \\
0 & 0 \\
\hline
\end{tabular}

方突

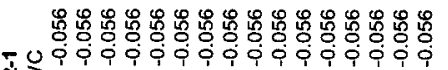
突荌

宫

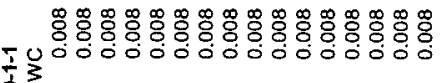
旁

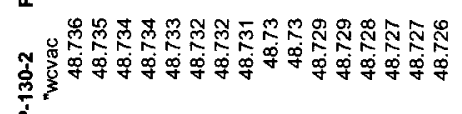

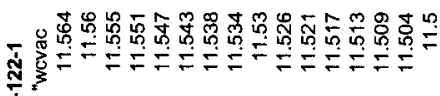

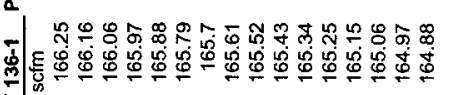

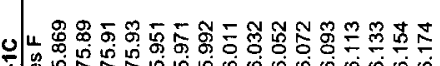

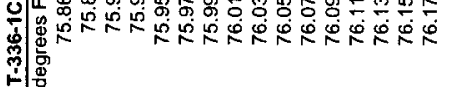

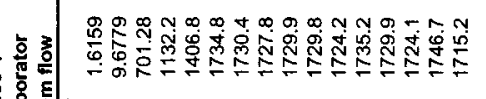
势部言

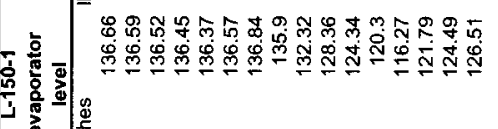

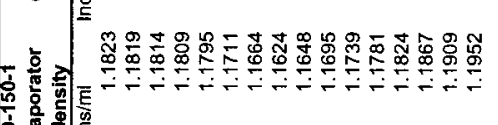

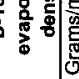

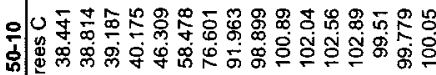
空兽

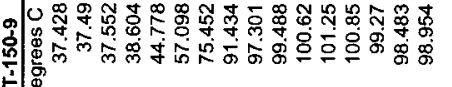

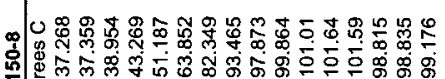

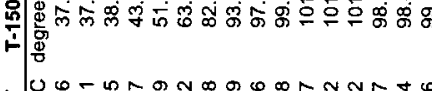

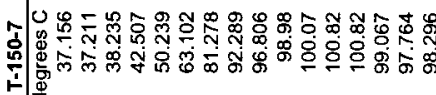

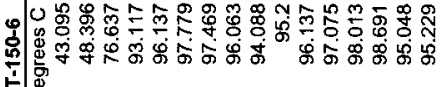

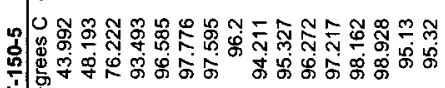

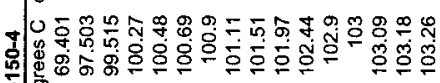
今)

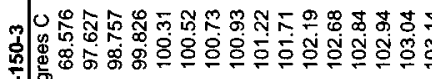
要

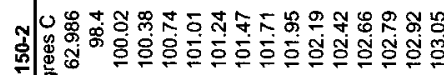

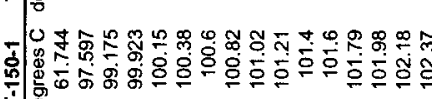

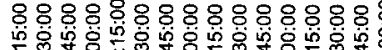
摛琄。

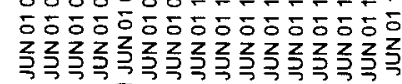

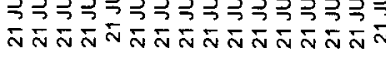

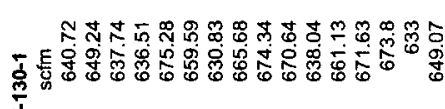

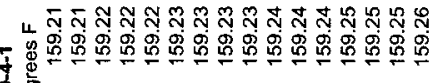
空

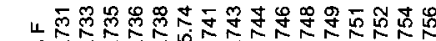

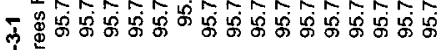
인

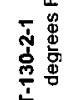

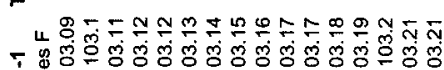
要要

4 作 î

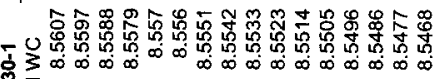
究

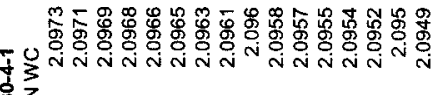

施总

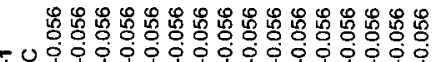

容

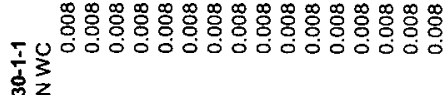
旁

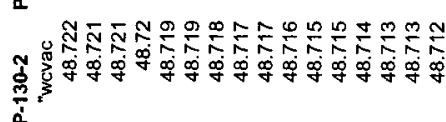

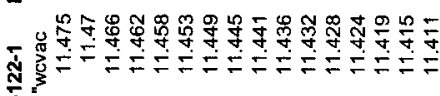

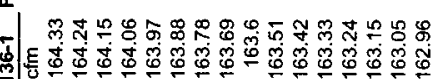

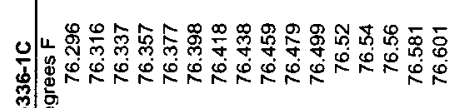

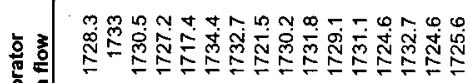

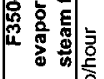

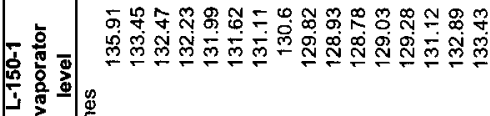

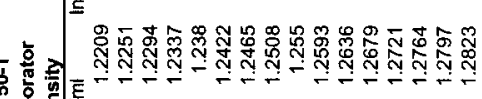

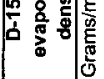

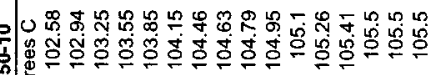

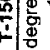

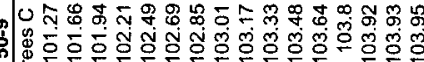

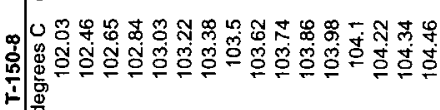

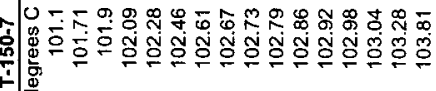

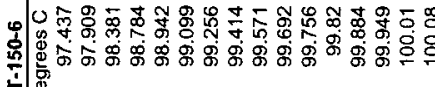

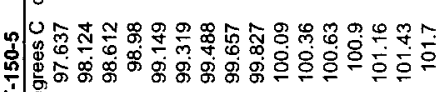

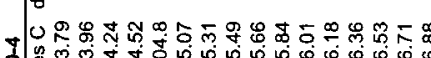

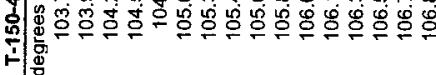

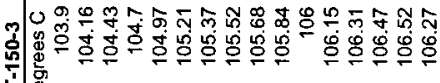
要

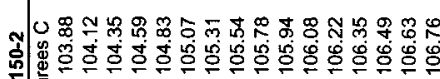

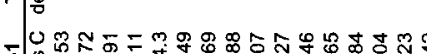

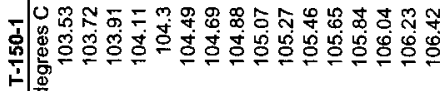

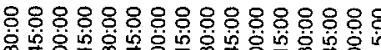

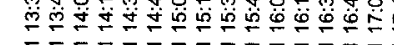

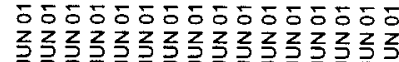
ลㅅำ 